\title{
Durability of Green Concrete as a Structural Material
}

\author{
by \\ ABDELGADIR AHMED ABBAS, B. Sc., M.A.Sc. \\ A thesis submitted to the Faculty of Graduate Studies and Research \\ in partial fulfillment of the Requirements for the Degree of
}

\section{Doctor of Philosophy}

\author{
Department of Civil and Environmental Engineering \\ Carleton University \\ Ottawa, Ontario, Canada
}

March 2007

The Doctor of Philosophy in Civil Engineering is a joint program with the University of Ottawa, administered by the Ottawa-Carleton Institute for Civil Engineering

(C) 2007, Abdelgadir Ahmed Abbas 


$\begin{array}{ll}\begin{array}{l}\text { Library and } \\ \text { Archives Canada }\end{array} & \begin{array}{l}\text { Bibliothèque et } \\ \text { Archives Canada }\end{array} \\ \begin{array}{l}\text { Published Heritage } \\ \text { Branch }\end{array} & \begin{array}{l}\text { Direction du } \\ \text { Patrimoine de l'édition }\end{array} \\ \begin{array}{l}\text { 395 Wellington Street } \\ \text { Ottawa ON K1A ON4 }\end{array} & \begin{array}{l}\text { 395, rue Wellington } \\ \text { Ottawa ON K1A ON4 } \\ \text { Canada }\end{array}\end{array}$

Your file Votre référence ISBN: 978-0-494-27085-1 Our file Notre référence ISBN: 978-0-494-27085-1

NOTICE:

The author has granted a nonexclusive license allowing Library and Archives Canada to reproduce, publish, archive, preserve, conserve, communicate to the public by telecommunication or on the Internet, loan, distribute and sell theses worldwide, for commercial or noncommercial purposes, in microform, paper, electronic and/or any other formats.

The author retains copyright ownership and moral rights in this thesis. Neither the thesis nor substantial extracts from it may be printed or otherwise reproduced without the author's permission.
AVIS:

L'auteur a accordé une licence non exclusive permettant à la Bibliothèque et Archives Canada de reproduire, publier, archiver, sauvegarder, conserver, transmettre au public par télécommunication ou par l'Internet, prêter, distribuer et vendre des thèses partout dans le monde, à des fins commerciales ou autres, sur support microforme, papier, électronique et/ou autres formats.

L'auteur conserve la propriété du droit d'auteur et des droits moraux qui protège cette thèse. $\mathrm{Ni}$ la thèse ni des extraits substantiels de celle-ci ne doivent être imprimés ou autrement reproduits sans son autorisation.
In compliance with the Canadian

Privacy Act some supporting forms may have been removed from this thesis.

While these forms may be included in the document page count, their removal does not represent any loss of content from the thesis.
Conformément à la loi canadienne sur la protection de la vie privée, quelques formulaires secondaires ont été enlevés de cette thèse.

Bien que ces formulaires aient inclus dans la pagination, il n'y aura aucun contenu manquant.

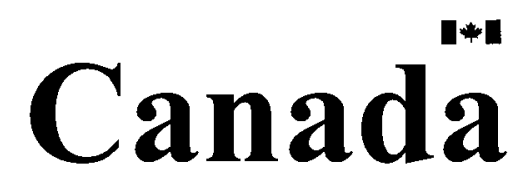




\begin{abstract}
Many of the buildings in Canada were built in the 50's or 60 's, and are near the end of their design life. It is expected that during the next two decades, a large amount of concrete, resulting from the demolition of these buildings and structures, will be available for either disposal or for being recycled for reuse as concrete aggregates. Currently, to the author's knowledge, there are no standards available in North America, which can be followed to consistently design and produce structural-grade concrete made specifically with recycled concrete aggregates (RCA). To overcome this problem, a comprehensive study investigating the durability of concrete produced with these aggregates (green concrete) as a structural material is presented in this thesis. The research program was developed around the identified gaps in the literature regarding the structural-grade concrete produced with RCA. The research was carried out in stages including: (1) the material characterization of two sources of RCA obtained from different geographical locations of Canada and showing significantly different properties; (2) the preliminary study investigating the fresh and hardened properties of concrete produced with these two types of RCA; (3) the development of mix design procedures for green concrete based on the results obtained from the preliminary study; (4) the investigation of the durability properties, more specifically, the resistance to freeze-and-thaw action, chloride penetration and carbonation, of green concrete produced with the developed mix design procedures. Material characterization testing of the two sources of RCA has demonstrated that the main factor affecting the RCA properties is the residual mortar content (RMC) and not the source of RCA. A quick RMC determination test was developed, and this technique was verified through a comprehensive image analysis study. The preliminary
\end{abstract}


study revealed that the main factor affecting the fresh and hardened properties of concrete produced with RCA is the residual mortar of RCA. It has been observed that the concrete produced by the developed mix design procedures have strong resistance to freeze-andthaw action, chloride penetration and carbonation. The investigation have demonstrated that the use of RCA in the production of structural-grade green concrete is a viable alternative to conventional concrete production using virgin aggregates. 


\section{ACKNOWLEDGEMENTS}

I gratefully acknowledge the technical and financial support of the Public Works and Government Services Canada and the Natural Resources Canada. I would like to thank my supervisor Prof. O. Burkan Isgor for the guidance and help he provided during this study. I also would like to express my appreciation to Prof. A. Ghani Razaqpur, Dr. Benoit Fournier and Dr. Simon Foo for their constructive suggestions and help during all the stages of this study. I can not thank enough to my friend, Gholamreza Fathifazl, for his help and many contributions to this study. The large portion of the testing programme presented in this thesis was carried out in CANMET Material Technology Laboratories. Therefore, I would like to express my sincere gratitude to the CANMET staff, Mr. Raymond Chevrier, Mr. Alain Bilodeau, Dr. Nabil Bouzoubaa, Dr. Pierre Nkinambubanzi, Mr. Steve Michaud and Mr. Vince Deacon. I also would like to thank to Renata Zavadi of CANMET (NRCAN) who provided her expertise during the image analysis study. Additional thanks go to Ken McMartin, Stanely Conley, and Dr. Marie Tudonet Chow of Civil and Environmental Engineering laboratories at Carleton University. It should also be mentioned that the RCA used in this study were donated by RichVan Holdings Ltd of Richmond, BC and Groupe Beauval, Montréal, PQ. The virgin aggregates from BC were provided by Lafarge Canada Inc. and Lehigh Northwest Materials. The cement was provided by Lafarge North America. Without the in-kind contributions of these companies, this research would be very difficult to complete.

This acknowledgement would not be complete without expressing heartfelt thank to my wife Hanaa for her unconditional support and encouragement, during my studies. 


\section{TABLE OF CONTENTS}

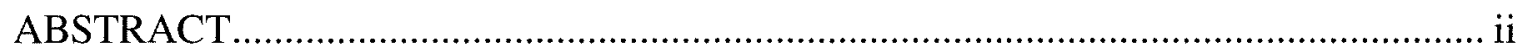

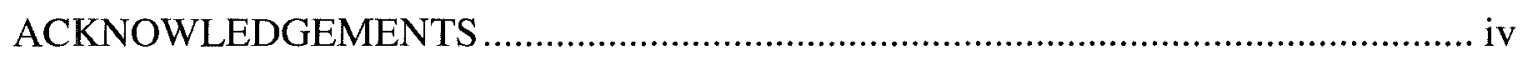

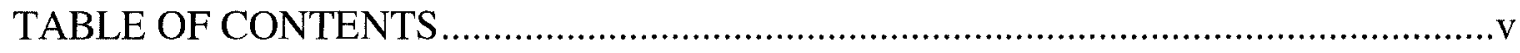

LIST OF TABLES ..........................................................................................

LIST OF FIGURES …....................................................................................

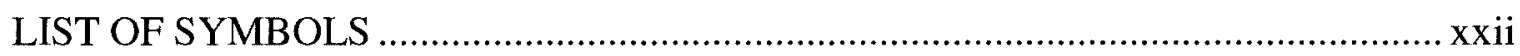

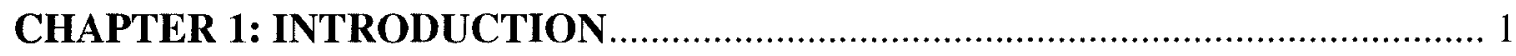

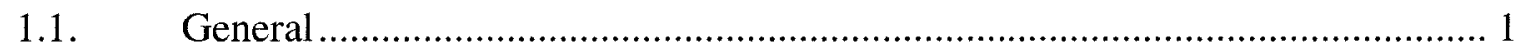

1.2. Economic and Environmental Benefits of GC......................................... 5

1.3. A Note on Important Definitions and Acronyms..................................... 8

1.4. Problem Definition, Objectives and Scope ............................................. 9

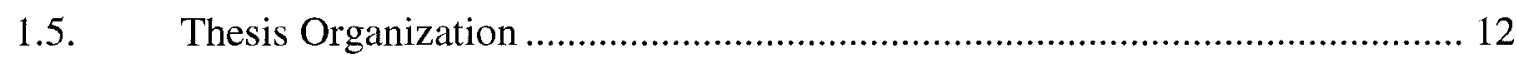

CHAPTER 2: LITERATURE REVIEW ..................................................... 16

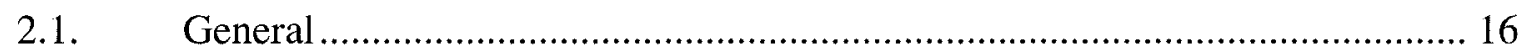

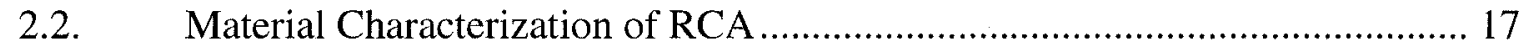

2.2.1. Particle Shape and Size...................................... 19

2.2.2. Specific Gravity, Density and Water Absorption Capacity.............. 20

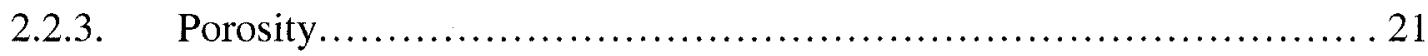

2.2.4. Mechanical Properties and Soundness of RCA......................... 22

2.3. Physical and Mechanical Properties of RAC .............................................. 23

2.3.1. The RCA Content.............................................. 24 


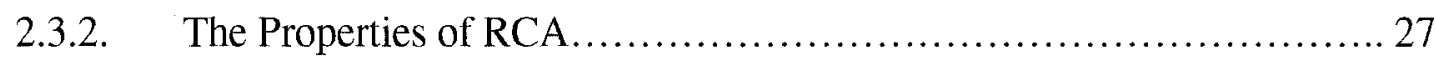

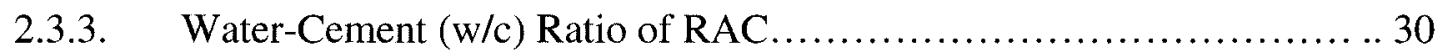

2.3.4. Effect of Other Binders............................................ 30

2.3.5. Effect of the Original Concrete Properties.............................. 32

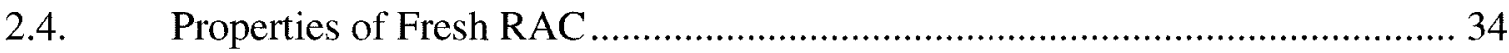

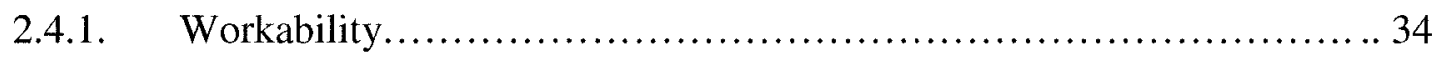

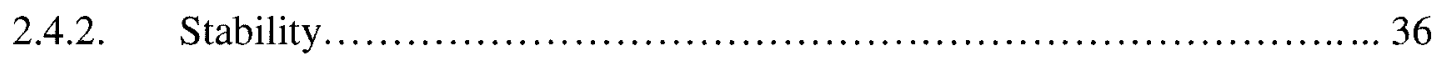

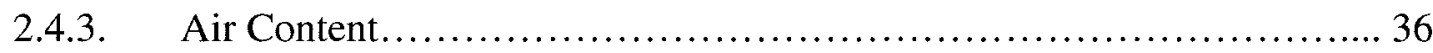

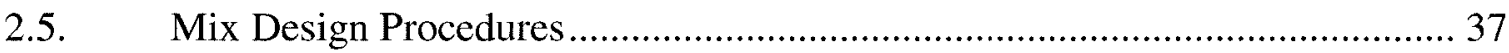

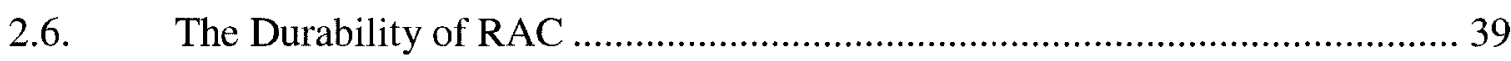

2.6.1. Freeze-and-Thaw Action.......................................... 40

2.6.2. Chloride Penetration............................................... 45

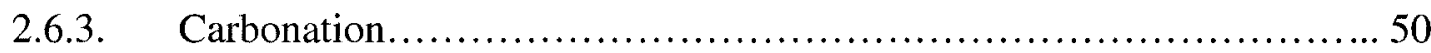

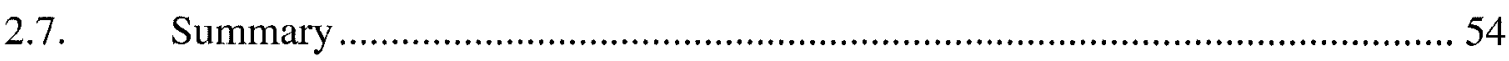

CHAPTER 3: MATERIAL CHARACTERIZATION OF RCA ……………...........61

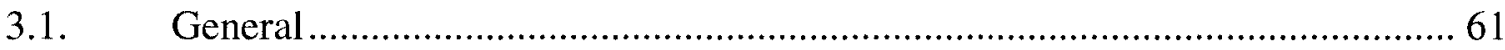

3.2. Preparation of RCA for Material Characterization Testing .............................. 63

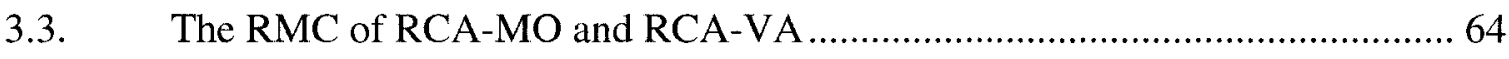

3.3.1. The Development of the Quick Test Procedure.......................... 65

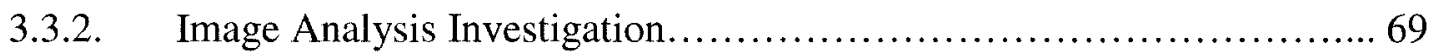

3.3.2.1. Preparation of Samples for the Image Analysis................................. 69

3.3.2.2. Process of Image Analysis .............................................................. 70

3.3.2.3. Results and Analysis of the Data from Image Analysis .................... 73 
3.4. Specific Gravity and Absorption Capacity Test ..................................... 76

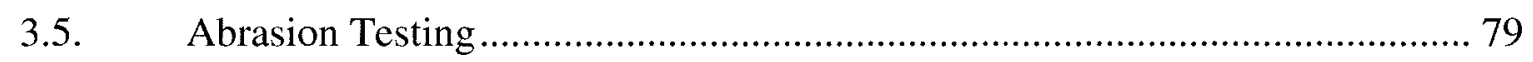

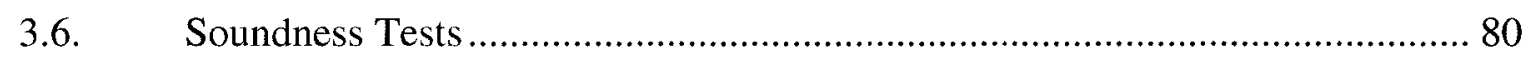

3.6.1. MTO Soundness Test.......................................... 81

3.6.1.1. Determination of Sieving Time for Quantitative Analysis .............. 81

3.6.1.2. MTO Soundness Test and Results for RCA …............................ 83

3.6.2. ASTM Soundness Test..................................... 84

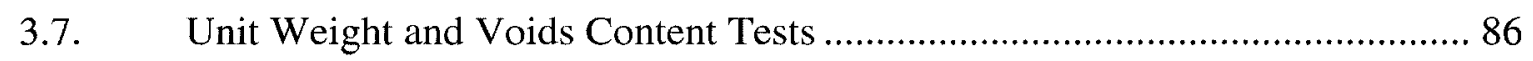

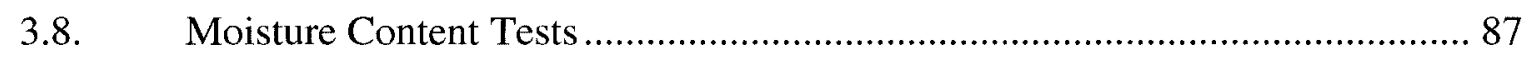

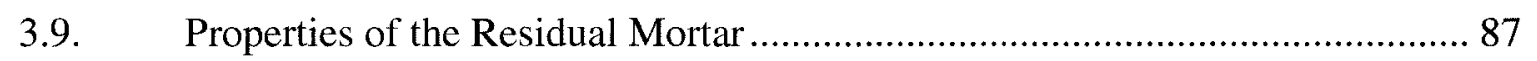

3.10. Summary and Discussion of Material Characterization Testing .................... 89

3.11. Rational Approach to Material Characterization of RCA ............................. 91

3.11.1. The Development of the Rational Approach...................... 92

3.11.1.1. Absorption Capacity ….................................................. 92

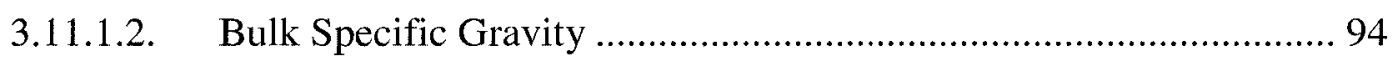

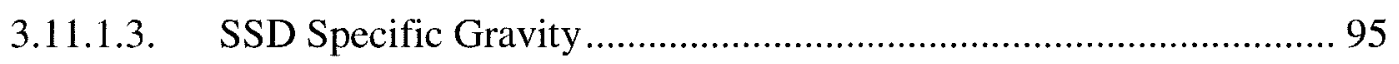

3.11.1.4. Apparent Specific Gravity ..................................................... 95

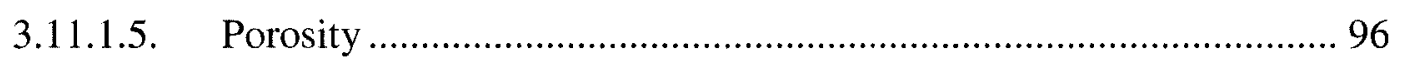

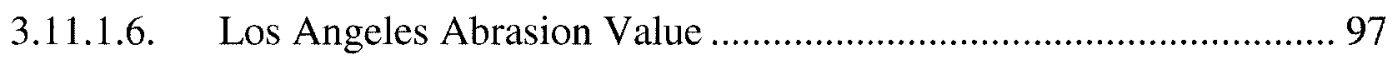

3.11.2. Comparison of the Rational Approach with Experimental Data.......... 98

\section{CHAPTER 4: PRELIMINARY STUDY ON CONCRETE PRODUCED WITH}

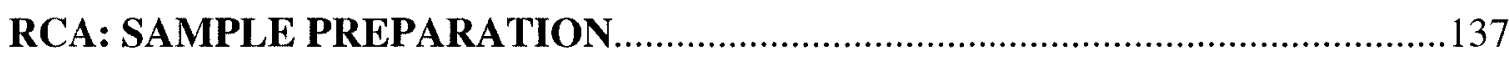

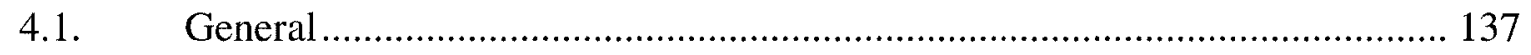




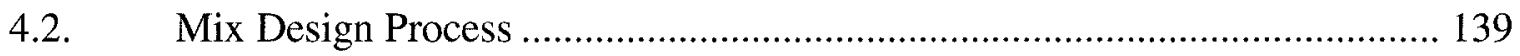

4.2.1. Estimation of Mixing Water and Air Content..........................140

4.2.2. Calculation of the Cement Content................................. 140

4.2.3. Calculation of Aggregate Content............................... 141

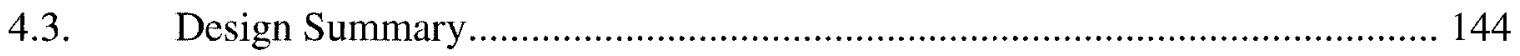

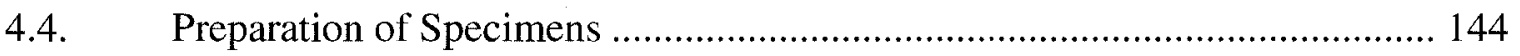

4.4.1. Preparation of Materials........................................ 144

4.4.2. Water Content Adjustment..................................... 145

4.4.3. Concrete Mixing Procedure......................................... 146

CHAPTER 5: PRELIMINARY STUDY ON CONCRETE PRODUCED WITH

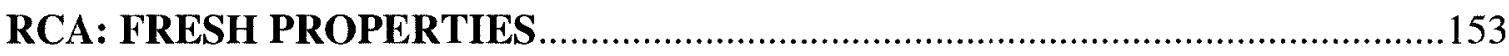

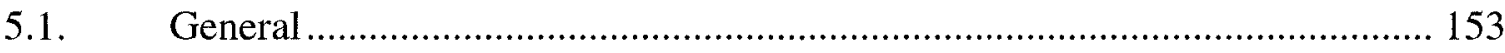

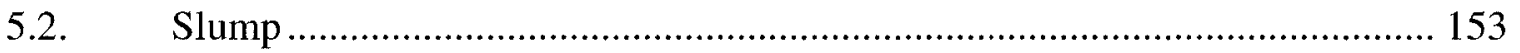

5.2.1. The Effect of Cement Content................................. 155

5.2.2. The Effect of TRMC .......................................... 156

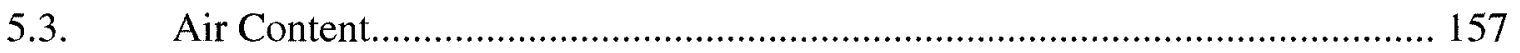

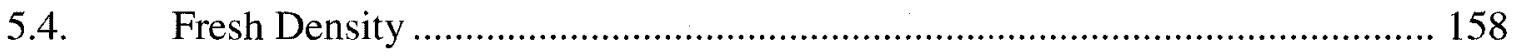

5.5. Summary of Fresh Properties of GC....................................................... 159

CHAPTER 6: PRELIMINARY STUDY ON CONCRETE PRODUCED WITH

RCA: HARDENED PROPERTIES …………….............................................171

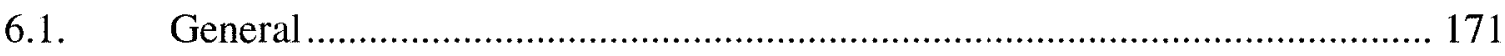

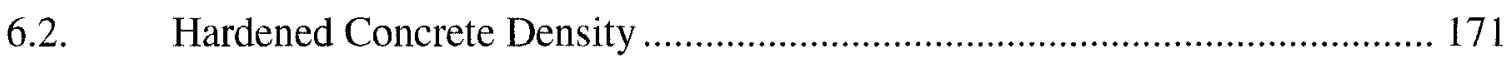

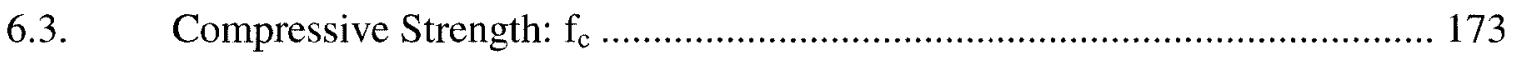

viii 


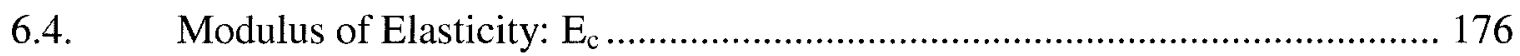

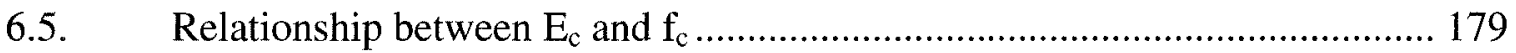

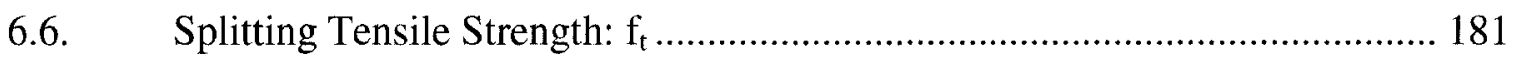

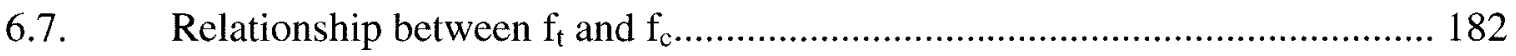

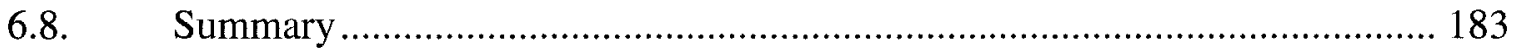

CHAPTER 7: MIX DESIGN OF GREEN CONCRETE....................................209

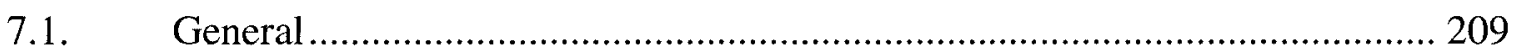

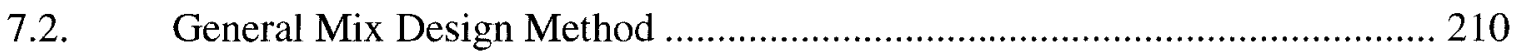

7.2.1. Estimation of Water Content................................... 211

7.2.2. Selection of $w / c$ Ratio and the Calculation of the Cement Content..... 211

7.2.3. Calculation of Aggregate Content............................... 212

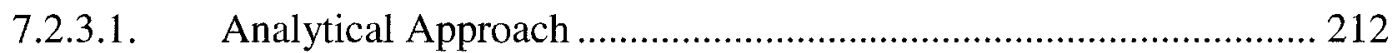

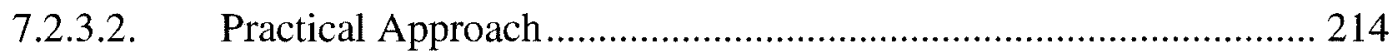

7.3. Equivalent Total Volume of Mortar Method .............................................. 215

7.3.1. Mix Design Procedure..................................... 216

CHAPTER 8: DURABILITY OF GREEN CONCRETE.....................................221

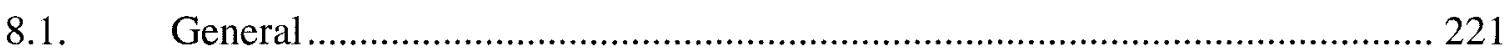

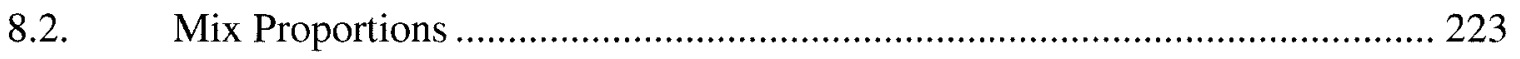

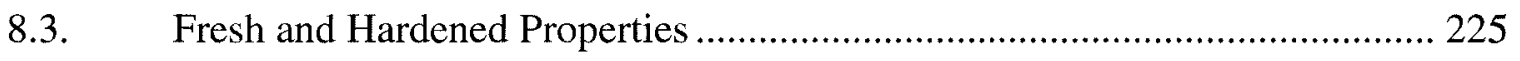

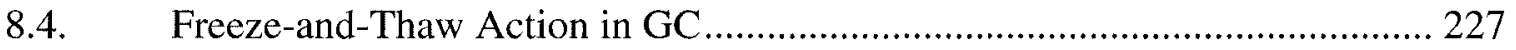

8.4.1. Relative Dynamic Modulus and the Durability Factor................2229

8.4.2. Length Change.......................................... 232

8.4.3. Pulse Velocity.............................................. 234 
8.4.4. Summary of the Freeze-and-Thaw Tests Results.................. 236

8.5. Chloride Penetration of GC ................................................................ 238

8.5.1. Acid Soluble Bulk Diffusion Test.............................. 239

8.5.2. Rapid Chloride Test..................................... 246

8.5.3. Bulk Diffusion vs. Rapid Chloride Test.......................... 248

8.5.4. Water Soluble Test (Preliminary Study on Chloride Binding).......... 250

8.5.5. Summary of the Chloride Tests Results.......................... 253

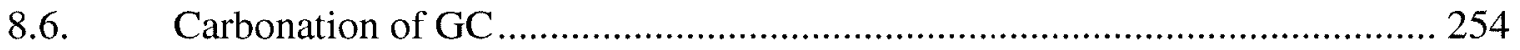

CHAPTER 9: CONCLUSIONS AND FUTURE WORK .......................................306

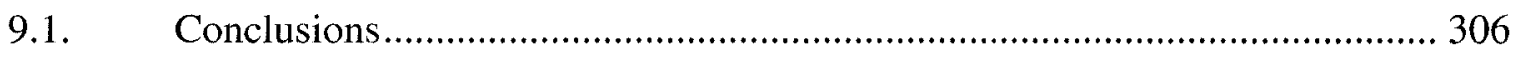

9.1.1. Material Characterization........................................ 306

9.1.2. Preliminary Study: Fresh Properties of GC ....................... 309

9.1.3. Preliminary Study: Mechanical Properties of GC.................... 310

9.1.4. Durability of GC.................................................. 311

9.1.4.1. Freeze-and-Thaw Action .................................................... 311

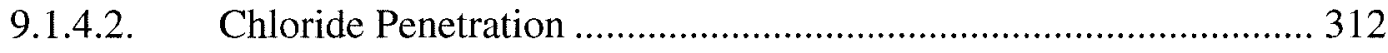

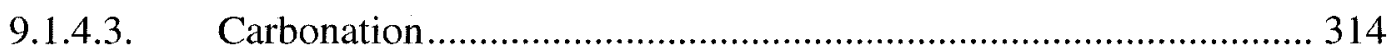

9.2. Recommendation for Future Work ...................................................... 315

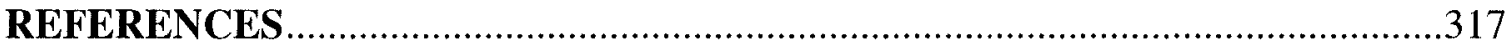

APPENDIX A: MATERIAL CHARACTERIZATION METHODS ......................326

A.1. Determination of the Residual Mortar Content ......................................... 326

A.2. Determination of Impurities of RCA ................................................. 329

A.3. Properties of Cementitious Materials...................................................... 333 
B.1. General Mix Design Examples .......................................................... 335

B.1.1. Example 1: Design of 100\% RCA Concrete for Severe Exposure...... 335

B.1.2. Example 2: Design of 50\% RCA Concrete for Moderate Exposure.... . 337

B.2. Equivalent Total Volume of Mortar Method …....................................... 340

B.2.1. Derivation the Equation of Equivalent Total Volume of Mortar Method....

B.2.1.1. Coarse Aggregate Content................................... 340

B.2.1.2. New Mortar Content........................................ 343

B.2.1.3. RCA Content ............................................. 345

B.2.2 Equivalent Total Volume of Mortar Method Example................ 346

APPENDIX C: DURABILITY EXPERIMENTS (RAW DATA) ..........................348

C.1. Freeze-and-Thaw Test (RAW DATA) ….......................................... 348

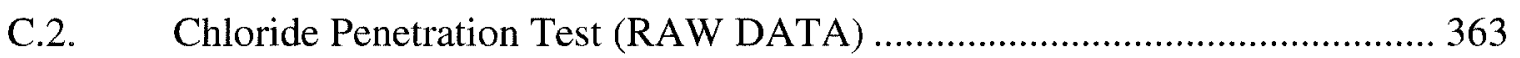

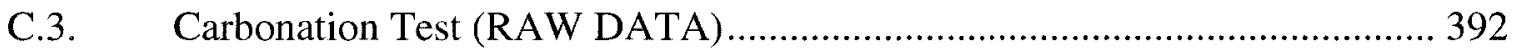




\section{LIST OF TABLES}

Table 2.1: Physical and mechanical properties of RCA from literature 56

Table 2.2: Factors that affect freeze-and-thaw resistance, chloride penetration, and carbonation in RAC 57

Table 3.1: Impurities in RCA-MO and RCA-VA ............................................... 100

Table 3.2: RMC for different fraction sizes of RCA-MO........................................ 100

Table 3.3: RMC for different fraction sizes of RCA-VA ........................................ 101

Table 3.4: Mix proportions used for the manufacture of the concrete prisms for image analysis 101

Table 3.5: Typical raw and processed data from image analysis (example from RCA$\mathrm{MO}, 12.7 \mathrm{~mm}$ fraction) 102

Table 3.6: Total area of RCA, OVA, residual mortar and RMC for RCA-MO and RCAVA (from image analysis work) 103

Table 3.7: Measured bulk specific gravities used for the calculation of RMC by image analysis 104

Table 3.8: The RMC by image analysis and the quick test method 104

Table 3.9: Measured properties of RCA-MO after 24-hr, 48-hr and 72-hr immersion . 105

Table 3.10: Measured properties of RCA-VA after 24-hr, 48-hr and 72-hr immersion 106

Table 3.11: Averaged properties of RCA-MO and RCA-VA specimens 107

Table 3.12: Los Angeles abrasion values for RCA-MO and RCA-VA samples 107

Table 3.13: Weight of the control aggregates on individual sieves as function of time in the MTO Soundness Test LS-614 108 
Table 3.14: Cumulative percentage loss for the control aggregates on individual sieves in the MTO Soundness Test LS-614 108

Table 3.15: Average weighted freeze-and-thaw loss for control aggregates in the MTO Soundness Test LS-614 109

Table 3.16: MTO Soundness Test LS-614 results for the RCA-MO and RCA-VA ..... 109

Table 3.17: Results for ASTM soundness test using $\mathrm{Na}_{2} \mathrm{SO}_{4}$ for RCA-MO and RCA-VA 110

Table 3.18: Soundness values for RCA-MO and RCA-VA samples from the external laboratory

Table 3.19: Loose and compacted unit weight and void content for RCA-MO and RCAVA.

Table 3.20: Moisture content for different factions of RCA-MO and RCA-VA 113

Table 3.21: Measured properties for the residual mortar of RCA-MO 113

Table 3.22: Measured properties for the residual mortar of RCA-VA 114

Table 3.23: Summary of material characterization testing of RCA-MO and RCA-VA 115 Table 3.24: Residual mortar and original natural aggregate properties of Nagataki et al. experiments used in the rational approach 115

Table 4.1: Approximate mixing water and air content requirements for different slumps up to the nominal maximum size of aggregate of $19 \mathrm{~mm}$ (reproduced from ACI 211-197) 148

Table 4.2: Volume of coarse aggregate per unit volume of concrete $\left(v_{c a}\right)$ (reproduced from ACI 211.1-97). 148

Table 4.3: Mix design proportions of air-entrained GC-MO mixes $\left(\mathrm{kg} / \mathrm{m}^{3}\right)$ 149 
Table 4.4: Mix design proportions of non-air-entrained GC-MO mixes $\left(\mathrm{kg} / \mathrm{m}^{3}\right) \ldots \ldots \ldots 150$

Table 4.5: Mix design proportions of air-entrained GC-VA mixes $\left(\mathrm{kg} / \mathrm{m}^{3}\right) \ldots \ldots \ldots \ldots \ldots 151$

Table 4.6: Mix design proportions of non-air-entrained GC-VA mixes $\left(\mathrm{kg} / \mathrm{m}^{3}\right)$......... 152

Table 5.1: Fresh property of air-entrained GC-MO mixes ..................................... 161

Table 5.2: Fresh property of non-air-entrained GC-MO mixes ................................. 161

Table 5.3: Fresh property of air-entrained GC-VA mixes ....................................... 162

Table 5.4: Fresh property of non-air-entrained GC-VA mixes................................ 162

Table 6.1: Hardened density of air-entrained GC-MO mixes................................... 185

Table 6.2: Hardened density of non air-entrained GC-MO mixes.............................. 186

Table 6.3: Hardened density of air-entrained GC-VA mixes .................................. 187

Table 6.4: Hardened density of non air-entrained GC-VA mixes ............................. 188

Table 6.5: Mechanical properties of air-entrained GC-MO mixes ............................. 189

Table 6.6: Mechanical properties of non air-entrained GC-MO mixes ...................... 190

Table 6.7: Mechanical properties of air-entrained GC-VA mixes ............................. 191

Table 6.8: Mechanical properties of non air-entrained GC-VA mixes........................ 192

Table 7.1: Volume of coarse aggregate per unit volume of concrete $\left(v_{c a}\right)$ (reproduced

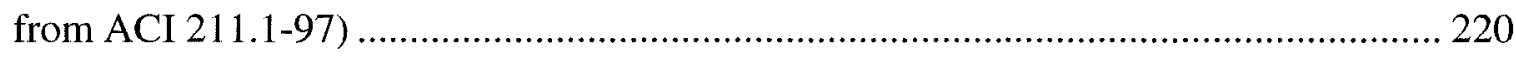

Table 7.2: Approximate total aggregate-to-cement ratio and fine-to-total aggregate ratio

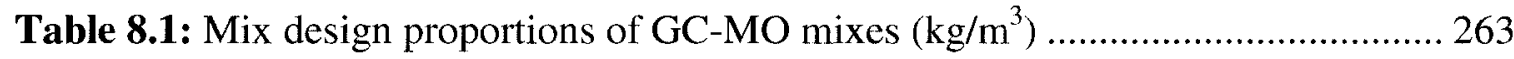

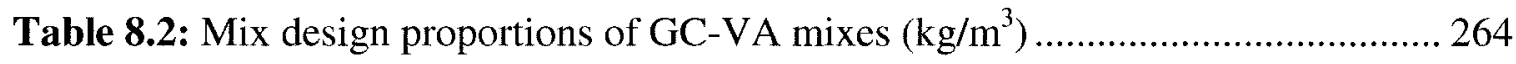

Table 8.3: Fresh concrete properties for GC-MO mixes ...................................... 265

Table 8.4: Fresh concrete properties for GC-VA mixes ....................................... 265 
Table 8.5: Hardened concrete properties for GC-MO specimens........................... 266

Table 8.6: Hardened concrete properties for GC-VA specimens .............................. 267

Table 8.7: Durability factor, pulse velocity, length change after 300 cycles of freeze-and-

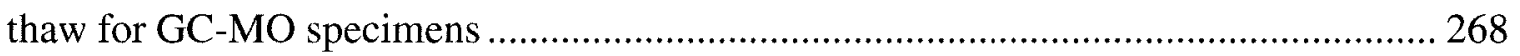

Table 8.8: Durability factor, pulse velocity, length change after 300 cycles of freeze-and-

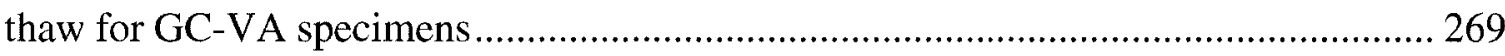

Table 8.9: Results of the acid soluble chloride content determination test (ASTM C

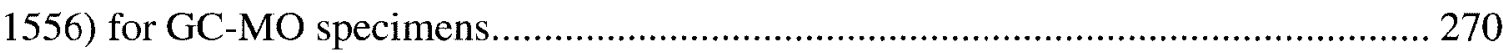

Table 8.10: Results of the acid soluble chloride content determination test (ASTM C

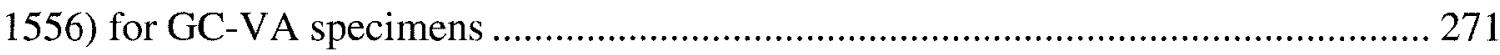

Table 8.11: Charge passed (Coulombs) for GC-MO specimens ............................... 272

Table 8.12: Charge passed (Coulombs) for GC-VA specimens ................................. 272

Table 8.13: The results of the water soluble chloride test results $(\%)$........................ 273

Table 8.14: Average carbonation depth and carbonation coefficient for GC-MO ....... 274

Table 8.15: Average carbonation depth and carbonation coefficient for GC-VA ........ 274 


\section{LIST OF FIGURES}

Figure 1.1: Schematic representation for recycled concrete aggregate (RCA)......... 15

Figure 1.2: Schematic representation for TRMC in GC........................... 15

Figure 2.1: Cylinder compressive strength for RAC with RCA from (a) laboratory, (b)demolished roof slab, and (c) demolished building........................... 58

Figure 2.2: Relationship between the grade of concrete and (a) $A / C$ ratio, (b) cementdemand (reproduced from Bairagi et al., 1990)............................ 59

Figure 2.3: (a) Effect of water saturation type on the relative dynamic modulus; (b) durability factor for complete water saturation (reproduced from Zaharieva et al., 2004)

.60

Figure 2.4: Carbonation depth versus RCA content (reproduced from Levy and

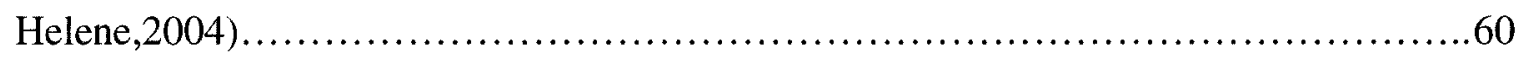

Figure 3.1: Screening and air drying of RCA................................... 116

Figure 3.2: Large impurities in RCA-MO and RCA-VA........................ 116

Figure 3.3: Small impurities in RCA-MO and RCA-VA.......................... 117

Figure 3.4: RCA-MO samples after 15 days immersion.......................... 117

Figure 3.5: RCA-MO samples after 5 freeze-and-thaw cycles...................117

Figure 3.6: Stages of removing of residual mortar by chemical and mechanical stresses

Figure 3.7: RCA-MO samples after full removal of the attached residual mortar...... 118

Figure 3.8: RCA-VA samples after full removal of the attached residual mortar.......119

Figure 3.9: Preparation of image analysis specimens (a) Sawing of concrete slabs, (b)

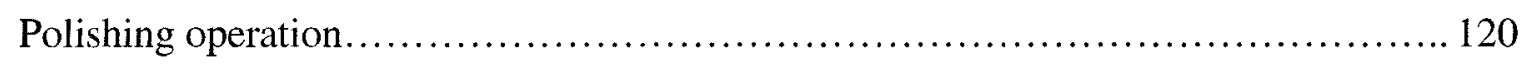


Figure 3.10: Polished sample for RCA-MO

Figure 3.11: Polished sample for RCA-VA.

Figure 3.12: Photoshop process (a) Original picture of the slab (1/3 of the total length of the slab analyzed) (b) Edited picture in Photoshop, green mask applied 122

Figure 3.13: Separation of the white cement binder from the RCA particle. 122

Figure 3.14: Binarization operation (a) Detail of RCA particle with the green mask representing the binder of white cement (b) Binarized image corresponding to Figure $3.14 \mathrm{a}$. 123

Figure 3.15: Visual results of some of the image analysis functions used in the routine 124

Figure 3.16: Average percentage frequency of particles for RCA-MO and RCA-VA... 125 Figure 3.17: Average weighted freeze-and-thaw loss vs. sieving time for control aggregate for the MTO soundness test......................................... 126

Figure 3.18: Absorption capacity vs. RMC for different immersion periods............127

Figure 3.19: Bulk specific gravity vs. RMC for different immersion periods..........128

Figure 3.20: SSD specific gravity vs. RMC for different immersion periods........... 129

Figure 3.21: Apparent specific gravity vs. RMC for different immersion periods...... 130

Figure 3.22: Porosity vs. RMC at different immersion periods.....................131

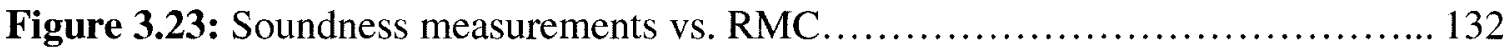

Figure 3.24: Comparison of the rational model with experimental data for the absorption capacity- RMC relationship.

Figure 3.25: Comparison of the rational model with experimental data for the specific gravity- $\mathrm{RMC}$ relationships (a) $\mathrm{SG}_{\mathrm{b}}-\mathrm{RMC}$, (b) $\mathrm{SG}_{\mathrm{SSD}}-\mathrm{RMC}$, (c) $\mathrm{SG}_{\mathrm{app}}-\mathrm{RMC}$ 133 
Figure 3.26: Comparison of the rational model with experimental data for the porosity RMC relationship. 134

Figure 3.27: Comparison of the rational model with experimental data for the abrasion value-RMC relationship.

Figure 3.28: Predicted and experimental average absorption capacity values for
(a) Nagatki-1, (b) Nagatki-2, (c) Nagatki-3,
(d) Nagatki-4 .135

Figure 3.29: Predicted and experimental average bulk specific gravity values for

(a) Nagatki-1, (b) Nagatki-2, (c) Nagatki-3,

(d) Nagatki-4 136

Figure 5.1: Variation of slump with cement content of GC-MO mixes.... 163

Figure 5.2: Variation of slump with cement content of GC-VA mixes .164

Figure 5.3: Variation of slump with TRMC of GC-MO mixes...................... 165

Figure 5.4: Variation of slump with TRMC of GC-VA mixes.......................166

Figure 5.5: Variation of air content with TRMC of GC-MO mixes..................167

Figure 5.6: Variation of air content with TRMC of GC-VA mixes..................... 168

Figure 5.7: Variation of fresh density with the TRMC of GC-MO mixes............ 169

Figure 5.8: Variation of fresh density with the TRMC of GC-VA mixes............. 170

Figure 6.1: Variation of hardened density with TRMC of GC-MO mixes............193

Figure 6.2: Variation of hardened density with TRMC of GC-VA mixes............ 194

Figure 6.3: Variation of compressive strength with the w/c ratio of GC-MO mixes.... 195

Figure 6.4 Variation of compressive strength with the w/c ratio of GC-VA mixes...... 196

Figure 6.5 Variation of 28-day compressive strength with TRMC of GC-MO mixes... 197

Figure 6.6: Variation of 28-day compressive strength with TRMC of GC-VA mixes.. 198

Figure 6.7: Variation of elastic modulus with w/c ratio of GC-MO mixes 199

Xviii 
Figure 6.8: Variation of elastic modulus with w/c ratio of GC-VA mixes.............200

Figure 6.9: Variation of elastic modulus with TRMC of GC-MO mixes..............201

Figure 6.10: Variation of elastic modulus with TRMC of GC-VA mixes............. 202

Figure 6.11: Relationship between elastic modulus and compressive strength for GC-MO

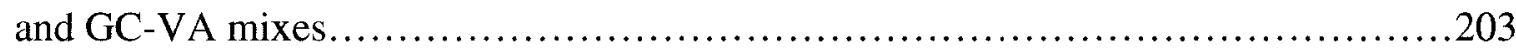

Figure 6.12: Variation of splitting tensile strength with w/c ratio of GC-MO mixes...204

Figure 6.13: Variation of splitting tensile strength with w/c ratio of GC-VA mixes... 205

Figure 6.14: Variation of splitting tensile strength with TRMC of GC-MO mixes...206

Figure 6.15: Variation of splitting tensile strength with TRMC of GC-VA mixes....207

Figure 6.16: Relationship between compressive strength and splitting tensile strength for

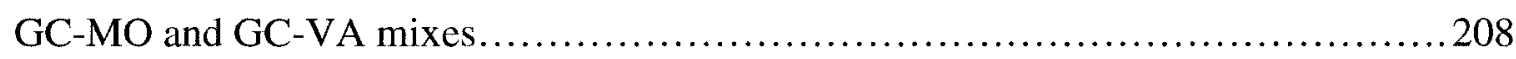

Figure 7.1: Flowchart for the general mix design procedure......................218

Figure 7.2: Relationship between w/c ratio and compressive strength for air-entrained and non-air-entrained concrete........................................... 219

Figure 8.1: The test grid for the durability study of GC....................... 275

Figure 8.2: Variation of fresh density with TRMC for GC-MO-D-C and GC-VA-D-C

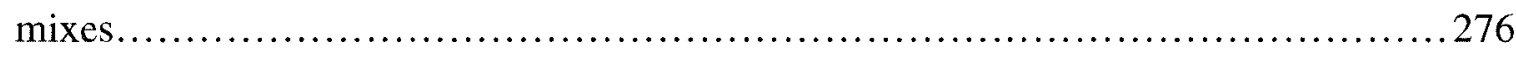

Figure 8.3: Apparatus for measuring properties in freeze-and-thaw tests.............277

Figure 8.4: Freezers used in the freeze-and-thaw tests.........................278

Figure 8.5: Variation of the relative dynamic modulus with number of freeze-and-thaw cycles for specimens with no SCM's

Figure 8.6: Variation of the relative dynamic modulus with number of freeze-and-thaw cycles for specimens with fly ash..... 280

xix 
Figure 8.7: Variation of the relative dynamic modulus with number of freeze-and- thaw cycles for specimens with bfs.

Figure 8.8: Summary of the $D F$ measurements of the freeze-and-thaw specimens......282

Figure 8.9: Variation of the length change with number of freeze-and-thaw cycles for specimens with fly ash. 283

Figure 8.10: Variation of the length change with number of freeze-and-thaw cycles for specimens with bfs 284

Figure 8.11: Variation of the length change with number of freeze-and-thaw cycles for specimens with no SCM's 285

Figure 8.12: Summary of the length change measurements of the freeze-and-thaw specimens 286

Figure 8.13: Variation of the pulse velocity with number of freeze-and-thaw cycles for specimens with fly ash. 287

Figure 8.14: Variation of the pulse velocity with number of freeze-and-thaw_Cycles for specimens with bfs 288

Figure 8.15: Variation of the pulse velocity with number of freeze-and-thaw cycles for specimens with no SCM's 289

Figure 8.16: Summary of the normalized PV 290

Figure 8.17: Visual appearance of GC-MO specimens during the freeze-and-thaw tests .291

Figure 8.18: Visual appearance of GC-VA specimens during the freeze-and-thaw tests .292

Figure 8.19: Summary of the performance of GC 293 
Figure 8.20: Samples for chloride penetration tests............................ 294

Figure 8.21: Preparation of the concrete powder for bulk diffusion test..............295

Figure 8.22: Variation of the apparent diffusion coefficient..................... 296

Figure 8.23: Variation of the apparent diffusion coefficient with TRMC for GC

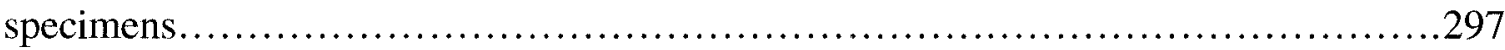

Figure 8.24: Rapid chloride test setup...................................... 298

Figure 8.25: Charge passed vs. apparent chloride diffusion coefficient...............299

Figure 8.26: Total, free and bound chloride (\%) measurements for GC-MO specimens .300

Figure 8.27: Total, free and bound chloride $(\%)$ measurements for GC-VA specimens 301

Figure 8.28: Carbonation test apparatus and process...............................302

Figure 8.29: Variation of the carbonation depth with exposure time...................303

Figure 8.30: Variation of the carbonation coefficient with specimens with/without

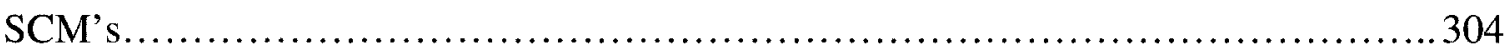

Figure 8.31: Measured and predicted values for the carbonation coefficient for specimens without SCM's. 305 


\section{LIST OF SYMBOLS}

$\alpha=$ binding capacity of concrete

$\alpha_{E c}=$ correlation factor for elastic modulus and compressive strength

$\alpha_{f t}=$ correlation factor for splitting tensile strength and compressive strength

$\gamma=$ hardened density of concrete, $\mathrm{kg} / \mathrm{m}^{3}$

$\Omega=$ fundamental transverse frequency at 0 cycles of freezing and thawing, $\mathrm{Hz}$

$\Omega_{1} \quad=$ fundamental transverse frequency after c cycles of freezing-and-thawing, $\mathrm{Hz}$

$\rho_{w}=$ density of water, $\mathrm{g} / \mathrm{cm}^{3}$

$A=$ air content in concrete, $\%$

$A_{R C A, i}=$ total area of each RCA particle in the image analysis study, $\mu \mathrm{m}^{2}$

$A_{O V A, i}=$ total area of OVA particles in the image analysis study, $\mu \mathrm{m}^{2}$

$A_{R M, i}=$ total area of residual mortar in the image analysis study, $\mu \mathrm{m}^{2}$

$A C=$ absorption capacity of aggregate, $\%$

$A C^{R C A}=$ absorption capacity of RCA, \%

$A C^{R M}=$ absorption capacity of residual mortar, $\%$

$A C^{O V A}=$ absorption capacity of original virgin aggregate, $\%$

$C_{0}=$ initial chloride-ion concentration of concrete, $\%$ by mass of concrete

$C_{s}=$ chloride concentration at the surface, $\%$ by mass of concrete

$C_{b}=$ bound chloride concentrations in concrete, $\%$ by mass of concrete

$C_{f}=$ free chloride concentrations in concrete, $\%$ by mass of concrete

$C_{t}=$ total chloride concentrations in concrete, $\%$ by mass of concrete

$C_{t}(x, t)=$ chloride concentration measured at depth $\mathrm{x}$ and exposure time $\mathrm{t}, \%$ by mass of concrete

$d=$ depth of carbonation front, $\mathrm{mm}$

$D_{a}=$ apparent chloride diffusion coefficient, $\mathrm{m}^{2} / \mathrm{s}$

$D_{e}=$ effective chloride diffusion coefficient, $\mathrm{m}^{2} / \mathrm{s}$

$D_{c}=$ coefficient for the progression of carbonation, $\mathrm{mm} / \mathrm{day}^{0.5}$

$D_{c}^{G C}=$ carbonation coefficient of GC, mm/day ${ }^{0.5}$

$D_{c}^{N A C}=$ carbonation coefficient of NAC, $\mathrm{mm} /$ day $^{0.5}$

$D F=$ durability factor, $\%$

$E_{c}=$ elastic modulus of concrete, GPa 
erf $=$ error function

$F M=$ fines modulus of fine aggregate

$f_{c}=28$-day compressive strength of concrete, $\mathrm{MPa}$

$f_{t}=$ splitting tensile strength of concrete, $\mathrm{MPa}$

$L=$ length between transmitting and receiving transducer, length of the specimen, $\mathrm{mm}$

$L_{c}=$ length change of the test specimen after c cycles of freezing-and-thawing,

$L_{I} \quad=$ length comparator reading after 0 cycles, $\mathrm{mm}$

$L_{2}=$ length comparator reading after $\mathrm{C}$ cycles, $\mathrm{mm}$

$L_{g} \quad=$ effective gage length between inner most ends of the gage studs, mm

$L A T=$ Los Angeles Test (abrasion) value of aggregate

$L A T^{R C A}=$ Los Angeles Test value of RCA

$L A T^{R M}=$ Los Angeles Test value of residual mortar

$L A T^{\text {OVA }}=$ Los Angeles Test value of original virgin aggregate

$\mathrm{M}=$ unit weight of oven dry-rodded coarse aggregate, $\mathrm{kg} / \mathrm{m}^{3}$

$m_{c}^{C A}=$ moisture content of coarse aggregate, $\%$

$n$ = porosity of the aggregate

$n^{R C A}=$ porosity of RCA

$n^{R M}=$ porosity of residual mortar

$n^{O V A}=$ porosity of original virgin aggregate

$N=$ number of cycle at which $P_{N}$ reaches the specified minimum value for discontinuing the test or the specified number of cycles to terminate test.

$N P V=$ normalized pulse velocity after 300 cycles of freezing and thawing, $\%$

$P V=$ pulse velocity, $\mathrm{m} / \mathrm{s}$

$P_{c} \quad=$ relative dynamic modulus of elasticity, after c cycles of freezing-andthawing, \%

$P_{N}=$ relative dynamic modulus of elasticity at $\mathrm{N}$ cycle, $\%$

$P V_{O}=$ initial pulse velocity at 0 freeze-and thaw cycles, $\mathrm{m} / \mathrm{s}$

$P V_{300}=$ pulse velocity at 300 freeze-and-thaw cycles, $\mathrm{m} / \mathrm{s}$

$R M C=$ residual mortar content of RCA by weight, \%

$\mathrm{RMC}_{\mathrm{v}}=$ residual mortar content by volume, $\%$

$S G_{\mathrm{c}} \quad=$ specific gravity of cement 


$$
\begin{aligned}
& S G_{b} \quad \text { = bulk specific gravity of aggregate } \\
& S G_{a}=\text { apparent specific gravity of aggregate } \\
& S G_{S S D}=\mathrm{SSD} \text { specific gravity of aggregate } \\
& S G_{S S D}^{C A}=\text { SSD specific gravity of coarse aggregate } \\
& S G_{b}^{C A}=\text { bulk specific gravity of coarse aggregate } \\
& S G_{S S D}^{F A} \quad=\text { SSD specific gravity of fine aggregate } \\
& S G_{a}^{O V A}=\text { apparent specific gravity of original virgin aggregate } \\
& S G_{a}^{R C A}=\text { apparent specific gravity of RCA } \\
& S G_{a}^{R M}=\text { apparent specific gravity of residual mortar } \\
& \mathrm{SG}_{\mathrm{SSD}, \mathrm{av}}=\text { average } \mathrm{SSD} \text { specific gravity of combined fine and coarse aggregate } \\
& S G_{b}^{R C A}=\text { bulk specific gravity of RCA } \\
& S G_{b}^{R M}=\text { bulk specific gravity of residual mortar } \\
& S G_{b}^{\text {OVA }}=\text { bulk specific gravity of original virgin aggregate } \\
& S G_{S S D}^{R C A}=\text { SSD specific gravity of RCA } \\
& S G_{S S D}^{R M}=\text { SSD specific gravity of residual mortar } \\
& S G_{S S D}^{O V A}=S S D \text { specific gravity of original virgin aggregate } \\
& t=\text { time, } \mathrm{s} \\
& t^{\prime}=\text { time, day } \\
& \text { TRMC = total residual mortar content, } \% \\
& U=\text { mass fresh density of concrete, } \mathrm{kg} / \mathrm{m}^{3} \\
& U_{G C}=\text { fresh density of the green concrete, } \mathrm{kg} / \mathrm{m}^{3} \\
& \mathrm{Va}=\text { ratio of the compacted volume of coarse aggregate to the absolute } \\
& \text { volume of concrete } \\
& V_{N M}=\text { volume of the new mortar in } \mathrm{GC}, \mathrm{m}^{3} \\
& V_{R M}=\text { volume of the residual mortar in } \mathrm{GC}, \mathrm{m}^{3} \\
& v_{c a}=\text { volume of dry-rodded coarse aggregate per unit volume of concrete. } \\
& v_{R C A}^{G C}=\text { volume of dry-rodded RCA per unit volume of GC } \\
& w_{e}=\text { evaporable water content in concrete } \\
& W_{c} \quad=\text { weight of cement in concrete, } \mathrm{kg} / \mathrm{m}^{3} \\
& W_{O D}=\text { weight of oven-dry test sample in air, } \mathrm{g} \\
& W_{S S D}=\text { weight of SSD test aggregate sample in air, } \mathrm{g} \\
& W_{w-s s d}=\text { weight of SSD test aggregate sample in water, } \mathrm{g}
\end{aligned}
$$




$$
\begin{aligned}
& W_{w}=\text { weight of water in concrete, } \mathrm{kg} / \mathrm{m}^{3} \\
& \mathrm{w} / \mathrm{c}=\text { water to cement ratio } \\
& W_{S S D}^{F A}=\text { weight of SSD fine aggregate in concrete, } \mathrm{kg} / \mathrm{m}^{3} \\
& W_{A D}^{C A}=\text { weight of air-dry coarse aggregate, } \mathrm{kg} / \mathrm{m}^{3} \\
& W_{O D}^{C A}=\text { weight of oven-dry coarse aggregate, } \mathrm{kg} / \mathrm{m}^{3} \\
& W_{S S D}^{C A}=\text { weight of SSD coarse aggregate in concrete, } \mathrm{kg} / \mathrm{m}^{3} \\
& W_{O D}^{R C A}=\text { oven-dry weight of RCA, g } \\
& W_{O D}^{R M}=\text { oven dry weight of residual mortar, } \mathrm{g} \\
& W_{O D}^{O V A}=\text { oven-dry weight of OVA, } \mathrm{g} \\
& W_{O V A, i}=\text { weight of the OVA in a given RCA particle in the image analysis study, } \mathrm{g} \\
& W_{R M, i}=\text { weight of the residual mortar in a given RCA particle in the image analysis } \\
& \text { study, } \mathrm{g} \\
& W_{S S D}^{R C A}=\text { weight of SSD RCA, } \mathrm{g} \\
& W_{S S D}^{R M} \quad=\text { weight of SSD residual mortar, } \mathrm{g} \\
& W_{S S D}^{O V A}=\text { weight of SSD original virgin aggregate, } \mathrm{g} \\
& W_{w-S S D}^{R C A} \quad=\text { weight of SSD RCA in water, } \mathrm{g} \\
& W_{w-S S D}^{R M} \quad=\text { weight of SSD residual mortar in water, } \mathrm{g} \\
& W_{w-S S D}^{O V A}=\text { weight of SSD original virgin aggregate in water, } \mathrm{g} \\
& W_{C}^{G C}=\text { weight of cement in } \mathrm{GC}, \mathrm{kg} / \mathrm{m}^{3} \\
& W_{W}^{G C}=\text { weight of water for } \mathrm{GC}, \mathrm{kg} / \mathrm{m}^{3} \\
& W_{S S D-F A}^{G C}=\text { weight of SSD natural fine aggregate in } \mathrm{GC}, \mathrm{kg} / \mathrm{m}^{3} \\
& W_{S S D-R C A}^{G C}=\text { weight of SSD RCA in GC, } \mathrm{kg} / \mathrm{m}^{3} \\
& W_{O D R C A}^{G C}=\text { weight of oven-dry RCA in } \mathrm{GC}, \mathrm{kg} / \mathrm{m}^{3} \\
& W_{O D N A}^{G C}=\text { weight of oven-dry natural aggregate in } \mathrm{GC}, \mathrm{kg} / \mathrm{m}^{3} \\
& W_{O D-N A}^{N A C}=\text { oven-dry weight of natural aggregate in NAC, } \mathrm{kg} / \mathrm{m}^{3} \\
& W_{W}^{N A C}=\text { weight of water in NAC, } \mathrm{kg} / \mathrm{m}^{3} \\
& W_{C}^{N A C}=\text { weight of cement in NAC, } \mathrm{kg} / \mathrm{m}^{3} \\
& W_{F A}^{N A C}=\text { weight of fine aggregate in NAC, } \mathrm{kg} / \mathrm{m}^{3}
\end{aligned}
$$




\section{CHAPTER 1: INTRODUCTION}

\subsection{General}

Concrete is the most common material used in the construction of civil engineering structures. Due to its environmental impact, it is also one of the most costly ones. Conventional concrete typically contains about $12 \%$ cement and $80 \%$ aggregate by mass (Neville, 1995a). Global construction industry uses approximately 1.6 billion tons of cement and 10 billion tons of sand, gravel, and crushed rock every year (Mehta, 2001). In Canada, the total aggregate supply is around 350.5 million tons per year in 2003 figures (Panagapko, 2003). The world's yearly cement production of 1.6 billion tons accounts for about $7 \%$ of the global loading of carbon dioxide $\left(\mathrm{CO}_{2}\right)$ into the atmosphere (Mehta,

2001). Producing a ton of portland cement requires about four giga-joules of energy. Mining large quantities of raw materials for the production of cement such as limestone and clay, and fuel such as coal, often results in extensive deforestation or denudation and top-soil loss (Mehta, 2001).

Concrete structures have service lives of several decades to more than a century. Those structures, which are no longer able to fulfill their original purpose after their service life will be demolished. The demolished materials are considered as construction waste, and concrete constitutes a large part of this waste. The annual worldwide output of concrete and masonry rubble has been estimated roughly as one billion tones (Mehta, 2001). In Canada, a large portion of the building stock and infrastructure are made of concrete. Many of these buildings were built in the 50 's or 60 's, and are near the end 
of their design life. It is expected that during the next two decades, a large amount of concrete, resulting from the demolition of these buildings and structures, will be available for either disposal or for being recycled for reuse as concrete aggregates. Currently, the construction waste in Canada is estimated as 11 million tons per year, and approximately $42 \%$ (by weight) of this amount is reused or recycled. Concrete constitutes $52 \%$ of construction waste, and approximately $73 \%$ of it is reused in low-value applications as filler material or as road sub-grade (George and Michael, 2001).

Nowadays, recycled concrete is widely used to produce aggregates for nonstructural construction, such as many types of general bulk fills, base or fill for drainage structures, pavement base and sub-base, lean-concrete bases, concrete blocks, and wall sound barriers in highways. Although the use of recycled concrete aggregates (RCA) in structural concrete is limited, various quantities of RCA were used successfully in the construction of concrete pavements and low rise buildings in United Kingdom (Collins, 1998) and in a small number of projects in Japan (Takenaka, 1999). The main factors behind the limited use of RCA in structural concrete are barriers such as (1) technical problems; (2) commercial barriers; (3) quality assurance (QA) and quality control (QC) problems; (4) lack of suitable regulations and experience; and (5) poor image of recycled materials.

The commercial barriers to the extensive use of RCA arise from the unsteady supply of structural-grade RCA, which cannot compete with efficient and stable supply of traditional concrete materials. The steady supply of normal concrete materials makes it very difficult for a new material such as RCA to enter the market. However, the continuous decline of the natural aggregate resources, and its consequence that the 
aggregates must be hauled from significantly long distances, is expected to make RCA a commercially viable alternative to natural aggregate in the near future. The early indications of this trend have started to emerge in large cities and in areas with limited natural resources. A recent newspaper article reporting aggregates being shipped to California from British Columbia is a good example of the current state of the problem (Stueck, April, 13, 2006).

The quality of RCA depends on the equipment used to produce the RCA, and it varies from one plant to another. This variation may cause concerns about the quality of structures using RCA. The poor image issue of RCA arises from the fact that the recycled materials in general have the problem of low quality. However, this poor image is based on the preconceived notion that RCA can not yield good quality concrete. In general, all these difficulties can be overcome by developing sound technical specifications and QC/QA methodologies for producing structural-grade concrete.

Currently, to the author's knowledge, there are no standards available in North America, which can be followed to consistently design and produce structural-grade concrete made specifically with RCA. Although a guideline on the use of RCA as a filling material exists in Quebec (BNQ, 2002), this guideline does not address the processes and methods to produce structural-grade concrete from RCA. CSA Standard A23.1 (2004), "Concrete Materials and Methods of Concrete Construction" included the use of RCA under Section 4.2.3.1 by stating that "RCA as aggregate should be evaluated in a manner similar to the evaluation of normal-density aggregate with particular attention to durability characteristics; deleterious materials; potential alkali-aggregate reactivity; chloride contamination; and the workability characteristics of concrete 
manufactured with the material". In the US, since 1982, ASTM C33-01 (ASTM, 2003) "Standard specifications for concrete aggregates" defines coarse aggregate as including crushed hydraulic cement concrete. ASTM C125-00 (ASTM, 2003) "Standard definitions of terms relating to concrete and concrete aggregates" defines manufactured sand as including hydraulic cement concrete. Furthermore, ACI Committee 555R-01 (2001) provided a report on the removal and reuse of the hardened concrete. This report provides some consideration from the literature for evaluating and processing waste concrete for the production of aggregates suitable for reuse in concrete construction. However, in all cases, concrete produced with RCA is mostly treated as conventional concrete, and little attention is placed on the particular properties of this new material.

In Europe and Japan concrete recycling is regulated and often mandated. In the UK, the use of construction and demolition waste as recycle aggregates has been permitted since the publication of BS EN 12620 "Aggregates for Concrete." A current research project in the UK is aimed at maximizing the use of RCA in concrete construction (Dhir et al., 2004). The purpose of the project is to overcome the main barriers preventing adoption of recycled aggregate for use in concrete. In Germany, the national standard, DIN 4226-1000, “Aggregates for Concrete and Mortar" sets the guidelines for recycled concrete content in concrete aggregates. In Netherlands, the Dutch standard, NEN 6720:95, "Construction Requirements and Calculating Methods", allows the use of concrete aggregates, provided the replacement percentage of natural aggregate is not higher than $20 \%$. Only a replacement of up to $10 \%$ is allowed for masonry aggregate (Corinaldesi et al., 2002). In Japan, the draft standard for use of recycled concrete was published in 1977, and the "Recycling Law" was established in 1991 to suppress the 
increase of waste and to promote recycling of useful resources (Noguchi and Tamura, 2001).

In summary, the use of RCA in new structural applications is limited by several technical and practical uncertainities and difficulties. There is still a strong global reluctance against using RCA and concrete produced from RCA as structural material. In addition, the unique circumstances imposed by Canada's environmental conditions, and the extensive use of de-icing salts that may cause pre-contamination of RCA, require comprehensive investigation of the properties of concrete produced with these aggregates. To the author's knowledge, in Canada, there are no major studies underway to evaluate the applicability and long-term performance of this type of concrete in structural applications. As a consequence of this lack of research, there are no established guidelines regarding the production of concrete using RCA and supplementary cementing materials (SCM's) as partial replacement for cement. There are, however, extensive guidelines for producing conventional concrete with supplementary materials, including fly ash.

\subsection{Economic and Environmental Benefits of GC}

The global demands for regulating and recycling of construction and demolition waste arise from the growth of environmental and economic issues. In the United States, according to Wilburn and Goonan (1998), the processing cost for RCA was around $\$ 10$ per metric ton depending on the size of the operation. The large operations produce cheaper products due to lower distribution cost over more units of output. The unit cost of producing natural sand and crushed stone in processing plants range from $\$ 6.5$ to $\$ 8$ (MOTH, 1999). It can be seen that the use of RCA will not create significantly high 
additional costs in the production stage as long as the quality-related issues regarding RCA and concrete produced with RCA can be addressed.

Currently, most of the natural aggregates are obtained from crushed stone processing plants since the natural sand and gravel sources, which do not require any processing, are limited. Therefore, the cost and transport distances of natural aggregates will continue to increase, as the natural sources grow scarce. As stated in the Environmental Council of Concrete Organization information sheet (ECCO, 1999), it is common for contractors to haul aggregates from distances 80 to $110 \mathrm{~km}$ away from the construction sites. Haul distances greater than $300 \mathrm{~km}$ are not uncommon in the United States. Therefore, the transportation cost constitutes a major part of the overall cost of commercially produced concrete in mixing plants. With RCA, there is potential for cost savings in hauling: it is estimated that the total transportation distance for hauling the aggregates can be reduced as much as $80 \%$ (Abbas et al., 2006). One of the best ways of achieving both economic savings and resource conservation is by using portable recycling equipment.

The continuous use of natural aggregates to produce conventional concrete has negative effects on the environment; therefore, the use of RCA has environmental benefits. The following list provides a summary of the negative impact of the extraction and the use of natural aggregates:

- The effect on the habitat: As stated by Winfield and Taylor (2005), the extraction of aggregates from pits and quarries results in the destruction of the natural habitats of many organisms.

- Quality of water resources: Impact of the natural aggregate production on the quality 
of the surface and groundwater resources is a significant concern. As stated by Winfield and Taylor (2005), the excavation of aggregate resources alters the slope of the land, and hence changes water drainage patterns. Also, aggregate deposits behave as underground water reservoirs. By excavating the aggregate, the water storage capacity of the ground is lost.

- Increased green house gas (GHG) emissions: The mining, processing, and transport operations involving such large quantities of aggregate consume considerable amount of energy, produce large quantities of GHG's, and adversely affect the ecology of the areas they are extracted from. As the hauling distances between the quarries to the processing plants and processing plants to construction sites increase, GHG emissions associated with the transportation of aggregates also increase.

Among these negative effects, GHG emissions associated with the production of cement, and the production and transportation of aggregates are particularly important. The GHG's are considered to be the main reason for the climate change that we have been experiencing over the past century. Although Canada accounts for only $2.5 \%$ percent of global emissions, it is the world's third largest per capita emitter of these gases, after the United States and Australia (IPCC-GC, 2005a and EC-GC, 2006c). According to the Kyoto Protocol, 38 industrialized countries, including Canada, are committed to cut their emissions of GHG by 5.2\% below 1990 levels by 2008 to 2012 . With Canada being a signatory to the Kyoto protocol, there is an urgent need for reducing GHG emissions. 


\subsection{A Note on Important Definitions and Acronyms}

Throughout this thesis, a number of acronyms were used to make the thesis more readable. Some of these acronyms have important meanings for the understanding of the thesis; therefore these are explained here for clarification.

- RCA (Recycled Concrete Aggregate) is coarse aggregate produced in aggregate production plants by crushing conventional concrete waste. It consists of two components: original virgin aggregate (OVA) and the residual mortar attached to it, as illustrated in Figure 1.1.

- NA (Natural Aggregate) is a coarse aggregate produced from natural resources by crushing of the rocks such as limestone or river gravel.

- OVA (Original Virgin Aggregate) is NA as it exists as part of RCA (see Figure 1.1).

- RMC (Residual Mortar Content) is the percentage of the residual mortar by weight in the RCA.

- NAC (Natural Aggregate Concrete) is conventional concrete produced with NA.

- RAC (Recycled Aggregate Concrete) is the generic definition for the concrete produced with recycled aggregates. In this thesis, this definition will only be used to refer to the works of other researchers in the context of the literature review presented in Chapter 2.

- GC (Green concrete) is the special definition for the concrete produced with RCA in the context of the present research, which can be produced with a combination of RCA and NA as coarse aggregate, sand as fine aggregate, OPC, SCM's and water. Recycled fine aggregates are not used as replacement for sand due to their proven deteriorative properties. 
- TRMC (Total Residual Mortar Content) is the residual mortar content of GC. Figure 1.2 illustrates the concept of the TRMC in GC and its difference from RMC. In this figure, RCA with $40 \% \mathrm{RMC}$ is shown as part of two different mixes of GC produced with (1) $100 \% \mathrm{RCA}+$ no NA, and (2) $50 \%$ RCA and $50 \%$ NA. The TRMC values of each mix are calculated as $40 \%$ for the first mix, $20 \%$ for the later.

\subsection{Problem Definition, Objectives and Scope}

As it will be presented in the literature review (Chapter 2), a number of studies have been carried out to investigate the use of RCA in structural-grade concrete. Although these studies cover a relatively wide range, they do not follow a comprehensive and systematic approach that can lead to the standardization of the practice. In general, the following gaps in the literature have been identified:

- Although the properties of RCA have been studied by a number of researchers, there is a lack of fundamental study investigating how these properties change with the presence of residual mortar.

- The quantification of the RMC of RCA is a crucial task in the prediction of RCA properties; therefore, a rapid and accurate RMC determination test is needed. This RMC determination test needs to be verified by accurate validation techniques such as digital imaging.

- Most studies in the literature were carried out with RCA produced in the laboratory. In a limited number of cases in which the RCA was obtained from commercial sources, no significant attempt was made to investigate the source effect which would produce varying material properties. Since Canada is a large country, there is an obvious need to investigate the source of the RCA by choosing materials from different 
geographical locations.

- Currently, to the author's knowledge, there is no mix design procedure specifically developed for structural-grade concrete. In the current practice, mix design procedures for conventional concrete are used to produce $\mathrm{GC}$ with some adjustments such as using increased cement content.

- In order to develop a mix design procedure for $\mathrm{GC}$, a comprehensive investigation of the fresh and hardened properties of concrete produced with RCA obtained from different commercial sources need to be carried out. Only such a comprehensive study can lead to the development of a special mix design procedure.

Structural-grade concrete must possess certain mechanical and physical properties in order to satisfy the strength and durability requirements. After the development of the mix design procedures, the durability properties of concrete produced with them need to be investigated to study the long-term performance. Among these properties, the resistance to freeze-and-thaw action, chloride penetration and carbonation can be considered to be of particular importance since these properties are significantly affected by the quality and the quantity of the mortar, whose characteristics will be different when RCA are used. It is understood that the durability problems associated with concrete are not limited to the three aspects; however, Canada's unique conditions bring these three issues to the forefront of the possible durability problems in GC. Although topics such as sulfate attack, alkali-silica reaction and acid attack are important, they will not be covered in this research.

The general objective of this research is to address these issues through a study following a systematic approach with the ultimate goal of paving the road towards the 
standardization of the use of RCA in structural-grade concrete in Canada. The research methodology that is used in this study consists of analytical and experimental investigation of each issue identified above as "gaps in research". In addition to these, there are also a number of specific problems that will be addressed in addition to the general objectives of this research. These are listed as follows:

- It has been observed in the literature regarding the determination of the freeze-andthaw resistance of concrete produced with RCA that the identification of the best testing procedure and the performance criterion to evaluate $\mathrm{GC}$ is an outstanding problem. To study this issue, three performance measures will be studied: relative dynamic modulus, length change and pulse velocity.

- Rapid chloride test method as per ASTM C 1202-97 (ASTM, 2003) is a quick and easy procedure which can give a good indication of the quality of conventional concrete against chloride penetration. This method is a measure of the electrical conductivity of conventional concrete in the penetration of chloride ions. Since the residual mortar in GC can be potentially contaminated with other ions, the electrical conductivity of GC may be not be directly correlated with the chloride concentration; therefore, the applicability of the rapid chloride test for GC needs to be studied further. For this purpose, the current study will carry out a comparative investigation between the results of rapid chloride test and the bulk diffusion (ASTM C 1556-04) test, which is based on titration and is considered to be a reliable measurement of chloride content of concrete.

- In order to model the chloride binding mechanism in conventional concrete, binding isotherms have been suggested by a number of researchers. These chloride binding 
isotherms provide the relationship between the free and bound chlorides in concrete at a given temperature. To the author's knowledge, chloride binding in GC is yet to be studied. In this study, a preliminary investigation on the binding properties of GC will be carried out.

It should be acknowledged that standardization is a difficult and a time-consuming process, which requires a more comprehensive investigation that needs to cover other aspects of durability, structural properties and QC/QA guidelines. The research presented in this thesis is part of a collaborative study that covers also other aspects of this large scope. Therefore, the following areas, which are related to the mechanical and structural behaviour of GC, are not covered in this study since they are investigated as part of a complementary research project:

- The creep and shrinkage of GC;

- The bond characteristics of GC with steel;

- The structural behaviour of slabs and beams produced with GC.

Upon the completion of these two major research projects, the QA/QC guidelines will be produced.

\subsection{Thesis Organization}

This thesis is organized in nine chapters and three appendices. In order to improve the readability of the thesis, the tables and figures are presented at the end of each chapter.

In Chapter 1, a general introduction on the use of the GC as structural material is presented, and the environmental and economic benefits of GC are addressed. The problem definition, the objectives and the scope of the study are outlined. 
Chapter 2 presents the existing literature on the use of RCA in the production of RAC. In the first part of the literature review, the research on the material characterization of RCA is discussed; this is followed by the review of studies that were carried out to determine the factors affecting the properties of RAC. The content and properties of RCA, w/c ratio, binder content and original concrete properties on the physical and mechanical properties of RAC are the considered factors. The section on durability discusses the studies that were carried out on freeze-and-thaw resistance, chloride penetration, and the carbonation of RAC.

In Chapter 3, the experimental and analytical investigations of the material characterization of the two types of RCA that are used in the current study are presented. The material characterization of RCA involves the determination of specific gravity, absorption capacity, moisture content, porosity, degradation resistance (using the Los Angeles Test), and soundness properties. The newly developed RMC determination test is also discussed in this chapter.

Chapters 4, 5 and 6 present the preliminary study to investigate the fresh and hardened properties of concrete produced with two types of RCA. In Chapter 4, the preparation of specimens for the preliminary study is explained. Chapter 5 presents the results of the preliminary investigation on the effect of the RCA on the fresh properties of concrete. In Chapter 6, the effect of the RCA on the hardened and mechanical properties is investigated.

Chapter 7 presents two GC mix design procedures (i.e., the general mix design method and the equivalent total volume of mortar method) that were developed based on the results of the preliminary study presented in Chapters 4-6. 
Chapter 8 presents the durability of GC produced with the two mix design methods with respect to freeze-and-thaw action, chloride penetration and carbonation. The conclusions and the recommendations for future work are presented in Chapter 9. 


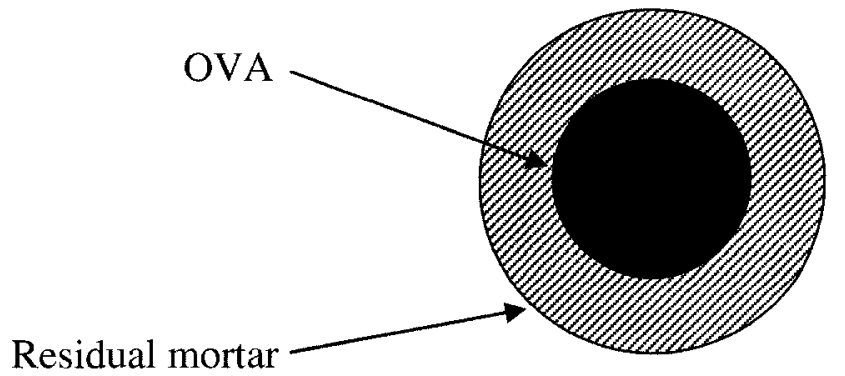

Figure 1.1: Schematic representation for recycled concrete aggregate (RCA)

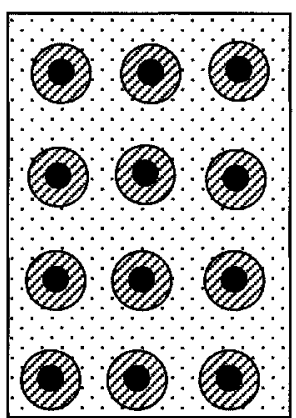

RCA Content: RMC of RCA: TRMC of GC:
$100 \%$ RCA

$40 \%$

$40 \%$

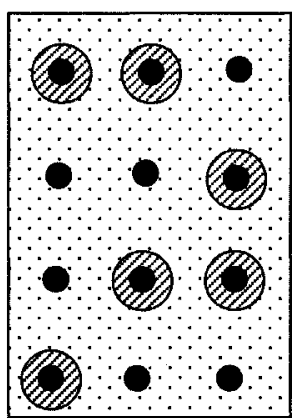

$50 \% \mathrm{RCA}+50 \% \mathrm{NA}$ $40 \%$

$0.5 \times 40 \%=20 \%$

Figure 1.2: Schematic representation for TRMC in GC 


\section{CHAPTER 2: LITERATURE REVIEW}

\subsection{General}

This chapter presents the review of the existing literature on the use of RCA in the production of RAC. During the past decade, the number of research studies on the use of RCA has increased significantly. Majority of the research, on the other hand, is mainly concerned with the use of RCA in non-structural applications; this literature review does not cover these studies whose main objective is the investigation of the use of RCA as a filler material. In addition to this, the review is presented by subject, not by chronology, and it focuses on the aspects of RAC as they are related to the scope of this research. Topics such as sulfate attack, alkali-silica reaction and acid attack are not included in the present review as they are not investigated in the present study.

In the first part of the literature review, the research on the material characterization of RCA is discussed; this is followed by the review of studies that were carried out to determine the factors affecting the properties of RAC. The content and properties of $\mathrm{RCA}, \mathrm{w} / \mathrm{c}$ ratio, binder content and original concrete properties on the physical and mechanical properties of RAC are the factors are considered. The section on durability discusses the studies that were carried out on the freeze-and-thaw action, chloride penetration, and the carbonation of RAC. 


\subsection{Material Characterization of RCA}

The quality of RCA has a direct influence on the quality of fresh concrete and the final product, i.e., RAC. One way of evaluating the quality of RCA is by measuring the amount of residual mortar attached to the OVA as suggested by Shayan and Xu (2003) and Otsuki et al. (2003). Different techniques have been reported by a number of authors for the measurement of the residual mortar of RCA. Ravindrarajah and Tam (1985) manufactured concrete cubes using RCA and a white cement binder; then they determined the attached mortar content in the RCA on traverses, through four slabs sliced from the cubes, using an electronic digital planimeter. Nagataki et al. (1999) used hydrochloric acid solution to disintegrate the mortar; however this technique is noted to be not suitable for the RCA containing acid-sensitive OVA, such as limestone, due to the partial dissolution of the aggregate material in the acid. Sanchez de Juan and Guttierrez (2004) reported a method in which the RCA is subjected to heat treatment, soaking in water (before and after heat treatment), and manual removal (through rubber hammering and scratching) of the residual mortar. However, none of these methods was validated to provide accurate RMC measurements.

Although Shayan and Xu (2003) and Otsuki et al. (2003) thought that the quality of RCA increased with the decreased quantities of attached mortar, based on the microstructural study by Nagataki et al. (2004), it has been reported that attached mortar content was not always the primary parameter determining the quality of RCA. In the study by Nagataki et al. (2004), microscopic analysis performed on the OVA showed defects in the form of voids and cracks. Sandstone as coarse aggregate was the most susceptible constituent of RCA in terms of micro-structural stability. While the double 
crushing of the source concrete by jaw and impact crusher considerably reduced the density of the cracks in the OVA by eliminating the particles with micro defects and irregular voids, continued processing by mechanical grinder reintroduced new cracks. Only very minor amounts of cracking were detected in the attached mortar or at the interfacial transition zone (ITZ). Extending the recycling process up to grinding enhanced the RCA properties by reducing the volume of attached mortar. In addition, Nagataki et al. (2004) pointed out that there is inadequate research focusing on the characteristics of RCA produced by other processing technologies aimed at improving the quality of the product.

Tavakoli and Soroushian (1996a) argued that the quality of original concrete restricted the quality achievable in RCA that is produced from it. Dhir et al. (1999) also studied the production procedure of RCA and the effect of the original concrete on the characteristics of RCA. In this study, RCA was derived from six concrete sources, ranging from airport pavements, demolished concrete structures, masonry concrete blocks, rejected precast structural elements, laboratory-cast and sidewalks. The analysis indicated that the sources had little effect on the RCA quality. In summary, there is significant variability in research with respect to the quality of RCA. Therefore, it is crucial to understand the effect of the residual mortar on the material properties of the RCA and to identify the material properties of RCA accurately.

Existing research on the material characterization of RCA mainly focuses on the determination of surface texture, particle shape, particle size distribution (gradation), density, specific gravity, water absorption, porosity, aggregate crushing value, abrasion value and soundness. In the following sections, the studies on these topics are presented. 


\subsubsection{Particle Shape and Size}

Ramamurthy and Gumaste (1998) investigated the particle shape of RCA obtained from demolished concrete and masonry with two types of bricks (wire-cut and table molded) by determining the elongation and flakiness indices. It was found that the elongation and flakiness indices of RCA from demolished concrete were closer to those of fresh granite aggregates. However, the elongation and flakiness indices for RCA from masonry were found to be higher than those of the RCA from demolished concrete. Chen et al. (2003) carried out a particle shape analysis using ASTM D 4791 specifications, and the results showed that RCA had a similar particle shape as crushed rock used in conventional concrete. In general, main observations about the surface texture and particle shape of RCA compared to NA from original concrete are that RCA is coarser, more porous and rougher in surface but more equidimensional (Dhir et al., 1999; Limbachiya et al., 2000; Krezel and McManus, 2000).

It has been demonstrated that crushing process plays an important role in the gradation of RCA. Sagoe-Crentsil and Brown (1998) found that the grading of RCA depended primarily on the crushing process, and the careful setting of sieve sizes was required to achieve recommended nominal uniform grading. Ramamurthy and Gumaste (1998) and Limbachiya et al. (2000) suggested that, with a properly designed crushing process, gradations that will satisfy the requirements of concrete design standards could be achieved with RCA. Katz (2003) demonstrated that the grading of RCA did not depend on the strength of the original concrete, but on the crushing process. 


\subsubsection{Specific Gravity, Density and Water Absorption Capacity}

Specific gravity, density and water absorption capacity are important properties, that are used in the mix design of concrete produced with RCA. The actual value of the specific gravity of aggregates is generally not considered to be a measure of quality except for the case of mass construction (e.g. gravity dams), in which a minimum density of concrete is essential for the stability of the structure. The water absorption capacity of aggregates influences the properties of the fresh concrete such as its workability (Neville, 1995a).

In general, the specific gravity of RCA in saturated surface dry (SSD) condition has been found to be lower than that of NA of original concrete. On the other hand, the water absorption capacity of RCA is higher than that of NA of original concrete. These observations can be attributed to the presence of the porous and less dense residual mortar, as well as other particles that adhere to RCA (Bairagi et al 1990, Tavakoli and Soroushian, 1996a; Ramamurthy and Gumaste, 1998; Sagoe-Crentsil and Brown, 1998; Dhir et al., 1999, Rakshvir and Barai, 2006). However, it has to be noted that there is significant variation in the reported values of these properties between RCA and NA of original concrete.

Dhir et al. (1999) reported that the RCA in the SSD state had 3-15\% lower specific gravity and 3-6 times higher water absorption capacity than NA that were used in the original concrete. Limbachiya et al. (2000) reported that the RCA had 7-9\% lower specific gravity and two times higher water absorption capacity than the properties observed in NA. Sagoe-Crentsil and Brown (1998) argued that the water absorption capacity of RCA is variable and generally higher than standard values observed in NA. 
However, no attempt was made from in the previous studies to rationally correlate the amount of the residual mortar with specific gravity and absorption capacity of the RCA.

\subsubsection{Porosity}

Since aggregates can constitute approximately $70-80 \%$ of the total volume of concrete, the porosity of the aggregates has a significant contribution to the overall porosity of concrete (Neville, 1995a). It is preferable to avoid aggregates with high porosity that may affect the durability of concrete (e.g. freeze-and-thaw resistance). The porosity of common NA for structural purposes is in the range of 0 to $5 \%$.

Different researchers have reported a wide range of values for the porosity of RCA. Krezel and McManus (2000) characterized RCA to be used in concrete noise barriers for urban freeways. They reported that the porosity of RCA was found to be in the range of 12 to $50 \%$ compared to $6 \%$ of NA, and the average amount of the old mortar paste in RCA was around $16 \%$. Zaharieva et al. (2003) showed that the porosity of the coarse RCA was around $13 \%$ compared to $0.3 \%$ for siliceous crushed rock. They also reported that the weight fraction of old mortar of RCA was around $30 \%$.

Gomez-Soberon (2003) determined the pore diameter size distribution in the capillary and gel zone of RAC by using $\mathrm{N}_{2}$ adsorption test and also found that RCA was more porous than NA from the original concrete. RCA provides an extra amount of mortar to RAC, which in turn leads to an increase in concrete porosity. Hence, it can be expected that RAC will be more susceptible to durability problems than regular concrete produced with NA. Due to this fact, it is very important to conduct additional research on the characteristics and microstructure of RCA in order to understand them better and to develop guidelines for producing durable concrete. 


\subsubsection{Mechanical Properties and Soundness of RCA}

Several researchers found that the RCA content affects the mechanical properties of RAC. One of the reasons of this correlation is the effect of mechanical properties of RCA on the mechanical properties of RAC. Limbachiya et al. (2000) suggested that coarse RCA that were obtained from rejected precast members could be used in high performance concrete without significant effect on the mechanical properties of RAC. In contrast to this study, Katz (2003) suggested that the mechanical properties of RAC produced from RCA obtained from partially-hydrated laboratory concrete specimens were very similar to those of lightweight aggregate concrete; hence it was suggested that RCA are used more cautiously.

The mechanical properties of NA such as toughness and strength can be measured by impact value and crushing value (Neville, 1995a). The abrasion resistance is measured by use of the Los Angeles abrasion test (Neville, 1995a). The aggregate abrasion value is the percentage of mass loss during the test; hence a high value denotes a low resistance (Neville, 1995a).

In general, the LA abrasion and impact values of RCA were found to be higher than those of NA (Tavakoli and Soroushian, 1996a; Shayan and Xu, 2003). The crushing values of RCA were also found to be higher than those of NA (Bairagi et al 1990, Ramamurthy and Gumaste, 1998, Limachiya et al., 2000, Shayan and Xu, 2003, Rakshvir and Barai, 2006). The high LA abrasion and crushing values were generally attributed to the attached residual mortar. Shayan and $\mathrm{Xu}$ (2003) suggested that it is possible to allow for the abrasion loss in the mixture proportion because the aggregate itself does not break down. 
The aggregate soundness test is used as indictor to predict its durability performance in concrete exposed to weathering condition. Zaharieva et al (2004) carried out soundness test for RCA using the French standard NF P 18-593 and ASTM C 88-99a. The frost resistance of the RCA was assessed as satisfactory by the French standard that is based on the change of the Los Angeles coefficient after 25 direct freezing-andthawing cycles on saturated samples. However, the ASTM sulphate soundness test showed that the threshold of $10 \%$ of weight loss was exceeded even after the first three cycles. Due to these contradictory results, further research is required to investigate the soundness of the RCA using different methods.

Table 2.1 presents a summary of the results for the material characterization of RCA reported in previous studies.

\subsection{Physical and Mechanical Properties of RAC}

In this section, research conducted on the physical and mechanical properties of RAC will be presented. The physical properties of RAC include absorption capacity, density, porosity and water permeability. The mechanical properties include compressive strength, tensile strength, flexural strength and elastic modulus.

Tavakoli and Soroushian (1996a) performed an experimental study to determine the compressive, splitting tensile and flexural strength of RAC and compared the results with the corresponding values obtained from conventional concrete (NAC). The results showed that relationships established for the strength of NAC were generally unconservative when applied to RAC. Contradicting this study, Sagoe-Crentsil et al. (2001) reported that for RAC without blast furnace slag (bfs), the ratio of splitting tensile strength to compressive strength was found to be in good agreement with established 
values derived for equivalent grade concrete made with normal weight NA.

According to Tavakoli and Soroushian (1996a), the mechanical properties of RAC are influenced by the following key factors: (1) the strength of the original concrete; (2) the ratio of coarse-to-fine aggregate in the original concrete; (3) the ratio of maximum size of aggregates in the original concrete to that of RCA; (4) the abrasion loss of RCA (based on the Los Angeles test); (5) the water absorption capacity of RCA. In addition to these, more studies were performed to evaluate the influence of other factors on the mechanical and physical properties of RAC. These factors include, but not limited to, the quality and gradation of RCA, water-to-binder ratio, and the percentage of RCA in the concrete mixture (RCA content). The influences of additional binding agents such as fly ash, bfs and silica fume have also been studied. In the following sections, a review of the existing research on these factors will be presented.

\subsubsection{The RCA Content}

The majority of the existing research reported that up to $30 \%$ coarse RCA, no significant effects were observed on the compressive strength of RAC, but there was a gradual reduction in the compressive strength with the increased RCA content beyond 30\% (Dhir et al., 1999; Limbachiya et al., 2000; Chini et al., 2001; Gomez-Soberon, 2002). Furthermore, Dhir et al. (1999) showed that up to $20 \%$ fine RCA replacement, there was no significant effect on compressive strength.

Various researchers investigated the effect of high RCA content (up to 100\%) on the mechanical properties of RAC. Dhir et al. (1999) reported that the compressive strength of RAC with $100 \%$ coarse and $50 \%$ fine RCA was found to be $20-30 \%$ lower than that of the corresponding NAC, and 15-20\% lower than that of RAC made with 
$100 \%$ coarse RCA and natural sand. There was also gradual reduction in the flexural strength and the modulus of elasticity when the RCA content increased. The reduction became more significant when $100 \%$ coarse and $50 \%$ fine RCA were used. However, Sagoe-Crentsil et al. (2001) study showed that for RAC and NAC having similar volumetric mixture proportion and workability, there was no significance difference in concrete compression and tensile strength of RAC made with $100 \%$ coarse RCA from commercially recycled aggregate and NAC made from basalt aggregate and fine sand.

Rakshvir and Barai (2006) also studied the effect of the RCA content on the compressive and tensile strengths of RAC. The RAC were prepared using three types of RCA which were obtained from laboratory specimens, a demolished roof slab and a demolished building. The RCA contents of $0 \%, 25 \%, 50 \%$ and $75 \%$ by weight were investigated. All mixes were designed for characteristic strength of $20 \mathrm{MPa}$ with a w/c ratio of 0.5 . The researchers found that the compressive strength decreased with the increase of the RCA content, and that the variation was from 5 to $15 \%$ (Figure 2.1). Similar values were observed for splitting tensile strength. The reduction in the strength was explained by the increase of the voids through increased RCA content. The elastic modulus is investigated for RAC produced with $25 \%$ replacement of RCA. The elastic modulus of RAC showed a reduction of up to $19 \%$ when compared with NAC values. In this study the amount of residual mortar of RCA is not reported. Hence, the effect of the total amount of the residual mortar in RAC on the mechanical properties was not investigated.

Zaharieva et al. (2002) found that the replacement of the fine and coarse NA by RCA doubles the air permeability as well as the water permeability of the RAC compared 
to control NAC. In addition, the surface permeability of the RAC was 10 to 25 times higher than the reference concrete. The common parameters of the RAC and NAC mixes considered were: similar aggregate mix density, equal cement content and an equal workability in terms of the slump value.

Gomez-Soberon (2002) carried out an experimental study to investigate the effect of the RCA content on the physical and mechanical properties of RAC. The results of the mechanical properties of RAC were used for comparison with the results of the mercury intrusion porosimetry test in which the distribution of the theoretical pore radius, critical pore ratio, the surface area of concrete, threshold ratio and average pore radius were studied at the ages of 7, 28 and 90 days. The results showed that the absorption capacity and the porosity of RAC increased proportionally with the RCA content, while the density decreased slightly. Compression and tensile strength also decreased with the increased of RCA content. Without reference to the age of the test and the amount of the total residual mortar of RAC, the minimum value of the modulus of elasticity of RAC was observed in specimens with 60\% RCA, closely followed by $100 \%$ RCA replacement. The tensile strength of RAC with $100 \%$ and $60 \%$ RCA was lower than that of the reference NAC specimens.

Gomez-Soberon (2002) also demonstrated that the replacement of NA by RCA showed a correlation between the total volume replaced and the pore size. This correlation was more pronounced at early ages and diminished as the concrete aged. This was attributed to the crystallization of new products that reduce both the number and the size of the pores. However, the replacement of NA by RCA did not seem to influence the critical pore radius. Correlation between the properties of RAC and total porosity was 
difficult to determine. However, the researcher suggested including the distribution of the pore radius to improve this correlation. In addition to these observations, Gomez-Soberon informed to include parameters that evaluate the formation and propagation of micro cracks in the interface zones as these zones increase in the RAC due to RCA replacement of NA.

\subsubsection{The Properties of RCA}

The quality and properties of RCA, such as water absorption capacity, LA abrasion value, shape, surface texture, maximum size and moisture condition may have a direct influence on the mechanical properties of RAC.

In several studies the reduction in the compression strength of RAC produced by RCA compared with control NAC was attributed to the volume of old attached residual mortar in RCA (Tavakoli and Soroushian, 1996a; Sagoe-Crentsil and Brown, 1998). In addition, Ramamurthy and Gumaste (1998) and Mandal et al. (2003) attributed the reduction of the compressive strength and the modulus of rupture of $\mathrm{RAC}$ to the inferior properties of RCA and the presence of the weaker bond areas between RCA and the fresh mortar of RAC. Ramamurthy and Gumaste (1998) suggested that further in depth research is necessary to investigate the bond characteristic between RCA and the mortar of RAC. Salem and Burdette (1998) attributed the reduction on the modulus of elasticity of RAC compared to NAC (with the same mix proportions) to the old mortar with a relatively low modulus of elasticity.

Salem and Burdette (1998) also compared the compressive strength and the modulus of rupture of RAC and control NAC at a medium w/c ratio of 0.47 . They found that the compressive strength of RAC increased by $16 \%$ and $2 \%$ at 7 and 28 days 
respectively, compared to control NAC. However, the modulus of rupture increased by $2 \%$ and decreased by $9 \%$ at 7 and 28 days, respectively. The increase in the compressive strength was attributed to the angular shape and rough texture of RCA that may have provided better bonding and interlocking between the RCA and the cement paste, or to the reduction of the w/c ratio during mixing, caused by the absorbent nature of the RCA. The increase in the flexural strength of RAC at early ages and decrease at later ages were also attributed to the rough texture of RCA. A stronger physical bond between the rough textured RCA and the cement paste was responsible for the increase in the strength at early age. However, at later ages when chemical interaction between the aggregate and the paste begins to take effect, the effect of the surface texture may not be as important.

Sagoe-Crentsil et al. (2001) indicated that the difference between the characteristics of hardened RAC produced from commercially available RCA and similar NAC were relatively narrower than the RAC produced from laboratory-produced RCA. Enhanced hardened RAC properties produced from commercial RCA as compared to the those produced in the laboratory were attributed to the improvement of RCA grading and quality in plant crushing operations.

Ryu (2002a, 2002b) and Otsuki et al. (2003) studied the effect of RCA on the ITZ and the mechanical characteristic of RAC. The effect of RCA on the ITZ was evaluated using Vickers hardness test. Hardness measurements were made at 14 points from 10-200 $\mu \mathrm{m}$ distances from the RCA surface. The mechanical properties were evaluated by tensile and compressive strength tests. The study reported that the strength of RAC was influenced by the quality of RCA when the w/c ratio is low $(w / c=0.25)$. The researchers illustrated that for low w/c ratio, the old ITZ was weaker than the new ITZ, and the 
strength characteristics of RAC depended on the quality of the old ITZ. Therefore, when the quality of RCA decreased, the compressive strength of RAC also decreased. However, the strength of RAC was not influenced by the quality of RCA when the w/c ratio was high. The study demonstrated that for high w/c ratio, the old ITZ was stronger than the new ITZ, and the strength characteristic of the RAC depended on the quality of the new ITZ.

Barra de Oliveira and Vazquez (1996) investigated the influence of the moisture condition of RCA on the physical and mechanical properties of RAC. These researchers studied the effects of three different moisture conditions (dry, saturated and semisaturated) on the strength of the concrete. The results showed that the RAC had similar compression strength irrespective of the degree of moisture of RCA. However, the flexural strength of the concrete made with the saturated aggregates showed a clear decrease when compared to control NAC.

Poon et al. (2004) also investigated the influence of moisture states of NA and RCA on the compressive strength of NAC and RAC, respectively. The moisture states of aggregates were controlled at air-dry (AD), oven-dry (OD) and SSD states prior to use. The ratio of cement to free water was kept constant for all mixes. The effect of the moisture states of the aggregates on the compressive strength of the concretes prepared with OD and an SSD state aggregate at early stages (i.e., 3 and 7 days) was noticeable. The concrete prepared with the $\mathrm{AD}$ aggregates achieved the highest average strength values at 3, 7 and 28 days. However, at 28 days, the concrete compressive strengths were similar. The results suggested that $\mathrm{AD}$ aggregates that contain no more than $50 \% \mathrm{RCA}$ are optimum for producing ordinary compressive strength of RAC. 


\subsubsection{Water-Cement (w/c) Ratio of RAC}

It is commonly understood that an increase in w/c ratio decreases the compressive strength of RAC (Ryu, 2002a; Salem et al., 2003; Otsuki et al., 2003). Depending on w/c ratio and dry mixing period, Tavakoli and Soroushian (1996a) reported that the splitting tensile and flexural strengths of RAC could be higher or lower than those of NAC. Ryu (2002a) and Otsuki et al. (2003) studied the effect of w/c ratio on the ITZ, and they demonstrated that for low $w / c$ ratio $(w / c=0.25)$, the ITZ of the original concrete was weaker than ITZ of RAC. In these cases, the strength characteristics of RAC depended on the quality of the ITZ of the original concrete, and the strength of RAC was less than that of the control NAC. For high w/c ratio (w/c $\geq 0.55$ ), the ITZ of the original concrete was stronger than the ITZ of RAC, and the strength characteristic of the RAC depended on the quality of the ITZ of RAC and was equal to that of control NAC. Ryu (2002b) and Otsuki et al. (2003) suggested that, for higher w/c ratios, double mixing method could be used to improve the strength of RAC. The double mixing method involves dividing the necessary water of concrete mixture into two parts and adding it at two different times during the mixing process. When double-mixing method was used, the quality of ITZ of RAC was improved by reducing w/c ratio in the aggregate vicinity. With this approach, the compressive and tensile strengths increased by $17 \%$ and $26 \%$, respectively.

\subsubsection{Effect of Other Binders}

Ryu (2002b) used $30 \%$ fly ash by weight of cement to improve the ITZ of RAC at higher $w / c$ ratios $(w / c=0.55)$ and observed that the compressive and tensile strengths of RAC increased by $8 \%$ and $17 \%$ respectively, compared with the control specimens of RAC without fly ash. Contradicting with Ryu's results, Salem and Burdette (1998) found 
that the increase of the fly ash content from $14 \%$ to $28 \%$ of $\mathrm{RAC}$ at w/c ratio of 0.47 , the compressive strength and modulus of rupture of RAC decreased by $9 \%$ and $14 \%$ respectively. However, Mandal et al. (2003) reported that the compressive strength and the overall quality of RAC improved when $10 \%$ fly ash was used. Because of these contradictory findings, more research is needed to evaluate the effect of fly ash on the mechanical properties of RAC.

Sagoe-Crentsil et al. (2001) studied the effect of using bfs on the compression and tensile strength of RAC. RAC was produced with $100 \%$ coarse commercially produced RCA and natural sand. Two RAC mixtures were prepared: one with $35 \%$ bfs and $65 \%$ OPC, and the other with OPC and without bfs. The results showed that the increase in compressive strength of RAC with bfs from $20.2 \mathrm{MPa}$ to $32.6 \mathrm{MPa}$ between 7 and 28 days was significantly higher than the equivalent nominal $6 \mathrm{MPa}$ average increase observed in the RAC without bfs. On the other hand, after one year, the tensile strength of the RAC with bfs was approximately $25 \%$ larger than that of the RAC without bfs, compared to an equivalent $15 \%$ difference at 28 days.

Shayan and $\mathrm{Xu}$ (2003) attempted to improve the strength of $50 \mathrm{MPa}$ RAC by using a different combinations of sodium silicate, lime, and silica fume to treat the surface of the RCA used to produce the RAC. RCA had been examined in the untreated form as well as after surface treatment with sodium silicate, sodium silicate and lime, sodium silicate and silica fume, a combination of sodium silicate, lime, and silica fume and finally with silica fume slurry alone. The results showed that even though the treatments improved the surface characteristics, no particular advantage in terms of strength was achieved with sodium silicate solution. However, silica fume was found important in 
achieving high strength. This conclusion obtained from the observation that the RCA treated with slurry of silica fume alone or with sodium silicate and silica fume developed higher strength values than untreated RCA in the RAC mixture containing the same amount of silica fume in the binder.

\subsubsection{Effect of the Original Concrete Properties}

Various researchers investigated the effect of the properties of the original concrete from which RCA was produced on the mechanical properties of the RAC. Tavakoli and Soroushian (1996a) reported that the basic trends with respect to the strength of the RAC produced with field-demolished RCA were not significantly different from those of RAC produced with laboratory-made RCA. Sagoe-Crentsil et al. (2001) carried out performance tests on fresh and hardened properties of concrete made with commercially produced coarse RCA and natural fine sand. Contrary to the findings of Tavakoli and Soroushian, RAC produced with commercially produced RCA showed better mechanical properties. It was suggested that better mechanical properties of fresh and hardened RAC made with commercially produced RCA arise from improved aggregate grading and quality in plant crushing operations.

Ramamurthy and Gumaste (1998) attributed the variation of the compressive strength of RAC, compared to control NAC specimens, on the strength of the original (demolished) concrete. Similarly Ajdukiewicz and Kliszczewicz (2002) and Salem et al. (2003) found that the properties of original concrete had significant influence on the mechanical properties of RAC. Tavakoli and Soroushian (1996a) concluded that the RAC could be made to have higher compressive strength than the NAC, if the compressive strength of the original concrete that is being recycled is higher than that of the control 
NAC made with NA in the laboratory. In other words, the researchers have found that the compressive strength of RAC could be lower than that of corresponding control concrete made with NA at the same w/c ratio when the compressive strength of such control concrete exceeds the strength of the original concrete from which the RAC is originated. In contrast to these studies, Dhir et al. (1999) reported that there was no significant difference in the strength of RAC produced from six different RCA sources. Contradicting research results demonstrate that further research on the topic is necessary.

Katz (2003) studied the properties of RAC made with RCA from partially hydrated original concrete. The physical properties of the original concrete and the presence of the unhydrated cement in RCA seemed to affect the mechanical properties of RAC. These effects were important when the new cement matrix was significantly stronger than the one in the original concrete. In such cases, the combination of strength and cementing capacity of RCA crushed at 3 days provided better strength over crushing ages of 1 or 28 days. In weaker new cement matrix, this effect was reversed and the new concrete made from RCA crushed at 3 days was slightly weaker than concrete made from RCA crushed at 1 or 28 days.

The main source to produce RCA is by crushing the original concrete. Therefore, the effect of the original concrete properties on the physical and mechanical characteristic of the RAC made from RCA must be clear. The contradicting studies on the effect of the original concrete on the properties of the RAC lead us to conduct further research in this subject. 


\subsection{Properties of Fresh RAC}

The main goal with respect to the properties of fresh concrete is that the consistency of the mix must be such that the concrete can be transported, placed, compacted and finished relatively easily and without segregation (Neville, 1995a). In this section, existing research on the main properties of fresh concrete such as workability, stability and air content will be presented.

\subsubsection{Workability}

Due to attached residual mortar, RCA has relatively high water absorption capacity compared to NA. Because of the high water absorption capacity, the research on the influence of the moisture content of RCA on the properties of fresh RAC has received some attention. A number of researchers studied the effect of the RCA content on the workability of RAC. They found that the workability reduced with the increase of the RCA content, which was attributed to the higher water absorption of RCA (Topcu, 1997, Rakshvir and Barai, 2006). However, pre-soaked and saturated RCA were used in several studies (Ramamurthy and Gumaste, 1998; Sagoe-Crentsil, et al., 2001; Zaharieva et al., 2002; Poon et al., 2004). Dhir et al. (1999) examined the effects of RCA conditions used in practice: wet and dry RCA pretreatments were investigated for their effect on the properties of fresh RAC. Pre-soaking showed a slight improvement in the fresh properties of RAC containing $100 \%$ coarse and $50 \%$ fine RCA, when compared with the corresponding mixes with dry RCA where supplementary water was added to allow for absorption. They suggested that the influence of the RCA preconditioning on the properties of the fresh RAC is relatively minor.

Dhir et al. (1999) investigated the effect of RCA content on the slump of RAC and 
compared it with the NAC with the same mix proportions. In general, results showed reduction in slump with increasing RCA content in the mix, but this remained within the specific tolerances of $\pm 25 \mathrm{~mm}$. In order to assess the effect of RCA on the retention of workability, compacting factor measurements were made at 30 minutes intervals up to 150 minutes after mixing. The results showed that the loss of workability was uniform and was found to be little less for concrete produced with $100 \%$ coarse RCA, or $100 \%$ coarse and 50\% fine RCA than that of NA mixes.

The effect of water-reducing admixtures on the workability of RAC was also investigated by various researchers. Rohi et al. (2003) reported that to achieve the same level of slump from RAC as NAC with the same prescribed physical and mechanical properties, a high-range water-reducing admixture, such as Type F per ASTM C494-99, could be added to the RAC mixture. However, for a high w/c ratio of 0.7 , Sagoe-Crentsil et al. (2001) reported no difficulty in achieving the desired consistency and subsequent compaction of RAC without using any admixtures.

Poon et al. (2004) also investigated the influence of moisture content of NA and RCA on the properties of fresh RAC using slump tests. The moisture contents of aggregates were brought to equilibrium at $\mathrm{AD}, \mathrm{OD}$ and $\mathrm{SSD}$ states prior to use. The tests results showed that the initial slump values of the concrete mixtures depended on the initial free water contents of the concrete mixes; and the slump loss of the mixture was related to the moisture states of the aggregates. Slump loss was significant when $100 \%$ AD or OD RCA were used.

In addition to the high water absorption capacity of RCA, Zaharieva et al. (2002) reported that coarse surface texture and the angularity of RCA have significant effects on 
the workability of the RAC. Sagoe-Crentsil and Brown (1998) reported that RAC was easy to batch, mix, transport, place and finish as conventional concrete. It was observed that the requirements for good finishability for RAC depended on the same factors as those of conventional concrete such as aggregate angularity, grading, the extent of bleeding and the mix design.

\subsubsection{Stability}

The stability of concrete mix refers to the cohesion of the mix; i.e., its resistance to segregation. The lack of cohesiveness of the concrete mix affects the integrity and homogeneity of the fresh concrete during casting, which would affect the mechanical and durability properties of the hardened concrete.

Dhir et al. (1999) investigated the stability of RAC mixes by visual observation and bleeding test in accordance with ASTM C232-92. The mixes with high proportions of RCA (more than $50 \%$ coarse or $20 \%$ fine) were found to be less cohesive and exhibited more bleeding than the corresponding NA concrete mixes. They used a filler material such as coarse pulverized-fuel ash to overcome the stability problem. It was also reported in this research and in Poon et al. (2004) that more than 50\% of fine RCA cause problems in the stability of fresh mix of RAC.

\subsubsection{Air Content}

Air-entraining admixtures are used in concrete mixes if freeze-and-thaw durability is required. Dhir et al. (1999) showed that the use of coarse RCA had no effect on the amount of air-entraining admixture to be used. However, to achieve $5.5 \%$ nominal air content in RAC containing high proportions of fine RCA, the amount of required airentraining agent was slightly less than the corresponding control conventional concrete 
with the same mix design. The testing on the maintenance of air content in fresh RAC with time showed negligible differences in air loss between control conventional concrete and RAC.

Rohi and Edwin (2003) and Katz (2003) reported that the natural (entrapped) air content of fresh RAC concrete was slightly higher than corresponding values in conventional concrete with the same mix design. Rohi and Edwin (2003) attributed the higher air content of the RAC to the higher void content of the RCA. However, Katz (2003) reported that the air in the RCA voids was taken into account through the bulk specific gravity. Therefore, the cause of the increased air content in the fresh RAC was not clear. The effect of the amount of residual mortar on the air content was not investigated; hence further study is needed in order to better understand this phenomenon.

In order to proportion the constituents of concrete, mix design procedures are needed. The following section will explain the studies carried out on the mix design procedures of RAC.

\subsection{Mix Design Procedures}

Currently, to the authors' knowledge, there is no standardized procedure in North America for designing concrete mixes with RCA. However, a number of studies investigated the suitability of applying the mix design procedures for conventional concrete to RAC (Sagoe-Crentsil and Brown, 1998; Dhir et al., 1999; Limbachiya et al., 2000; ACI committee 555R-01, 2001; Olorunsogo and Padayachee, 2002; Ajdukiewicz and Kliszczewicz, 2002; Rakshvir and Barai, 2006). Most research focused on the development of trial mixes of RAC, in which physical and mechanical properties were 
tested for optimum performance. The trial mixing was mostly implemented by slightly changing the coarse and fine aggregate contents to maintain the fresh properties. The reduction in the strength of $\mathrm{RAC}$ was compensated by adjusting w/c ratio or by increasing the cement content (Bairagi et al., 1990, Dhir et al., 1999; Limbachiya et al., 2000; ACI committee 555R-01, 2001; Ajdukiewicz and Kliszczewicz , 2002). ACI committee 555R (2001) suggested that, to determine a target mean strength on the basis of a required strength, a higher standard deviation of $4.83 \mathrm{MPa}$ for compressive strength should be used when designing concrete with RCA of variable quality. This standard deviation is higher than the cases where RCA of uniform quality or NA are used in the mix.

Bairagi et al. (1990) investigated four mix design procedures to produce RAC: Indian standard code method, ACI method, RRL method and surface and angularity index method. Of the four methods of the mix design, ACI method was found to be more appropriate for the design of RAC. It should be noted that in Bairagi et al. the RCA used to produce the RAC was obtained from crushing three-month-old $15 \mathrm{MPa}$ concrete produced in the laboratory. Modified aggregate over cement ratio $(\mathrm{A} / \mathrm{C})$ for various grades of RAC was suggested (Figure 2.2a). As a consequence of the modified A/C ratio, additional $8 \%$ to $13 \%$ cement was suggested for RAC to achieve designed target strength; the higher value meant for higher grade of concrete (Figure 2.2b).

The total volume of mortar in concrete produced with RCA has two components: (1) residual mortar; (2) new mortar. When conventional mix design procedures such as the general mix design method are used, the RCA is considered as coarse aggregate, and no consideration is given to the volume of attached residual mortar. Because of this, 
concrete produced with RCA has larger volumes of total mortar (residual and new), which can lead to problems in serviceability (e.g. low modulus of elasticity, high creep and shrinkage) and in durability (low freeze-and-thaw resistance, high permeability, etc.)

An alternative mix design procedure to eliminate this issue was developed by Fathifazl et al (2007b). This new technique was developed based on the observations of the preliminary study presented in Chapters 4-6, and considers the residual mortar as part of the total concrete mortar, not as part of the RCA. The method involves the determination of the appropriate amount of RCA to be used in the mix in relation to the amount of coarse NA that would be used in a conventional concrete mix with the same specified properties. This approach guarantees that the volume of total mortar (residual and new) in concrete produced with RCA be equivalent to that in the conventional concrete. From a different way of thinking, the total volume of coarse NA (OVA plus $\mathrm{NA}$ ) in GC is equal to the volume of NA in conventional concrete with the same specified properties. The details of this method presented in Fathifazl et al. (2007) will be explained further in Chapter 7.

The suitability of the mix design is judged by producing concrete that satisfies the required mechanical and durability properties. The following section will discuss the literature on the durability of the RAC.

\subsection{The Durability of RAC}

Durability is the capacity of concrete to resist weathering action, chemical attack, abrasion, and other deteriorative processes under service. The deterioration of reinforced concrete structures, primarily due to the corrosion of steel reinforcement, has become a major concern of infrastructure owners and operators. Within the high alkaline 
environment provided by concrete, reinforcing steel is covered with an insoluble film of ferric oxides (passive layer), which generally provides adequate protection against corrosion. The partial or complete loss of the passive layer, known as depassivation, may result in the loss of this protection and lead to the corrosion of steel (Bertolini et al., 2004). The key factors affecting the depassivation of steel in concrete are the presence of chloride ions and carbonation of concrete (i.e., the reduction of alkalinity). Furthermore, freeze-and-thaw action can damage and crack the concrete structure, which in turn, may lead to further ingress of deteriorative agents such as chlorides. This section will discuss the research carried out on the factors affecting the durability of concrete, specifically freeze-and-thaw action, chloride penetration, and carbonation from the perspective of RAC.

\subsubsection{Freeze-and-Thaw Action}

The repeated cycles of freezing and thawing of the water present in concrete can cause damage in concrete. When the pore water in the capillary pore freezes it occupies a larger volume than the original water volume. This compressed water pressurizes the remaining liquid, which can damage the cement paste and the porous aggregate. The damage can be aggravated by the use of de-icing salts due to increase of the osmotic pressure. The salt are absorbed by the upper layers of the concrete producing an osmotic pressure gradient in the direction of the surface concrete. Some of the symptoms of the damage in concrete structures due to freeze-and-thaw action are pop-outs, cracking scaling, and D-cracking (Mailvaganam N.P, 1992). More information on the mechanism of freeze-and-thaw damage in concrete can be obtained from Mehta and Monterio, 2006.

The main factors affecting the freeze-and-thaw resistance for conventional concrete 
are air entrainment, w/c ratio, the degree of saturation, and tensile strength (Mehta and Monterio, 2006). Frost resistance is greatly influenced by the degree of saturation of the pore. The critical value of saturation occurs generally when $80-90 \%$ of the total pores volume is water filled (Bertolini et al., 2004). Below the critical value concrete is capable of withstanding a high number of freeze-and-thaw cycles; while above it only a few cycles might be sufficient to damage it.

Air entrainment in concrete provides empty space for excess water move and freeze without damage. The efficiency of the air entrainment depends on the spacing factor, which can be defined as the average distance water must travel to reach free space. Spacing factor of $100 \mu \mathrm{m}$ to $200 \mu \mathrm{m}$ is necessary for the protection of concrete against freeze-and-thaw action (Mehta and Monterio, 2006). The volume of entrained air in concrete can vary between 4 to $7 \%$ of the volume of concrete; however, for every mixture, there is a minimum content of entrained air below which the presence of air bubbles is not effective (Neville, 1995a).

Porous aggregates are susceptible to freeze-and-thaw damage due to build up of hydraulic pressure as water freezes in the pores. The damage depends on the distance (i.e., aggregate size) the water needs to travel to find an escape boundary. Therefore the pressure build-up depends on the size of the aggregate: larger aggregates are more susceptible to damage than smaller ones. The aggregates can be classified according to their permeability of water as low, medium, and high permeability aggregates. The low permeability (or high strength) aggregates are generally strong enough to support hydraulic pressure. In the medium permeability aggregates, the aggregate size is the most critical factor affecting the freeze-and-thaw resistance. In the high permeability 
aggregates, the potential of damage is higher around the ITZ (Mehta and Monterio, 2006).

The high porosity and permeability of the residual mortar attached to RCA will play a role by increasing the porosity of RAC. The increased porosity of RAC can allow more water fill in the pores of the concrete; hence more damage can be expected by freeze-and-thaw action. In addition, the general practice of pre-immersion of RCA until saturation before using in RAC can increase the amount of freezable water in RAC. It should also be noted that RCA has higher absorption capacity than NA; therefore RCA particles can absorb more water than its surroundings. This may cause additional pressure development on the surrounding cement paste and on the ITZ.

The research on the freeze-and thaw susceptibility of RAC is quite limited. The effect of the of w/c ratio, fly ash content, RCA content and air entrainment on the freezeand-thaw resistance of RAC were studied by a number of researchers. Salem and Burdette (1998) and Salem et al. (2003) investigated the effect of the air entrainment on the freeze-and-thaw resistance of RAC. They found that the use of 5\% air entrainment in the RAC mixtures was the most efficient method for improving the freeze-and-thaw resistance.

Dhir et al. (1999) investigated the effect of RCA content in a mixture of RAC contained $5.5 \%$ air entrainment. The coarse RCA content was varied in the mixtures from 0 to $100 \%$, and no fine RCA was used. The results indicate that across the range of RCA content, both NAC and RAC exhibited similar freeze and thaw resistance in water. In addition, the inclusion of RCA had no significant influence on the dosage of admixture required. 
Salem and Burdette (1998) investigated the freeze-and-thaw resistance of a RAC and NAC with a w/c ratio of 0.47 (the base mix), and a fly ash content ranging from 14 to $28 \%$ by the weight of cement. $100 \%$ of the coarse aggregates were replaced with RCA. It was observed that the use of RCA with w/c ratio of 0.47 reduced the freeze-and-thaw resistance of RAC. This was attributed to the existence of a significant amount of freezable water in the capillary pores in the cement paste, which upon freezing produced expansive internal pressure that damaged the concrete. The use of higher fly ash content in both the RAC and NAC resulted in significant improvements in their freeze-and-thaw durability.

Salem et al. (2003) investigated the effect of using high performance RAC, produced with reduced w/c of 0.29 , on the resistance to freeze-and-thaw effect. The RAC resistance to freeze-and-thaw action was carried according to ASTM C 666 - Procedure A, rapid freezing and thawing in water. Lowering the w/c ratio was reported to be highly beneficial to the development of the resistance to freezing-and-thawing of NAC. However, at a w/c ratio of 0.29 , while the resistance to freeze-and-thaw action of the NAC specimens was extremely high, the RAC specimens failed to provide acceptable resistance. Contrary to this observation, Ajdukiewicz and Kliszczewicz (2002) found that high strength or performance RAC had similar or better freeze-and-thaw durability than the NAC with similar properties. This result was supported by standard tests with 50 - to 24-hour cycles of air freezing and water thawing.

In addition to these studies, the quality of the original concrete used to produce RCA and the quality of RCA itself were also investigated. Gokce et al. (2004) investigated the freeze-and-thaw resistance of air entrained RAC for which air-entrained 
and non-air entrained original concrete were used to produce the RCA. The laboratory produced air-entrained and non-air-entrained concretes with $w / c$ ratio of 0.45 were recycled at the crushing age of one year to obtain the coarse aggregates used in the investigations. The RCA were produced by using jaw crusher, impact crusher and mechanical grinding equipment. The results showed that the RCA produced from non-air entrained concrete caused poor freeze-and-thaw resistance in RAC even when proper air entrainment was used in the production of RAC. Micro-structural studies indicated that non-air-entrained adhered mortar caused disintegration of the RCA and disrupted the surrounding new mortar after a limited number of freeze-and-thaw cycles. Minimizing non-air-entrained adhered mortar or enhancing the performance of new surrounding matrix could not give satisfactory results for long freeze-and-thaw exposures.

Zaharieva et al (2004) studied the effect of the degree of water saturation in the frost resistance of RAC. RCA from industrial demolished waste was used to produce RAC with w/c ratio of 0.34 . Complete and cyclic water saturation procedures were used; the cyclic water saturation simulates the effect of drying and wetting cycles. They concluded that the degree of saturation has effect on the frost resistance of the RAC: RAC with complete saturation had lower relative dynamic modulus than RAC with cyclic water saturation (Figure 2.3a). However, the RAC with complete water saturation presents higher frost resistance with durability factor of $82 \%$ (Figure $2.3 \mathrm{~b}$ ), and it was concluded that it could be used even in severe climatic conditions.

Despite these studies, further research is needed in order to better understand the freeze-and-thaw of RAC produced by using of RCA. Furthermore, relating the freezeand-thaw resistance to the RCA content (e.g. 100\% or 50\% RCA replacement) may not 
be appropriate to define the vulnerability of RAC. Different types of RCA may have different amounts of residual mortar; therefore it is important to relate the freeze-andthaw resistance to the total residual mortar.

\subsubsection{Chloride Penetration}

Chlorides in concrete can be found in three forms: (1) free in the pore solution, (2) physically adsorbed by the pore walls, and (3) chemically bound by the hydrated cement products (Martin-Perez, 1999). The physically bound chlorides are adsorbed to the pore walls of concrete by calcium silicate hydrate (C-S-H) (Bertolini et al., 2004). The main form of the chemical bound chloride in concrete is by reaction with tri-calcium aluminate $\left(\mathrm{C}_{3} \mathrm{~A}\right)$ in portland cement to form calcium chloro-alumiante hydrate (3CaO. $\mathrm{Al}_{2} \mathrm{O}_{3} \cdot \mathrm{CaCl}_{2} \cdot 10 \mathrm{H}_{2} \mathrm{O}$ ), normally known as Friedel's salt (Neville, 1995a). The free chlorides are dissolved in the pore solution and penetrate through the concrete cover; free chlorides are considered to be the main contributor to the corrosion risk of steel reinforcement.

Chloride ions may be introduced into the concrete through mix ingredients such as aggregates, mixing water and accelerating admixture, at the time of concrete production or from external sources such as de-icing salts and sea water. Recent concrete production standards limit the amount of chlorides that can be introduced during mixing, and in conventional concrete production, it is possible to avoid chlorides by choosing clean constituents and non-chloride-based admixtures. However, since RCA are produced from old concrete structures, they have the potential of being pre-contaminated with chloride ions.

Chloride penetrates the concrete from the external sources by a combination of 
several transport mechanism such as ionic diffusion, capillary sorption, permeation, dispersion and wick action. The details of these processes will not be discussed here; however a comprehensive review can be found in (Martin-Perez, 1999). Diffusion-driven flow (as in the case of saturated concrete) of chloride ions in one-dimension $(x)$ is governed by Fick's second law:

$$
\frac{\partial C_{t}}{\partial t}=D_{a} \frac{\partial^{2} C_{t}}{\partial x^{2}}
$$

where $C_{t}$ ( $\%$ by mass of concrete) is the total concentration of chloride ions, $D_{a}$ is the apparent diffusion coefficient $\left(\mathrm{m}^{2} / \mathrm{s}\right)$ and $t$ is the time (s). The closed-form solution of Eq.2.1 is possible and can be given as:

$$
C_{t}(x, t)=C_{s}-\left(C_{s}-C_{0}\right) \operatorname{erf}\left(\frac{x}{\sqrt{4 D_{a} t}}\right)
$$

where $C_{s}$ (\% by mass of concrete) is the chloride concentration on the exposed surface, $C_{0}(\%$ by mass of concrete)is the initial chloride concentration and erf is the error function. Since Eq.2.1 is formulated in terms of total chloride concentration, it is important to calculate the amount of binding during the diffusion process. The total chloride concentration, $C_{t}(\%$ by mass of concrete $)$, the bound chloride concentration, $C_{b}$ ( $\%$ by mass of concrete), and the free chloride concentration, $C_{f}(\%$ by mass of concrete) in concrete are related by:

$$
C_{t}=C_{b}+w_{e} C_{f}
$$

where $w_{e}$ is the evaporable water content of concrete. In order to model the chloride binding mechanism in conventional concrete, binding isotherms have been suggested by a number of researchers. Chloride binding isotherms provide the relationship between the 
free and bound chlorides in concrete at a given temperature. Among these isotherms, one can list: (1) linear binding isotherm (Nilsson et al., 1994); (2) Langmuir isotherm (Sergi et al., 1992); and (3) Freundlich isotherm (Tang and Nilsson, 1993). More information on these isotherms can be obtained from the associated references. Here, as an example, the linear isotherm is presented in Eq. 2.4:

$$
C_{b}=\alpha C_{f}
$$

where $C_{b}$ is the concentration of bound chlorides, $\alpha$ is the binding capacity which depends on the concrete type. It should be noted that, to the author's knowledge, chloride binding in RAC is yet to be studied in detail.

Nilsson et al. (1994) suggested that the effect of chloride binding on Eq. 2.1 can be included by using an effective diffusion coefficient, which can be represented as:

$$
D_{e}=\frac{D_{a}}{1+\frac{1}{w_{e}} \frac{\partial C_{b}}{\partial C_{f}}}
$$

where $D_{e}$ is the effective diffusion coefficient $\left(\mathrm{m}^{2} / \mathrm{s}\right), \alpha=\partial C_{b} / \partial C_{f}$ is the binding capacity, which can be calculated from the binding isotherm. Because of the possible precontamination of RCA with chlorides, the binding capacity of RAC can be lower than uncontaminated concrete, increasing the apparent diffusion coefficient. However, this hypothesis is yet to be examined in detail.

A number of researchers have proposed different models for effective chloride diffusion coefficient, $D_{e}$. In general, the diffusivity of conventional concrete depends on a number of factors including temperature, the relative humidity of concrete, w/c ratio (or properties associated with the w/c ratio such as porosity, compressive strength, etc.) In addition, previous research has demonstrated that concrete produced with OPC and 
SCM's (e.g. fly ash and bfs) has lower chloride diffusivity compared to concrete produced with OPC alone (Neville, 1995b). The conventional concrete classified by Concrete Society (1988), as high permeability concrete when the chloride diffusion coefficient is greater than $5 \times 10^{-12} \mathrm{~m}^{2} / \mathrm{s}$; as moderate when the chloride diffusion coefficient ranges from $1 \times 10^{-12}$ to $5 \times 10^{-12} \mathrm{~m}^{2} / \mathrm{s}$; and as low when the chloride diffusion coefficient is lower than $1 \times 10^{-12} \mathrm{~m}^{2} / \mathrm{s}$.

A number of researchers studied the effect of the quality and quantity of RCA on the chloride penetration of RAC. Limbachiya et al. (2000) reported that the use of up to $100 \%$ coarse RCA had no negative influence on the chloride diffusivity of RAC. Similarly, Shayan and Xu (2003) reported that small difference exists between chloride penetration into NAC and into RAC with $100 \%$ coarse RCA. In contrast to the observations of Limbachiya et al. (2000) and Shayan and Xu (2003), Ryu (2002b) and Otsuki et al. (2003) showed that, for the same w/c ratio, the chloride iron penetration depth of RAC produced with $100 \%$ coarse RCA was greater than that of control NAC with the same mixture proportions. Also, Olorunsogo and Padayachee (2002) showed that chloride permeability increased with an increase in RCA content for a given curing duration. However, at a fixed RCA content, it was found that the longer the duration of curing, the lower the observed chloride permeability of RAC. Because of the contradictions among these findings, more research is needed to better understand the chloride permeability of RAC. Furthermore, in the previous studies the amount of residual mortar of RCA by weight or volume was not determined. This property is a major factor affecting the amount and quality of the total RAC mortar, which in turn, will have an effect on the chloride penetration of RAC. 
There are methods proposed to improve the chloride ion penetration of RAC. Ryu (2002b) and Otsuki et al. (2003) suggested the double mixing method to improve the chloride penetration of RAC, especially at higher w/c ratios. As discussed previously, the double mixing method involves dividing the necessary water of concrete mixture into two parts and adding it at two different times during the mixing process. In addition to this method, Ryu (2002b) used $30 \%$ fly ash as a method to decrease the chloride permeability of RAC with successful results. Shayan and Xu (2003) attempted to improve the surface properties of RCA by chemical treatment and investigated its effect on the chloride penetration on high strength RAC. RCA was chemically treated by sodium silicate solution, lime, silica fume, which were used at different proportions for several RAC mixtures. The rapid chloride permeability test results showed that mixture with silica fume had high resistance to chloride penetration. Otherwise, the chemical treatment of the RCA material did not provide any advantage in terms of reducing chloride penetration.

In the rapid chloride test, the total charged passed from a concrete specimen in coulombs is measured and is related to the resistance of the specimen to chloride ion penetration. It is important to mention that, the rapid chloride permeability test method is applicable to types of concrete where correlation have been established between this test procedure and reliable tests that are based on long-term chloride ponding and the determination of the amount of chloride ions in concrete using titration, such as the one described in AASHTO T 259, ASTM C 1202-97 (ASTM, 2003) and ASTM 1556-04. However, there is no established correlation between the rapid chloride permeability test method and the results obtained from long-term chloride ponding for RAC. Therefore a 
comparative study needs to be carried out if one is to use and interpret the results of rapid chloride testing of RAC properly. To the author's knowledge, no study has been conducted to measure the chloride diffusion coefficient of RAC using methods that are based on ponding and titration. However, Dhir et al. (2004) demonstrated that an acidsoluble test gave the most accurate indication of the chloride content in RCA. This test procedure has been adopted in the British Standard, BS EN 8500-02.

\subsubsection{Carbonation}

Carbonation is a chemical reaction between carbon dioxide from air and the hydration products of cement in concrete that causes a reduction in the alkalinity $(\mathrm{pH})$ of concrete, and consequently, its ability to protect the steel reinforcement from corrosion. Therefore, it is important to know the carbonation susceptibility of RAC. Carbonation can simply be defined as the reaction of carbon dioxide $\left(\mathrm{CO}_{2}\right)$ with calcium hydroxide, $\mathrm{Ca}(\mathrm{OH})_{2}$, in the hardened cement paste, resulting in the production of calcium carbonate $\left(\mathrm{CaCO}_{3}\right)$. Carbonation consumes $\mathrm{Ca}(\mathrm{OH})_{2}$, a product which endows concrete with high $\mathrm{pH}$; hence reducing the $\mathrm{pH}$ of the pore solution as it progresses (Neville, 1995a).

As stated by Neville (1995a), the carbonation in conventional concrete is affected mainly by the following factors: (1) $\mathrm{CO}_{2}$ diffusivity; (2) reserve alkalinity; (3) $\mathrm{CO}_{2}$ concentration; (4) temperature; and (5) relative humidity of concrete. The reserve alkalinity is a function of the cementitious system used in concrete. Concrete produced with OPC has high reserve alkalinity compared to concrete with OPC and SCM's such as fly ash or bfs. Consequently, concrete produced with OPC has lower carbonation depth compared to concrete with SCM's under the same exposure conditions. 
The carbonation reactions occur ideally when the relative humidity of the concrete is between $50 \%$ and $70 \%$ (Neville, 1995a). If the humidity is less than $50 \%, \mathrm{CO}_{2}$ ions cannot be dissolved completely due to inadequate water in the pores, while if is larger than $70 \%$, the water inside the pores inhibits the $\mathrm{CO}_{2}$ diffusion, resulting in very slow rates of carbonation.

The $\mathrm{CO}_{2}$ concentration in the atmosphere may vary from $0.03 \%$ in rural environments to more than $0.1 \%$ in urban environments. Accelerated tests carried out in the laboratory to compare the resistance to carbonation in different types of concrete show that, one week of exposure to an atmosphere containing $4 \%$ will cause the same amount of carbonation as a year of exposure to normal atmospheric conditions (Ho and Lewis, 1987).

The carbonation depth is often modeled by the following parabolic formula (Neville, 1995a):

$$
d=D_{c} \sqrt{t^{\prime}}
$$

where $d$ is the depth of carbonation front $(\mathrm{mm}), t^{\prime}$ is the time (day) and $D_{c}$ is the coefficient for the progression of carbonation $\mathrm{mm} /$ day $^{0.5}$. The value $D_{c}$ is depends on the factors presented above and thus changes as a function of concrete properties and environmental conditions. The value $D_{c}$ for real structures exposed to atmospheric conditions, but protected from rain, vary from 2 to $15 \mathrm{~mm} /$ year $^{0.5}$ (i.e., 0.1 to 0.79 $\mathrm{mm} /$ day $^{0.5}$ ) (Bertolini et al., 2004).

In RAC, there are also other factors that need to be considered regarding the carbonation of concrete. The reserve alkalinity from the hydrated residual mortar attached 
to RCA has the potential to reduce the carbonation of the RAC. However, there is also the possibility of RCA containing SCM's (such as fly ash), which potentially increase the rate of carbonation in RAC. Furthermore, the residual mortar can be pre-carbonated. In addition, the high porosity of RCA compared to NA may increase the $\mathrm{CO}_{2}$ diffusivity of the RAC, which in turn may result in increase of the carbonation depth.

The effects of RCA content and its quality as well as the $w / c$ ratio on the carbonation of RAC were investigated by a number of researchers. Dhir et al. (1999) and Levy and Helene (2004) studied the effect of the RCA content on the carbonation depths of RAC specimens exposed to $\mathrm{CO}_{2}$. Results by Dhir et al. showed that up to $50 \%$ coarse RCA had negligible effect on the carbonation rates of RAC, and thereafter a slight increase in carbonation depth was observed. However, carbonation resistance improved with an increase in $\mathrm{RCA}$ content by reducing the w/c ratio. This improvement was attributed to the increase in calcium hydroxide from the hydrated cement paste attached to the RCA, and to the increase in the alkalinity from the reduction of the w/c ratio. Levy and Helene (2004) demonstrated that the carbonation depth decreases when the amount of RCA increases presenting a better behavior when this replacement was 20 or $50 \%$ (Figure 2.4). As shown in Figure 2.4, when using 100\% RCA replacement, the carbonation depth was still lower than reference concrete with NA. This was attributed by increasing the cement content of RAC to achieve the same compressive strength as NAC. Sagoe-Crentsil et al. (2001) showed that the carbonation rate of RAC made from commercially produced coarse RCA and natural fine sand was $10 \%$ higher than that of control NAC. In contrast, they reported that there was a $65 \%$ increase in carbonation rate relative to the control NAC specimens from Hansen study. 
Ryu (2002a) and Otsuki et al. (2003) examined the effect of the quality of RCA and w/c ratio of RAC on the carbonation rate. They observed that the carbonation rate of $\mathrm{RAC}$ was affected by the w/c ratio and the quality of the RCA. They found that when w/c ratio increased, the carbonation depth of RAC also increased. For the same w/c ratio, the carbonation depths of RAC were slightly higher than the ones of NAC with the same properties. In addition, Ryu (2002a) reported that the carbonation depth increased when the strength of the residual mortar decreased. Otsuki et al. (2003) attributed the higher carbonation depth of the RAC compared to NAC to the residual mortar in RCA.

Zaharieva et al (2003) evaluated the depth of carbonation of the RAC by using accelerated carbonation test. RAC specimens were exposed to $50 \% \mathrm{CO}_{2}, 50 \%$ air, in $65 \%$ relative humidity. They reported that more permeable concrete presents a higher depth of carbonation. The carbonation of RAC was faster than the control NAC with the same mixture.

A few researchers proposed methods to improve the carbonation resistance of RAC. Otsuki et al. (2003) used a double mixing method to improve the carbonation resistance of RAC especially for high w/c ratios. They observed that the carbonation resistance improved for RAC specimens prepared by this method. Shayan and Xu (2003) reported that the RAC mixtures with RCA chemically treated with sodium silicate solution had higher carbonation depths; and the RAC mixtures with no chemical treatment had smaller carbonation depths. These results were attributed to the greater tendency of the mixture containing sodium silicate solution to absorb more $\mathrm{CO}_{2}$.

It should be noted that, in the previous studies, the effect of the amount of the residual mortar on the carbonation process of the RAC was not studied. As discussed 
before, the hydrated cement in the residual mortar may increase change the reserve alkalinity, which may reduce the carbonation depth of RAC. Furthermore, relating the carbonation to the RCA content (e.g. $100 \%$ or $50 \%$ RCA replacement) may not be appropriate to define the vulnerability of the RAC to carbonation. Different types of RCA may have different amounts of residual mortar; therefore it is important to relate the carbonation to the total residual mortar.

\subsection{Summary}

As presented in this literature review, a number of studies have been carried out to investigate the use of RCA in structural-grade concrete. Although these studies cover a relatively wide range, they do not follow a comprehensive and systematic approach that can lead to the standardization of the practice. In general, the following gaps in the literature have been identified:

- Although the properties of RCA have been studied by a number of researchers, there is a lack of fundamental study investigating how these properties change with the presence of residual mortar;

- The quantification of the RMC of RCA is a crucial task in the prediction of the RCA properties; therefore, a rapid and accurate RMC determination test is needed.

- Most studies in the literature were carried out with RCA produced in the laboratory. In a limited number of cases in which the RCA was obtained from commercial sources, no significant attempt was made to investigate the sources effect. There is an obvious need to investigate the source of the RCA by choosing materials from different geographical locations. Especially for a country like Canada, this very important.

- Currently, to the author's knowledge, there is no mix design procedure specifically 
developed for structural-grade RAC.

- In order to develop such a mix design procedure, a comprehensive investigation of the fresh and hardened properties of concrete produced with RCA obtained from different commercial sources need to be carried out. Only such a comprehensive study can lead to the development of special mix design procedures for RAC.

- After the development of the mix design procedures, the durability properties of RAC produced with them need to be investigated to study the long-term performance of the structures produced. Among these properties, the effects of freeze-and-thaw action, chloride penetration and carbonation can be considered to be of particular importance.

A summary of the factors that may affect the freeze-and-thaw action, chloride penetration and carbonation of RAC are presented in Table 2.2. In general, these factors are similar to the ones affecting conventional concrete, with a number of additional complications introduced by the attached residual mortar. 
Table 2.1: Physical and mechanical properties of RCA from literature

\begin{tabular}{|c|c|c|c|}
\hline \multicolumn{3}{|c|}{ RCA Properties } & Range \\
\hline \multicolumn{3}{|l|}{ Specific Gravity, SSD } & $2.1 \sim 2.64$ \\
\hline \multirow{2}{*}{\multicolumn{2}{|c|}{ Unit weight $\left(\mathrm{kg} / \mathrm{m}^{3}\right)$}} & Compacted & $1220 \sim 2557$ \\
\hline & & Loose & $880 \sim 1432$ \\
\hline \multirow{2}{*}{\multicolumn{2}{|c|}{ Voids (\%) }} & Compacted & $33 \sim 39$ \\
\hline & & Loose & $44 \sim 49$ \\
\hline \multirow{2}{*}{\multicolumn{2}{|c|}{ Water Absorption (\%) }} & Coarse & $3.2 \sim 11$ \\
\hline & & Fine RCA & $5.4 \sim 19.5$ \\
\hline \multicolumn{3}{|l|}{ Total Porosity (\%) } & $10.5 \sim 20$ \\
\hline \multicolumn{3}{|l|}{ Longitudinal Index (\%) } & $15.6 \sim 45$ \\
\hline \multicolumn{3}{|l|}{ Flakiness Index (\%) } & $4.6 \sim 22.1$ \\
\hline \multicolumn{3}{|l|}{ Fineness Modulus (\%) } & $2.44 \sim 6.69$ \\
\hline \multicolumn{3}{|l|}{ Moisture Content (\%) } & $3.2 \sim 5.32$ \\
\hline \multicolumn{3}{|l|}{ Crushing Value $(\%)$} & $14 \sim 38$ \\
\hline \multicolumn{3}{|l|}{ Abrasion Value (\%) } & $26 \sim 42.7$ \\
\hline \multicolumn{3}{|l|}{ Impact Value (\%) } & $10 \sim 42.7$ \\
\hline \multirow{3}{*}{ Chemical Contaminant (\%) } & \multirow{2}{*}{$\mathrm{Cl}^{-}$} & Water & $\sim 0.05$ \\
\hline & & Acid & $\sim 0.15$ \\
\hline & \multicolumn{2}{|c|}{ Sulfate } & $\sim 0.35$ \\
\hline \multicolumn{3}{|l|}{ Soundness } & $8.1 \sim 49.1$ \\
\hline
\end{tabular}


Table 2.2: Factors that affect freeze-and-thaw resistance, chloride penetration, and carbonation in RAC

\begin{tabular}{|c|c|}
\hline Durability Aspect & Parameter \\
\hline \multirow{6}{*}{ Freeze-and-thaw } & w/c of the new mortar \\
\hline & Air entrainment \\
\hline & Tensile strength of concrete \\
\hline & Degree of saturation of the RCA \\
\hline & Quality and amount of the residual mortar \\
\hline & Durability and permeability of RCA \\
\hline \multirow{6}{*}{ Chloride Penetration } & Chloride diffusivity of the new mortar and residual mortar \\
\hline & w/c of new mortar \\
\hline & Type of the cementitious system \\
\hline & Contamination of the residual mortar \\
\hline & Chloride binding ability of the residual mortar \\
\hline & Initial binding chloride in residual mortar \\
\hline \multirow{5}{*}{ Carbonation } & Diffusivity of $\mathrm{CO}_{2}$ \\
\hline & w/c of new mortar \\
\hline & $\begin{array}{l}\text { Type of the cementitious system of the residual and new } \\
\text { mortars }\end{array}$ \\
\hline & Micro-cracking in residual mortar \\
\hline & Reserve alkalinity in residual and new mortars \\
\hline
\end{tabular}




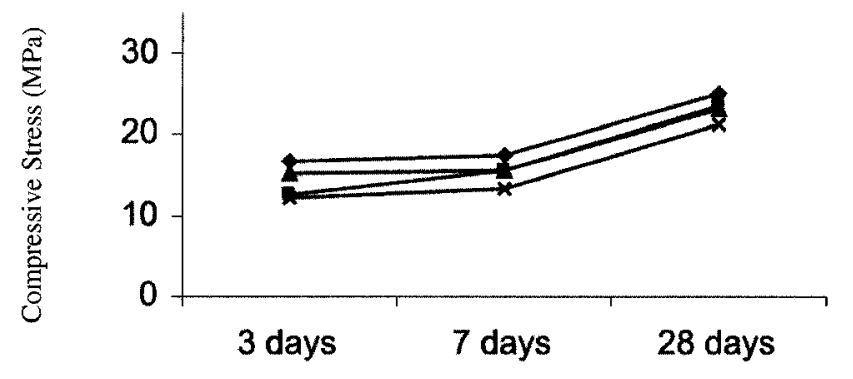

Number of days

(a)

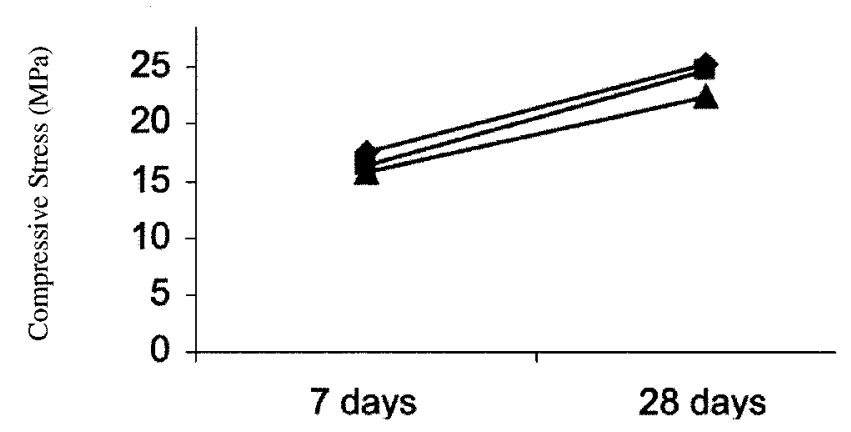

Number of days

(c)

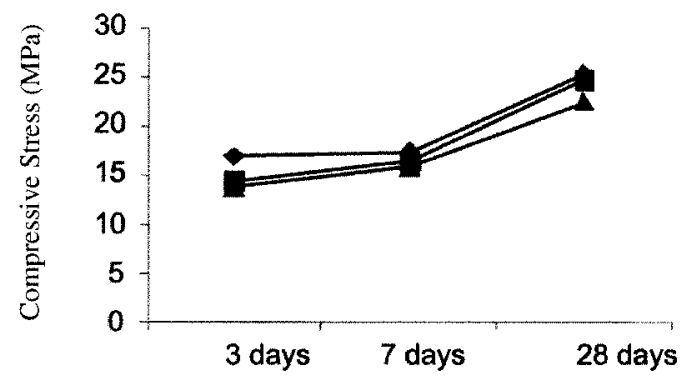

Number of days

(b)

$\longrightarrow 0 \% \mathrm{RCA} \longrightarrow-25 \% \mathrm{RCA} \longrightarrow 50 \% \mathrm{RCA} \longrightarrow 75 \% \mathrm{RCA}$

Figure 2.1: Cylinder compressive strength for RAC with RCA from (a) laboratory, (b) demolished roof slab, and (c) demolished building 


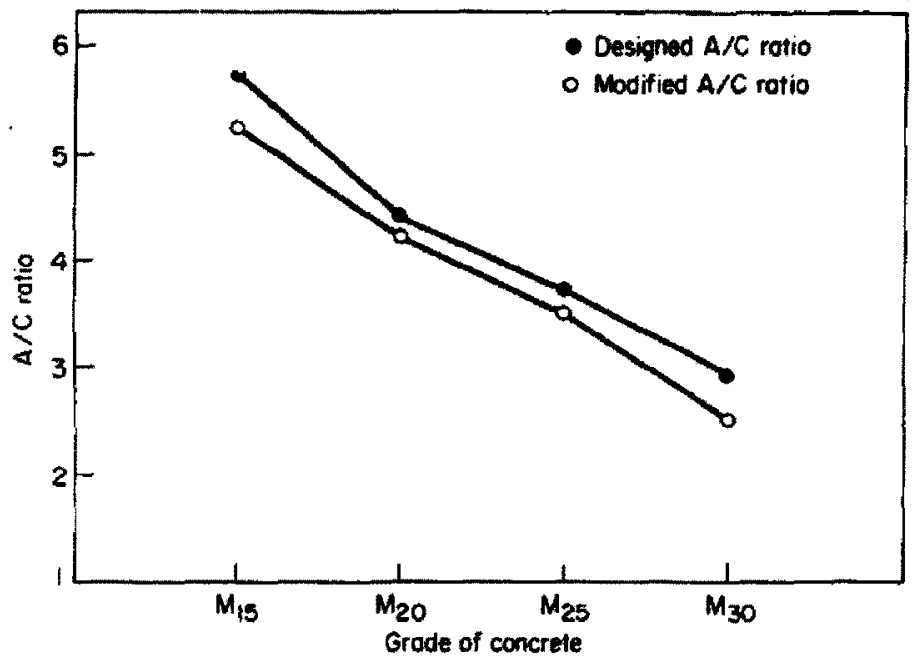

(a)

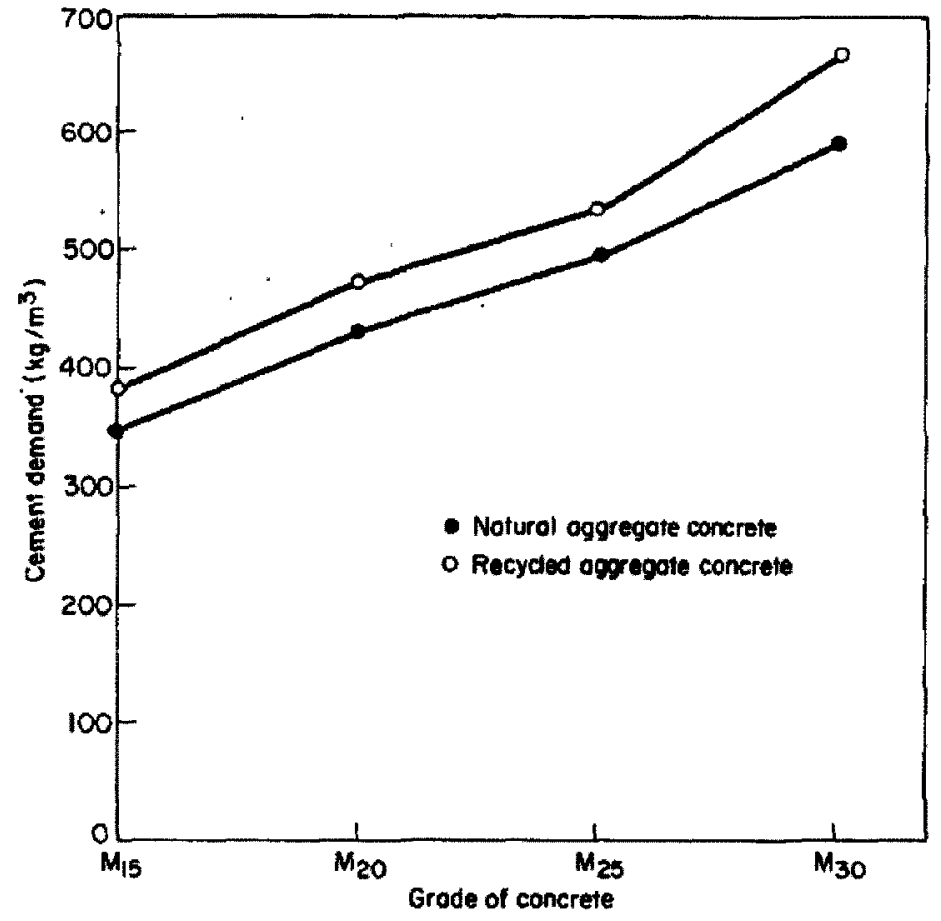

(b)

Figure 2.2: Relationship between the grade of concrete and (a) A/C ratio, (b) cement demand (reproduced from Bairagi et al., 1990) 

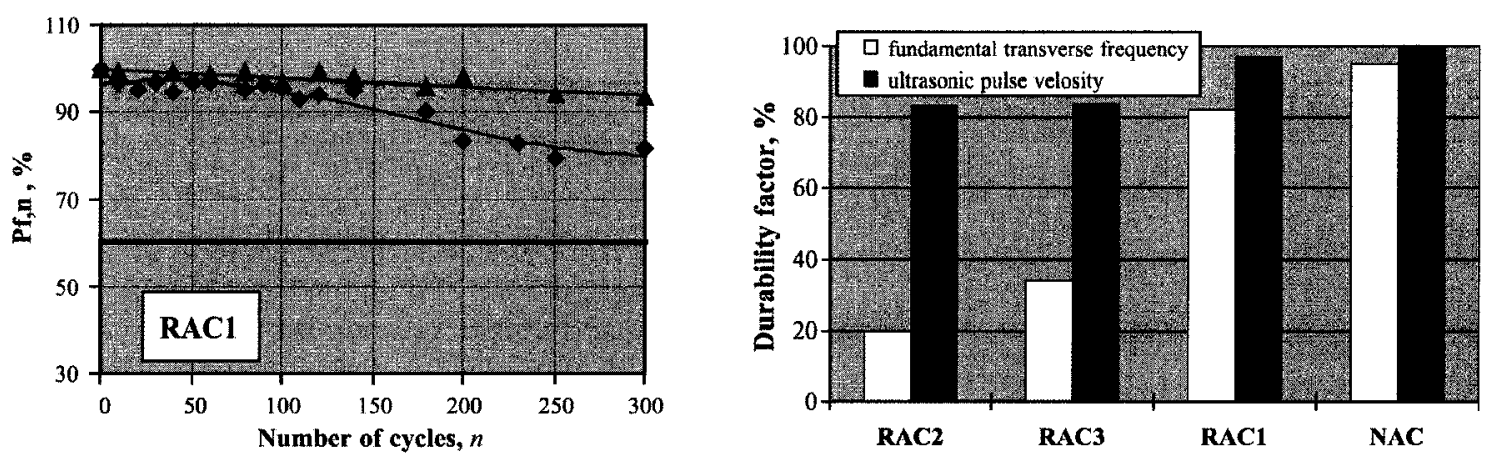

- complete 4 cyclic $\longrightarrow$ threshold

(a)

(b)

Figure 2.3: (a) Effect of water saturation type on the relative dynamic modulus; (b) durability factor for complete water saturation (reproduced from Zaharieva et al., 2004)

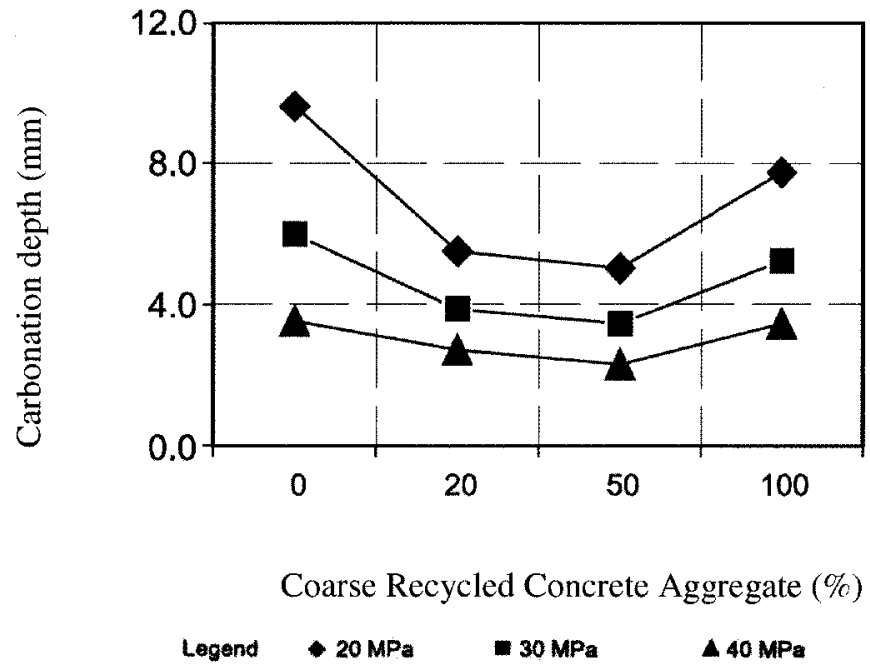

Figure 2.4: Carbonation depth versus RCA content (reproduced from Levy and Helene, 2004) 


\section{CHAPTER 3: MATERIAL CHARACTERIZATION OF RCA}

\subsection{General}

Specified mechanical and durability properties of concrete are achieved through mix design procedures, which involve the proportioning of main constituents of concrete; i.e., cementitious materials, fine and coarse aggregates, water and chemical admixtures. The computations that are carried out during the mix design procedures require precise characterization of the material properties of the constituents. The material properties of coarse aggregates are particularly important in the design of mixes since they occupy approximately $75 \%$ of the total volume of concrete. The material characterization of $\mathrm{RCA}$, when used as coarse aggregates in new concrete, requires special attention since its properties may vary depending on the source, the production method and the amount of attached residual mortar it contains.

In this chapter, the material characterization of the two types of RCA that are used in the current study is presented. The recycling plants that provided the RCA for the present study receive concrete from various demolition projects, and the material that they produce incorporates a blend of virgin aggregates from the different sources. The first batch of RCA was obtained from a recycled aggregate processing plant located in Montreal, Québec, and the second was from a plant in Vancouver, British Columbia. The RCA from Montreal was obtained from concrete in which the natural aggregates were crushed limestone, the most widely used aggregate in the St. Lawrence Lowlands of the Province of Québec, where the main urban areas are located. The RCA from British Columbia was obtained from demolition concrete made predominantly with well-rounded 
river-bed gravel. Although the RCA from both locations likely included concrete from various demolition projects, and they were probably a blend of several different concrete mixtures, possibly fly-ash concrete in the case of the RCA from Vancouver, no attempt was made to separate or to quantify the constituent mixtures. In terms of their shape and surface characteristics, the two types of RCA showed differences as well. The RCA particles from Montreal showed more equi-dimensional and angular shape characteristics and had rougher surface texture than RCA-VA particles. The aggregates had in fact the general shape of their corresponding OVA particles; i.e., limestone and river gravel, respectively. Both types were observed to be rougher in surface texture than their corresponding OVA due to the apparent presence of attached residual mortar. For brevity, henceforth, the RCA from Montreal and Vancouver will be referred to as RCA-MO and RCA-VA, respectively.

The material characterization of RCA involves the determination of specific gravity, absorption capacity, moisture content, porosity, degradation resistance (Los Angeles Test), and soundness properties. The specific gravity and absorption capacity tests were performed as per ASTM C 127-01 (ASTM, 2003). To evaluate the soundness of RCA specimens, two tests were carried out: (1) ASTM C 88-99a (ASTM, 2003) using sodium sulfate and magnesium sulfate solutions; (2) MTO Test Method LS-614 (MTO, 2001) using repeated freeze-and-thaw cycles for RCA immersed in sodium chloride solution for 24 hours. The abrasion value was determined using the Los Angeles Test as specified in ASTM C 131-89 (ASTM, 2003).

Since the aggregates from both sources were received unscreened and ungraded, before starting the material characterization testing, they were screened from impurities 
and fines, and were separated according to their size. The following section discusses this preparation process of the aggregates before material characterization testing.

\subsection{Preparation of RCA for Material Characterization Testing}

In order to eliminate the fines from the coarse aggregates and to clean RCA from impurities such as wood, plastics, steel bars, asphalt particles, etc., a large sieve machine, which is shown in Figure 3.1, was used. As illustrated in Figure 3.2, a variety of large impurities were found in RCA-MO and RCA-VA. In addition to these obvious impurities, visual inspection has also demonstrated that there were less noticeable and smaller impurities in the form of asphalt, metal, plastic and wood particles in the RCA. Since the presence of these impurities will affect the properties of concrete produced with $\mathrm{RCA}$, it was decided that a more comprehensive investigation on the amount of impurities be carried out as part of the material characterization testing. It should be noted that there is no standard test for determination of impurities; therefore the test, for which details are provided in Appendix A, was developed for this specific purpose.

The impurity test was performed on RCA-MO and RCA-VA for different fraction sizes. Figure 3.3 shows the impurities in typical samples of RCA-MO and RCA-VA. Impurities such as bitumen-coated aggregates, tiles, bricks, wood, metal, and other materials (e.g. nylon, rubber, fibres, etc.) were quantified as the weight percentage of the RCA. The raw data collected from this test is also summarized in Appendix A. Summary of the results is provided in Table 3.1 , which shows the percentage of the impurities for RCA-MO and RCA-VA. In general, the impurities of RCA-VA are higher than RCAMO; however, in both cases, the amounts of impurities were not considered to be a major concern for producing structural grade concrete. 
Following the sieving process, sieves with opening sizes of $4.75 \mathrm{~mm}(\# 4), 9.5 \mathrm{~mm}$ (3/8 in), $12.7 \mathrm{~mm}(1 / 2 \mathrm{in}), 19 \mathrm{~mm}(3 / 4 \mathrm{in})$, and $25 \mathrm{~mm}(1 \mathrm{in})$ were used to separate RCA into different grades. Throughout this thesis, RCA size indicates the size of aggregate retained on the corresponding sieve. To avoid damage to the RCA, the sieving time was fixed to 30 seconds for each batch. RCA were washed, air dried in mesh drum (Figure 3.1), and stored in solid drums that had been clearly marked with aggregate size information.

\subsection{The RMC of RCA-MO and RCA-VA}

Existing literature demonstrates that the amount and properties of the residual mortar in RCA significantly affect its mechanical and durability properties. For example, the lower specific gravity, the higher absorption capacity and the higher Los Angeles abrasion value of RCA, compared to NA, can be qualitatively ascribed to its RMC. Therefore, it is important to quantify RMC of the RCA that is used in the present investigation.

Currently, there is no standard method that can be used to determine the RMC; hence as part of this research, a comprehensive investigation to obtain a "quick" test method to quantify this property was carried out. The developed method uses mechanical and chemical stresses to separate the residual mortar from the OVA to which it is bonded. The mechanical stresses are induced through freeze-and-thaw cycles while the chemical stresses through repeated exposure of RCA to sodium sulphate solution. The validation of the developed procedure was done by a detailed image analysis study. In the following sections, the development of the quick RMC determination test and the image analysis study to validate the developed method are presented. A step-by-step procedure of the 
developed "quick-and-dirty" test method is provided in Appendix A.

\subsubsection{The Development of the Quick Test Procedure}

Exploratory investigations on the applicability of the existing soundness test methods, which are based on the immersion of aggregates in sulphate solutions (i.e., chemical stresses) or on the freeze-and-thaw cycles (i.e., mechanical stresses), revealed that similar methods could be used to remove the residual mortar from the aggregates.

To further investigate the effects of chemical stresses on the loss of residual mortar, RCA-MO samples were prepared and tested using sodium sulfate, magnesium sulfate and magnesium chloride solutions. The concentration of all the solutions was kept $26 \%$ by weight, which reflects approximately the same concentration used in ASTM soundness test. Immersion time and storage temperature of each specimen were kept constant at 15 days and $23 \pm 2{ }^{\circ} \mathrm{C}$, respectively. Figure 3.4 shows the appearance of the RCA-MO samples after immersion in magnesium sulphate, magnesium chloride and sodium sulphate solutions for 15 days. As it can be observed from the figure, the samples immersed in sodium sulfate solution experienced noticeable disintegration, while there were no significant signs of residual mortar crumbling from the samples immersed in magnesium sulfate and magnesium chloride solutions.

To investigate the combined effect of mechanical and chemical stresses, freezing and thawing of the samples immersed in the three solutions was used to accelerate the degradation of the attached residual mortar. For this purpose, representative RCA-MO samples were immersed in magnesium sulphate, magnesium chloride and sodium sulphate solutions for 6 hours, or about one third of the period suggested by the ASTM soundness test. Then, the RCA samples (in the solutions) were placed in a freezer at -18 
${ }^{\circ} \mathrm{C}$ for 12 hours (overnight), and later they were left out to thaw at room temperature during the day. This process was repeated five times over 5 days. The RCA-MO samples immersed in sodium sulphate solution started to disintegrate during the second cycle. Even after five freeze-and-thaw cycles, the same aggregates immersed in the other two solutions showed no significant signs of mortar degradation. Figure 3.5 shows the RCAMO samples initially immersed in the three solutions and after being subjected to five freeze-and-thaw cycles. Notice that the combined method was found to be more effective in removing attached residual mortar from RCA than using freeze-and-thaw cycles or chemical immersion alone.

To ensure quick and complete removal of the attached residual mortar, the method was further modified by performing the thaw cycle in an oven at $80{ }^{\circ} \mathrm{C}$. In addition, the time of immersion of RCA samples in $26 \%$ sodium sulfate solution was increased to 24 hours before starting the freeze-and-thaw cycles. This refinement allowed total removal of the residual mortar from RCA without damaging the OVA. The refined approach was used to calculate the residual mortar content of RCA-MO and RCA-VA samples of different sizes retained on $4.75 \mathrm{~mm}, 9.5 \mathrm{~mm}, 12.7 \mathrm{~mm}$ and $19 \mathrm{~mm}$ sieves. Ten samples were tested to determine the average residual mortar content of each aggregate size to confirm repeatability. The oven-dried sample sizes used in the test were $1000 \mathrm{~g}$ for the $4.75 \mathrm{~mm}$ aggregates and $2000 \mathrm{~g}$ for the larger aggregates. During the freeze-and-thaw cycles, the samples were examined continuously to determine the number of cycles required to completely/efficiently separate the attached residual mortar from the natural aggregates. From these observations, 5 cycles were found to be adequate for the $4.75,9.5$, $12.7 \mathrm{~mm}$ aggregates, and four cycles were satisfactory for the $19 \mathrm{~mm}$ aggregates. The 
same study carried out on RCA-VA samples showed that only three cycles were needed to adequately remove the attached residual mortar for all aggregate sizes. The difference is associated to the surface characteristics of the OVA particles of the RCA-VA specimens. Figure 3.6 shows the stages of residual mortar removal from RCA-MO samples by using chemical and mechanical stresses. Furthermore, Figures 3.7 and 3.8 illustrate different sizes of RCA-MO and RCA-VA samples, respectively, after the removal of the attached residual mortar. A small amount of remaining residual mortar (3 to $49 \mathrm{~g}$ in average) was observed in some of the RCA particles, and these were removed by gently hitting the RCA with hammer. After the process, the samples were oven-dried and weighed to compute their RMC using:

$$
R M C=\left[\frac{W_{O D}^{R C A}-W_{O D}^{O V A}}{W_{O D}^{R C A}}\right] \times 100
$$

where RMC is the residual mortar content $(\%), W_{O D}^{R C A}$ is oven-dry weight of RCA before test $(\mathrm{g}), W_{O D}^{O V A}$ is oven-dry weight of OVA after full removal of the residual mortar $(\mathrm{g})$. Tables 3.2 and 3.3 summarize the RMC for different factions of RCA-MO and RCA-VA samples, respectively. On an average, the RMC of the different fractions of RCA-MO and RCA-VA samples vary between $39-46 \%$ and $21-26 \%$, respectively. In both cases, the RMC of $4.75 \mathrm{~mm}$ samples was found to be slightly higher than that of $9.5 \mathrm{~mm}$ and 12.7 mm RCA, for which RMC values are relatively similar to each other. The weighted average RMC for RCA-MO and RCA-VA with combined fraction size of $4.75 \mathrm{~mm}, 9.5$ $\mathrm{mm}$ and $12.7 \mathrm{~mm}$ are $41 \%$ and $23 \%$, respectively. Standard deviation (STD) and coefficient of variation $(\mathrm{CoV})$ of the results among different samples for each size were found to be within the acceptable limits. 
Since it will be necessary in Chapter 7 (mix design chapter), it is also important to represent the RMC in terms of volume. The volume-based $\mathrm{RMC}, R M C_{V}$, can be obtained as:

$$
R M C_{V}=\frac{V_{R M}}{V_{R C A}}=\frac{\left(\frac{W_{O D}^{R M}}{S G_{b}^{R M}}\right)}{\left(\frac{W_{O D}^{R C A}}{S G_{b}^{R C A}}\right)}=\frac{S G_{b}^{R C A}}{S G_{b}^{R M}} R M C
$$

By knowing the RMC (by weight) and the bulk specific gravity for RCA and OVA, the $S G_{R M}$ for RCA-MO and RCA-VA can be calculated by the weighted average method as follows.

$$
\frac{1}{S G_{b}^{R C A}}=\frac{R M C}{S G_{b}^{R M}}+\frac{1-R M C}{S G_{b}^{O V A}}
$$

The $\left(S G_{b}^{R M}\right.$ ) for RCA-MO and RCA-VA are calculated using Eq.3.3 as 1.91 and 1.79, respectively. This will be discussed in detail in the development of the rational approach (Section 3.11). Furthermore, it is important to mention that there is no standard test method available to measure the specific gravity of the residual mortar. However, an attempt was made to measure the residual mortar specific gravity by using the test procedure developed for fine aggregates. The results of this test will be discussed in Section 3.9. From Eq.3.2, the $R M C_{v}$ (volume-base) for RCA-MO and RCA-VA is equal to 1.21 and 1.35 times the (weight-base) $R M C$, respectively. Therefore, the average $R M C_{v}$ for RCA-MO and RCA-VA is equal $49.6 \%$ (vol.\%) and $31.0 \%$ (vol.\%), respectively. The detailed testing protocol developed for the determination of the RMC of RCA is presented in Appendix A. To verify the results and to validate the quick RMC determination method developed in this study, an image analysis study was performed. 
The following section discusses the image analysis investigation.

\subsubsection{Image Analysis Investigation}

The image analysis is used as a method to verify the results of the "quick-anddirty" RMC determination tests described in the previous section (also, see Appendix A for the detailed procedure). Preparation of the samples for the image analysis is explained in the following section.

\subsubsection{Preparation of Samples for the Image Analysis}

Representative samples of RCA-MO and RCA-VA were obtained to make polished slabs required for image analysis. A cement paste was first prepared using a laboratorysize Hobart mixer and white cement. The various amounts of RCA-MO and RCA-VA were then added to the paste and mixed by hand (Table 3.4 ). The "no-fines" concrete thus produced was then placed and gently consolidated in plastic moulds, $100 \times 100 \times 400 \mathrm{~mm}$ in size, using vibration table. The moulds were then covered and placed in the moist curing room $\left(23^{\circ} \mathrm{C}, 100 \%\right.$ R.H. $)$ for 24 hours. The moulds were then stripped and the prisms were placed in plastic pails at $60^{\circ} \mathrm{C}$ and $100 \%$ R.H. for a period of seven days. This was done in order to accelerate strength development.

After curing, the prisms were cut in 30 -mm-thick slabs using an automated slowspeed concrete saw (Figure 3.9a). The latter was used because of the good-quality surface it generates during the cutting operation; also, it operates in special cutting oil (water-free system) without significantly raising heat in the sample. In order to establish the proper polishing requirements for assuring high quality results of image analysis, tests were performed on a number of companion slabs using a portable wet stone grinder using different diamond-impregnated wet resin polishing pads (no free grit material). Polished 
samples resulting from the following polishing processes were thus compared: (1) grinding using a single row cupwheel to simply eliminate cutting grooves, if any; (2) step 1 followed by 400 -grit pad; and (3) steps $1+2$ plus 3500-grit pad (Figure 3.9b).

Cut and polished slabs were then photographed using a Bencher copy stand and four lights. The lighting was carefully adjusted until all corners and middle area of the slab reflected the same intensity of light. After various tests, it was found that appropriate contrast and definition could be obtained from samples that were obtained from the operation no. 1 described above, i.e., without any further polishing, provided that a thin layer of water was applied to each surface just prior to exposure. Figures 3.10 and 3.11 illustrate "no-fine concrete" slabs incorporating the different size fractions of the RCAMO and RCA-VA aggregates. After that, the image analysis is carried out; the following section explains the image analysis process.

\subsubsection{Process of Image Analysis}

After the capture phase through digital photographic process, each basic colour image of the concrete slabs was carefully edited and sized using Photoshop software to specific resolution for consistency. Several resolutions (i.e., image sizes ranging from $100 \mathrm{~KB}$ to $3 \mathrm{MB}$ ) were tested to determine the one suitable for the operator to use, i.e., that would allow to binarize the image with adequate details of the structure in a manner that would differentiate phases and allow measuring of tested features with acceptable accuracy within reasonable time period (i.e., acceptable image treatment time). The photo-digital technique adopted had to allow differentiating and select separately the following parameters in the images: (1) the original coarse aggregates (dark-grey, angular, fine-grained limestone in the case of RCA-MO; multi-colored, well-rounded, 
river gravel for RCA-VA)(see Figures 3.10 and 3.11), (2) the residual or attached mortar phase, and (3) the binder of white cement. It can be observed from these figures that the RCA-VA particles are characterized by their relatively round shape; however, the RCAMO particles are more equi-dimensional and angular.

Many selection tools available in Photoshop were able to select the background tone (i.e., binder of white cement) accurately, but air bubbles in the white-cement matrix proved to be more difficult. The pockets created by trapped air naturally caused shadows when lit and these shadows were of similar tonal quality to some of the aggregate particles. As a result, a fully-automatic image analysis solution was not available and significant manual editing was required for accuracy. The technique used was thus to first select the background tone (white cement binder) in Photoshop, fine tune that selection with a layer mask (green) (Figure 3.12), and then use the lasso tool to manually add each entrapped air pocket in the white cement paste to the overall selection. In order to maintain accuracy (i.e., in the nature of the elements to be incorporated in the matrix and which would not be quantified as RCA particles), the original concrete slab was used as a reference during this process of manual editing.

Clemex Vision PE image analysis software application was then used to develop custom image analysis. A routine was built based on the specifics of the project. The goal of the routine was to separate individual segments of the recycled concrete and measure the areas corresponding to the original river-bed gravel or crushed limestone particles, the attached mortar (consisting of sand, cement and air voids) and the new binder (white cement). In order to analyse images without specified magnification, a calibration factor was created based on the actual length of the slab and the initial resolution of the image. 
The calibration factor was $217.519421 \mu \mathrm{m}$ per pixel.

The applied bright green mask from Photoshop treatment of the original digital pictures was found to be easily differentiated by the Clemex software package and could easily be binarized to separate the new matrix or binder of white cement and each individual RCA particle (Figures 3.13). In the early stages of the routine developed to analyse the images, a bitplane erase tool in the Clemex Vision software application was used to separate particles that were not entirely surrounded by the binder of white cement, i.e. when two or several RCA particles were touching each other (Figure 3.13).

Successive binarization and binary operations were then used that allowed separating, on tonal fractioning basis, the various parameters of interest, i.e., total area of each RCA particle, the residual mortar fraction and the OVA particles, limestone or riverbed gravel (Figures 3.14a and b). Figures 3.15 (a) to (f) illustrate typical results of some of those binarization steps.

The accuracy of the analysis performed on tonal basis was verified for each particle by referring to the original concrete slab. The "automatic" binarization was generally more accurately accomplished in the case of the RCA incorporating the dark-grey crushed limestone aggregate particles. However, in the case of the RCA-VA, i.e., those incorporating river-bed gravel particles, manual editing (using the lasso tool in the software package) was often required to accurately identify the quantifiable elements, as some of the OVA particles had tonal characteristics close to those of the residual mortar. Also, once again in the case of the RCA-VA, which incorporated fine and coarse aggregates from natural sand \& gravel mining operations, a size-fractioning limit had to be incorporated in the routine to allow separating fine aggregate particles (i.e., to be 
considered as part of the residual mortar) from coarse aggregate particles.

\subsubsection{Results and Analysis of the Data from Image Analysis}

The analysis of the images using the software provides the following data for each RCA particle in the specimen: (1) the total area of each RCA particle, $A_{R C A, i}\left(\mu \mathrm{m}^{2}\right)(2)$ the total area of OVA particles, $A_{O V A, i}\left(\mu \mathrm{m}^{2}\right)$, (3) the total area of residual mortar, $A_{R M, i}\left(\mu \mathrm{m}^{2}\right)$. The subscript, $i$, denotes that these quantities are for an individual RCA particle. All three measurements were recorded and tabulated; Table 3.5 gives an example of the data obtained. Table 3.6 summarizes the total area of RCA, OVA and residual mortar for each fraction size for RCA-MO and RCA-VA slabs obtained from image analysis.

Since, the RMC is weight based, analytical equations are developed to convert the surface area data provided by the image analysis to weight-based quantities. For a unit thickness of the specimen and assuming uniform shape, the total weight of the OVA in a given RCA particle, $W_{O V A, i}$ can be calculated using:

$$
W_{O V A, i}=S G_{b}^{O V A} A_{O V A, i}
$$

where $S G_{b}^{\text {OVA }}$ is the bulk specific gravity of OVA. The weight of the residual mortar in a given RCA particle, $W_{R M, i}$, can be determined by:

$$
W_{R M, i}=S G_{b}^{R M} A_{R M, i}
$$

where $S G_{b}^{R M}$ is the bulk specific gravity of residual mortar. By substituting Eqs.3.4 and 3.5 in Eq.3.1, and assuming that the total weight of the RCA is equal to the sum of the weights of the OVA and the residual mortar, the RMC of an individual particle, $R M C_{i}$, can be calculated as: 


$$
R M C_{i}=\left[\frac{S G_{b}^{R M} A_{R M, i}}{S G_{b}^{R M} A_{R M, i}+S G_{b}^{\text {OVA }} A_{\text {OVA }, i}}\right] \times 100
$$

A similar expression can also be derived for the entire specimen, which contains a large number of RCA particles. Overall, bulk specific gravity of the RCA in the specimen, $S G_{b}^{R C A}$, can be calculated as:

$$
S G_{b}^{R C A}=\frac{\sum\left(W_{O V A, i}+W_{R M, i}\right)}{\sum A_{R C A, i}}
$$

where the denominator of the equation represents the total volume of the RCA for the unit thickness of the specimen. In addition, the bulk specific gravity of OVA, $S G_{b}^{O V A}$ can be calculated by:

$$
S G_{b}^{O V A}=\frac{\sum W_{O V A, i}}{\sum A_{O V A, i}}
$$

Using Eqs. 3.7 and 3.8, the weight of the residual mortar per unit thickness in a specimen can be calculated as:

$$
W_{R M}=S G_{b}^{R C A} \sum A_{R C A, i}-S G_{b}^{O V A} \sum A_{O V A, i}
$$

Combining Eqs. 3.1, 3.7 and 3.9, the RMC of the RCA that are present in a given slab specimen can be determined as:

$$
R M C=\left[\frac{S G_{b}^{R C A} \sum A_{R C A, i}-S G_{b}^{O V A} \sum A_{O V A, i}}{S G_{b}^{R C A} \sum A_{R C A, i}}\right] \times 100
$$

Furthermore, the $R M C_{v}$ (by volume) in the slab specimen can be calculated from the image analysis:

$$
R M C_{V}=\left[\frac{\sum A_{R M_{i}}}{\sum A_{R C A_{i}}}\right] \times 100
$$


The specific gravities of the RCA and OVA that are used in Eq.3.10 for the present study are shown in Table 3.7; they were obtained by testing representative samples of RCA-MO and RCA-VA in the laboratory. Table 3.6 shows the total area of the RCA, residual mortar (RM), and OVA recorded from image analysis and the calculated RMC by weight and volume for different fraction size for RCA-MO and RCA-VA. From Table 3.6 the calculated weighted average RMC (\%) by weight from image analysis for RCA$\mathrm{MO}$ and RCA-VA are $42 \%$ and $24 \%$, respectively, which are very close to the value of $41 \%$ and $23 \%$ obtained from the quick RMC determination test method developed in this study. In addition, the weighted average $\mathrm{RMC}_{\mathrm{v}}$ (by volume) for RCA-MO and RCA-VA are $50 \%$ and $32 \%$, respectively. These values are close to the values of $49.6 \%$ and $31 \%$ calculated from the developed method.

Table 3.8 compares the RMC of RCA-MO and RCA-VA for each fraction size as obtained by image analysis and by the developed quick test method. The standard deviation (STD) and the coefficient of variation $(\mathrm{CoV})$ of the image analysis results vary between 1.7 to $4.2 \%$ and from 4 to $20 \%$, respectively. The results obtained through image analysis confirmed that the quick laboratory test provides an accurate measurement of the residual mortar content in RCA. Actually, the RMC determination through image analysis showed a larger variability than that obtained by the quick laboratory test.

In addition to validating data obtained through the quick RMC determination test method, image analysis study also provided valuable information about the particle distribution of the RCA used in the present research. Figure 3.16 illustrates the average frequency of the RCA particles in different size fractions for RCA-MO and RCA-VA. In plotting these frequency diagrams, Eq. 3.6 was used with the assumption that $S G_{R M}$ for 
RCA-MO and RCA-VA were 1.91 and 1.79 , respectively. The $S G_{R M}$ for RCA-MO and RCA-VA are calculated using Eq.3.3 (Section 3.3.1). The results in Figure 3.16 show that in the case of the RCA-MO, there are no concentrations in the proportions of particles with unexpectedly large amounts of residual mortar, with the exception of the 4.75-9.5 $\mathrm{mm}$ size fraction where about $20 \%$ of the particles are composed of between 95 and $100 \%$ of residual mortar. On the other hand, in the case of the RCA-VA, there is an obvious trend where more than $50 \%$ of the particles in each of the three size fractions investigated are composed by less than $15 \%$ residual mortar. This is related to the nature of the RCA-VA, for which processing (crushing / sieving operations) results in increased separation of the attached mortar from the well-rounded river-bed gravel particles compared to angular limestone particles in RCA-MO.

\subsection{Specific Gravity and Absorption Capacity Test}

The specific gravity and absorption capacity tests were performed as per ASTM C 127-88 (ASTM, 2003). For both aggregate sources, ten samples were prepared for each aggregate size of $4.75 \mathrm{~mm}, 9.5 \mathrm{~mm}$, and $12.7 \mathrm{~mm}$. The sample size for $4.75-\mathrm{mm}$ RCA was $1000 \mathrm{~g}$, while for $9.5-\mathrm{mm}$ and $12.7-\mathrm{mm}$ aggregates, it was $2000 \mathrm{~g}$. In addition to the standard 24-hour soaking period required by ASTM C 127-88 for NA, the RCA samples were immersed in water for 48 and 72 hours to investigate the effect of the immersion time on the specific gravity values and absorption capacity. The same samples were also used to determine the RMC of each fraction using the newly developed method, which is described in Section 3.3.1.

When RCA is fully saturated, its porosity, $n$, was calculated using the data obtained from the specific gravity tests and the following relation: 


$$
n=\left[\left(1-\frac{W_{O D}}{W_{S S D}}\right) S G_{S S D}\right] \times 100
$$

where, $n$ is the porosity of the aggregates $(\%), W_{O D}$ is oven dry weight of the aggregate $(\mathrm{g}), W_{S S D}$ is weight of SSD aggregate in air $(\mathrm{g}), S G_{S S D}$ is $\mathrm{SSD}$ specific gravity of aggregate. Equation 3.12 is derived from the definition of porosity:

$$
n=\left[\frac{V_{v}}{V_{T}}\right] \times 100
$$

where $V_{v}$ is the volume of voids and $V_{T}$ is the total volume of the aggregate particle. When the aggregates are in SSD condition, the volume of voids is equal to volume of water assuming all voids are accessible by water. The volume of water in the voids of SSD aggregates is given by Eq. 3.14:

$$
V_{v}=\left[\frac{W_{S S D}-W_{O D}}{\rho_{w}}\right]
$$

where, $\rho_{w}$ is the density of the water $\left(\mathrm{g} / \mathrm{cm}^{3}\right)$. The total volume of SSD aggregate is specified by:

$$
V_{T}=\left[\frac{W_{S S D}-W_{S S D-W}}{\rho_{w}}\right]
$$

where $W_{S S D-W}$ is weight of SSD aggregate in water (g). Substitution of Eqs. 3.14 and 3.15 in Eq. 3.13 yields:

$$
\begin{gathered}
n=\left[\frac{W_{S S D}-W_{O D}}{W_{S S D}-W_{S S D-W}}\right] \times 100 \\
n=\left[\frac{W_{S S D}-W_{O D}}{W_{S S D}}\right]\left[\frac{W_{S S D}}{W_{S S D}-W_{S S D-W}}\right] \times 100
\end{gathered}
$$

The second term in Eq. 3.17 is the definition of the SSD specific gravity of the aggregate 
$\left(S G_{S S D}\right)$. Rearranging Eq. 3.17 yields Eq. 3.12.

Tables 3.9 and 3.10 present the measured absorption capacity $(A C)$, bulk specific gravity $\left(S G_{b}^{R C A}\right)$, SSD specific gravity $\left(S G_{S S D}^{R C A}\right)$ and apparent specific gravity $\left(S G_{a p p}^{R C A}\right)$, as well as the porosity $(n)$ after 24,48 and 72 hours immersion for RCA-MO and RACAVA test samples, respectively. Table 3.11 compares the averaged values of the parameters listed above for the RCA samples, natural limestone and river-bed gravel from the same regions.

It can be observed from Tables 3.9 and 3.10 that the porosity ( $n$ ) of RCA-MO and RCA-VA samples vary between $10.8-15.0 \%$ and $6.6-9.5 \%$, respectively. In both types, the RMC of $4.75 \mathrm{~mm}$ samples was found to be slightly higher than that of $9.7 \mathrm{~mm}$ and $12.7 \mathrm{~mm}$ RCA (Table 3.8), whose porosity values are relatively similar to each other. Similar differences were observed between the RMC values of the RCA-MO (39-43\%) and RCA-VA (21-26\%), as well as between the $4.75 \mathrm{~mm}$ and the other two size fractions. The 24-hour absorption capacity of the RCA-MO and RCA-VA aggregate samples vary from 4.91 to $5.92 \%$ and from 2.83 to $3.96 \%$, respectively. In both cases, the absorption is slightly higher for the $4.75 \mathrm{~mm}$ size fraction than with for the other two size fractions. For 48 and 72 hours, although a slight increase in absorption capacity can be observed in some of the RCA-MO specimens, which are more porous than RCA-VA, in general, it can be stated that after 24 hours of immersion, absorption capacity reaches a fairly stable value.

The higher absorption capacity and porosity of the RCA-MO (compared to those of the RCA-VA) and of the $4.75 \mathrm{~mm}$ size fraction (compared to those of $9.7 \mathrm{~mm}$ and 12.7 
mm samples), can be attributed to the relatively higher RMC observed in the RCA-MO as well as in the smaller $(4.75 \mathrm{~mm})$ size fractions. Table 3.11 shows that the absorption capacity and the porosity values of the RCA are significantly higher than those of the corresponding OVA, i.e., about 16x higher in the case of RCA-MO and about 3-4x higher in the case of the RCA-VA.

Again, because of their attached residual mortar (with associated higher porosity), the RCA aggregates have slightly lower specific gravity values compared to the OVA (e.g. 2.31 vs. 2.70 for the bulk SG of the RCA-MO as shown in Table 3.11 ); it is important to note, however, that specific gravities of different fractions are very close to each other for both sources. The within-laboratory reproducibility of the $A C, S G$ and $n$ measurements, based on ten repeat RCA samples, was found to be very good, with coefficient of variations less than about $7 \%$.

\subsection{Abrasion Testing}

Following ASTM C 131-89 (ASTM, 2003), RCA-MO and RCA-VA were classified as grade $\mathrm{B}$, which predominantly includes a combination of $9.5 \mathrm{~mm}$ and 12.7 $\mathrm{mm}$ aggregates. The RCA samples were prepared by mixing $2500 \mathrm{~g}$ of $9.5 \mathrm{~mm}$ and 2500 $\mathrm{g}$ of $12.7 \mathrm{~mm}$. The RCA Los Angeles abrasion values are presented in Table 3.12. The abrasion values of RCA-MO and RCA-VA were determined to be $42 \%$ and $24.5 \%$, respectively. The abrasion values of the RCA used in this study are lower than the $50 \%$ allowable abrasion value for coarse aggregates to be used in structural concrete specified by ASTM C33-93 (ASTM, 2003). The coefficient of variation in both types is less than or equal to 2\%, which is the limit specified by ASTM C 131-89 (ASTM, 2003).

It can be observed from Table 3.12 that the Los Angeles abrasion values are 
directly correlated with the RMC of the RCA. Actually, $42.0 \%$ and $24.5 \%$ loss values are numerically very close to the measured RMC values of RCA-MO (39\% to 43\%) and RCA-VA (21\% to 26\%) specimens, respectively. This suggests that Los Angeles test removes the vast majority of the residual mortar from the original virgin aggregate particles. Visual examination of the samples after each test also confirmed that original virgin aggregates were mostly free of residual mortar. However, whether Los Angeles test can be used for the determination of the RMC of RCA requires more detailed investigation. On the other hand, since conducting Los Angeles test is easier than the quick RMC determination test presented in the previous section, it could possibly be used as a tool to quickly obtain an idea about the RMC of RCA. Additional testing is needed to confirm this relationship, and this study is highly recommended for future work.

\subsection{Soundness Tests}

To evaluate the soundness of RCA specimens, two tests were carried out: (1) ASTM C 88-90 (ASTM, 2003) using sodium sulfate and magnesium sulfate solutions; (2) MTO unconfined freeze-and-thaw test method LS-614 (MTO, 2001) using repeated freeze-and-thawing for aggregate sample immersed for $24 \mathrm{hr}$ in a sodium chloride solution. For each test, three repeat samples for each size and source were prepared in accordance with the specifications of the test method. The following section will explain the MTO soundness test. 


\subsubsection{MTO Soundness Test}

Prior to testing the aggregate samples under investigation by MTO unconfined freeze-and-thaw test method LS-61 (MTO, 2001), a control aggregate is required to adjust the sieving time for quantitative analysis of samples for a given set of equipment. The Brechin Quarry No.2 control aggregates are used to determine the sieving time for the quantitative analysis. The sieving time depends on the type of the mechanical shaker and the diameter of the sieve. The control aggregates is obtained from Ontario Ministry of Transportation (MTO); they were tested for soundness following test method LS-614 (MTO, 2001) to determine the sieving time for quantitative analysis. In the following section this process is elaborated further.

\subsubsection{Determination of Sieving Time for Quantitative Analysis}

As stated by MTO test method LS-614 (MTO, 2001), the determination of the sieving time is important to reduce inter-laboratory variation of the weighted percent loss of an individual sample due to differences that may arise from the use of different types of laboratory equipment. The amount of time required for sieving is established through incremental timed-testing of the control aggregate. Sieving time is interpolated from the mean weighted freeze-thaw loss of $20.8 \%$ for the control aggregate specified by the test method. This time is then used for testing the samples for the RCA. The following describes this process, as per MTO (2001).

The control aggregates were washed and oven dried. Then, they were sieved to fraction sizes of $4.75 \mathrm{~mm}, 9.5 \mathrm{~mm}$, and $12.7 \mathrm{~mm}$. A minimum of three control aggregate samples were prepared and tested to find the sieving time. Each sample was sieved for 20 seconds and the mass retained in each individual sieve fraction was weighed and 
recorded. Each fraction was returned to the corresponding sieve and shaking was continued in the sieve shaker for additional 40 seconds. The process was repeated for 1 , 2, 3, 4 and 5 minutes. Table 3.13 summarizes the weight of the control aggregates retained in each sieve as function of time. The cumulative percentage loss for each individual sieve for the three control aggregate samples was calculated by subtracting the mass retained after each test from the original mass, and dividing this value by the original mass. Table 3.14 shows the cumulative percent loss for individual sieves.

The weighted average loss is calculated by multiplying the retained percentage for each fraction by the percentage loss for that fraction. The retained percentages for the control aggregates specified by MTO are $45 \%, 30 \%$ and $25 \%$ for sieve size of $12.7 \mathrm{~mm}$, $9.5 \mathrm{~mm}$, and $4.75 \mathrm{~mm}$, respectively. The sum of the weighted average loss divided by 100 is the weighted average percentage freeze-and-thaw loss for the sample. Table 3.15 summarizes the calculated average weighted freeze-and-thaw losses with respect to time. Figure 3.17 illustrates the freeze-and-thaw loss $(\%)$ versus sieving time for the Brechin Quarry No.2 aggregates. From this plot, the sieving time is interpolated as 36 seconds, which is the corresponding sieving time for the mean weighted freeze-and-thaw loss of 20.8\%. This sieving time is subsequently used for testing RCA-MO and RCA-VA samples. The following section discusses the MTO soundness tests and the results for RCA. 


\subsubsection{MTO Soundness Test and Results for RCA}

For RCA-MO and RCA-VA, three samples for each fraction size of $4.75 \mathrm{~mm}$, $9.5 \mathrm{~mm}$ and $12.7 \mathrm{~mm}$ weighing $500 \mathrm{~g}, 1000 \mathrm{~g}$ and $1250 \mathrm{~g}$ were prepared. The RCA fraction size of $9.5 \mathrm{~mm}$ and $12.7 \mathrm{~mm}$ were placed in a one liter plastic mason jar; and the RCA fraction size $4.75 \mathrm{~mm}$ were placed in a $500 \mathrm{~mL}$ jar. The RCA samples were immersed for $24 \mathrm{hr}$ in $3 \%$ sodium chloride solution. Then, the sodium chloride solution was drained, and RCA samples were subjected to five cycles of freezing and thawing, by placing the RCA samples in the freezer at $-18.0 \pm 2.0^{\circ} \mathrm{C}$ for $16 \pm 2$ hours (usually overnight), and removing them in the morning to allow approximately 8 hours to thaw out at room temperature.

Table 3.16 summarizes the results of MTO soundness test for RCA-MO and RCAVA. For RCA-MO, the MTO unconfined freeze-and-thaw test resulted in losses ranging from about 20 to $30 \%$, being slightly higher for larger size fractions. The CoV observed between the three repeats for each size fractions ranged from about 5 to $12 \%$. In the case of RCA-VA, the MTO unconfined freeze-and-thaw test resulted in losses ranging from about 12 to $20 \%$; however, no clear trend could be established between the various size fractions. The $\mathrm{CoV}$ observed between the three repeats for each size fractions (i.e. 6 to 13\%) was similar to that observed in that test with the RCA-MO.

The retained percentage or grading for the RCA-MO and RCA-VA used in the calculations of weighted average freeze-and-thaw loss are $40 \%, 25 \%$ and $35 \%$ for 12.7 $\mathrm{mm}, 9.5 \mathrm{~mm}, 4.75 \mathrm{~mm}$, respectively. The weighted average loss values are $26.7 \%$ for RCA-MO, and $16 \%$ for RCA-VA. From Test Method LS-614, the allowable limit for average weighted freeze-and-thaw loss is $20.8 \%$. Therefore, RCA-VA satisfies the limit 
while RCA-MO loss is 1.3 times higher than the specified loss value.

\subsubsection{ASTM Soundness Test}

The ASTM C 88-90 (ASTM, 2003) soundness first test involved immersion of the aggregates in sodium sulfate or magnesium sulfate solutions for 16 to 18 hours. After the immersion, the aggregates were removed from the solution and oven dried to constant weight. Following this, the aggregates were subjected to five cycles of immersion and drying. The sodium sulfate solution was prepared by dissolving of $350 \mathrm{~g}$ of anhydrous sodium sulfate in one liter of water. Table 3.17 summarizes the ASTM test results using sodium sulfate solutions for RCA-MO and RCA-VA. As it can be seen in this table, the weighted percentage loss for RCA-MO and RCA-VA are $53.9 \%$ and $22.5 \%$, respectively.

Due to high value of the weighted percentage loss of RCA, and to confirm the results, the ASTM soundness test using sodium sulfate was also sent to an external (commercial) laboratory. Furthermore, the ASTM soundness test using magnesium sulfate solution was carried out for RCA-MO and RCA-VA in the same external laboratory. Table 3.18 summarizes soundness test results for RCA-MO and RCA-VA received from the external laboratory. It can be observed from Table 3.17 and 3.18 , there is a variation in the loss values using sodium sulfate solutions; this difference was attributed to the different laboratory conditions.

For RCA-MO tested with the ASTM method, the sodium sulfate losses were found to increase with increasing size of aggregate, reaching more than $40 \%$ loss in the case of the $12.7 \mathrm{~mm}$ fraction. The coefficient of variation between the three repeats for each size fractions ranged from about 3 to $13 \%$. Because of the much lower losses and the relatively high variability observed, significantly higher $\mathrm{CoV}$ were observed in the case 
of magnesium sulfate testing (up to $65 \%$ ).

In the case of the RCA-VA, the sodium sulfate losses for fractions 4.75 and $9.5 \mathrm{~mm}$ were found to range from 20 to $29 \%$, similar to those of the smallest $(4.75 \mathrm{~mm})$ fraction of RCA-MO; however, at the opposite of the RCA-MO, the losses observed for the RCAVA were found to decrease significantly (to $12-16 \%$ ) for the $12.7 \mathrm{~mm}$ fraction. The CoV between the three repeats for each size fractions ranged from about 7 to $16 \%$. Similar to the RCA-MO, the immersion in the magnesium sulfate solution induced small losses with the RCA-VA, which were not much different from one size fraction to another.

The weighted percentage losses for RCA-MO and RCA-VA using sodium sulfate solution were $34.9 \%$ and $19.8 \%$, respectively. The tests that were performed on RCAMO samples using magnesium sulfate solution yielded $2.1 \%$ total weighted percentage loss, which is approximately 16 times lower than the losses obtained from the tests that used sodium sulfate solution. The total weighted percentage loss for RCA-VA using magnesium sulfate solution is $1.5 \%$, which is approximately 13 times lower than the losses obtained from the tests that used sodium sulfate solution. The total weighted percentage loss of RCA-MO using magnesium sulfate solution is 1.4 times the percentage loss of RCA-VA. These observations contradict the common understanding about the effects of $\mathrm{Na}_{2} \mathrm{SO}_{4}$ and $\mathrm{MgSO}_{4}$ on the NA. This may be due to the presence of a protective layer of magnesium hydroxide (brucite) around the RCA particles, which can be more effectively damaged by applying mechanical stresses. Further investigation is necessary to fully explain this phenomenon; however, such an investigation is not within the scope of the present study. It should be noted that the allowable weighted percentage loss value specified by ASTM C 33-93 (ASTM, 2003) for sodium and magnesium sulfate 
solutions are $12 \%$ and $18 \%$, respectively. Therefore using sodium sulfate solution fail to satisfy the ASTM standards for both RCA-MO and RCA-VA. On the other hand, using magnesium sulfate solution for RCA-MO and RCA-VA satisfied the ASTM standard. These controversial results show that ASTM soundness test is not conclusive and require further investigation so that it is calibrated for RCA. In general, it can be concluded from the inconsistencies of the results of different methods that the determination of the soundness of RCA is a topic that requires further investigation.

\subsection{Unit Weight and Voids Content Tests}

Oven-dry unit weight of RCA-MO and RCA-VA in compacted (rodded) and loose (shoveled) conditions are determined using ASTM C29/C29M-91 (ASTM, 2003). Furthermore, the amount of voids is calculated for the compacted and loose condition. For this purpose, the RCA samples were prepared by combining 35\% (by weight) of 4.75 $\mathrm{mm}, 25 \%$ (by weight) of $9.5 \mathrm{~mm}$, and $40 \%$ (by weight) of $12.7 \mathrm{~mm}$ aggregates. Table 3.19 shows the compacted and loose unit weight and void for RCA-MO and RCA-VA. The average loose unit weight for RCA-MO and RCA-VA were $1268 \mathrm{~kg} / \mathrm{m}^{3}$ and 1359 $\mathrm{kg} / \mathrm{m}^{3}$, respectively. The average loose void content for RCA-MO and RCA-VA were found to be $45 \%$ and $44 \%$, respectively. The average compacted unit weight for RCAMO and RCA-VA were $1372 \mathrm{~kg} / \mathrm{m}^{3}$ and $1468 \mathrm{~kg} / \mathrm{m}^{3}$, respectively. Furthermore, the average compacted void content for RCA-MO and RCA-VA were found to be $40 \%$ and $39 \%$, respectively. In general, the average loose and compacted unit weight for RCA-VA is higher than those of RCA-MO. This can be attributed to the lower RMC of RCA-VA compared to that of RCA-MO. However, there was no significant difference between the average loose and compacted voids content for RCA-MO and RCA-VA. The standard 
deviation for the unit weight for RCA-MO and RCA-VA was found to be within in the limit of $30 \mathrm{~kg} / \mathrm{m}^{3}$ specified by the ASTMC29/C29M-91 (ASTM, 2003).

\subsection{Moisture Content Tests}

The moisture content of the coarse aggregate is used in the adjustment of the mixing water for GC (Section 4.5). The moisture content tests of the RCA-MO and RCAVA were performed using ASTM C 566-96 (ASTM, 2003). Three representative samples for each air dry fraction size of $4.75 \mathrm{~mm}, 9.5 \mathrm{~mm}$, and $12.7 \mathrm{~mm}$ were prepared and tested for moisture content. Table 3.20 summarizes the moisture content for air dry RCA-MO and RCA-VA fractions of $4.75 \mathrm{~mm}, 9.5 \mathrm{~mm}$, and $12.7 \mathrm{~mm}$. It can be seen from this table, the average moisture content for RCA-MO and RCA-VA were found to be $1.1 \%$ and $1.3 \%$, respectively.

\subsection{Properties of the Residual Mortar}

There is no standard to measure the specific gravity and absorption capacity of the residual mortar. Hence, the specific gravity and absorption capacity of the residual mortar were measured by Fathifazl (2007a) following ASTM C 128-97 (ASTM, 2003), the standard test method for absorption capacity and specific gravity for fine aggregates. Due to the specific nature of the residual mortar, the measurements were not expected to be accurate; however these tests were intended to provide a general idea about these properties of residual mortar.

The residual mortar samples were mostly obtained from a single size fraction of RCA (i.e., $4.75 \mathrm{~mm}$ ) by hand picking particles of mortar only. Other parts of the residual mortar samples that were tested as fine aggregates were prepared by breaking them off 
from the OVA that they were attached to. The removed residual mortar samples were dried in an oven (at $105^{\circ} \mathrm{C}$ ) to constant weight. The oven dry weights were recorded. After the residual mortar samples were immersed in water for 24 hours to achieve SSD conditions, the water film was removed from the residual mortar surface. Then, ASTM C128 test procedure was followed. Le Chatelier flask method was used to measure and calculated the specific gravity and absorption capacity of the residual mortar.

Tables 3.21 and 3.22 show the measured physical properties for residual mortar from RCA-MO and RCA-VA, respectively. In general, no significant difference was observed between the measured properties of the residual mortar of RCA-MO and RCAVA. The absorption capacity of the residual mortar of RCA-MO and RCA-VA are $9.97 \%$ and $10.08 \%$, respectively, showing no significant difference. The SSD specific gravity of the residual mortar of RCA-MO and RCA-VA were 2.31 and 2.32, respectively. The bulk specific gravity of the residual mortar of RCA-MO and RCA-VA were 2.10 and 2.11, respectively.

It should be noted that the bulk specific gravity of the residual mortar for RCA-MO and RCA-VA calculated by Eq.3.3 were 1.91 and 1.79, respectively. It is obvious from this that the measured and calculated residual mortar properties for RCA-MO and RCAVA show difference: the measured bulk specific gravity for residual mortar is higher than the calculated one. This can be attributed to the breaking of the voids in the residual mortar during sample preparation while separating them from the OVA particles, which would lead to the decrease of the volume of the residual mortar and would increase the bulk specific gravity. Furthermore, it was observed during the test that the residual mortar still partially contained OVA particles with higher specific gravity and low porosity. This 
also contributed to the observed increase in bulk specific gravity of the residual mortar using the ASTM C 128-97 method. As a result of these observations, it was decided that it is more appropriate to use the calculated residual mortar properties in this research.

\subsection{Summary and Discussion of Material Characterization Testing}

The summary of material characterization testing explained in this chapter is provided in Table 3.23. The RMC of RCA-MO and RCA-VA were found to be $41 \%$ and $23 \%$, respectively. The Los Angeles abrasion values were directly correlated with the RMC of the RCA. Actually, $42.0 \%$ and $24.5 \%$ loss values are numerically very close to the measured RMC values of RCA-MO $41 \%$ and RCA-VA $23 \%$ specimens, respectively. The abrasion value of RCA was lower than 50\%, the allowable abrasion value for coarse aggregates to be used in structural concrete specified by ASTM C33-93. The soundness losses for RCA-MO and RCA-VA using magnesium sulfate were found to be lower than the allowable limit of $18 \%$, specified by the ASTM standards.

The change in absorption capacity, specific gravity, and porosity are plotted against RMC in Figures 3.18 to 3.22, respectively. These relationships are plotted at 24-, 48- and 72-hours of water immersion. The data points that are accumulated around 40\% RMC are from RCA-MO and the ones around 25\% RMC are from RCA-VA samples. Data points corresponding to $0 \% \mathrm{RMC}$ are for OVA. As per Table 3.11, the absorption capacity, bulk specific gravity, SSD specific gravity, apparent specific gravity and porosity of virgin limestone aggregate were determined to be $0.34 \%, 2.70,2.72,2.65$, and 0.9 , respectively; the same properties for river-bed gravel were measured as $0.89 \%, 2.72,2.75,2.79$, and 2.4 , respectively.

It can be concluded from Figures 3.18 to 3.22 that the immersion period had no 
significant effect on the relationship between the measured property and the RMC of the RCA samples, which implies that the RCA become largely saturated within 24 hours. The absorption capacity and porosity plots presented in Figures 3.18 and 3.22 , respectively, illustrate this observation clearly. Specific gravity plots presented in Figure 3.19 to 3.21 do not provide any significant observation related to the effect of immersion time since these quantities vary within a very narrow range.

The empirical equations that are presented in Figures 3.18 to 3.22 correlate RCA properties to RMC, and they were obtained by regression analysis. It can be observed from these figures that there is a direct relationship between RCA properties and RMC. While the absorption capacity and porosity show a linear relationship, specific gravities are hyperbolically related to RMC. An interesting observation is that apparent specific gravity is only slightly affected by RMC. This can be attributed to the fact that apparent specific gravity does not include the presence of voids in its determination. When void space is not considered as part of specific gravity calculation, whether RCA has large RMC or not will not affect the results.

Figure 3.23 illustrates the relationship between soundness values measured using ASTM and MTO methods and RMC. From this figure, it can be observed that a relationship between the soundness and RMC cannot readily be established, especially when the ASTM method is used. As mentioned previously, the low correlation can be attributed to the testing methods, which are basically developed for NA.

Since each plot in Figures 3.18 to 3.22 are based on data obtained from two RCA sources and from OVA, the relationship between RCA properties and RMC observed in these figures is independent of the source; i.e. it can be stated that, for the two sources 
used in this study, the main factor affecting the RCA properties is the RMC, regardless of the source. Since this observation is valid for RCA obtained from two sources only, further investigation is necessary to verify this claim. To further study this relationship, a rational approach to correlate the $\mathrm{RCA}$ properties to the $\mathrm{RMC}$ is presented in the following section.

\subsection{Rational Approach to Material Characterization of RCA}

The experimental study presented in this chapter revealed that there is a direct relationship between most properties of RCA used in this study and the RMC. It was observed that while the absorption capacity and porosity show a linear relationship, specific gravities are hyperbolically related to RMC. Soundness measurements by ASTM and MTO methods did not correlate well with the RMC of the RCA investigated; however it was noted that these soundness test methods were developed for NA, which might be considered as a factor influencing the results. The relationships between the RCA properties and RMC have been shown to be independent of the source of RCA; i.e., for the two sources used in the study, the main factor affecting the RCA properties was found to be RMC, not the source. Since this observation is valid for the RCA obtained from two sources investigated, it was concluded that further investigation was necessary to verify this claim.

In addition to this goal, in order to determine in practice, without excessive laboratory testing, some key properties of RCA that are normally required in the design of GC mixtures made with RCA, it is beneficial to develop a rational approach that could be used to relate those properties to the RMC of a given RCA. In the following sections, the details of such an approach are presented. In the proposed approach, RCA is treated 
as a composite of the residual mortar and the OVA and the required relationships are developed for expressing certain properties of RCA in terms of the corresponding properties of its constituents. First, the approach is applied to relate the absorption capacity, specific gravity and porosity of RCA to its RMC and the results of the derived relationships are validated by the experimental data. After the validation, the same rational approach is extended to predict the abrasion value properties of RCA.

\subsubsection{The Development of the Rational Approach}

If the RCA is treated as a composite of the residual mortar and the OVA, a rational approach can be used to develop the relationships between RCA properties and RMC. In the following sections, these relationships will be derived for absorption capacity, specific gravity, porosity and Los Angeles abrasion value. Since there is currently no appropriate test to measure the soundness of RCA, this property is not related to RMC rationally.

\subsubsection{Absorption Capacity}

ASTM C127-88 (ASTM, 2003) defines the absorption capacity, AC (\%), of aggregates as:

$$
A C=\left(\frac{W_{S S D}-W_{O D}}{W_{O D}}\right) \times 100
$$

where, $W_{S S D}$ is the weight of the SSD aggregate test sample in air and $W_{\mathrm{OD}}$ is its OD weight in air. Based on Eq. 3.18, the absorption capacity of RCA, $A C^{R C A}$, of the OVA, $A C^{O V A}$, and of the residual mortar, $A C^{R M}$, can be written in the following format:

$$
A C^{i}=\left(\frac{W_{S S D}{ }^{i}-W_{O D}{ }^{i}}{W_{O D}{ }^{i}}\right) \times 100
$$


where $i$ is the placeholder for superscripts $R C A, O V A$ and $R M$. Note that for brevity, the absorption relationships are expressed as fraction rather than percent in the following expressions. Since RCA is considered to be a composite material, the following weight balance relationships can be written:

$$
\begin{aligned}
& W_{O D}^{O V A}+W_{O D}^{R M}=W_{O D}^{R C A} \\
& W_{S S D}^{O V A}+W_{S S D}^{R M}=W_{S S D}^{R C A}
\end{aligned}
$$

Eq.3.1 used to determine the RMC of RCA, can be written in fraction format as follows:

$$
R M C=\frac{W_{O D}^{R M}}{W_{O D}^{R C A}}
$$

For a unit weight of oven-dried RCA; i.e., $W_{O D}^{R C A}=1$ :

$$
\begin{gathered}
W_{O D}^{R M}=R M C \\
W_{O D}^{O V A}=1-R M C
\end{gathered}
$$

By subtracting Eq. 3.20 from Eq. 3.21 and using Eqs. 3.19, 3.23, and 3.24, the absorption capacity of RCA can be expressed as:

$$
A C^{R C A}=\left(A C^{R M}-A C^{O V A}\right) R M C+A C^{O V A}
$$

Equation 3.25 can be re-arranged to solve for $R M C$ as:

$$
R M C=\frac{A C^{R C A}-A C^{O V A}}{A C^{R M}-A C^{O V A}}
$$

Equation 3.26 indicates that the RMC of RCA can be indirectly determined by performing absorption tests on the RCA and its constituent components separately. 


\subsubsection{Bulk Specific Gravity}

According to ASTM C127-88 (ASTM, 2003), bulk specific gravity is defined as:

$$
S G_{b}^{i}=\frac{W_{O D}^{i}}{V^{i}}=\frac{W_{O D}{ }^{i}}{W_{S S D}{ }^{i}-W_{S S D-W}{ }^{i}}
$$

where $W_{S S D-W}$ is the weight of saturated test sample in water, $W_{S S D}$ is the weight of SSD test sample in air, and $V^{i}$ is the volume of the test sample. The superscript, $i$, is a placeholder for superscripts $R C A, R M$ or $O V A$.

The volume of RCA is the sum of the volumes of its constituents, viz.

$$
V^{O V A}+V^{R M}=V^{R C A}
$$

By combining, Eq. 3.27 and 3.28, and using Eqs. 3.23 and 3.24, $S G_{b}^{R C A}$ can be written as:

$$
\frac{1}{S G_{b}^{R C A}}=\left[\frac{1}{S G_{b}^{R M}}-\frac{1}{S G_{b}^{O V A}}\right] R M C+\frac{1}{S G_{b}^{O V A}}
$$

Equation 3.29 relates the specific gravity of RCA to its RMC and the specific gravities of the OVA and the residual mortar comprising RCA. Alternatively, Eq. 3.29 can be rearranged to yield RMC in terms of the specific gravities of RCA and its constituents as shown below:

$$
R M C=\frac{\left[\frac{1}{S G_{b}^{R C A}}-\frac{1}{S G_{b}^{O V A}}\right]}{\left[\frac{1}{S G_{b}^{R M}}-\frac{1}{S G_{b}^{O V A}}\right]}
$$




\subsubsection{SSD Specific Gravity}

According to ASTM C127-88 (ASTM Committee C09, 2003), SSD specific gravity can be determined as:

$$
S G_{S S D}=\frac{W_{S S D}}{V}=\frac{W_{S S D}}{W_{S S D}-W_{S S D-W}}
$$

Rearranging Eq. 3.18 gives:

$$
W_{S S D}^{i}=\left(1+A C^{i}\right) W_{O D}^{i}
$$

By combining Eqs. 3.27, 3.31 and 3.32, we can write:

$$
S G_{S S D}{ }^{i}=\left(1+A C^{i}\right) S G_{b}{ }^{i}
$$

By combining Eqs. 3.29, and 3.33, $S G_{S S D}^{R C A}$ can be derived as:

$$
\frac{1}{S G_{S S D}^{R C A}}=\left\{\frac{\left(\frac{1+A C^{R M}}{S G_{S S D}^{R M}}-\frac{1+A C^{O V A}}{S G_{S S D}^{O V A}}\right) R M C+\left(\frac{1+A C^{O V A}}{S G_{S S D}^{O V A}}\right)}{\left(A C^{R M}-A C^{O V A}\right) R M C+\left(1+A C^{O V A}\right)}\right\}
$$

Equation 3.34 can be rearranged to solve for RMC as:

$$
R M C=\left\{\frac{\left[\frac{1+A C^{R C A}}{S G_{S S D}^{R C A}}-\frac{1+A C^{O V A}}{S G_{S S D}^{O V A}}\right]}{\left[\frac{1+A C^{R M}}{S G_{S S D}^{R M}}-\frac{1+A C^{O V A}}{S G_{S S D}^{O V A}}\right]}\right\}
$$

\subsubsection{Apparent Specific Gravity}

According to ASTM C127-88, the apparent specific gravity, $S G_{a}$ is defined as:

$$
S G_{a}=\frac{W_{O D}}{W_{O D}-W_{S S D-W}}
$$

By using Eqs. 3.27, 3.32, and 3.36, the apparent specific gravity can be calculated as a function of bulk specific gravity and absorption capacity using: 


$$
A C=\left[\frac{1}{S G_{b}}-\frac{1}{S G_{a}}\right]
$$

By substituting Eqs. 3.25 and 3.29 in Eq. 3.37, the apparent specific gravity of $\mathrm{RCA}, S G_{a}^{R C A}$, can be expressed as:

$$
\frac{1}{S G_{a}^{R C A}}=\left[\frac{1}{S G_{a}^{R M}}-\frac{1}{S G_{a}^{O V A}}\right] R M C+\frac{1}{S G_{a}^{O V A}}
$$

By rearranging Eq. 3.38, RMC can be obtained as:

$$
R M C=\frac{\left[\frac{1}{S G_{a}^{R C A}}-\frac{1}{S G_{a}^{O V A}}\right]}{\left[\frac{1}{S G_{a}^{R M}}-\frac{1}{S G_{a}^{O V A}}\right]}
$$

\subsubsection{Porosity}

Porosity is defined in Eq.3.13 section 3.4 and will be rewritten in Eq.3.40 as:

$$
n^{i}=\frac{V_{V}^{i}}{V_{T}^{i}}
$$

where, $V_{v}^{i}$ is the volume of the voids; and $V_{T}$ is the total volume of the constituent materials of RCA, which can be written as:

$$
V_{T}^{i}=\frac{W_{O D}{ }^{i}}{S G_{b}^{i}}
$$

By combining Eqs. 3.23, 3.29, and 3.41, $n^{R C A}$ can be expressed as:

$$
n^{R C A}=\left\{\frac{\left[\left(\frac{n^{R M}}{S G_{b}^{R M}}-\frac{n^{\text {OVA }}}{S G_{b}^{\text {OVA }}}\right) R M C+\left(\frac{n^{\text {OVA }}}{S G_{b}^{\text {OVA }}}\right)\right]}{\left[\left(\frac{1}{S G_{b}^{R M}}-\frac{1}{S G_{b}^{\text {OVA }}}\right) R M C+\left(\frac{1}{S G_{b}^{\text {OVA }}}\right)\right]}\right\}
$$

Alternatively, by rearranging Eq. 3.42, RMC can be presented as: 


$$
R M C=\frac{\left[\frac{n^{R C A}}{S G_{b}^{R C A}}-\frac{n^{O V A}}{S G_{b}^{O V A}}\right]}{\left[\frac{n^{R M}}{S G_{b}^{R M}}-\frac{n^{O V A}}{S G_{b}^{O V A}}\right]}
$$

When the aggregates are in SSD condition, and the volume of voids assumed to be filled with water and is equal to the volume of water, Eq.3.12 can be used to find the porosity of the RCA. By substituting, Eqs.3.32 and 3.33 in Eq. 3.12 and rearranging the porosity can be found as follows:

$$
n^{i}=A C^{i} S G_{b}^{i}
$$

\subsubsection{Los Angeles Abrasion Value}

Since Los Angeles abrasion value is measured through a destructive test which is not based on measuring intrinsic properties of the aggregates, it is not possible to utilize a rational theoretical approach to relate it to RMC. However, if it is postulated that this relationship is similar to the relationships of absorption capacity, specific gravity or porosity with RMC, the following relationship can be proposed Los Angeles abrasion ( $L A T)$ value and the RMC:

$$
R M C=\frac{L A T^{R C A}-L A T^{O V A}}{L A T^{R M}-L A T^{O V A}}
$$

If one assumes that the $L A T$ value for mortar is 1 ( $100 \%$ loss), Eq. 3.45 can be rewritten as:

$$
R M C=\frac{L A T^{R C A}-L A T^{O V A}}{1-L A T^{O V A}}
$$

From the preceding relationships, it can be observed that certain physical properties of RCA can be written functions of the same properties of its constituents and of its RMC. Consequently, RMC plays an important role in the determination of the properties 
of RCA and its effect can be quantified using the relationships derived above.

\subsubsection{Comparison of the Rational Approach with Experimental Data}

In order to verify the applicability of the developed approach, the predictions from the equations are compared with experimental data. Firstly, the experimental data obtained from this study is used. This is followed by comparison with the experimental results obtained by Nagataki et al. (1999).

The comparisons with the present experimental results for RCA-MO and RCA-VA are shown in Figures 3.24 to 3.27 . The predicted absorption capacity in Figure 3.24 is based on Eq. 3.25; the predicted specific gravities in Figure 3.25 are based on Eqs. 3.29, 3.34 and 3.38; the predicted porosity in Figure 3.26 is based on Eq. 3.42; and the predicted Los Angeles abrasion value in Figure 3.27 is based on Eq. 3.46. It should be noted that the absorption capacity and specific gravity of the residual mortar that are required in these equations were estimated. The estimation for absorption capacity of residual mortar was carried out using the measured values of $R M C, A C^{R C A}$ and $A C^{O V A}$, and solving Eq. 3.25 for $A C^{R M}$ for each test sample. For the specific gravities of the residual mortar, they were estimated using the measured values of $R M C, S G^{R C A}$ and $\mathrm{SG}^{O V A}$, and solving Eqs. $3.30,3.35,3.38$ for $S G^{R M}$ for RCA-MO and RCA-VA. The values of $A C^{R M}$ and $S G^{R M}$ for RCA-MO and RCA-VA were used in plotting $A C$ vs. $R M C$ and $1 / S G$ vs. $R M C$ plots, given in Figures 3.24 and 3.25 , respectively.

As it can be seen in Figures 3.24 to 3.27 , there is a direct relationship between the predicted values of aforementioned properties and the RMC of both sources of RCA. The favorable comparison between the predicted and measured values validates the fundamental assumptions of the proposed approach and its accuracy. The lines that 
correspond to RCA-VA and RCA-MO are very close to each other with similar slopes, which also confirm that RMC is the major factor which affects the properties of RCA, and the source of a particular RCA is not as significant as its RMC. Practically, this finding diminishes the concern about the particular blend of concretes within an aggregate batch. In other words, variations in mortar content would have greater influence on the properties of RCA than variations in the specific properties of the mortar. However, more research is necessary to further confirm this statement, especially for concrete with pastes of very different composition.

Figures 3.28 and 3.29 , respectively, show the comparison of the absorption capacity and bulk specific gravity predictions of the rational approach with the experimental data from Nagataki et al. (1999). Four types of RCA are used for comparison: Nagataki-1, Nagataki-2, Nagataki-3, and Nagataki-4. Nagataki 1, 2, and 3 specimens refer to the air entrained specimens with low, medium and high w/c ratio, respectively. Nagataki-4 specimens are non-air entrained with medium w/c ratio. The residual mortar and OVA properties that are used in the rational approach are presented in Table 3.24. Since the residual mortar properties were not specified in the original paper, the absorption capacity and specific gravity values for the residual mortar were estimated using the recycled fine aggregate properties. Further information on this experimental study can be obtained from Nagataki et al. (1999) study. Based on the close agreement between the predicted and experimental values in Figures 3.28 and 3.29, it can be once again concluded that the rational approach predicts the RCA properties accurately. 
Table 3.1: Impurities in RCA-MO and RCA-VA

\begin{tabular}{|c|c|c|}
\hline \multirow{2}{*}{ Impurities } & \multicolumn{2}{|c|}{ Percentage by Weight } \\
\cline { 2 - 3 } & RCA-VA & RCA-MO \\
\hline Bitumen Coating & 2 & 0.01 \\
\hline Tiles & 0.07 & 0.00 \\
\hline Brick & 0.26 & 0.02 \\
\hline Wood & 0.04 & 0.01 \\
\hline Metal & 0.27 & - \\
\hline Other & 0.03 & 0.04 \\
\hline
\end{tabular}

Table 3.2: RMC for different fraction sizes of RCA-MO

\begin{tabular}{|c|c|c|c|c|c|c|c|c|c|c|c|c|}
\hline \multirow{3}{*}{$\begin{array}{c}\text { Sample } \\
\text { No. }\end{array}$} & \multicolumn{4}{|c|}{ Initial weight } & \multicolumn{4}{|c|}{ Final Weight } & \multicolumn{4}{|c|}{$\operatorname{RMC~(\% )~}$} \\
\hline & \multicolumn{4}{|c|}{ RCA-MO size (mm) } & \multicolumn{4}{|c|}{ "RCA-MO size (mm) } & \multicolumn{4}{|c|}{ RCA-MO size $(\mathrm{mm})$} \\
\hline & 4.75 & 9.5 & 12.7 & 19 & 4.75 & 9.5 & 12.7 & 19 & 4.75 & 9.5 & 12.7 & 19 \\
\hline 1 & 991 & 1973 & 1981 & 1974 & 581 & 1223 & 1223 & 1091 & 41 & 38 & 38 & 45 \\
\hline 2 & 978 & 1979 & 1989 & 1993 & 550 & 1216 & 1186 & 1107 & 44 & 39 & 40 & 44 \\
\hline 3 & 992 & 1975 & 1977 & 1982 & 577 & 1212 & 1196 & 1079 & 42 & 39 & 40 & 46 \\
\hline 4 & 983 & 1930 & 2104 & 1993 & 557 & 1209 & 1258 & 1082 & 43 & 37 & 40 & 46 \\
\hline 5 & 982 & 1968 & 1993 & 1992 & 561 & 1203 & 1191 & 1056 & 43 & 39 & 40 & 47 \\
\hline 6 & 972 & 1981 & 1986 & 1982 & 558 & 1219 & 1199 & 1042 & 43 & 38 & 40 & 47 \\
\hline 7 & 975 & 1982 & 1986 & 1990 & 562 & 1219 & 1186 & 1097 & 42 & 38 & 40 & 45 \\
\hline 8 & 971 & 1982 & 1981 & 1995 & 554 & 1195 & 1158 & 1093 & 43 & 40 & 42 & 45 \\
\hline 9 & 976 & 1973 & 1977 & 1996 & 561 & 1185 & 1177 & 1079 & 43 & 40 & 40 & 46 \\
\hline 10 & 979 & 1932 & 1951 & 1988 & 563 & 1156 & 1193 & 1092 & 42 & 40 & 39 & 45 \\
\hline Average & & & & & & & & & 43 & 39 & 40 & 46 \\
\hline Std. Dev. & & & & & & & & & 0.69 & 0.88 & 0.92 & 0.97 \\
\hline $\operatorname{CoV}(\%)$ & & & & & & & & & 1.6 & 2.3 & 2.3 & 2.1 \\
\hline
\end{tabular}


Table 3.3: RMC for different fraction sizes of RCA-VA

\begin{tabular}{|c|c|c|c|c|c|c|c|c|c|c|c|c|}
\hline \multirow{3}{*}{$\begin{array}{c}\text { Sample } \\
\text { No. }\end{array}$} & \multicolumn{4}{|c|}{ Initial weight } & \multicolumn{4}{|c|}{ Final Weight } & \multicolumn{4}{|c|}{$\overline{\mathrm{RMC}(\%)}$} \\
\hline & \multicolumn{4}{|c|}{ RCA-VA size $(\mathrm{mm})$} & \multicolumn{4}{|c|}{ RCA-VA size $(\mathrm{mm})$} & \multicolumn{4}{|c|}{ RCA-VA size $(\mathrm{mm})$} \\
\hline & 4.75 & 9.5 & 12.7 & 19 & 4.75 & 9.5 & 12.7 & 19 & 4.75 & 9.5 & 12.7 & 19 \\
\hline 1 & 1050 & 2173 & 2172 & 2039 & 756 & 1702 & 1743 & 1653 & 28 & 22 & 20 & 19 \\
\hline 2 & 1028 & 2040 & 2102 & 2083 & 766 & 1604 & 1672 & 1641 & 25 & 21 & 20 & 21 \\
\hline 3 & 1046 & 2060 & 2079 & 2034 & 771 & 1687 & 1612 & 1557 & 26 & 18 & 22 & 23 \\
\hline 4 & 1096 & 2045 & 2089 & 2060 & 842 & 1609 & 1649 & 1609 & 23 & 21 & 21 & 22 \\
\hline 5 & 1047 & 2002 & 2106 & 2137 & 767 & 1537 & 1641 & 1650 & 27 & 23 & 22 & 23 \\
\hline 6 & 1091 & 2018 & 2127 & 2107 & 835 & 1564 & 1691 & 1653 & 23 & 22 & 20 & 22 \\
\hline 7 & 1034 & 2106 & 2096 & 2293 & 749 & 1681 & 1686 & 1848 & 28 & 20 & 20 & 19 \\
\hline 8 & 1020 & 2096 & 2178 & 2121 & 741 & 1598 & 1721 & 1676 & 27 & 24 & 21 & 21 \\
\hline 9 & 1058 & 2061 & 2067 & 2028 & 797 & 1620 & 1608 & 1560 & 25 & 21 & 22 & 23 \\
\hline 10 & 1072 & 2159 & 2097 & 2020 & 782 & 1688 & 1647 & 1595 & 27 & 22 & 21 & 21 \\
\hline Average & & & & & & & & & 26 & 22 & 21 & 21 \\
\hline Std. Dev. & & & & & & & & & 1.71 & 1.58 & 1.01 & 1.48 \\
\hline $\operatorname{CoV}(\%)$ & & & & & & & & & 6.6 & 7.3 & 4.8 & 6.9 \\
\hline
\end{tabular}

Table 3.4: Mix proportions used for the manufacture of the concrete prisms for image analysis

\begin{tabular}{|c|c|c|c|c|}
\hline RCA size fractions & Water $(\mathbf{k g})$ & Cement $(\mathbf{k g})$ & $\begin{array}{c}\text { RCA } \\
(\mathbf{k g})\end{array}$ & w/c \\
\hline $12.7 \mathrm{~mm}$ & 1.3 & 3.8 & 4.60 & 0.35 \\
$9.5 \mathrm{~mm}$ & 1.3 & 3.8 & 3.60 & 0.35 \\
$4.75 \mathrm{~mm}$ & 1.3 & 3.8 & 2.60 & 0.35 \\
\hline
\end{tabular}


Table 3.5: Typical raw and processed data from image analysis (example from RCAMO,12.7 mm fraction)

\begin{tabular}{|c|c|c|c|c|c||c|}
\hline \multirow{2}{*}{$\begin{array}{c}\text { Particle } \\
\text { number }\end{array}$} & \multicolumn{3}{|c|}{ Area $\left(\mathbf{H m}^{2}\right)$} & \multicolumn{2}{c|}{$\begin{array}{c}\text { Weight } \\
\text { (Kg/unit thickness) }\end{array}$} & $\begin{array}{c}\text { RM } / \\
\text { Particle } \\
\text { \% }\end{array}$ \\
\cline { 2 - 6 } & RCA & OVA & RM & RM & OVA & 62 \\
\hline 1 & 236289600 & 70972048 & $1.65 \mathrm{E}+08$ & 0.315837 & 0.191837 & 62 \\
2 & 86775160 & 60326240 & 26401602 & 0.050454 & 0.163062 & 24 \\
4 & 71350568 & 21954020 & 49396544 & 0.094398 & 0.059342 & 61 \\
5 & 112561672 & 97988744 & 14572927 & 0.027849 & 0.264864 & 10 \\
6 & 20913096 & 5725079 & 15188018 & 0.029025 & 0.015475 & 65 \\
7 & 123254792 & 92642184 & 30612610 & 0.058502 & 0.250412 & 19 \\
8 & 68180480 & 58622912 & 9557569 & 0.018265 & 0.158458 & 10 \\
9 & 52424688 & 46368404 & 6056282 & 0.011574 & 0.125334 & 8 \\
10 & 52945148 & 46084516 & 6860632 & 0.013111 & 0.124566 & 10 \\
11 & 61698368 & 59663836 & 2034532 & 0.003888 & 0.161271 & 2 \\
12 & 55736716 & 29855574 & 25881140 & 0.04946 & 0.0807 & 38 \\
13 & 145729264 & 24083182 & $1.22 \mathrm{E}+08$ & 0.23247 & 0.065097 & 78 \\
14 & 76129352 & 38892684 & 37236668 & 0.07116 & 0.105127 & 40 \\
15 & 111000280 & 40170180 & 70830104 & 0.135359 & 0.10858 & 55 \\
16 & 29855574 & 27158638 & 2696938 & 0.005154 & 0.07341 & 7 \\
17 & 83131928 & 37094724 & 45895256 & 0.087707 & 0.100267 & 47 \\
18 & 149703712 & $1.14 \mathrm{E}+08$ & 35864540 & 0.068538 & 0.307707 & 18 \\
19 & 48734140 & 18641992 & 30092148 & 0.057507 & 0.050389 & 53 \\
20 & 104991320 & 95055232 & 9936087 & 0.018988 & 0.256934 & 7 \\
$\ldots$ & 141802144 & $1.3 \mathrm{E}+08$ & 11686731 & 0.022334 & 0.351702 & 6 \\
170 & $\ldots$ & $\ldots$ & $\ldots$ & $\ldots$ & $\ldots$ & $\ldots$ \\
171 & 108965752 & 75135744 & 33830008 & 0.06465 & 0.203092 & 24 \\
172 & 18358104 & 2933511 & 15424592 & 0.029477 & 0.007929 & 79 \\
173 & 67565392 & 26874748 & 40690640 & 0.077761 & 0.072642 & 52 \\
174 & 24792902 & 16654774 & 8138128 & 0.015552 & 0.045018 & 26 \\
175 & 35107508 & 9368310 & 25739196 & 0.049188 & 0.025323 & 66 \\
Total & 21007726 & 0 & 21007726 & 0.040146 & 0 & 100 \\
\hline
\end{tabular}


Table 3.6: Total area of RCA, OVA, residual mortar and RMC for RCA-MO and RCAVA (from image analysis work)

\begin{tabular}{|c|c|c|c|c|c|c|c|c|c|c|c|}
\hline \multirow{3}{*}{$\begin{array}{l}\text { Size } \\
(\mathbf{m m})\end{array}$} & \multirow{3}{*}{$\#$} & \multicolumn{5}{|c|}{ RCA-MO } & \multicolumn{5}{|c|}{ RCA-VA } \\
\hline & & \multicolumn{3}{|c|}{ Area $\times 10^{9}\left(\mu \mathrm{m}^{2}\right)$} & \multicolumn{2}{|c|}{$\begin{array}{l}\text { RMC } \\
(\%)^{*} \\
\end{array}$} & \multicolumn{3}{|c|}{ Area $\times 10^{9}\left(\mu \mathrm{m}^{2}\right)$} & \multicolumn{2}{|c|}{$\begin{array}{l}\text { RMC } \\
(\%)^{*}\end{array}$} \\
\hline & & RCA & OVA & $\mathbf{R M}$ & Wt. & Vol. & RCA & OVA & $\mathbf{R M}$ & Wt. & Vol. \\
\hline \multirow{3}{*}{4.75} & $\overline{1} 1$ & 99.37 & 4.50 & 4.87 & 44 & 52 & 10.5 & 6.49 & $\overline{44}$ & 30 & 38 \\
\hline & 2 & 8.96 & 4.58 & 4.38 & 40 & 49 & 10.1 & 6.49 & 3.58 & 27 & 36 \\
\hline & 3 & 8.97 & 4.21 & 4.75 & 45 & 53 & 10.9 & 6.49 & 4.36 & 33 & 40 \\
\hline \multicolumn{2}{|c|}{ Average } & & & & 43 & 51 & & & & 30 & 38 \\
\hline \multicolumn{2}{|c|}{ Std. Dev. } & & & & 2.5 & 2.2 & & & & 2.6 & 2.3 \\
\hline \multicolumn{2}{|c|}{$\operatorname{Cov}(\%)$} & & & & 6 & 4 & & & & 9 & 6 \\
\hline \multirow{3}{*}{9.50} & 1 & 14.2 & 7.32 & 6.89 & 39 & 48 & 13.3 & 9.64 & 3.66 & 20 & 27 \\
\hline & 2 & 14.4 & 7.72 & 6.7 & 37 & 46 & 14.1 & 9.73 & 4.32 & 23 & 31 \\
\hline & 3 & 14.1 & 7.15 & 6.94 & 40 & 49 & 14.2 & 10.2 & 4.02 & 21 & 28 \\
\hline \multicolumn{2}{|c|}{ Average } & & & & 39 & 48 & & & & 21 & 29 \\
\hline \multicolumn{2}{|c|}{ St. Dev. } & & & & 1.7 & 1.5 & & & & 1.8 & 1.7 \\
\hline \multicolumn{2}{|c|}{$\operatorname{Cov}(\%)$} & & & & 4 & 3 & & & & 9 & 6 \\
\hline \multirow{3}{*}{12.70} & 1 & 19.2 & 9.74 & 9.45 & 40 & $\overline{499}$ & 18.0 & 12.4 & 5.6 & 23 & 31 \\
\hline & 2 & 18.2 & 9.28 & 8.9 & 40 & 49 & 18.1 & 13.4 & 4.65 & 17 & 26 \\
\hline & 3 & 19.8 & 9.21 & 10.5 & 45 & 53 & 17.9 & 12.0 & 5.86 & 25 & 33 \\
\hline \multicolumn{2}{|c|}{ Average } & & & & 42 & 50 & & & & 21 & 30 \\
\hline \multicolumn{2}{|c|}{ St. Dev. } & & & & 2.9 & 2.4 & & & & 4.2 & 3.7 \\
\hline \multicolumn{2}{|c|}{$\operatorname{CoV}(\%)$} & & & & 7 & 5 & & & & 20 & 12 \\
\hline \multicolumn{3}{|c|}{ Weighted Average } & & & 42 & 50 & & & & 24 & 32 \\
\hline
\end{tabular}

*RMC, Wt. (by weight), Vol. (by volume) 
Table 3.7: Measured bulk specific gravities used for the calculation of RMC by image analysis

\begin{tabular}{|c|c|c|c|c|c|}
\hline \multicolumn{6}{|c|}{$\mathbf{S G}_{\mathbf{R C A}}$} \\
\hline & RCA-MO & & & RCA- & \\
\hline \multicolumn{6}{|c|}{ Fraction size $(\mathrm{mm})$} \\
\hline 4.75 & 9.5 & 12.7 & 4.75 & 9.5 & 12.7 \\
\hline 2.31 & 2.30 & 2.30 & 2.41 & 2.46 & 2.42 \\
\hline \multicolumn{6}{|c|}{ SGOVA* } \\
\hline \multicolumn{3}{|c|}{ Limestone } & \multicolumn{3}{|c|}{ River Gravel } \\
\hline \multicolumn{3}{|c|}{$\overline{2.70}$} & \multicolumn{3}{|c|}{2.72} \\
\hline
\end{tabular}

* The values for the OVA were obtained from various sources from the Vancouver area (for RCA-VA) and from limestone aggregate similar to RCA-MO that were available at CANMET laboratories (for RCA$\mathrm{MO})$.

Table 3.8: The RMC by image analysis and the quick test method

\begin{tabular}{|c||c|c|c||c||c|c|}
\hline \multirow{2}{*}{\multicolumn{1}{|c|}{ Sample }} & \multicolumn{3}{|c|}{ RCA-MO } & \multicolumn{3}{c|}{ RCA-VA } \\
\cline { 2 - 7 } & \multicolumn{5}{|c|}{ Fraction size (mm) } \\
\cline { 2 - 7 } & $\mathbf{4 . 7 5}$ & $\mathbf{9 . 5}$ & $\mathbf{1 2 . 7}$ & $\mathbf{4 . 7 5}$ & $\mathbf{9 . 5}$ & $\mathbf{1 2 . 7}$ \\
\cline { 2 - 7 } & \multicolumn{5}{|c|}{ Image Analysis } \\
\hline 1 & 44 & 39 & 40 & 30 & 20 & 23 \\
2 & 40 & 37 & 40 & 27 & 23 & 17 \\
3 & 45 & 40 & 45 & 32 & 21 & 25 \\
\hline Average & $\mathbf{4 3}$ & $\mathbf{3 9}$ & $\mathbf{4 2}$ & $\mathbf{3 0}$ & $\mathbf{2 1}$ & $\mathbf{2 2}$ \\
Std. Dev. & 2.5 & 1.7 & 2.9 & 2.6 & 1.8 & 4.2 \\
CoV (\%) & 6 & 4 & 7 & 9 & 9 & 20 \\
\hline \hline \multicolumn{6}{|c|}{ Quick Test Method } \\
\hline Average (n=10) & 43 & 39 & 40 & 26 & 22 & 21 \\
Std. Dev. & 0.7 & 0.9 & 0.9 & 1.7 & 1.6 & 1 \\
CoV (\%) & 2 & 2 & 2 & 7 & 7 & 5 \\
\hline
\end{tabular}


Table 3.9: Measured properties of RCA-MO after 24-hr, 48-hr and 72-hr immersion

\begin{tabular}{|c|c|c|c|c|c|c|c|c|c|c|c|c|c|c|c|c|}
\hline \multirow{3}{*}{$\begin{array}{l}\text { Size } \\
\text { mm }\end{array}$} & \multirow{3}{*}{ \# } & \multicolumn{5}{|c|}{ 24-hr } & \multicolumn{5}{|c|}{ 48-hr } & \multicolumn{5}{|c|}{ 72- hr } \\
\hline & & \multirow{2}{*}{$\begin{array}{c}\mathrm{AC} \\
\%\end{array}$} & \multicolumn{3}{|c|}{ SG } & \multirow{2}{*}{$\begin{array}{l}\mathrm{n} \\
\%\end{array}$} & \multirow{2}{*}{$\begin{array}{c}\mathrm{AC} \\
\%\end{array}$} & \multicolumn{3}{|c|}{ SG } & \multirow{2}{*}{$\begin{array}{l}\mathbf{n} \\
\%\end{array}$} & \multirow{2}{*}{$\begin{array}{c}\mathrm{AC} \\
\%\end{array}$} & \multicolumn{3}{|c|}{ SG } & \multirow{2}{*}{$\begin{array}{l}\mathrm{n} \\
\%\end{array}$} \\
\hline & & & "Bulk & SSD & App & & & Bulk & SSD & App & & & Bulk & SSD & App & \\
\hline \multirow{10}{*}{4.75} & 1 & 5.44 & 2.37 & 2.50 & 2.72 & 12.9 & 5.42 & 2.22 & 2.34 & 2.52 & 12.1 & 5.41 & 2.29 & 2.41 & 2.61 & 12.5 \\
\hline & 2 & 5.72 & 2.14 & 2.26 & 2.44 & 12.2 & 5.73 & 2.32 & 2.46 & 2.68 & 13.3 & 5.52 & 2.29 & 2.41 & 2.61 & 12.6 \\
\hline & 3 & 5.63 & 2.24 & 2.36 & 2.56 & 12.6 & 5.61 & 2.26 & 2.39 & 2.59 & 12.8 & 5.56 & 2.28 & 2.41 & 2.61 & 12.6 \\
\hline & 4 & 5.57 & 2.23 & 2.36 & 2.55 & 12.4 & 5.63 & 2.22 & 2.34 & 2.53 & 12.4 & 5.75 & 2.19 & 2.31 & 2.50 & 12.5 \\
\hline & 5 & 5.69 & 2.41 & 2.55 & 2.79 & 13.7 & 5.62 & 2.26 & 2.39 & 2.59 & 12.6 & 5.77 & 2.29 & 2.42 & 2.63 & 13.1 \\
\hline & 6 & 5.63 & 2.33 & 2.46 & 2.68 & 13.1 & 5.74 & 2.28 & 2.41 & 2.62 & 13.1 & 5.94 & 2.30 & 2.44 & 2.66 & 13.5 \\
\hline & 7 & 5.61 & 2.68 & 2.83 & 3.15 & 15.0 & 5.52 & 2.26 & 2.39 & 2.59 & 12.5 & 5.77 & 2.32 & 2.45 & 2.68 & 13.3 \\
\hline & 8 & 5.85 & 2.30 & 2.44 & 2.66 & 13.5 & 5.76 & 2.18 & 2.30 & 2.49 & 12.3 & 5.85 & 2.33 & 2.47 & 2.70 & 13.5 \\
\hline & 9 & 5.92 & 2.17 & 2.30 & 2.49 & 12.8 & 5.85 & 2.37 & 2.51 & 2.75 & 13.8 & 5.73 & 2.32 & 2.45 & 2.67 & 13.3 \\
\hline & 10 & 5.60 & 2.28 & 2.41 & 2.61 & 12.8 & 5.51 & 2.21 & 2.33 & 2.51 & 12.2 & 5.67 & 2.31 & 2.44 & 2.65 & 13.0 \\
\hline \multicolumn{2}{|c|}{ Average } & 5.67 & 2.32 & 2.45 & 2.67 & 13.1 & 5.63 & 2.26 & 2.39 & 2.59 & 12.7 & 5.66 & 2.29 & 2.42 & 2.63 & 13.0 \\
\hline \multicolumn{2}{|c|}{ Std. Dev. } & 0.13 & 0.15 & 0.15 & 0.19 & 0.77 & 0.12 & 0.06 & 0.06 & 0.08 & 0.55 & 0.13 & 0.04 & 0.04 & 0.06 & 0.41 \\
\hline \multicolumn{2}{|c|}{$\mathrm{CoV}(\%)$} & 2.3 & 6.5 & 6.1 & 7.1 & 5.9 & 2.12 & 2.52 & 2.60 & 3.14 & 4.35 & 2.26 & 1.75 & 1.78 & 2.11 & 3.15 \\
\hline \multirow{10}{*}{9.5} & 1 & 5.07 & 2.16 & 2.27 & 2.43 & 11.0 & 5.13 & 2.16 & 2.27 & 2.43 & 11.0 & 5.26 & 2.33 & 2.46 & 2.66 & 12.2 \\
\hline & 2 & 5.17 & 2.31 & 2.43 & 2.62 & 12.0 & 5.25 & 2.31 & 2.43 & 2.62 & 11.9 & 5.24 & 2.28 & 2.40 & 2.59 & 11.9 \\
\hline & 3 & 5.37 & 2.27 & 2.39 & 2.58 & 12.2 & 5.42 & 2.27 & 2.39 & 2.58 & 12.2 & 5.24 & 2.42 & 2.54 & 2.77 & 12.7 \\
\hline & 4 & 5.06 & 2.34 & 2.46 & 2.66 & 11.9 & 5.17 & 2.34 & 2.46 & 2.66 & 11.9 & 5.26 & 2.34 & 2.46 & 2.66 & 12.1 \\
\hline & 5 & 5.37 & 2.23 & 2.35 & 2.53 & 12.0 & 5.45 & 2.23 & 2.35 & 2.53 & 12.0 & 5.44 & 2.30 & 2.43 & 2.63 & 12.3 \\
\hline & 6 & 5.18 & 2.34 & 2.46 & 2.66 & 12.1 & 5.28 & 2.34 & 2.46 & 2.66 & 12.1 & 5.32 & 2.33 & 2.46 & 2.66 & 12.3 \\
\hline & 7 & 4.95 & 2.34 & 2.46 & 2.65 & 11.6 & 5.00 & 2.34 & 2.46 & 2.65 & 11.6 & 5.57 & 2.32 & 2.44 & 2.65 & 12.7 \\
\hline & 8 & 5.05 & 2.33 & 2.45 & 2.65 & 11.8 & 5.10 & 2.33 & 2.45 & 2.65 & 11.8 & 3.05 & 2.38 & 2.45 & 2.56 & 7.2 \\
\hline & 9 & 5.22 & 2.33 & 2.45 & 2.65 & 12.1 & 5.25 & 2.33 & 2.45 & 2.65 & 12.1 & 5.67 & 2.31 & 2.44 & 2.66 & 13.0 \\
\hline & 10 & 5.28 & 2.33 & 2.45 & 2.65 & 12.3 & 5.34 & 2.33 & 2.45 & 2.65 & 12.3 & 8.23 & 2.26 & 2.45 & 2.78 & 18.6 \\
\hline \multicolumn{2}{|c|}{ Average } & 5.20 & 2.30 & 2.42 & 2.61 & 11.9 & 5.20 & 2.30 & 2.42 & 2.61 & 11.9 & 5.40 & 2.33 & 2.45 & 2.66 & 12.5 \\
\hline Std. & & 0.14 & 0.06 & 0.06 & 0.08 & 0.38 & 0.14 & 0.06 & 0.06 & 0.08 & 0.38 & 1.23 & 0.04 & 0.04 & 0.07 & 2.71 \\
\hline $\mathrm{CoV}$ & & 2.7 & 2.6 & 2.5 & 3.1 & 3.2 & 2.72 & 2.64 & 2.60 & 2.90 & 3.23 & 2.94 & 1.93 & 1.50 & 2.55 & 2.65 \\
\hline & 1 & 4.91 & 2.20 & 2.31 & 2.47 & 10.8 & 5.14 & 2.34 & 2.46 & 2.65 & 11.9 & 5.43 & 2.32 & 2.45 & 2.65 & 12.4 \\
\hline & 2 & 5.10 & 2.32 & 2.44 & 2.63 & 11.8 & 5.26 & 2.31 & 2.43 & 2.63 & 12.1 & 5.65 & 2.30 & 2.43 & 2.65 & 13.0 \\
\hline & 3 & 5.06 & 2.35 & 2.47 & 2.67 & 11.9 & 5.18 & 2.33 & 2.44 & 2.64 & 11.8 & 5.53 & 2.31 & 2.44 & 2.65 & 12.8 \\
\hline & 4 & 5.30 & 2.27 & 2.40 & 2.59 & 12.1 & 5.36 & 2.26 & 2.38 & 2.56 & 12.0 & 5.67 & 2.30 & 2.43 & 2.64 & 12.9 \\
\hline 12.7 & 5 & 5.31 & 2.31 & 2.44 & 2.64 & 12.3 & 5.35 & 2.27 & 2.39 & 2.58 & 12.0 & 5.67 & 2.30 & 2.43 & 2.64 & 12.8 \\
\hline & 6 & 5.37 & 2.31 & 2.43 & 2.63 & 12.4 & 5.44 & 2.33 & 2.45 & 2.66 & 12.6 & 5.67 & 2.30 & 2.43 & 2.64 & 12.9 \\
\hline & 7 & 5.19 & 2.32 & 2.44 & 2.63 & 12.0 & 5.74 & 2.30 & 2.43 & 2.64 & 13.0 & 5.99 & 2.28 & 2.41 & 2.63 & 13.5 \\
\hline & 8 & 5.41 & 2.30 & 2.43 & 2.63 & 12.4 & 5.83 & 2.29 & 2.42 & 2.64 & 13.3 & 6.05 & 2.36 & 2.50 & 2.75 & 14.1 \\
\hline & 9 & 5.31 & 2.32 & 2.44 & 2.65 & 12.3 & 5.74 & 2.30 & 2.43 & 2.64 & 13.2 & 5.82 & 2.16 & 2.28 & 2.47 & 12.5 \\
\hline & 10 & 5.23 & 2.32 & 2.44 & 2.64 & 12.1 & 5.58 & 2.32 & 2.45 & 2.66 & 12.8 & 5.77 & 2.30 & 2.43 & 2.64 & 13.1 \\
\hline Ave & & $\overline{5.22}$ & 2.30 & 2.42 & 2.62 & 12.0 & 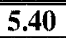 & 2.30 & 2.43 & 2.63 & 12.5 & 5.67 & 2.29 & 2.42 & 2.64 & 13.0 \\
\hline Std. & ev. & 0.16 & 0.04 & $\overline{0.04}$ & 0.06 & 0.47 & 0.26 & 0.03 & $\overline{0.03}$ & $\overline{\overline{0.03}}$ & 0.58 & 0.19 & 0.05 & 0.06 & 0.07 & 0.48 \\
\hline $\mathrm{CoV}$ & & 3.1 & 1.7 & 1.7 & 2.3 & 3.9 & 4.9 & 1.1 & 1.1 & 1.2 & 4.7 & 3.4 & 2.3 & 2.3 & $2 . \overline{2.6}$ & 3.7 \\
\hline
\end{tabular}


Table 3.10: Measured properties of RCA-VA after 24-hr, 48-hr and 72-hr immersion

\begin{tabular}{|c|c|c|c|c|c|c|c|c|c|c|c|c|c|c|c|c|}
\hline \multirow{3}{*}{$\begin{array}{l}\text { Size } \\
\text { mm }\end{array}$} & \multirow{3}{*}{$\#$} & \multicolumn{5}{|c|}{ 24-hr } & \multicolumn{5}{|c|}{ 48-hr } & \multicolumn{5}{|c|}{ 72-hr } \\
\hline & & \multirow{2}{*}{$\begin{array}{c}\mathbf{A C} \\
\%\end{array}$} & \multicolumn{3}{|c|}{ SG } & \multirow{2}{*}{$\begin{array}{l}\mathbf{n} \\
\%\end{array}$} & \multirow{2}{*}{$\begin{array}{c}\mathbf{A C} \\
\%\end{array}$} & \multicolumn{3}{|c|}{ SG } & \multirow{2}{*}{ n } & \multirow{2}{*}{$\begin{array}{c}\mathbf{A C} \\
\%\end{array}$} & \multicolumn{3}{|c|}{ SG } & \multirow{2}{*}{$\begin{array}{l}\mathbf{n} \\
\%\end{array}$} \\
\hline & & & Bulk & SSD & App & & & Bulk & SSD & App & & & Bulk & SSD & App & \\
\hline \multirow{10}{*}{4.75} & 1 & 3.91 & 2.43 & 2.53 & 2.69 & 9.5 & 4.11 & 2.44 & 2.54 & 2.71 & 10.0 & 3.94 & 2.41 & 2.50 & 2.66 & 9.4 \\
\hline & 2 & 3.73 & 2.46 & 2.55 & 2.70 & 9.1 & 3.82 & 2.42 & 2.52 & 2.67 & 9.2 & 3.87 & 2.42 & 2.51 & 2.67 & 9.2 \\
\hline & 3 & 3.75 & 2.41 & 2.50 & 2.64 & 8.9 & 3.93 & 2.44 & 2.53 & 2.70 & 9.6 & 3.84 & 2.31 & 2.40 & 2.54 & 8.8 \\
\hline & 4 & 3.33 & 2.47 & 2.55 & 2.69 & 8.1 & 3.42 & 2.49 & 2.57 & 2.71 & 8.4 & 3.52 & 2.44 & 2.52 & 2.67 & 8.4 \\
\hline & 5 & 3.82 & 2.41 & 2.50 & 2.65 & 9.2 & 3.85 & 2.43 & 2.53 & 2.68 & 9.3 & 3.76 & 2.41 & 2.50 & 2.64 & 8.9 \\
\hline & 6 & 3.64 & 2.41 & 2.49 & 2.63 & 8.6 & 3.62 & 2.46 & 2.55 & 2.70 & 8.8 & 3.52 & 2.38 & 2.46 & 2.60 & 8.3 \\
\hline & 7 & 3.96 & 2.44 & 2.53 & 2.69 & 9.4 & 4.05 & 2.47 & 2.57 & 2.74 & 9.8 & 3.95 & 2.41 & 2.50 & 2.66 & 9.3 \\
\hline & 8 & 3.85 & 2.36 & 2.45 & 2.60 & 9.0 & 3.87 & 2.37 & 2.46 & 2.61 & 9.1 & 3.77 & 2.40 & 2.49 & 2.63 & 8.9 \\
\hline & 9 & 3.59 & 2.50 & 2.59 & 2.74 & 8.7 & 3.75 & 2.39 & 2.48 & 2.63 & 8.8 & 3.58 & 2.56 & 2.65 & 2.81 & 8.9 \\
\hline & 10 & 3.86 & 2.22 & 2.31 & 2.43 & 8.5 & 3.83 & 2.43 & 2.52 & 2.68 & 9.3 & 3.85 & 2.43 & 2.53 & 2.68 & 9.3 \\
\hline \multicolumn{2}{|c|}{ Average } & 3.72 & 2.41 & 2.50 & 2.65 & 8.9 & 3.80 & 2.44 & 2.53 & 2.68 & 9.2 & 3.72 & 2.42 & 2.51 & 2.65 & 9.0 \\
\hline \multicolumn{2}{|c|}{ Std. Dev. } & 0.18 & 0.07 & 0.07 & 0.08 & 0.41 & 0.20 & 0.03 & 0.03 & 0.04 & 0.48 & 0.17 & 0.06 & 0.06 & 0.07 & 0.37 \\
\hline \multicolumn{2}{|c|}{$\mathrm{CoV}(\%)$} & 4.9 & 2.9 & 2.8 & 3.0 & 4.6 & 5.4 & 1.4 & 1.4 & 1.5 & 5.2 & 4.5 & 2.5 & 2.5 & 2.6 & 4.1 \\
\hline \multirow{10}{*}{9.5} & 1 & 3.14 & 2.40 & 2.48 & 2.59 & 7.4 & 3.37 & 2.42 & 2.50 & 2.62 & 7.9 & 3.22 & 2.52 & 2.60 & 2.74 & 8.1 \\
\hline & 2 & 3.07 & 2.55 & 2.63 & 2.76 & 7.6 & 3.15 & 2.41 & 2.49 & 2.61 & 7.6 & 3.05 & 2.52 & 2.60 & 2.73 & 7.6 \\
\hline & 3 & 2.96 & 2.60 & 2.67 & 2.81 & 7.5 & 3.04 & 2.47 & 2.54 & 2.66 & 7.4 & 2.95 & 2.47 & 2.55 & 2.67 & 7.2 \\
\hline & 4 & 3.24 & 2.47 & 2.55 & 2.68 & 7.8 & 3.33 & 2.41 & 2.48 & 2.61 & 7.9 & 3.38 & 2.46 & 2.54 & 2.67 & 8.0 \\
\hline & 5 & 3.24 & 2.44 & 2.52 & 2.65 & 7.9 & 3.47 & 2.41 & 2.49 & 2.63 & 8.2 & 3.35 & 2.46 & 2.55 & 2.68 & 8.2 \\
\hline & 6 & 3.56 & 2.46 & 2.54 & 2.68 & 8.5 & 3.54 & 2.41 & 2.49 & 2.63 & 8.4 & 3.43 & 2.43 & 2.52 & 2.66 & 8.3 \\
\hline & 7 & 3.33 & 2.41 & 2.49 & 2.62 & 7.9 & 3.28 & 2.39 & 2.47 & 2.59 & 7.8 & 3.21 & 2.45 & 2.53 & 2.65 & 7.8 \\
\hline & 8 & 3.56 & 2.49 & 2.58 & 2.73 & 8.8 & 3.53 & 2.43 & 2.51 & 2.66 & 8.6 & 3.44 & 2.44 & 2.53 & 2.67 & 8.4 \\
\hline & 9 & 3.38 & 2.39 & 2.47 & 2.60 & 7.9 & 3.25 & 2.47 & 2.55 & 2.68 & 8.0 & 3.26 & 2.47 & 2.55 & 2.68 & 8.0 \\
\hline & 10 & 3.25 & 2.34 & 2.41 & 2.53 & 7.6 & 3.29 & 2.38 & 2.46 & 2.58 & 7.8 & 3.38 & 2.41 & 2.49 & 2.61 & 7.9 \\
\hline \multicolumn{2}{|c|}{ Average } & 3.22 & 2.46 & 2.53 & 2.67 & 7.9 & 3.30 & 2.42 & 2.50 & 2.63 & 8.0 & 3.24 & 2.46 & 2.54 & 2.68 & 8.0 \\
\hline Std. & & 0.19 & 0.08 & 0.08 & 0.09 & 0.44 & 0.15 & 0.03 & 0.03 & 0.03 & 0.35 & 0.16 & 0.03 & 0.03 & 0.04 & 0.35 \\
\hline $\mathrm{CoV}$ & & 5.9 & 3.3 & 3.1 & 3.4 & 5.6 & 4.6 & 1.2 & 1.2 & 1.2 & 4.4 & 5.0 & 1.4 & 1.3 & 1.3 & 4.4 \\
\hline & 1 & 2.83 & 2.33 & 2.40 & 2.50 & 6.6 & 2.94 & 2.41 & 2.48 & 2.59 & 7.0 & 2.99 & 2.50 & 2.57 & 2.69 & 7.2 \\
\hline & 2 & 3.06 & 2.45 & 2.53 & 2.65 & 7.4 & 3.16 & 2.41 & 2.49 & 2.61 & 7.6 & 3.07 & 2.46 & 2.53 & 2.66 & 7.5 \\
\hline & 3 & 3.24 & 2.31 & 2.38 & 2.49 & 7.3 & 3.24 & 2.42 & 2.50 & 2.63 & 7.8 & 3.24 & 2.44 & 2.52 & 2.65 & 7.7 \\
\hline & 4 & 3.08 & 2.48 & 2.56 & 2.68 & 7.5 & 3.06 & 2.44 & 2.51 & 2.63 & 7.4 & 3.04 & 2.50 & 2.57 & 2.70 & 7.5 \\
\hline 12.7 & 5 & 3.07 & 2.44 & 2.51 & 2.63 & 7.4 & 3.04 & 2.48 & 2.55 & 2.68 & 7.5 & 3.02 & 2.46 & 2.54 & 2.66 & 7.5 \\
\hline & 6 & 3.03 & 2.40 & 2.47 & 2.58 & 7.1 & 3.08 & 2.46 & 2.54 & 2.66 & 7.3 & 3.07 & 2.46 & 2.53 & 2.65 & 7.3 \\
\hline & 7 & 2.96 & 2.46 & 2.53 & 2.65 & 7.1 & 3.06 & 2.43 & 2.50 & 2.62 & 7.2 & 2.95 & 2.48 & 2.55 & 2.67 & 7.2 \\
\hline & 8 & 3.08 & 2.43 & 2.51 & 2.62 & 7.2 & 3.02 & 2.48 & 2.55 & 2.68 & 7.5 & 3.03 & 2.46 & 2.54 & 2.66 & 7.3 \\
\hline & 9 & 3.14 & 2.42 & 2.49 & 2.61 & 7.5 & 3.16 & 2.45 & 2.52 & 2.65 & 7.7 & 3.16 & 2.50 & 2.57 & 2.70 & 7.7 \\
\hline & 10 & 3.07 & 2.47 & 2.54 & 2.66 & 7.4 & 3.08 & 2.46 & 2.54 & 2.66 & 7.4 & 3.09 & 2.47 & 2.55 & 2.67 & 7.3 \\
\hline Aver & & 3.00 & 2.42 & 2.49 & 2.61 & 7.3 & 3.00 & 2.44 & 2.52 & 2.64 & 7.4 & 3.01 & 2.47 & 2.55 & 2.67 & 7.4 \\
\hline Std. & & 0.11 & 0.06 & 0.06 & 0.07 & 0.27 & 0.10 & 0.03 & 0.03 & 0.03 & 0.24 & 0.09 & 0.02 & 0.02 & 0.02 & 0.19 \\
\hline $\mathrm{CoV}$ & & 3.7 & 2.5 & 2.4 & 2.7 & 3.7 & 3.3 & 1.0 & 1.0 & 1.1 & 3.2 & 2.8 & 0.8 & 0.8 & 0.8 & 2.6 \\
\hline
\end{tabular}


Table 3.11: Averaged properties of RCA-MO and RCA-VA specimens

\begin{tabular}{|c|c|c|c|c|c|}
\hline \multirow{2}{*}{$\begin{array}{c}\text { Coarse } \\
\text { Aggregate }\end{array}$} & \multirow{2}{*}{ AC (\%) } & \multicolumn{3}{|c|}{ SG } & \multirow{2}{*}{$\begin{array}{c}\text { n } \\
\text { (\%) }\end{array}$} \\
\cline { 3 - 6 } & & Bulk & SSD & App. & \\
\hline RCA-MO & 5.36 & 2.31 & 2.42 & 2.64 & 12.3 \\
\hline RCA-VA & 3.30 & 2.42 & 2.50 & 2.64 & 8.1 \\
\hline Limestone & 0.34 & 2.70 & 2.72 & 2.65 & 0.9 \\
\hline Gravel & 0.89 & 2.72 & 2.75 & 2.79 & 2.4 \\
\hline
\end{tabular}

Table 3.12: Los Angeles abrasion values for RCA-MO and RCA-VA samples

\begin{tabular}{|c|c|c|c|c|c|c|}
\hline \multirow{3}{*}{ Sample } & \multicolumn{3}{|c|}{ RCA-MO } & \multicolumn{3}{|c|}{ RCA-VA } \\
\hline & \multicolumn{2}{|c|}{ Weight (g) } & \multirow[t]{2}{*}{ \% Loss } & \multicolumn{2}{|c|}{ Weight (g) } & \multirow[t]{2}{*}{ \% Loss } \\
\hline & Original & Final & & Original & Final & \\
\hline 1 & 5000 & 2900 & 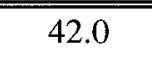 & 5003 & 3773 & 24.6 \\
\hline 2 & 5003 & 2863 & 42.8 & 5004 & 3777 & 24.5 \\
\hline 3 & 5001 & 2945 & 41.1 & 5004 & 3777 & 24.5 \\
\hline \multirow{2}{*}{\multicolumn{3}{|c|}{$\begin{array}{l}\text { Average } \\
\text { Std. Dev. }\end{array}$}} & 42.0 & & & 24.5 \\
\hline & & & 0.83 & & & 0.04 \\
\hline \multicolumn{3}{|c|}{$\operatorname{CoV}(\%)$} & 2 & & & 1.5 \\
\hline
\end{tabular}


Table 3.13: Weight of the control aggregates on individual sieves as function of time in the MTO Soundness Test LS-614

\begin{tabular}{|c|c|c|c|c|c|c|c|c|c|}
\hline \multirow{2}{*}{ Sample } & \multirow{2}{*}{ Sieve $(\mathbf{m m})$} & \multirow{2}{*}{ Initial Weight (g) } & \multicolumn{7}{|c|}{ Time (min.) } \\
\hline & & & 0.33 & 0.67 & 1 & 2 & 3 & 4 & 5 \\
\hline \multirow{3}{*}{1} & 12.7 & 1250 & 1087 & 1015 & 985 & 934 & 902 & 887 & 857 \\
\hline & 9.5 & 1001 & 795 & 747 & 719 & 685 & 649 & 620 & 599 \\
\hline & 4.75 & 500 & 387 & 365 & 317 & 303 & 293 & 280 & 255 \\
\hline \multirow{3}{*}{2} & 12.7 & 1252 & 1122 & 1064 & 1022 & 937 & 872 & 814 & 780 \\
\hline & 9.5 & 1001 & 791 & 736 & 691 & 659 & 626 & 607 & 586 \\
\hline & 4.75 & 500 & 413 & 382 & 366 & 348 & 338 & 332 & 325 \\
\hline \multirow{3}{*}{3} & 12.7 & 1250 & 1065 & 1002 & 968 & 922 & 862 & 839 & 809 \\
\hline & 9.5 & 1000 & 802 & 740 & 706 & 660 & 620 & 586 & 561 \\
\hline & 4.75 & 500 & 417 & 399 & 385 & 366 & 356 & 330 & 309 \\
\hline
\end{tabular}

Table 3.14: Cumulative percentage loss for the control aggregates on individual sieves in the MTO Soundness Test LS-614

\begin{tabular}{|c|c|c|c|c|c|c|c|c|}
\hline \multirow{3}{*}{ Sample } & \multirow{2}{*}{ Sieve $(\mathbf{m m})$} & \multicolumn{7}{|c|}{ Time (min.) } \\
\cline { 3 - 9 } & & $\mathbf{0 . 3 3}$ & $\mathbf{0 . 6 7}$ & $\mathbf{1}$ & $\mathbf{2}$ & $\mathbf{3}$ & $\mathbf{4}$ & $\mathbf{5}$ \\
\hline \hline \multirow{3}{*}{1} & 12.7 & 13.0 & 18.8 & 21.2 & 25.3 & 27.8 & 29.0 & 31.4 \\
& 9.5 & 20.6 & 25.4 & 28.2 & 31.6 & 35.2 & 38.1 & 40.2 \\
& 4.75 & 22.6 & 27.0 & 36.6 & 39.4 & 41.4 & 44.0 & 49.0 \\
\hline \multirow{3}{*}{2} & 12.7 & 10.4 & 15.0 & 18.4 & 25.2 & 30.4 & 35.0 & 37.7 \\
& 9.5 & 21.0 & 26.5 & 31.0 & 34.2 & 37.5 & 39.4 & 41.5 \\
& 4.75 & 17.4 & 23.6 & 26.8 & 30.4 & 32.4 & 33.6 & 35.0 \\
\hline \multirow{3}{*}{3} & 12.7 & 14.8 & 19.8 & 22.6 & 26.2 & 31.0 & 32.9 & 35.3 \\
& 9.5 & 19.8 & 26.0 & 29.4 & 34.0 & 38.0 & 41.4 & 43.9 \\
& 4.75 & 16.6 & 20.2 & 23.0 & 26.8 & 28.8 & 34.0 & 38.2 \\
\hline
\end{tabular}


Table 3.15: Average weighted freeze-and-thaw loss for control aggregates in the MTO Soundness Test LS-614

\begin{tabular}{|c|c|c|c||c|}
\hline \multirow{2}{*}{ Time (min) } & \multicolumn{3}{|c|}{ Sample } & \multirow{2}{*}{ Average } \\
\cline { 2 - 4 } & $\mathbf{1}$ & $\mathbf{2}$ & $\mathbf{3}$ & \\
\hline 0.33 & 17.7 & 15.3 & 16.8 & 16.6 \\
\hline 0.67 & 22.8 & 20.6 & 21.8 & 21.7 \\
\hline 1 & 27.1 & 24.3 & 24.7 & 25.4 \\
\hline 2 & 30.7 & 29.2 & 28.7 & 29.5 \\
\hline 3 & 33.4 & 33.0 & 32.6 & 33.0 \\
\hline 4 & 35.5 & 36.0 & 35.7 & 35.7 \\
\hline 5 & 38.4 & 38.2 & 38.6 & 38.4 \\
\hline
\end{tabular}

Table 3.16: MTO Soundness Test LS-614 results for the RCA-MO and RCA-VA

\begin{tabular}{|c|c|c|c|c|c|c|c|c|c|}
\hline \multirow{3}{*}{ Sample \# } & \multicolumn{3}{|c|}{ Initial Weight } & \multicolumn{3}{|c|}{ Final Weight } & \multicolumn{3}{|c|}{$(\%)$ loss } \\
\hline & \multicolumn{3}{|c|}{ RCA-MO size (mm) } & \multicolumn{3}{|c|}{ RCA-MO size $(\mathrm{mm})$} & \multicolumn{3}{|c|}{ RCA-MO size (mm) } \\
\hline & 4.75 & 9.5 & 12.7 & 4.75 & 9.5 & 12.7 & 4.75 & 9.5 & 12.7 \\
\hline 1 & 501 & 1001 & 1251 & 397 & 690 & 929 & 20.8 & 31.1 & 25.7 \\
\hline 2 & 501 & 1001 & 1250 & 384 & 722 & 914 & 23.4 & 27.9 & 26.9 \\
\hline 3 & 501 & 1002 & 1252 & 369 & 708 & 871 & 26.3 & 29.3 & 30.4 \\
\hline \multicolumn{7}{|c|}{ Average } & 23.5 & 29.4 & 27.7 \\
\hline \multicolumn{7}{|c|}{ Std. Dev. } & 2.80 & 1.60 & 2.45 \\
\hline \multicolumn{7}{|c|}{$\operatorname{CoV}(\%)$} & 11.9 & 5.4 & 8.8 \\
\hline \multicolumn{7}{|c|}{ Weighted Average } & \multicolumn{3}{|c|}{26.7} \\
\hline \multirow{2}{*}{ Sample \# } & \multicolumn{3}{|c|}{ RCA-VA size (mm) } & \multicolumn{3}{|c|}{ RCA-VA size (mm) } & \multicolumn{3}{|c|}{ RCA-VA size $(\mathrm{mm})$} \\
\hline & 4.75 & 9.5 & 12.7 & 4.75 & 9.5 & 12.7 & 4.75 & 9.5 & $\overline{12.7}$ \\
\hline 1 & 500 & 1000 & 1252 & 425 & 798 & 1063 & 15.0 & 20.2 & 15.1 \\
\hline 2 & 500 & 1000 & 1250 & 427 & 797 & 1054 & 14.6 & 20.3 & 15.7 \\
\hline 3 & 500 & 1001 & 1251 & 417 & 820 & 1098 & 16.6 & 18.1 & 12.2 \\
\hline \multicolumn{7}{|c|}{ Average } & 15.4 & 19.5 & 14.3 \\
\hline \multicolumn{7}{|c|}{ Std. Dev. } & 1.06 & 1.25 & 1.85 \\
\hline \multicolumn{7}{|c|}{$\operatorname{CoV}(\%)$} & 6.9 & 6.4 & 12.9 \\
\hline \multicolumn{7}{|c|}{ Weighted Average } & \multicolumn{3}{|c|}{16.0} \\
\hline
\end{tabular}


Table 3.17: Results for ASTM soundness test using $\mathrm{Na}_{2} \mathrm{SO}_{4}$ for RCA-MO and RCA-VA

\begin{tabular}{|c|c|c|c|c|c|c|c|c|c|c|c|c|}
\hline \multirow{3}{*}{ Sample \# } & \multicolumn{4}{|c|}{ Initial Weight (g) } & \multicolumn{4}{|c|}{ Final Weight (g) } & \multicolumn{4}{|c|}{ Loss $(\%)$} \\
\hline & \multicolumn{4}{|c|}{ RCA-MO size (mm) } & \multicolumn{4}{|c|}{ RCA-MO size (mm) } & \multicolumn{4}{|c|}{ RCA-MO size (mm) } \\
\hline & 4.75 & 9.5 & 12.7 & 19 & 4.75 & 9.5 & 12.7 & 19 & 4.75 & 9.5 & 12.7 & 19 \\
\hline 1 & 302 & 331 & 678 & 508 & 151 & 144 & 279 & 93 & 50.0 & 56.5 & 58.8 & 81.7 \\
\hline 2 & 299 & 331 & 676 & 513 & 144 & 128 & 300 & 82 & 51.8 & 61.3 & 55.6 & 84.0 \\
\hline 3 & 300 & 331 & 671 & 509 & 155 & 147 & 334 & 117 & 48.3 & 55.6 & 50.2 & 77.0 \\
\hline \multicolumn{9}{|c|}{ Average } & 50.1 & 57.8 & 54.9 & 80.9 \\
\hline \multicolumn{9}{|c|}{ Weighted Average } & \multicolumn{3}{|c|}{53.9} & \\
\hline \multirow{2}{*}{ Sample \# } & \multicolumn{4}{|c|}{ RCA-VA size (mm) } & \multicolumn{4}{|c|}{ RCA-VA size (mm) } & \multicolumn{4}{|c|}{ RCA-VA size (mm) } \\
\hline & 4.75 & 9.5 & 12.7 & 19 & 4.75 & 9.5 & 12.7 & 19 & 4.75 & 9.5 & 12.7 & 19 \\
\hline 1 & 332 & 331 & 668 & 551 & 232 & 211 & 469 & 145 & 30.1 & 36.3 & 29.8 & 73.7 \\
\hline 2 & 331 & 332 & 676 & 503 & 249 & 227 & 471 & 183 & 24.8 & 31.6 & 30.3 & 63.6 \\
\hline 3 & 331 & 331 & 677 & 518 & 221 & 236 & 417 & 255 & 33.2 & 28.7 & 38.4 & 50.8 \\
\hline \multicolumn{9}{|c|}{ Average } & 29.4 & 32.2 & 32.8 & 62.7 \\
\hline \multicolumn{9}{|c|}{ Weighted Average } & & 22.5 & & \\
\hline
\end{tabular}


Table 3.18: Soundness values for RCA-MO and RCA-VA samples from the external laboratory

\begin{tabular}{|c|c|c|c|c|c|}
\hline \multirow{3}{*}{$\begin{array}{c}\text { RCA-size } \\
\text { (mm) }\end{array}$} & \multirow{3}{*}{$\underset{\#}{\text { Sample }}$} & \multicolumn{2}{|c|}{ RCA-MO } & \multicolumn{2}{|c|}{ RCA-VA } \\
\hline & & \multicolumn{2}{|c|}{ ASTM } & \multicolumn{2}{|c|}{ ASTM } \\
\hline & & $\mathrm{Na}_{2} \mathrm{SO}_{4}$ & $\mathrm{MgSO}_{4}$ & $\mathrm{Na}_{2} \mathrm{SO}_{4}$ & $\mathrm{MgSO}_{4}$ \\
\hline \multirow{3}{*}{4.75} & 1 & 24.9 & 4.3 & 22.1 & 1.3 \\
\hline & 2 & 23.8 & 3.4 & 20.7 & 1.6 \\
\hline & 3 & 25.5 & 2.1 & 23.7 & 1.7 \\
\hline \multicolumn{2}{|c|}{ Average } & 24.7 & 3.3 & 22.2 & 1.5 \\
\hline \multicolumn{2}{|c|}{ Std. Dev. } & 0.86 & 1.11 & 1.50 & 0.21 \\
\hline \multicolumn{2}{|c|}{$\operatorname{CoV}(\%)$} & 3.5 & 33.6 & 6.8 & 14.0 \\
\hline \multirow{3}{*}{9.5} & 1 & 32.9 & 5.4 & 28.9 & 2.9 \\
\hline & 2 & 42.5 & 3.1 & 27.9 & 1.0 \\
\hline & 3 & 37.5 & 1.2 & 22.6 & 3.1 \\
\hline \multicolumn{2}{|c|}{ Average } & 37.6 & 3.2 & 26.5 & 2.3 \\
\hline \multicolumn{2}{|c|}{ Std. Dev. } & 4.80 & 2.10 & 3.39 & 1.16 \\
\hline \multicolumn{2}{|c|}{$\operatorname{CoV}(\%)$} & 12.8 & 65.6 & 12.8 & 50.4 \\
\hline \multirow{3}{*}{12.7} & 1 & 40.5 & 0.4 & 15.9 & 1.3 \\
\hline & 2 & 44.2 & 0.2 & 13.0 & 1.3 \\
\hline & 3 & 41.6 & 0.2 & 11.8 & 0.2 \\
\hline \multicolumn{2}{|c|}{ Average } & 42.1 & 0.3 & 13.6 & 0.9 \\
\hline \multicolumn{2}{|c|}{ Std. Dev. } & 1.90 & 0.12 & 2.11 & 0.64 \\
\hline \multicolumn{2}{|c|}{$\operatorname{CoV}(\%)$} & 4.5 & 40 & 15.5 & 71.1 \\
\hline
\end{tabular}


Table 3.19: Loose and compacted unit weight and void content for RCA-MO and RCAVA

\begin{tabular}{|c|c|c|c|c|c|c|}
\hline \multirow{3}{*}{ Sample \# } & \multicolumn{3}{|c|}{ Loose Coarse Aggregate } & \multicolumn{3}{|c|}{ Compacted Coarse Aggregate } \\
\hline & $\begin{array}{c}\text { Mass } \\
(\mathrm{Kg})\end{array}$ & $\begin{array}{c}\mathbf{U W} \\
\left(\mathbf{K g} / \mathbf{m}^{3}\right)\end{array}$ & $\begin{array}{l}\mathrm{VC} \\
(\%)\end{array}$ & $\begin{array}{c}\text { Mass } \\
(\mathbf{K g})\end{array}$ & $\begin{array}{c}\mathbf{U W} \\
\left(\mathbf{K g} / \mathbf{m}^{3}\right)\end{array}$ & $\begin{array}{l}\text { VC } \\
(\%)\end{array}$ \\
\hline & \multicolumn{6}{|c|}{ RCA-MO } \\
\hline 1 & 17.973 & 1285 & 44 & 19.073 & 1363 & 41 \\
\hline 2 & 17.527 & 1253 & 46 & 19.16 & 1370 & 41 \\
\hline 3 & 17.707 & 1266 & 45 & 19.348 & 1383 & 40 \\
\hline $\begin{array}{l}\text { Average } \\
\text { Std. Dev. }\end{array}$ & & $\begin{array}{c}1268 \\
16\end{array}$ & $\begin{array}{l}45 \\
0.7\end{array}$ & & $\begin{array}{c}1372 \\
10\end{array}$ & $\begin{array}{l}40 \\
0.4\end{array}$ \\
\hline Sample \# & \multicolumn{6}{|c|}{ RCA-VA } \\
\hline 1 & 18.774 & 1342 & 44 & 20.536 & 1468 & 39 \\
\hline 2 & 19.287 & 1379 & 43 & 20.499 & 1465 & 39 \\
\hline 3 & 18.985 & 1357 & 44 & 20.568 & 1470 & 39 \\
\hline Average & & 1359 & 44 & & 1468 & 39 \\
\hline Std. Dev. & & 18 & 0.8 & & 2 & 0.1 \\
\hline
\end{tabular}


Table 3.20: Moisture content for different factions of RCA-MO and RCA-VA

\begin{tabular}{|c|c|c|c|c|c|c|c|c|c|}
\hline \multirow{3}{*}{$\begin{array}{c}\text { Sample } \\
\#\end{array}$} & \multicolumn{3}{|c|}{ Initial (Stock) weight (g) } & \multicolumn{3}{|c|}{$\begin{array}{c}\text { Final Weight (OD) } \\
(\mathrm{g})\end{array}$} & \multicolumn{3}{|c|}{$\begin{array}{c}\text { Moisture Content } \\
(\%)\end{array}$} \\
\hline & \multicolumn{3}{|c|}{ RCA-MO size (mm) } & \multicolumn{3}{|c|}{ RCA-MO size (mm) } & \multicolumn{3}{|c|}{ RCA-MO size (mm) } \\
\hline & 4.75 & 9.5 & 12.7 & 4.75 & 9.5 & 12.7 & 4.75 & 9.5 & 12.7 \\
\hline 1 & 1000 & 2002 & 2001 & 990 & 1981 & 1978 & 1.0 & 1.1 & 1.2 \\
\hline 2 & 1001 & 2000 & 2003 & 990 & 1978 & 1982 & 1.1 & 1.1 & 1.1 \\
\hline 3 & 1003 & 2001 & 2004 & 992 & 1980 & 1983 & 1.1 & 1.1 & 1.1 \\
\hline \multicolumn{7}{|c|}{ Average } & 1.1 & 1.1 & 1.1 \\
\hline \multicolumn{7}{|c|}{ Weighted Average } & \multicolumn{3}{|c|}{1.1} \\
\hline \multirow{2}{*}{ Sample \# } & \multicolumn{3}{|c|}{ RCA-VA size (mm) } & \multicolumn{3}{|c|}{ RCA-VA size (mm) } & \multicolumn{3}{|c|}{ RCA-VA size (mm) } \\
\hline & 4.75 & 9.5 & 12.7 & 4.75 & 9.5 & 12.7 & 4.75 & 9.5 & 12.7 \\
\hline 1 & 1280 & 2252 & 2369 & 1263 & 2221 & 2341 & 1.3 & 1.4 & 1.2 \\
\hline 2 & 1193 & 2596 & 2027 & 1177 & 2563 & 2002 & 1.4 & 1.3 & 1.2 \\
\hline 3 & 1377 & 2455 & 2611 & 1359 & 2425 & 2580 & 1.3 & 1.2 & 1.2 \\
\hline \multicolumn{7}{|c|}{ Average } & 1.3 & 1.3 & 1.2 \\
\hline \multicolumn{7}{|c|}{ Weighted Average } & \multicolumn{3}{|c|}{1.3} \\
\hline
\end{tabular}

Table 3.21: Measured properties for the residual mortar of RCA-MO

\begin{tabular}{|c|c|c|c|c|c|c|c|c|c|}
\hline \multirow{2}{*}{ Sample \# } & \multirow{2}{*}{$\begin{array}{c}\mathbf{W}_{\text {OD }} \\
(\mathrm{g})\end{array}$} & \multirow{2}{*}{$\begin{array}{c}\mathbf{W}_{\mathrm{SSD}} \\
(\mathrm{g})\end{array}$} & \multicolumn{2}{|c|}{$\begin{array}{l}\text { Reading of water level } \\
\text { in Le Chatelier flask }\end{array}$} & \multirow{2}{*}{$\begin{array}{l}\mathrm{AC} \\
\%\end{array}$} & \multirow{2}{*}{$\begin{array}{l}\mathbf{n} \\
\%\end{array}$} & \multicolumn{3}{|c|}{ SG } \\
\hline & & & Initial & Final & & & Bulk & SSD & App \\
\hline 1 & 48.78 & 54.00 & 0.10 & 23.90 & 10.70 & 21.99 & 2.05 & 2.27 & 2.63 \\
\hline 2 & 45.00 & 49.48 & 0.10 & 21.50 & 9.96 & 20.99 & 2.11 & 2.32 & 2.67 \\
\hline 3 & 45.00 & 49.35 & -0.10 & 21.30 & 9.67 & 20.38 & 2.11 & 2.31 & 2.65 \\
\hline 4 & 45.00 & 49.30 & -0.10 & 21.20 & 9.56 & 20.24 & 2.12 & 2.32 & 2.66 \\
\hline Average & & & & & 9.97 & 20.90 & 2.10 & 2.31 & 2.65 \\
\hline Std. Dev. & & & & & 0.52 & 0.80 & 0.03 & 0.02 & 0.01 \\
\hline$\overline{C o V}(\%)$ & & & & & 5.2 & 3.8 & $\overline{0.9}$ & 1.4 & $\overline{0.5}$ \\
\hline
\end{tabular}


Table 3.22: Measured properties for the residual mortar of RCA-VA

\begin{tabular}{|c|c|c|c|c|c|c|c|c|c|}
\hline \multirow{2}{*}{ Sample \# } & \multirow{2}{*}{$\begin{array}{c}\mathbf{W}_{\mathrm{OD}} \\
(\mathrm{g})\end{array}$} & \multirow{2}{*}{$\begin{array}{c}\mathbf{W}_{\text {SSD }} \\
(\mathrm{g})\end{array}$} & \multicolumn{2}{|c|}{$\begin{array}{l}\text { Reading of water level } \\
\text { in Le Chatelier flask }\end{array}$} & \multirow{2}{*}{$\underset{\%}{\mathrm{AC}}$} & \multirow{2}{*}{$\begin{array}{c}\mathbf{n} \\
\%\end{array}$} & \multicolumn{3}{|c|}{ SG } \\
\hline & & & Initial & Final & & & Bulk & SSD & App \\
\hline 1 & 49.22 & 54.40 & 0.00 & 23.70 & 10.52 & 21.91 & 2.08 & 2.30 & 2.67 \\
\hline 2 & 45.00 & 49.55 & 0.50 & 21.90 & 10.11 & 21.31 & 2.11 & 2.32 & 2.68 \\
\hline 3 & 45.00 & 49.45 & 0.00 & 21.30 & 9.89 & 20.94 & 2.12 & 2.33 & 2.68 \\
\hline 4 & 45.00 & 49.41 & -0.10 & 21.10 & 9.80 & 20.85 & 2.13 & 2.34 & 2.69 \\
\hline Average & & & & & 10.08 & 21.26 & 2.11 & 2.32 & 2.68 \\
\hline Std. Dev. & & & & & 0.32 & 0.48 & 0.02 & 0.02 & 0.01 \\
\hline $\operatorname{CoV}(\%)$ & & & & & 3.2 & 2.3 & 0.6 & 0.9 & 0.3 \\
\hline
\end{tabular}


Table 3.23: Summary of material characterization testing of RCA-MO and RCA-VA

\begin{tabular}{|c|c|c|c|c|c|}
\hline \multirow{2}{*}{\multicolumn{3}{|c|}{ RCA Properties }} & \multicolumn{2}{|c|}{ RCA } & \multirow{2}{*}{$\begin{array}{c}\text { Allowable } \\
\text { Limit }\end{array}$} \\
\hline & & & \multirow{2}{*}{$\frac{\text { MO }}{1268}$} & \multirow{2}{*}{$\frac{\mathbf{V A}}{1359}$} & \\
\hline \multirow{2}{*}{\multicolumn{2}{|c|}{ Unit weight $\left(\mathrm{Kg} / \mathrm{m}^{3}\right)$}} & Loose & & & \\
\hline & & Compacted & 1372 & 1468 & \\
\hline \multirow{2}{*}{\multicolumn{2}{|c|}{ Air Content (\%) }} & Loose & 45 & 44 & \\
\hline & & Compacted & 40 & 39 & \\
\hline \multicolumn{3}{|c|}{ SSD Specific gravity } & 2.42 & 2.50 & - \\
\hline \multicolumn{3}{|c|}{ Bulk Specific gravity } & 2.31 & 2.42 & - \\
\hline \multicolumn{3}{|c|}{ Apparent Specific gravity } & 2.64 & 2.64 & - \\
\hline \multicolumn{3}{|c|}{ Absorption Capacity (\%) } & 5.4 & 3.3 & - \\
\hline \multicolumn{3}{|c|}{ Moisture Content (\%) } & 1.1 & 1.3 & \\
\hline \multicolumn{3}{|c|}{ Porosity } & 12.3 & 8.1 & - \\
\hline \multirow{3}{*}{$\begin{array}{l}\text { Weighted } \\
\text { percentage } \\
\text { loss }\end{array}$} & \multirow[b]{2}{*}{ ASTM } & $\mathrm{Na}_{2} \mathrm{SO}_{4}$ & 34.9 & 19.8 & 12 \\
\hline & & $\mathrm{MgSO}_{4}$ & 2.1 & 1.5 & 18 \\
\hline & \multicolumn{2}{|c|}{ MTO } & 26.7 & 16.0 & 20.8 \\
\hline \multicolumn{3}{|c|}{ Abrasion (\%) } & 42 & 25 & 50 \\
\hline \multicolumn{3}{|c|}{ Residual Mortar content (RMC) } & 41 & 23 & - \\
\hline
\end{tabular}

Table 3.24: Residual mortar and original natural aggregate properties of Nagataki et al. experiments used in the rational approach

\begin{tabular}{||c|c|c||c|c||}
\hline \multirow{2}{*}{ Specimen } & \multicolumn{2}{|c|}{ Residual Mortar } & \multicolumn{2}{c|}{ OVA } \\
\cline { 2 - 4 } & $\begin{array}{c}\text { AC } \\
(\%)\end{array}$ & SG & \multirow{2}{*}{$\begin{array}{c}\text { AC } \\
(\%)\end{array}$} & SG \\
\hline Nagataki-1 & 8.01 & 2.17 & & \\
\hline Nagataki-2 & 9.12 & 2.13 & \multirow{2}{*}{0.68} & \multirow{2}{*}{2.65} \\
\hline Nagataki-3 & 9.86 & 2.09 & & \\
\hline Nagataki-4 & 10.1 & 2.11 & & \\
\hline
\end{tabular}




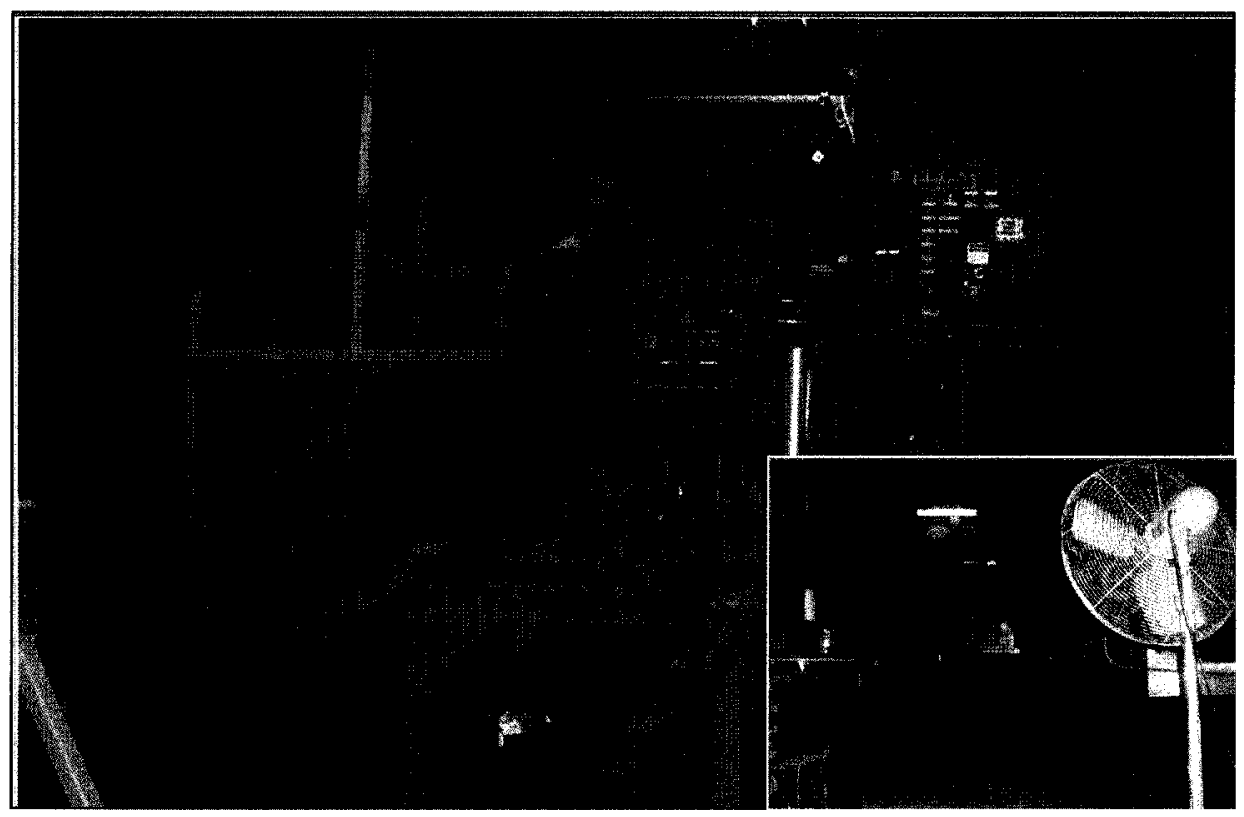

Figure 3.1: Screening and air drying of RCA
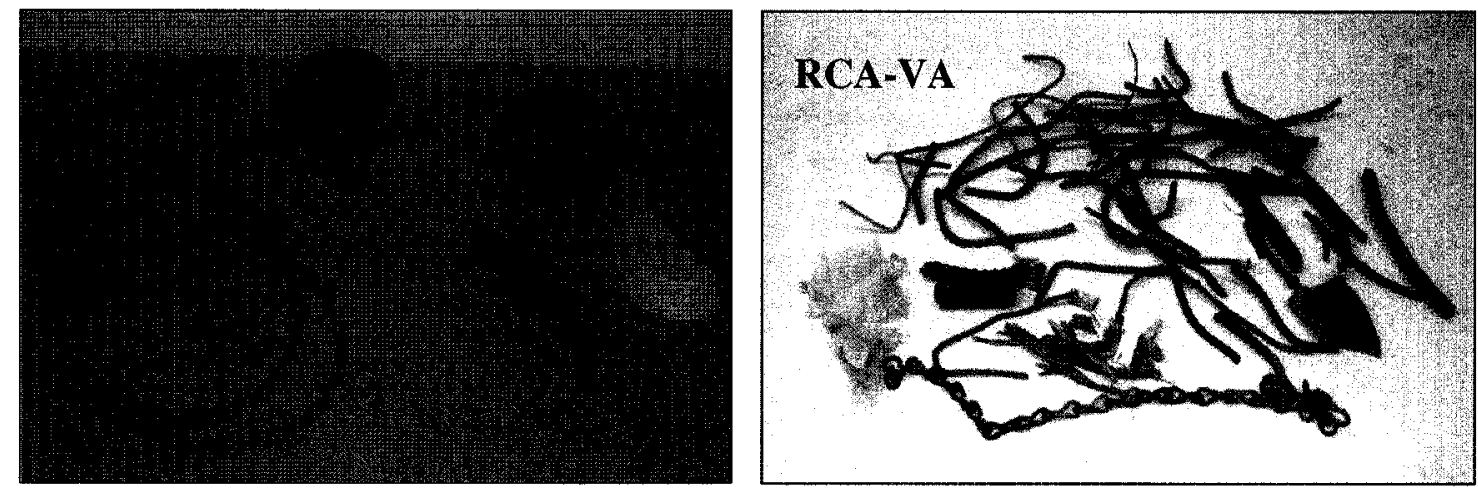

Figure 3.2: Large impurities in RCA-MO and RCA-VA 


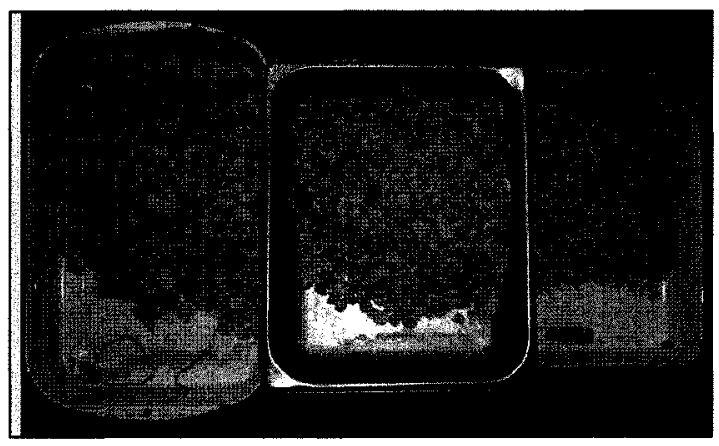

RCA-MO

(Right to left: $4.75,9.5$, and $12.7 \mathrm{~mm}$ )

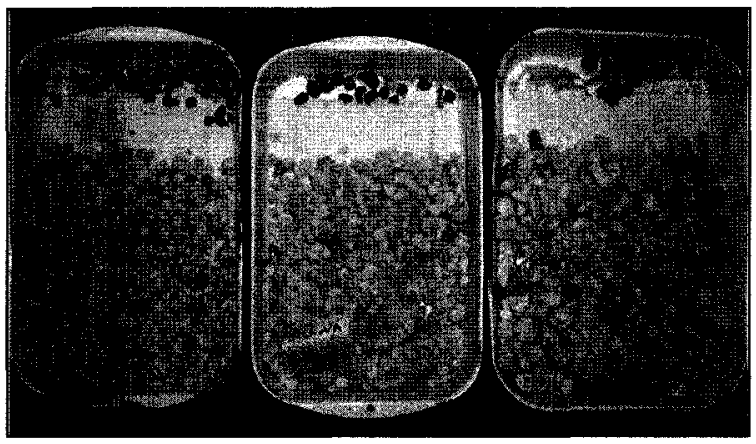

RCA-VA

Figure 3.3: Small impurities in RCA-MO and RCA-VA

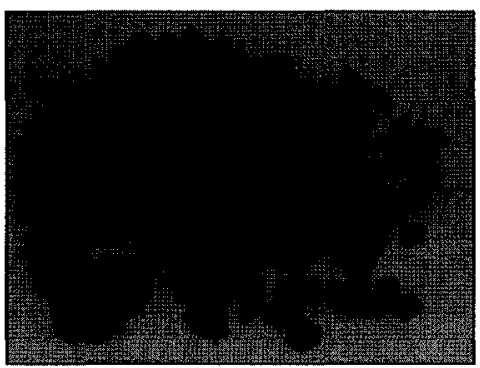

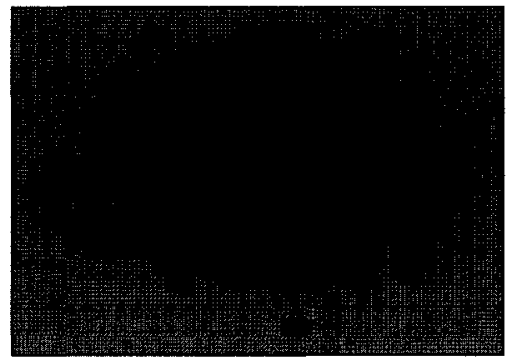

RCA in $\mathrm{MgCl}_{2}$ (no significant effect)

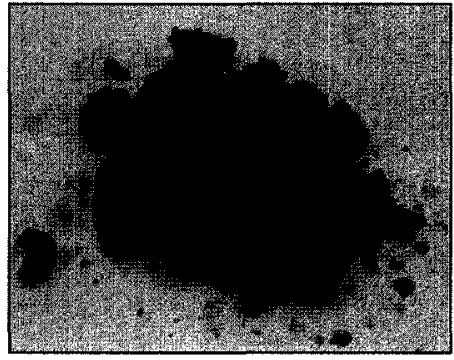

$\mathrm{RCA}$ in $\mathrm{Na}_{2} \mathrm{SO}_{4}$

(significant degradation) (no significant effect)

Figure 3.4: RCA-MO samples after 15 days immersion
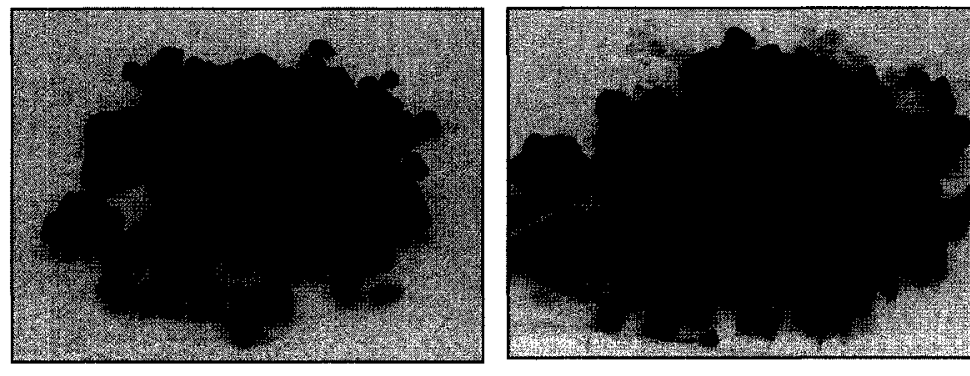

RCA in $\mathrm{MgSO}_{4}$ (no significant effect)
$\mathrm{RCA}$ in $\mathrm{MgCl}_{2}$ (no significant effect)

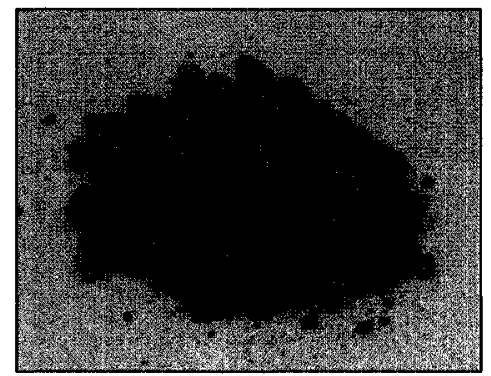

$\mathrm{RCA}$ in $\mathrm{Na}_{2} \mathrm{SO}_{4}$

(significant degradation)

Figure 3.5: RCA-MO samples after 5 freeze-and-thaw cycles 


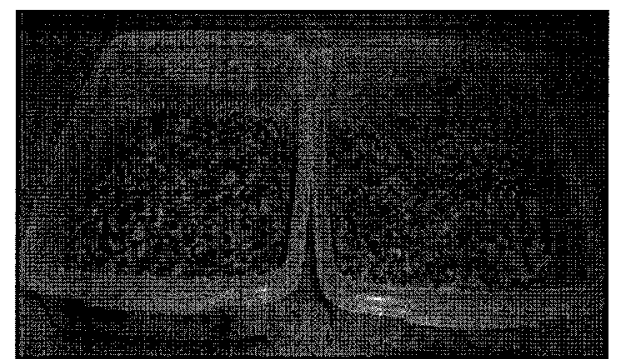

Before the test

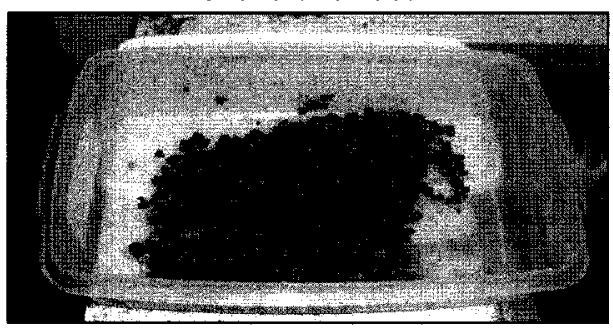

After washing by water

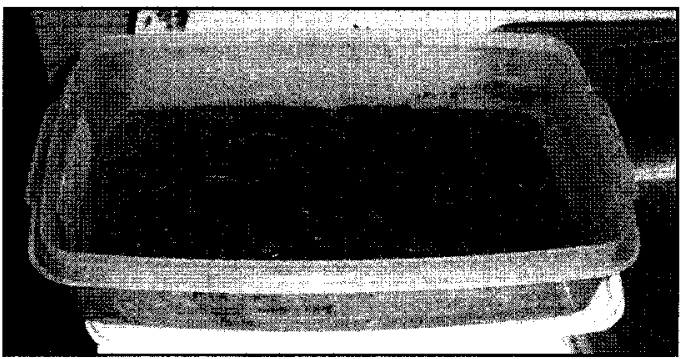

After 5 cycles of freeze-and-thaw

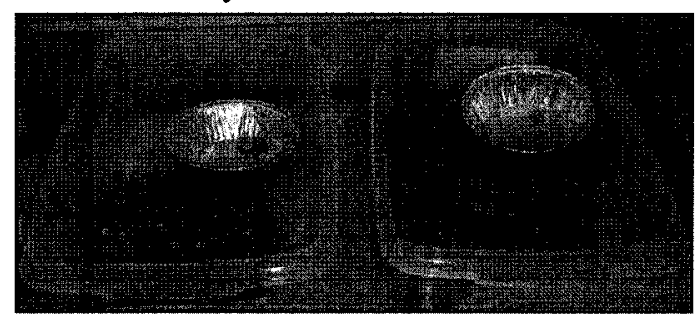

After test

Figure 3.6: Stages of removing of residual mortar by chemical and mechanical stresses

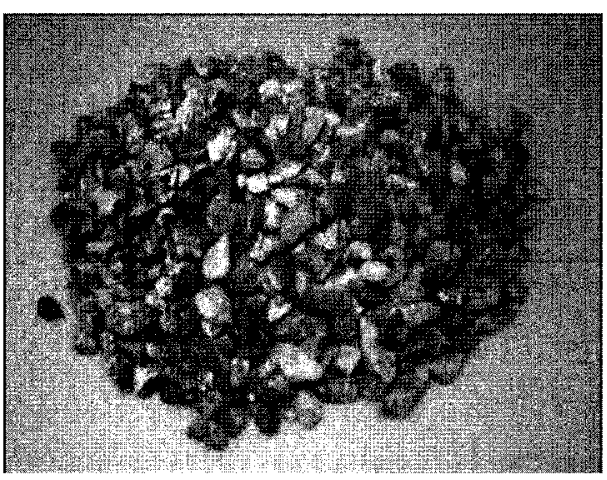

$(4.75 \mathrm{~mm})$

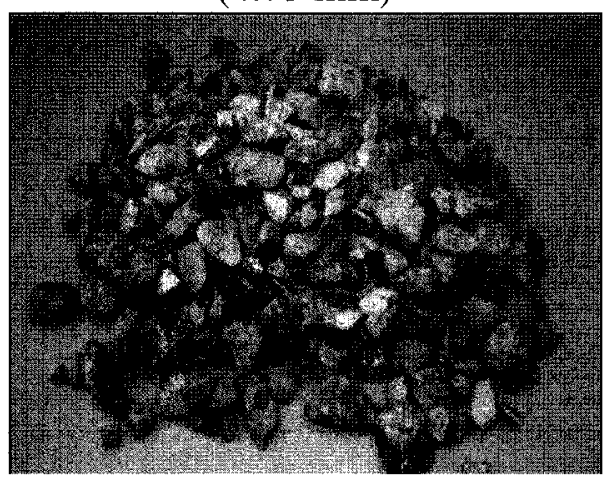

$(12.7 \mathrm{~mm})$

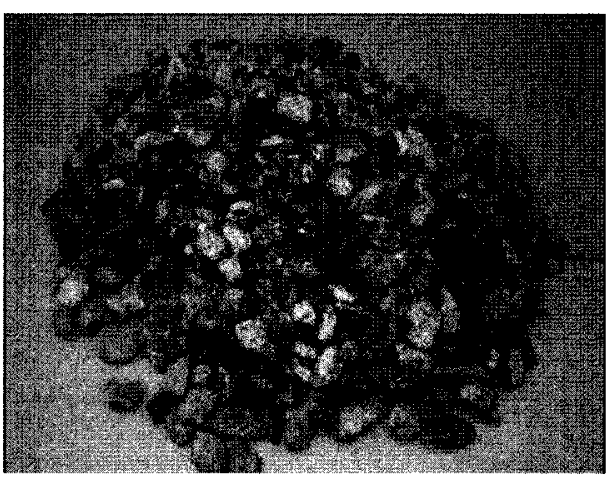

$(9.5 \mathrm{~mm})$

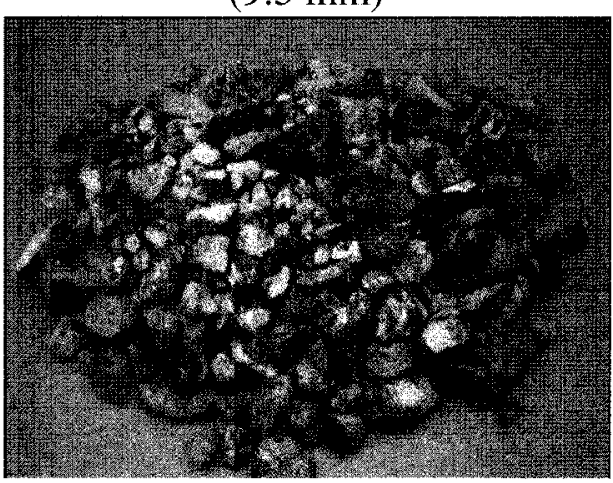

$(19 \mathrm{~mm})$

Figure 3.7: RCA-MO samples after full removal of the attached residual mortar 


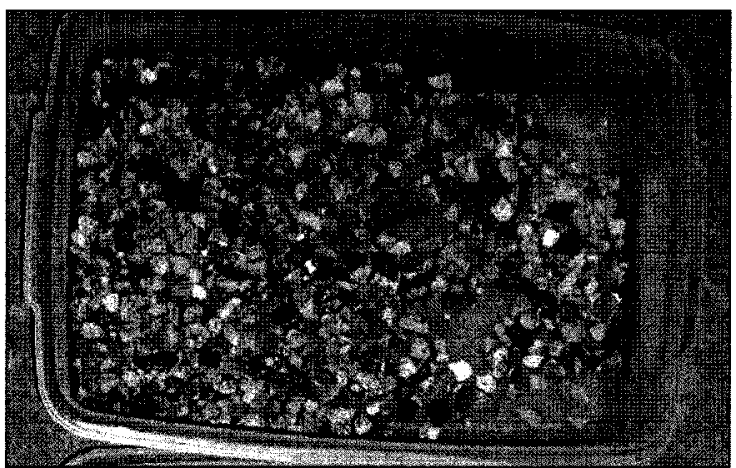

$(4.75 \mathrm{~mm})$

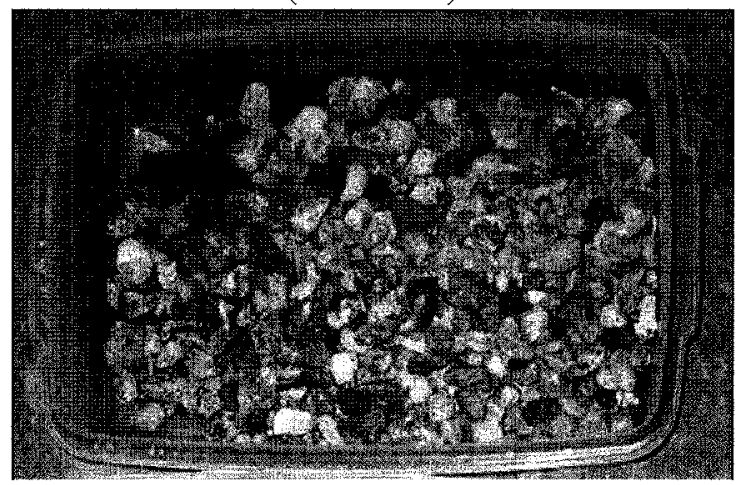

$(12.7 \mathrm{~mm})$

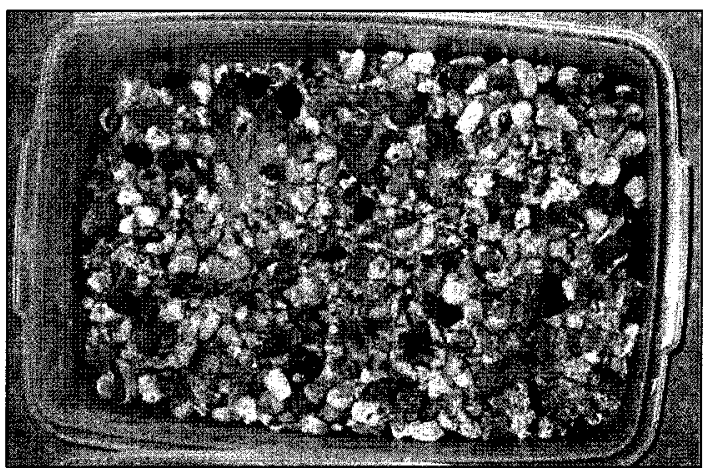

$(9.5 \mathrm{~mm})$

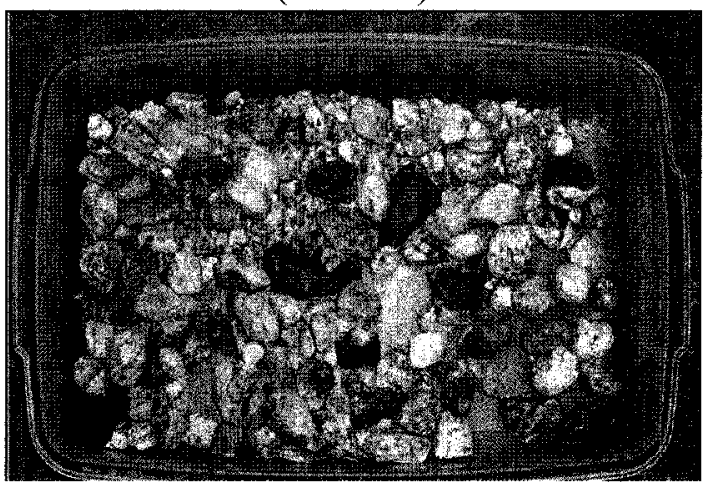

$(19 \mathrm{~mm})$

Figure 3.8: RCA-VA samples after full removal of the attached residual mortar 


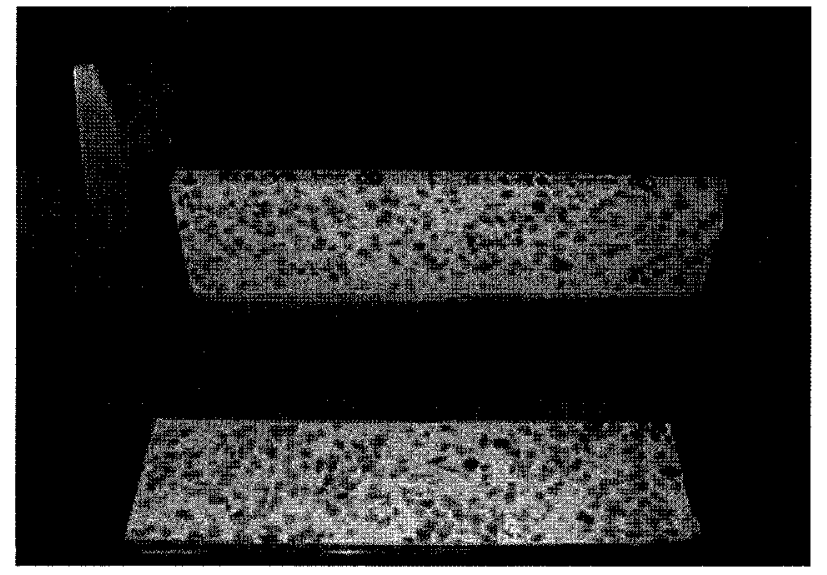

(a)

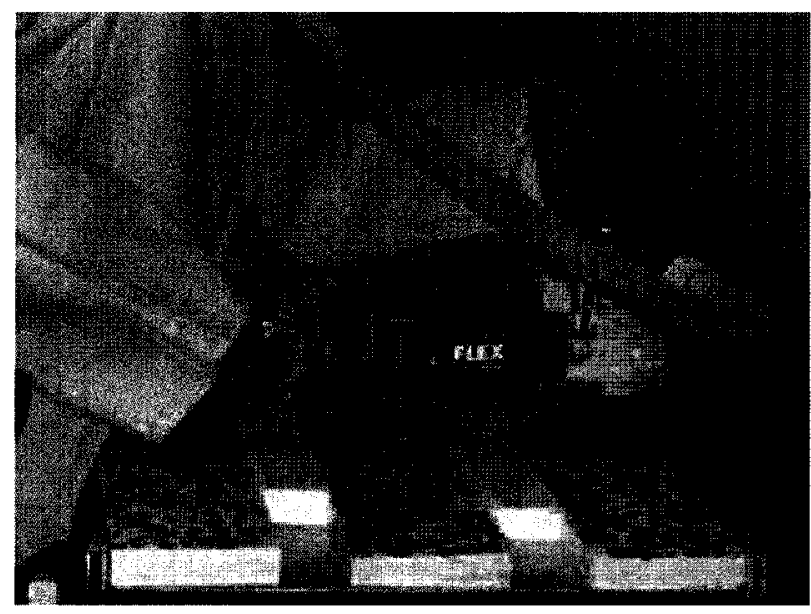

(b)

Figure 3.9: Preparation of image analysis specimens (a) Sawing of concrete slabs, (b) Polishing operation 


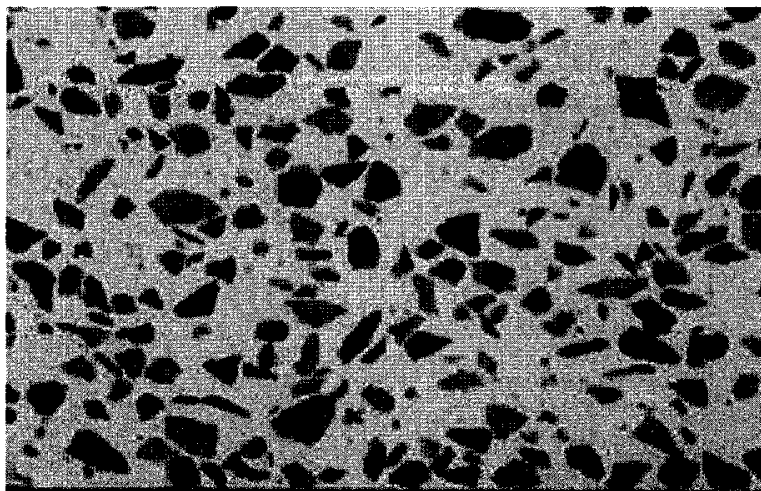

(a) $4.75 \mathrm{~mm}$ size fraction

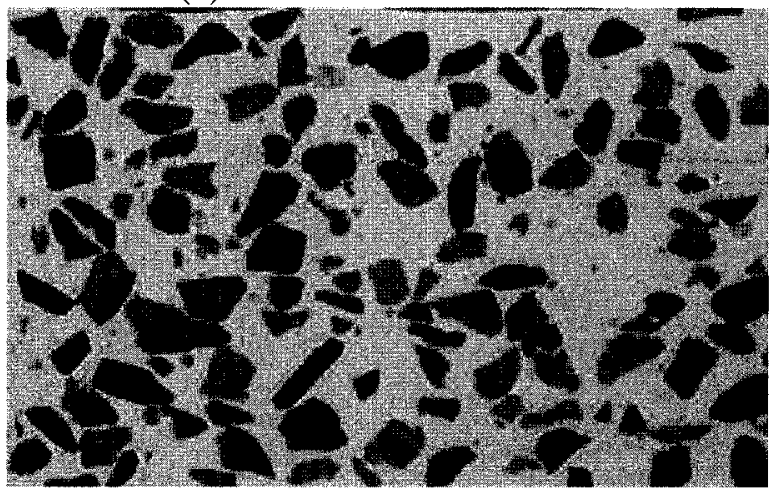

(b) $9.5 \mathrm{~mm}$ size fraction

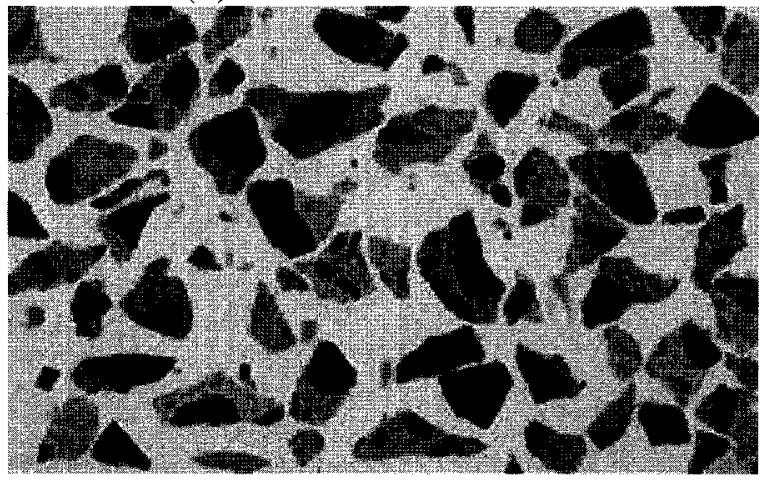

(c) 12.7 size fraction

Figure 3.10: Polished sample for RCA-MO

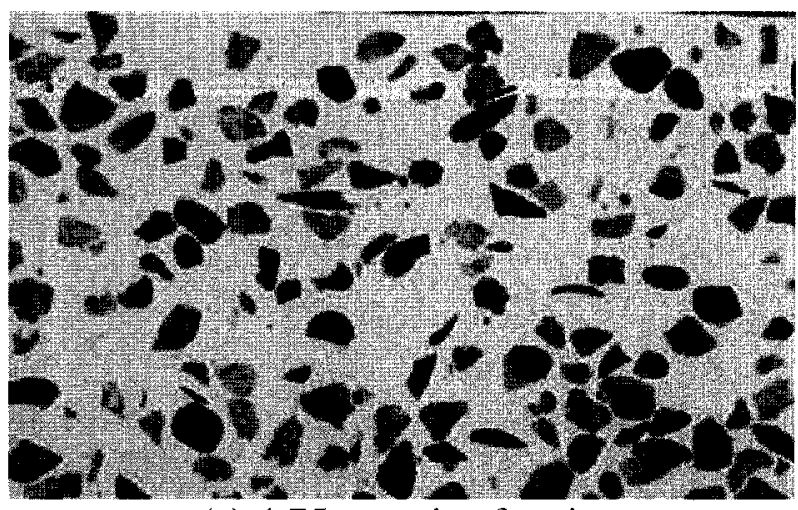

(a) $4.75 \mathrm{~mm}$ size fraction

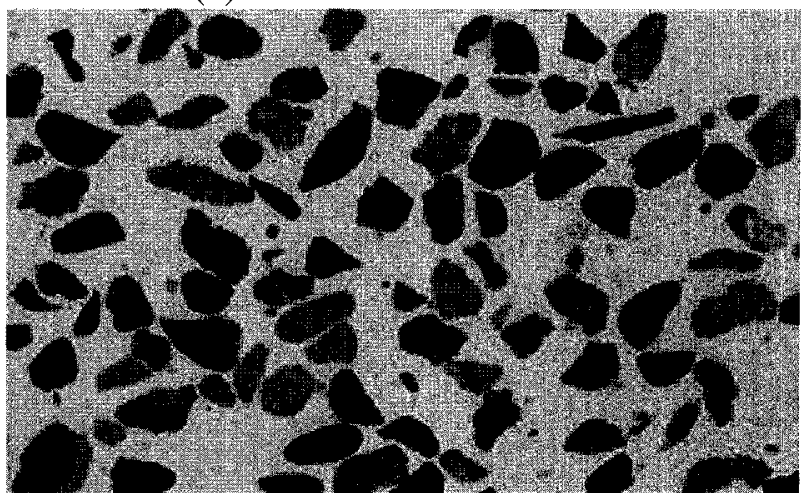

(b) $9.5 \mathrm{~mm}$ size fraction

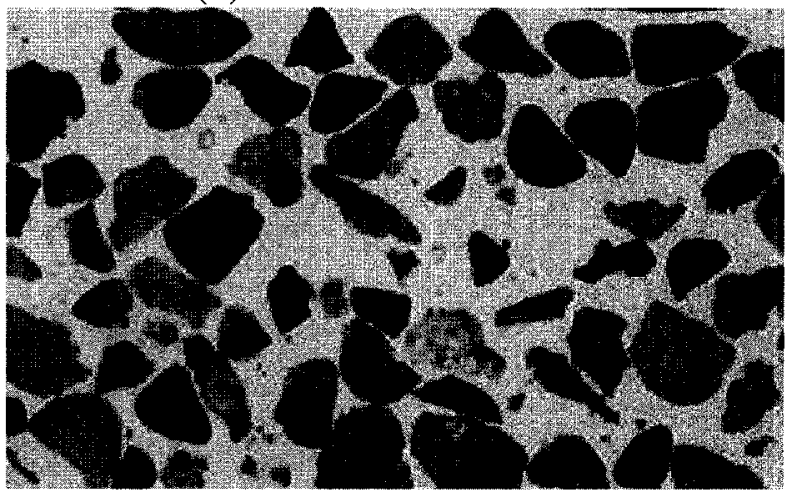

(c) $12.7 \mathrm{~mm}$ size fraction

Figure 3.11: Polished sample for RCA-VA 

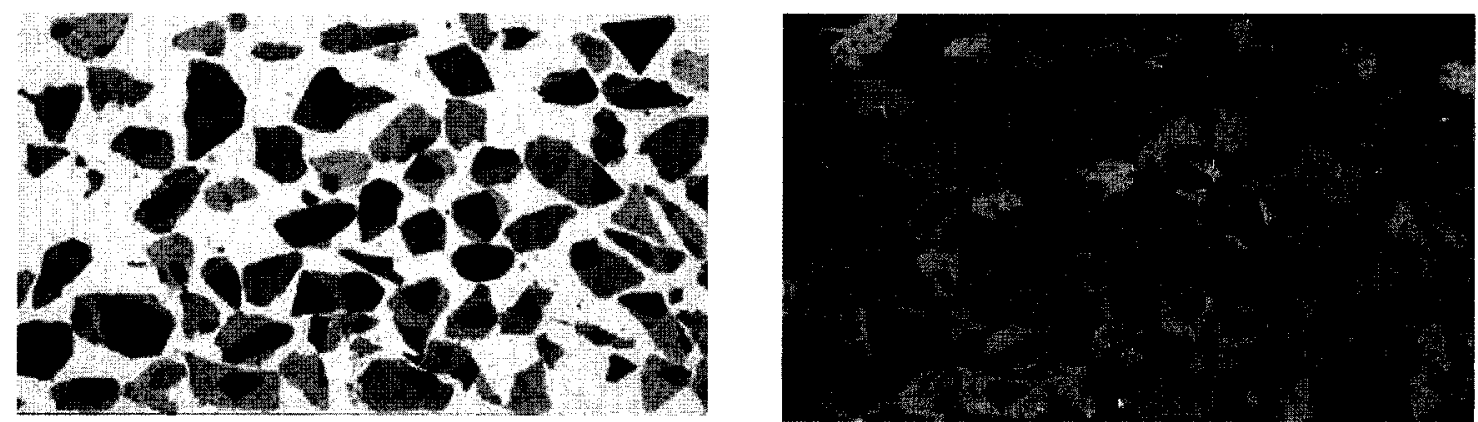

Figure 3.12: Photoshop process (a) Original picture of the slab (1/3 of the total length of the slab analyzed) (b) Edited picture in Photoshop, green mask applied

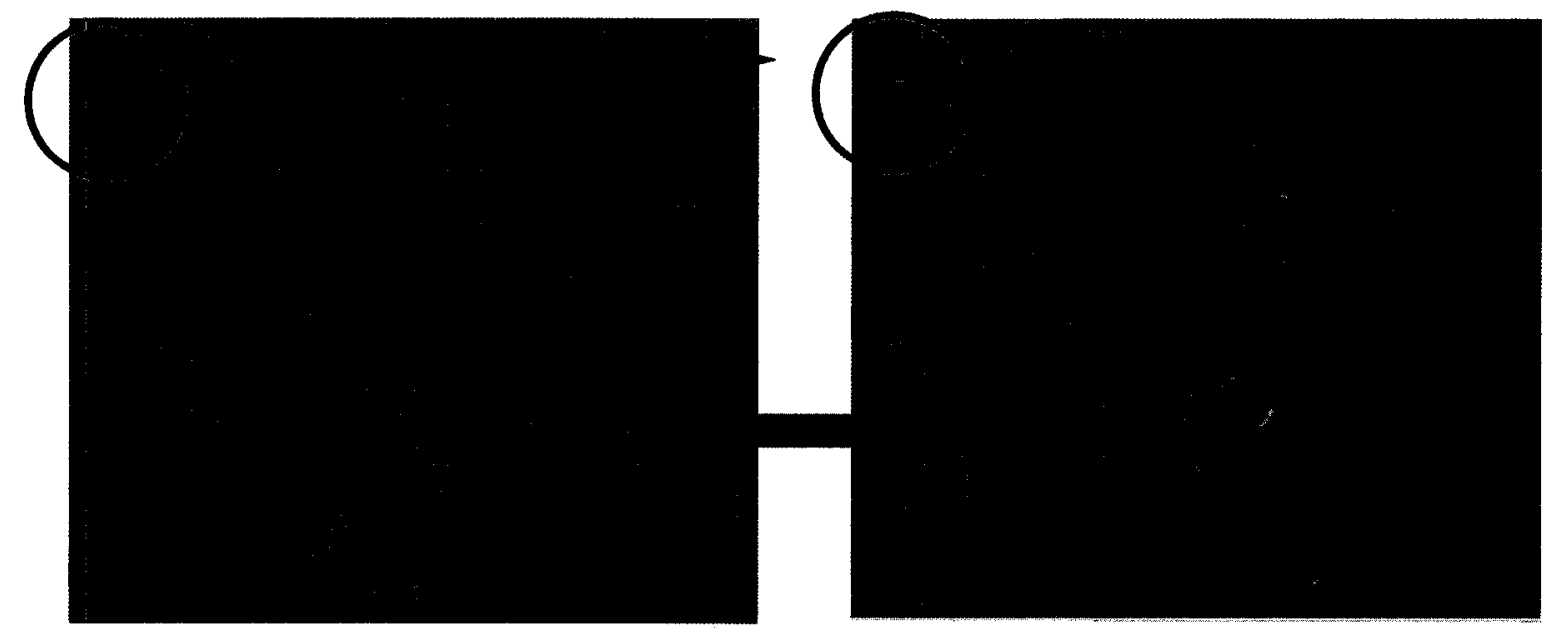

Figure 3.13: Separation of the white cement binder from the RCA particle 


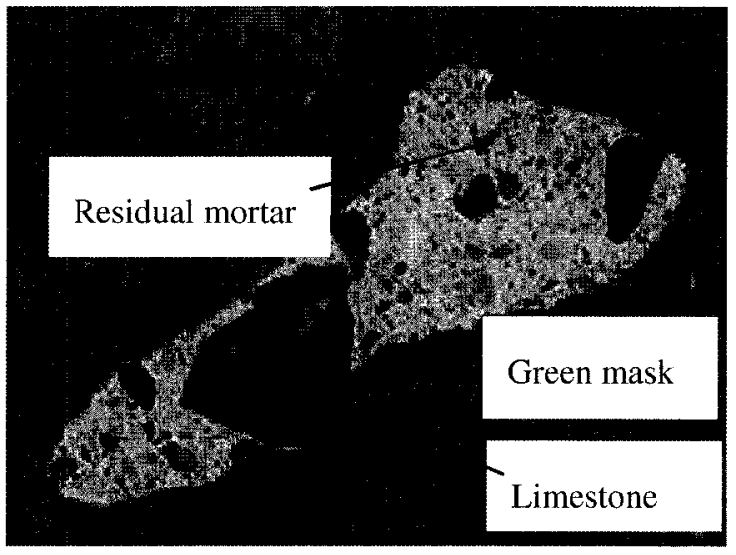

(a)

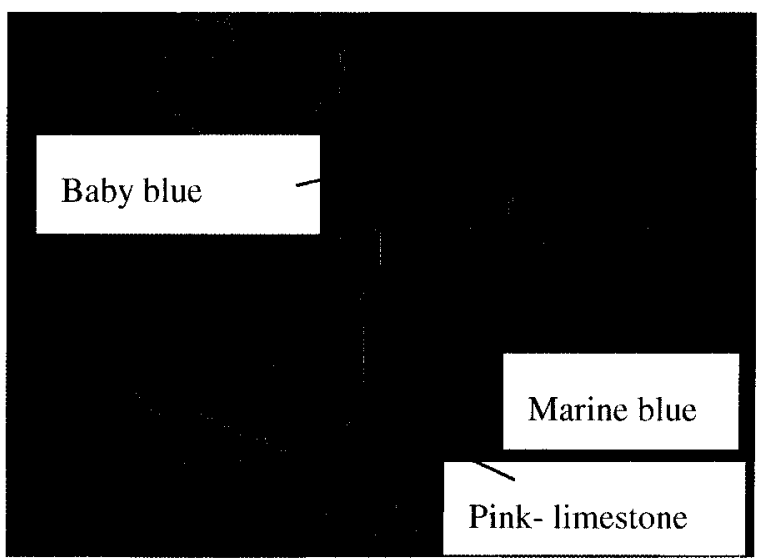

(b)

Figure 3.14: Binarization operation (a) Detail of RCA particle with the green mask representing the binder of white cement (b) Binarized image corresponding to Figure 3.14a 


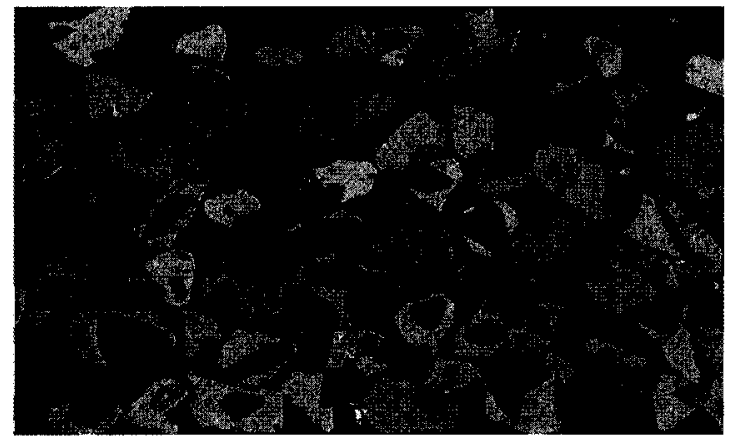

(a) Binarization by colour threshold, ranges of hue, saturation and intensity are defining the bitplane.

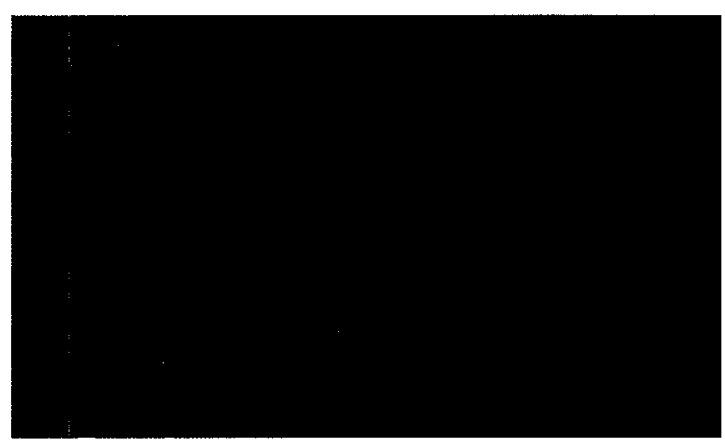

(c) Successful separation of recycled concrete segments - Binarized image ready for the first measurement - count and volume fraction of RCA particles.

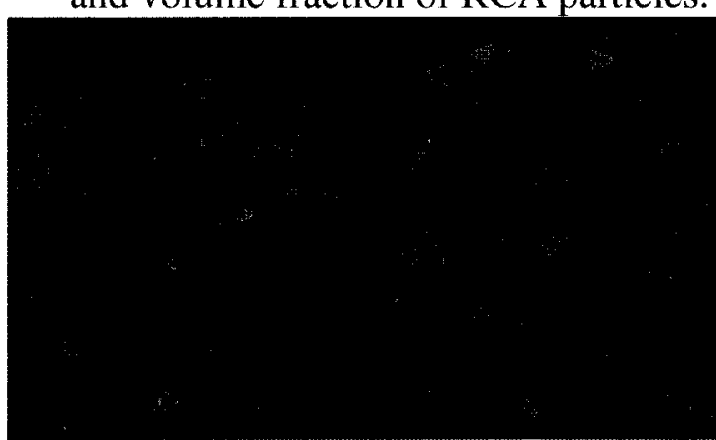

(e) Light blue area, i.e. residual mortar, was obtained in the routine by subtracting the area occupied by OVA from the total area of each individual segment of RCA.

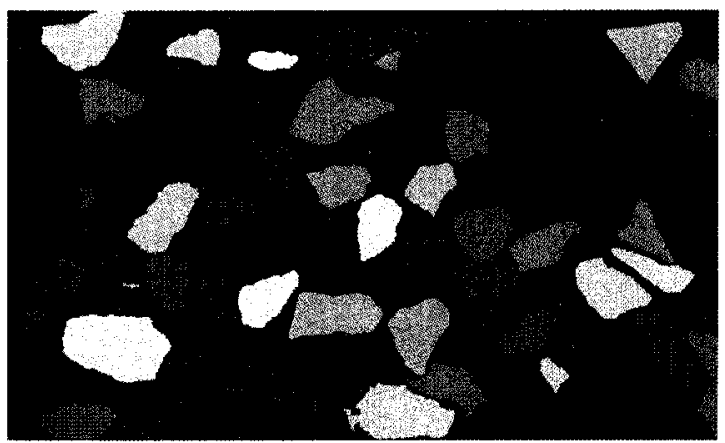

(b) Visual confirmation - each separate individual segment of the recycled concrete will be painted in different colour if properly separated. Touching segments will appear in identical colour.

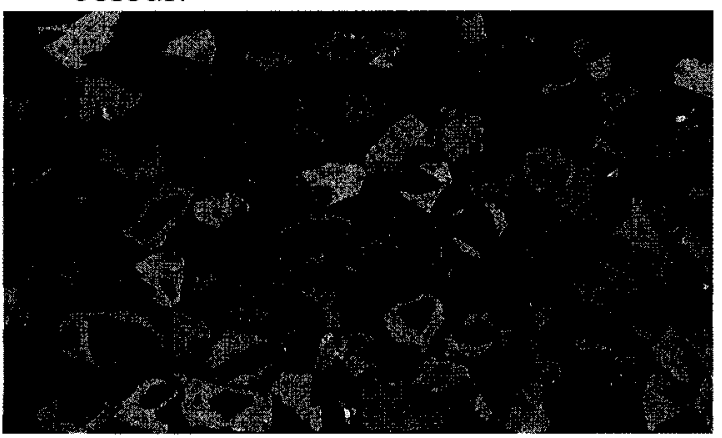

(d) Colour threshold and manual editing was applied to isolate original virgin aggregate (OVA) particles.

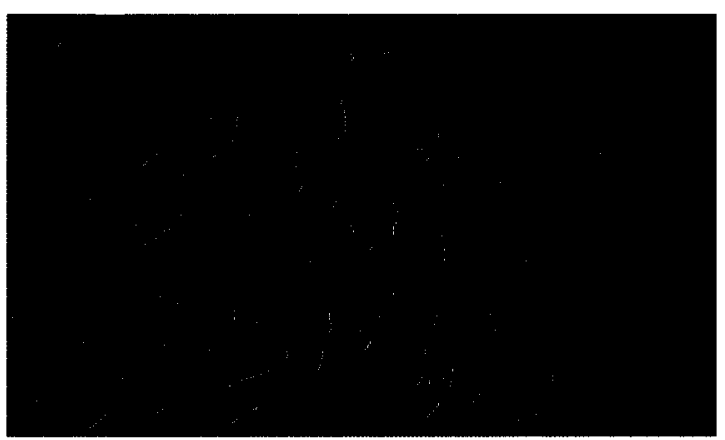

(f) Final image - volume fraction of all phases separated and measured

Figure 3.15: Visual results of some of the image analysis functions used in the routine 


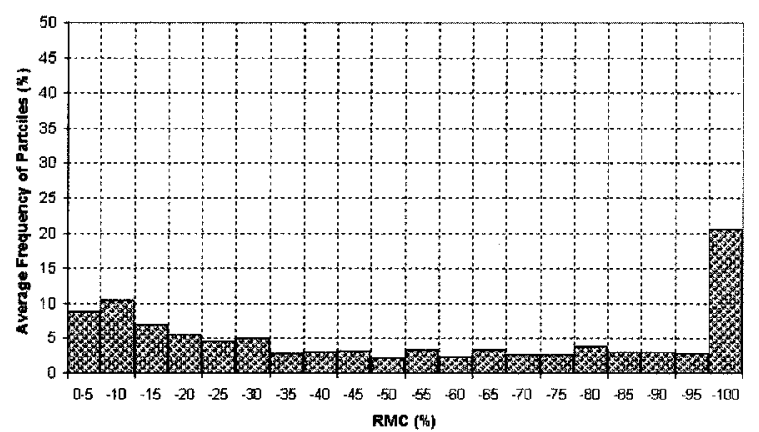

(a) RCA-MO (4.75 $\mathrm{mm}$ fraction)

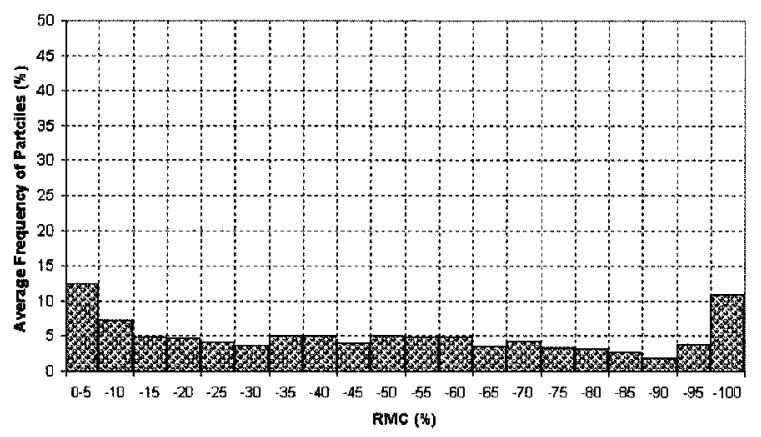

(b) RCA-MO (9.5 $\mathrm{mm}$ fraction)

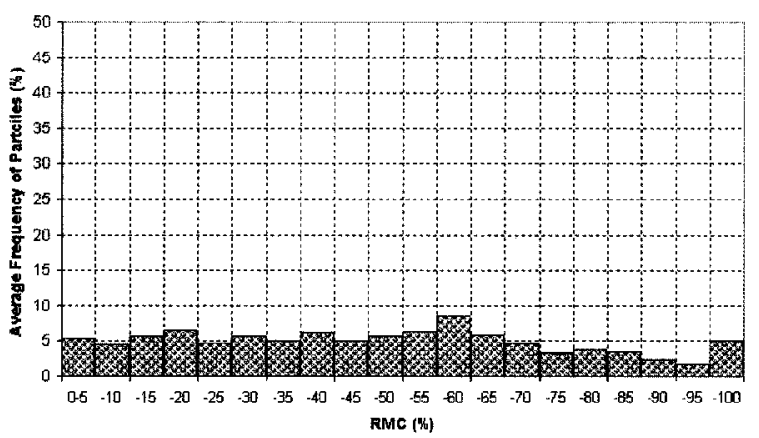

(c) RCA-MO (12.7 mm fraction)

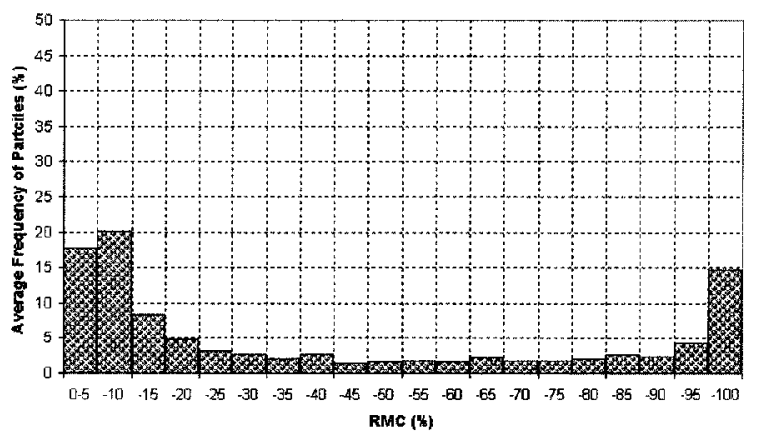

(d) RCA-VA (4.75 mm fraction)

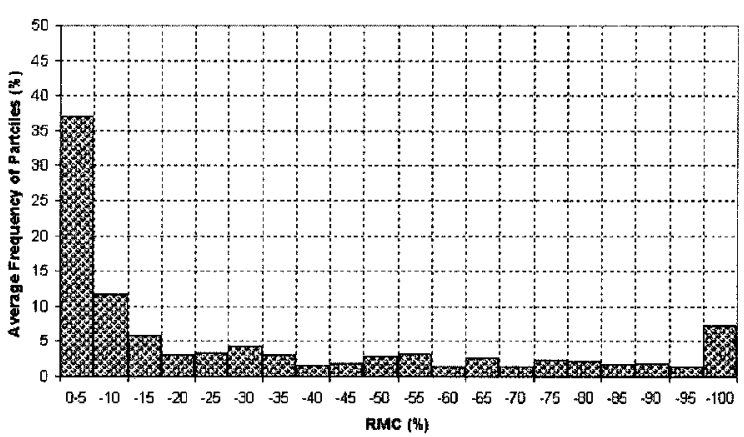

(e) RCA-VA (9.5 mm fraction)

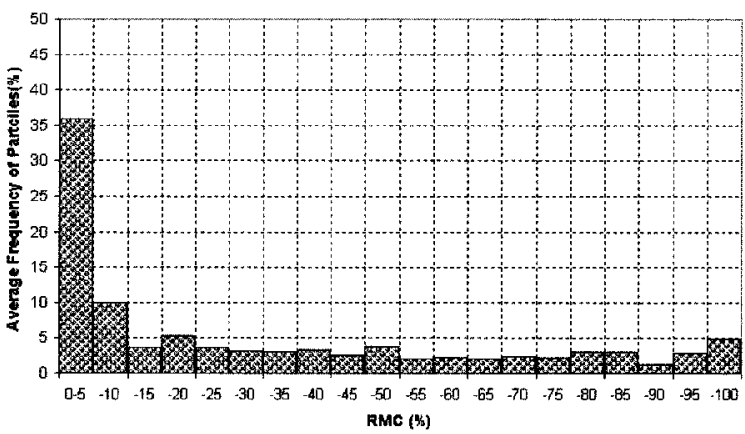

(f) RCA-VA (12.7 mm fraction)

Figure 3.16:Average percentage frequency of particles for RCA-MO and RCA-VA 


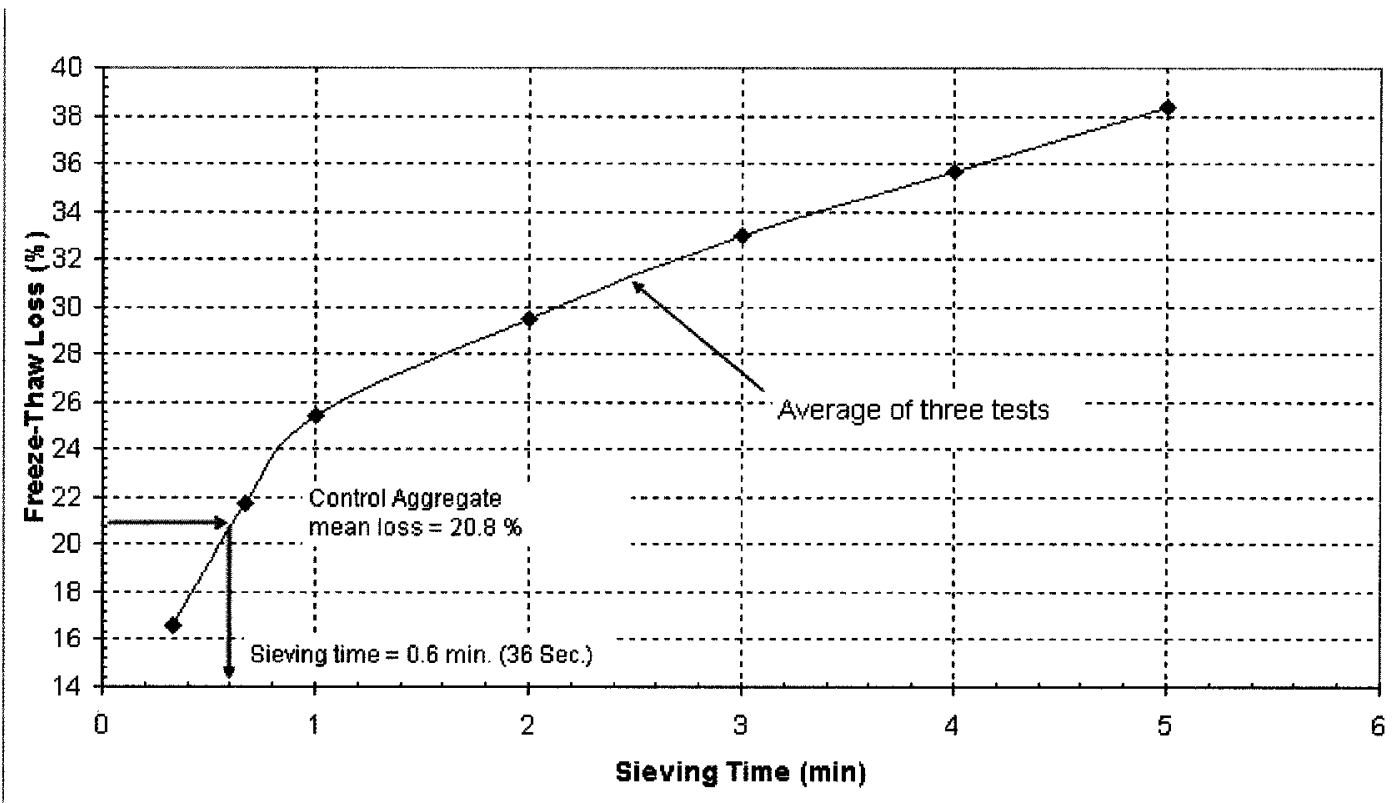

Figure 3.17: Average weighted freeze-and-thaw loss vs. sieving time for control aggregate for the MTO soundness test 


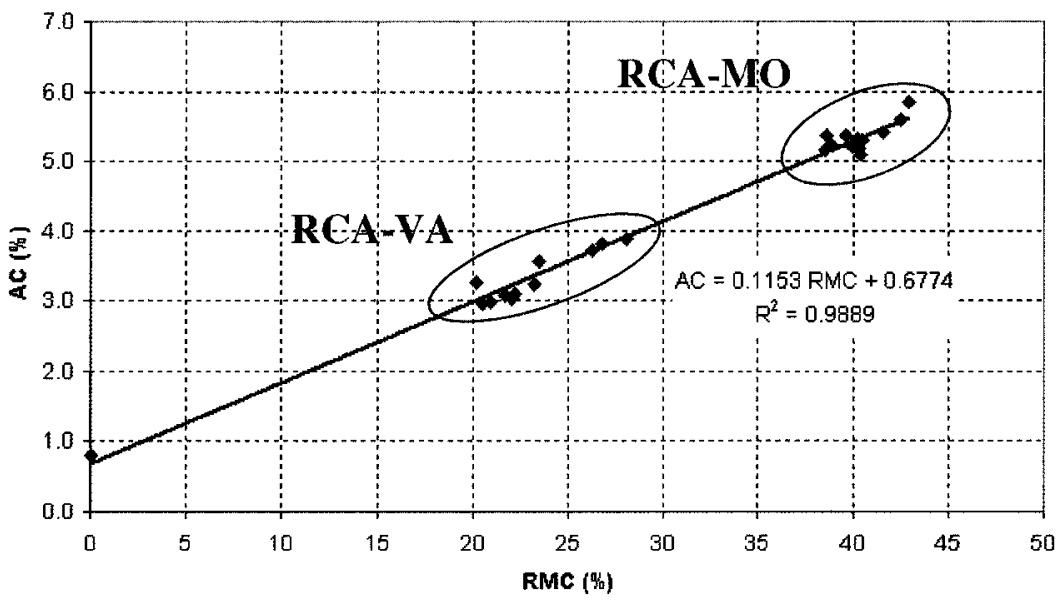

(a) 24-h immersion

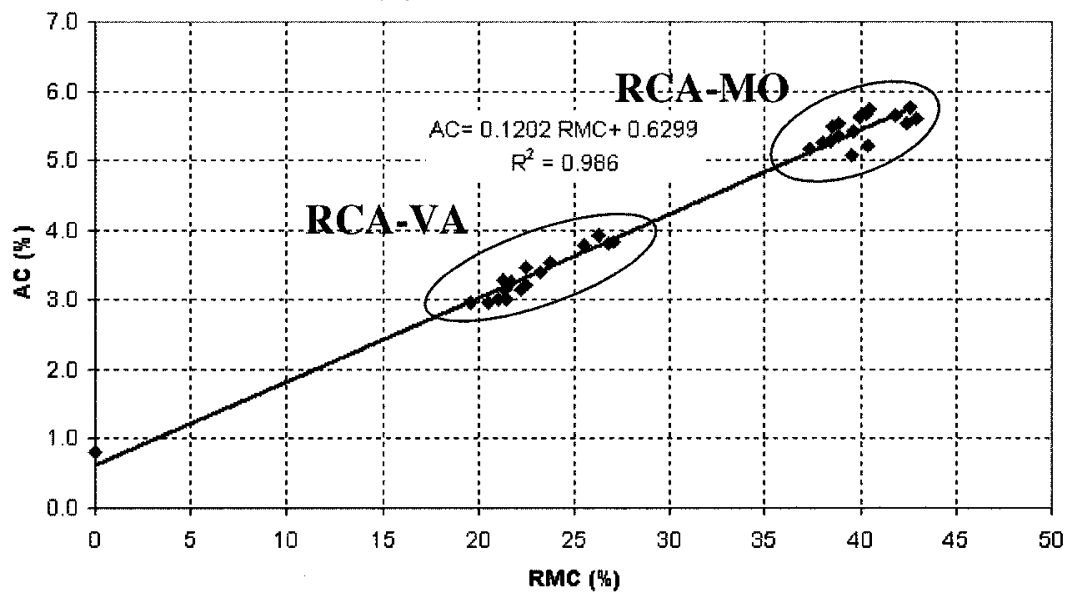

(b) 48-h immersion

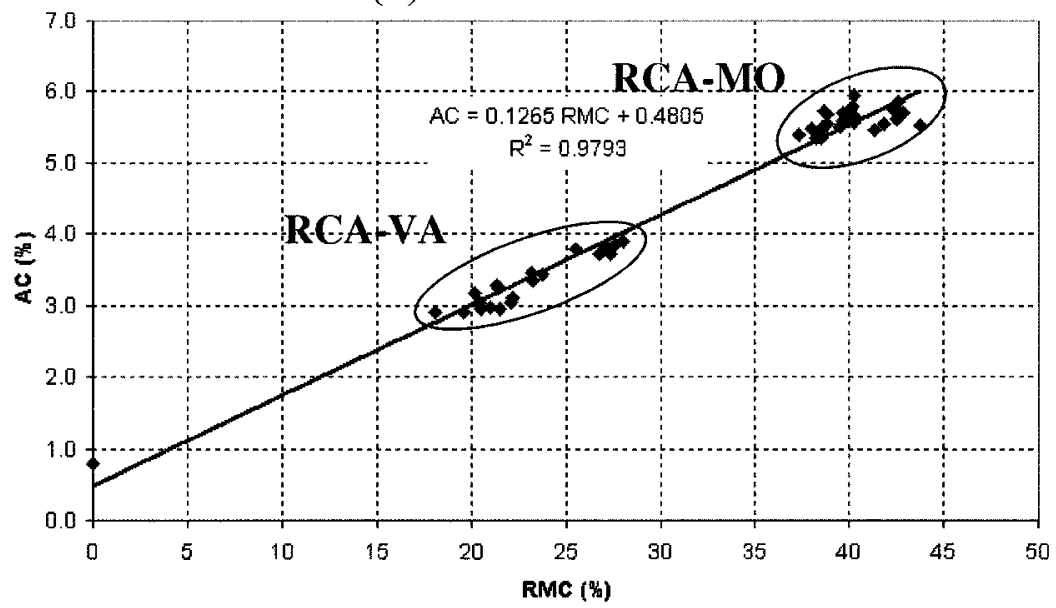

(c) 72-h immersion

Figure 3.18: Absorption capacity vs. RMC for different immersion periods 


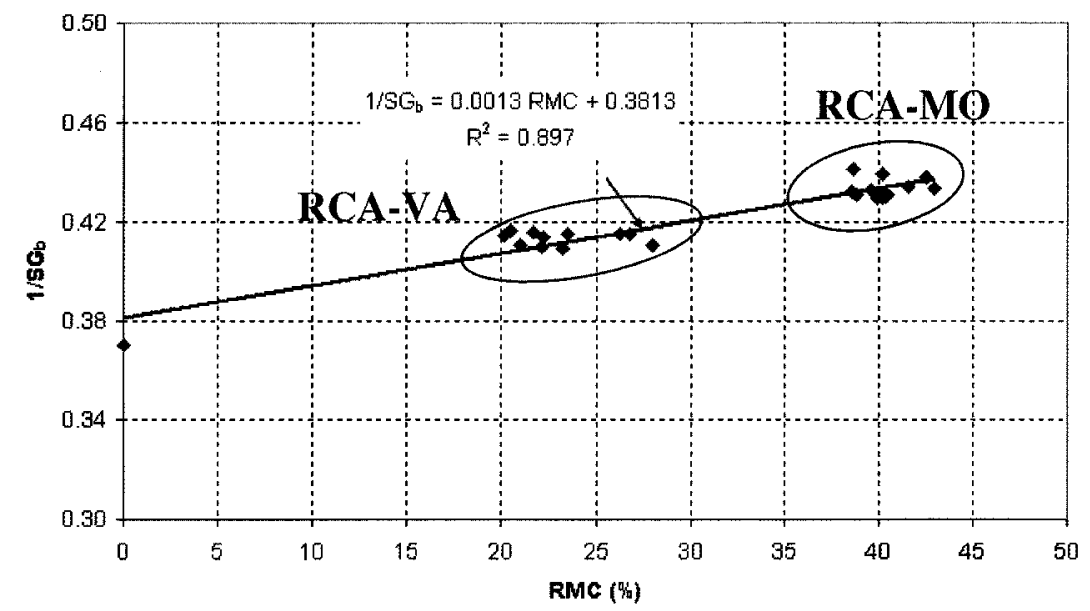

(a) 24-h immersion

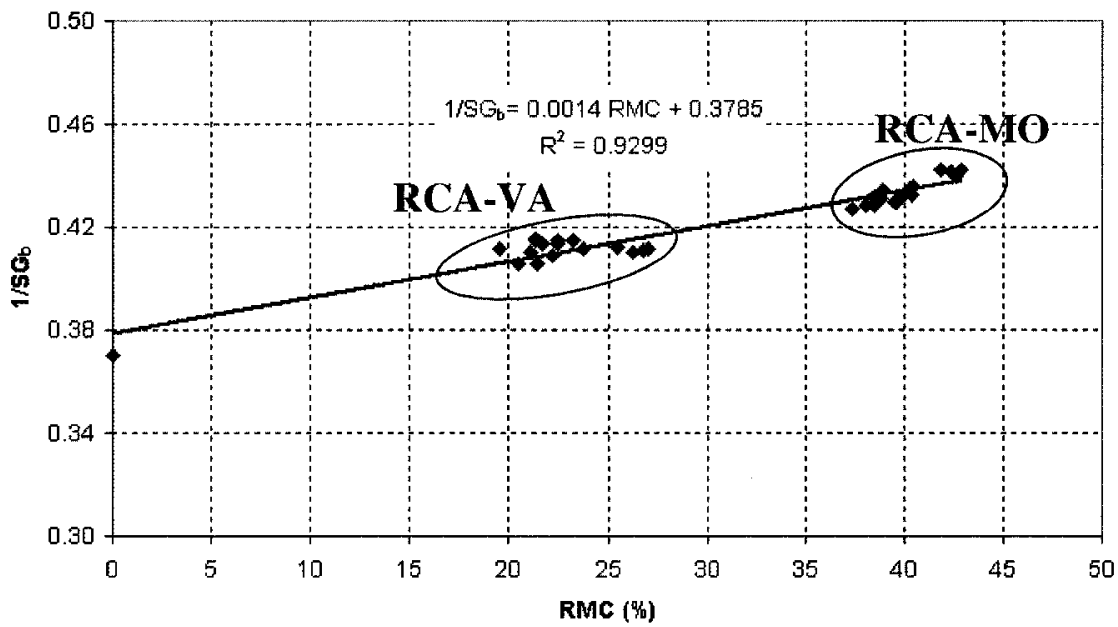

(b) 48-h immersion

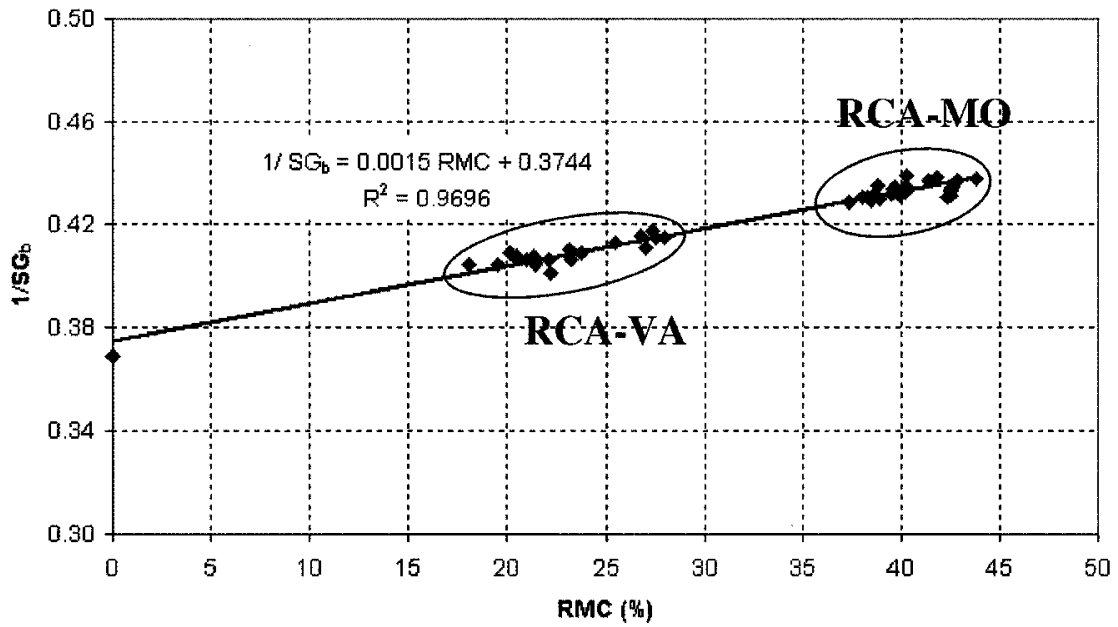

(c) 72-h immersion

Figure 3.19: Bulk specific gravity vs. RMC for different immersion periods 


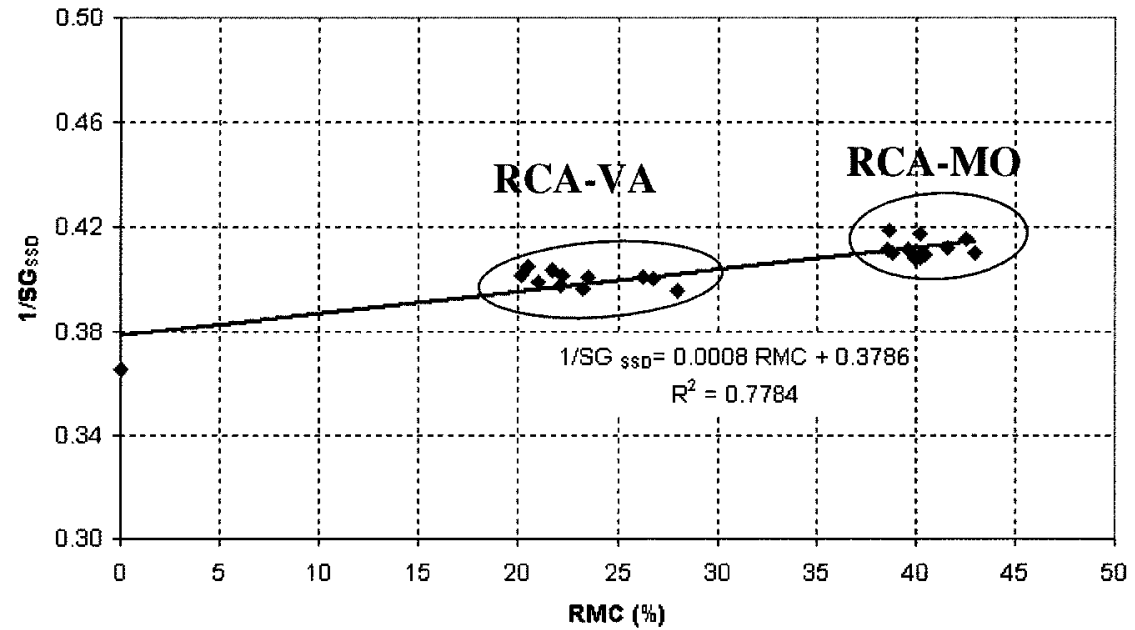

(a) 24-h immersion

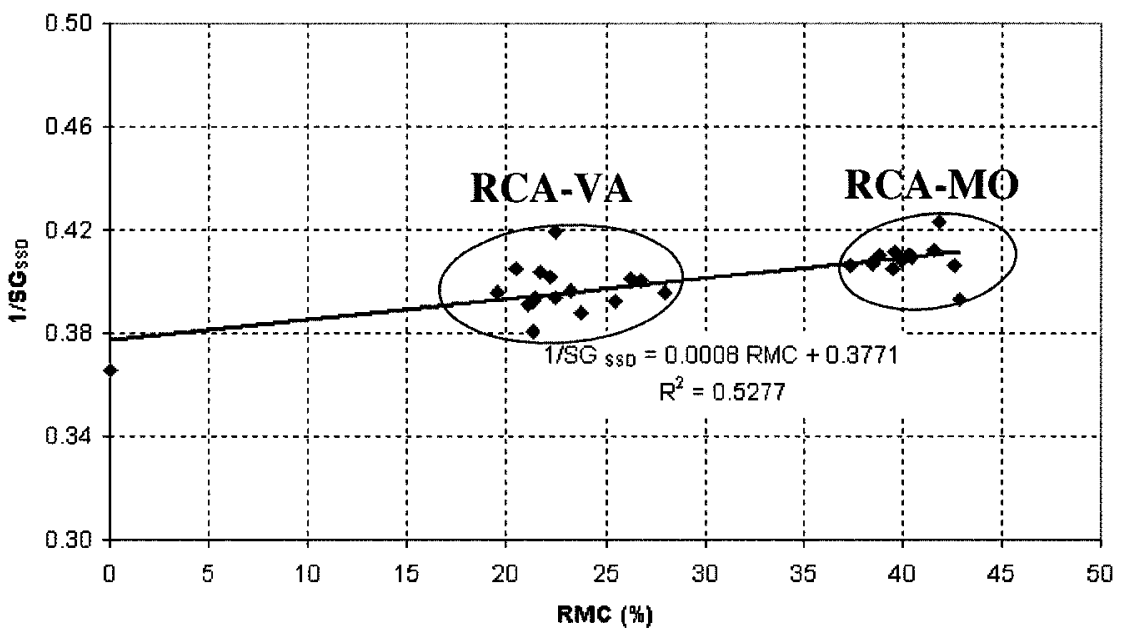

(b) 48-h immersion

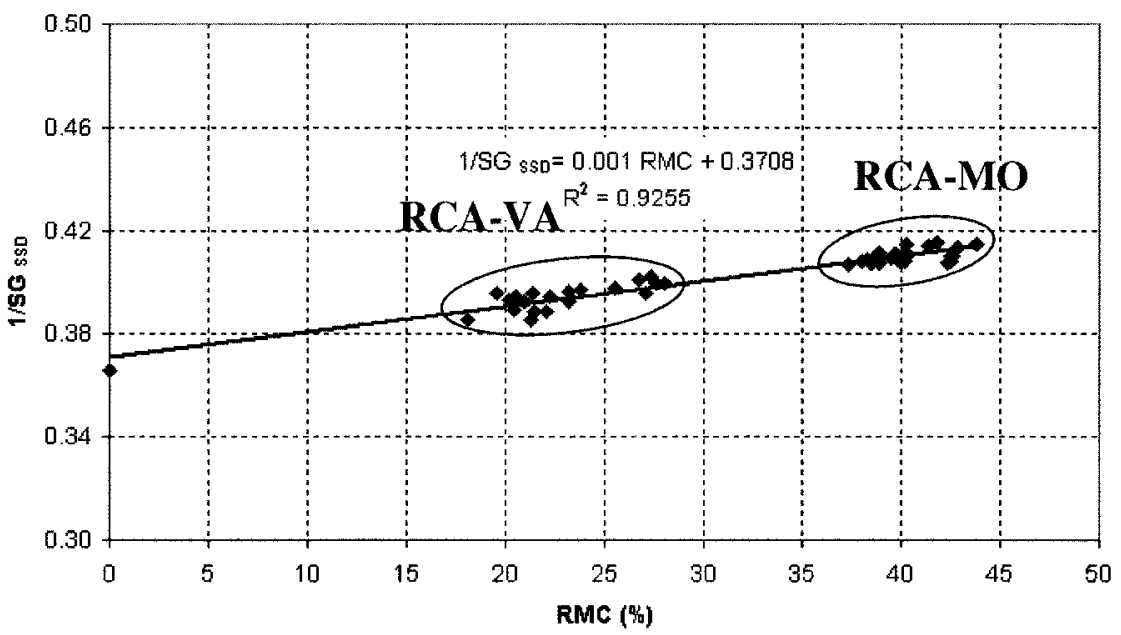

(c) 72-h immersion

Figure 3.20: SSD specific gravity vs. RMC for different immersion periods 


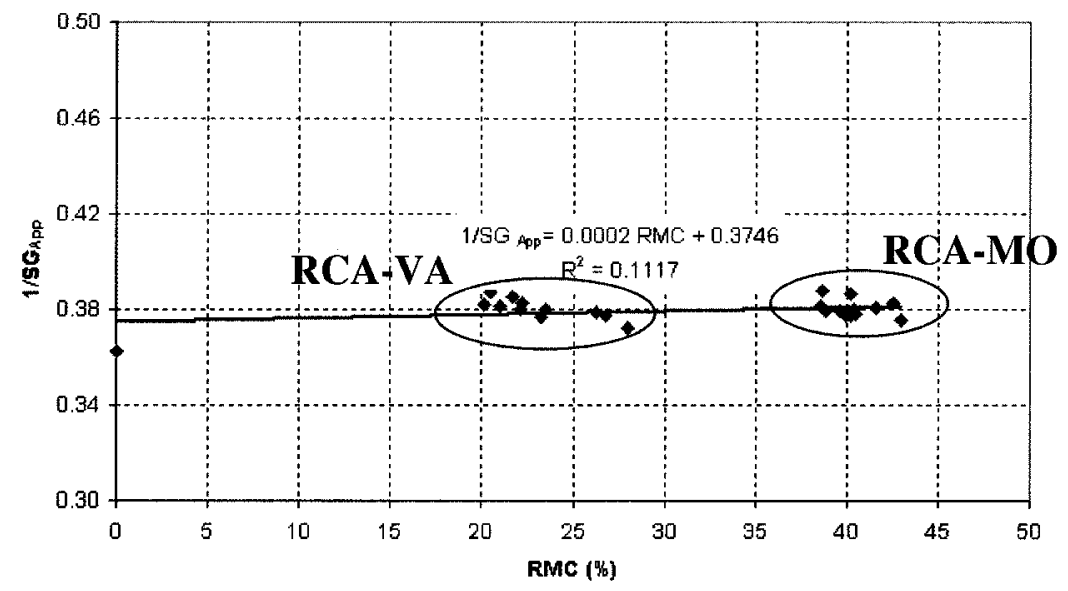

(a) 24-h immersion

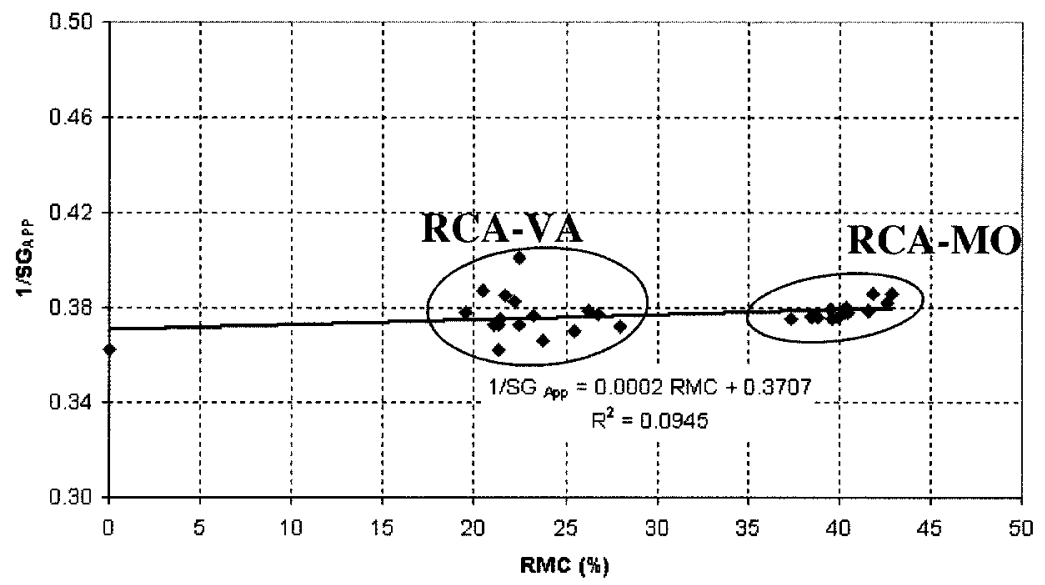

(b) 48-h immersion

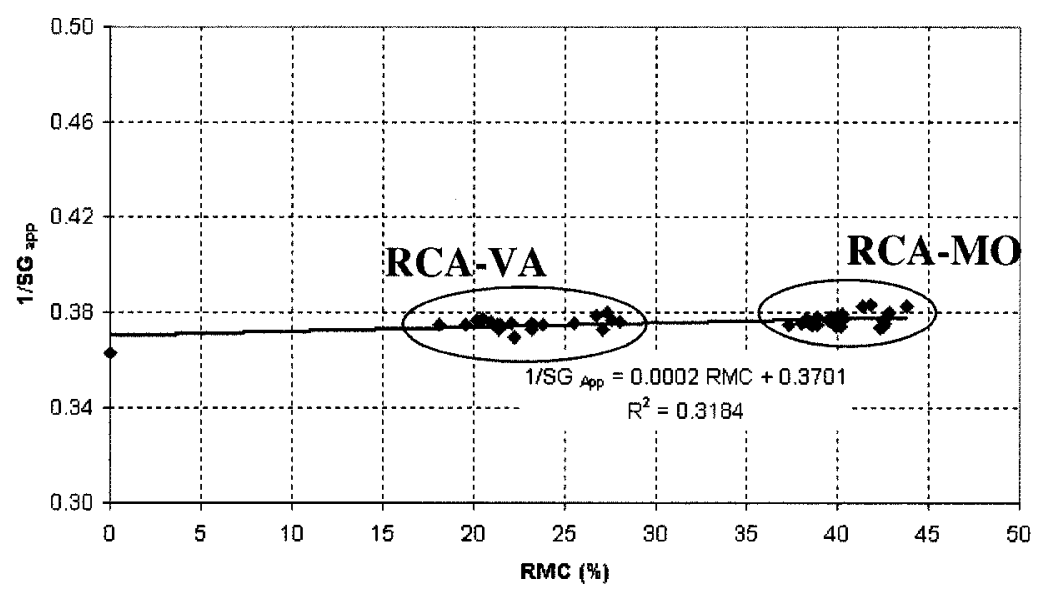

(c) 72-h immersion

Figure 3.21: Apparent specific gravity vs. RMC for different immersion periods 


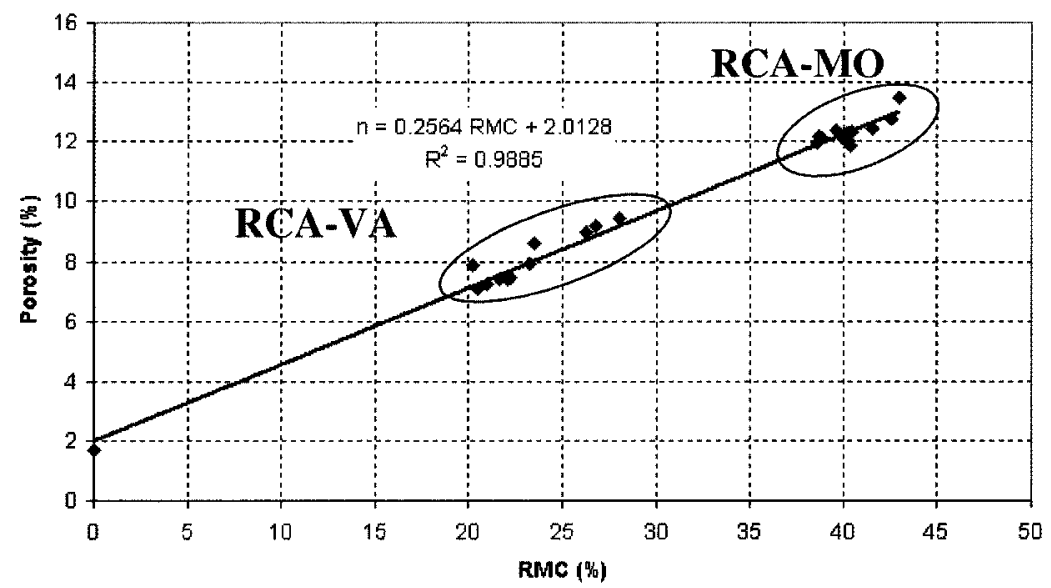

(a) 24-h immersion

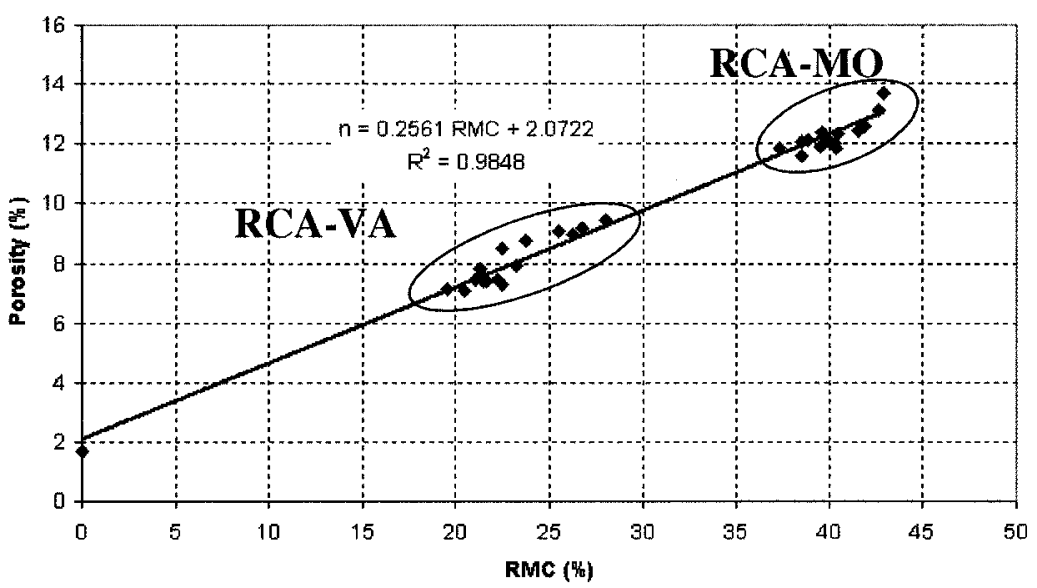

(b) 48-h immersion

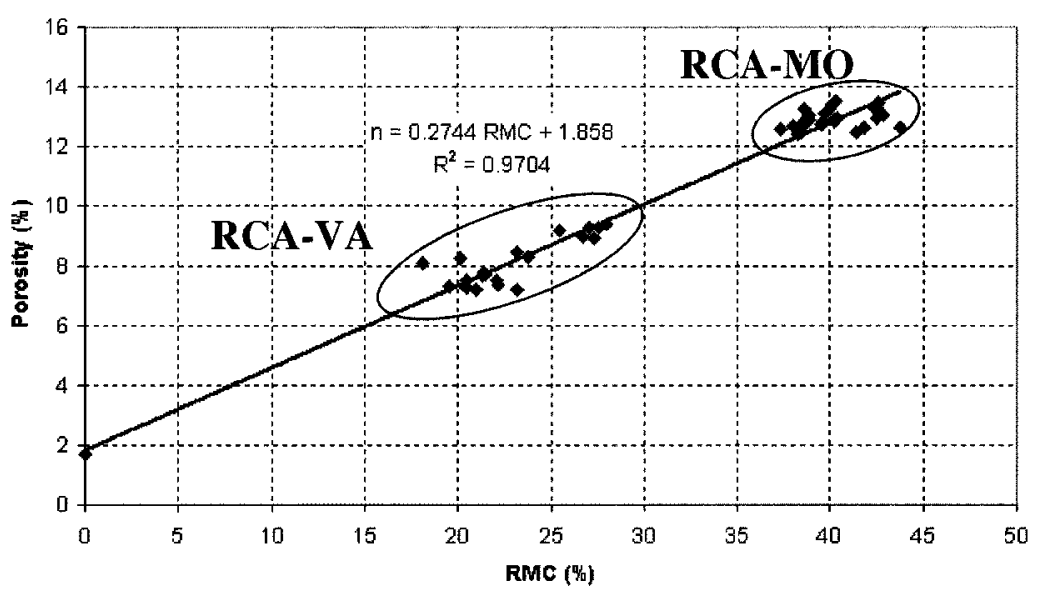

(c) 72-h immersion

Figure 3.22: Porosity vs. RMC at different immersion periods 


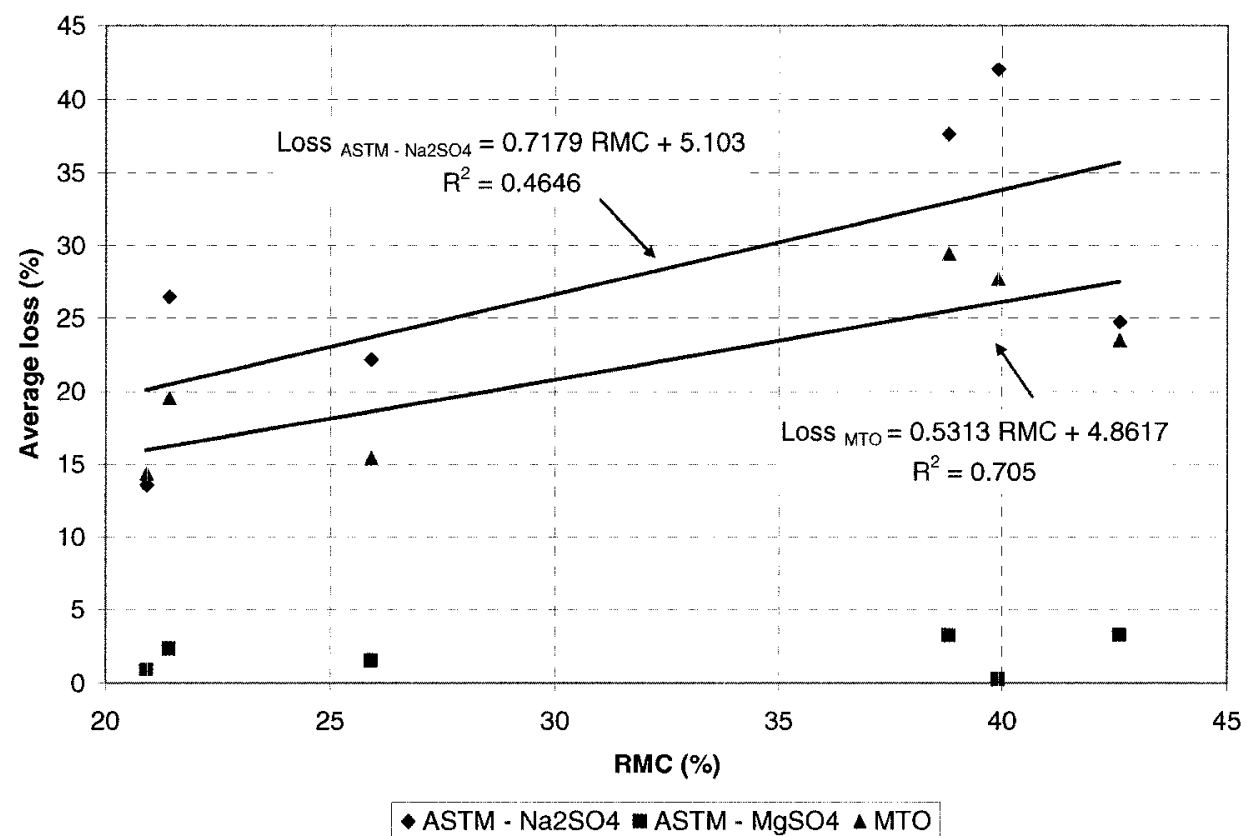

Figure 3.23: Soundness measurements vs. RMC

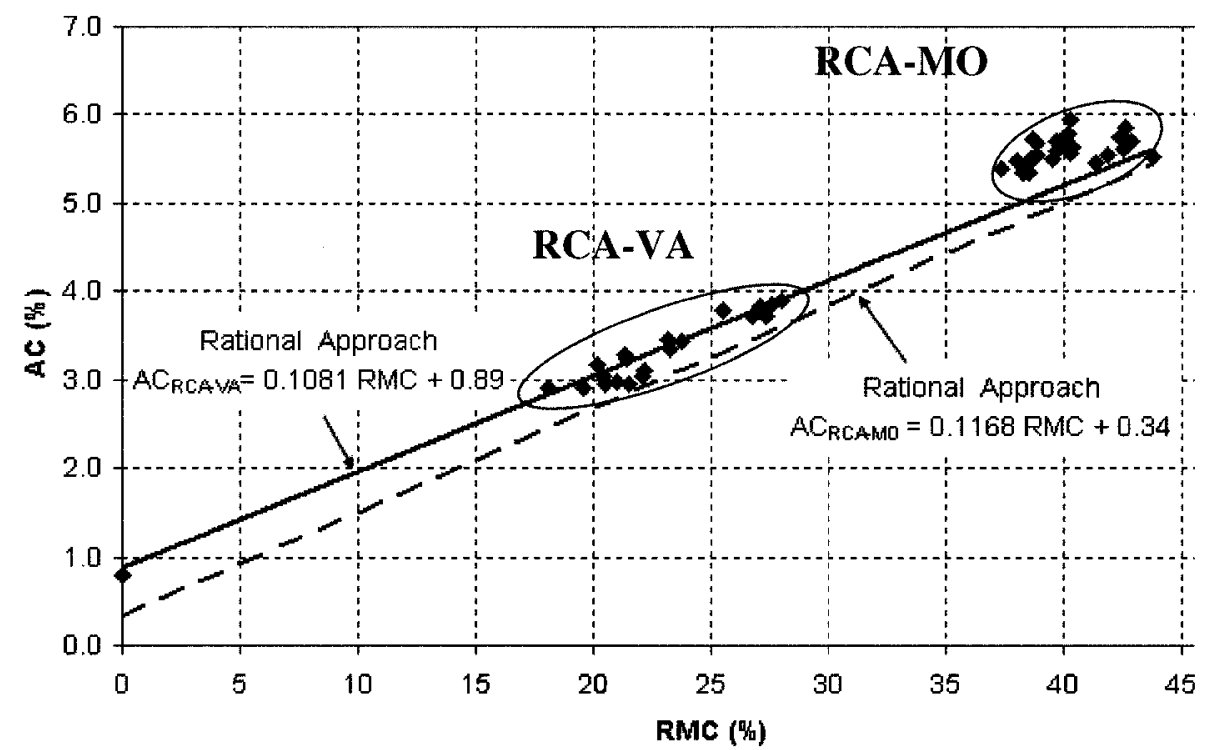

Figure 3.24: Comparison of the rational model with experimental data for the absorption capacity- RMC relationship 


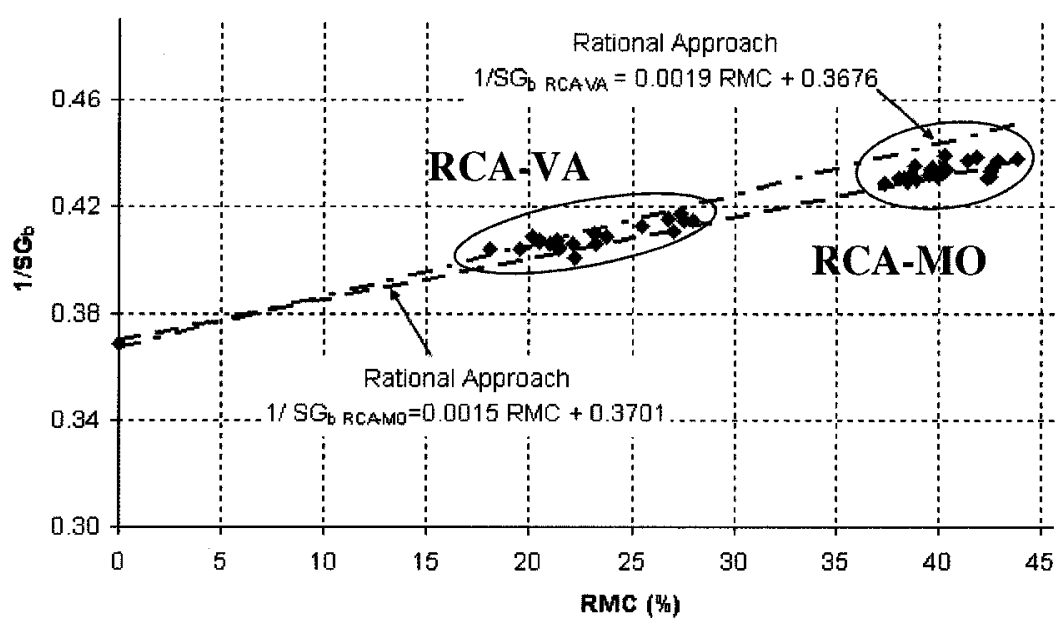

(a)

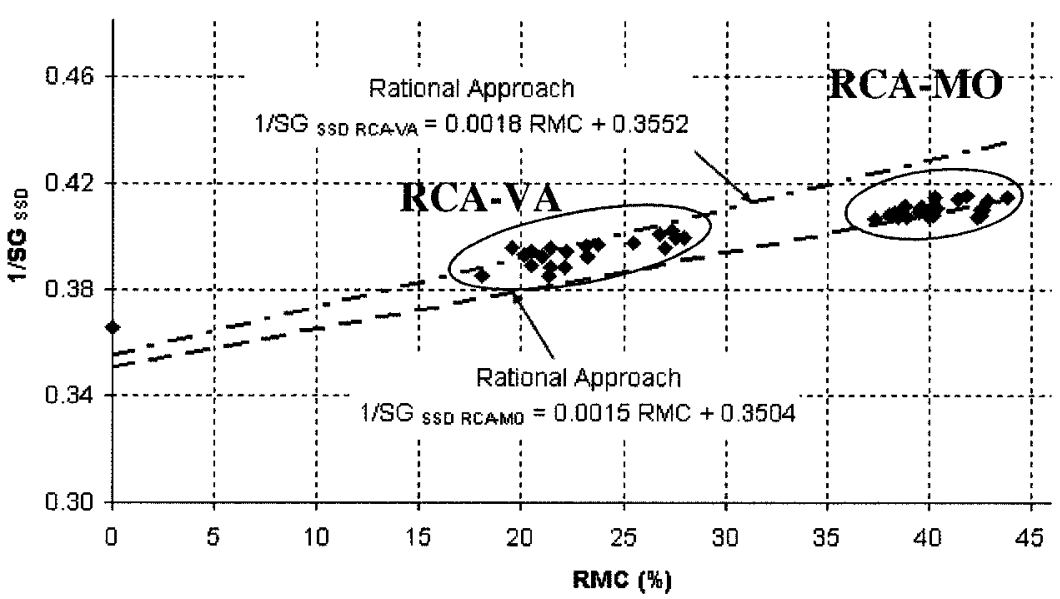

(b)

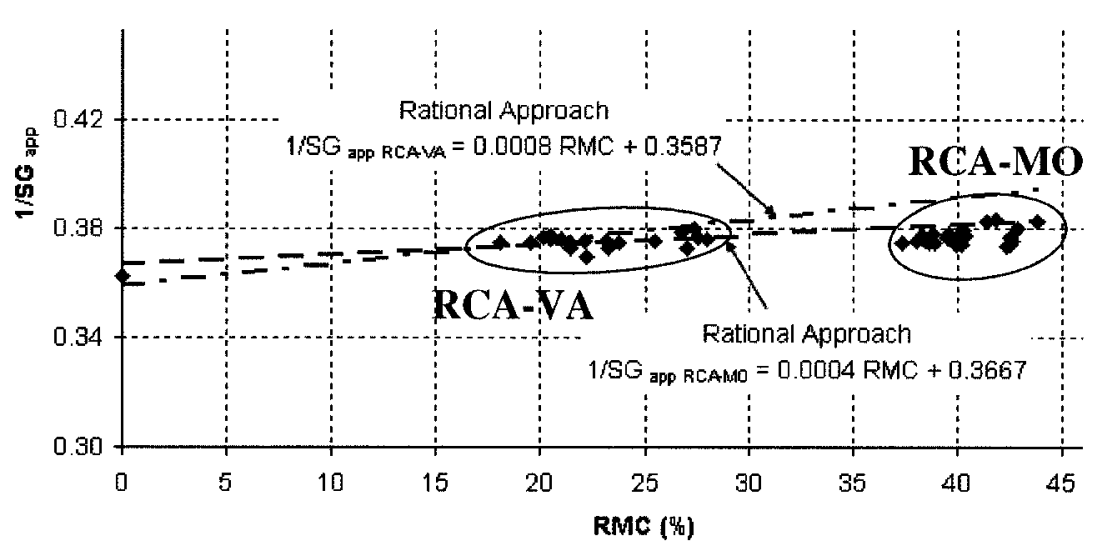

(c)

Figure 3.25: Comparison of the rational model with experimental data for the specific gravity- RMC relationships (a) $S_{6}-R M C$, (b) $S G_{S S D}-R M C$, (c) $S_{a p p}$ RMC 


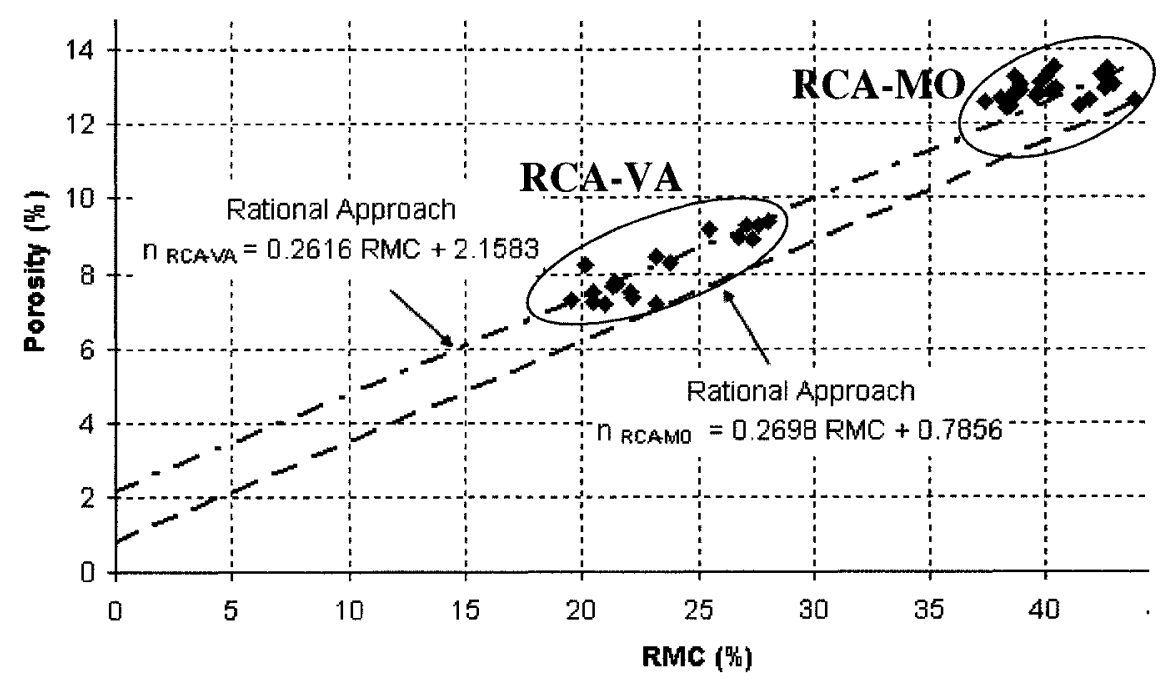

Figure 3.26: Comparison of the rational model with experimental data for the porosity $\mathrm{RMC}$ relationship

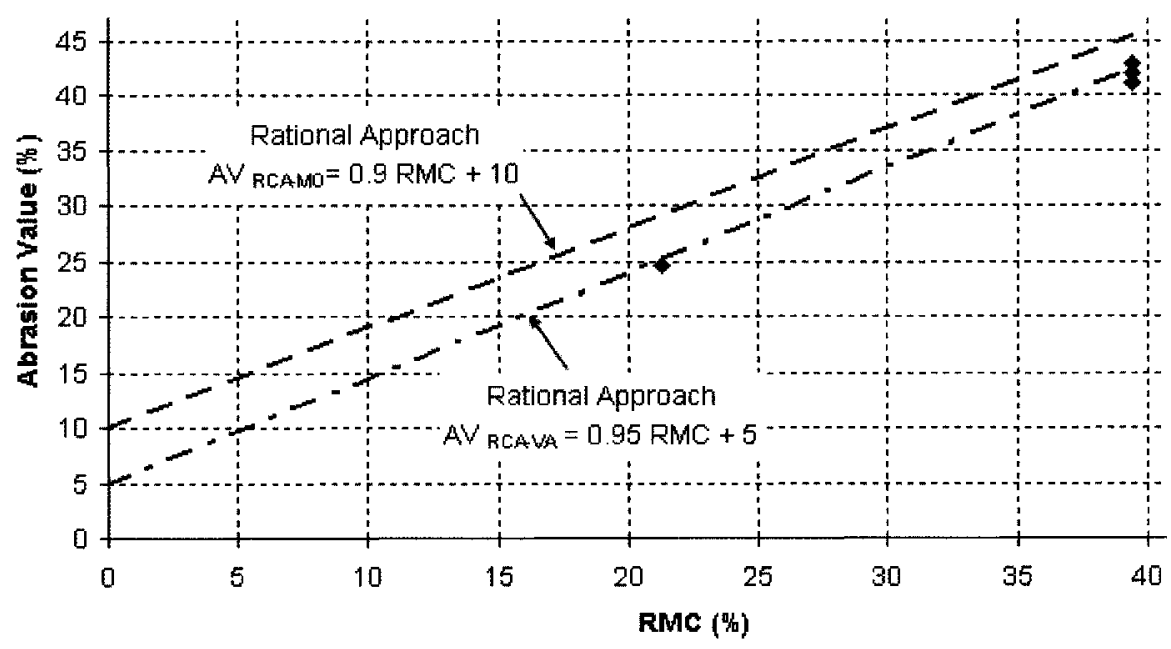

Figure 3.27: Comparison of the rational model with experimental data for the abrasion value-RMC relationship 


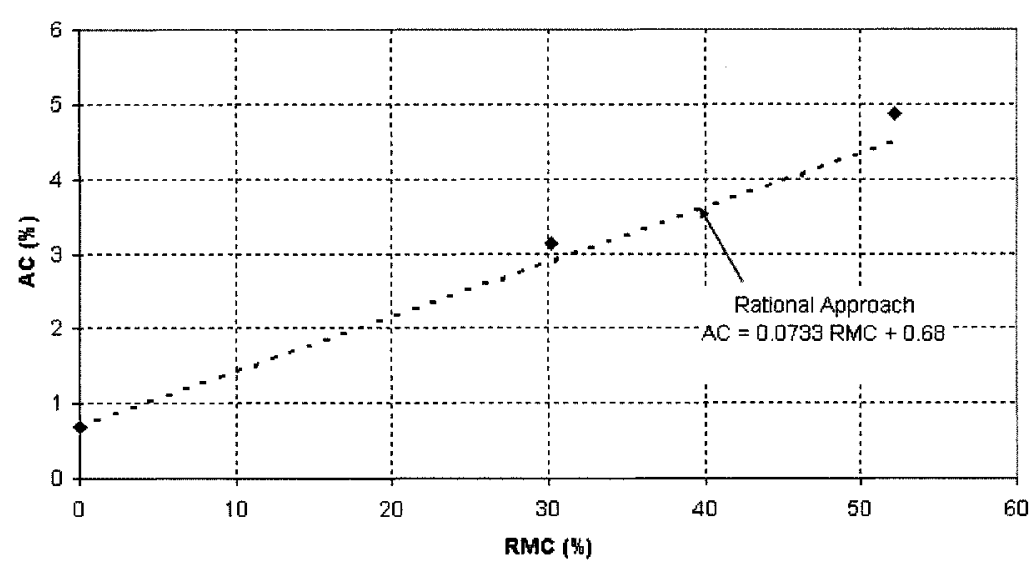

(a)

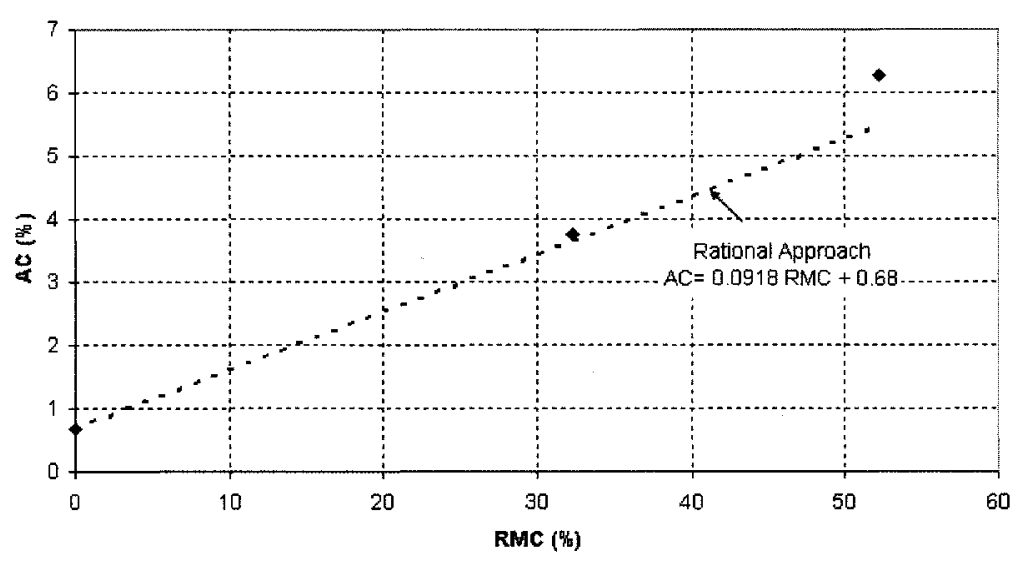

(c)

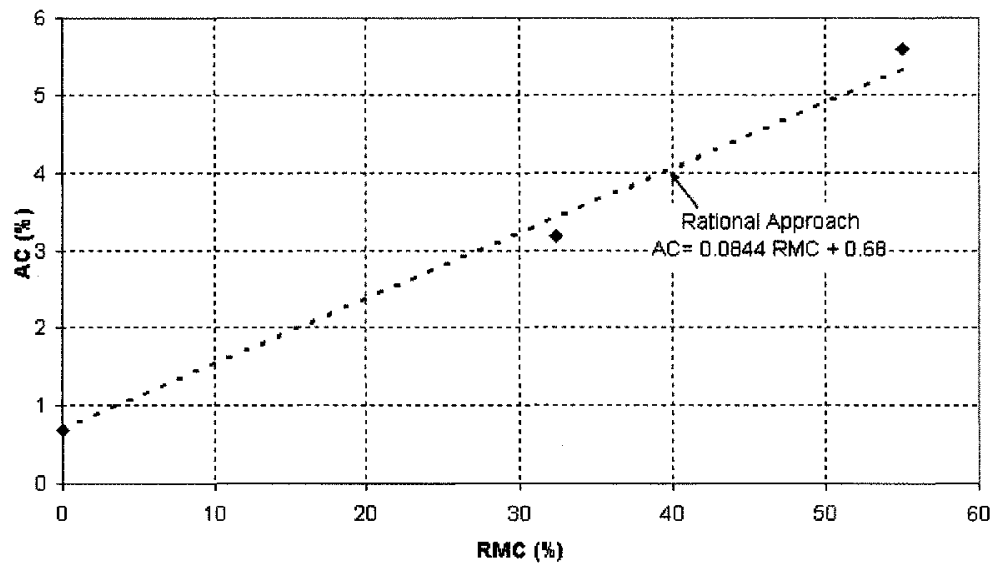

(b)

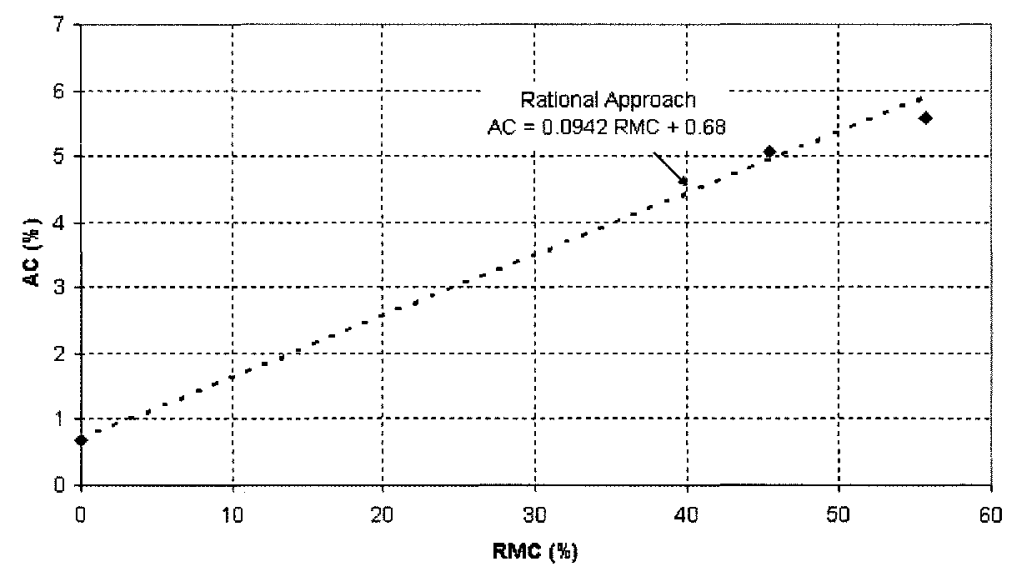

(d)

Figure 3.28: Predicted and experimental average absorption capacity values for (a) Nagatki-1, (b) Nagatki-2, (c) Nagatki-3, (d) Nagatki-4 


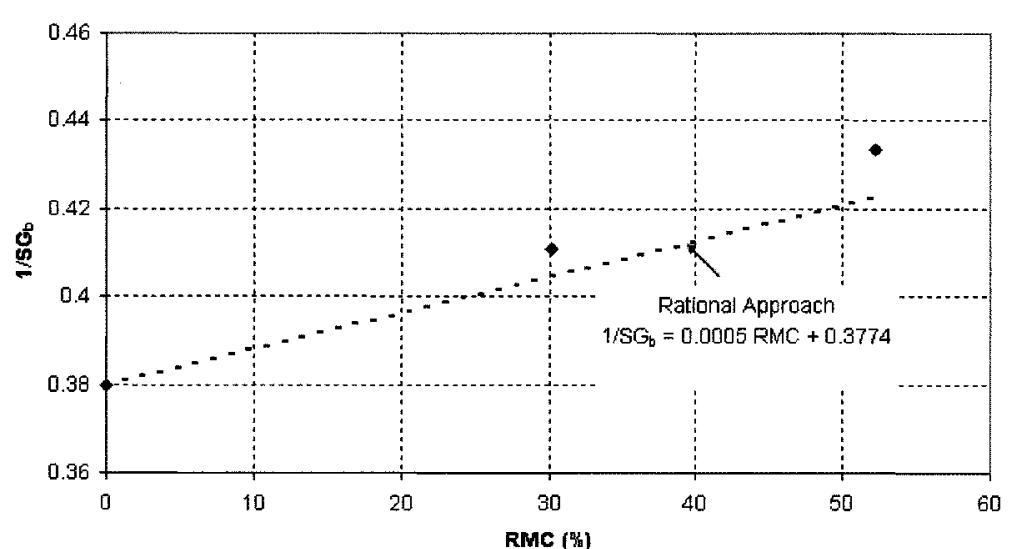

(a)

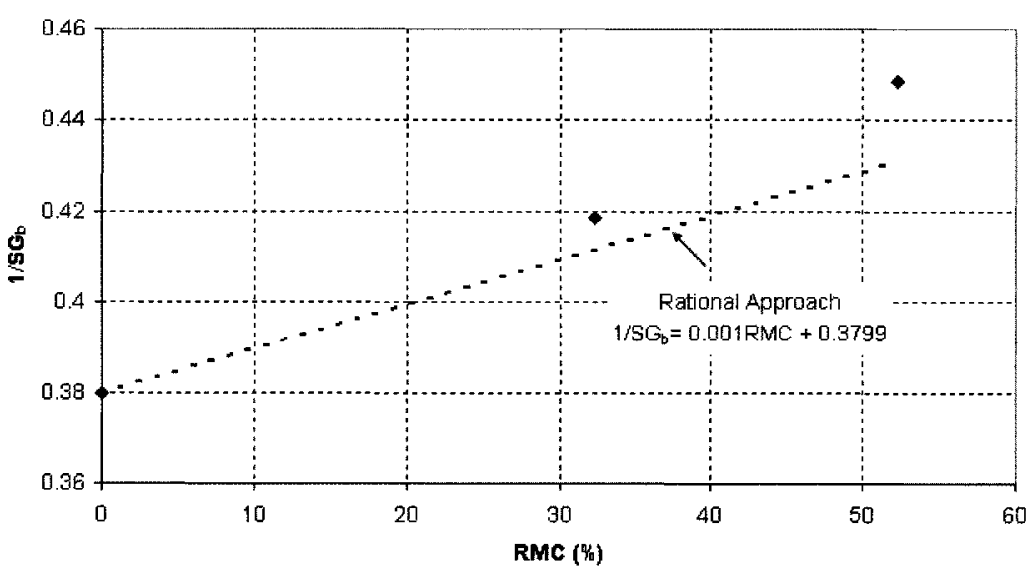

(c)

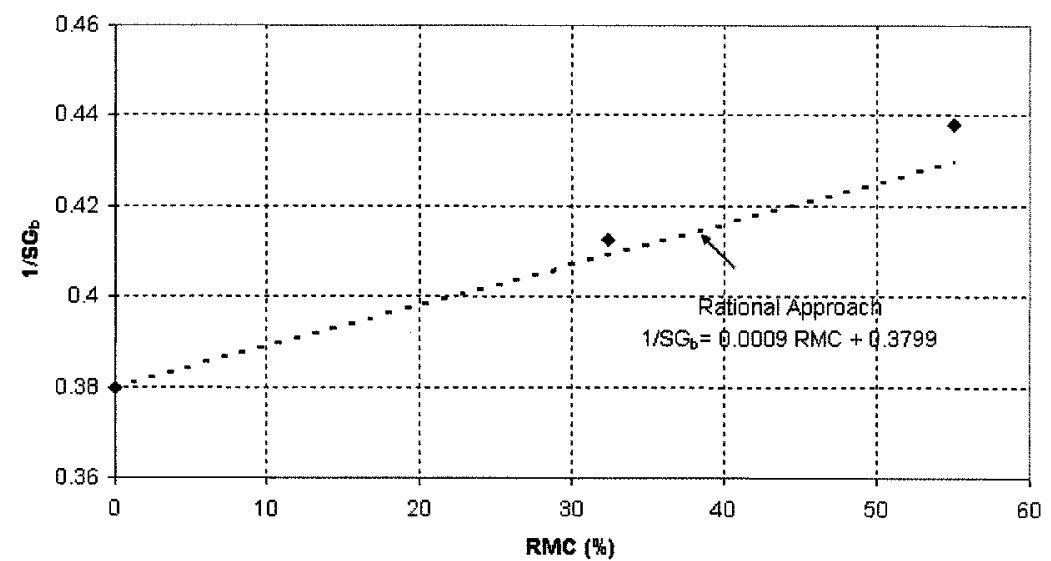

(b)

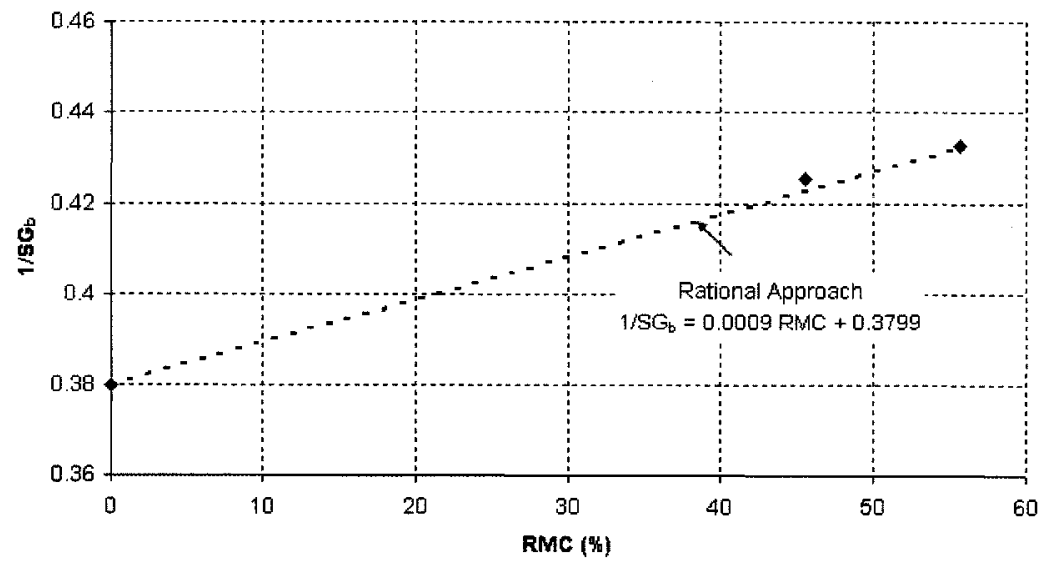

(d)

Figure 3.29: Predicted and experimental average bulk specific gravity values for (a) Nagatki-1, (b) Nagatki-2, (c) Nagatki-3, (d) Nagatki-4 


\section{CHAPTER 4: PRELIMINARY STUDY ON CONCRETE PRODUCED WITH RCA: SAMPLE PREPARATION}

\subsection{General}

The main difference between RCA and NA is the residual mortar attached to the former. As concluded in Chapter 3, the attached residual mortar leads to the observed lower specific gravity and higher absorption capacity of RCA in comparison to NA. Unless it is pre-saturated, RCA with high absorption capacity may reduce the workability of fresh GC by absorbing the mixing water. Low specific gravity can affect the fresh and hardened density of the GC. The presence of residual mortar also affects the shape and surface texture of the RCA, which, in turn, can influence the fresh and hardened properties of GC. It is, therefore, crucial to carry out a preliminary study that investigates the effect of residual mortar on the fresh and hardened properties of GC so that mix design procedures that can produce structural-grade concrete can be developed. Since there is no standard method describing how structural-grade durable concrete can be produced using RCA, this investigation will provide valuable information for the durability phase of this research by providing insight to how GC mixes should be designed.

The RCA types used in the preliminary study were RCA-MO and RCA-VA, whose properties were provided in Chapter 3. The NA used as replacement for RCA-MO and RCA-VA were Navan limestone (from Ottawa, ON) and river gravel (from Vancouver, BC), respectively; their physical properties are given in Table 3.11. For simplicity, GC produced by using RCA-MO and RCA-VA are called GC-MO and GC-VA, respectively. 
In order to investigate the effect of the attached residual mortar on the fresh and hardened concrete properties, several GC specimens with different combinations of RCA replacement and w/c ratio were produced. As a start, the GC was produced by $100 \%$ RCA content. In the following two trials, the RCA is partially replaced by NA $(50 \%$ and $70 \%$ replacement by weight). The replacement of the RCA with NA allows concrete to be produced with different total residual mortar content (TRMC). Control specimens that were prepared with $100 \%$ NA (no RCA) were also prepared.

As noted in Chapter 1, the TRMC of GC is calculated from the amount of RCA content and its RMC. In other words, GC produced by using $100 \%$ RCA will have TRMC equal to the RMC. Therefore, it is rational to study the effect of the TRMC rather than the RCA content, i.e., the effect of $100 \%$ RCA content has no meaning if the RMC of RCA is not specified. Furthermore, the study of the average frequency of the RCA particles with respect to the amount of the RMC (Chapter 3, Section 3.3.2.3) shows that some particles have no residual mortar (i.e. OVA). This means that the RCA batches obtained from plant can contain some OVA particles. Therefore, only the TRMC of GC with partial RCA replacement can simulate the RMC of RCA. By this approach, the problem of the contradictory results observed in the literature about the effect of the RCA content on the mechanical and durability properties of concrete produced with RCA can be resolved.

Since RCA-MO and RCA-VA have averaged RMC values of $41 \%$ and $23 \%$, respectively, GC-MO and GC-VA produced by using 100\% RCA will have TRMC of $41 \%$ and $23 \%$. Similarly, GC-MO and GC-VA specimens produced by $50 \%$ NA replacement will have TRMC of $21 \%$ and $12 \%$, respectively. With the same logic, it can 
be stated that GC-MO and GC-VA produced by using 30\% RCA and 70\% NA will have the TRMC of $12 \%$ and $7 \%$, respectively. The specimens with no RCA (100\% NA) have a TRMC value of $0 \%$.

As a summary, four types of GC specimens with $100 \%, 50 \%, 30 \%$ and $0 \%$ RCA content were produced. For each type, three levels of w/c ratio $(0.35,0.45$ and 0.55$)$ were used in order to study the effect of w/c ratio on the physical and mechanical properties of GC. Since there is no standard mix design procedure for producing structural-grade durable GC, the mixes were produced using a slightly modified version of ACI standard mix design procedure (ACI committee $211.1,1997$ ) that was developed for conventional concrete produced with NA.

\subsection{Mix Design Process}

The main steps for proportioning the constituents of GC are: (1) estimation of mixing water and air content, (2) computation of the cement content, and (3) calculation of the aggregate content. The coarse aggregate content of the mixes were calculated based on 100\% RCA content; and all other replacement cases were designed by weight replacement of RCA with NA. The estimation of the mixing water depends on the slump required to produce $\mathrm{GC}$ with suitable workability for the specified job. In the preliminary study, the slump for GC was selected from the recommendations provided for conventional concrete, by the ACI mix design method. The selected slump provides the estimation of mixing water content. The computation of the cement content depends on the selection of water-to-cementitious materials ratio. In the preliminary study no supplementary cementing materials in addition to OPC was used. Three levels of w/c ratio $0.35,0.45$ and 0.55 were considered to produce air-entrained and non-air-entrained 
concrete mixes. The fine aggregate and RCA contents were calculated using an analytical approach. The details of each step described above are provided in the following sections.

\subsubsection{Estimation of Mixing Water and Air Content}

A maximum slump range of 75 to $100 \mathrm{~mm}$ is suitable for most types of construction of structural members such as reinforced foundation, beams, slabs and columns (ACI $211.1,1997)$; therefore, this slump range is selected for the GC specimens that are investigated in this preliminary study. The water content of GC mixes was determined using $75 \mathrm{~mm}$ to $100 \mathrm{~mm}$ targeted slump and the nominal maximum aggregate size of 19 $\mathrm{mm}$ from Table 4.1, which is a reproduction of Table A1.5.3.3 of ACI 211-1-97 (ACI committee 211 , 1997). From Table 4.1, the water content of non-air-entrained and airentrained concrete with $19 \mathrm{~mm}$ nominal maximum size of aggregate and with a slump of 75 to $100 \mathrm{~mm}$ are $205 \mathrm{~kg} / \mathrm{m}^{3}$ and $184 \mathrm{~kg} / \mathrm{m}^{3}$, respectively. GC with $6 \%$ air entrainment is expected to withstand the freeze-and-thaw action and the severe exposure conditions in Canada.

\subsubsection{Calculation of the Cement Content}

The required cement can be calculated by dividing the estimated mixing water content by the $\mathrm{w} / \mathrm{c}$ ratio. As mentioned before, three levels of $w / \mathrm{c}$ ratio are considered in this study $0.35,0.45$ and 0.55 . Therefore, three cement contents for each air-entrained and non-air-entrained specimen were calculated. After the calculation of the cement content, the aggregate content can be calculated. The calculation of the aggregate content is explained in the following section. 


\subsubsection{Calculation of Aggregate Content}

An analytical approach was used to calculate the natural fine aggregate and the coarse aggregate content (i.e., RCA). Firstly, the RCA content was determined by relating the compacted volume of the coarse aggregate to the absolute volume of concrete. The ratio, $V_{a}$, of the compacted, or absolute, volume of coarse aggregate to the absolute volume of concrete can be expressed using (Lydon, 1982):

$$
V_{a} \approx \frac{\frac{W_{S S D}^{C A}}{S G_{S S D}^{C A}}}{\frac{W_{c}}{S G^{C}}+\frac{W_{S S D}^{F A}}{S G_{S S D}^{F A}}+\frac{W_{S S D}^{C A}}{S G_{S S D}^{C A}}+W_{w}}
$$

where, $W_{S S D}^{C A}$ is the weight of the SSD coarse aggregate, $W_{S S D}^{F A}$ is the weight of the SSD fine aggregate, $W_{c}$ is the weight of the cement, $W_{w}$ is the weight of the water, $S G_{S S D}^{C A}$ and $S G_{S S D}^{F A}$ are the SSD specific gravity of coarse and fine aggregates, respectively, $S G^{C}$ is the specific gravity of cement.

According to ACI-211.1-97 (ACI committee 211, 1997), the weight of coarse aggregate can be found from the volume of the dry-rodded coarse aggregate per unit volume, $v_{c a}$, as per:

$$
W_{O D}^{C A}=M \times v_{c a}
$$

where, $W_{O D}^{C A}$ and $M$ are the weight and the unit weight of the OD coarse aggregate, respectively. Furthermore, the coarse aggregate weight can be assumed to be equal to the ratio of the compacted volume of coarse aggregate, $V_{a}$, multiplied by the density of the coarse aggregate (i.e., $1000 S G_{b}^{C A}$ ): 


$$
W_{O D}^{C A}=1000 S G_{b}^{C A} \times V_{a}
$$

Combining and re-arranging Eqs. 4.2 and 4.3 yields:

$$
V_{a}=\left(\frac{M}{1000 S G_{b}^{C A}}\right) \times v_{c a}
$$

The volume of dry-rodded coarse aggregate per unit volume of concrete, $v_{c a}$, can be found from Table 4.2, which is reproduced from Table A1.5.3.6 provided by ACI 211.197 (ACI committee 211,1997 ). The selection of the volume of dry-rodded coarse aggregate per unit volume of concrete is based on the fineness modulus of the fine aggregate $(F M)$, and the nominal maximum size of the coarse aggregate.

It should be noted that the $v_{c a}$ values in Table A1.5.3.6 (ACI 211.1, 1997) are obtained from empirical relationships to produce conventional concrete with a degree of workability suitable for usual reinforced concrete construction. However, to produce more workable concrete, the volume of coarse aggregate provided in Table A1.5.3.6 can be reduced up to $10 \%$ (ACI 211.1, 1997). Since the absorption capacity of RCA is generally larger than that of NA due to the presence of the attached residual mortar, additional water may be necessary to achieve the required workability. Therefore, the $v_{c a}$ values from Table 4.2 can be reduced by the absorption capacity of RCA, which are $5.4 \%$ and $3.3 \%$ for RCA-MO and RCA-VA, respectively. The reduction of coarse aggregate volume is within the $10 \%$ limit specified by ACI (ACI committee 211,1997 ).

From Table 4.2, for sand with fineness modulus of 2.6 (the value used in this study), the volume of dry-rodded coarse aggregate per unit volume of concrete $\left(v_{c a}\right)$ can be determined as 0.64 . This value was reduced by $5.4 \%$ and $3.3 \%$ for mixes produced with RCA-MO and RCA-VA, respectively. 
The natural fine aggregate weight, $W_{S S D}^{F A}$, can be determined from:

$$
W_{S S D}^{F A}=U-W_{c}-W_{S S D}^{C A}-W_{w}
$$

where $U$ is the density of fresh concrete, which can be calculated from Eq. 4.6, which is adopted from ACI 211.1 (ACI committee 211, 1997):

$U=10 S G_{S S D . a v}(100-A)+W_{c}\left(1-\frac{S G_{S S D, a v}}{S G^{c}}\right)-W_{w}\left(S G_{S S D, a v}-1\right)$

Where, $S G_{S S D, a v}$ is the weighted average SSD specific gravity of combined fine and coarse aggregates, and $A$ is the air content. By substitution of $V_{a}$ and $W_{S S D}^{F A}$ from Eqs. 4.4 and 4.5, respectively, in Eq. 4.1, the weight of coarse RCA can be found using:

$$
W_{S S D}^{C A}=\frac{V_{a}\left[W_{c}\left(\frac{1}{S G^{c}}-\frac{1}{S G_{S S D}^{F A}}\right)+\frac{\left(U-W_{w}\right)}{S G_{F A}}+W_{w}\right]}{\left[\frac{1}{S G_{S S D}^{C A}}-\left(\frac{1}{S G_{S S D}^{C A}}-\frac{1}{S G_{S S D}^{F A}}\right) V_{a}\right]}
$$

Equations 4.5 and 4.7 can be used to calculate the fine and coarse aggregate (i.e. RCA and NA) for GC mixes. All parameters used in these two equations were determined in the material characterization study presented in Chapter 3. The specific gravity of the cement was measured as 3.15 . The measured data as well as the chemical composition of the cementitious materials used in this study are presented in Appendix A. The summary of the design of GC-MO and GC-VA mixes are presented in the following section. 


\subsection{Design Summary}

Tables 4.3 to 4.6 present the mix proportions of air-entrained and non-air-entrained GC-MO and GC-VA specimens as determined per the method described in Section 4.2. It should be noted that each mix in these tables is identified with unique designation. For example, air entrained GC-MO produced with w/c ratio of 0.35 and with $100 \%$ RCA-MO (or $41 \%$ TRMC) is designated as GC-MO-AE-P1-41. For non-air-entrained GC, the designation "AE" is replaced with "NE." The terms P1, P2 and P3 stand for w/c ratios of $0.35,0.45$ and 0.55 , respectively ("P" stands for "Preliminary Study"). The last term designates the TRMC value of the mix.

\subsection{Preparation of Specimens}

The GC-MO and GC-VA specimens were produced using a three step process: (1) preparation of the materials, (2) adjustment of the mixing water, (3) mixing. These stages are discussed in the following sections.

\subsubsection{Preparation of Materials}

The materials used in the batches were prepared 24 hours prior to the time of mixing. The material preparation includes the following steps:

- Weigh the air-dried coarse aggregate in individual size fractions, and recombine the individual fraction size in the proper proportions to produce the desired grading. Respectively, $35 \%, 25 \%, 40 \%$ of the RCA for the fraction size of $4.75 \mathrm{~mm}, 9.5 \mathrm{~mm}$ and $12.5 \mathrm{~mm}$ were used in this study,

- Weigh the air-dried fine aggregate;

- Pre-soak recombined RCA in water $24 \mathrm{~h}$ prior to use; 
- Moisten the fine aggregate by water of about $4 \%$ weight of fine aggregate 24 hours prior to use and cover the container by plastic sheet;

- Weigh and store cementitious materials in dry plastic pail.

\subsubsection{Water Content Adjustment}

Since the coarse aggregate (RCA and NA) used in the GC mixes were pre-soaked (i.e., in wet condition), an adjustment in the mixing water content was necessary just prior to the concrete mixing. The weight of the surface water is equal to the weight of the coarse aggregate in wet condition minus the SSD weight of the coarse aggregate (RCA and NA). The weight of the surface water is subtracted from the designed mixing water of each batch.

The weight of coarse aggregate in SSD condition $W_{S S D}^{C A}$ can be calculated as:

$$
W_{S S D}^{C A}=\frac{W_{A D}^{C A}}{\left(\frac{1+m_{c}^{C A}}{1+A C^{C A}}\right)}
$$

where, $W_{A D}^{C A}$ is the weight of the coarse aggregate in air dry condition, $A C^{C A}$ is the absorption capacity of the coarse aggregate, and $m_{c}^{C A}$ is the moisture content of of the coarse aggregate. The factor to convert the air dry weight of the coarse aggregate to SSD condition can be calculated by substituting $m_{c}^{C A}$ and $A C^{C A}$ values in Eq.4.8. The $m_{c}^{C A}$ and $A C^{C A}$ of the RCA-MO, RCA-VA, limestone and river gravel were previously presented in Chapter 3. Using these values, to calculate the weight of RCA in SSD condition, the air-dry weight of coarse aggregate was divided by 0.9593 and 0.9806 for RCA-MO and RCA-VA, respectively. Similarly, for limestone and river gravel, the air 
dry weights were divided by 0.998 and 0.993 to find the corresponding weights in SSD condition. On the other hand, the fine aggregate has absorption capacity of $0.8 \%$ (Chapter 3). Hence, the weight of fine aggregate in SSD condition is equal to the weight of dry fine aggregate multiplied by 1.008 . The water adjustment for the coarse and fine aggregates was performed as follows:

- Remove pre-soaked coarse aggregate from water and drain excess water, weigh the aggregate in the wet condition. The surface water is estimated by subtracting the calculated SSD weight of aggregate (Eq.4.8) from the wet weight of aggregate. The surface water is subtracted from the estimated mixing water.

- Remove the plastic sheet over the fine aggregate container and weigh the moist fine aggregate. Subtract the difference between wet weight and SSD weight from mixing water.

\subsubsection{Concrete Mixing Procedure}

A pan mixer was used for mixing the GC batches. The mixing process of GC was performed using ASTM C 192/C 192 M-00 (ASTM, 2003) procedure as follows:

- Grease the mixer by mixing a mortar batch closely proportioned to the test batch;

- Add the coarse aggregate and the air entrainment agent (if used);

- Start the mixer and add the fine aggregate;

- Add the cement and water while mixer is running;

- Mix the concrete for 3 minutes;

- Stop the mixer for 3 minutes; while at rest, keep the mixer covered;

- Remix the concrete for another 2 minutes; 
- Measure the slump, temperature, air content, and weight (for the fresh concrete density).

After the mixing process, concrete specimens were cast and cured following the procedures described in ASTM C 192/C 192 M-00 (ASTM, 2003). Six cylindrical specimens (100 x $200 \mathrm{~mm})$ were prepared for 28 -day compressive strength ( 3 specimens) and splitting tensile strength tests ( 3 specimens). Three cylindrical specimens $(150 \times 300$ $\mathrm{mm}$ ) were prepared for the determination of elastic modulus. Specimens were stored for 28 days in a moist room in accordance with the requirements of ASTM C 511-98 (ASTM, 2003).

The fresh and hardened properties of the mixes prepared in this preliminary study are presented in Chapters 5 and 6, respectively. 
Table 4.1: Approximate mixing water and air content requirements for different slumps up to the nominal maximum size of aggregate of $19 \mathrm{~mm}$ (reproduced from ACI 211-1-97)

\begin{tabular}{|c|c|c|c|}
\hline \multirow{2}{*}{ Slump (mm) } & \multicolumn{3}{|c|}{$\begin{array}{c}\text { Water, } \mathbf{~ k g} \mathbf{m}^{3} \text { of concrete for indicated } \\
\text { nominal maximum size of aggregate }\end{array}$} \\
\cline { 2 - 4 } & 9.5 & 12.5 & 19 \\
\hline \multicolumn{3}{|c|}{ Non-air-entrained Concrete } \\
\hline 25 to 50 & 207 & 199 & 190 \\
\hline 75 to 100 & 228 & 216 & 205 \\
\hline 150 to 175 & 243 & 228 & 216 \\
\hline $\begin{array}{c}\text { Approximate amount of entrapped } \\
\text { air in non-air entrained concrete, (\%) }\end{array}$ & 3 & 2.5 & 2 \\
\hline \multicolumn{3}{|c|}{ Air-entrained Concrete } \\
\hline \hline 25 to 50 & 181 & 175 & 168 \\
\hline 75 to 100 & 202 & 193 & 184 \\
\hline 150 to 175 & 216 & 205 & 197 \\
\hline $\begin{array}{c}\text { Recommended average \& total air } \\
\text { content, (\%) for level of exposure }\end{array}$ & 4.5 & 4.0 & 3.5 \\
\hline Mild exposure & 6.0 & 5.5 & 5.0 \\
\hline Moderate exposure & 7.5 & 7.0 & 6.0 \\
\hline Extreme exposure
\end{tabular}

Table 4.2: Volume of coarse aggregate per unit volume of concrete $\left(v_{c a}\right)$ (reproduced from ACI 211.1-97).

\begin{tabular}{|c|c|c|c|c||}
\hline \multirow{2}{*}{$\begin{array}{c}\text { Aggregate Nominal maximum size } \\
(\mathbf{m m})\end{array}$} & \multicolumn{4}{c|}{$v_{c a}$ for different (FM) } \\
\cline { 2 - 6 } & $\mathbf{2 . 4 0}$ & $\mathbf{2 . 6 0}$ & $\mathbf{2 . 8 0}$ & $\mathbf{3 . 0}$ \\
\hline 9.5 & 0.5 & 0.48 & 0.46 & 0.44 \\
\hline 12.5 & 0.59 & 0.57 & 0.55 & 0.53 \\
\hline 19 & 0.66 & 0.64 & 0.62 & 0.60 \\
\hline
\end{tabular}


Table 4.3: Mix design proportions of air-entrained GC-MO mixes $\left(\mathrm{kg} / \mathrm{m}^{3}\right)$

\begin{tabular}{|c|c|c|c|c|c|}
\hline \multirow{2}{*}{ Materials } & \multicolumn{4}{|c|}{ RCA (\%) / TRMC (\%) } & \multirow{2}{*}{ Sample Designation } \\
\hline & $100 / 41$ & $50 / 21$ & $30 / 12$ & $\mathbf{0}$ & \\
\hline $\mathrm{C}\left(\mathrm{kg} / \mathrm{m}^{3}\right)$ & 526 & 526 & 526 & 526 & \multirow{9}{*}{ GC-MO-AE-P1 } \\
\hline $\mathrm{W}\left(\mathrm{kg} / \mathrm{m}^{3}\right)$ & 184 & 184 & 184 & 184 & \\
\hline$w / c$ & 0.35 & 0.35 & 0.35 & 0.35 & \\
\hline $\operatorname{OVA}^{1}\left(\mathrm{~kg} / \mathrm{m}^{3}\right)$ & 0 & 398 & 558 & 796 & \\
\hline $\operatorname{RCA}^{2}\left(\mathrm{~kg} / \mathrm{m}^{3}\right)$ & 796 & 398 & 239 & 0 & \\
\hline $\operatorname{Sand}^{3}\left(\mathrm{~kg} / \mathrm{m}^{3}\right)$ & 673 & 673 & 673 & 673 & \\
\hline $\operatorname{AEA}^{4}(\mathrm{ml})$ & 158 & 158 & 158 & 158 & \\
\hline $\mathrm{FA} / \mathrm{A}_{\mathrm{c}}{ }^{5}$ & 0.46 & 0.46 & 0.46 & 0.46 & \\
\hline $\mathrm{A}_{\mathrm{c}} / \mathrm{c}^{6}$ & 2.8 & 2.8 & 2.8 & 2.8 & \\
\hline $\mathrm{C}\left(\mathrm{kg} / \mathrm{m}^{3}\right)$ & 409 & 409 & 409 & 409 & \multirow{9}{*}{ GC-MO-AE-P2 } \\
\hline $\mathrm{W}\left(\mathrm{kg} / \mathrm{m}^{3}\right)$ & 184 & 184 & 184 & 184 & \\
\hline$w / c$ & 0.45 & 0.45 & 0.45 & 0.45 & \\
\hline $\operatorname{OVA}^{1}\left(\mathrm{~kg} / \mathrm{m}^{3}\right)$ & 0 & 397 & 556 & 794 & \\
\hline $\mathrm{RCA}^{2}\left(\mathrm{~kg} / \mathrm{m}^{3}\right)$ & 794 & 397 & 238 & 0 & \\
\hline Sand $^{3}\left(\mathrm{~kg} / \mathrm{m}^{3}\right)$ & 769 & 769 & 769 & 769 & \\
\hline $\mathrm{AEA}^{4}(\mathrm{ml})$ & 123 & 123 & 123 & 123 & \\
\hline $\mathrm{FA} / \mathrm{A}_{\mathrm{c}}{ }^{5}$ & 0.49 & 0.49 & 0.49 & 0.49 & \\
\hline $\mathrm{A}_{\mathrm{c}} / \mathrm{c}^{6}$ & 3.8 & 3.8 & 3.8 & 3.8 & \\
\hline $\mathrm{C}\left(\mathrm{kg} / \mathrm{m}^{3}\right)$ & 335 & 335 & 335 & 335 & \multirow{9}{*}{ GC-MO-AE-P3 } \\
\hline $\mathrm{W}\left(\mathrm{kg} / \mathrm{m}^{3}\right)$ & 184 & 184 & 184 & 184 & \\
\hline $\mathrm{w} / \mathrm{c}$ & 0.55 & 0.55 & 0.55 & 0.55 & \\
\hline $\operatorname{OVA}^{1}\left(\mathrm{~kg} / \mathrm{m}^{3}\right)$ & 0 & 397 & 556 & 794 & \\
\hline $\operatorname{RCA}^{2}\left(\mathrm{~kg} / \mathrm{m}^{3}\right)$ & 794 & 397 & 238 & 0 & \\
\hline $\operatorname{Sand}^{3}\left(\mathrm{~kg} / \mathrm{m}^{3}\right)$ & 830 & 831 & 831 & 830 & \\
\hline $\mathrm{AEA}^{4}(\mathrm{ml})$ & 100 & 100 & 100 & 100 & \\
\hline $\mathrm{FA} / \mathrm{A}_{\mathrm{c}}{ }^{5}$ & 0.51 & 0.51 & 0.51 & 0.51 & \\
\hline $\mathrm{A}_{\mathrm{c}} / \mathrm{c}^{6}$ & 4.9 & 4.9 & 4.9 & 4.9 & \\
\hline
\end{tabular}

${ }^{1}$ OVA used for GC-MO mixes is limestone

${ }^{2} \mathrm{RCA}$ used for GC-MO mixes is RCA-MO

${ }^{3}$ Cantley (Quebec) natural sand derived from granite

${ }^{4}$ AEA: air entraining agent (Micro Air 940) $30 \mathrm{ml}$ per $100 \mathrm{~kg} / \mathrm{m}^{3}$ was used.

${ }^{5}$ Fine aggregate to total aggregate ratio.

${ }^{6}$ Total aggregate to cement ratio. 
Table 4.4: Mix design proportions of non-air-entrained GC-MO mixes $\left(\mathrm{kg} / \mathrm{m}^{3}\right)$

\begin{tabular}{|c|c|c|c|c|c|}
\hline \multirow{2}{*}{ Materials } & \multicolumn{4}{|c|}{ RCA (\%) / TRMC (\%) } & \multirow{2}{*}{ Sample Designation } \\
\hline & $100 / 41$ & $50 / 21$ & $30 / 12$ & $\mathbf{0}$ & \\
\hline $\mathrm{C}\left(\mathrm{kg} / \mathrm{m}^{3}\right)$ & $\overline{586}$ & $\overline{5886}$ & $\overline{586}$ & 586 & \multirow{9}{*}{ GC-MO-NE-P1 } \\
\hline $\mathrm{W}\left(\mathrm{kg} / \mathrm{m}^{3}\right)$ & 205 & 205 & 205 & 205 & \\
\hline$w / c$ & 0.35 & 0.35 & 0.35 & 0.35 & \\
\hline $\operatorname{OVA}^{1}\left(\mathrm{~kg} / \mathrm{m}^{3}\right)$ & 0 & 424 & 594 & 848 & \\
\hline $\operatorname{RCA}^{2}\left(\mathrm{~kg} / \mathrm{m}^{3}\right)$ & 848 & 424 & 254 & 0 & \\
\hline $\operatorname{Sand}^{3}\left(\mathrm{~kg} / \mathrm{m}^{3}\right)$ & 670 & 671 & 671 & 670 & \\
\hline $\mathrm{AEA}^{4}(\mathrm{ml})$ & 0 & 0 & 0 & 0 & \\
\hline $\mathrm{FA} / \mathrm{A}_{\mathrm{c}}{ }^{5}$ & 0.44 & 0.44 & 0.44 & 0.44 & \\
\hline$A_{c} / c^{6}$ & 2.6 & 2.6 & 2.6 & 2.6 & \\
\hline $\mathrm{C}\left(\mathrm{kg} / \mathrm{m}^{3}\right)$ & 456 & 456 & 456 & 456 & \multirow{9}{*}{ GC-MO-NE-P2 } \\
\hline $\mathrm{W}\left(\mathrm{kg} / \mathrm{m}^{3}\right)$ & 205 & 205 & 205 & 205 & \\
\hline $\mathrm{w} / \mathrm{c}$ & 0.45 & 0.45 & 0.45 & 0.45 & \\
\hline $\operatorname{OVA}^{1}\left(\mathrm{~kg} / \mathrm{m}^{3}\right)$ & 0 & 423 & 592 & 846 & \\
\hline $\operatorname{RCA}^{2}\left(\mathrm{~kg} / \mathrm{m}^{3}\right)$ & 846 & 423 & 254 & 0 & \\
\hline $\operatorname{Sand}^{3}\left(\mathrm{~kg} / \mathrm{m}^{3}\right)$ & 778 & 778 & 778 & 777 & \\
\hline $\mathrm{AEA}^{4}(\mathrm{ml})$ & 0 & 0 & 0 & 0 & \\
\hline $\mathrm{FA} / \mathrm{A}_{\mathrm{c}}{ }^{5}$ & 0.48 & 0.48 & 0.48 & 0.48 & \\
\hline $\mathrm{A}_{\mathrm{c}} / \mathrm{c}^{6}$ & 3.6 & 3.6 & 3.6 & 3.6 & \\
\hline $\mathrm{C}\left(\mathrm{kg} / \mathrm{m}^{3}\right)$ & 373 & 373 & 373 & 373 & \multirow{9}{*}{ GC-MO-NE-P3 } \\
\hline $\mathrm{W}\left(\mathrm{kg} / \mathrm{m}^{3}\right)$ & 205 & 205 & 205 & 205 & \\
\hline $\mathrm{w} / \mathrm{c}$ & 0.55 & 0.55 & 0.55 & 0.55 & \\
\hline $\operatorname{OVA}^{1}\left(\mathrm{~kg} / \mathrm{m}^{3}\right)$ & 0 & 423 & 592 & 845 & \\
\hline $\operatorname{RCA}^{2}\left(\mathrm{~kg} / \mathrm{m}^{3}\right)$ & 845 & 423 & 254 & 0 & \\
\hline $\operatorname{Sand}^{3}\left(\mathrm{~kg} / \mathrm{m}^{3}\right)$ & 846 & 846 & 846 & 845 & \\
\hline $\mathrm{AEA}^{4}(\mathrm{ml})$ & 0 & 0 & 0 & 0 & \\
\hline $\mathrm{FA} / \mathrm{A}_{\mathrm{c}}{ }^{5}$ & 0.50 & 0.50 & 0.50 & 0.50 & \\
\hline $\mathrm{A}_{\mathrm{c}} / \mathrm{c}^{6}$ & 4.5 & 4.5 & 4.5 & 4.5 & \\
\hline
\end{tabular}

${ }^{1}$ OVA used for GC-MO mixes is limestone

${ }^{2} \mathrm{RCA}$ used for GC-MO mixes is RCA-MO

${ }^{3}$ Cantley (Quebec) natural sand derived from granite

${ }^{4}$ AEA: air entraining agent (Micro Air 940$) 30 \mathrm{ml}$ per $100 \mathrm{~kg} / \mathrm{m}^{3}$ was used.

${ }_{6}^{5}$ Fine aggregate to total aggregate ratio.

${ }^{6}$ Total aggregate to cement ratio. 
Table 4.5: Mix design proportions of air-entrained GC-VA mixes $\left(\mathrm{kg} / \mathrm{m}^{3}\right)$

\begin{tabular}{|c|c|c|c|c|c|}
\hline \multirow{2}{*}{ Materials } & \multicolumn{4}{|c|}{ RCA (\%)/ TRMC (\%) } & \multirow{2}{*}{ Sample Designation } \\
\hline & $100 / 23$ & $50 / 12$ & $30 / 7$ & 0 & \\
\hline $\mathrm{C}\left(\mathrm{kg} / \mathrm{m}^{3}\right)$ & 526 & 526 & 526 & 526 & \multirow{9}{*}{ GC-VA-AE-P1 } \\
\hline $\mathrm{W}\left(\mathrm{kg} / \mathrm{m}^{3}\right)$ & 184 & 184 & 184 & 184 & \\
\hline $\mathrm{w} / \mathrm{c}$ & 0.35 & 0.35 & 0.35 & 0.35 & \\
\hline $\operatorname{OVA}^{1}\left(\mathrm{~kg} / \mathrm{m}^{3}\right)$ & 0 & 435 & 610 & 871 & \\
\hline $\mathrm{RCA}^{2}\left(\mathrm{~kg} / \mathrm{m}^{3}\right)$ & 871 & 435 & 261 & 0 & \\
\hline $\operatorname{Sand}^{3}\left(\mathrm{~kg} / \mathrm{m}^{3}\right)$ & 640 & 640 & 640 & 640 & \\
\hline $\mathrm{AEA}^{4}(\mathrm{ml})$ & 158 & 158 & 158 & 158 & \\
\hline FA/A ${ }_{c}^{5}$ & 0.42 & 0.42 & 0.42 & 0.42 & \\
\hline$A_{d} / c^{6}$ & 2.9 & 2.9 & 2.9 & 2.9 & \\
\hline $\mathrm{C}\left(\mathrm{kg} / \mathrm{m}^{3}\right)$ & 409 & $\overline{409}$ & 409 & 4409 & \multirow{9}{*}{ GC-VA-AE-P2 } \\
\hline $\mathrm{W}\left(\mathrm{kg} / \mathrm{m}^{3}\right)$ & 184 & 184 & 184 & 184 & \\
\hline $\mathrm{w} / \mathrm{c}$ & 0.45 & 0.45 & 0.45 & 0.45 & \\
\hline OVA $^{1}\left(\mathrm{~kg} / \mathrm{m}^{3}\right)$ & 0 & 435 & 609 & 870 & \\
\hline $\mathrm{RCA}^{2}\left(\mathrm{~kg} / \mathrm{m}^{3}\right)$ & 870 & 435 & 261 & 0 & \\
\hline $\operatorname{Sand}^{3}\left(\mathrm{~kg} / \mathrm{m}^{3}\right)$ & 737 & 737 & 737 & 737 & \\
\hline $\mathrm{AEA}^{4}(\mathrm{ml})$ & 123 & 123 & 123 & 123 & \\
\hline $\mathrm{FA} / \mathrm{A}_{\mathrm{c}}{ }^{5}$ & 0.46 & 0.46 & 0.46 & 0.46 & \\
\hline $\mathrm{A}_{\mathrm{c}} / \mathrm{c}^{6}$ & 3.9 & 3.9 & 3.9 & 3.9 & \\
\hline $\mathrm{C}\left(\mathrm{kg} / \mathrm{m}^{3}\right)$ & 335 & 335 & 335 & 335 & \multirow{9}{*}{ GC-VA-AE-P3 } \\
\hline $\mathrm{W}\left(\mathrm{kg} / \mathrm{m}^{3}\right)$ & 184 & 184 & 184 & 184 & \\
\hline $\mathrm{w} / \mathrm{c}$ & 0.55 & 0.55 & 0.55 & 0.55 & \\
\hline $\operatorname{OVA}^{1}\left(\mathrm{~kg} / \mathrm{m}^{3}\right)$ & 0 & 434 & 608 & 869 & \\
\hline $\operatorname{RCA}^{2}\left(\mathrm{~kg} / \mathrm{m}^{3}\right)$ & 869 & 434 & 261 & 0 & \\
\hline $\operatorname{Sand}^{3}\left(\mathrm{~kg} / \mathrm{m}^{3}\right)$ & 799 & 799 & 799 & 799 & \\
\hline $\mathrm{AEA}^{4}(\mathrm{ml})$ & 100 & 100 & 100 & 100 & \\
\hline $\mathrm{FA}^{\prime} / \mathrm{A}_{\mathrm{c}}^{5}$ & 0.48 & 0.48 & 0.48 & 0.48 & \\
\hline $\mathrm{A}_{\mathrm{d}} / \mathrm{c}^{6}$ & 5.0 & 5.0 & 5.0 & 5.0 & \\
\hline
\end{tabular}

OVA used for GC-VA mixes is river gravel

${ }^{2}$ RCA used for GC-VA mixes is RCA-VA

${ }^{3}$ Cantley (Quebec) natural sand derived from granite

${ }_{5}^{4}$ AEA: air entraining agent (Micro Air 940) $30 \mathrm{ml} \mathrm{per} 100 \mathrm{~kg} / \mathrm{m}^{3}$ was used.

${ }_{6}^{5}$ Fine aggregate to total aggregate ratio.

${ }^{6}$ Total aggregate to cement ratio. 
Table 4.6: Mix design proportions of non-air-entrained GC-VA mixes $\left(\mathrm{kg} / \mathrm{m}^{3}\right)$

\begin{tabular}{|c|c|c|c|c|c|}
\hline \multirow{2}{*}{ Materials } & \multicolumn{4}{|c|}{ RCA (\%)/ TRMC (\%) } & \multirow{2}{*}{ Sample Designation } \\
\hline & $100 / 23$ & $50 / 12$ & $30 / 7$ & $\mathbf{0}$ & \\
\hline$\overline{C\left(\mathrm{~kg} / \mathrm{m}^{3}\right)}$ & 586 & $\overline{586}$ & 586 & 586 & \multirow{9}{*}{ GC-VA-NE-P1 } \\
\hline $\mathrm{W}\left(\mathrm{kg} / \mathrm{m}^{3}\right)$ & 205 & 205 & 205 & 205 & \\
\hline $\mathrm{w} / \mathrm{c}$ & 0.35 & 0.35 & 0.35 & 0.35 & \\
\hline $\mathrm{OVA}^{1}\left(\mathrm{~kg} / \mathrm{m}^{3}\right)$ & 0 & 464 & 649 & 927 & \\
\hline $\operatorname{RCA}^{2}\left(\mathrm{~kg} / \mathrm{m}^{3}\right)$ & 927 & 464 & 278 & 0 & \\
\hline $\operatorname{Sand}^{3}\left(\mathrm{~kg} / \mathrm{m}^{3}\right)$ & 634 & 634 & 634 & 634 & \\
\hline $\mathrm{AEA}^{4}(\mathrm{ml})$ & 0 & 0 & 0 & 0 & \\
\hline $\mathrm{FA} / \mathrm{A}_{\mathrm{c}}{ }^{5}$ & 0.41 & 0.41 & 0.41 & 0.41 & \\
\hline $\mathrm{A}_{\mathrm{c}} / \mathrm{c}^{6}$ & 2.7 & 2.7 & 2.7 & 2.7 & \\
\hline $\mathrm{C}\left(\mathrm{kg} / \mathrm{m}^{3}\right)$ & $\overline{4566}$ & $4 \overline{456}$ & 456 & 4456 & \multirow{9}{*}{ GC-VA-NE-P2 } \\
\hline $\mathrm{W}\left(\mathrm{kg} / \mathrm{m}^{3}\right)$ & 205 & 205 & 205 & 205 & \\
\hline$w / c$ & 0.45 & 0.45 & 0.45 & 0.45 & \\
\hline $\mathrm{OVA}^{1}\left(\mathrm{~kg} / \mathrm{m}^{3}\right)$ & 0 & 463 & 648 & 926 & \\
\hline $\operatorname{RCA}^{2}\left(\mathrm{~kg} / \mathrm{m}^{3}\right)$ & 926 & 463 & 278 & 0 & \\
\hline $\operatorname{Sand}^{3}\left(\mathrm{~kg} / \mathrm{m}^{3}\right)$ & 743 & 743 & 743 & 743 & \\
\hline $\mathrm{AEA}^{4}(\mathrm{ml})$ & 0 & 0 & 0 & 0 & \\
\hline $\mathrm{FA} / \mathrm{A}_{\mathrm{c}}^{5}$ & 0.45 & 0.45 & 0.45 & 0.45 & \\
\hline $\mathrm{A}_{\mathrm{c}} / \mathrm{c}^{6}$ & 3.7 & 3.7 & 3.7 & 3.7 & \\
\hline $\bar{C}\left(\mathrm{~kg} / \mathrm{m}^{3}\right)$ & 373 & 373 & 373 & 373 & \multirow{9}{*}{ GC-VA-NE-P3 } \\
\hline $\mathrm{W}\left(\mathrm{kg} / \mathrm{m}^{3}\right)$ & 205 & 205 & 205 & 205 & \\
\hline$w / c$ & 0.55 & 0.55 & 0.55 & 0.55 & \\
\hline $\operatorname{OVA}^{1}\left(\mathrm{~kg} / \mathrm{m}^{3}\right)$ & 0 & 462 & 647 & 925 & \\
\hline $\operatorname{RCA}^{2}\left(\mathrm{~kg} / \mathrm{m}^{3}\right)$ & 925 & 462 & 277 & 0 & \\
\hline $\operatorname{Sand}^{3}\left(\mathrm{~kg} / \mathrm{m}^{3}\right)$ & 811 & 811 & 811 & 811 & \\
\hline $\mathrm{AEA}^{4}(\mathrm{ml})$ & 0 & 0 & 0 & 0 & \\
\hline $\mathrm{FA} / \mathrm{A}_{\mathrm{c}}{ }^{5}$ & 0.47 & 0.47 & 0.47 & 0.47 & \\
\hline$A_{c} / c^{6}$ & 4.7 & 4.7 & 4.7 & 4.7 & \\
\hline
\end{tabular}

${ }^{1}$ OVA used for GC-VA mixes is river gravel

${ }^{2} \mathrm{RCA}$ used for GC-VA mixes is RCA-VA

${ }^{3}$ Cantley (Quebec) natural sand derived from granite

${ }^{4}$ AEA: air entraining agent (Micro Air 940) $30 \mathrm{ml}$ per $100 \mathrm{~kg} / \mathrm{m}^{3}$ was used.

${ }^{5}$ Fine aggregate to total aggregate ratio.

${ }^{6}$ Total aggregate to cement ratio. 


\section{CHAPTER 5: PRELIMINARY STUDY ON CONCRETE PRODUCED WITH RCA: FRESH PROPERTIES}

\subsection{General}

In this chapter, the results of the slump, air content and density measurements of the fresh concrete mixes produced in Chapter 4 are presented. The design parameters of each mix, whose test results are reported here, can be found in Tables 4.3 to 4.6. The slump, air content and fresh density were measured once for each mix. Tables 5.1 to 5.4 present the summary of the fresh property results of the tests presented in this chapter. In the following sections, these results are explained in further detail.

\subsection{Slump}

The conventional slump test as per ASTM C143-00 (ASTM, 2003) was used to determine the workability of the prepared concrete mixes. Table 5.1 to 5.4 present the slump results for GC-MO and GC-VA. It can be observed from Table 5.1 that the slump of GC-MO-AE mixes with partial RCA replacement range from $60 \mathrm{~mm}$ (GC-MO-AEP1-30/12) to $200 \mathrm{~mm}$ (GC-MO-AE-P3-50/21). The slump of the conventional concrete produced with limestone is in the range of $40 \mathrm{~mm}$ (GC-MO-AE-P1-0/0) to $180 \mathrm{~mm}$ (GCMO-AE-P3-0/0). From Table 5.2, it can be observed that the slump of GC-MO-NE mixes with partial RCA replacement range from $55 \mathrm{~mm}$ (GC-MO-NE-P1-50/21) to $205 \mathrm{~mm}$ (GC-MO-NE-P3-100/41). The slump of the conventional concrete produced with limestone is in the range of $90 \mathrm{~mm}$ (GC-MO-NE-P1-0/0) to $200 \mathrm{~mm}$ (GC-MO-NE-P3$0 / 0)$. 
Similarly, it can be observed from Table 5.3 that the slump of GC-VA-AE mixes with partial RCA replacement range from $50 \mathrm{~mm}$ (GC-VA-AE-P1-100/23) to $215 \mathrm{~mm}$ (GC-VA-AE-P3-50/12). The slump of the conventional concrete produced with river gravel is in the range of $130 \mathrm{~mm}$ (GC-VA-AE-P1-0/0) to $230 \mathrm{~mm}$ (GC-VA-AE-P3-0/0). From Table 5.4, it can be observed that the slump of GC-VA-NE mixes with partial RCA replacement range from $100 \mathrm{~mm}$ (GC-VA-NE-P1-100/23) to $225 \mathrm{~mm}$ (GC-VA-NE-P330/7). The slump of the conventional concrete produced with river gravel is in the range of $160 \mathrm{~mm}$ (GC-VA-NE-P1-0/0) to $230 \mathrm{~mm}$ (GC-VA-AE-P3-0/0).

Furthermore, the minimum slumps for conventional concrete with limestone and river gravel were $40 \mathrm{~mm}$ (GC-MO-AE-P1-0/0) and $130 \mathrm{~mm}$ (GC-VA-AE-P1-0/0), respectively. The minimum slump for GC-MO and GC-VA samples with RCA were 60 $\mathrm{mm}$ (GC-MO-AE-P1-30/12) and $50 \mathrm{~mm}$ (GC-VA-AE-P1-100/23). These minimum slump values are in the range of $\pm 25 \mathrm{~mm}$ of the $75 \mathrm{~mm}$ minimum targeted slump. Therefore, GC produced with RCA can be considered to be as workable as conventional concrete.

It is well known that the workability of concrete is mainly affected by: (1) water content; (2) shape and texture of aggregates; and (3) cement content. As described in Tables 4.3 to 4.6 , the water content is fixed for air-entrained and non-air-entrained mixes as $184 \mathrm{~kg} / \mathrm{m}^{3}$ and $205 \mathrm{~kg} / \mathrm{m}^{3}$, respectively. Since the residual mortar attached to the OVA particles affects the shape and surface texture of RCA, the change in the aggregate properties on the slump of the fresh mixes are reflected through different levels of RCA replacement $(100 \%, 50 \%, 30 \%$ and $0 \%)$ that results in varying TRMC for each batch of GC-MO $(41 \%, 21 \%, 12 \%, 0 \%)$ and GC-VA $(23 \%, 12 \%, 7 \%, 0 \%)$. The cement content of 
the mixes was varied for each level of RCA replacement to study its effect on the slump of fresh concrete. In the following sections, the effects of these factors on the slump of concrete produced with RCA are presented.

\subsubsection{The Effect of Cement Content}

Figures 5.1 and 5.2 illustrate the relationship between the cement content and the slump for GC-MO and GC-VA mixes, respectively. It is evident from these figures that the slump is linearly correlated with the cement content: an increase in cement content results in a decrease in the slump of the fresh concrete mix (with fixed total water content). This can be attributed to the increase in the total surface area of cement in the mix, which in turn, results in larger water consumption. As it can be observed from these figures, in all cases, the linear correlations between slump and the cement content have $\mathrm{R}^{2}$ values larger than $88 \%$. This linear trend is valid for all TRMC values and for both air entrained (Figures 5.1a and 5.2a) and non-air entrained mixes (Figures 5.1b and 5.2b).

For GC-MO-AE mixes, the slope of the trend lines corresponding to the TRMC values of $41 \%, 21 \%$ and $12 \%$ are $-0.57,-0.54$ and -0.57 , respectively, showing slight to no difference. For GC-MO-NE mixes, the slope of the trend lines corresponding to $41 \%$, $21 \%$ and $12 \%$ are $-0.48,-0.59,-0.52$, respectively, once again pointing out no marked difference. Similarly, for GC-VA-AE mixes, the slope of the trend lines corresponding to the TRMC values of $23 \%, 12 \%$ and $7 \%$ are $-0.74,-0.65$ and -0.63 , respectively, showing slight but no significant difference. For GC-VA-NE mixes, the slope of the trend lines corresponding to $23 \%, 12 \%$ and $7 \%$ are $-0.47,-0.49,-0.58$, respectively, once again pointing out no marked difference. These observations show that when RCA is used, the amount of RCA replacement (i.e. TRMC) is not a significant factor in how cement 
content affects the slump.

In general, it can be observed that air-entrained mixes experience higher slump changes with varying cement content than non-air entrained mixes. This can be attributed to the lubrication effect of the air bubbles in the fresh concrete mix.

\subsubsection{The Effect of TRMC}

Figures 5.3 and 5.4 illustrate the variation of slump with TRMC for air-entrained and non-air-entrained GC-MO and GC-VA mixes at different w/c ratios. As it can be observed from Figure 5.3, the TRMC does not seem to be correlated with the slump of the fresh mix for GC-MO samples. This observation was expected since the shape and the texture of the RCA-MO and NA (limestone) particles are quite similar to each other. Since the amount of residual mortar that is attached to NA (limestone) does not change the shape and texture of the aggregates significantly, the TRMC does not have an effect on the slump for these mixes.

In the case of GC-VA mixes, on the other hand, as it is illustrated in Figure 5.4, there is a slight, but recognizable decrease in the slump of the mixes as the TRMC increases. This observation is true for both air-entrained and non-air-entrained samples; however the $\mathrm{R}^{2}$ for the linear regression analysis show that the correlation is less obvious for non-air-entrained samples. This observation can be explained by the difference between the RCA-VA and NA (river gravel) particles in terms of surface texture and the shape. As described in Chapter 3, river gravel has smooth surface and round shape. On the other hand, attached residual mortar on the river gravel particles makes the surface texture of the aggregates rougher and their shape relatively more irregular and angular. It 
can be observed from Figure 5.4 that increasing TRMC values result in decreasing values of slump for both air-entrained and non-air-entrained GC-VA mixes.

\subsection{Air Content}

The air content of fresh concrete mixes is measured by the standard pressure method using type B meter; according to ASTM C 231-97 (ASTM, 2003). Figures 5.5 and 5.6 illustrate the variation of air content measurements with the TRMC for GC-MO and GC-VA mixes at different levels of w/c, respectively. As it can be observed from these figures, the air content of GC-MO-AE and GC-VA-AE mixes were in the range of $5-8 \%$, which is close to the $6 \%$ goal that was set out during the preparation of mixes as presented in Chapter 4. The air content of the GC-MO-NE and GC-VA-NE mixes were around $2 \%$ or lower, which is also expected from typical fresh concrete batches.

From Figures 5.5a and 5.6a, it is clear that the TRMC does not have a marked effect on the air content of fresh mixes. A similar observation can also be made from Figures $5.5 \mathrm{~b}$ and $5.6 \mathrm{~b}$ that the TRMC is not correlated well with the measured air content. Although the air content of the non-air entrained GC-MO mixes with no RCA replacement is slightly lower than the air content of the mixes with RCA replacement, whether this observation can be attributed to the presence of residual mortar is not conclusive. The fluctuations in the measurement can be the usual noise in the experimental results. 


\subsection{Fresh Density}

The density of fresh concrete mixes is measured according to ASTM C 138-01a (ASTM Committee C09, 2003). The fresh concrete samples were prepared in the same bowl for air content determination in fresh concrete $\left(0.0071 \mathrm{~m}^{3}\right)$ (Section 5.3$)$ and before the air content tests were carried out. The fresh density was obtained by dividing the net weight of the fresh concrete by the volume of the bowl.

Figures 5.7 and 5.8 illustrate the variation of fresh concrete density with the TRMC of the GC-MO and GC-VA mixes at different levels of w/c ratio, respectively. In general, it can be observed from these figures that, for both GC-MO and GC-VA mixes, the fresh density decreases by the increasing values of TRMC. This observation is valid for both air-entrained and non-air-entrained mixes. This observation can be attributed to the fact that residual mortar has lower density than NA particles; therefore, increasing values of TRMC will lower the fresh density of the mix.

In all cases, the fresh density of the mixes is linearly correlated with the TRMC with the following format:

$$
U_{G C}=\alpha_{u}(T R M C)+U_{N A C}
$$

where, $U_{G C}$ and $U_{N A C}$ are the fresh density of the (GC) and conventional concrete (NAC), respectively; $\alpha_{u}$ is the slope of the trend line which depends on the w/c ratio. In general, the range of $\alpha_{l l}$ for GC-MO and GC-VA mixes were found to be between -1.5 and -3.7 . To generalize the relationship, a multi-variable regression analysis was carried out to relate the fresh density with TRMC and w/c ratio. Equations 5.2 and 5.3 are the results of this analysis for air-entrained and non-air entrained mixes, respectively: 


$$
\begin{aligned}
& U_{G C}=70.4\left(\frac{1}{\left(\frac{w}{c}\right)}\right)-2.9(\text { TRMC })+2154.2 \\
& U_{G C}=12.4\left(\frac{1}{\left(\frac{w}{c}\right)}\right)-2.3(T R M C)+2394.6
\end{aligned}
$$

The variance $\mathrm{R}^{2}$ for the regression analysis for both air-entrained (Eq. 5.2) and non-airentrained concrete (Eq. 5.3) is 0.84 . The standard errors of estimate for the fresh density are $22.4 \mathrm{Kg} / \mathrm{m}^{3}$ and $13.9 \mathrm{Kg} / \mathrm{m}^{3}$ for air-entrained and non-air-entrained, respectively. The TRMC coefficients for air-entrained and non-air-entrained concrete are -2.9 and -2.3 , respectively, showing slight difference. The $w / c$ ratio is inversely proportioned to the fresh concrete density, with coefficients of 70.4 and 12.4 for air-entrained and non-airentrained concrete, respectively. It is obvious from Eqs. 5.2 and 5.3, an increase in the TRMC or w/c ratio results in the decrease of the fresh concrete density.

\subsection{Summary of Fresh Properties of GC}

- It has been demonstrated that the ACI guidelines for achieving workable structuralgrade concrete (i.e., Table 4.1) are applicable to GC produced with RCA.

- It has been observed that the main factors affecting the slump of the GC mixes are the shape and surface characteristics of the RCA.

- TRMC does not seem to be correlated with the slump of the fresh mix for GC-MO samples. This observation was expected since the shape and the texture of the RCAMO and NA (limestone) particles are quite similar to each other. Since the amount of residual mortar that is attached to NA (limestone) does not change the shape and 
texture of the aggregates significantly, the TRMC does not have an effect on the slump for these mixes.

- In the case of GC-VA mixes, on the other hand, there is a slight, but recognizable decrease in the slump of the mixes as the TRMC increases. This observation is true for both air-entrained and non-air-entrained samples and can be explained by the difference between the RCA-VA and NA (river gravel) particles in terms of surface texture and shape. River gravel has smooth surface and round shape. The attached residual mortar on the river gravel particles makes the surface texture of the aggregates rougher and their shape more irregular and angular.

- The TRMC has clear effect on the fresh density of the concrete produced with RCA. The increases in the TRMC decrease the fresh density of GC. General expressions that relate the fresh density of air-entrained and non-air-entrained concrete to the TRMC and $w / c$ ratio were developed. 
Table 5.1: Fresh property of air-entrained GC-MO mixes

\begin{tabular}{|c||c|c|c|c||c||}
\hline \multirow{2}{*}{ Fresh Property } & \multicolumn{4}{|c||}{ RCA (\%)/ TRMC (\%) } & \multirow{2}{*}{ Sample Designation } \\
\cline { 2 - 5 } & $\mathbf{1 0 0 / 4 1}$ & $\mathbf{5 0 / 2 1}$ & $\mathbf{3 0 / 1 2}$ & $\mathbf{0}$ & \\
\hline Slump (mm) & 85 & 95 & 60 & 40 & \multirow{2}{*}{ GC-MO-AE-P1 } \\
Air Content (\%) & 5.8 & 6.0 & 5.0 & 4.5 & \\
Density $\left(\mathrm{kg} / \mathrm{m}^{3}\right)$ & 2227 & 2281 & 2338 & 2366 & \\
\hline Slump $(\mathrm{mm})$ & 150 & 145 & 150 & 110 & \multirow{2}{*}{ GC-MO-AE-P2 } \\
Air Content (\%) & 7.4 & 8.0 & 7.2 & 6.4 & \\
Density $\left(\mathrm{kg} / \mathrm{m}^{3}\right)$ & 2181 & 2238 & 2238 & 2295 & \\
\hline Slump $(\mathrm{mm})$ & 195 & 200 & 165 & 180 & \multirow{2}{*}{ GC-MO-AE-P3 } \\
Air Content (\%) & 6.6 & 7.0 & 7.9 & 6.4 & \\
Density $\left(\mathrm{kg} / \mathrm{m}^{3}\right)$ & 2181 & 2210 & 2210 & 2267 & \\
\hline
\end{tabular}

Table 5.2: Fresh property of non-air-entrained GC-MO mixes

\begin{tabular}{|c|c|c|c|c|c||}
\hline \multirow{2}{*}{ Fresh Property } & \multicolumn{3}{|c|}{ RCA (\%)/ TRMC (\%) } & \multirow{2}{*}{ Sample Designation } \\
\cline { 2 - 6 } & $\mathbf{1 0 0 / 4 1}$ & $\mathbf{5 0 / 2 1}$ & $\mathbf{3 0} / \mathbf{1 2}$ & $\mathbf{0}$ & \\
\hline Slump (mm) & 105 & 55 & 90 & 90 & \multirow{2}{*||}{ GC-MO-NE-P1 } \\
Air Content (\%) & 2.2 & 2.0 & 2.1 & 1.8 & \\
Density $\left(\mathrm{kg} / \mathrm{m}^{3}\right)$ & 2338 & 2395 & 2381 & 2409 & \\
\hline Slump $(\mathrm{mm})$ & 185 & 140 & 165 & 155 & \multirow{2}{*}{ GC-MO-NE-P2 } \\
Air Content (\%) & 2.0 & 1.9 & 1.7 & 1.3 & \\
Density $\left(\mathrm{kg} / \mathrm{m}^{3}\right)$ & 2324 & 2366 & 2381 & 2409 & \\
\hline Slump $(\mathrm{mm})$ & 205 & 180 & 200 & 200 & \multirow{2}{*}{ GC-MO-NE-P3 } \\
Air Content $(\%)$ & 1.4 & 1.6 & 2.0 & 1.0 & \\
Density $\left(\mathrm{kg} / \mathrm{m}^{3}\right)$ & 2324 & 2352 & 2366 & 2409 & \\
\hline
\end{tabular}


Table 5.3: Fresh property of air-entrained GC-VA mixes

\begin{tabular}{|c|c|c|c|c|c|}
\hline \multirow{2}{*}{ Fresh Property } & \multicolumn{3}{|c|}{ RCA (\%)/ TRMC (\%) } & \multirow{2}{*}{ Sample Designation } \\
\cline { 2 - 5 } & $\mathbf{1 0 0 / 2 3}$ & $\mathbf{5 0 / 1 2}$ & $\mathbf{3 0 / 7}$ & $\mathbf{0}$ & \\
\hline Slump (mm) & 50 & 90 & 95 & 130 & \multirow{2}{*}{ GC-VA-AE-P1 } \\
Air Content (\%) & 5.0 & 5.2 & 5.0 & 5.4 & \\
Density (kg/m $\left.{ }^{3}\right)$ & 2315 & 2335 & 2339 & 2349 & \\
\hline Slump (mm) & 150 & 165 & 170 & 175 & \multirow{2}{*}{ GC-VA-AE-P2 } \\
Air Content (\%) & 6.9 & 6.0 & 6.2 & 6.4 & \\
Density (kg/m $\left.{ }^{3}\right)$ & 2224 & 2272 & 2284 & 2290 & \\
\hline Slump (mm) & 190 & 215 & 215 & 230 & \multirow{2}{*}{ GC-VA-AE-P3 } \\
Air Content (\%) & 6.6 & 5.6 & 6.0 & 4.8 & \\
Density $\left(\mathrm{kg} / \mathrm{m}^{3}\right)$ & 2238 & 2278 & 2277 & 2331 & \\
\hline
\end{tabular}

Table 5.4: Fresh property of non-air-entrained GC-VA mixes

\begin{tabular}{|c|c|c|c|c|c|}
\hline \multirow{2}{*}{ Fresh Property } & \multicolumn{4}{|c|}{ RCA $(\%) /$ TRMC $(\%)$} & \multirow{2}{*}{ Sample Designation } \\
\hline & $100 / 23$ & $50 / 12$ & $30 / 7$ & 0 & \\
\hline Slump (mm) & 100 & 120 & 105 & 160 & \multirow{3}{*}{ GC-VA-NE-P1 } \\
\hline Air Content (\%) & 1.8 & 1.8 & 1.8 & 1.7 & \\
\hline Density $\left(\mathrm{kg} / \mathrm{m}^{3}\right)$ & 2381 & 2404 & 2422 & 2438 & \\
\hline Slump (mm) & 160 & 215 & 200 & 220 & \multirow{3}{*}{ GC-VA-NE-P2 } \\
\hline Air Content (\%) & 1.8 & 1.0 & 1.1 & 1.0 & \\
\hline Density $\left(\mathrm{kg} / \mathrm{m}^{3}\right)$ & 2366 & 2393 & 2427 & 2446 & \\
\hline Slump (mm) & 200 & 220 & 225 & 230 & \multirow{3}{*}{ GC-VA-NE-P3 } \\
\hline Air Content (\%) & 1.2 & 0.6 & 0.6 & 0.4 & \\
\hline Density $\left(\mathrm{kg} / \mathrm{m}^{3}\right)$ & 2381 & 2394 & 2409 & 2429 & \\
\hline
\end{tabular}




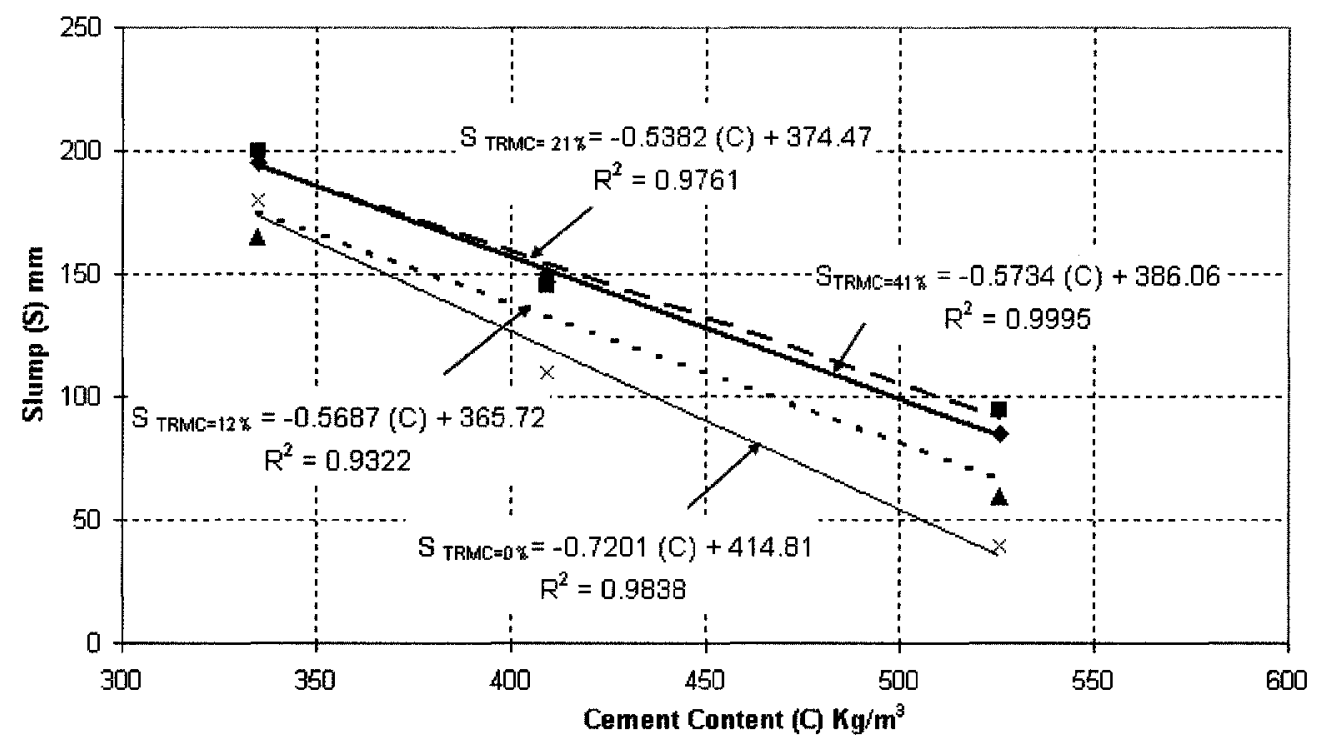

- TRMC $=41 \%$ TRMC $=21 \% \triangle$ TRMC $=12 \% \times$ TRRAC $=0 \%$

(a) GC-MO-AE mixes

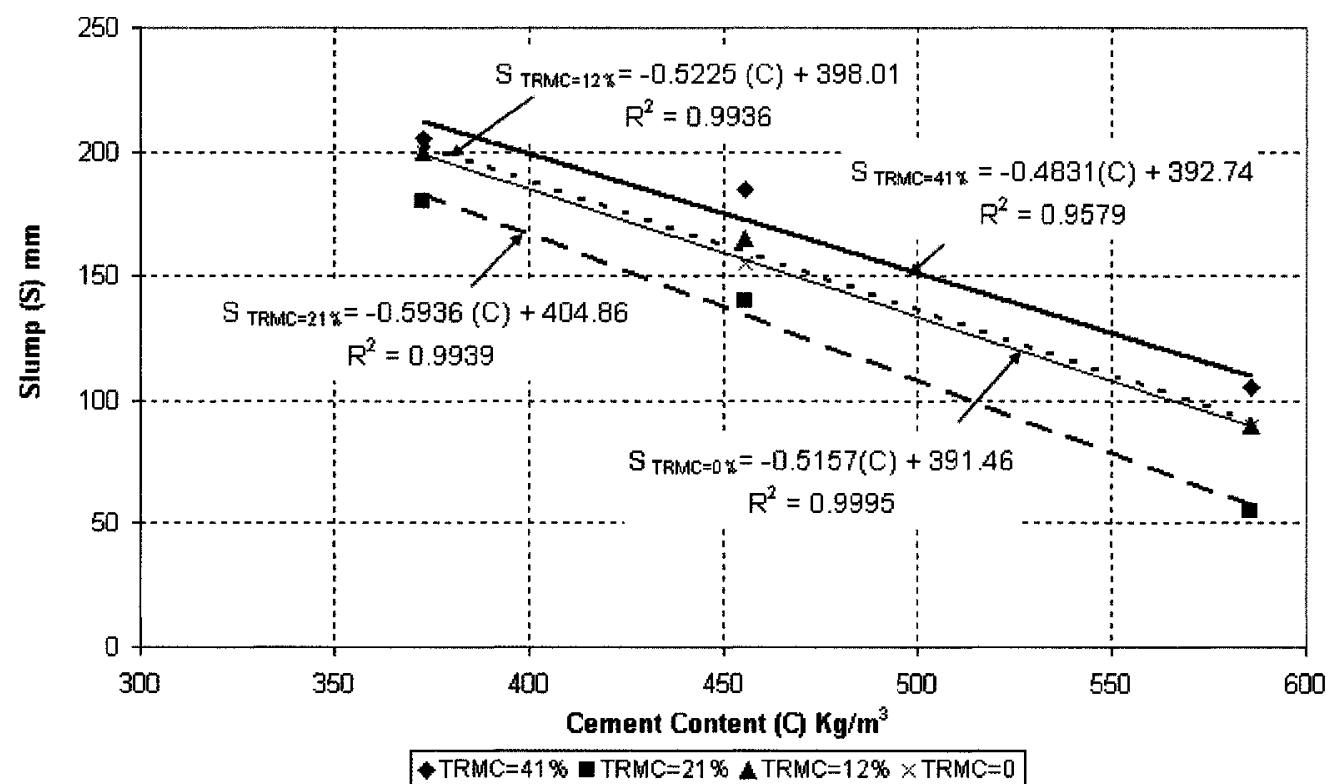

(b) GC-MO-NE mixes

Figure 5.1: Variation of slump with cement content of GC-MO mixes 


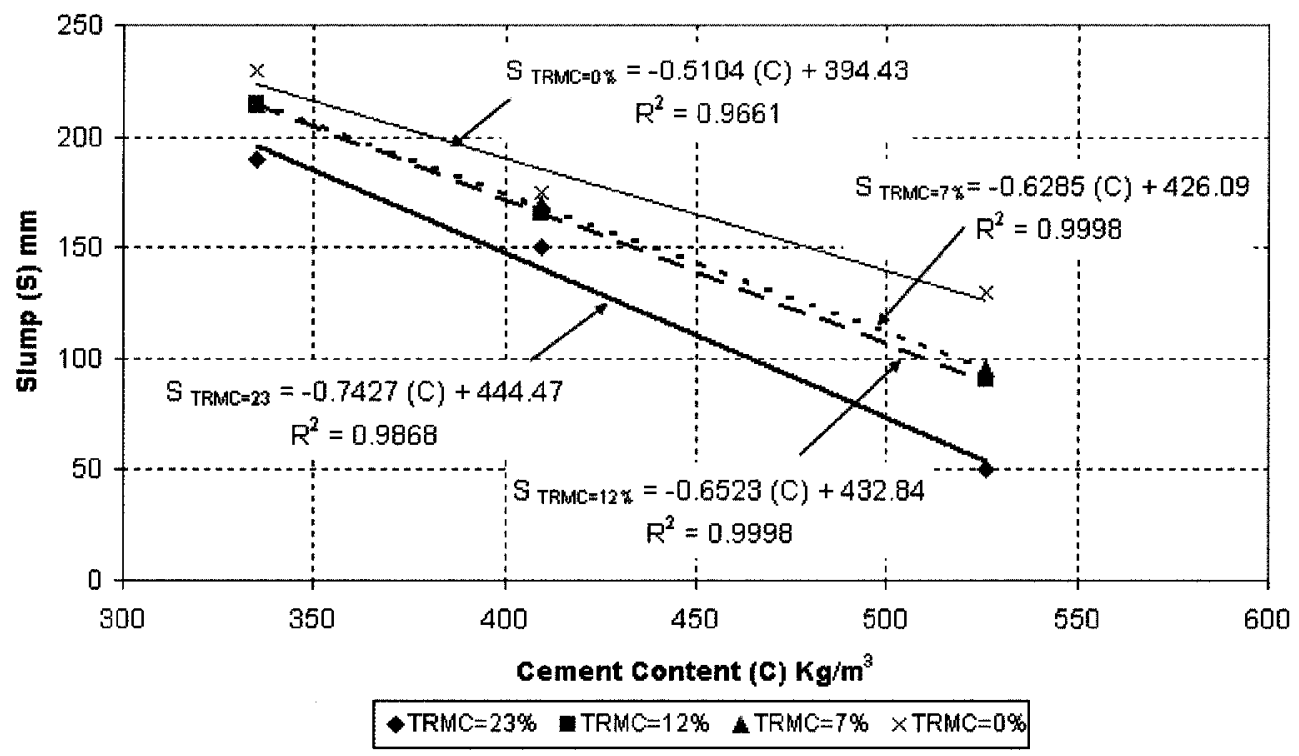

(a) GC-VA-AE mixes

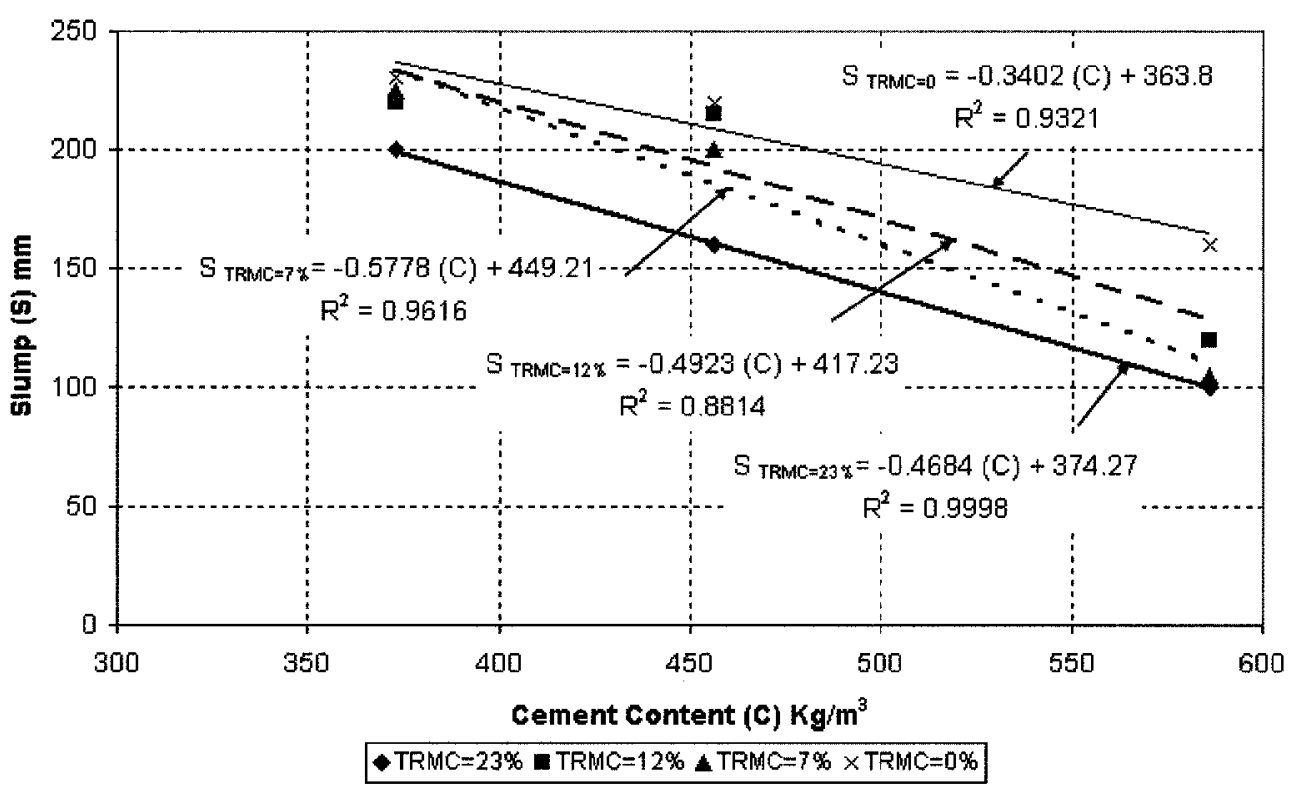

(b) GC-VA-NE mixes

Figure 5.2: Variation of slump with cement content of GC-VA mixes 


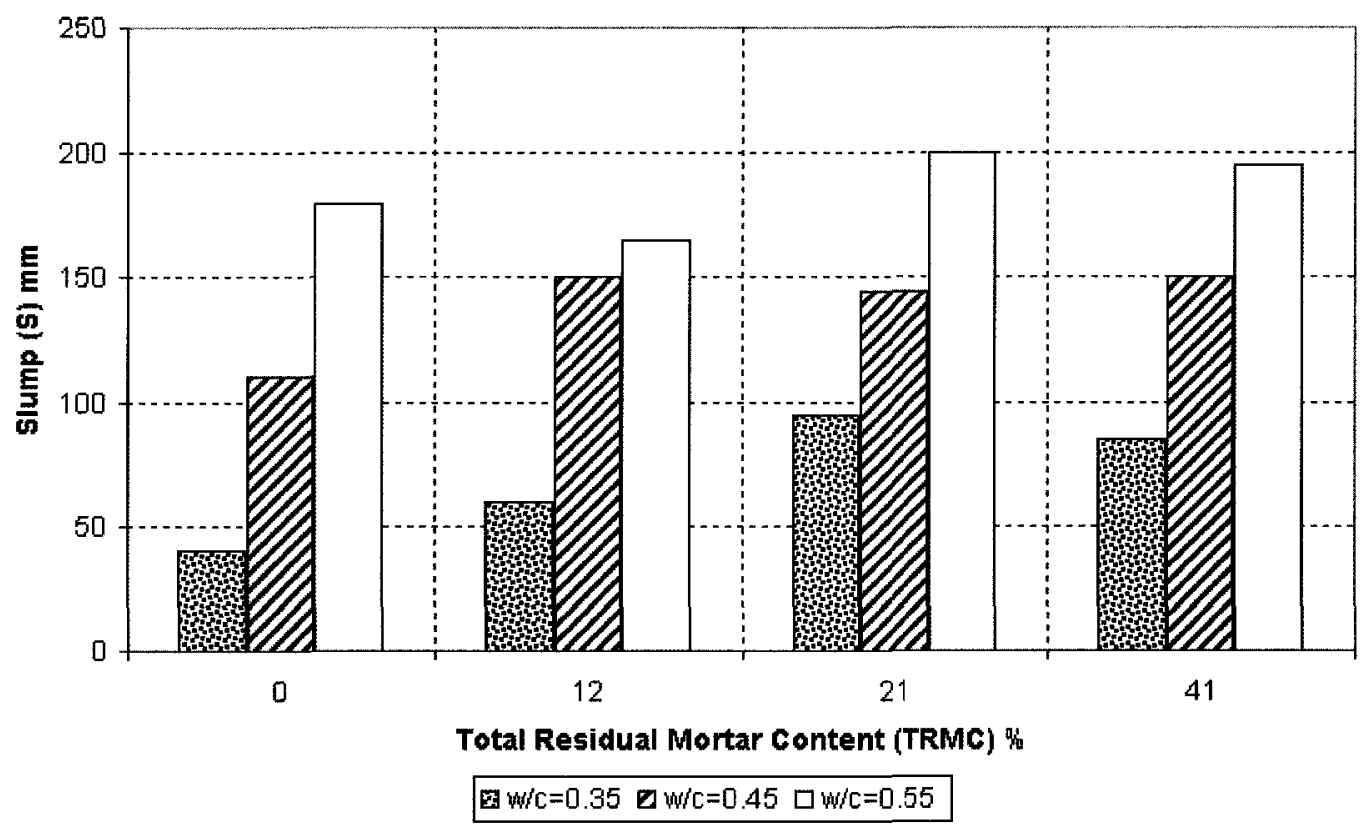

(a) GC-MO-AE mixes

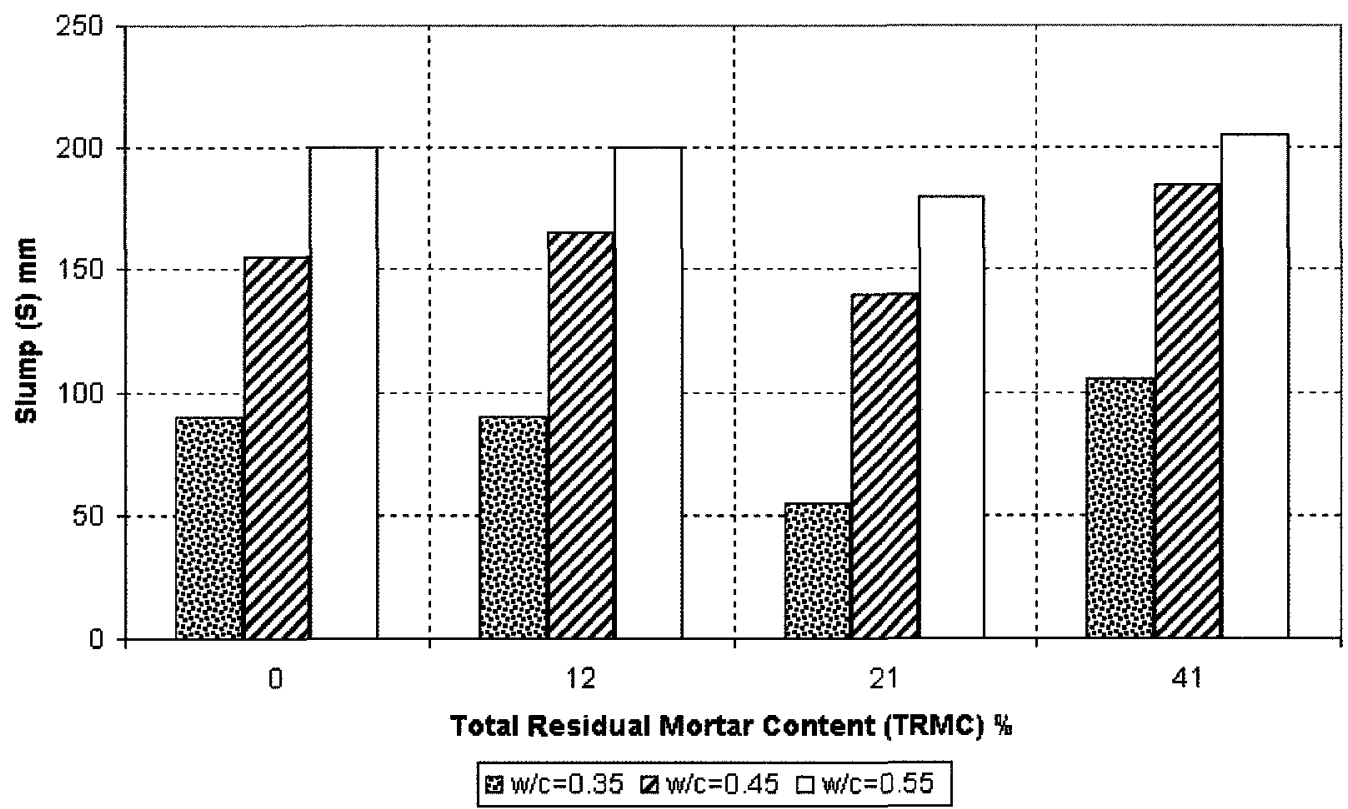

(b) GC-MO-NE mixes

Figure 5.3: Variation of slump with TRMC of GC-MO mixes 


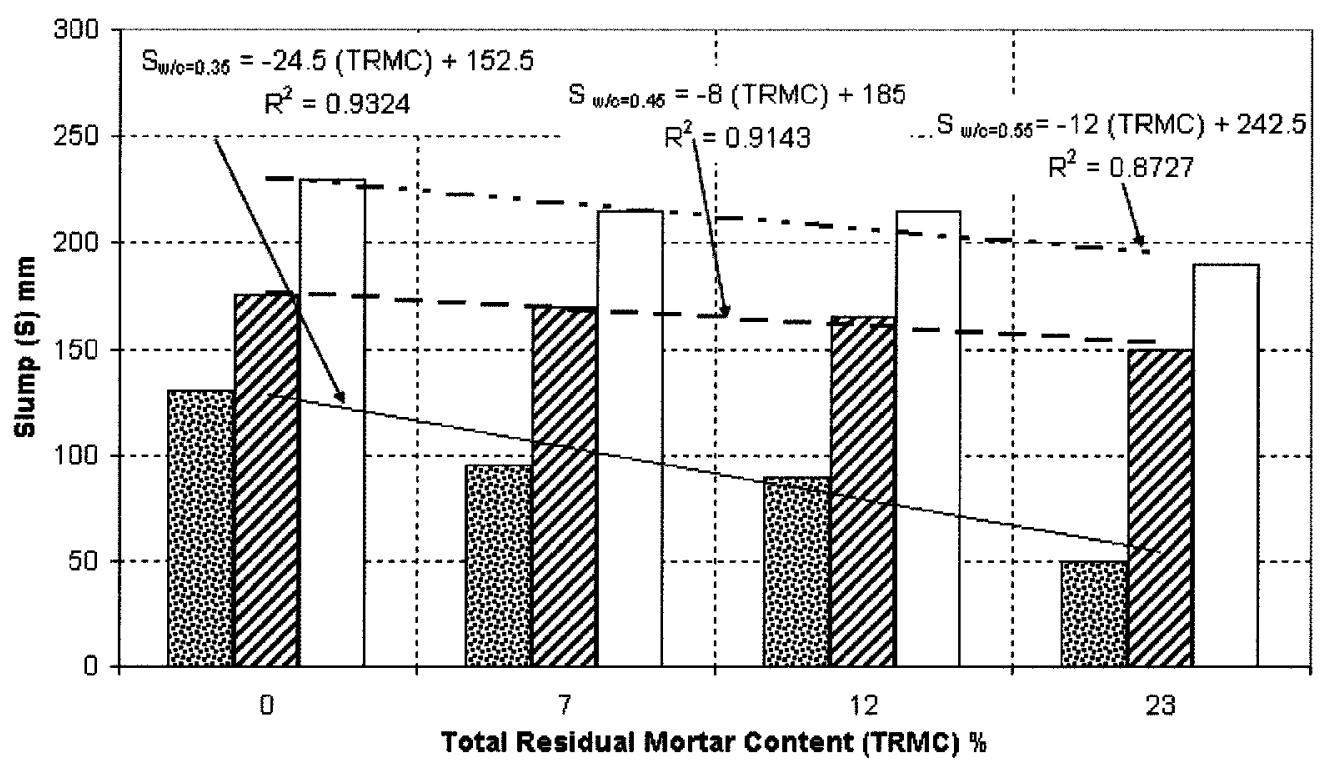

F $w / C=0.35 \quad \square w / C=0.45 \square w / C=0.55$

(a) GC-VA-AE mixes

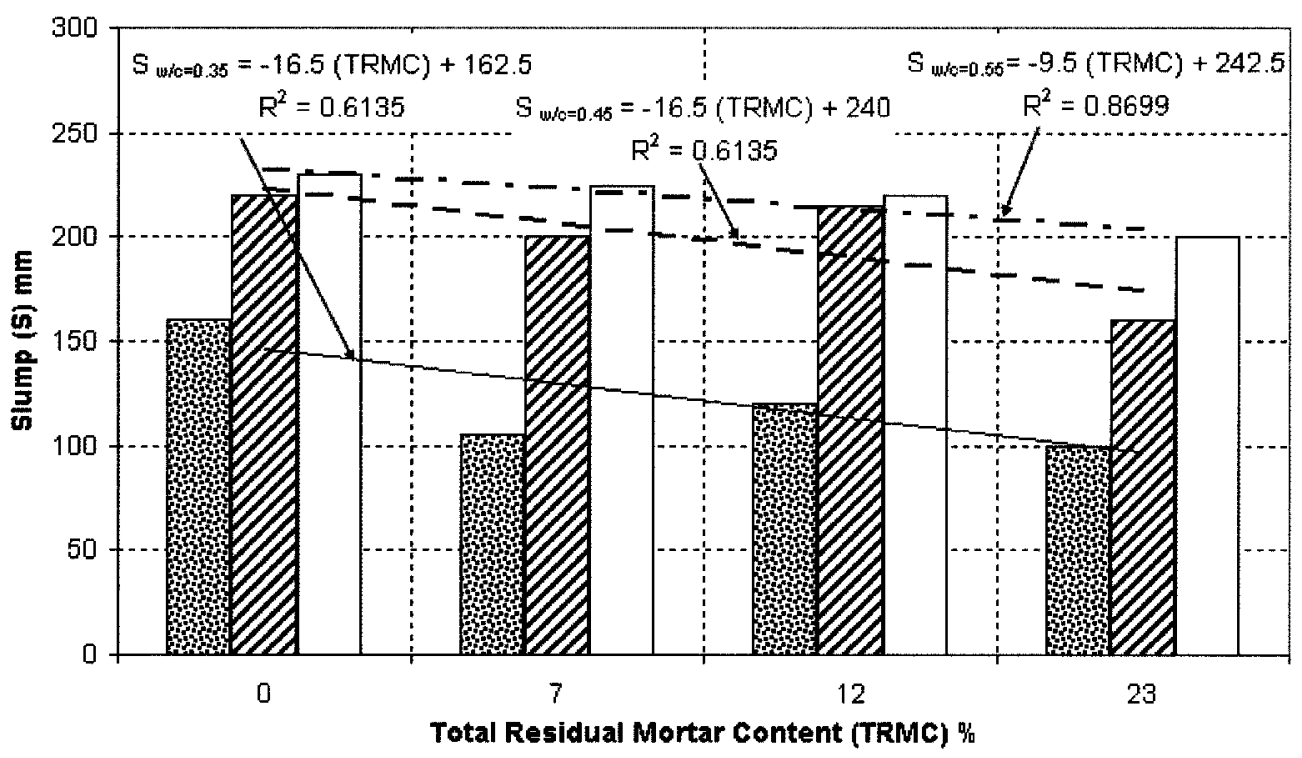

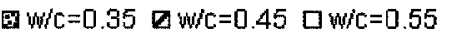

(b) GC-VA-NE mixes

Figure 5.4: Variation of slump with TRMC of GC-VA mixes 


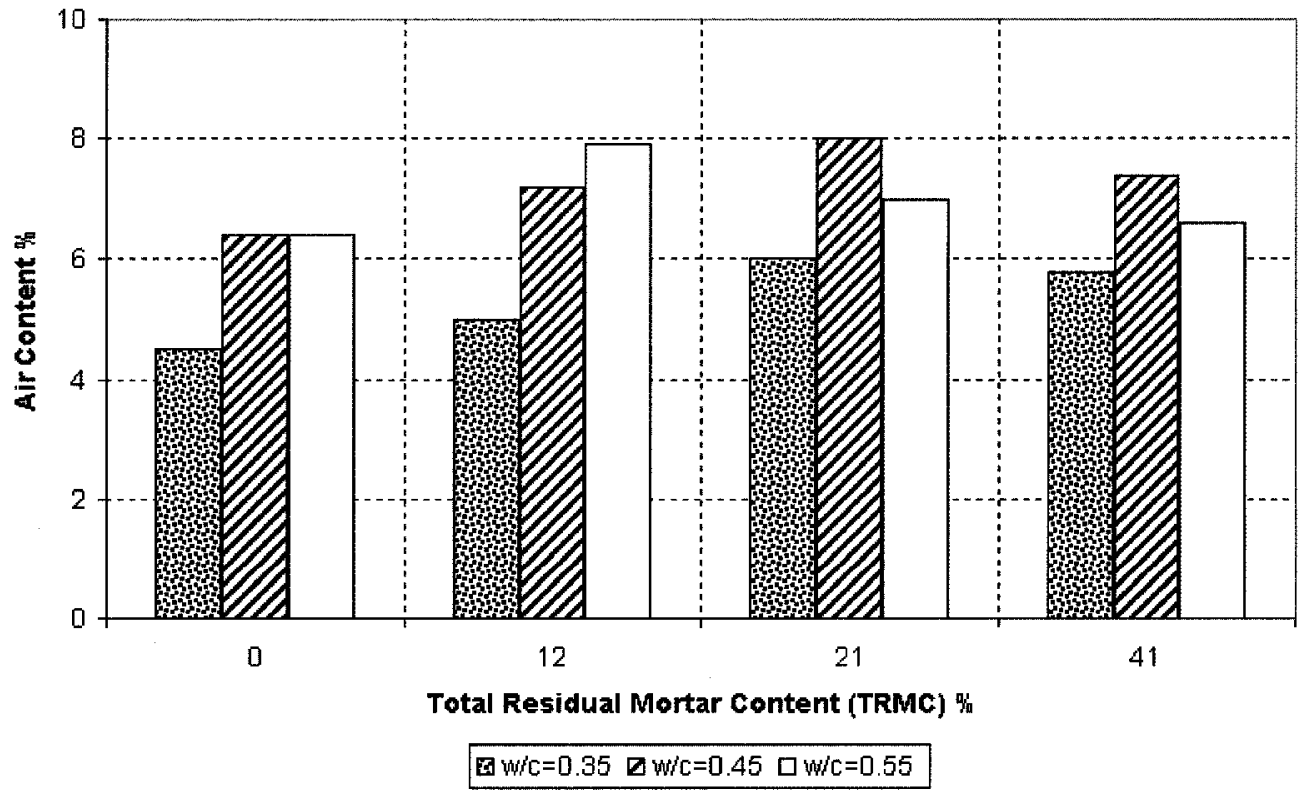

(a) GC-MO-AE mixes

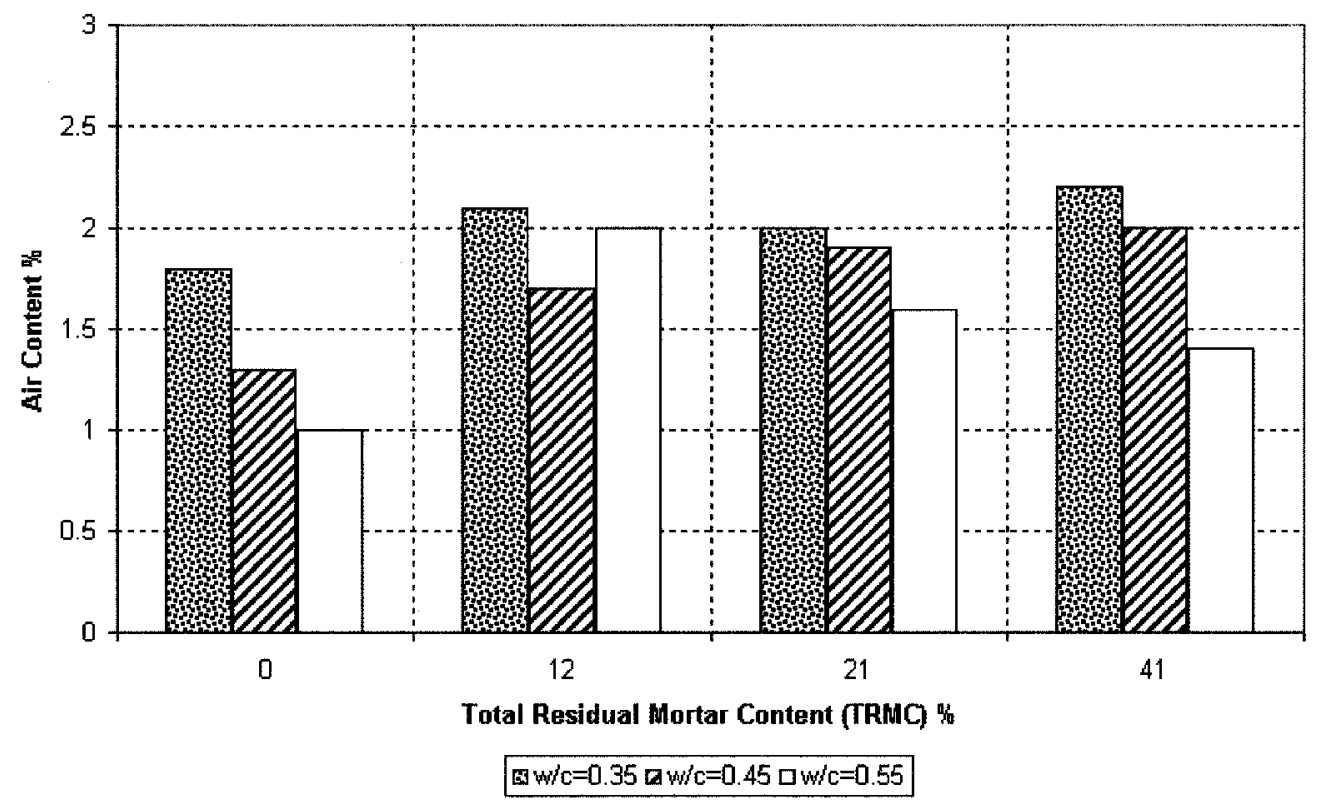

(b) GC-MO-NE mixes

Figure 5.5: Variation of air content with TRMC of GC-MO mixes 


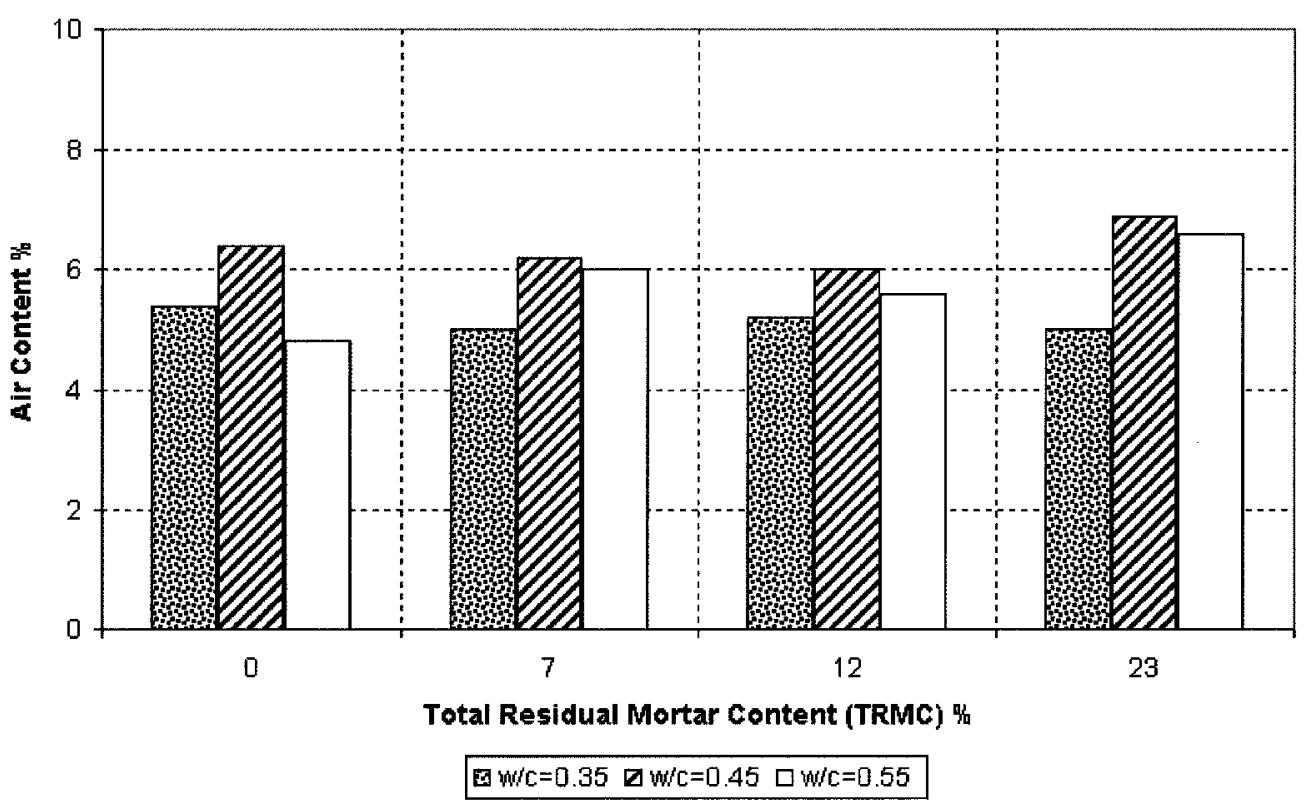

(a) GC-VA-AE mixes

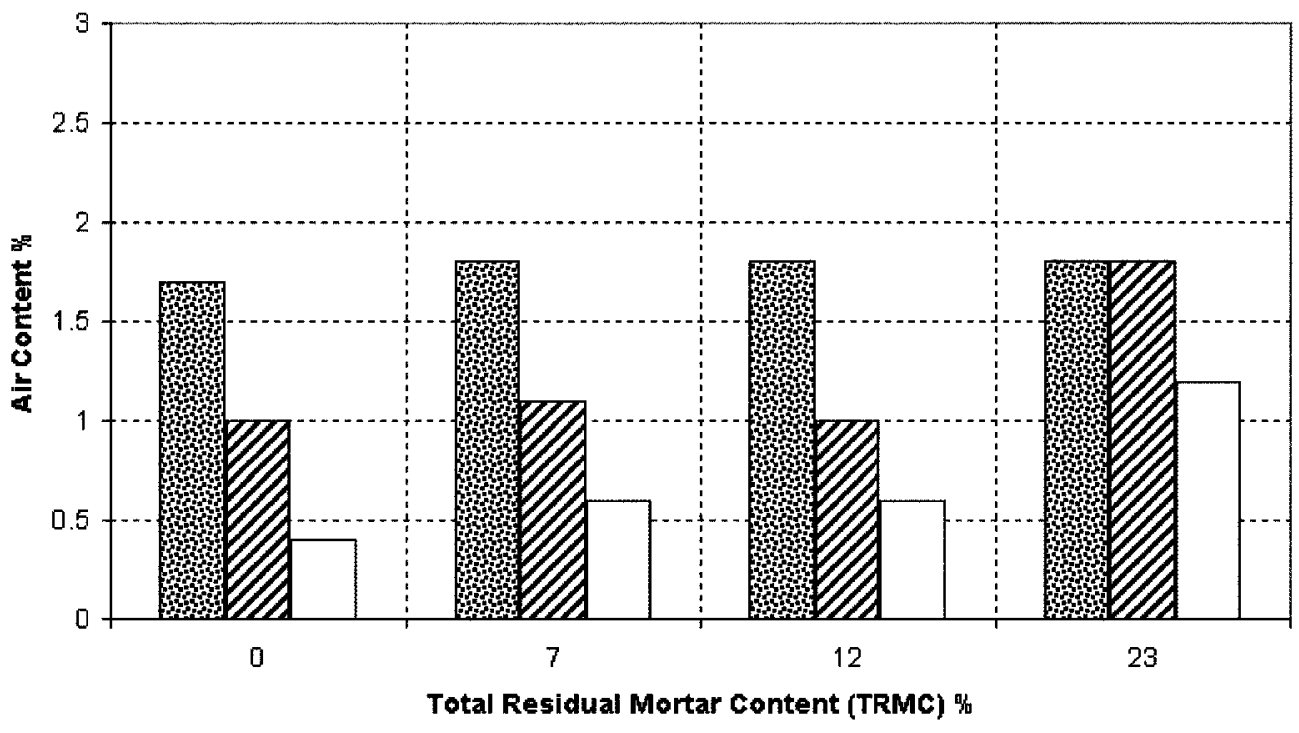

国 $w / c=0.35 \square w / c=0.45 \square w / c=0.55$

(b) GC-VA-NE mixes

Figure 5.6: Variation of air content with TRMC of GC-VA mixes 


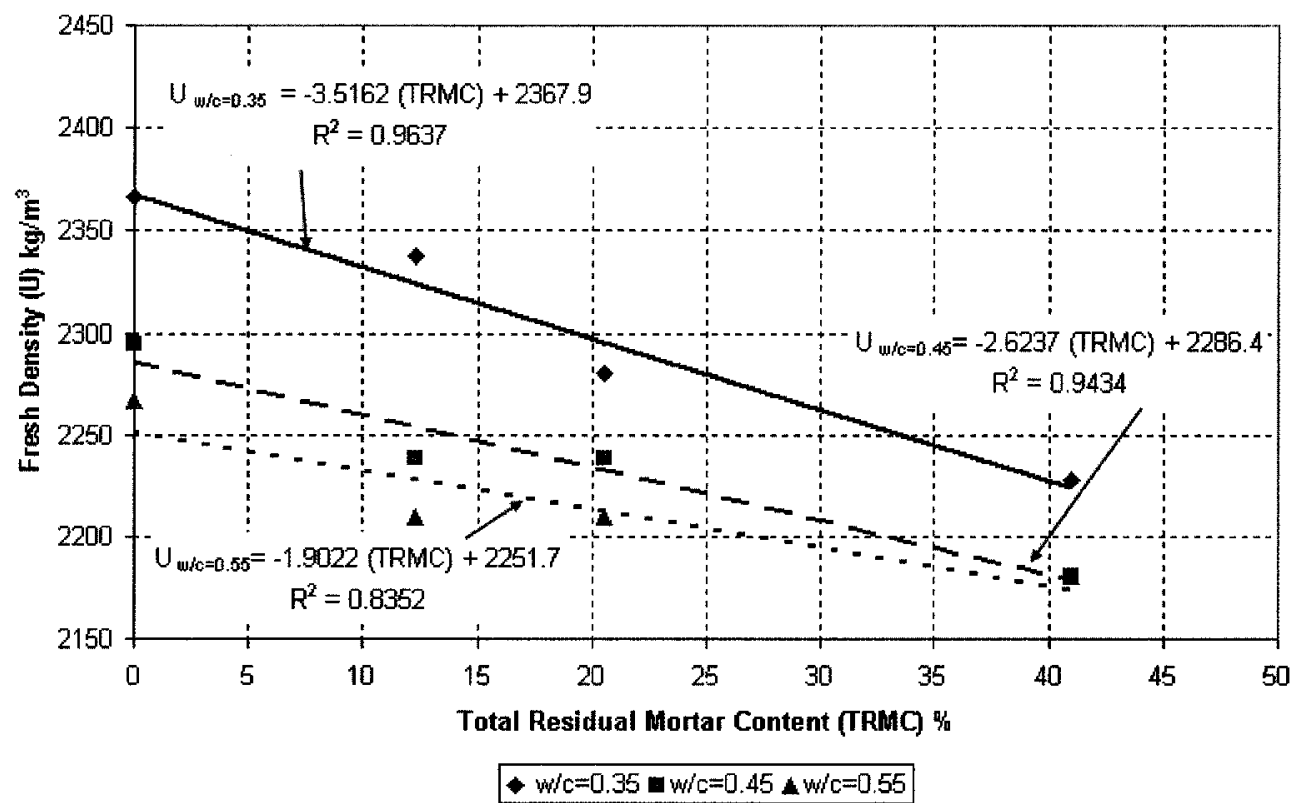

(a) GC-MO-AE mixes

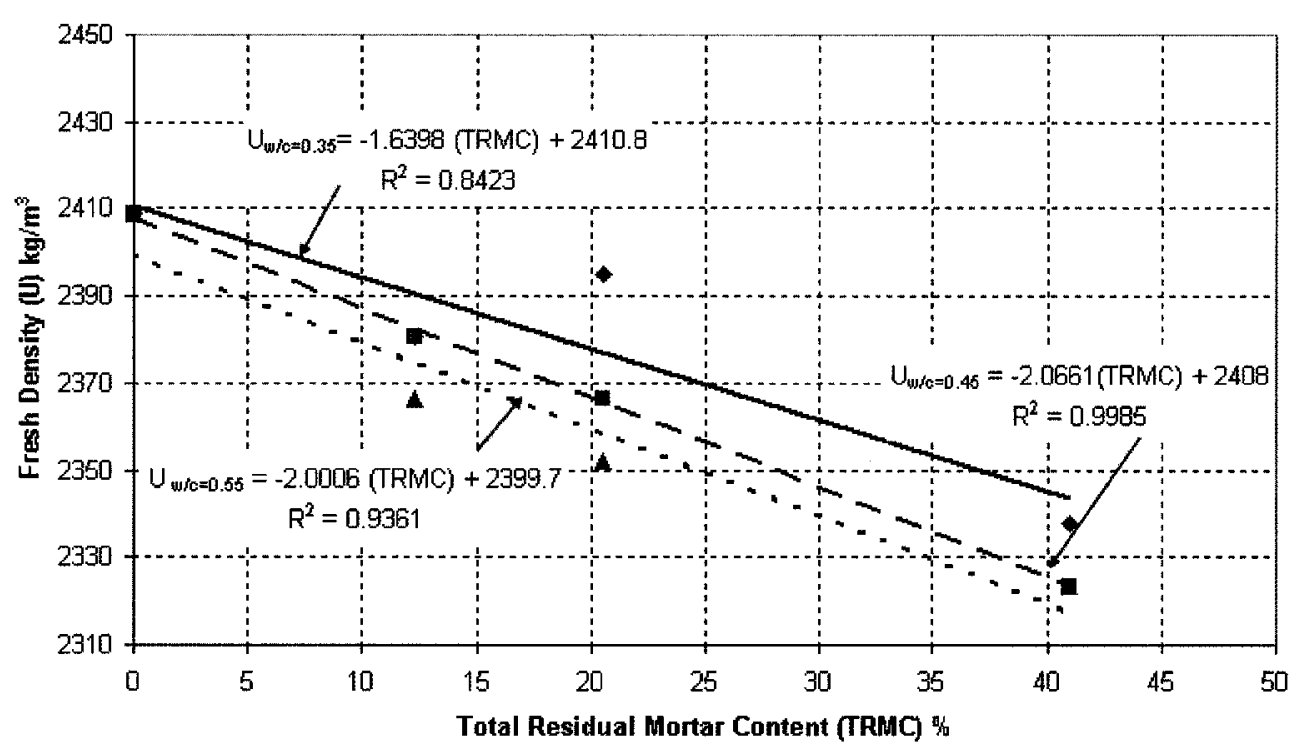

- $w / c=0.35 \backsim w / c=0.45$ A $w / c=0.55$

(b) GC-MO-NE mixes

Figure 5.7: Variation of fresh density with the TRMC of GC-MO mixes 


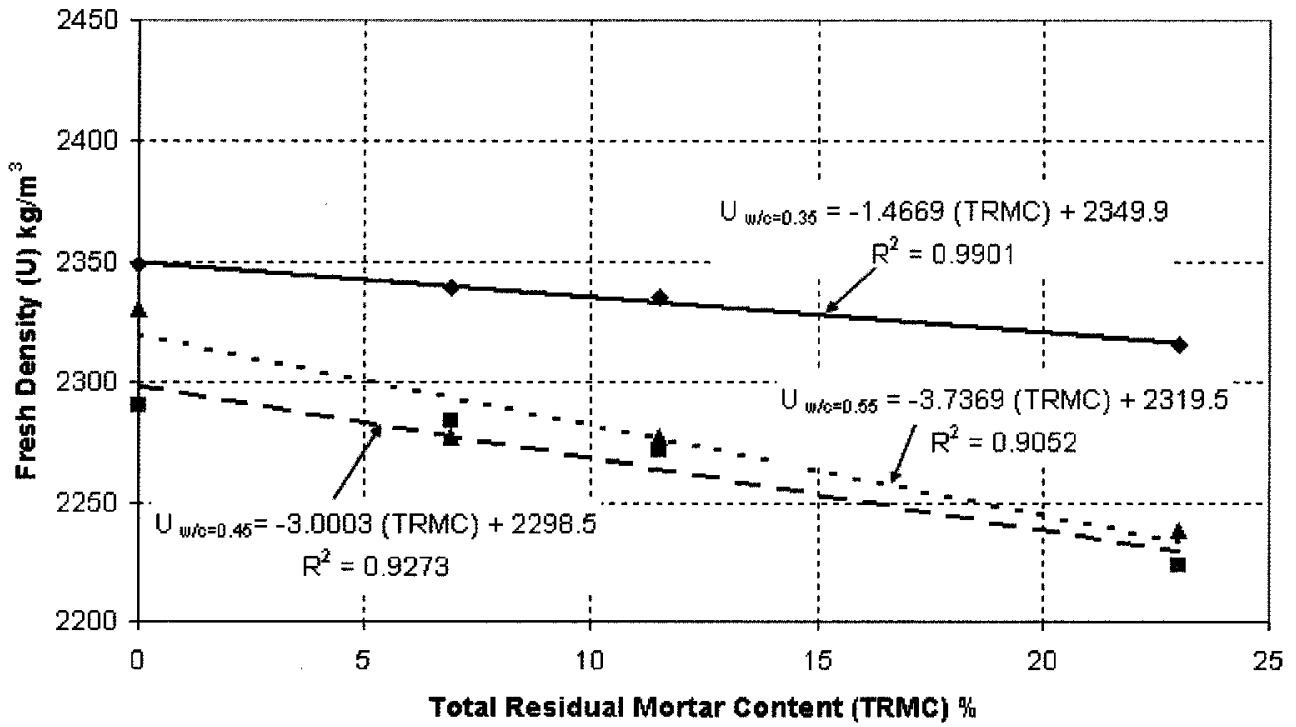

$-w / c=0.35 \mathrm{w} / \mathrm{c}=0.45$ A $w / c=0.55$

(a) GC-VA-AE mixes

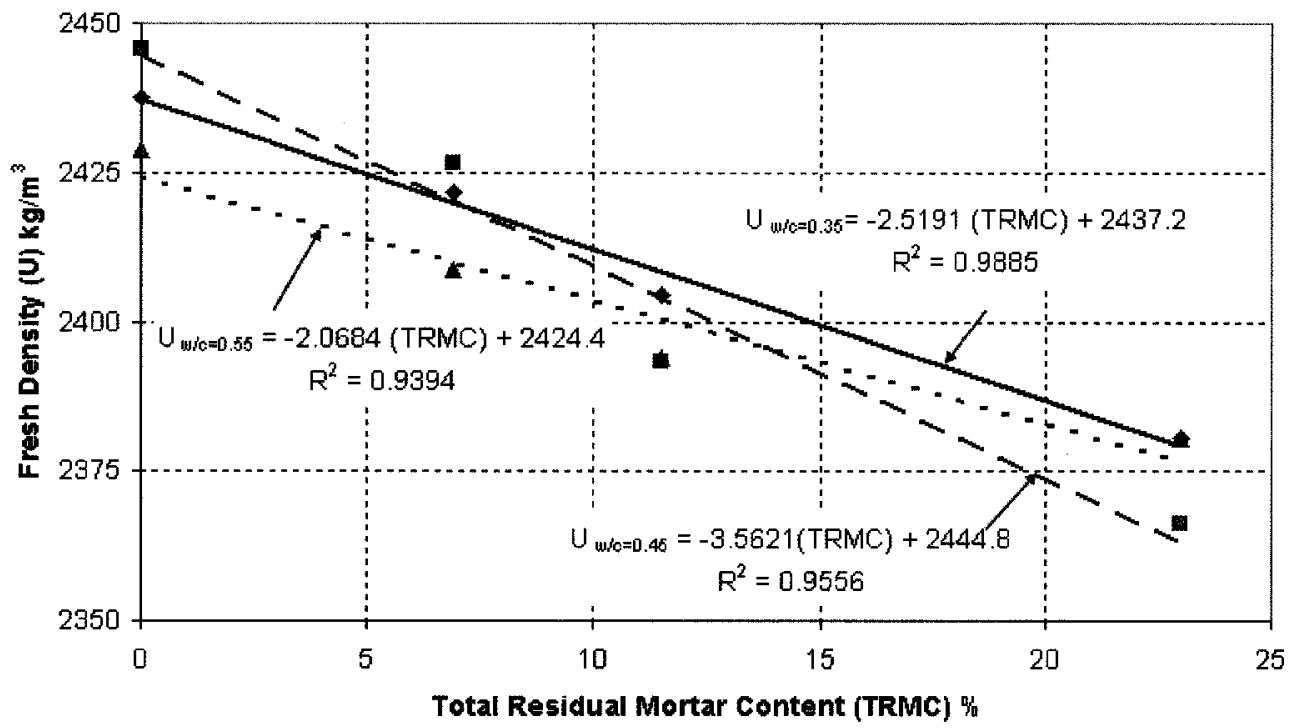

$\bullet w / c=0.35 \square w / c=0.45 \wedge w / c=0.55$

(b) GC-VA-NE mixes

Figure 5.8: Variation of fresh density with the TRMC of GC-VA mixes 


\section{CHAPTER 6: PRELIMINARY STUDY ON CONCRETE PRODUCED WITH RCA: HARDENED PROPERTIES}

\subsection{General}

In this chapter, the results of the hardened density $(\gamma)$, compressive strength $\left(f_{c}\right)$, elastic modulus $\left(E_{c}\right)$, and splitting tensile strength $\left(f_{t}\right)$ measurements of the hardened concrete mixes described in Chapter 4 are presented. The mix design parameters of each mix, whose test results are reported here, can be found in Tables 4.3 to 4.6 . Table 6.1 to 6.4 present the hardened density results for GC-MO and GC-VA mixes. Tables 6.5 to 6.8 present the mechanical properties of the GC-MO and GC-VA mixes. In all results reported here, the mean values, standard deviations and the CoV values are for three repeat test samples. In the following sections, the results of the hardened properties of the preliminary mixes will be discussed further in detail

\subsection{Hardened Concrete Density}

The hardened density of GC was obtained by weighing the $100 \times 200 \mathrm{~mm}$ cylinders in air and in water after 24 hours from casting of the specimens. The density was calculated by dividing the weight of the specimen in the air by the volume of the volume of the specimen. In general, as it can be observed in Tables 6.1 to 6.4 , the hardened density of the specimens tested are in the range of normal density concrete. 
For air-entrained GC-MO mixes, the range of hardened density values was from $2138 \mathrm{~kg} / \mathrm{m}^{3}$ (mean value for GC-MO-AE-P2-100/41 samples) to $2322 \mathrm{~kg} / \mathrm{m}^{3}$ (mean value for GC-MO-AE-P1-0/0 samples). For non-air-entrained GC-MO mixes, the range was from $2298 \mathrm{~kg} / \mathrm{m}^{3}$ (mean value for GC-MO-NE-P3-100/41 samples) to $2388 \mathrm{~kg} / \mathrm{m}^{3}$ (mean value for GC-MO-NE-P1-0/0 samples). The range for air-entrained GC-VA specimens was found to be between $2196 \mathrm{~kg} / \mathrm{m}^{3}$ (mean value for GC-VA-NE-P3-100/23 samples) and $2329 \mathrm{~kg} / \mathrm{m}^{3}$ (mean value for GC-VA-AE-P1-30/12 samples). For non-air entrained GC-VA specimens, the range was from $2281 \mathrm{~kg} / \mathrm{m}^{3}$ (mean value for GC-VA-NE-P3$100 / 23$ samples) to $2417 \mathrm{~kg} / \mathrm{m}^{3}$ (mean value for GC-VA-NE-P1-0/0 samples). The standard deviation calculations were found to be well within the acceptable range of hardened density measurements.

Figures 6.1 and 6.2 illustrate the variation of hardened concrete density with the TRMC of the GC-MO and GC-VA mixes at different levels of w/c ratio, respectively. In general, it can be observed from these figures that, for both GC-MO and GC-VA mixes, the hardened density decreases by the increasing values of TRMC. This observation is valid for most air-entrained and non-air-entrained mixes. This observation can be attributed to the fact that residual mortar has lower density than NA particles (Chapter 3); therefore, increasing values of TRMC will lower the hardened density of the mix. It should also be noted that for air-entrained GC-VA mixes with w/c ratio of 0.35 , there is no change observed in the hardened density with the increase of the TRMC. 


\subsection{Compressive Strength: $f_{c}$}

The conventional compressive strength test as per ASTM C 39- 01 (ASTM, 2003) was used to determine the 28-day compressive strength of the preliminary GC mixes. In general, as it can be observed in Tables 6.5 to 6.8 , the compressive strengths of the specimens tested were in the range expected from structural-grade concrete. For airentrained GC-MO mixes, the range was from 19.8 MPa (mean value for GC-MO-AE-P3100/41 samples) to $47.8 \mathrm{MPa}$ (mean value for GC-MO-AE-P1-30/12 samples). For nonair-entrained GC-MO mixes, the range was from 27.0 MPa (mean value for GC-MO-NEP3-100/41 samples) to 57.5 MPa (mean value for GC-MO-NE-P1-30/12 samples). Airentrained GC-VA specimens performed well with compressive strengths ranging from 26.8 $\mathrm{MPa}$ (mean value for GC-VA-AE-P3-100/23 samples) to $44.9 \mathrm{MPa}$ (mean value for GC-VA-AE-P1-100/23 samples). For non-air entrained GC-VA specimens, the range was from 37.9 $\mathrm{MPa}$ (mean value for GC-VA-NE-P3-100/23 samples) to $54.7 \mathrm{MPa}$ (mean value for GC-VA-NE-P1-100/23 samples).

It can be seen in Tables 6.5 and 6.6 that the standard deviation of the compressive strength values for GC-MO specimens with partial RCA replacement, excluding GCMO-AE-P1-100/41 samples (6.5 MPa), range from 0.3 to $4.4 \mathrm{MPa}$. Concretes with increasing proportion of RCA generally show increasing standard deviations, which demonstrates that GC with high TRMC can show larger standard deviation for compressive strength when compared with specimens with lower TRMC. With this rational is mind, as expected, concrete mixes produced with no RCA replacement (i.e., limestone as NA only) showed standard deviation for compressive strength in the range 
of 0.7 to $2.1 \mathrm{MPa}$, which is lower than the 0.3 to $6.5 \mathrm{MPa}$ range of GC-MO mixes with RCA replacement.

From Tables 6.7 and 6.8 , it can be observed that the standard deviation of the compressive strength results for GC-VA specimens with partial RCA replacement ranged from 0.2 to $3.3 \mathrm{MPa}$. Similarly, GC mixes produced with no RCA replacement (i.e., river gravel as NA only) showed standard deviation for compressive strength in the range of 0.5 to $3.5 \mathrm{MPa}$. The lower standard deviation observed in the compressive strength values of GC-VA specimens compared to those of GC-MO mixes can also be attributed to the TRMC.

It should be noted that the standard deviation results presented in Tables 6.5 to 6.8 are mostly within the allowable limits of $4.83 \mathrm{MPa}$ for variable quality RCA, recommended by ACI 555R-01 (ACI committee 555R, 2001). However, it should also be noted that the standard deviation of the test results increases as the TRMC of the GC mixes increases. Therefore, as a good quality control practice, it might be necessary to carry out larger number of compressive tests when GC has large TRMC values.

One of the main factors affecting the compressive strength of GC is the w/c ratio of the new mortar. Figures 6.3 and 6.4 demonstrate the relationship between the w/c ratio and 28-day compressive strength for GC-MO and GC-VA mixes, respectively. It is clear from these figures that the compressive strength is linearly correlated with the w/c ratio: an increase in $w / c$ ratio results in a decrease in the compressive strength of GC. As it can be observed from these figures, in all cases, the linear correlations between compressive strength and the $\mathrm{w} / \mathrm{c}$ ratio have $\mathrm{R}^{2}$ values larger than $92 \%$ (mostly above $95 \%$ ), except for GC-VA-AE with TRMC of $0 \%$ (no RCA replacement). The $\mathrm{R}^{2}$ values for GC-VA-AE 
with $0 \%$ TRMC is $76 \%$, and this low correlation can possibly be attributed to the experimental noise. Therefore, this linear trend is valid for all TRMC values and for both air entrained (Figures 6.3a and 6.4a) and non-air entrained mixes (Figures 6.3b and 6.4b).

As it can be noted from the figures, the slope and the intercept of each trend line are different but not significantly; the largest slope (i.e., the largest change in compressive strength with w/c ratio) is observed exclusively in specimens with $100 \% \mathrm{RCA}$. Therefore it can be concluded that these constants are functions of the TRMC and w/c ratio of each mix. These observations lead to the conclusion that the effect of TRMC on the compressive strength should be investigated. In the following section this investigation is presented.

The total mortar of GC contains residual mortar attached to the RCA and the new mortar. In this section the effect of the total residual mortar on the compressive strength is studied. Figures 6.5 and 6.6 illustrate the variation of 28-day compressive strength with the TRMC of GC-MO and GC-VA mixes, respectively. As it can be observed from theses figures, in general, the compressive strength does not appear to be affected by the TRMC as much as by the w/c ratio. For GC-MO mixes, in which the TRMC range is quite large ( 0 to $41 \%$ ), a modest decrease in the compressive strength can be observed as the TRMC value increases towards $41 \%$; however, for GC-VA specimens no obvious conclusions of TRMC affecting the compressive strength can be made.

To study the combined effect of the w/c ratio and the TRMC on the compressive strength of GC, a multi-variable regression analysis was carried out. Equations 6.1 and 6.2 are the results of this analysis for air-entrained and non-air-entrained mixes, respectively: 


$$
\begin{gathered}
f_{c}=\left[\frac{16.4}{\left(\frac{w}{c}\right)}\right]-0.1 \text { TRMC }-1.5 \\
f_{c}=\left[\frac{14.5}{\left(\frac{w}{c}\right)}\right]-0.07 T R M C+13.2
\end{gathered}
$$

The $\mathrm{R}^{2}$ values of the regression analyses for air-entrained and non-air-entrained concrete were found to be equal to 0.92 and 0.85 , respectively. The standard errors of estimate for compressive strength of air-entrained and non-air-entrained specimens are 2.3 MPa and 2.8 $\mathrm{MPa}$, respectively, showing only a slight difference. The coefficients in front of the TRMC term ( -0.1 for air-entrained and -0.07 for non-air-entrained mixes) are quite small, which indicates that the TRMC does not have a significant effect on the compressive strength (e.g. a TRMC of $41 \%$ (i.e., concrete made with $100 \%$ RCA-MO) will affect the compressive strength by $-4.1 \mathrm{MPa}$ for air-entrained mixes and $-2.87 \mathrm{MPa}$ for non-airentrained mixes). It should be noted that Eqs.6.1 and 6.2 will be used for developing the general mix design procedure for GC in Chapter 7.

\subsection{Modulus of Elasticity: $E_{c}$}

The modulus of elasticity test for concrete was carried out following the method described in ASTM C469-94 (ASTM, 2003). As it can be observed in Tables 6.5 to 6.8, for air-entrained GC-MO mixes, the range of elastic modulus was from $22.1 \mathrm{GPa}$ (mean value for GC-MO-AE-P2-100/41 samples) to 34.2 GPa (mean value for GC-MO-AE-P10/0 samples). For non-air-entrained GC-MO mixes, the range was from $27.0 \mathrm{GPa}$ (mean value for GC-MO-NE-P3-100/41 samples) to $36.3 \mathrm{GPa}$ (mean value for GC-MO-NE-P1- 
0/0 samples). Air-entrained GC-VA specimens performed slightly better with elastic modulus ranging from 24.0 GPa (mean value for GC-VA-AE-P3-100/23 samples) to 33.0 GPa (mean value for GC-VA-AE-P1-30/7 samples). For non-air entrained GC-VA specimens, the range was from 29.3 MPa (mean value for GC-VA-NE-P3-100/23 samples) to $36.4 \mathrm{GPa}$ (mean value for GC-VA-NE-P1-30/7 samples). From Table 6.5 to 6.8, the standard deviation of the elastic modulus test for both GC-MO and GC-VA mixes was found to be in the range of 0 to $1.7 \mathrm{GPa}$. This indicates that the standard deviation is low for all mixes.

It is well known that there are a number of factors that may affect the modulus of elasticity of concrete. Among these the following can be considered as important: (1) the higher the modulus of elasticity of coarse aggregates the greater the elastic modulus of the concrete (Popovics, 1998); (2) volume of the concrete mortar phase: the higher the volume of mortar the lower the elastic modulus; (3) since the mortar quality depends on the $\mathrm{w} / \mathrm{c}$ ratio, the lower the w/c the higher is the elastic modulus.

It is therefore important to study effect of the RCA addition (i.e., TRMC) on the modulus of elasticity of concrete. Furthermore, the effect of the $w / c$ ratio on the elastic modulus of GC needs to be investigated. In the current building codes, the modulus of elasticity is generally related to the compressive strength and the unit weight of concrete. Therefore, the applicability of the relationship between $f_{c}$ and $E_{c}$ that is observed in conventional concrete needs to be studied for GC. The following sections will discuss these in detail.

Figures 6.7 and 6.8 demonstrate the relationship between the w/c ratio and elastic modulus for GC-MO and GC-VA mixes, respectively. From these figures, it can be 
observed that the elastic modulus is linearly correlated with the w/c ratio: an increase in w/c ratio results in a decrease in the elastic modulus of concrete. In most cases, the linear correlations between elastic modulus and the w/c ratio have $\mathrm{R}^{2}$ values larger than 0.90 . However, there are few cases which did not show high correlation (e.g. GC-VA-AE and GC-VA-NE specimens with TRMC of $0 \%$ (conventional concrete with NA only), and GC-VA-MO specimens with $41 \%$ TRMC). However, in these cases the range of the modulus of elasticity measurements are quite small, and the low correlation observed in the regression analysis is not significant.

For GC-VA-AE mixes, the slope of the trend lines corresponding to the TRMC values of $23 \%, 12 \%$ and $7 \%$ are $-24.8,-25.7$ and -21.6 , respectively, showing slight difference. For GC-VA-NE mixes, the slope of the trend lines corresponding to $23 \%$, $12 \%$ and $7 \%$ are $-33.8,-30.1,-34.1$, respectively, once again pointing out no marked difference. This shows that when RCA-VA (i.e. $\mathrm{RMC}=23 \%$ ) is used, the amount of RCA replacement (i.e., TRMC) is not a significant factor in how w/c ratio affects the elastic modulus for GC-VA. However, it should be noticed that, for air-entrained mixes and nonair-entrained mixes produced without RCA addition (i.e. river gravel as NA only), there is a marked increase in the (slope $=-7.9$ for $\mathrm{AE}$ and slope $=-4.1$ for $\mathrm{NE}$ ), pointing out that a change in the w/c ratio for these mixes will have a lower effect on the elastic modulus than the behaviour observed in GC-VA-AE and GC-VA-NE mixes with RCA replacement. These observations are not valid for GC-MO mixes (Figure 6.7) with RCA replacement or without RCA replacement (i.e., limestone as NA only). This shows that when RCA-MO (i.e., $\mathrm{RMC}=41 \%$ ) is used, the amount of RCA replacement (i.e. TRMC) is a factor in how w/c ratio affects the elastic modulus for GC-MO. 
Figures 6.9 and 6.10 show the variation of the elastic modulus with TRMC for GCMO and GC-VA mixes at different w/c ratios. As it can be observed from theses figures, the TRMC is linearly correlated with the elastic modulus of GC-MO mixes. For the GCVA, the TRMC does not have a significant effect on the elastic modulus.

To establish a general relationship between the modulus of elasticity, the TRMC and w/c ratio, a multi-variable regression analysis was carried out. Equations 6.3 and 6.4 are the results of this analysis for air-entrained and non-air-entrained GC mixes, respectively:

$$
\begin{aligned}
& E_{c}=\left[\frac{5.2}{\left(\frac{w}{c}\right)}\right]-0.13 \text { TRMC }+17.96 \\
& E_{c}=\left[\frac{4.0}{\left(\frac{w}{c}\right)}\right]-0.13 T R M C+25.25
\end{aligned}
$$

The $\mathrm{R}^{2}$ values for the regression analyses for air-entrained and non-air-entrained GC are 0.80 and 0.90 , respectively. The standard errors of estimate for the elastic modulus of air-entrained and non-air-entrained are $1.51 \mathrm{GPa}$ and $0.88 \mathrm{GPa}$, respectively. The TRMC coefficients for both air-entrained and non-air-entrained GC specimens are 0.13 , indicating that the TRMC has the same effect on both types.

\subsection{Relationship between $E_{c}$ and $f_{c}$}

In the current building codes (e.g. CSA A23.3-04, 2004), the modulus of elasticity is generally related to the compressive strength and the unit weight of concrete. Assuming normal weight concrete, the relationship can be simplified as: 


$$
E_{c}=\alpha_{E c} \sqrt{f_{c}}
$$

where $\alpha_{\mathrm{Ec}}$ is the correlation factor; which equals to 4.5 and 4.7 for CSA (CSA A23.3-04, 2004) and $\mathrm{ACI}$ (ACI committee $318 \mathrm{M}, 1995$ ) standards, respectively, when $E_{c}$ is represented in $\mathrm{GPa}$ and $f_{c}$ is in MPa. To study the applicability of Eq.6.5 to the GC mixes, a regression analysis on the two parameters was carried out. For the analysis, the experimental data of the elastic modulus and the compressive strength for GC-MO and GC-VA specimens presented in Tables 6.1 to 6.4 was used. From the regression analysis, $\alpha_{E c}$ for GC-MO and GC-VA were found to be equal to 4.6 and 4.9 , respectively, showing slightly higher values than the CSA standard value of 4.5. The $\mathrm{R}^{2}$ values of the regression analysis for GC-MO and GC-VA mixes were found to be 0.71 and 0.78 , respectively. The standard error between the predicted values and the experimental observations for GC-MO and GC-VA was $2.0 \mathrm{GPa}$ and $1.5 \mathrm{GPa}$, respectively.

Another multi-variable regression analysis was also carried out to incorporate the effect of the TRMC on the elastic modulus of GC-MO and GC-VA specimens; Eq.6.6 demonstrates the results of this analysis:

$$
E_{c}=4.5(1.1-0.004 T R M C) \sqrt{f_{c}}
$$

In this analysis, the $\mathrm{R}^{2}$ value and the standard error of estimate for the regression analysis were found to be 0.80 and $1.6 \mathrm{GPa}$, respectively. It should be noted that when the TRMC value is not large, Eqs. 6.5 and 6.6 are very similar to each other.

Figure 6.11 illustrates the experimental data along with the predictions provided by Eq.6.5 using $\alpha_{\mathrm{Ec}}$ values of 4.5 (i.e., CSA prediction), 4.6 (i.e,. for GC-MO) and 4.9 (i.e., GC-VA), and Eq.6.6 using the TRMC of 41\%. It is evident from this figure that the CSA equation is underestimating the modulus of elasticity of GC-VA specimens; however, 
some of the GC-MO specimens are overestimated. On the other hand, Eq.6.6 provides a more conservative prediction of the modulus of elasticity of the GC-MO specimens. Therefore, it can be concluded that the CSA equation for conventional concrete that relate the compressive strength to its elastic modulus can be used for GC when the TRMC values are not large (e.g. lower than 25\%). However, Eq.6.6 will provide more accurate result as the TRMC of the GC becomes larger.

\subsection{Splitting Tensile Strength: $f_{t}$}

The splitting tensile tests were carried out following the method described in ASTM 496-96 (ASTM, 2003). In general, as it can be observed in Tables 6.5 to 6.8, the range of the splitting tensile strengths of the all mixes specimens tested were not significantly different. For air-entrained GC-MO mixes, the range was from $3.3 \mathrm{MPa}$ (mean value for GC-MO-AE-P3-50/21 samples) to 4.5 MPa (mean value for GC-MOAE-P1-100/41 samples). For non-air-entrained GC-MO mixes, the range was from 3.4 $\mathrm{MPa}$ (mean value for GC-MO-NE-P3-30/12 samples) to $4.9 \mathrm{MPa}$ (mean value for GCMO-NE-P1-50/21 samples). The range for air-entrained GC-VA specimens was from 2.5 $\mathrm{MPa}$ (mean value for GC-VA-AE-P3-100/23 samples) to 5.3 MPa (mean value for GCVA-AE-P1-30/7 samples). For non-air entrained GC-VA specimens, the range was from 3.0 $\mathrm{MPa}$ (mean value for GC-VA-NE-P3-100/23 samples) to 5.6 MPa (mean value for GC-VA-NE-P2-30/7 samples). In general, as it can be observed from Table 6.5 to 6.8 , the standard deviation of splitting tensile strength is low for all mixes. The splitting tensile strength to compressive strength ratio $\left(f_{t} / f_{c}\right)$ ranges from $8 \%$ to $18 \%$ for GC-MO mixes, and $7 \%$ to $14 \%$ for GC-VA mixes.

As carried out for the compressive strength and the modulus of elasticity, the 
effects of the w/c ratio and the TRMC on the splitting tensile strength of concrete is further investigated. Figures 6.12 and 6.13 illustrate the variation of $f_{t}$ with the w/c ratio for GC-MO and GC-VA specimens, respectively. For GC-MO specimens, it can be observed from Figure 6.12 that the splitting tensile strength values are mostly linearly correlated with the w/c ratio: an increase in the w/c ratio results in a decrease in the splitting tensile strength of concrete. However, it can be observed for GC-MO-NE specimens that with $41 \%$ TRMC, the w/c ratio has no effect on the splitting tensile strength; i.e., the splitting tensile strength is constant. It should also be noted from Figure 6.13 that GC-VA specimens do not show meaningful correlation with the w/c ratio.

Figures 6.14 and 6.15 illustrate the variation of splitting tensile strength with the TRMC of air-entrained and non-air-entrained GC-MO and GC-VA specimens at different w/c ratios. As it can be observed from these figures, the TRMC does not seem to be strongly correlated with the splitting tensile strength.

\subsection{Relationship between $f_{t}$ and $f_{c}$}

In the current building codes (e.g. CSA A23.3-04, 2004), the splitting tensile strength is generally related to the compressive strength of concrete in the following format:

$$
f_{t}=\alpha_{f t} \sqrt{f_{c}}
$$

where $\alpha_{\mathrm{ft}}$ is the correlation factor, which is equal to 0.6 and 0.56 for CSA and ACI standards, respectively. To study the applicability of Eq.6.7 for GC, a regression analysis was carried out to relate the experimental data presented in Tables 6.1 to 6.4. From the regression analysis, $\alpha_{\mathrm{ft}}$ for GC-MO and GC-VA were found to be equal to 0.63 and 0.64 , 
respectively, showing slight difference with the CSA standard value of 0.6. However, the standard errors for any observation for GC-MO and GC-VA were 0.31 and $0.64 \mathrm{MPa}$, respectively.

Figure 6.16 illustrates the relationship between the measured compressive strength and the splitting tensile strength for GC-MO and GC-VA specimens, as well as the predictions of Eq.6.7 using $\alpha_{\mathrm{ft}}$ of 0.6. It is evident from the figure that the experimental data for the GC-MO specimens are well correlated with Eq.6.7; however, the GC-VA specimens show some scatter around the CSA equation. It can be concluded that the CSA equation for conventional concrete that relate the compressive strength and the splitting tensile strength can be also be used for GC.

\subsection{Summary}

The following conclusions can be drawn from the results obtained in this study:

- The hardened density of the specimens tested has been observed to be in the range of normal density concrete.

- The hardened density of GC has been shown to decrease by the increasing values of TRMC.

- As a good quality control practice, it might be necessary to carry out larger number of compressive tests than the usual two when GC has large TRMC values (TRMC> $40 \%)$.

- The main factors affecting the compressive strength and elastic modulus of GC are the TRMC, w/c ratio and air content. General expressions relating the compressive strength and elastic modulus of air-entrained and non-air-entrained GC to the TRMC and $w / c$ ratio were developed. 
- The main factor affecting the mechanical properties of GC is the residual mortar content of the RCA, and not the RCA content.

- A relationship between the modulus of elasticity and compressive strength for GC was developed, in which the effect of the TRMC was also incorporated. It was found that the CSA standard equation for conventional concrete that relate the compressive strength to its elastic modulus can be used for GC instead of the developed equation when the TRMC values are not large (e.g. lower than 25\%).

- The CSA equation for conventional concrete that relates the compressive strength and the splitting tensile strength can also be used for GC. 
Table 6.1: Hardened density of air-entrained GC-MO mixes

\begin{tabular}{|c|c|c|c|c|c|c|c|c|c|c|c|c|c|}
\hline \multirow{3}{*}{ Sample \# } & \multicolumn{12}{|c|}{ RCA (\%)/ TRMC (\%) } & \multirow{3}{*}{$\begin{array}{c}\text { Sample } \\
\text { Designation }\end{array}$} \\
\hline & \multicolumn{3}{|c|}{$100 / 41$} & \multicolumn{3}{|c|}{$50 / 21$} & \multicolumn{3}{|c|}{$30 / 12$} & \multicolumn{3}{|c|}{0} & \\
\hline & 1 & 2 & 3 & 1 & 2 & 3 & 1 & 2 & 3 & 1 & 2 & 3 & \\
\hline $\mathrm{W}^{\mathrm{A} 1}(\mathrm{~g})$ & 3755 & 3698 & 3641 & 3758 & 3760 & 3751 & 3873 & 3862 & 3895 & 3938 & 3818 & 3839 & \\
\hline$W^{w 2}(g)$ & 2071 & 2046 & 2008 & 2077 & 2066 & 2059 & 2195 & 2194 & 2211 & 2263 & 2136 & 2202 & \\
\hline Density & 2230 & 2238 & 2230 & 2236 & 2220 & 2217 & 2308 & 2315 & 2313 & 2351 & 2270 & 2345 & GC-MO-AE-P1 \\
\hline Average & & 2233 & & & 2224 & & & 2312 & & & 2322 & & \\
\hline Std. Dev. & & 5.07 & & & 10.09 & & & 3.69 & & & 45.23 & & \\
\hline $\mathrm{CoV} \%$ & & 0.23 & & & 0.45 & & & 0.16 & & & 1.95 & & \\
\hline $\mathrm{W}^{\mathrm{A} \perp}(\mathrm{g})$ & 3577 & 3545 & 3583 & 3664 & 3598 & 3700 & 3723 & 3719 & 3721 & 3764 & 3748 & 3733 & \\
\hline$W^{w 2}(g)$ & 1906 & 1882 & 1910 & 1982 & 1942 & 2007 & 2055 & 2056 & 2056 & 2100 & 2081 & 2082 & \\
\hline Density & 2141 & 2132 & 2142 & 2178 & 2173 & 2185 & 2232 & 2236 & 2235 & 2262 & 2248 & 2261 & GC-MO-AE-P2 \\
\hline Average & & 2138 & & & 2179 & & & 2234 & & & 2257 & & \\
\hline Std. Dev. & & 5.48 & & & 6.40 & & & 2.19 & & & 7.63 & & \\
\hline $\mathrm{CoV} \%$ & & 0.26 & & & 0.29 & & & 0.10 & & & 0.34 & & \\
\hline $\mathrm{W}^{\mathrm{A}}(\mathrm{g})$ & 3587 & 3578 & 3604 & 3522 & 3660 & 3662 & 3682 & 3569 & 3645 & 3714 & 3735 & 3729 & \\
\hline $\mathrm{W}^{\mathrm{w} 2}(\mathrm{~g})$ & 1911 & 1909 & 1926 & 1890 & 1974 & 1976 & 2016 & 1953 & 1992 & 2051 & 2061 & 2066 & \\
\hline Density & 2140 & 2144 & 2148 & 2158 & 2171 & 2172 & 2210 & 2209 & 2205 & 2233 & 2231 & 2242 & GC-MO-AE-P3 \\
\hline Average & & 2144 & & & 2167 & & & 2208 & & & 2236 & & \\
\hline Std. Dev. & & 3.79 & & & 7.72 & & & 2.56 & & & 5.92 & & \\
\hline $\mathrm{CoV} \%$ & & 0.18 & & & 0.36 & & & 0.12 & & & 0.26 & & \\
\hline
\end{tabular}

$\mathrm{W}^{\mathrm{A}}$ : weight of the specimen in the Air

${ }^{2} \mathrm{~W}^{\mathrm{w}}$ : weight of the specimen in the water 
Table 6.2: Hardened density of non air-entrained GC-MO mixes

\begin{tabular}{|c|c|c|c|c|c|c|c|c|c|c|c|c|c|}
\hline \multirow{3}{*}{ Sample \# } & \multicolumn{12}{|c|}{$\operatorname{RCA}(\%) / \operatorname{TRMC}(\%)$} & \multirow{3}{*}{$\begin{array}{c}\text { Sample } \\
\text { Designation }\end{array}$} \\
\hline & \multicolumn{3}{|c|}{$100 / 41$} & \multicolumn{3}{|c|}{$50 / 21$} & \multicolumn{3}{|c|}{$30 / 12$} & \multicolumn{3}{|c|}{0} & \\
\hline & 1 & 2 & 3 & 1 & 2 & 3 & 1 & 2 & 3 & 1 & 2 & 3 & \\
\hline $\mathrm{W}^{\wedge 1}(\mathrm{~g})$ & 3856 & 3863 & 3747 & 3948 & 3822 & 3927 & 3942 & 3955 & 3963 & - & 3995 & 3871 & \\
\hline $\mathrm{W}^{\mathrm{W} 2}(\mathrm{~g})$ & 2174 & 2177 & 2128 & 2250 & 2178 & 2238 & 2280 & 2293 & 2292 & - & 2325 & 2247 & \\
\hline Density & 2293 & 2291 & 2314 & 2325 & 2325 & 2325 & 2372 & 2380 & 2372 & - & 2392 & 2384 & GC-MO-NE-PI \\
\hline Average & & 2299 & & & 2325 & & & 2374 & & & 2388 & & \\
\hline Std. Dev. & & 13.02 & & & 0.15 & & & 4.58 & & & 6.08 & & \\
\hline $\mathrm{CoV} \%$ & & 0.57 & & & 0.01 & & & 0.19 & & & 0.25 & & \\
\hline $\mathrm{W}^{\mathrm{A} 1}(\mathrm{~g})$ & 3818 & 3810 & 3850 & 3808 & 3928 & 3812 & 3953 & 3931 & 3842 & 3975 & 3968 & 3978 & \\
\hline$W^{w^{2}}(g)$ & 2158 & 2153 & 2180 & 2192 & 2260 & 2187 & 2286 & 2273 & 2224 & 2155 & 2302 & 2301 & \\
\hline Density & 2300 & 2299 & 2305 & 2356 & 2355 & 2346 & 2371 & 2371 & 2375 & 2184 & 2382 & 2372 & GC-MO-NE-P2 \\
\hline Average & & 2302 & & & 2352 & & & 2372 & & & 2313 & & \\
\hline Std. Dev. & & 3.32 & & & 5.73 & & & 1.98 & & & 111.5 & & \\
\hline $\operatorname{CoV} \%$ & & 0.14 & & & 0.24 & & & 0.08 & & & 4.82 & & \\
\hline $\mathrm{W}^{\mathrm{A}}(\mathrm{g})$ & 3814 & 3693 & 3743 & 3822 & 3796 & 3872 & 3848 & 3952 & 3829 & 3963 & 3945 & 3959 & \\
\hline $\mathrm{W}^{\mathrm{w} 2}(\mathrm{~g})$ & 2150 & 2087 & 2118 & 2195 & 2185 & 2225 & 2219 & 2278 & 2203 & 2293 & 2285 & 2297 & \\
\hline Density & 2292 & 2300 & 2303 & 2349 & 2356 & 2351 & 2362 & 2361 & 2355 & 2373 & 2377 & 2382 & GC-MO-NE-P3 \\
\hline Average & & 2298 & & & 2352 & & & 2359 & & & 2377 & & \\
\hline Std. Dev. & & 5.75 & & & 3.74 & & & 3.89 & & & 4.55 & & \\
\hline $\mathrm{CoV} \%$ & & 0.25 & & & 0.16 & & & 0.17 & & & 0.19 & & \\
\hline
\end{tabular}

$\mathrm{W}^{\wedge}$ : weight of the specimen in the Air

${ }^{2} \mathrm{~W}^{\mathrm{w}}:$ weight of the specimen in the water 
Table 6.3: Hardened density of air-entrained GC-VA mixes

\begin{tabular}{|c|c|c|c|c|c|c|c|c|c|c|c|c|c|}
\hline \multirow{3}{*}{ Sample \# } & \multicolumn{12}{|c|}{ RCA (\%) / TRMC (\%) } & \multirow{3}{*}{$\begin{array}{c}\text { Sample } \\
\text { Designation }\end{array}$} \\
\hline & \multicolumn{3}{|c|}{$100 / 41$} & \multicolumn{3}{|c|}{$50 / 21$} & \multicolumn{3}{|c|}{$30 / 12$} & \multicolumn{3}{|c|}{$\mathbf{0}$} & \\
\hline & 1 & 2 & 3 & 1 & 2 & 3 & 1 & 2 & 3 & 1 & 2 & 3 & \\
\hline $\mathrm{W}^{\mathrm{A} 1}(\mathrm{~g})$ & 3751 & 3884 & 3896 & 3703 & 3840 & 3831 & 3833 & 3840 & 3838 & 3799 & 3820 & 3761 & \\
\hline $\mathrm{W}^{\mathrm{w} 2}(\mathrm{~g})$ & 2121 & 2201 & 2211 & 2107 & 2189 & 2187 & 2189 & 2190 & 2189 & 2157 & 2168 & 2125 & \\
\hline Density & 2301 & 2308 & 2312 & 2320 & 2326 & 2330 & 2332 & 2327 & 2327 & 2314 & 2312 & 2299 & GC-VA-AE-P1 \\
\hline Average & & 2307 & & & 2325 & & & 2329 & & & 2308 & & \\
\hline Std. Dev. & & 5.51 & & & 5.07 & & & 2.39 & & & 8.16 & & \\
\hline $\mathrm{CoV} \%$ & & 0.24 & & & 0.22 & & & 0.10 & & & 0.35 & & \\
\hline $\mathrm{W}^{\mathrm{A} 1}(\mathrm{~g})$ & 3750 & 3737 & 3708 & 3729 & 3752 & 3708 & 3740 & 3756 & 3622 & 3648 & 3749 & 3724 & \\
\hline $\mathrm{W}^{\mathrm{w} 2}(\mathrm{~g})$ & 2075 & 2068 & 2038 & 2083 & 2110 & 2073 & 2092 & 2114 & 2030 & 2052 & 2106 & 2088 & \\
\hline Density & 2239 & 2239 & 2220 & 2265 & 2285 & 2268 & 2269 & 2287 & 2275 & 2286 & 2282 & 2276 & GC-VA-AE-P2 \\
\hline Average & & 2233 & & & 2273 & & & 2277 & & & 2281 & & \\
\hline Std. Dev. & & 10.73 & & & 10.65 & & & 9.22 & & & 4.74 & & \\
\hline $\operatorname{CoV} \%$ & & 0.48 & & & 0.47 & & & 0.40 & & & 0.21 & & \\
\hline$W^{A}(g)$ & 3730 & 3669 & 3689 & 3703 & 3683 & 3611 & 3597 & 3720 & 3720 & 3810 & 3713 & 3683 & \\
\hline$W^{w^{2}}(g)$ & 2056 & 2009 & 1972 & 2066 & 2043 & 2011 & 1996 & 2071 & 2070 & 2178 & 2127 & 2063 & \\
\hline Density & 2228 & 2210 & 2149 & 2262 & 2246 & 2257 & 2247 & 2256 & 2255 & 2335 & 2341 & 2273 & GC-VA-AE-P3 \\
\hline Average & & 2196 & & & 2255 & & & 2252 & & & 2316 & & \\
\hline Std. Dev. & & 41.80 & & & 8.35 & & & 4.96 & & & 37.31 & & \\
\hline $\operatorname{Cov} \%$ & & 1.90 & & & 0.37 & & & 0.22 & & & 1.61 & & \\
\hline
\end{tabular}

$\mathrm{W}^{\mathrm{A}}$ : weight of the specimen in the Air

${ }^{2} \mathrm{~W}^{\mathrm{w}}:$ weight of the specimen in the water 
Table 6.4: Hardened density of non air-entrained GC-VA mixes

\begin{tabular}{|c|c|c|c|c|c|c|c|c|c|c|c|c|c|}
\hline \multirow{3}{*}{ Sample \# } & \multicolumn{12}{|c|}{ RCA (\%)/ TRMC (\%) } & \multirow{3}{*}{$\begin{array}{c}\text { Sample } \\
\text { Designation }\end{array}$} \\
\hline & \multicolumn{3}{|c|}{$100 / 41$} & \multicolumn{3}{|c|}{$50 / 21$} & \multicolumn{3}{|c|}{$30 / 12$} & \multicolumn{3}{|c|}{0} & \\
\hline & 1 & 2 & 3 & 1 & 2 & 3 & 1 & $\overline{2}$ & 3 & 1 & 2 & 3 & \\
\hline$\overline{\mathrm{W}^{\mathrm{A} 1}(\mathrm{~g})}$ & 3840 & 3850 & 3931 & 3941 & 3934 & 3938 & 3968 & 3943 & 3977 & 3976 & 3983 & 3942 & \\
\hline$W^{w^{2}}(g)$ & 2209 & 2212 & 2267 & 2288 & 2286 & 2293 & 2311 & 22.98 & 2317 & 2332 & 2335 & 2308 & \\
\hline Density & 2354 & 2350 & 2362 & 2384 & 2387 & 2394 & 2395 & 2397 & 2396 & 2418 & 2417 & 2412 & GC-VA-NE-P1 \\
\hline Average & & 2356 & & & 2388 & & & 2396 & & & 2416 & & \\
\hline Std. Dev. & & 6.09 & & & 5.01 & & & 1.14 & & & 3.11 & & \\
\hline $\mathrm{CoV} \%$ & & 0.26 & & & 0.21 & & & 0.05 & & & 0.13 & & \\
\hline $\mathrm{W}^{\mathrm{A} 1}(\mathrm{~g})$ & 3796 & 3929 & 3936 & 3935 & 3933 & 3908 & 3922 & 3910 & 3928 & 3861 & 3948 & 3958 & \\
\hline $\mathrm{W}^{\mathrm{w} 2}(\mathrm{~g})$ & 2175 & 2259 & 2125 & 2292 & 2289 & 2271 & 2285 & 2277 & 2284 & 2264 & 2316 & 2319 & \\
\hline Density & 2342 & 2353 & 2173 & 2395 & 2392 & 2387 & 2396 & 2394 & 2389 & 2418 & 2419 & 2415 & GC-VA-NE-P2 \\
\hline Average & & 2289 & & & 2392 & & & 2393 & & & 2417 & & \\
\hline Std. Dev. & & 100.52 & & & 3.92 & & & 3.44 & & & 2.15 & & \\
\hline $\mathrm{CoV} \%$ & & 4.39 & & & 0.16 & & & 0.14 & & & 0.09 & & \\
\hline $\mathrm{W}^{\mathrm{Al}}(\mathrm{g})$ & 3935 & 3775 & 3840 & 3929 & 3826 & 3940 & 3905 & 3919 & 3908 & 3919 & 3947 & 3927 & \\
\hline $\mathrm{W}^{\mathrm{w} 2}(\mathrm{~g})$ & 2265 & 2170 & 2041 & 2286 & 2228 & 2294 & 2265 & 2284 & 2278 & 2290 & 2313 & 2300 & \\
\hline Density & 2356 & 2352 & 2135 & 2391 & 2394 & 2394 & 2381 & 2397 & 2398 & 2406 & 2416 & 2414 & GC-VA-NE-P3 \\
\hline Average & & 2281 & & & 2393 & & & 2392 & & & 2412 & & \\
\hline Std. Dev. & & 126.83 & & & 1.53 & & & 9.33 & & & 5.18 & & \\
\hline $\mathrm{CoV} \%$ & & 5.56 & & & 0.06 & & & 0.39 & & & 0.21 & & \\
\hline
\end{tabular}

$\mathrm{W}^{\wedge}$ : weight of the specimen in the Air

${ }^{2} \mathrm{~W}^{\mathrm{w}}$ : weight of the specimen in the water 
Table 6.5: Mechanical properties of air-entrained GC-MO mixes

\begin{tabular}{|c|c|c|c|c|c|c|c|c|c|c|c|c|c|}
\hline \multirow{3}{*}{ Sample \# } & \multicolumn{12}{|c|}{ RCA $(\%) / T R M C(\%)$} & \multirow{3}{*}{$\begin{array}{c}\text { Sample } \\
\text { Designation }\end{array}$} \\
\hline & \multicolumn{3}{|c|}{$100 / 41$} & \multicolumn{3}{|c|}{$50 / 21$} & \multicolumn{3}{|c|}{$30 / 12$} & \multicolumn{3}{|c|}{$\mathbf{0}$} & \\
\hline & 1 & 2 & 3 & 1 & 2 & 3 & 1 & 2 & 3 & 1 & 2 & 3 & \\
\hline $\begin{array}{l}\mathbf{f}_{\mathrm{c}}(\mathbf{M P a}) \\
\text { Average } \\
\text { Std. Dev. } \\
\text { CoV }(\%)\end{array}$ & 49.5 & $\begin{array}{c}47.4 \\
44.8 \\
6.5 \\
14.5\end{array}$ & 37. & 38.6 & \begin{tabular}{c|}
42.1 \\
41.3 \\
2.4 \\
5.8 \\
\end{tabular} & 43.1 & 48.1 & $\begin{array}{c}48.6 \\
47.8 \\
0.9 \\
1.9\end{array}$ & 46.8 & 47.6 & $\begin{array}{c}45.5 \\
46.6 \\
1.5 \\
3.2 \\
\end{array}$ & - & \\
\hline $\begin{array}{l}\mathbf{f}_{\mathbf{t}} \text { (MPa) } \\
\text { Average } \\
\text { Std. Dev. } \\
\operatorname{CoV}(\%)\end{array}$ & 3.6 & $\begin{array}{c}5.3 \\
4.5 \\
1.3 \\
28.9\end{array}$ & (a) & 4.0 & $\begin{array}{l}4.3 \\
4.2 \\
0.2 \\
4.8\end{array}$ & 4.4 & 4.3 & $\begin{array}{l}3.9 \\
4.2 \\
0.3 \\
7.1\end{array}$ & 4.4 & 4.6 & $\begin{array}{c}3.4 \\
4.2 \\
0.7 \\
16.7\end{array}$ & 4.6 & GC-MO-AE-P1 \\
\hline $\begin{array}{c}\mathbf{E}_{\mathrm{c}}(\mathbf{G P a}) \\
\text { Average } \\
\text { Std. Dev. } \\
\mathrm{CoV}(\%)\end{array}$ & 27.8 & \begin{tabular}{c|}
26.3 \\
27.4 \\
1.0 \\
3.6 \\
\end{tabular} & $\begin{array}{c}28 . \\
1\end{array}$ & 28.4 & $\begin{array}{c}27.2 \\
27.9 \\
0.6 \\
2.2 \\
\end{array}$ & 28.0 & 30.9 & $\begin{array}{c}31.7 \\
31.3 \\
0.4 \\
1.3 \\
\end{array}$ & 31.3 & 34.2 & $\begin{array}{c}34.3 \\
34.2 \\
0.0 \\
0.00 \\
\end{array}$ & 34.2 & \\
\hline $\mathbf{f}_{\mathrm{t}} / \mathbf{f}_{\mathbf{c}}(\%)$ & & 10 & & & 10 & & & 9 & & & 9 & & \\
\hline $\begin{array}{l}\mathbf{f}_{\mathbf{c}}(\mathbf{M P a}) \\
\text { Average } \\
\text { Std. Dev. } \\
\text { CoV }(\%)\end{array}$ & 33.0 & $\begin{array}{l}31.2 \\
30.9 \\
2.2 \\
7.1 \\
\end{array}$ & 28. & 34.8 & $\begin{array}{c}30.6 \\
32.5 \\
2.1 \\
6.5 \\
\end{array}$ & 32.0 & 32.8 & \begin{tabular}{c|}
32.1 \\
33.1 \\
1.2 \\
3.6 \\
\end{tabular} & 34.5 & 35.5 & $\begin{array}{c}35.7 \\
36.2 \\
1.1 \\
3.0 \\
\end{array}$ & 37.5 & \\
\hline $\begin{array}{l}\mathbf{f}_{\mathbf{t}} \text { (MPa) } \\
\text { Average } \\
\text { Std. Dev. } \\
\text { CoV }(\%)\end{array}$ & 3.6 & $\begin{array}{c}3.3 \\
3.8 \\
0.6 \\
15.8 \\
\end{array}$ & 4.4 & 3.8 & $\begin{array}{l}3.9 \\
3.8 \\
0.1 \\
2.6 \\
\end{array}$ & 3.6 & 3.9 & $\begin{array}{l}3.9 \\
3.9 \\
0.0 \\
1.0\end{array}$ & 3.8 & 3.7 & $\begin{array}{l}3.6 \\
3.5 \\
0.3 \\
8.6\end{array}$ & 3.2 & GC-MO-AE-P2 \\
\hline $\begin{array}{c}\mathbf{E}_{\mathrm{c}}(\mathbf{G P a}) \\
\text { Average } \\
\text { Std. Dev. } \\
\mathrm{CoV}(\%)\end{array}$ & 21.5 & \begin{tabular}{c|}
22.6 \\
22.1 \\
0.8 \\
3.6
\end{tabular} & (b) & 25.0 & $\begin{array}{c}25.4 \\
25.5 \\
0.6 \\
2.4 \\
\end{array}$ & 26.1 & 26.5 & $\begin{array}{c}26.9 \\
26.4 \\
0.5 \\
1.9 \\
\end{array}$ & 25.9 & 31.0 & $\begin{array}{l}-(b) \\
30.7 \\
0.4 \\
1.3 \\
\end{array}$ & 30.5 & \\
\hline $\mathbf{f}_{\mathrm{t}} / \mathbf{f}_{\mathrm{c}}(\%)$ & & 12 & & & 12 & & & 12 & & & 10 & & \\
\hline $\begin{array}{l}\mathbf{f}_{\mathbf{c}}(\mathbf{M P a}) \\
\text { Average } \\
\text { Std. Dev. } \\
\mathrm{CoV}(\%)\end{array}$ & 21.2 & $\begin{array}{c}18.4 \\
19.8 \\
2.0 \\
10.1\end{array}$ & & 24.4 & $\begin{array}{c}26.6 \\
25.2 \\
1.2 \\
4.8\end{array}$ & 24.6 & 29.0 & $\begin{array}{c}27.8 \\
28.4 \\
0.6 \\
2.1 \\
\end{array}$ & 28.3 & 28.8 & $\begin{array}{c}28.2 \\
28.8 \\
0.7 \\
2.4 \\
\end{array}$ & 29.5 & \\
\hline $\begin{array}{l}\mathbf{f}_{\mathbf{t}}(\mathbf{M P a}) \\
\text { Average } \\
\text { Std. Dev. } \\
\text { CoV }(\%) \\
\end{array}$ & 3.1 & $\begin{array}{c}3.7 \\
3.6 \\
0.4 \\
11.1 \\
\end{array}$ & 3.8 & 3.0 & $\begin{array}{l}3.3 \\
3.3 \\
0.2 \\
6.1 \\
\end{array}$ & 3.4 & 3.5 & $\begin{array}{l}3.2 \\
3.4 \\
0.2 \\
5.9 \\
\end{array}$ & 3.5 & 3.5 & $\begin{array}{l}3.6 \\
3.5 \\
0.1 \\
2.9 \\
\end{array}$ & 3.4 & GC-MO-AE-P3 \\
\hline $\begin{array}{c}\mathbf{E}_{\mathrm{c}}(\mathbf{G P a}) \\
\text { Average } \\
\text { Std. Dev. } \\
\mathrm{CoV}(\%)\end{array}$ & 24.3 & $\begin{array}{c}25.3 \\
24.5 \\
0.7 \\
2.9 \\
\end{array}$ & $\begin{array}{c}23 \\
9\end{array}$ & 23.5 & $\begin{array}{c}23.9 \\
24.0 \\
0.6 \\
2.5 \\
\end{array}$ & 24.7 & 24.7 & $\begin{array}{c}24.4 \\
24.6 \\
0.2 \\
0.8\end{array}$ & 24.8 & 26.4 & $\begin{array}{c}24.3 \\
25.6 \\
1.2 \\
4.7 \\
\end{array}$ & 26.2 & \\
\hline $\mathbf{f}_{\mathrm{t}} / \mathbf{f}_{\mathrm{c}}(\boldsymbol{\%})$ & & 18 & & & 13 & & & 12 & & & 12 & & \\
\hline
\end{tabular}

${ }^{a}$ Sample discarded due to high rate of loading

${ }^{\mathrm{b}}$ Sample discarded because it failed in compression, i.e. not splitting 
Table 6.6: Mechanical properties of non air-entrained GC-MO mixes

\begin{tabular}{|c|c|c|c|c|c|c|c|c|c|c|c|c|c|}
\hline \multirow{3}{*}{ Sample \# } & \multicolumn{12}{|c|}{ RCA $(\%) /$ TRMC $(\%)$} & \multirow{3}{*}{$\begin{array}{c}\text { Sample } \\
\text { Designation }\end{array}$} \\
\hline & \multicolumn{3}{|c|}{$100 / 41$} & \multicolumn{3}{|c|}{$50 / 21$} & \multicolumn{3}{|c|}{$30 / 12$} & \multicolumn{3}{|c|}{0} & \\
\hline & $\overline{11}$ & 2 & $\overline{3}$ & $\overline{11}$ & 2 & $\overline{3}$ & $\bar{~} 1$ & 2 & 3 & $\overline{11}$ & 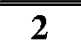 & $\overline{3}$ & \\
\hline $\mathbf{f}_{\mathrm{c}}(\mathbf{M P a})$ & 46.4 & 54.5 & 53.5 & 55.9 & 49.3 & 57.3 & 60.6 & 55.0 & 56.8 & 55.0 & 56.1 & 52.0 & \\
\hline Average & & 51.5 & & & 54.2 & & & 57.5 & & & 54.4 & & \\
\hline Std. Dev. & & 4.4 & & & 4.3 & & & 2.9 & & & 2.1 & & \\
\hline $\operatorname{CoV}(\%)$ & & 8.5 & & & 7.9 & & & 5.0 & & & 3.9 & & \\
\hline $\mathbf{f}_{\mathbf{t}}(\mathbf{M P a})$ & 4.6 & 4.2 & 3.8 & 4.9 & 5.1 & 4.6 & 4.6 & 4.4 & 4.6 & 4.8 & 4.7 & 4.0 & \\
\hline Average & & 4.2 & & & 4.9 & & & 4.5 & & & 4.5 & & GC-MO-NF-P1 \\
\hline Std. Dev. & & 0.4 & & & 0.3 & & & 0.1 & & & 0.4 & & UL-MUT-NE-FI \\
\hline $\operatorname{Cov}(\%)$ & & 9.5 & & & 6.1 & & & 2.2 & & & 8.9 & & \\
\hline $\mathrm{E}_{\mathrm{c}}(\mathrm{GPa})$ & 30.9 & 31.1 & 30.9 & 33.0 & 34.3 & 34.0 & 33.7 & 32.7 & 35.1 & 36.0 & 37.3 & 35.7 & \\
\hline Average & & 30.9 & & & 33.8 & & & 33.8 & & & 36.3 & & \\
\hline Std. Dev. & & 0.1 & & & 0.7 & & & 1.2 & & & 0.8 & & \\
\hline $\operatorname{Cov}(\%)$ & & 0.32 & & & 2.1 & & & 3.6 & & & 2.2 & & \\
\hline $\mathbf{f}_{\mathrm{t}} / \mathbf{f}_{\mathrm{c}}(\%)$ & & 8 & & & 9 & & & 8 & & & 8 & & \\
\hline $\mathbf{f}_{\mathrm{c}}(\mathrm{MPa})$ & 41.6 & 44.8 & 42.7 & 50.0 & 49.5 & 49.4 & 47.5 & 47.6 & 49.1 & 45.7 & 45.9 & 44.4 & \\
\hline Average & & 43.0 & & & 49.6 & & & 48.1 & & & 45.3 & & \\
\hline Std. Dev. & & 1.6 & & & 0.3 & & & 0.9 & & & 0.8 & & \\
\hline $\operatorname{CoV}(\%)$ & & 3.7 & & & 0.6 & & & 1.9 & & & 1.8 & & \\
\hline $\mathrm{f}_{\mathrm{t}}(\mathrm{MPa})$ & 3.5 & 4.0 & 4.4 & 4.0 & 4.5 & 4.5 & 4.6 & 4.5 & 4.2 & 4.6 & 3.4 & 4.0 & \\
\hline Average & & 4.0 & & & 4.3 & & & 4.4 & & & 4.0 & & $C C$ \\
\hline Std. Dev. & & 0.5 & & & 0.3 & & & 0.2 & & & 0.6 & & $\mathrm{GC}-1$ \\
\hline $\operatorname{CoV}(\%)$ & & 12.5 & & & 7.0 & & & 4.6 & & & 15.0 & & \\
\hline $\mathrm{E}_{\mathrm{c}}(\mathrm{GPa})$ & 27.3 & 29.2 & 28.3 & 31.4 & 31.3 & 31.6 & 32.7 & 31.8 & 31.8 & 33.9 & 33.9 & 35.0 & \\
\hline Average & & 28.3 & & & 31.4 & & & 32.1 & & & 34.3 & & \\
\hline Std. Dev. & & 0.9 & & & 0.1 & & & 0.5 & & & 0.6 & & \\
\hline $\operatorname{Cov}(\%)$ & & 3.2 & & & 0.3 & & & 1.6 & & & 1.8 & & \\
\hline $\mathbf{f}_{\mathrm{t}} / \mathbf{f}_{\mathrm{c}}(\%)$ & & 9 & & & 9 & & & 9 & & & 9 & & \\
\hline $\mathbf{f}_{\mathrm{c}}(\mathbf{M P a})$ & 32.6 & 29.9 & 32.1 & 42.8 & 43.3 & 41.1 & 38.0 & 38.3 & 39.8 & 38.2 & 36.3 & 37.9 & \\
\hline Average & & 31.5 & & & 42.4 & & & 38.7 & & & 37.5 & & \\
\hline Std. Dev. & & 1.4 & & & 1.2 & & & 1.0 & & & 1.0 & & \\
\hline $\operatorname{CoV}(\%)$ & & 4.4 & & & 2.8 & & & 2.6 & & & 2.7 & & \\
\hline $\mathrm{f}_{\mathrm{t}}(\mathrm{MPa})$ & $-(a)$ & 4.4 & 4.0 & 4.2 & 4.0 & 4.1 & 3.5 & 3.2 & 3.6 & 4.1 & 3.7 & 4.2 & \\
\hline Average & & 4.2 & & & 4.1 & & & 3.4 & & & 4.0 & & $\mathrm{CC}_{-} \mathrm{O}_{-\mathrm{NE}} \mathrm{P} 3$ \\
\hline Std. Dev. & & 0.3 & & & 0.1 & & & 0.2 & & & 0.2 & & 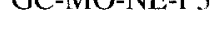 \\
\hline $\operatorname{CoV}(\%)$ & & 7.1 & & & 2.4 & & & 5.9 & & & 5.0 & & \\
\hline $\mathbf{E}_{\mathrm{c}}(\mathrm{GPa})$ & 27.3 & 26.8 & 26.9 & 28.8 & 27.3 & 27.2 & 30.7 & 30.0 & 30.6 & 30.0 & 33.0 & 33.1 & \\
\hline Average & & 27.0 & & & 27.7 & & & 30.4 & & & 32.0 & & \\
\hline Std. Dev. & & 0.2 & & & 0.9 & & & 0.4 & & & 1.7 & & \\
\hline $\operatorname{CoV}(\%)$ & & 0.7 & & & 3.3 & & & 1.3 & & & 5.3 & & \\
\hline $\mathbf{f}_{\mathrm{t}} / \mathbf{f}_{\mathrm{c}}(\%)$ & & 13 & & & 10 & & & 9 & & & 11 & & \\
\hline
\end{tabular}

${ }^{a}$ Sample discarded because it failed in compression, i.e. not splitting 
Table 6.7: Mechanical properties of air-entrained GC-VA mixes

\begin{tabular}{|c|c|c|c|c|c|c|c|c|c|c|c|c|c|}
\hline \multirow{3}{*}{ Sample \# } & \multicolumn{12}{|c|}{$\operatorname{RCA}(\%) / \mathrm{TRMC}(\%)$} & \multirow{3}{*}{$\begin{array}{c}\text { Sample } \\
\text { Designation }\end{array}$} \\
\hline & \multicolumn{3}{|c|}{$100 / 23$} & \multicolumn{3}{|c|}{$50 / 12$} & \multicolumn{3}{|c|}{$30 / 7$} & \multicolumn{3}{|c|}{0} & \\
\hline & 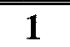 & 2 & $\overline{17}$ & $\overline{1} 1$ & 2 & 3 & 1 & 2 & 3 & 1 & 2 & 3 & \\
\hline $\mathbf{f}_{\mathfrak{c}}(\mathbf{M P a})$ & 45.0 & 45.7 & 44.1 & 46.0 & 44.1 & 44.0 & 40.0 & 38.1 & 44.5 & 41.7 & 40.4 & 40.3 & \\
\hline Average & & 44.9 & & & 44.7 & & & 40.9 & & & 40.8 & & \\
\hline Std. Dev. & & 0.8 & & & 1.1 & & & 3.3 & & & 0.8 & & \\
\hline $\operatorname{CoV}(\%)$ & & 1.8 & & & 2.5 & & & 8.1 & & & 2.0 & & \\
\hline $\mathbf{f}_{\mathbf{t}}(\mathbf{M P a})$ & 4.2 & 4.7 & 3.8 & 3.6 & 3.3 & 4.6 & 5.8 & 4.8 & 5.3 & 4.1 & 3.8 & - & \\
\hline Average & & 4.2 & & & 3.8 & & . & 5.3 & & & 3.9 & & GC-VA-AE-PI \\
\hline Std. Dev. & & 0.5 & & & 0.7 & & & 0.5 & & & 0.2 & & \\
\hline $\operatorname{CoV}(\%)$ & & 11.9 & & & 18.4 & & & 9.4 & & & 5.1 & & \\
\hline $\mathbf{E}_{\mathbf{c}}(\mathrm{GPa})$ & $-(b)$ & 30.8 & 30.7 & 32.9 & 32.0 & 33.8 & 34.0 & 31.0 & 33.9 & 29.8 & 31.1 & 28.9 & \\
\hline Average & & 30.8 & & & 32.9 & & & 33.0 & & & 29.9 & & \\
\hline Std. Dev. & & 0.1 & & & 0.9 & & & 1.7 & & & 1.1 & & \\
\hline $\operatorname{CoV}(\%)$ & & 0.32 & & & 2.7 & & & 5.2 & & & 3.7 & & \\
\hline $\mathbf{f}_{\mathbf{t}} / \mathbf{f}_{\boldsymbol{c}}(\boldsymbol{\%})$ & & 9 & & & 9 & & & 13 & & & 10 & & \\
\hline $\mathbf{f}_{\mathrm{c}}(\mathbf{M P a})$ & 33.5 & 34.3 & 35.2 & 37.1 & 36.4 & 36.2 & 35.1 & 34.9 & 34.1 & 33.8 & 34.8 & 32.4 & \\
\hline Average & & 34.3 & & & 36.6 & & & 34.7 & & & 33.7 & & \\
\hline Std. Dev. & & 0.9 & & & 0.5 & & & 0.5 & & & 1.2 & & \\
\hline $\operatorname{CoV}(\%)$ & & 2.6 & & & 1.4 & & & 1.4 & & & 3.6 & & \\
\hline $\mathbf{f}_{\mathrm{t}}(\mathbf{M P a})$ & 3.3 & 3.4 & - & 4.7 & 4.5 & 2.7 & 3.1 & 4.3 & 3.8 & - & 3.7 & 3.8 & \\
\hline Average & & 3.4 & & & 4.0 & & & 3.7 & & & 3.8 & & GC-VA-AE-P2 \\
\hline Std. Dev. & & 0.1 & & & 1.1 & & & 0.6 & & & 0.1 & & \\
\hline $\operatorname{CoV}(\%)$ & & 2.9 & & & 25.0 & & & 16.2 & & & 2.6 & & \\
\hline $\mathbf{E}_{\mathrm{c}}(\mathrm{GPa})$ & 27.0 & 26.6 & 28.0 & 29.7 & 29.8 & 28.7 & 28.4 & 29.0 & 28.7 & 29.0 & 28.5 & 28.9 & \\
\hline Average & & 27.2 & & & 29.4 & & & 28.7 & & & 28.8 & & \\
\hline Std. Dev. & & 0.7 & & & 0.6 & & & 0.3 & & & 0.2 & & \\
\hline $\operatorname{CoV}(\%)$ & & 2.6 & & & 2.0 & & & 1.1 & & & 0.7 & & \\
\hline $\mathbf{f}_{\mathbf{t}} / \mathbf{f}_{c}(\boldsymbol{\%})$ & & 7 & & & 11 & & & 11 & & & 11 & & \\
\hline $\mathbf{f}_{\mathrm{c}}(\mathbf{M P a})$ & 26.8 & 27.5 & 26.0 & 29.4 & 29.4 & 26.6 & 27.7 & 27.4 & 26.0 & 31.5 & 31.1 & 27.4 & \\
\hline Average & & 26.8 & & & 28.5 & & & 27.0 & & & 30.0 & & \\
\hline Std. Dev. & & 0.8 & & & 1.6 & & & 0.9 & & & 2.3 & & \\
\hline $\operatorname{CoV}(\%)$ & & 3.0 & & & 5.6 & & & 3.3 & & & 7.7 & & \\
\hline $\mathbf{f}_{\mathrm{t}}(\mathbf{M P a})$ & 2.6 & 2.2 & 2.7 & 3.0 & 3.8 & 3.6 & 2.7 & 3.3 & 4.5 & 5.4 & 2.8 & 4.2 & \\
\hline Average & & 2.5 & & & 3.5 & & & 3.5 & & & 4.1 & & GC-VA-AE-P3 \\
\hline Std. Dev. & & 0.3 & & & 0.4 & & & 0.9 & & & 1.3 & & G \\
\hline $\operatorname{CoV}(\%)$ & & 12.0 & & & 11.4 & & & 25.7 & & & 31.7 & & \\
\hline $\mathbf{E}_{\mathrm{c}}(\mathbf{G P a})$ & 24.2 & 23.0 & 24.9 & 27.2 & 26.0 & 27.5 & 23.7 & 27.6 & 27.2 & 29.4 & 28.7 & 29.2 & \\
\hline Average & & 24.0 & & & 26.9 & & & 26.1 & & & 29.1 & & \\
\hline Std. Dev. & & 1.0 & & & 0.8 & & & 2.1 & & & 0.3 & & \\
\hline $\operatorname{Cov}(\%)$ & & 4.2 & & & 3.0 & & & 8.1 & & & 1.0 & & \\
\hline $\mathbf{f}_{\mathbf{t}} / \mathbf{f}_{\mathbf{c}}(\%)$ & & 9 & & & 12 & & & 13 & & & 14 & & \\
\hline
\end{tabular}

${ }^{\mathrm{b}}$ Sample discarded because it was failed by compression during test. 
Table 6.8: Mechanical properties of non air-entrained GC-VA mixes

\begin{tabular}{|c|c|c|c|c|c|c|c|c|c|c|c|c|c|}
\hline \multirow{3}{*}{ Sample \# } & \multicolumn{12}{|c|}{ RCA (\%)/ TRMC (\%) } & \multirow{3}{*}{$\begin{array}{c}\text { Sample } \\
\text { Designation }\end{array}$} \\
\hline & \multicolumn{3}{|c|}{$100 / 23$} & \multicolumn{3}{|c|}{$50 / 12$} & \multicolumn{3}{|c|}{$30 / 7$} & \multicolumn{3}{|c|}{0} & \\
\hline & $\overline{1}$ & 2 & 3 & 1 & $\overline{2}$ & $\overline{13}$ & $\overline{1}$ & $\overline{2}$ & 3 & 1 & $\overline{2}$ & $\overline{17}$ & \\
\hline $\mathrm{f}_{\mathrm{c}}(\mathrm{MPa})$ & 55.0 & 52.5 & 56.5 & 48.4 & 48.8 & 48.5 & 56.4 & 52.7 & 51.5 & 56.2 & 53.6 & 49.4 & \\
\hline Average & & 54.7 & & & 48.6 & & & 53.5 & & & 53.1 & & \\
\hline Std. Dev. & & 2.0 & & & 0.2 & & & 2.6 & & & 3.4 & & \\
\hline $\operatorname{CoV}(\%)$ & & 3.7 & & & 0.4 & & & 4.9 & & & 6.4 & & \\
\hline $\mathbf{f}_{\mathrm{t}}(\mathbf{M P a})$ & 3.9 & 3.9 & 4.2 & 4.2 & 4.4 & 3.6 & 4.2 & 5.3 & 5.1 & 3.6 & 4.0 & 3.6 & \\
\hline Average & & 4.0 & & & 4.1 & & & 4.9 & & & 3.8 & & GC-VA-NE-PI \\
\hline Std. Dev. & & 0.2 & & & 0.4 & & & 0.6 & & & 0.3 & & \\
\hline $\operatorname{CoV}(\%)$ & & 5.0 & & & 9.8 & & & 12.2 & & & 7.9 & & \\
\hline $\mathrm{E}_{\mathrm{c}}(\mathrm{GPa})$ & 34.5 & 33.6 & 34.6 & 35.4 & 35.5 & 35.2 & 34.9 & 37.6 & 36.7 & 35.1 & 34.5 & 35.6 & \\
\hline Average & & 34.2 & & & 35.3 & & & 36.4 & & & 35.1 & & \\
\hline Std. Dev. & & 0.6 & & & 0.2 & & & 1.3 & & & 0.6 & & \\
\hline $\operatorname{CoV}(\%)$ & & 1.8 & & & 0.6 & & & 3.6 & & & 1.7 & & \\
\hline $\mathbf{f}_{\mathbf{t}} / \mathbf{f}_{\mathrm{c}}(\%)$ & & 7 & & & 8 & & & 9 & & & 7 & & \\
\hline $\mathbf{f}_{\mathrm{c}}(\mathrm{MPa})$ & 44.1 & 43.8 & 43.8 & 40.1 & 44.0 & 43.6 & 46.2 & 45.3 & 47.8 & 37.8 & 44.6 & 39.7 & \\
\hline Average & & 43.9 & & & 42.6 & & & 46.4 & & & 40.7 & & \\
\hline Std. Dev. & & 0.2 & & & 2.1 & & & 1.3 & & & 3.5 & & \\
\hline $\operatorname{CoV}(\%)$ & & 0.5 & & & 4.9 & & & 2.8 & & & 8.6 & & \\
\hline $\mathbf{f}_{\mathbf{t}}(\mathbf{M P a})$ & 4.1 & 4.3 & 4.7 & 4.3 & 5.4 & 4.0 & 4.7 & 6.2 & 6.0 & 3.8 & 3.9 & (a) & \\
\hline Average & & 4.4 & & & 4.6 & & & 5.6 & & & 3.9 & & GC-VA-NE-P2 \\
\hline Std. Dev. & & 0.3 & & & 0.7 & & & 0.8 & & & 0.0 & & \\
\hline $\operatorname{CoV}(\%)$ & & 6.8 & & & 15.2 & & & 14.3 & & & 1.0 & & \\
\hline $\mathrm{E}_{\mathrm{c}}(\mathrm{GPa})$ & 31.4 & 31.5 & 31.2 & 32.1 & 31.7 & 33.1 & 32.4 & 32.9 & 33.0 & 37.0 & 34.3 & 34.8 & \\
\hline Average & & 31.4 & & & 32.3 & & & 32.8 & & & 35.4 & & \\
\hline Std. Dev. & & 0.2 & & & 0.7 & & & 0.3 & & & 1.4 & & \\
\hline $\operatorname{CoV}(\%)$ & & 0.6 & & & 2.2 & & & 0.9 & & & 4.0 & & \\
\hline $\mathbf{f}_{\mathbf{t}} / \mathbf{f}_{\mathrm{c}}(\%)$ & & 10 & & & 11 & & & 12 & & & 9 & & \\
\hline $\mathbf{f}_{c}(\mathbf{M P a})$ & 37.6 & 37.5 & 38.6 & 38.2 & 39.2 & 36.8 & 39.3 & 38.7 & 39.3 & 39.9 & 40.8 & 40.8 & \\
\hline Average & & 37.9 & & & 38.1 & & & 39.1 & & & 40.5 & & \\
\hline Std. Dev. & & 0.6 & & & 1.2 & & & 0.3 & & & 0.5 & & \\
\hline $\operatorname{CoV}(\%)$ & & 1.6 & & & 3.2 & & & 0.8 & & & 1.2 & & \\
\hline $\mathbf{f}_{\mathfrak{t}}(\mathbf{M P a})$ & 3.1 & 3.0 & 3.0 & 3.4 & 5.1 & 4.6 & 4.6 & 4.8 & 4.9 & 4.1 & 4.2 & 5.1 & \\
\hline Average & & 3.0 & & & 4.3 & & & 4.8 & & & 4.1 & & GC-YA-NE-P3 \\
\hline Std. Dev. & & 0.1 & & & 0.8 & & & 0.1 & & & 0.6 & & \\
\hline $\operatorname{CoV}(\%)$ & & 3.3 & & & 18.6 & & & 2.1 & & & 14.6 & & \\
\hline $\mathbf{E}_{c}(\mathrm{GPa})$ & 29.0 & 28.8 & 30.1 & 31.6 & 29.4 & 29.6 & 32.8 & 33.0 & 30.4 & 34.6 & 32.7 & 33.1 & \\
\hline Average & & 29.3 & & & 30.2 & & & 32.1 & & & 33.5 & & \\
\hline Std. Dev. & & 0.7 & & & 1.2 & & & 1.4 & & & 1.0 & & \\
\hline $\operatorname{CoV}(\%)$ & & 2.4 & & & 4.0 & & & 4.4 & & & 3.0 & & \\
\hline $\mathbf{f}_{\mathrm{t}} / \mathbf{f}_{\mathrm{c}}(\%)$ & & 8 & & & 11 & & & 12 & & & 10 & & \\
\hline
\end{tabular}

${ }^{a}$ Sample discarded because it failed in compression, i.e. not splitting 


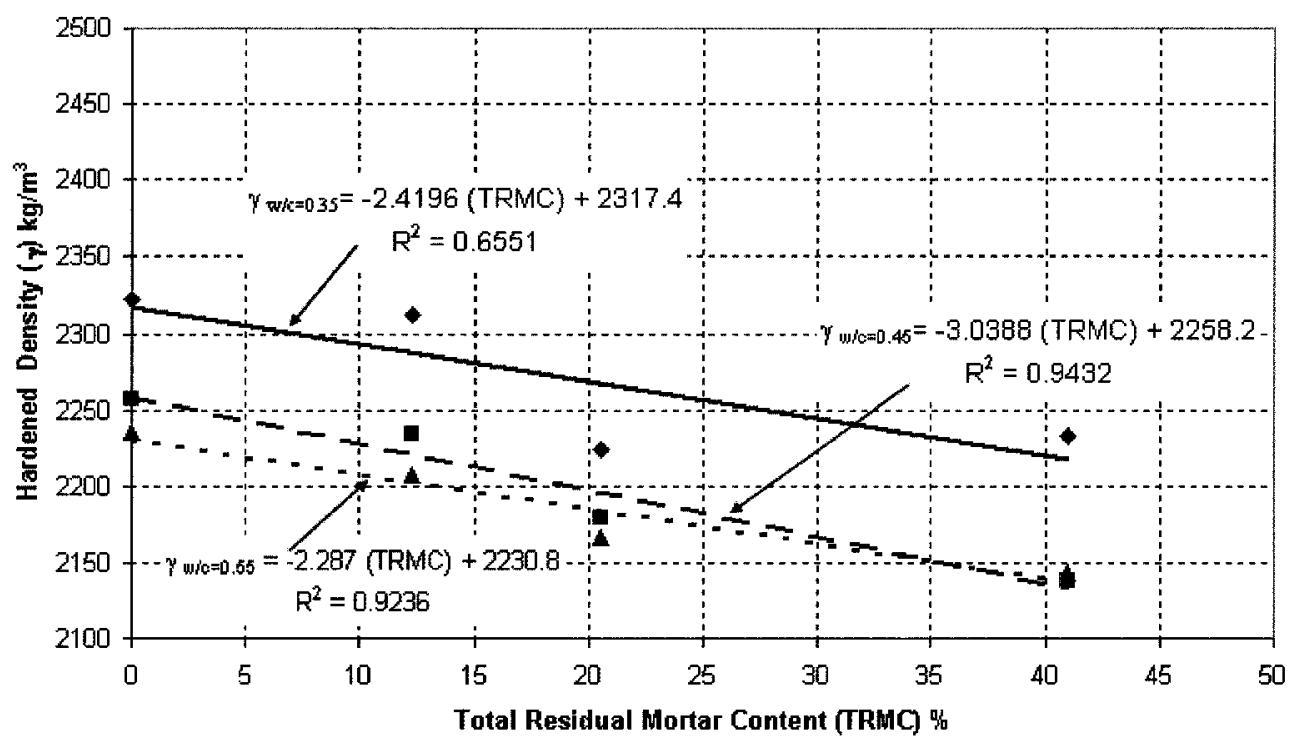

$-w / c=0.35 \mathrm{~m} w / c=0.45 \Delta w / c=0.55$

(a)GC-MO-AE mixes

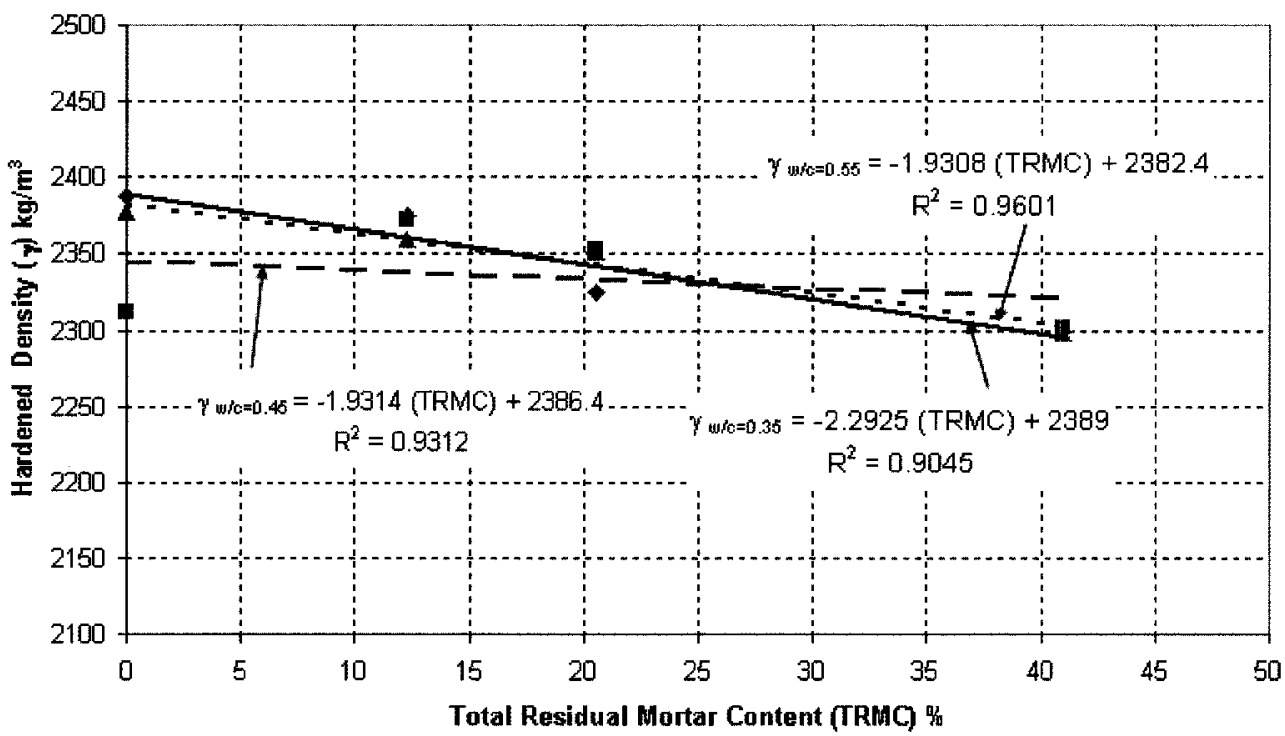

- $w / c=0.35 w / c=0.45 \wedge w / c=0.55$

(b) GC-MO-NE mixes

Figure 6.1: Variation of hardened density with TRMC of GC-MO mixes 


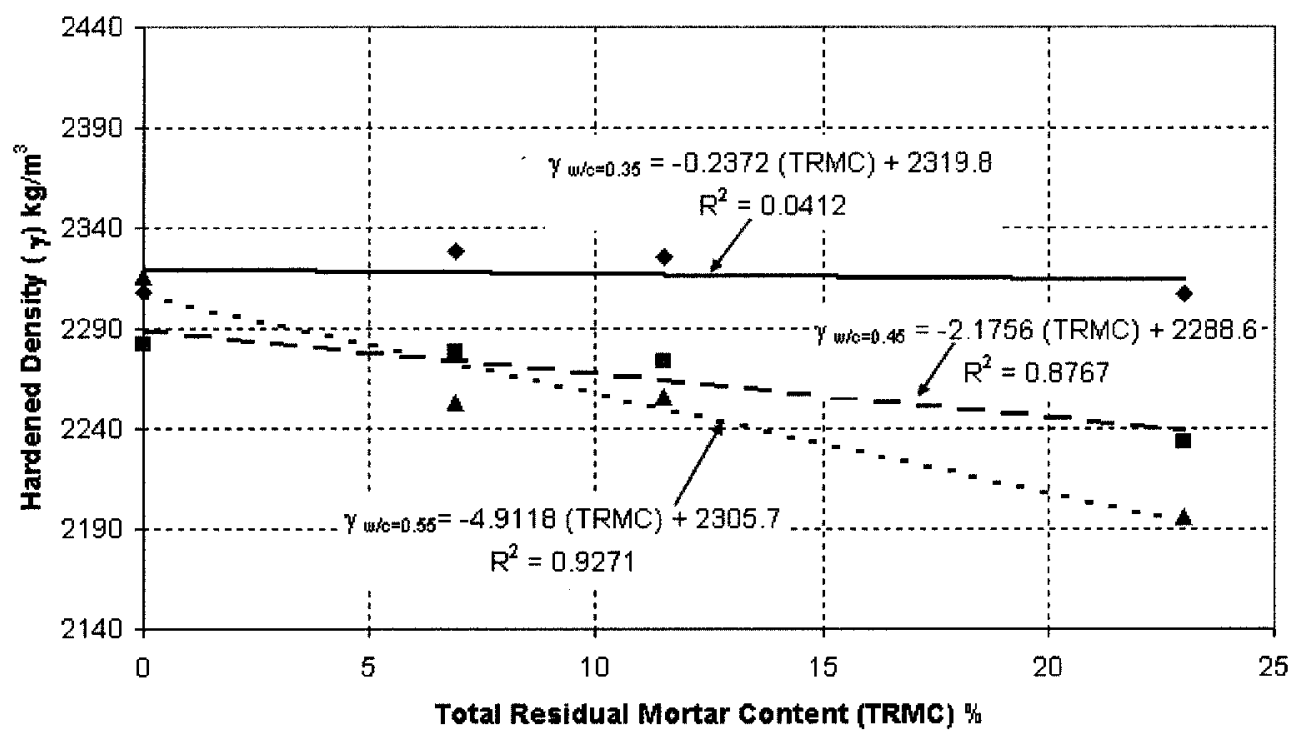

$+w / c=0.35 \square w / c=0.45 \wedge w / c=0.55$

(a) GC-VA-AE mixes

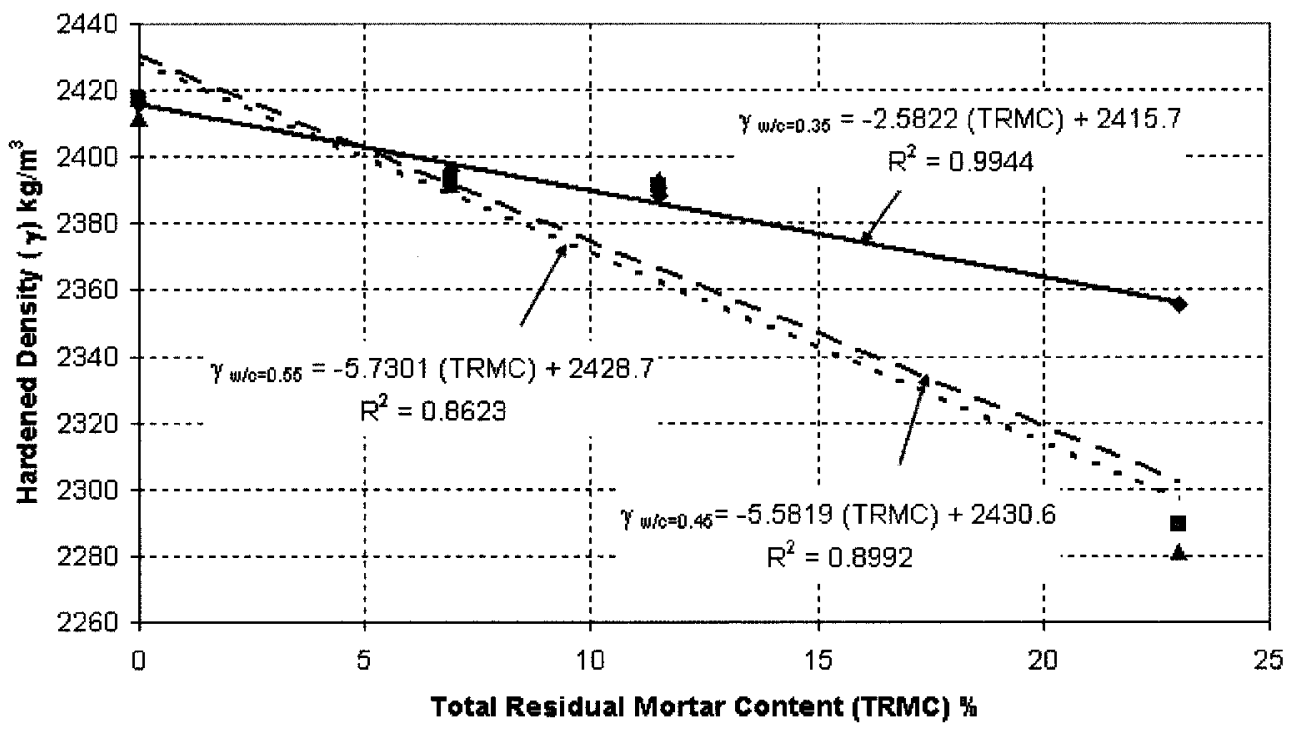

- $w / c=0.35 \square w / c=0.45 \wedge w / c=0.55$

(b) GC-VA-NE mixes

Figure 6.2: Variation of hardened density with TRMC of GC-VA mixes 


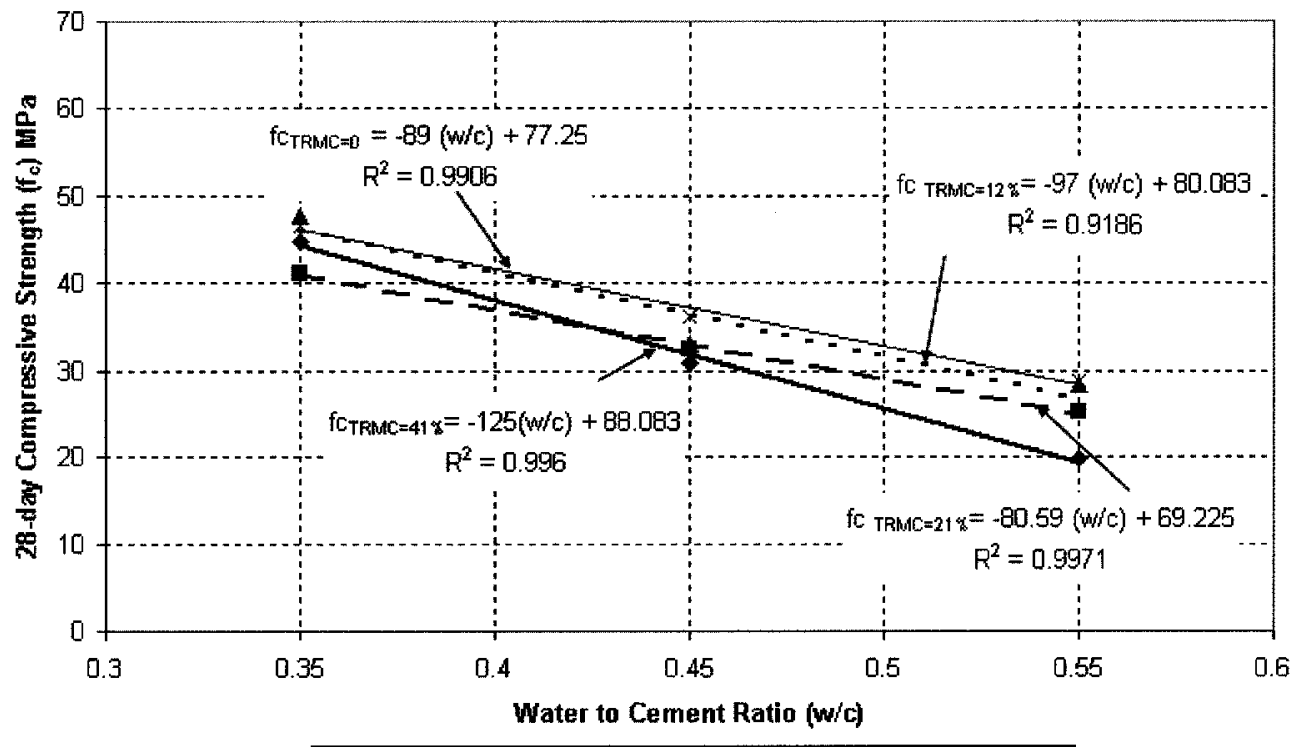

- $\mathrm{TRMC}=41 \%$ - TRMC $=21 \% \wedge \mathrm{TRMC}=12 \% \times \mathrm{TRMC}=0 \%$

(a) GC-MO-AE mixes

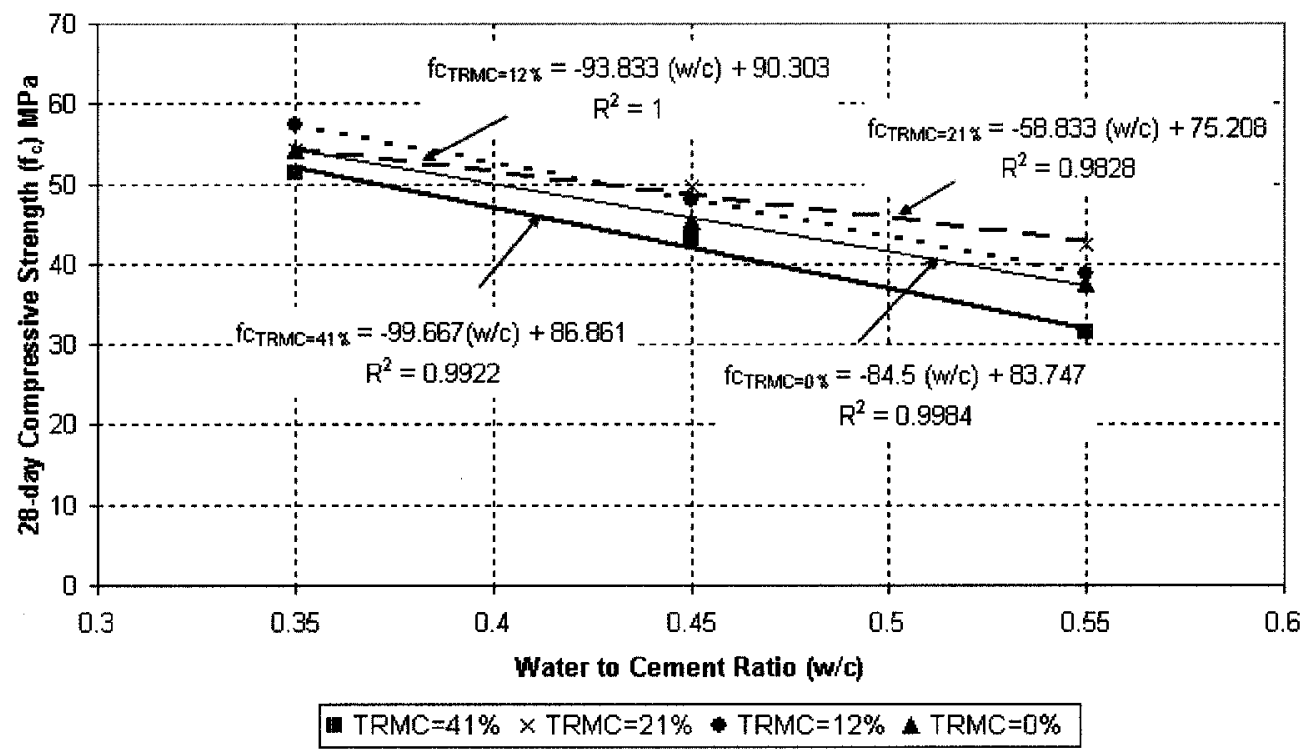

(b) GC-MO-NE mixes

Figure 6.3: Variation of compressive strength with the w/c ratio of GC-MO mixes 


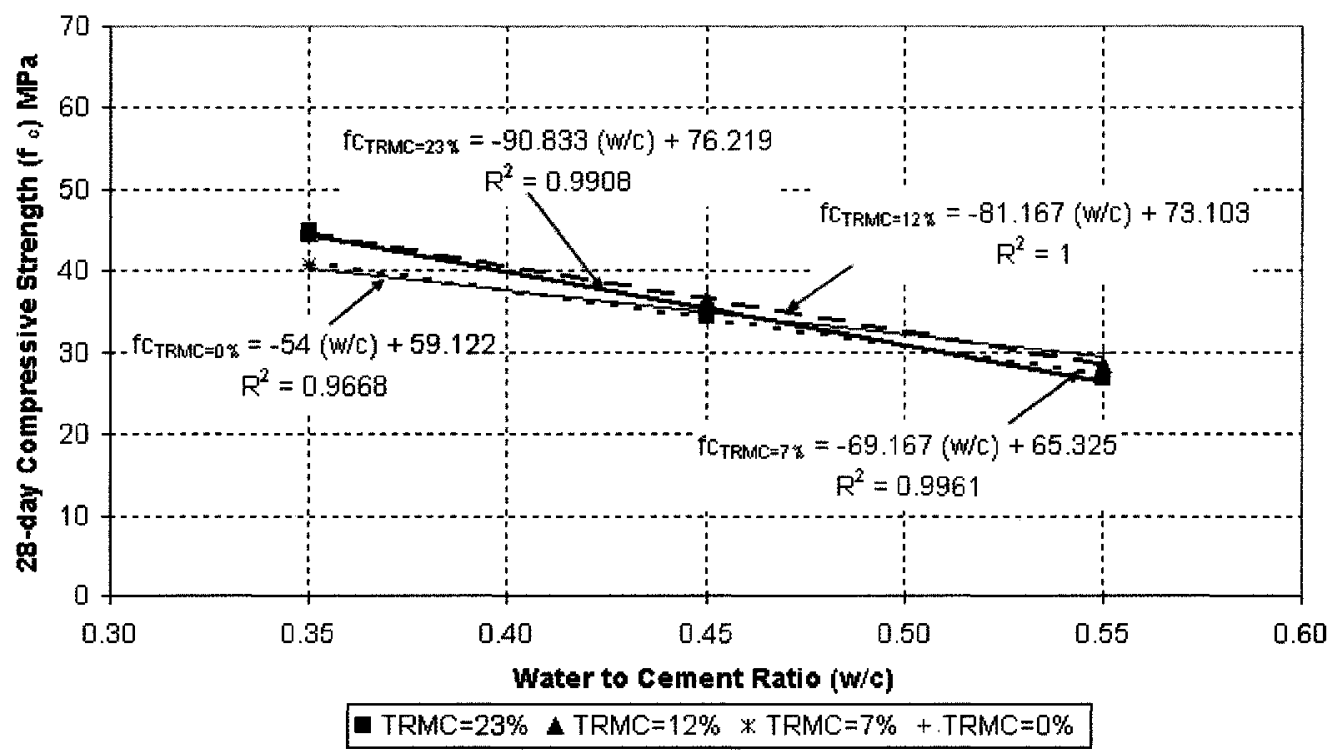

(a) GC-VA- AE mixes

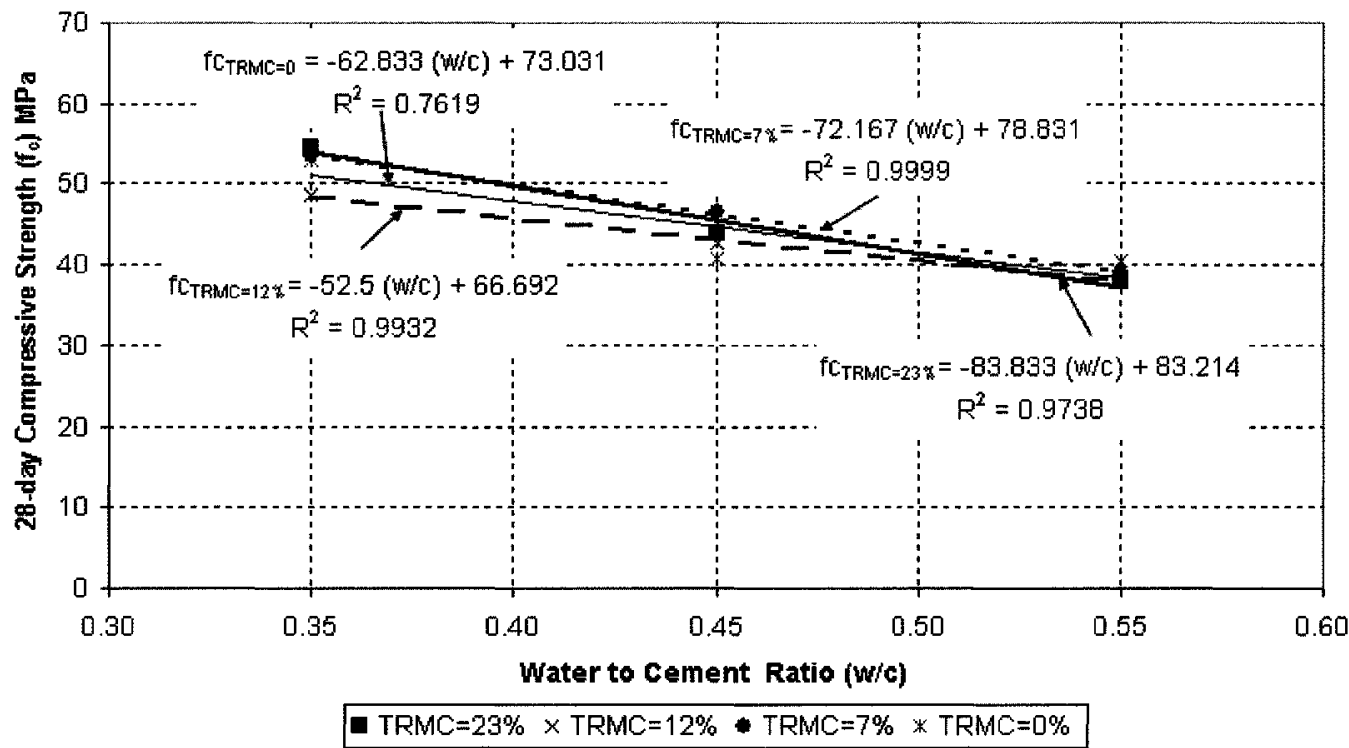

(b) GC-VA-NE mixes

Figure 6.4: Variation of compressive strength with the w/c ratio of GC-VA mixes 


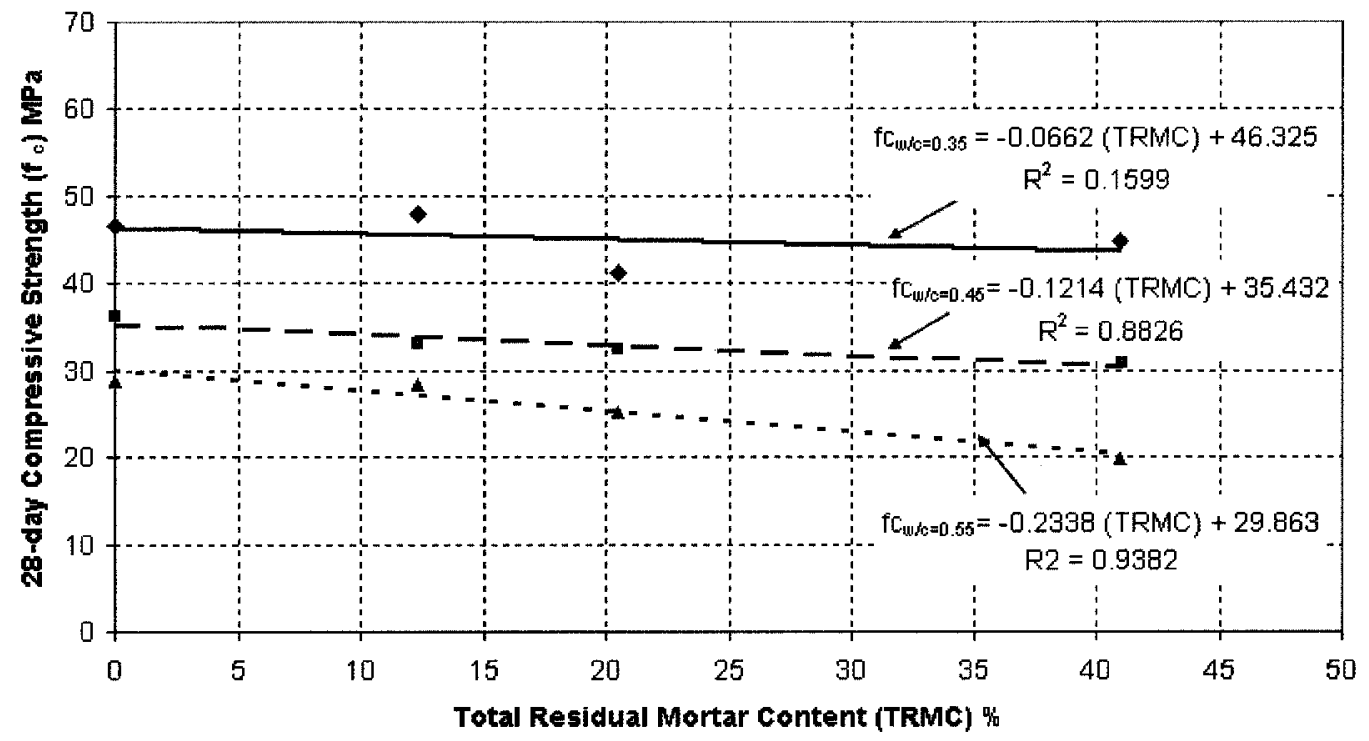

- $w / C=0.35 \mathrm{w} / \mathrm{C}=0.45 \propto w / \mathrm{c}=0.55$

(a) GC-MO-AE mixes

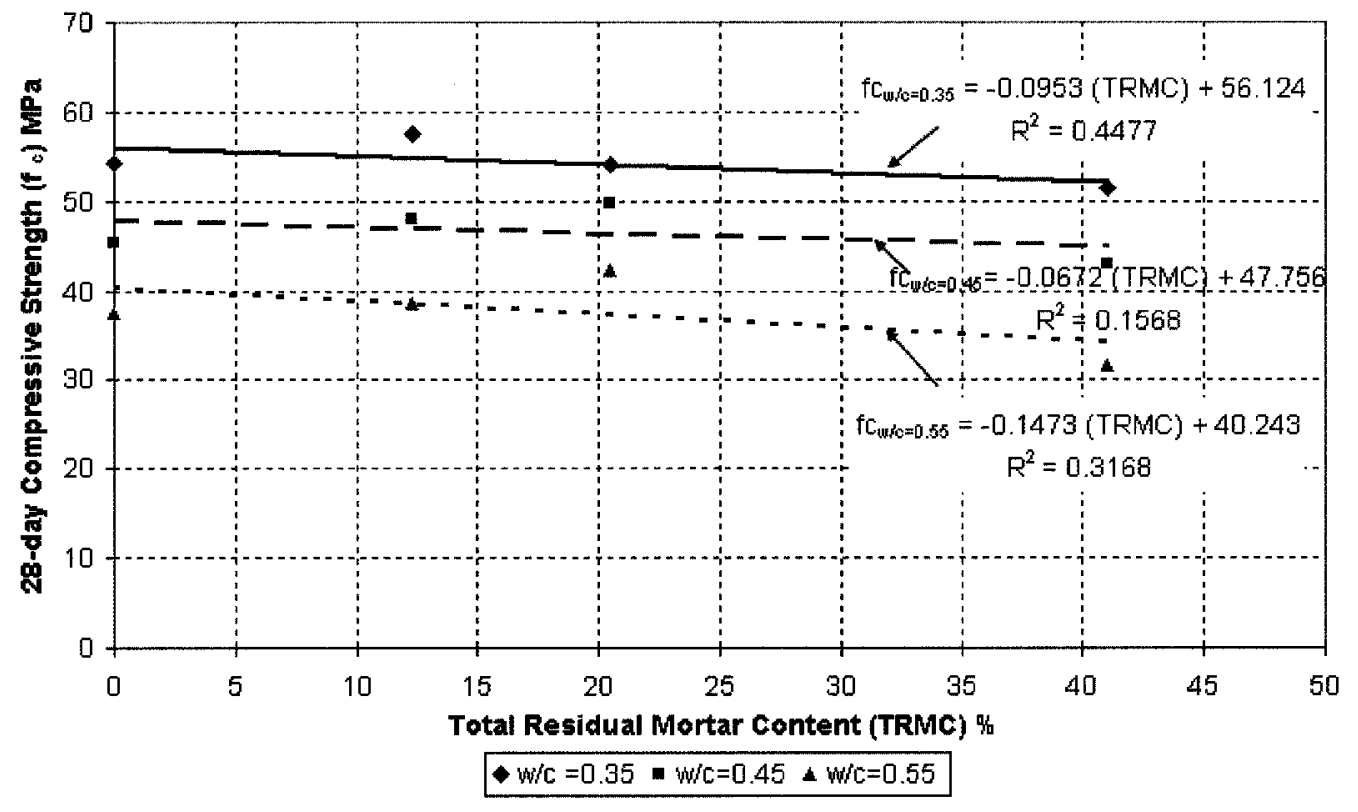

(b) GC-MO-NE mixes

Figure 6.5: Variation of 28-day compressive strength with TRMC of GC-MO mixes 


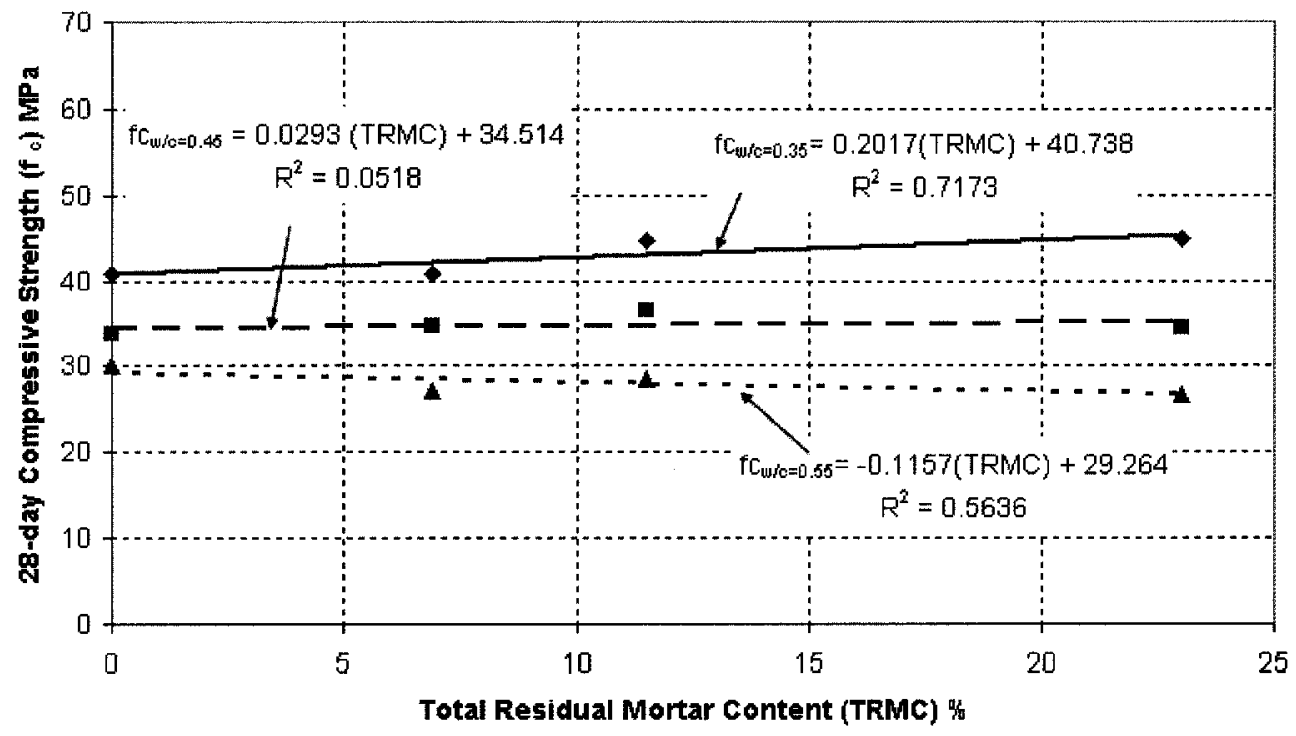

$-w / c=0.35 \quad w / c=0.45+w / c=0.55$

(a) GC-VA- AE mixes

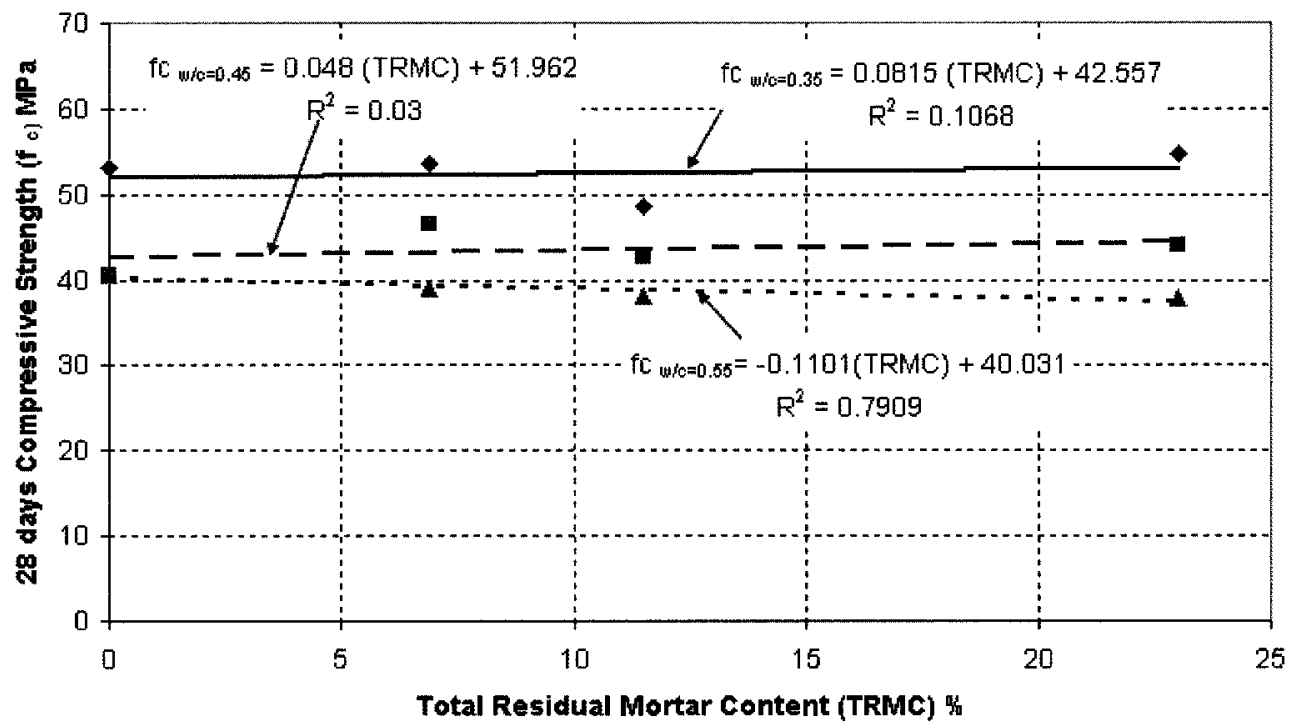

$-w / C=0.35 \quad w / C=0.45+w / C=0.55$

(b) GC-VA-NE mixes

Figure 6.6: Variation of 28-day compressive strength with TRMC of GC-VA mixes 


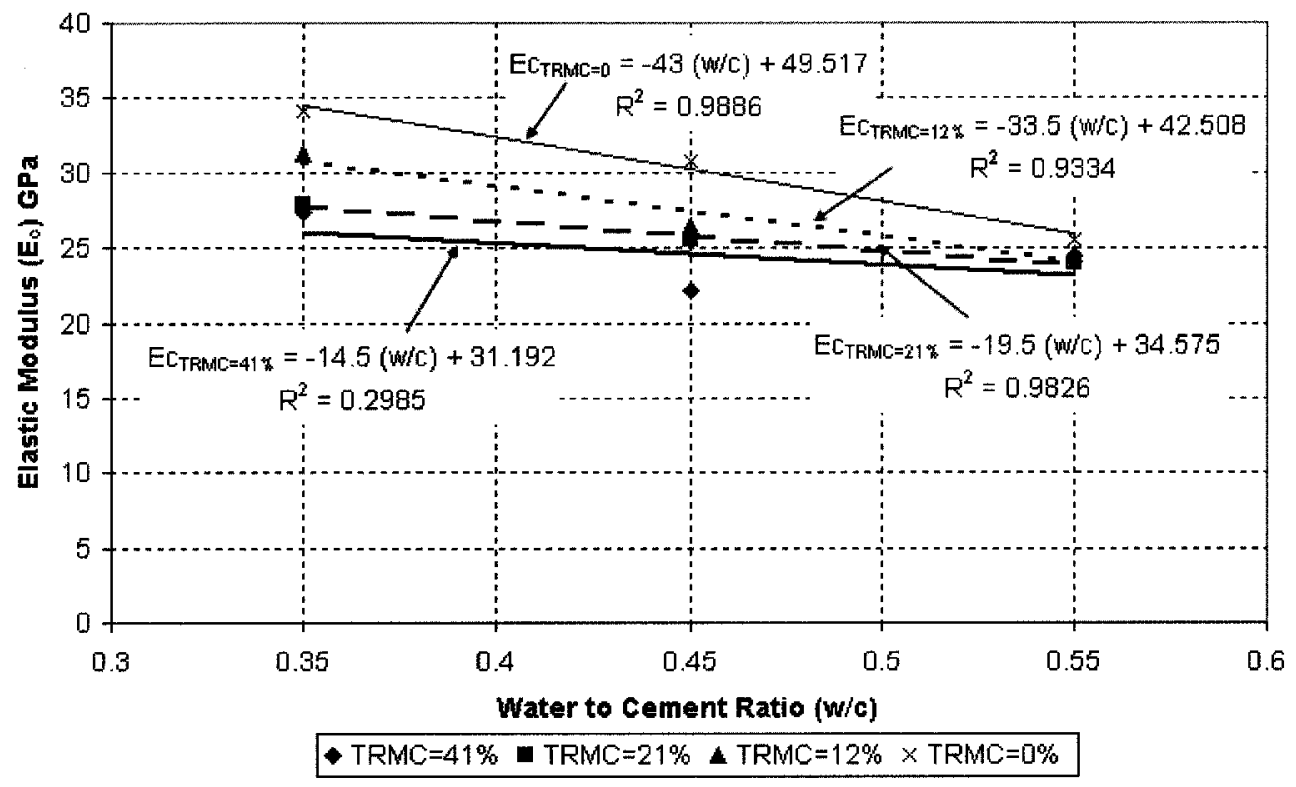

(a) GC-MO-AE mixes

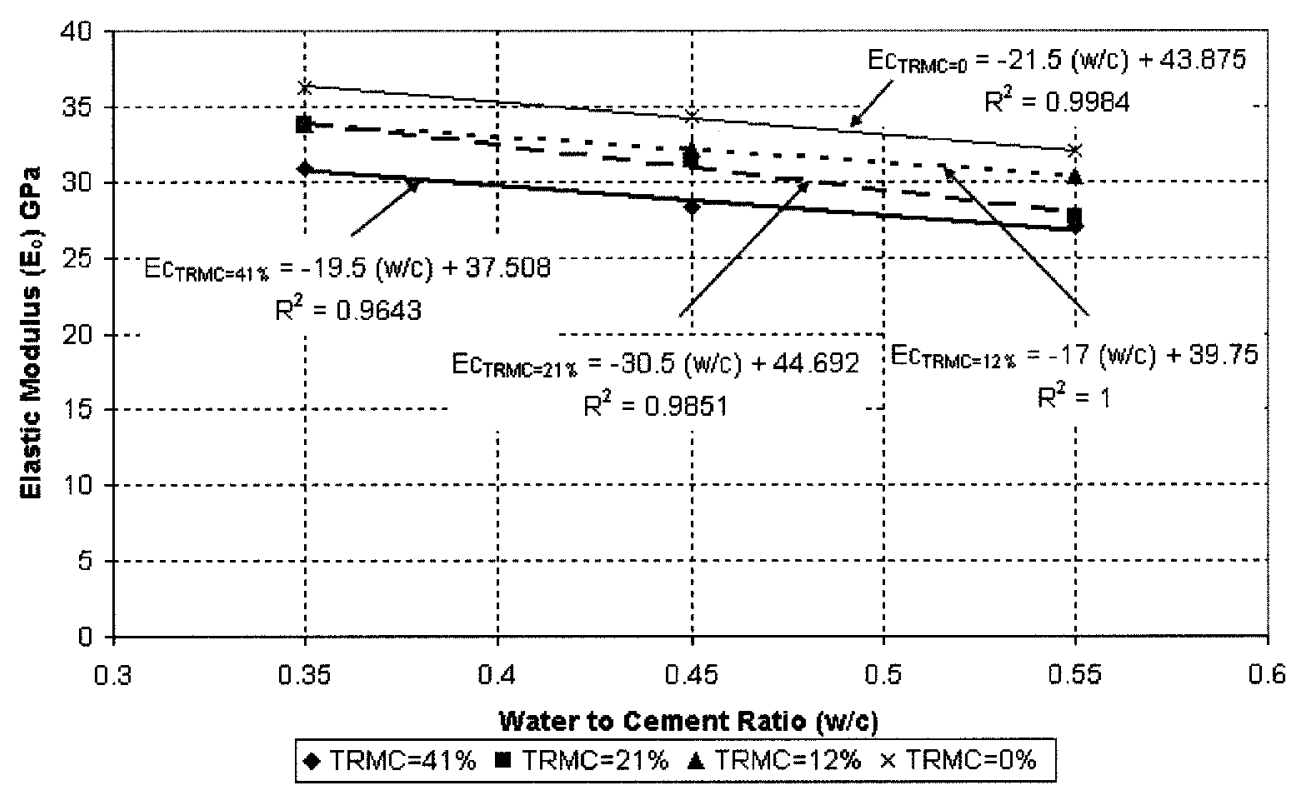

(b) GC-MO-NE mixes

Figure 6.7: Variation of elastic modulus with w/c ratio of GC-MO mixes 


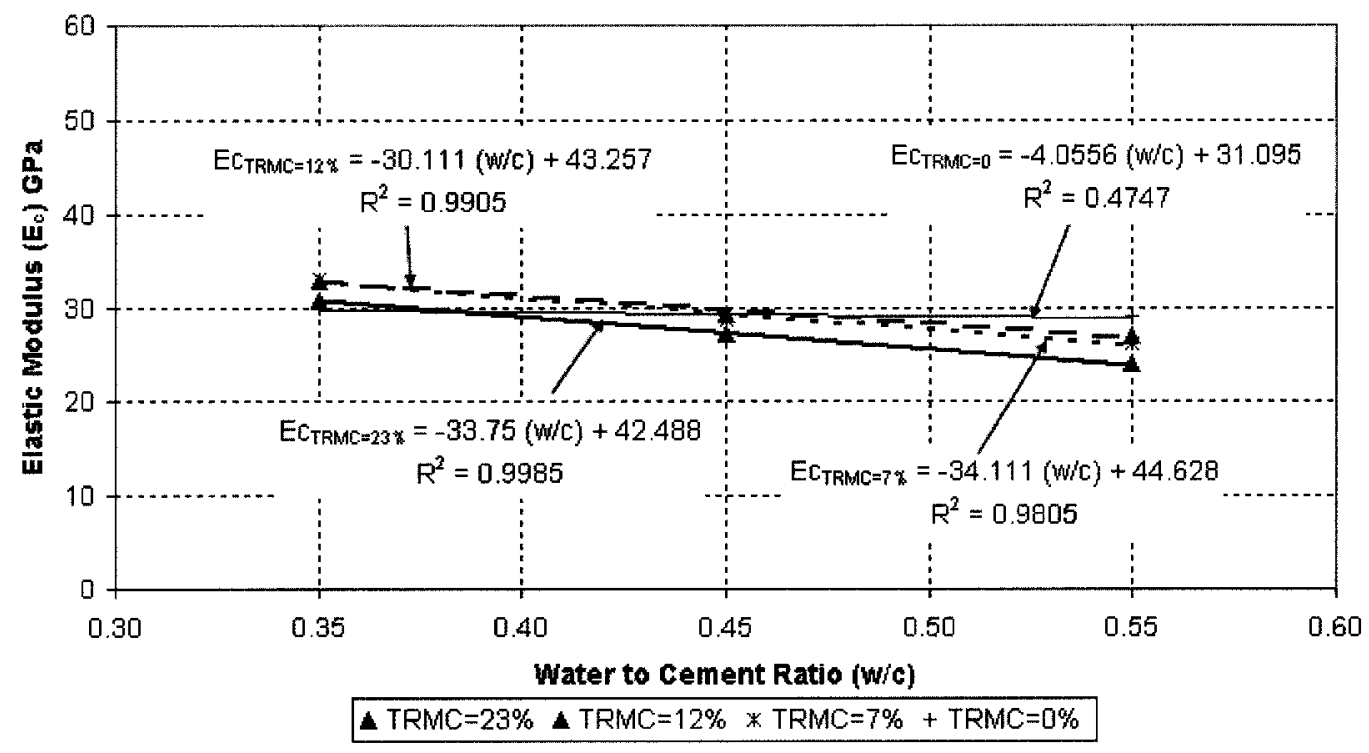

(a) GC-VA-AE mixes

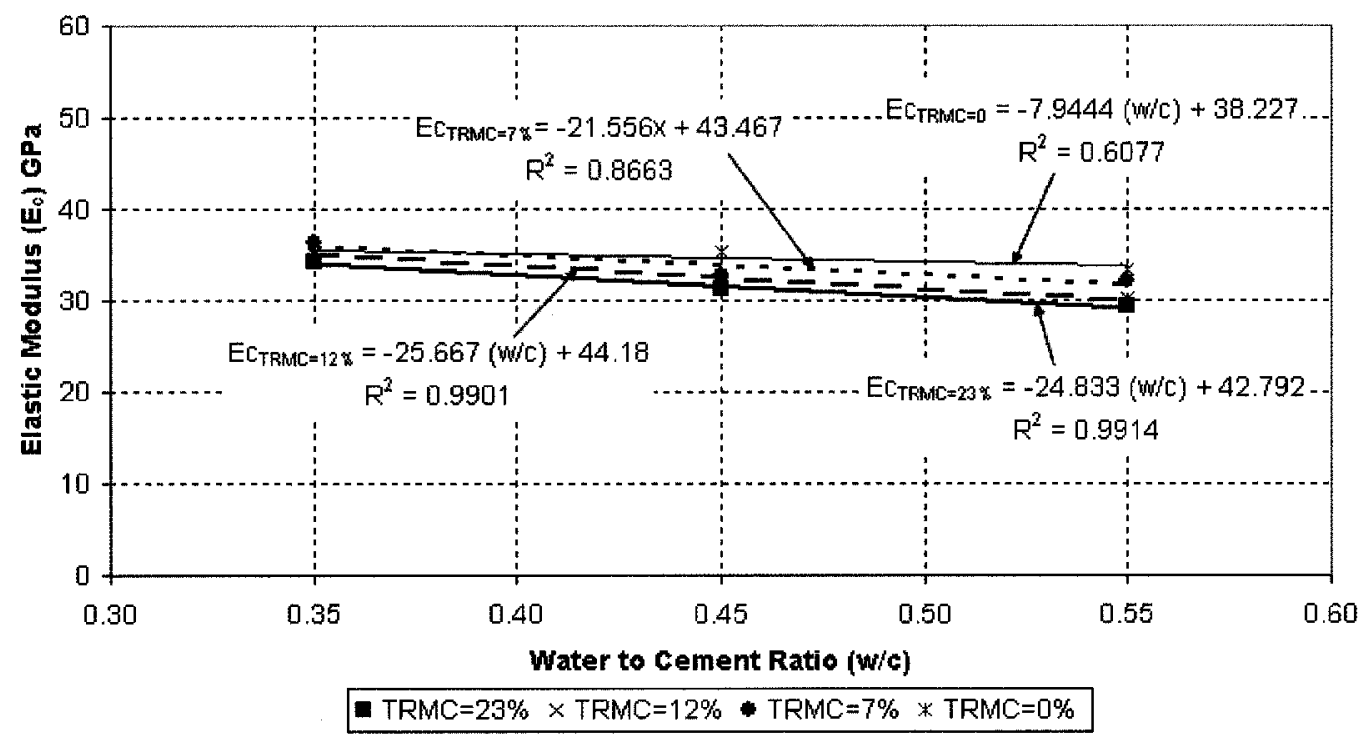

(b) GC-VA-NE mixes

Figure 6.8: Variation of elastic modulus with w/c ratio of GC-VA mixes 


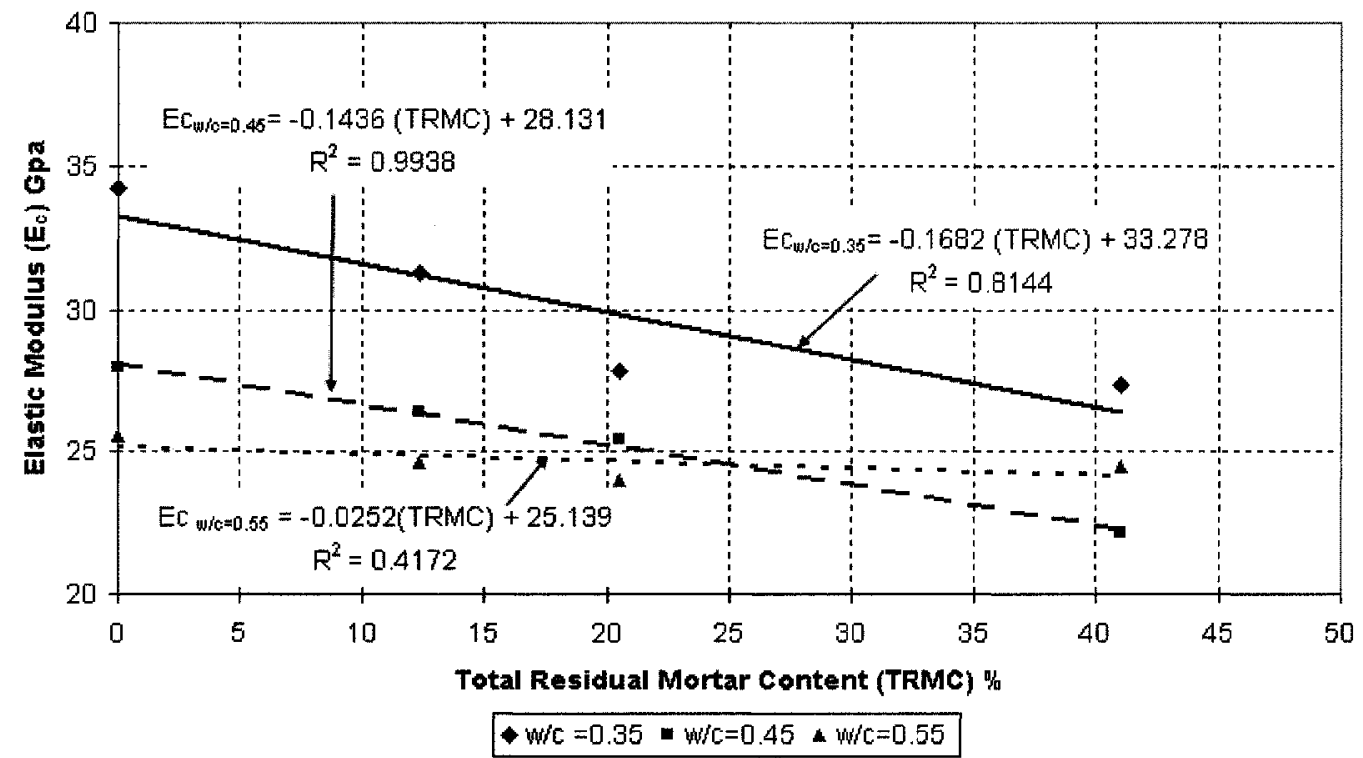

(a) GC-MO-AE mixes

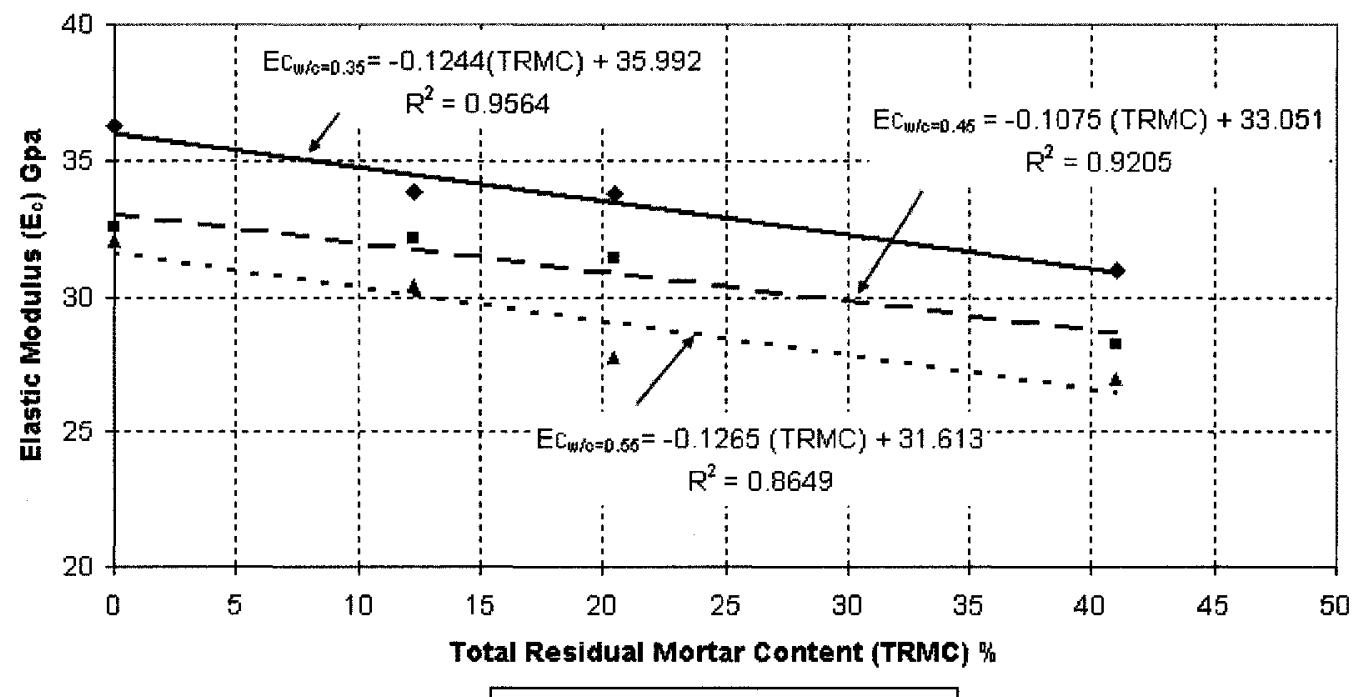

$\bullet w / c=0.35-w / c=0.45+w / c=0.55$

(b) GC-MO-NE mixes

Figure 6.9: Variation of elastic modulus with TRMC of GC-MO mixes 


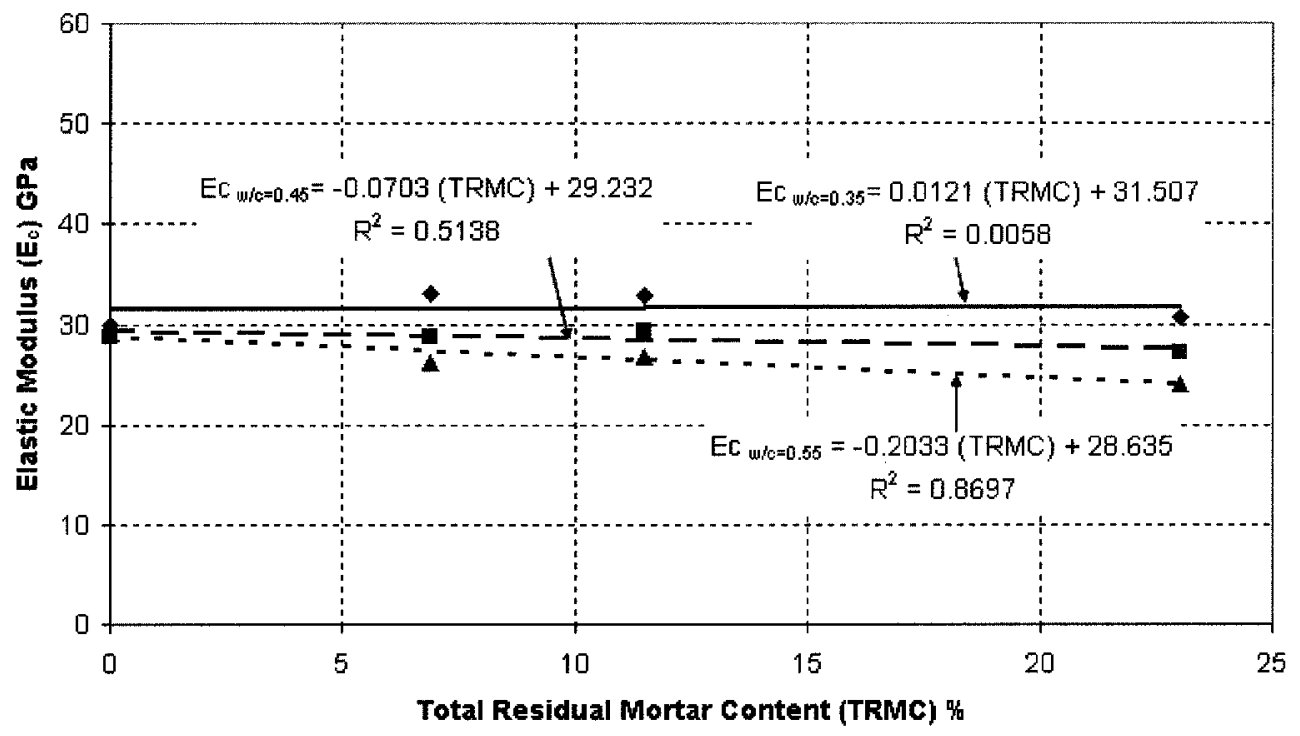

- $w / c=0.35 \square w / c=0.45 \wedge w / c=0.55$

(a) GC-VA-AE mixes

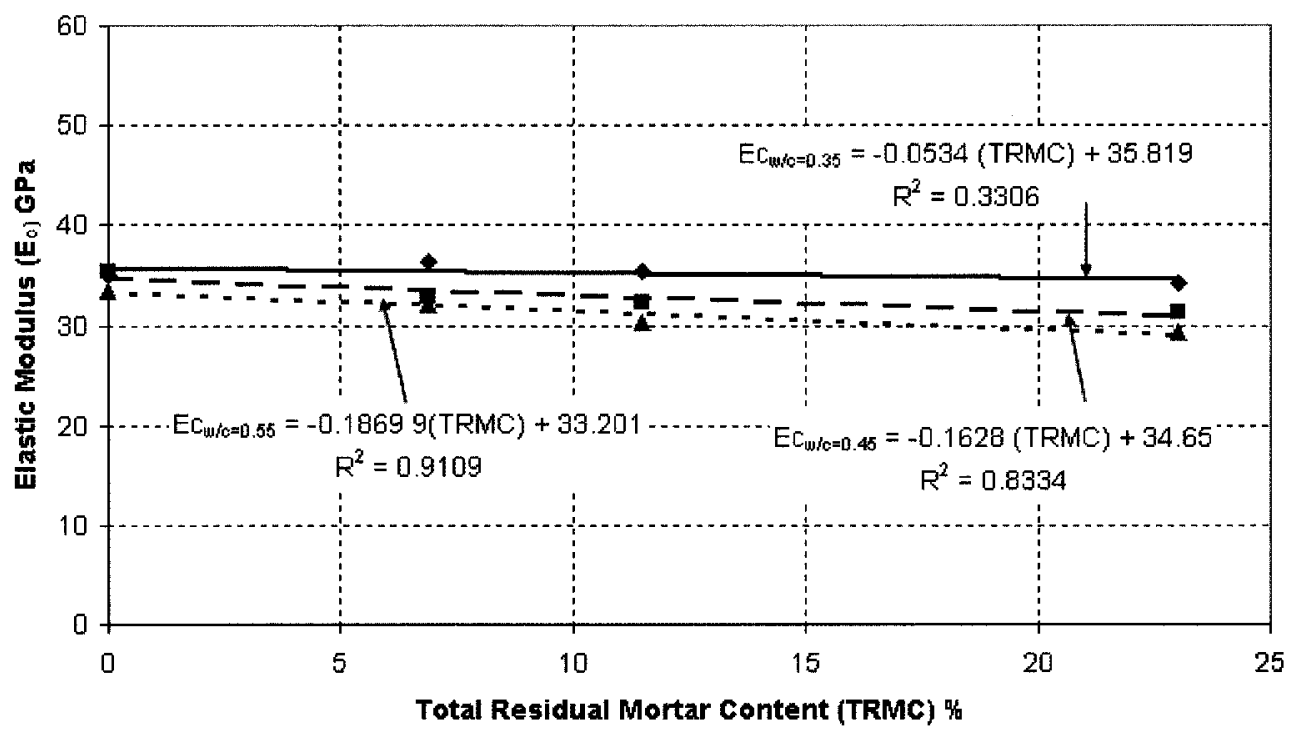

$\bullet w / c=0.35 \backsim w / c=0.45 \& w / c=0.55$

(b) GC-VA-NE mixes

Figure 6.10: Variation of elastic modulus with TRMC of GC-VA mixes 


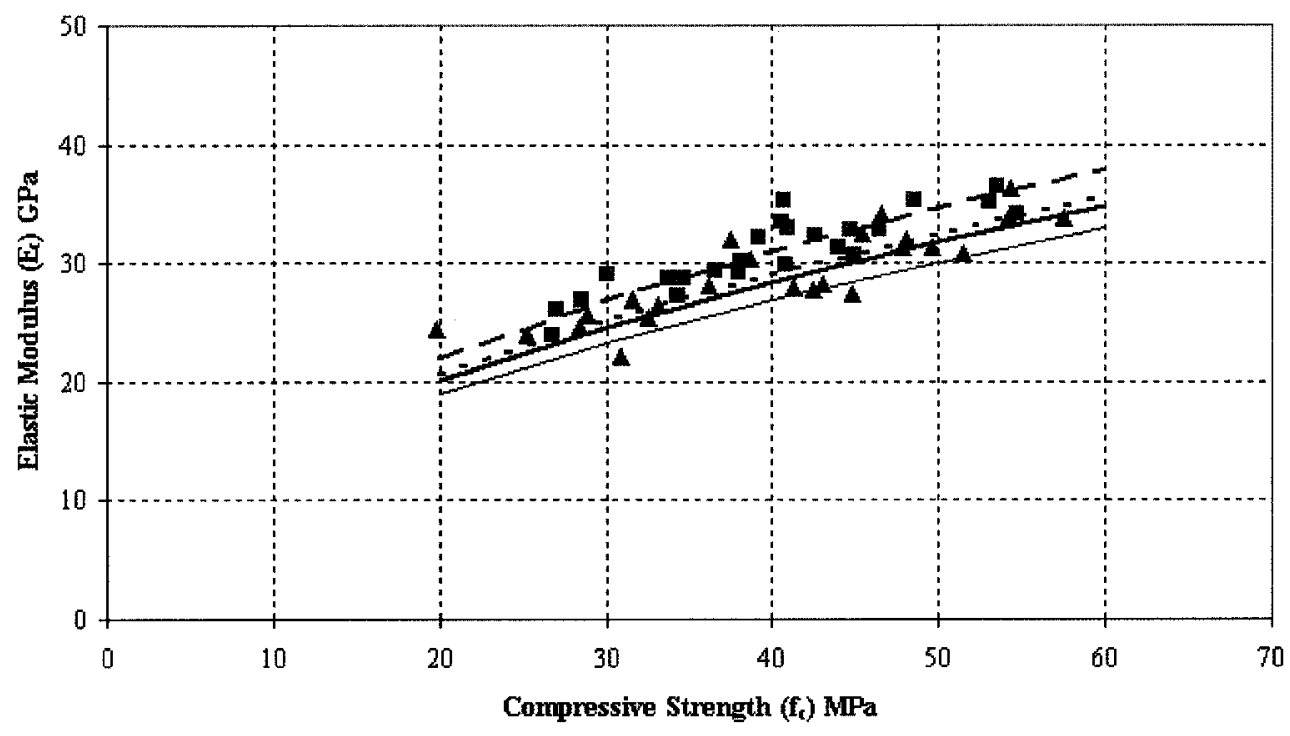

4 GC-MO (Exp) GC-VA (Exp.) C CSA - - GC-MO (Eq.) - -GC-VA (Eq.)-TRMC=41\%

Figure 6.11: Relationship between elastic modulus and compressive strength for GC-MO and GC-VA mixes. 


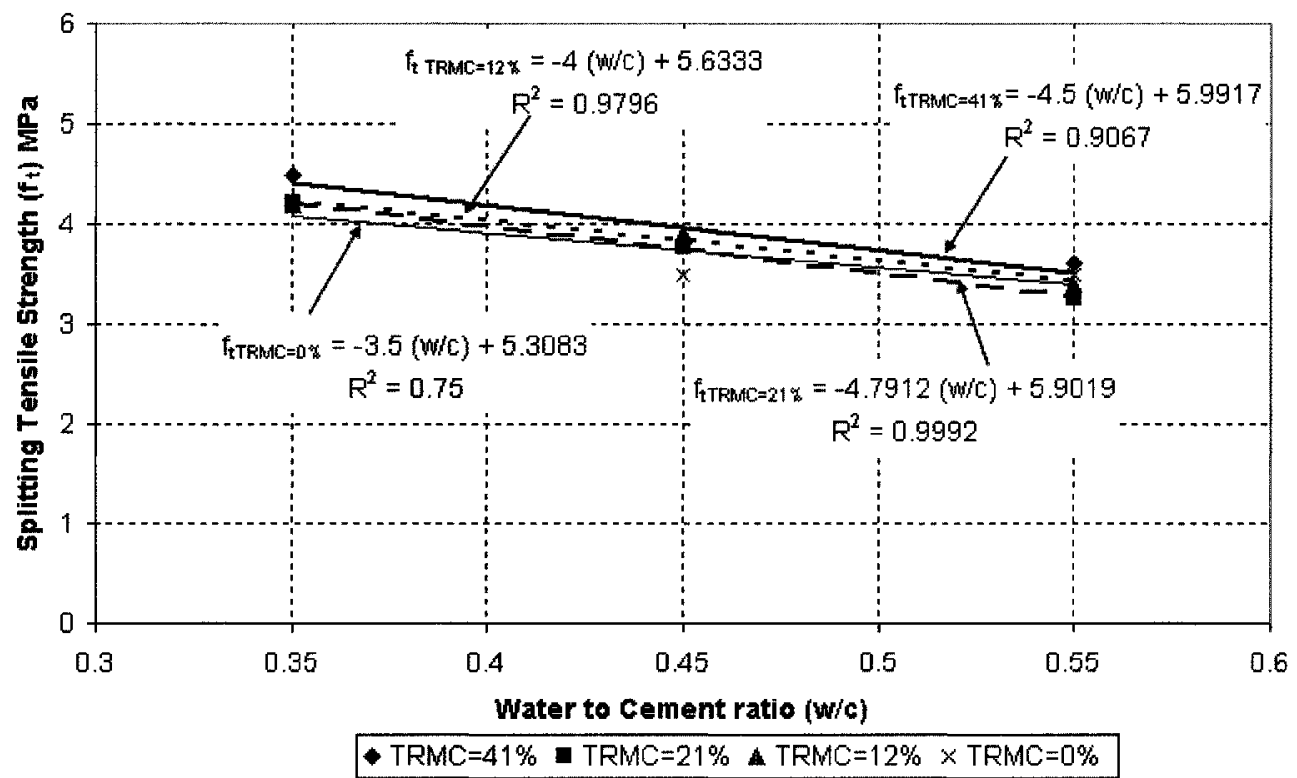

(a)GC-MO-AE mixes

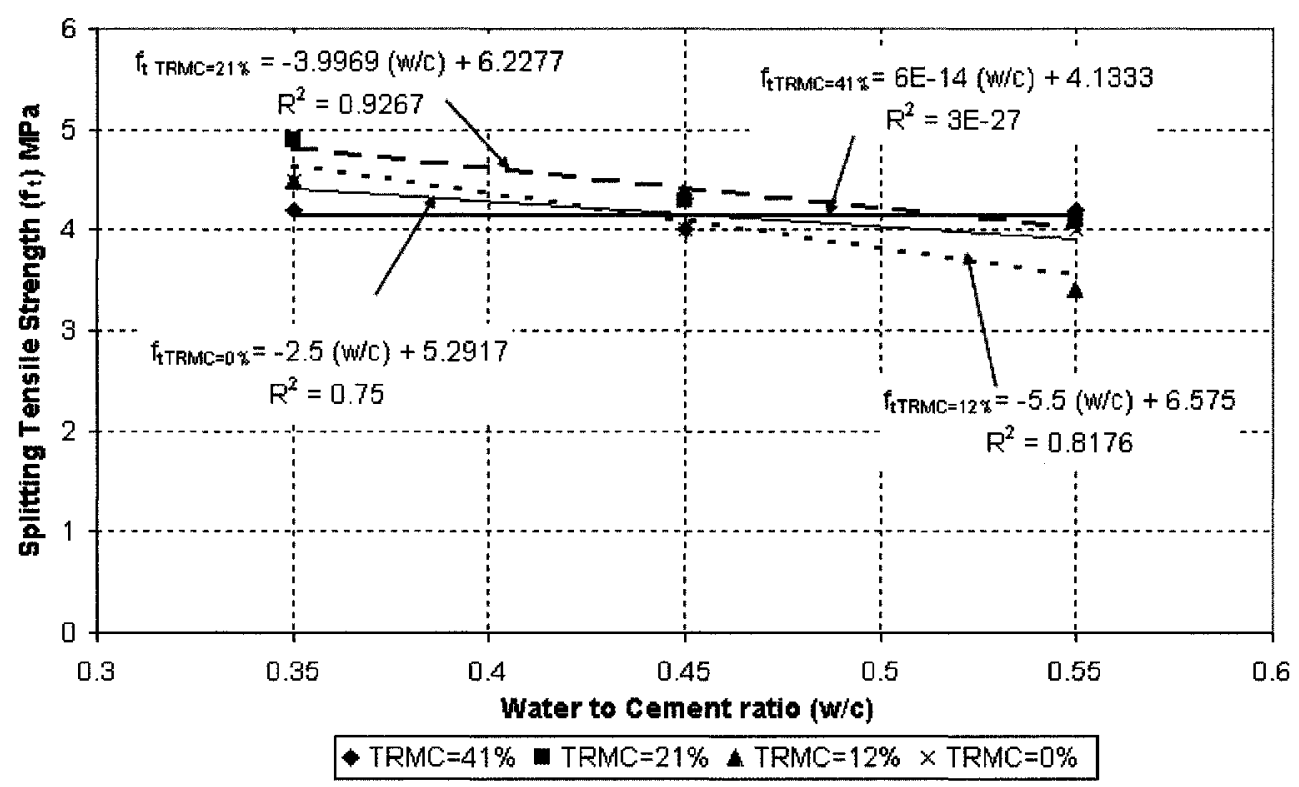

(b) GC-MO-NA mixes

Figure 6.12: Variation of splitting tensile strength with w/c ratio of GC-MO mixes 


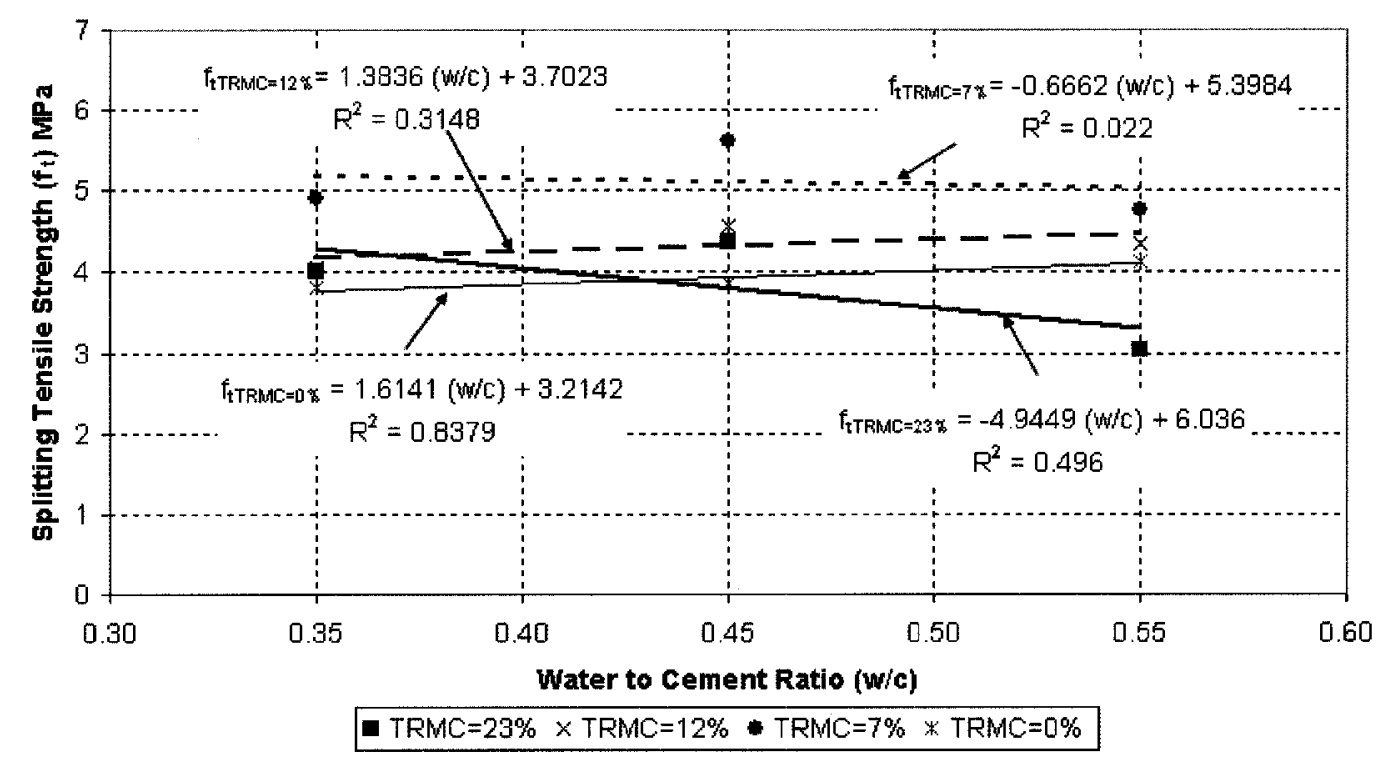

(a) GC-VA-AE mixes

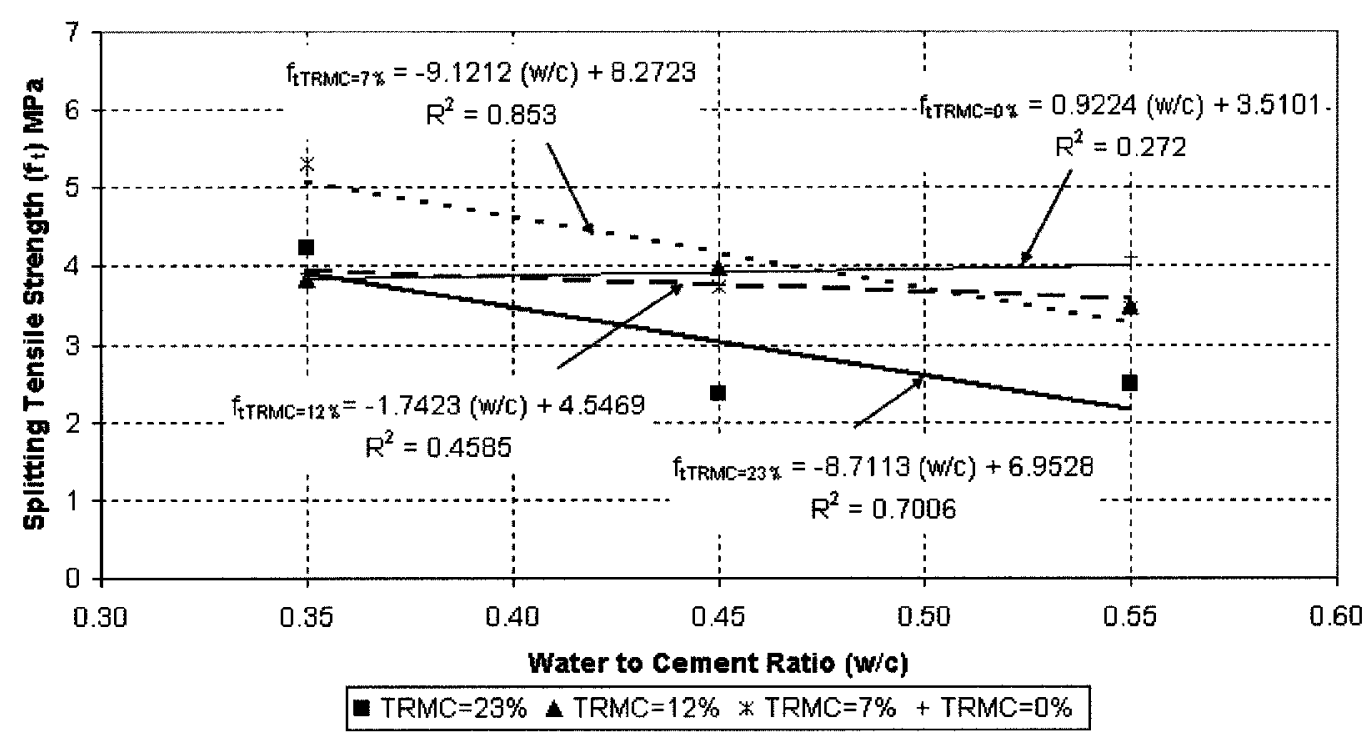

(b) GC-VA NE mixes

Figure 6.13: Variation of splitting tensile strength with w/c ratio of GC-VA mixes 


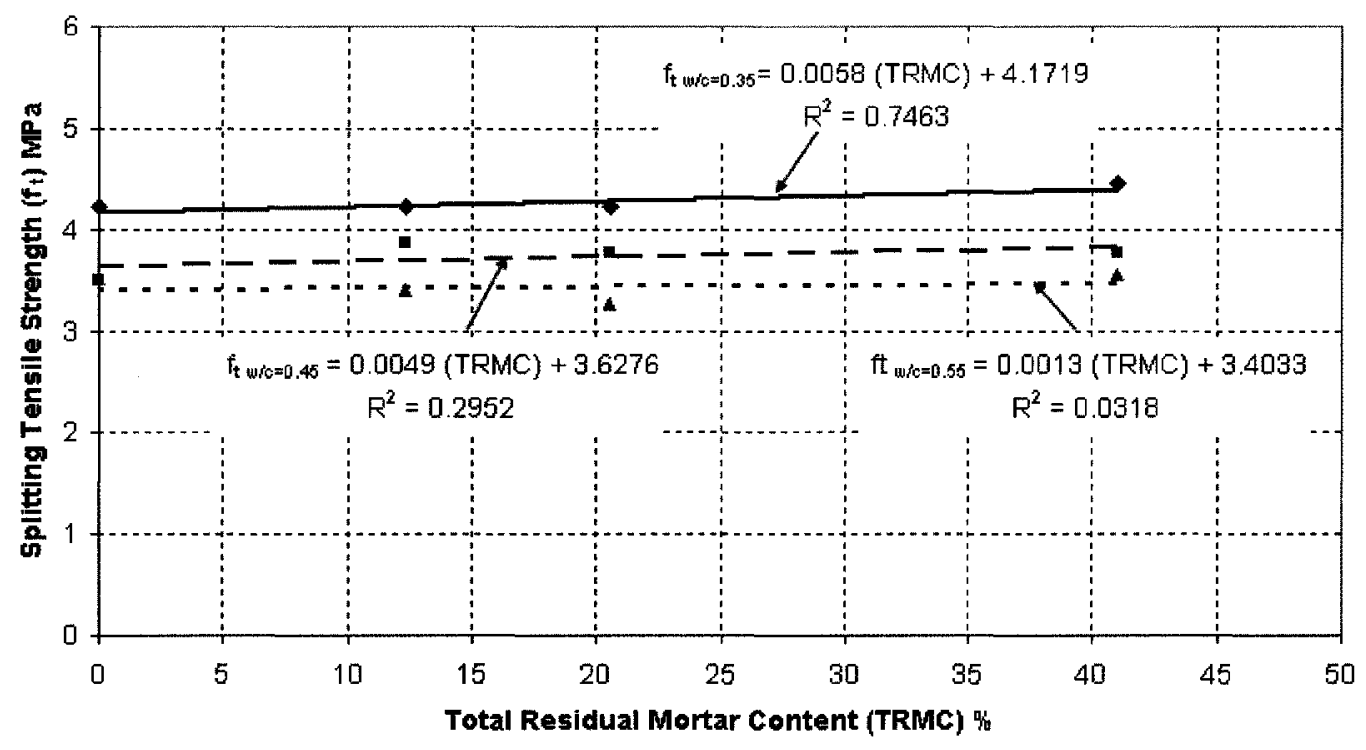

$\bullet w / c=0.35-w / c=0.45 \star w / c=0.55$

(a)GC-MO-AE mixes

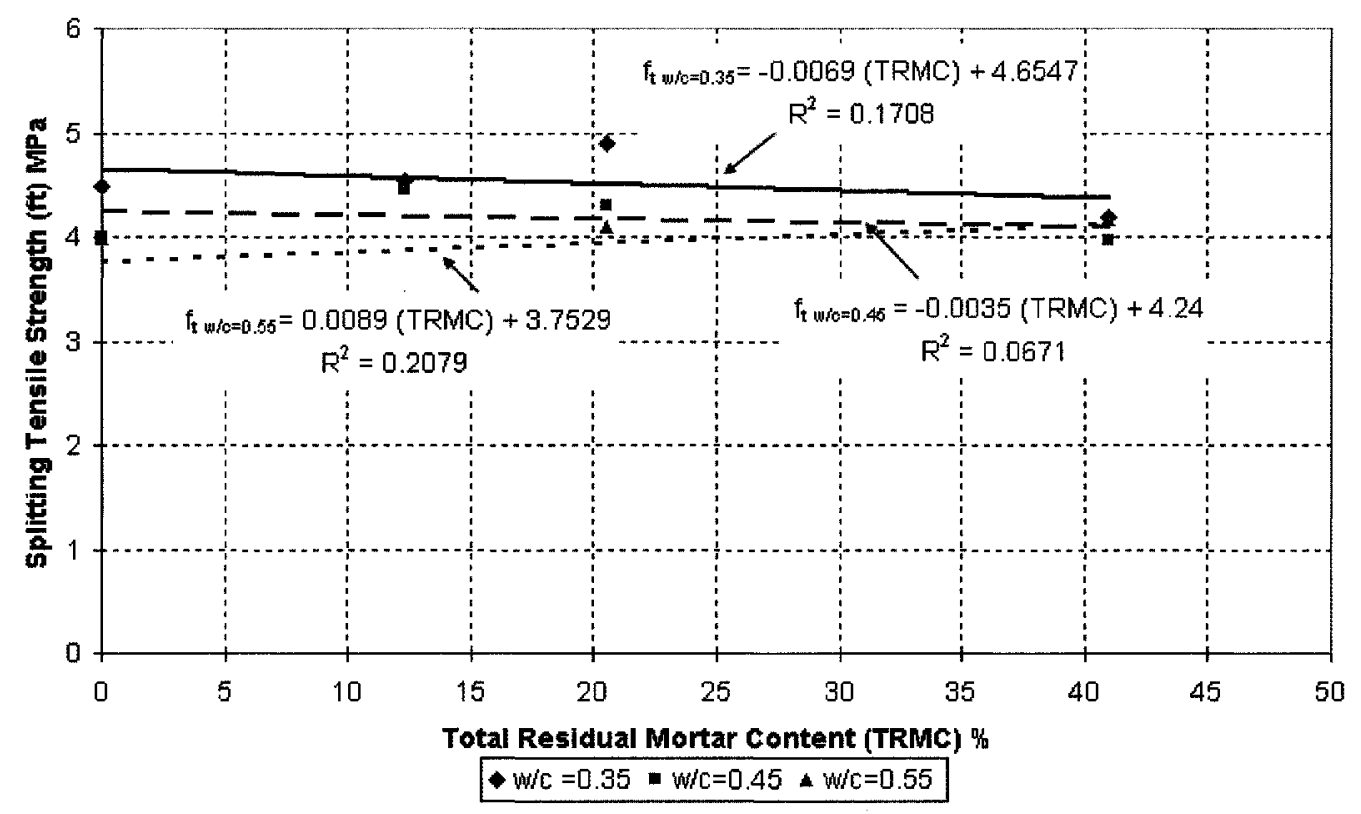

(b) GC-MO-NE mixes

Figure 6.14: Variation of splitting tensile strength with TRMC of GC-MO mixes 


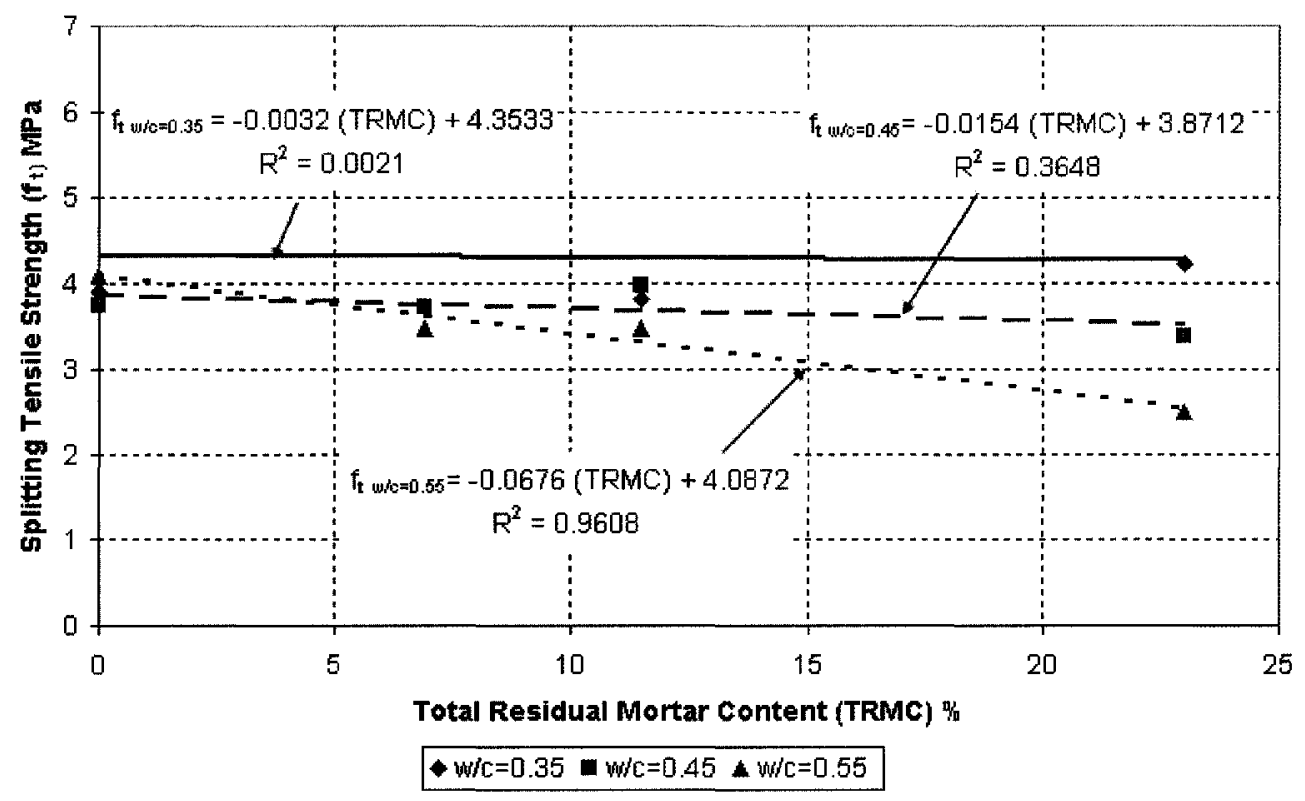

(a) GC-VA-AE mixes

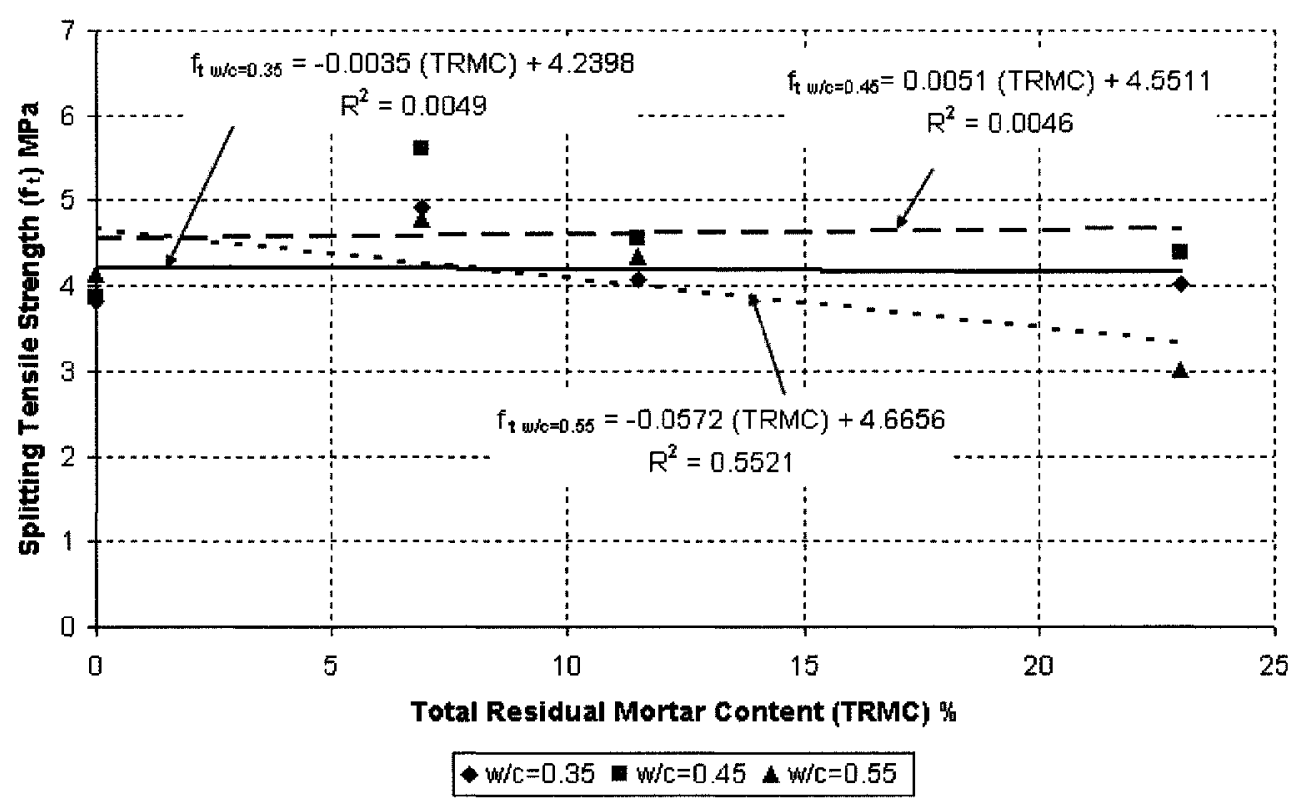

(b) GC-VA-NE mixes

Figure 6.15: Variation of splitting tensile strength with TRMC of GC-VA mixes 


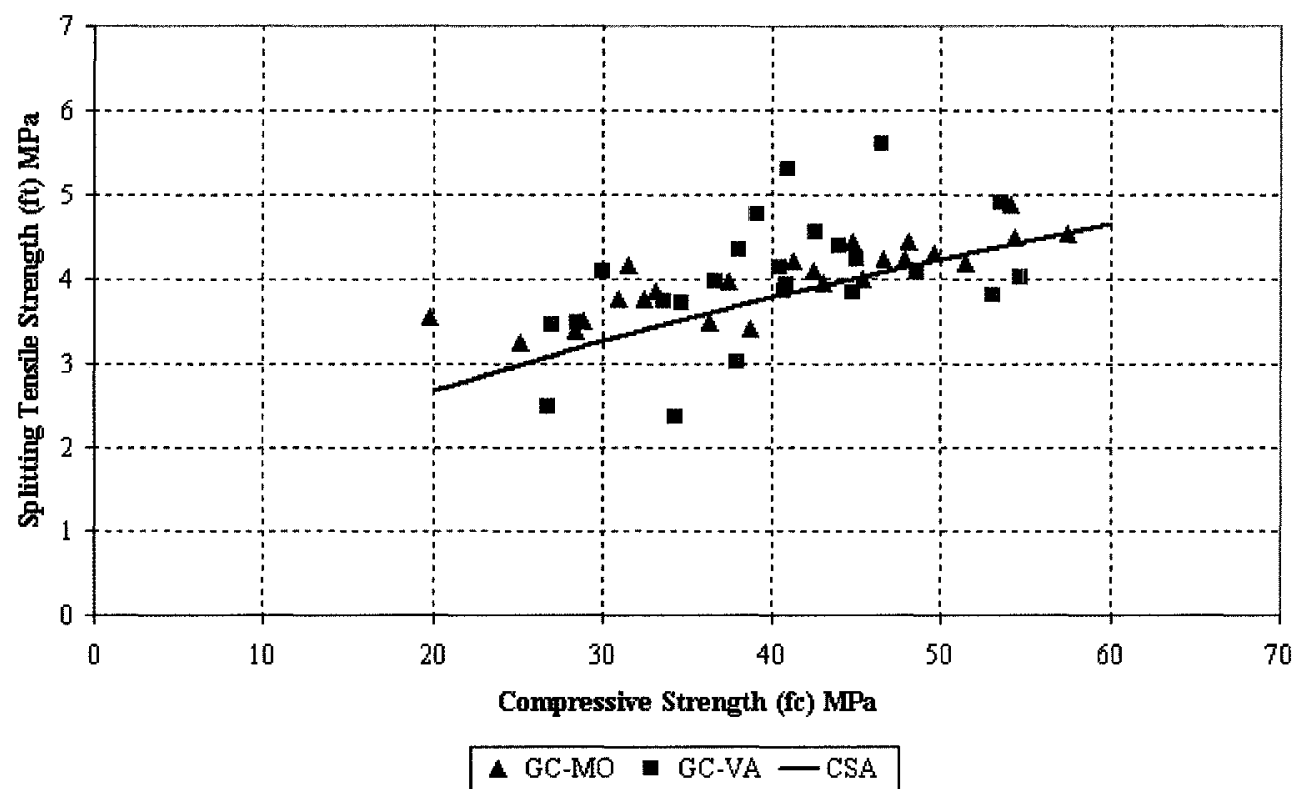

Figure 6.16: Relationship between compressive strength and splitting tensile strength for GC-MO and GC-VA mixes. 


\section{CHAPTER 7: MIX DESIGN OF GREEN CONCRETE}

\subsection{General}

In general, the mix design procedures for conventional concrete require the targeted slump and the compressive strength to be specified. In these procedures, the w/c ratio of the conventional concrete is obtained from the targeted compressive strength as the two are related by empirical relationships. The required slump is used to select the water content of the concrete mix. The amount of cement is calculated by dividing the water content by the w/c ratio. The quantities of the remaining constituents of the concrete, namely the fine and coarse aggregates, are determined by analytical procedures. Unfortunately, the existing mix design procedures for conventional concrete are not ideal for structural-grade GC since most relationships used in these methods (e.g. $f_{c}$ vs. w/c) are only valid for NA, not for RCA.

In this chapter, a general mix design method, which follows the similar steps of the existing conventional mix design procedures but developed specifically for structuralgrade GC, is presented. In addition to the general mix design procedure, an alternative mix design procedure developed by Fathifazl et al. (2007b), called "equivalent total volume of mortar method" is also presented. This new technique was developed based on the observations of the preliminary study presented in Chapters $4-6$, and considers the residual mortar as part of the total concrete mortar, not as part of the RCA.

Both methods that are presented in this chapter are used to produce the specimens that were tested for durability (see Chapter 8). 
Example of mix designs using both methods can be found in Appendix B.

\subsection{General Mix Design Method}

The general mix design method was developed using the experimental data obtained during the materials characterization of RCA presented in Chapter 3 and from the fresh and hardened properties of the preliminary study that is presented in Chapters 46. The method follows the well-known steps of the conventional mix design procedures and allows the designer to specify the required slump and compressive strength. In fact, conventional concrete mix design procedures (ACI 211.1-97) are the subset of the general mix design method presented here.

Figure 7.1 illustrates the basic steps of the general mix design procedure. The goal of the design approach is obvious: to obtain the required compressive strength and slump. Before the design, the following material properties of the constituents need to be previously determined:

- RMC of the RCA

- Specific gravity and absorption capacity of the RCA

- Specific gravity and absorption capacity of the NA (if used)

- Unit weight of the RCA

- Unit weight of the NA (if used)

- Fineness modulus of the fine aggregate

- Specific gravity of the cement

The design steps are explained in detail in the following sections. 


\subsubsection{Estimation of Water Content}

As it can be followed from the flowchart in Figure 7.1, the water content of the mix can be selected from the required slump, nominal maximum aggregate size and the air content using Table 4.1, which is the same table provided in ACI mix design method for conventional concrete. It has been demonstrated in Chapter 5 that as long as SSD condition are used (i.e., RCA were pre-soaked), Table 4.1 provides a good estimate for the initial trial batch.

\subsubsection{Selection of w/c Ratio and the Calculation of the Cement Content}

The w/c ratio is determined from the required compressive strength using Figure 7.2, which is plotted using Eqs.6.1 and 6.2 obtained in Chapter 6 of the preliminary study. This figure illustrates the relationship between the 28 -day compressive strength and the w/c ratio for air-entrained and non-air-entrained GC with varying TRMC values. Non-air entrained GC in these plots were assumed to be concrete containing no more than $2.2 \%$ air . Air-entrained concrete contains $5 \%$ to $8 \%$ of air. The air content is specified based on the preliminary study results of air content in Section 5.3.

The cement content is equal to the estimated mixing water content divided by the selected w/c ratio. The remaining constituents of the GC are the fine and coarse aggregate. The following step discusses the calculation of the fine and coarse aggregate contents. 


\subsubsection{Calculation of Aggregate Content}

To calculate the aggregate content of GC, two approaches are provided: (1) analytical approach; (2) practical approach. Analytical approach is based on solving the equations which originate from the ACI method as discussed in Chapter 4 (Section 4.2). The practical approach is based on the selection of fine-to-total aggregate ratio and total aggregate-to-cement ratio.

\subsubsection{Analytical Approach}

The aggregate content of the GC trial batch can be calculated analytically as follows:

(1) Estimate the fresh concrete density, $U$, using Eq. 7.1a or Eq.7.1b for air-entrained and non-air-entrained concrete, respectively.

$$
\begin{aligned}
& U=70.4\left(\frac{1}{\left(\frac{w}{c}\right)}\right)-2.9(\text { TRMC })+2154.2 \\
& U=12.4\left(\frac{1}{\left(\frac{w}{c}\right)}\right)-2.3(\text { TRMC })+2394.6
\end{aligned}
$$

These equations were developed in the preliminary study and are independent of the source of the coarse aggregates as long as the SSD specific gravities of the coarse aggregates (RCA and NA) are larger than 2.4 and the water content is calculated as per section 7.2.1. Alternatively, the traditional ACI method to estimate the fresh concrete density can also be used from: 


$$
U=10 S G_{S S D, a v}(100-A)+W_{C}^{G C}\left(1-\frac{S G_{S S D, a v}}{S G^{c}}\right)-W_{W}^{G C}\left(S G_{S S D, a v}-1\right)
$$

where $\mathrm{SG}_{\mathrm{SSD} \text {,av }}$ is the weighted average $\mathrm{SSD}$ specific gravity of combined fine and coarse aggregate (RCA and NA), $A$ is the air content, $S G^{C}$ is the specific gravity of cement, $W_{C}^{G C}$ is the weight of required cement in $\mathrm{GC}\left(\mathrm{kg} / \mathrm{m}^{3}\right)$, and $W_{W}^{G C}$ is the weight of mixing water $\left(\mathrm{kg} / \mathrm{m}^{3}\right)$.

(2) Calculate RCA weight in SSD condition using Eq. 7.2:

$$
W_{S S D-R C A}^{G C}=\frac{V_{a}\left[W_{C}^{G C}\left(\frac{1}{S G^{C}}-\frac{1}{S G_{S S D}^{F A}}\right)+\frac{\left(U-W_{W}^{G C}\right)}{S G_{S S D}^{F A}}+W_{W}^{G C}\right]}{\left[\frac{1}{S G_{S S D}^{R C A}}-\left(\frac{1}{S G_{S S D}^{R C A}}-\frac{1}{S G_{S S D}^{F A}}\right) \times V_{a}\right]}
$$

where $V_{a}$ is the ratio of the compacted or absolute volume of coarse aggregate to the absolute volume of concrete which can be calculated as follows:

$$
V_{a}=\left(\frac{M}{1000 \cdot S G_{b}^{R C A}}\right) \times v_{R C A}^{C C}
$$

where $M$ is the unit weight of OD-rodded coarse aggregate and $v_{R C A}^{G C}$ is the volume of dry-rodded RCA per unit volume of GC. $v_{R C A}^{G C}$ can be obtained from Table 7.1 given that the fineness modulus of the fine aggregate and the nominal maximum size and of the RCA are known. In the case of blended aggregate (i.e., partial replacement of RCA by NA), the weighted average unit weight, weighted average specific gravity, weighted average absorption capacity, and TRMC values of the aggregate mix should be calculated and the same procedure as per items (1) and (2) should be followed.

(3) Calculate the natural fine aggregate weight ( $W_{S S D-F A}^{G C}$ ) by using: 


$$
W_{S S D-F A}^{G C}=U-W_{C}^{G C}-W_{S S D-R C A}^{G C}-W_{W}^{G C}
$$

The alterative method to calculate the aggregate content by using practical approach is explained as follows.

\subsubsection{Practical Approach}

In the practical approach, the aggregate content is calculated based on the selection of fine-to-total aggregate ratio and aggregate-to-cement ratio.

Selection of the aggregate-to-cement ratio: The selection of the tota aggregate-tocement ratio $\left(A_{d} / C\right)$ depends on the $w / c$ ratio or concrete grade required for the specific job. Table 7.2, which was obtained in Chapter 4 , shows the approximate $A_{c} / C$ ratio and fine-to- total aggregate ratio by weight for air-entrained and non-air entrained concrete. When the $A_{c} / C$ ratio is selected from the required w/c ratio, and the cement content is found as per Section 7.2.2, the total aggregate content can be calculated as the cement content multiplied by the $A_{d} / C$ ratio.

Selection of the Fine-to-Total Aggregate Ratio: By knowing the w/c ratio or the corresponding compressive strength, the fine aggregate-to-total aggregate ratio $\left(\mathrm{FA} / \mathrm{A}_{\mathrm{c}}\right)$ can be found by using Table 7.2. The fine aggregate content can be calculated by multiplying the $\mathrm{FA} / \mathrm{A}_{\mathrm{c}}$ ratio by the total aggregate content.

To illustrate the application of the general mix design procedures to proportion the material for GC, two example problems are presented in Appendix B. The first example illustrates the design of a $100 \%$ RCA replacement, and the second one describes the mix design of concrete using a combination of RCA and NA. 


\subsection{Equivalent Total Volume of Mortar Method}

The total volume of mortar in concrete produced with RCA has two components: (1) residual mortar; (2) new mortar. When conventional mix design procedures such as the general mix design method described in Section 7.2 are used, the RCA is considered as coarse aggregate, and no consideration is given to the volume of attached residual mortar. Because of this, concrete produced with RCA has larger volumes of total mortar (residual and new), which can lead to potential problems in serviceability (e.g. low modulus of elasticity, high creep and shrinkage) and in durability (low freeze-and-thaw resistance, high permeability, etc.)

An alternative mix design procedure to eliminate this issue was developed by Fathifazl et al (2007b). The method involves the determination of the appropriate amount of RCA to be used in the mix in relation to the amount of coarse NA that would be used in a conventional concrete mix with the same specified properties. This approach guarantees that the volume of total mortar (residual and new) in concrete produced with RCA be equivalent to that in the conventional concrete. From a different way of thinking, the total volume of coarse NA (OVA plus NA) in GC is equal to the volume of NA in conventional concrete with the same specified properties.

The derivation of the equations of the equivalent total volume of mortar method can be found in Appendix B and also in Fathifazl et al (2007b). In the following section, the basic steps of the method are presented. 


\subsubsection{Mix Design Procedure}

The first step in the equivalent total volume of mortar method is the determination of the coarse aggregate content. The second step is the determination of the water, cement and fine aggregate contents. The following steps will describe the proportioning of GC by using equivalent total volume of mortar method.

Step 1: The mix proportions (in $\mathrm{kg} / \mathrm{m}^{3}$ ) of conventional (NAC) concrete (cement, sand, water, coarse NA) with required properties are designed using the existing procedures (e.g. ACI 211.1-97).

Step 2: The weight of OD RCA in GC, $W_{O D-R C A}^{G C}\left(\mathrm{~kg} / \mathrm{m}^{3}\right)$, and the weight of OD NA in $\mathrm{GC}, W_{O D-N A}^{G C}\left(\mathrm{~kg} / \mathrm{m}^{3}\right)$, are calculated using Eqs.7.1 and 7.2 respectively:

$$
\begin{gathered}
W_{O D-R C A}^{G C}=\frac{W_{O D-N A}^{N A C}\left(1-\frac{S G_{b}^{R C A}}{S G_{b}^{R M}} R M C\right)}{(1-R M C)} \\
W_{O D-N A}^{G C}=W_{O D-N A}^{N A C} \frac{S G_{b}^{R C A}}{S G_{b}^{R M}} R M C
\end{gathered}
$$

where $W_{O D-N A}^{N A C}$ is the oven dry weight of NA in NAC $\left(\mathrm{kg} / \mathrm{m}^{3}\right), S G_{b}^{R C A}$ is the bulk specific gravity of RCA, $S G_{b}^{R M}$ is the bulk specific gravity of residual mortar, which can be calculated as follows (See Eq.3.3 in Chapter 3) in the absence of a more accurate experimental procedure:

$$
S G_{b}^{R M}=\frac{R M C}{\frac{1}{S G_{b}^{R C A}}-\frac{1-R M C}{S G_{b}^{O V A}}}
$$

It is important to mention that, the values of RMC, $S G_{b}^{R C A}$, and $S G_{b}^{O V A}$ are obtained experimentally. The RMC value can be determined by using the quick method. The value 
of $S G_{b}^{\text {OVA }}$ can be determined from the OVA sample after removing of the residual mortar by the quick test. Furthermore, the bulk specific gravity of the OVA can be assumed equal to the bulk specific gravity of the NA obtained from the same source.

Step 3: The weight mix proportions of water, $W_{W}^{G C}\left(\mathrm{~kg} / \mathrm{m}^{3}\right)$ cement, $W_{C}^{N A C}\left(\mathrm{~kg} / \mathrm{m}^{3}\right)$, and fine aggregate, $W_{F A}^{G C}\left(\mathrm{~kg} / \mathrm{m}^{3}\right)$, contents in the new mortar of GC can be found by Eqs.7.4, 7.5 , and 7.6 , respectively:

$$
\begin{aligned}
& W_{W}^{G C}=W_{W}^{N A C}\left(\frac{V_{N M}^{G C}}{V_{M}^{N A C}}\right) \\
& W_{C}^{G C}=W_{C}^{N A C}\left(\frac{V_{N M}^{G C}}{V_{M}^{N A C}}\right) \\
& W_{F A}^{G C}=W_{F A}^{N A C}\left(\frac{V_{N M}^{G C}}{V_{M}^{N A C}}\right)
\end{aligned}
$$

where $W_{W}^{N A C}, W_{C}^{N A C}$ and $W_{F A}^{N A C}$ are the water, cement and fine aggregate contents $\left(\mathrm{kg} / \mathrm{m}^{3}\right)$ in NAC, respectively. The ratio of $\frac{V_{N M}^{G C}}{V_{M}^{N A C}}$ can be calculated using:

$$
\frac{V_{N M}^{G C}}{V_{M}^{N A C}}=\left[1-\left(\frac{\frac{W_{O D-R C A}^{G C}}{1000 S G_{b}^{R M}} R M C}{\left(\frac{W_{W}^{N A C}}{1}+\frac{W_{F A}^{N A C}}{S G_{b}^{F A}}+\frac{W_{C}^{N A C}}{S G^{C}}\right) \frac{1}{1000}+A}\right)\right]
$$

where $V_{N M}^{G C}$ is the volume of new mortar in GC and $V_{M}^{N A C}$ is the volume of the mortar in NAC. An example mix design using equivalent total volume of mortar method is presented in Appendix B. 


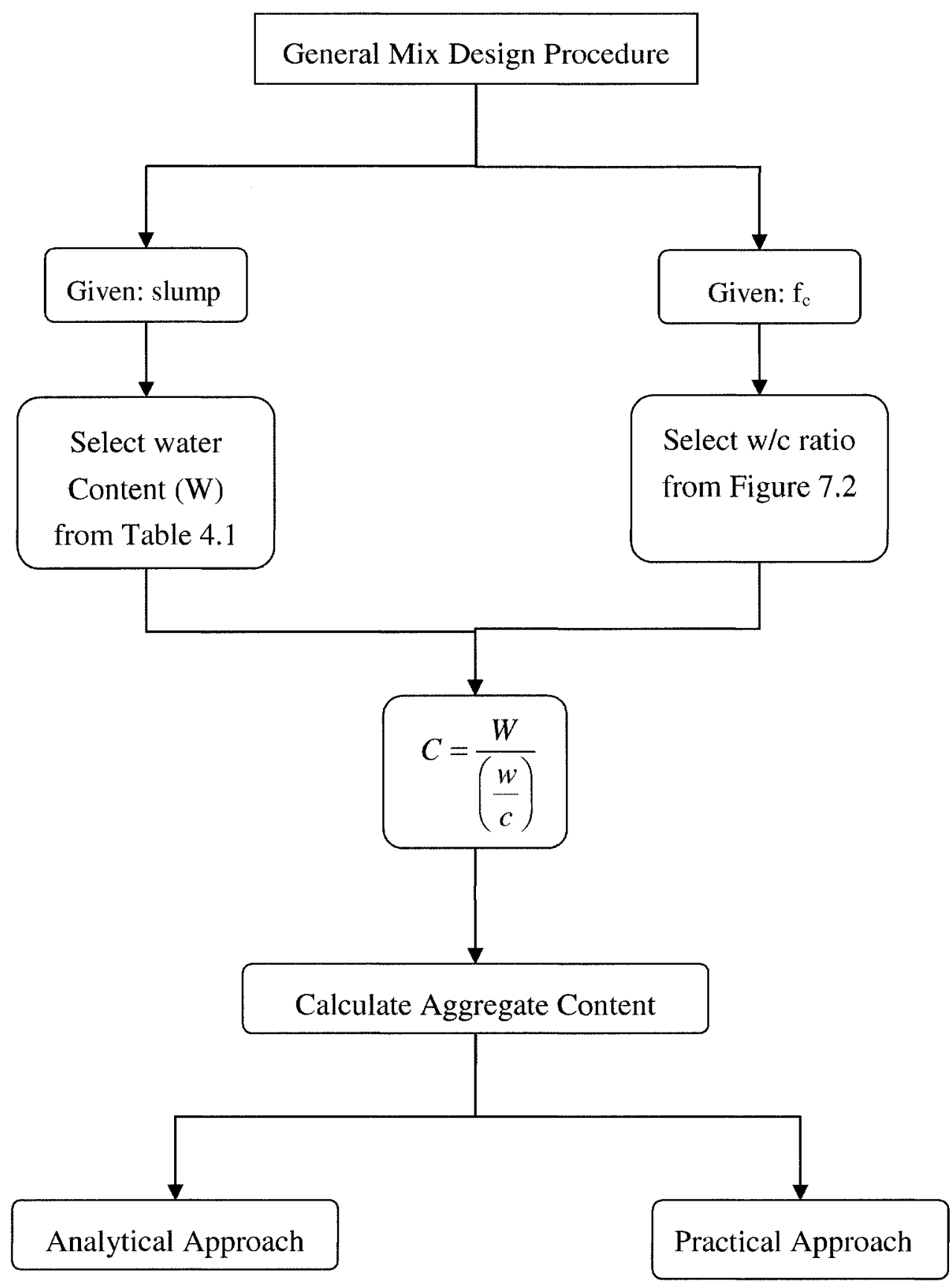

Figure 7.1: Flowchart for the general mix design procedure 


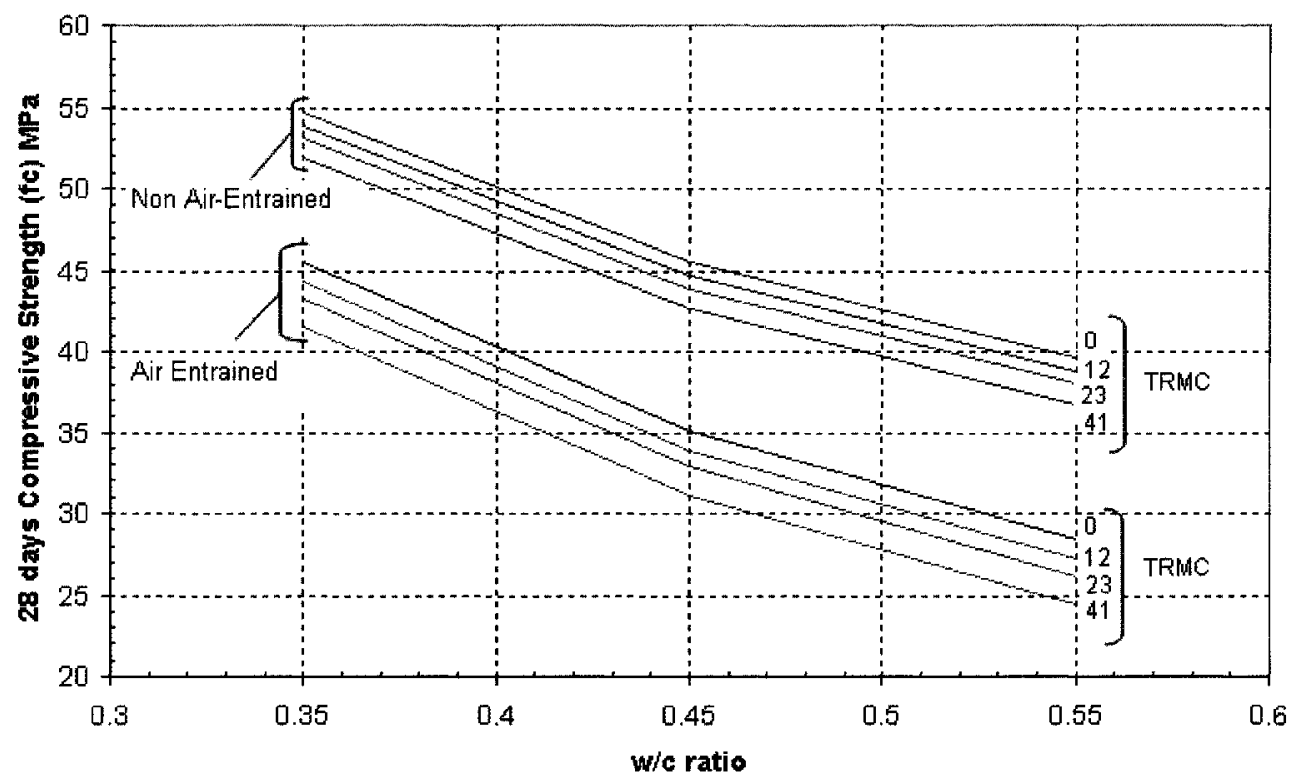

Figure 7.2: Relationship between $w / c$ ratio and compressive strength for air-entrained and non-air-entrained concrete 
Table 7.1: Volume of coarse aggregate per unit volume of concrete $\left(v_{c a}\right)$ (reproduced from ACI 211.1-97)

\begin{tabular}{|c|c|c|c|c|}
\hline \multirow{2}{*}{$\begin{array}{c}\text { Aggregate Nominal maximum size } \\
(\mathbf{m m})\end{array}$} & \multicolumn{4}{|c|}{$v_{c a} *$ for different fineness moduli } \\
\cline { 2 - 5 } & $\mathbf{2 . 4 0}$ & $\mathbf{2 . 6 0}$ & $\mathbf{2 . 8 0}$ & $\mathbf{3 . 0}$ \\
\hline 9.5 & 0.50 & 0.48 & 0.46 & 0.44 \\
\hline 12.5 & 0.59 & 0.57 & 0.55 & 0.53 \\
\hline 19 & 0.66 & 0.64 & 0.62 & 0.60 \\
\hline
\end{tabular}

*The volume of coarse aggregate per unit volume of $\mathrm{GC}\left(v_{R C A}^{G C}\right)$ should be calculated by reducing the volume of coarse aggregate $\left(v_{c a}\right)$ in this table by the absorption capacity of RCA. The volume of coarse aggregate in this table can be reduced up to $10 \%$ as specified by $\mathrm{ACI} 211.1$ for more workable concrete.

Table 7.2: Approximate total aggregate-to-cement ratio and fine-to-total aggregate ratio

\begin{tabular}{|c|c|c|c|c|}
\hline \multirow{2}{*}{ w/c } & \multicolumn{2}{|c|}{ Total-aggregate-to-cement Ratio } & \multicolumn{2}{c|}{ Fine-to-total aggregate Ratio } \\
\cline { 2 - 5 } & A.E.GC & Non A.E. GC & A.E.GC & Non A.E. GC \\
\hline 0.35 & 2.8 & 2.6 & 0.46 & 0.44 \\
\hline 0.45 & 3.8 & 3.6 & 0.49 & 0.48 \\
\hline 0.55 & 4.9 & 4.5 & 0.51 & 0.50 \\
\hline
\end{tabular}




\section{CHAPTER 8: DURABILITY OF GREEN CONCRETE}

\subsection{General}

One of the main objectives of the present study is to investigate the durability properties of GC. Specifically, the following durability properties are investigated: (1) chloride penetration; (2) carbonation; and (3) freeze-and-thaw resistance. To achieve this objective, an extensive experimental study was carried out. In this chapter the results of this comprehensive study are presented.

The investigation of durability of concrete in general is a challenging task. There are a number of factors that may affect the durability properties of concrete. Among these factors the following can be considered to be important: (1) w/c; (2) compressive strength; (3) aggregate type and content; (4) binder type (e.g. OPC and SCM's) and content; and (5) the air content. GC is different from conventional concrete (i.e., NAC) because of the use of RCA, hence, the attached residual mortar. Therefore, the present study focuses on the effect of the use of RCA from different sources (RCA-MO and RCA-VA) with different properties on the durability of GC. For this purpose, the experimental study was designed such that the effects of $w / c$ and compressive strength were eliminated by keeping these parameters constant $\left(\mathrm{w} / \mathrm{c}=0.45\right.$ and $f_{c}=30 \mathrm{MPa}$ to $35 \mathrm{MPa}$ ). However, the effect of the binder type, specifically the addition of SCM's (i.e., fly ash or ground granulated blast furnace slag (bfs)), on the durability properties were investigated. The percentages of SCM replacement used in the study were $25 \%$ for fly ash and $35 \%$ for bfs (by weight of cement), which are typical values of replacement used 
in the current practice. In addition, all mixes were prepared as air-entrained (targeting 6\% air content) to eliminate the effect of air content on the durability properties of GC.

As discussed in Chapter 7, GC can be designed using two mix design procedures: (1) the general mix design method; and (2) the equivalent total volume of mortar method. The former procedure follows the similar steps of conventional mix design methods; the later considers the volume of the old residual mortar as part of the total volume of mortar, which is equal to the volume of NAC designed with conventional mix design methods. Since the total volume of mortar resulting from the general mix design method is larger than the total volume of mortar from the equivalent total volume of mortar method, it is hypothesized that the second approach may provide improved durability properties. In order to test this hypothesis, three types of mixes were prepared:

(1) GC produced with $100 \%$ RCA using the general mix design method;

(2) NAC produced with $100 \%$ NA designed with conventional mix design;

(3) GC designed with the equivalent total volume of mortar method such that its total volume of mortar is equivalent to the second mix type (i.e., NAC);

The objective of the first mix type is threefold: (1) to investigate the durability of GC produced with $100 \%$ RCA (i.e., the worst-case scenario); (2) to study the effect of TRMC on the durability of GC; (3) to compare the durability properties of GC designed by the general mix design method to those of GC designed by the equivalent total volume of mortar method. The main purpose of the second mix type (i.e., NAC) is to provide a benchmark for the mixes designed by the equivalent total volume of mortar method since both mix types will have the same volume of total mortar. By comparing the two mix 
types, the effect of residual mortar on the durability of GC can be studied by isolating it from all other possible factors. Figure 8.1 illustrates the test grid for the durability study of GC. Further details of this study grid are presented in the following section.

\subsection{Mix Proportions}

Tables 8.1 and 8.2 present the mix proportions of the three types of mixes that were described in the previous section. It should be noted that each mix in these tables is identified with unique designation. For example, GC-MO produced with fly-ash and with $100 \%$ RCA-MO (or 41\% TRMC) is designated as GC-MO-D-F-G41 ("D" stands for "durability study", "F" stands for fly ash, and "G" stands for the general mix design method). Similarly, the GC-VA produced with bfs and the equivalent total volume of mortar method is designated as GC-VA-D-B-E17 ("B" stands for bfs and "E" stands for the equivalent total volume of mortar method.) The quantity following the letters "G", "E" or "N" (for conventional, NAC, concrete) represent the TRMC of the mix. The following summarizes the designation:

- Part 1: GC (Green concrete) -

- Part 2: MO or VA (produced with RCA-MO or RCA-VA) -

- Part 3: C or F or B (using OPC, or fly ash, or bfs) -

- Part 4: $\mathrm{G}$ or $\mathrm{E}$ or $\mathrm{N}$ (using the general mix design method, or equivalent total volume of mortar method, or conventional mix design) -

- Part 5: TRMC of the mix.

It is also important to note that the following points:

(1) All the mixes were air-entrained and had a w/c ratio of 0.45 ;

(2) $25 \%$ fly-ash and $35 \%$ bfs by weight of cement (replacement) were used to produce 
GC-MO and GC-VA with SCM's;

(3) NAC was produced by using OPC only with no SCM addition;

(4) Limestone was used as NA in NAC mixes that are used to design GC-MO specimens with the equivalent total volume of mortar method;

(5) River gravel was used as NA in NAC mixes that are used to design GC-VA specimens with the equivalent total volume of mortar method;

(6) A low-range water reducing admixture was used in all types of concrete except for NAC and GC with fly-ash, for which the additive was not found to be necessary;

(7) For GC designed by the general mix design method, a cementitious materials content of $350 \mathrm{~kg} / \mathrm{m}^{3}$ is targeted;

(8) For mixes produced with the equivalent total volume of mortar method, the cementitious material content varies between $335 \mathrm{~kg} / \mathrm{m}^{3}$ and $358 \mathrm{~kg} / \mathrm{m}^{3}$, depending on the RMC of the RCA.

Notice that the equivalent total volume of mortar method has an added advantage in terms of reducing the cement content of the mix. As it can be seen from Tables 8.1 and 8.2, the cement content of NAC (with 0\% RCA) designed using the conventional mix design procedures is above $400 \mathrm{~kg} / \mathrm{m}^{3}$. When $\mathrm{GC}$ with the same total volume of mortar is designed using the equivalent total volume of mortar method, the cement content goes down to between $218 \mathrm{~kg} / \mathrm{m}^{3}$ and $358 \mathrm{~kg} / \mathrm{m}^{3}$.

After the mixing process, the concrete was cast and cured for 24 hours following the procedures described in ASTM C 192/C 192 M-00 (ASTM, 2003). For each mix proportion, three prism specimens (with the dimensions of $75 \times 100 \times 400 \mathrm{~mm}$ ) were prepared for freeze-and-thaw testing as per ASTM C666-97 (ASTM, 2003). Three prism 
specimens (with the dimensions of $100 \times 100 \times 406 \mathrm{~mm}$ ) were prepared for the accelerated carbonation testing. Three cylindrical specimens (with the dimensions of 100 x $200 \mathrm{~mm}$ ) for chloride penetration test. In addition to these, three specimens were cast for measuring the 28-day compressive strength of all cases; additional 2 specimens were prepared for testing the 56-day compressive strength of mixes with SCM's. In summary, following the test grid of the durability study presented in Figure 8.1, it can calculated that a total of 226 specimens (in the form of cylinders and prisms) were prepared. Specimens were stored for 28 days in a moist room in accordance with the requirements of ASTM C 511-98 (ASTM, 2003). The following section will discuss the fresh and hardened properties of the mixes prepared for the durability study.

\subsection{Fresh and Hardened Properties}

There is direct correlation between the fresh and hardened properties (particularly $f_{c}$ ) of concrete and its durability. In general, concrete with proper air entrainment, slump, and compressive strength, showing no segregation or bleeding, is expected to be durable. Tables 8.3 and 8.4 present the fresh properties for GC-MO and GC-VA mixes, respectively.

As it can be seen from Table 8.3, in general, all GC-MO mixes designed with the general mix design method and the equivalent total volume of mortar method were found to be workable, with a slump range from $55 \mathrm{~mm}$ (GC-MO-D-B-G41) to $130 \mathrm{~mm}$ (GCMO-D-F-G41). The slump of the NAC mixes produced with limestone was quite large at $175 \mathrm{~mm}$. The low slump of $55 \mathrm{~mm}$ for GC-MO-D-B-G41 can be attributed to the combined effect of the use of bfs and the large TRMC (i.e., 41\%). However, this slump is within $\pm 25 \mathrm{~mm}$ of the lower limit of the targeted range $(75-100 \mathrm{~mm})$. From Table 8.4 , the 
slump for GC-VA mixes designed with the general mix design method and the equivalent total volume of mortar method were found to be in the range from $70 \mathrm{~mm}$ (GC-VA-D-CG23) to $150 \mathrm{~mm}$ (GC-VA-D-B-E17), respectively. The slump of the NAC mixes produced with river gravel is $210 \mathrm{~mm}$. From Tables 8.3 and 8.4 , it can be observed that in most of the cases, GC mixes with the highest TRMC (TRMC $=41 \%$ for GC-MO and TRMC $=23 \%$ for GC-VA) have the lowest slump, which can be attributed to the surface characteristics of the RCA.

It can also be seen in Tables 8.3 and 8.4 that the air content for all concrete mixes were measured to be in the range from 5.5\% (GC-VA-D-F-E17) to 7.4\% (GC-VA-D-CG23 and GC-MO-D-F-G41). These results are in line with the targeted air content of 6\% and are within the range of 5-8\% specified by CSA 23.2-04 for severe exposure conditions. From the same tables, the fresh concrete density for GC-MO and GC-VA mixes designed with the general mix design method and the equivalent total volume of mortar method ranged from $2226 \mathrm{~kg} / \mathrm{m}^{3}$ (GC-MO-D-F-G41) to $2328 \mathrm{~kg} / \mathrm{m}^{3}$ (GC-VA-DC-E17). The fresh density of GC-MO-C-NO and GC-VA-C-NO (NAC mixes) were determined as $2332 \mathrm{~kg} / \mathrm{m}^{3}$ and $2339 \mathrm{~kg} / \mathrm{m}^{3}$, respectively. It can be observed that the fresh density of GC-MO-D-C mixes and GC-VA-D-C mixes slightly decreases with increasing values of TRMC. The fresh densities of two mixes with similar TRMC values (e.g. GCMO-D-C-E26 and GC-VA-D-C-G23) are very similar. Figure 8.2 illustrates the variation of the fresh density with the TRMC for GC-MO-D-C mixes and GC-VA-D-C mixes, reiterating the same observations made in Chapter 5 regarding the effect of TRMC on fresh density.

Tables 8.5 and 8.6 present the hardened properties of the GC-MO and GC-VA 
specimens, respectively. The 28-day compressive strengths of all specimens tested were higher than $30 \mathrm{MPa}$, which is the minimum targeted strength. The compressive strength specimens with SCM's at 56 days were around $40 \mathrm{MPa}$ and higher. The hardened density of the specimens ranged from $2217 \mathrm{~kg} / \mathrm{m}^{3}$ (GC-MO-D-F-G41) to $2325 \mathrm{~kg} / \mathrm{m}^{3}$ (GC-VAD-B-E17), which is the range for normal density concrete.

It is important to notice that the compressive strength of specimens designed with the general mix design and the equivalent total volume of mortar methods are slightly higher than that of the NAC mixes. Although this difference is not significant (2 to 5 $\mathrm{MPa}$ ), it shows that GC produced with RCA does not have inferior hardened properties. The compressive strength measurements of specimens designed with the general mix design and the equivalent total volume of mortar methods did not show significant difference. Both design methods produced structural-grade concrete with the targeted parameters. The following section discusses the freeze-and-thaw testing of GC.

\subsection{Freeze-and-Thaw Action in GC}

The freeze-and-thaw tests were carried out following the Procedure A with rapid freezing and thawing in water described in ASTM C 666-97 (ASTM, 2003). The freezeand-thaw investigation is conducted on $75 \times 100 \times 400 \mathrm{~mm}$ prisms by monitoring the following three properties over a maximum of 300 cycles: (1) relative dynamic modulus;

(2) length change; (3) pulse velocity. The first two measurements are specified by ASTM C666-97 (length change measurements are specified as optional); however, pulse velocity measurements (ASTM C 597-97) were done additionally to support our conclusions since this parameter can also give an indication of the durability of concrete against freeze-andthaw action. All three measurements were taken for each specimen at the end of intervals 
not exceeding 36 cycles. Although the measurement techniques used in these tests are standardized, and their details can be found in the corresponding references, in the following, a brief description of each test is provided.

The relative dynamic modulus is determined by measuring the transverse frequency of the specimens (Figure 8.3a). The specimen under consideration was vibrated at the middle, and the frequency was picked up at the end of the specimen by an accelerometer and was recorded. Figure $8.3 \mathrm{~b}$ demonstrates the length change apparatus. During each measurement, the reference bar was placed in the comparator, and the comparator dial was set to zero. Then, the specimen was placed in the comparator and the comparator dial reading was recorded. The pulse velocity was measured following ASTM C 597-97 (ASTM, 2003). Figure 8.3c illustrates the pulse velocity instrument. The transmitting transducer and receiving transducer were longitudinally placed at each end in contact with the specimen. The transmitting transducer transmits a wave into the concrete specimen and the receiving transducer receives the pulse through the concrete at the end. The pulse velocity instrument display gives the time that takes for the wave pulse to travel through the concrete specimen in microseconds.

Three standard freeze-and-thaw cabinets (designated as A1, A2 and A3), as shown in Figure $8.4 \mathrm{a}$, were used to conduct the tests. The nominal freeze-and-thaw cycle of this test consist of repeatedly changing the temperature between $4.4 \pm 1.7^{\circ} \mathrm{C}$ and $-17.8 \pm$ $1.7^{\circ} \mathrm{C}$ in no less than $2-$ and no more than 5 -hour periods. Figure $8.4 \mathrm{~b}$ shows the typical freeze-and-thaw cycles in 24 hours as measured at the center of a dummy concrete sample placed in each of the three cabinets used in this study. It can be observed that for all freezers the freeze-and-thaw cycle periods are within the range of 2 to 5 hours as 
specified by the ASTM standard. Furthermore, the temperatures are within the lower and upper limits specified by ASTM, with the exception of freezer A2, which slightly exceeded the lower limit.

After 28 days of curing, the prisms were brought to the target thaw temperature of $4.0^{\circ} \mathrm{C}$ by placing them in a container with cold water. Then, the length, cross-sectional dimensions, and the weight of specimens were measured. The initial length of the comparator reading, initial transverse frequency, and initial pulse time were also recorded.

The freeze-and-thaw tests were initiated by placing the specimens in freezer's containers with thawing water. At the end of each measurement period (not exceeding 36 cycles), the specimens were removed from the freezer in a thawed condition, and the fundamental transverse frequency, length comparator reading, pulse time, weight, and cross sectional dimensions were measured. The raw data of these measurements are presented in Appendix C. The following sections will discuss the results of the measurements.

\subsubsection{Relative Dynamic Modulus and the Durability Factor}

The relative dynamic modulus, $P_{C}$, is calculated based on the formula given by the ASTM C 666-97 (ASTM, 2003) as follows:

$$
P_{c}=\left(\frac{\Omega_{1}^{2}}{\Omega^{2}}\right) 100
$$

where $P_{c}(\%)$ is the relative dynamic modulus of elasticity after $c$ (subscript) cycles of freezing and thawing, $\Omega$ is the initial fundamental transverse frequency, $\Omega_{1}$ is the fundamental transverse frequency after $c$ cycles of freezing and thawing. 
The durability factor, $D F(\%)$, can be calculated as:

$$
D F=\frac{P_{N} N}{M}
$$

where, $D F$ is the durability factor of the test specimen; $P_{N}(\%)$ is the relative dynamic modulus of elasticity at the $N^{\text {th }}$ cycle; $N$ is the number of cycles at which the relative dynamic modulus of elasticity reaches the specified minimum value for discontinuing the test or the specified number of cycles at which the exposure is to be terminated, whichever is less; and $M$ is the specified number of cycles at which the exposure is to be terminated ( 300 cycles for this study). Conventional air entrained concrete is considered satisfactory if it can withstand 300 cycles (Malhotra et al., 2005). For good quality concrete for a bridge deck (i.e., severe conditions), a durability factor of $90 \%$ and higher is considered to be adequate (Taylor et al., 2004).

Tables 8.7 and 8.8 summarize the $D F$ measurements after 300 cycles of exposure for GC-MO and GC-VA specimens. In general, the ASTM C 666 requires that the freezeand thaw test be terminated when (1) the relative dynamic modulus is lower than $60 \%$ of the initial value; (2) the number of cycles reach 300 . In none of the cases in this study, the relative dynamic moduli were lower than $60 \%$; therefore the tests were continued until 300 cycles. It can also be stated that the range of $D F$ for GC-MO and GC-VA are not significantly different and are within the expected range of performance from typical structural-grade concrete. As it can be seen in Table 8.7, for GC-MO specimens, the $D F$ ranged from $89 \%$ (GC-MO-D-F-G41) to 97\% (GC-MO-D-C-N0). From Table 8.8, it can be observed that the $D F$ for GC-VA specimens were in the range from $88 \%$ (GC-VA-DB-G23) to $96 \%$ (GC-VA-D-C-N0). For both series of control mixes, the specimens incorporating the natural aggregate only showed only a slightly higher $D F$ than those 
made with RCA. All cases showed low standard deviations and $\mathrm{CoV}$ values. A more detailed discussion on the results is provided hereafter.

Figure 8.5 illustrates the variation of the relative dynamic modulus with the number of freeze-and-thaw cycles for GC-MO-D-C and GC-VA-D-C specimens (no SCM's). In general, for all GC-MO-D-C and GC-VA-D-C specimens, the relative dynamic modulus is in a range from 90 to $100 \%$, which demonstrates that all specimens performed well against freeze-and-thaw action. However, during most freeze-and-thaw cycles, the relative dynamic moduli for GC-MO-D-C-NO specimens (i.e., NAC with limestone) were slightly higher than GC-MO-D-C-E26 and GC-MO-D-G41 specimens. Similarly, the relative dynamic moduli of GC-VA-D-C-NO specimens (i.e., NAC with river gravel) were slightly higher than GC-VA-D-C-E17 and GC-VA-D-C-G23 specimens. This slight difference can likely be attributed to the residual mortar attached to the RCA.

\subsubsection{Effect of SCM's}

Figures 8.6 and 8.7 illustrate the effects of fly ash and bfs on the relative dynamic modulus of GC-MO and GC-VA specimens respectively. It can be observed from Figure 8.6a that the relative dynamic moduli for GC-MO-D-F (with fly ash) specimens are lower than GC-MO-D-C specimens (no SCM's). Similarly, it can be observed in Figure 8.6b that the relative dynamic moduli for GC-VA-D-F (with fly ash) specimens are lower than GC-VA-D-C (no SCM's). It can be stated that the freeze-and-thaw resistance of GC is not improved by using fly ash as a partial replacement for cement. However, it should also be noted that these tests were carried out at 28 days, and it is expected that the properties of the mixes with fly ash would improve as the curing period for these specimens becomes longer. Similar observations can be made in Figure 8.7 for the 
addition of bfs: There seems to be a slight decrease in the relative dynamic modulus reading of the specimens prepared with bfs; however, this difference can also be attributed to the fact that the tests were conducted after 28 days of curing. Further curing of the specimens with bfs is expected to produce better results. It is important to emphasize that slightly lower readings observed in specimens with SCM's does not mean that these specimens are vulnerable to freeze-and-thaw action. As discussed before, all specimens tested here have showed high relative dynamic modulus (and durability factor) readings.

\subsubsection{2 . Effect of Mix Design Method}

A summary of the durability factors for the specimens are presented in Figure 8.8. A comparison of the DF values in this figure with respect to the mix design methods, namely general mix design method (G) vs. equivalent total volume of mortar method (E), shows that the later mixes perform better than the former: The DF for mixes with equivalent total volume of mortar method is slightly higher than the corresponding mixes with general mix design method. This difference can likely be attributed to the lower amounts of residual (i.e., TRMC) and total mortar of the mixes designed with the equivalent total volume of mortar method.

\subsubsection{Length Change}

The length change of the specimens tested is calculated as:

$$
L_{c}=\left[\frac{L_{2}-L_{1}}{L_{g}}\right] 100
$$

where $L_{c}(\%)$, is the length change of the test specimen after $c$ (subscript) cycles of freezing and thawing, $L_{1}$ is the initial comparator reading, $L_{2}$ is the comparator reading 
after $c$ cycles, and $L_{g}$ is the effective gage length between the inner most ends of the gage studs. As it is presented in Tables 8.7 and 8.8 respectively, the length change for GC-MO and GC-VA specimens is lower than $0.03 \%$ after 300 cycles. From Table 8.7 it can be observed that the length change for GC-MO specimens ranged from 0.007\% (GC-MO-DC-N0) to $0.027 \%$ (GC-MO-D-C-E26). Similarly, from Table 8.8 , the length change of GC-VA specimens were in the range from $0.001 \%$ (GC-VA-D-C-N0) to $0.026 \%$ (GCVA-D-C-E17). These values are very small; therefore it is difficult to use them to carry out a comparative investigation on the freeze-and-thaw resistance of GC. Despite this fact, in the following, an attempt is made to study the effects of SCM's and the mix design methods on the freeze-and-thaw resistance of GC.

\subsubsection{Effect of SCM's}

Figure 8.9 shows the variation of the length change with the number of freeze-andthaw cycles for specimens with fly ash. It can be observed in Figure 8.9a for GC-MO specimens that the addition of fly ash did not make any difference in the length change readings. Although observed to a smaller degree, as illustrated in Figure $8.9 \mathrm{~b}$, a similar conclusion can be made for GC-VA specimens. The specimens designed by the equivalent total volume of mortar method showed larger (and always expansive) length change than those observed in the specimens designed with the general mix design method.

Figure 8.10 illustrates the variation of the length change with the number of freezeand-thaw cycles for specimens with bfs. It can be observed from Figure 8.10a for GCMO specimens that the addition of bfs did not make any difference in the length changes readings. As illustrated in Figure $8.10 \mathrm{~b}$, a similar conclusion can be made for GC-VA 
specimens with general mix design method. On the other hand, lower expansion was observed for GC-VA specimens incorporating bfs and designed with equivalent total volume of mortar method.

\subsubsection{Effect of Mix Design Method}

As it can be observed in Figure 8.11 the length change of the mixes designed by the equivalent total volume of mortar method was found to be the highest. The mixes with no RCA replacement (NAC mixes) showed the lowest length change; the mixes designed with the general mix design method were in between the two. It is interesting to notice that the specimens designed with the equivalent total volume of mortar method did not show any contractions during the early freezing cycles.

A summary of the length change for the specimens are presented in Figure 8.12. A comparison of the length change values in this figure with respect to the mix design methods, namely general mix design method $(\mathrm{G})$ vs. equivalent total volume of mortar method $(\mathrm{E})$, shows that most of the former mixes perform better than the later. However, the mixes designed with the equivalent total volume of mortar method with SCM and TRMC of 17\% (i.e. GC-VA-D-F-E17 and GC-VA-D-B-E17) shows lower length change than the corresponding mixes designed by the general mix design method.

\subsubsection{Pulse Velocity}

The compressive wave pulse velocity, $P V$ is calculated as follows:

$$
P V=\frac{L}{t}
$$

where $L$ is the length between transmitting and receiving transducer (i.e., length of the specimen). As it is presented in Tables 8.7 and 8.8 respectively, the pulse velocity for 
GC-MO and GC-VA specimens were in the range from $4144 \mathrm{~m} / \mathrm{s}$ to $4654 \mathrm{~m} / \mathrm{s}$ after 300 cycles, which is higher than the typical range expected from conventional concrete 3700 to $4200 \mathrm{~m} / \mathrm{s}$ (Malhotra and Carino, 2004). It can be observed from Table 8.7 for GC-MO; the pulse velocity ranged from $4144 \mathrm{~m} / \mathrm{s}$ (GC-MO-D-F-G41) to $4686 \mathrm{~m} / \mathrm{s}$ (GC-MO-D-CN0); from Table 8.8, for GC-VA specimens, the pulse velocity was in the range from $4387 \mathrm{~m} / \mathrm{s}$ (GC-VA-D-F-G23) to $4654 \mathrm{~m} / \mathrm{s}$ (GC-VA-D-B-E17).

\subsubsection{Effect of SCM's}

Figures 8.13 and 8.14 illustrate the effects of fly ash and bfs on pulse velocity of GC-MO and GC-VA specimens, respectively. It can be observed from Figure 8.13a that the pulse velocity for GC-MO-D-F (with fly ash) specimens are lower than GC-MO-D-C specimens (no SCM's). Similarly, it can be observed to a lesser degree, as illustrated in Figure $8.13 \mathrm{~b}$, that the pulse velocity for GC-VA-D-F (with fly ash) specimens are lower than GC-VA-D-C (no SCM's). It can be stated that the freeze-and-thaw resistance of GC is not improved by using fly ash a partial replacement of cement. However, it should again be noted that these tests were carried out at 28 days, and it is expected that the properties of the mixes with fly ash would improve as the curing period for these specimens becomes longer. On the other hand, it can be observed from Figure 8.14 for the addition of bfs: There seems to be a slight increase in the pulse velocity reading for most of the specimens prepared with bfs. However, the pulse velocity of GC-VA-D-BG23 (with bfs) specimens is lower than GC-VA-D-C-G23 (no SCM's) this difference can also be attributed to the fact that the tests were conducted after 28 days of curing. Further curing of the specimens with bfs is expected to produce better results for this specimen. 


\subsubsection{Effect of Mix Design Method}

Figure 8.15 illustrates the variation of the pulse velocity with the number of freezeand- thaw cycle for specimens with no SCM's. As it can be observed in this figure, the pulse velocity of the NAC (no RCA replacement) was found to be the highest. The mixes with the general mix design method showed the lowest pulse velocity; the mixes designed by the equivalent total volume of mortar method were in between the two. These observations, which are in line with the relative dynamic modulus readings, can also be attributed to the higher total volume of the mortar in the mixes designed with the general mix design method. To summarize the measurements for the pulse velocity for the specimens, the normalized pulse velocity is calculated as follows:

$$
N P V=\left(\frac{P V_{300}^{2}}{P V_{0}^{2}}\right) 100
$$

where $N P V(\%)$ is the normalized pulse velocity after 300 cycles of freezing and thawing, $P V_{0}$ is the initial pulse velocity, $P V_{300}$ is the pulse velocity at 300 freeze-and-thaw cycles. Figure 8.16 presents a summary of the normalized pulse velocity for the specimens. It can be observed from this figure that, the normalize pulse velocity for all GC specimens is higher than $88 \%$.

\subsubsection{Summary of the Freeze-and-Thaw Tests Results}

- The visual examination of GC-MO and GC-VA specimens was carried out through out 300 freeze-and-thaw cycles. Figures 8.17 and 8.18 illustrate the defects observed during and at the end of the freeze-and-thaw tests for GC-MO and GC-VA specimens, respectively. In general, the defects range from minor scaling to scarcely distributed pop outs. 
- The performance of GC against freeze-and-thaw action was represented by the durability factor, normalized pulse velocity and length change values. Figure 8.19 illustrates the summary of these performance indicators for the freeze-and-thaw resistance of GC specimens. It can be observed from this figure that, the durability factors for all GC specimens were higher than $88 \%$, higher than $60 \%$ specified by the ASTM C 666-97 to terminate the freeze-and-thaw test. The maximum length change for all specimens was lower than $0.032 \%$. The normalized pulse velocity measurements were also found to be higher than $88 \%$.

- It can be observed from Figure 8.19 that, among the performance indicators, the relative dynamic modulus and pulse velocity are correlated well with each other; therefore, either method can be used to study the effect of freeze and-thaw action in GC. However, since the relative dynamic modulus technique is standardized, and the pulse velocity measurements do not provide additional information, it can be stated that ASTM C 666-97 (ASTM, 2003) method is more appropriate to study the freezeand-the resistance of GC.

- Length change measurements can be used to have a general idea about the freeze-andthaw resistance of GC; however, since the measurements are very small and are sensitive to the measurement conditions such as the temperature of the specimen, it can be stated that it should be used cautiously.

- It can be stated that, when the same cementitious material is used, the freeze-and-thaw resistance of GC decreases with increasing values of TRMC. Furthermore, the mixes with no RCA replacement (NAC, TRMC $=0$ ) showed higher durability factor than GC 
specimens designed with the equivalent total volume of mortar method; this is attributed to the presence of residual mortar in GC.

- The durability factor of GC was not improved by using SCM's as a partial replacement for OPC. However, as mentioned earlier, it should be noted that these tests were carried out at 28 days, and it is expected that the properties of the mixes with SCM's would improve as the curing period for these specimens becomes longer.

- From all performance indicators, it can be concluded that GC produced with either general mix design method (100\% RCA replacement) or with the equivalent total volume of mortar method (partial RCA replacement) have strong resistance against freeze-and-thaw action, a resistance expected from structural-grade concrete. However, since lower TRMC values can be achieved using the equivalent total volume of mortar method, this method could be preferred for RCA with very large RMC values and when freeze-and-thaw resistance is considered to be a serious problem for the structure under consideration.

\subsection{Chloride Penetration of GC}

The chloride penetration of GC is investigated by conducting three tests on the specimens described in Figure 8.1: (1) the acid soluble bulk diffusion test as per ASTM C 1556-04; (2) the rapid chloride test as per ASTM C 1202-97 (ASTM, 2003); and (3) the determination of water-soluble free chloride content of GC to study the effect of chloride binding as per ASTM C 1218-99 (ASTM, 2003). The acid soluble bulk diffusion is used to determine the apparent chloride diffusion coefficient of GC. The rapid chloride test provides a quick indication of the resistance of $\mathrm{GC}$ to the penetration of chloride ions. The main objective of this test is to investigate the correlation of its results with the bulk 
diffusion test. It should be noted that the water-soluble test was conducted only on specimens without SCM's.

Each $100 \times 200 \mathrm{~mm}$ concrete cylinder ( 3 repeat cylinders) produced for chloride testing was used to prepare the samples for the three tests described above. As illustrated in Figure 8.20, after 28-days of curing, from each of the $100 \times 200 \mathrm{~mm}$ cylinder specimens, a 75-mm-thick slice was cut parallel to the top finished surface. These samples were sealed by epoxy resin in all sides except for the finished surface (Figure 8.21a). From the remaining part of the each $100 \times 200 \mathrm{~mm}$ cylinder specimen, a $20-\mathrm{mm}$ thick slice (Figure 8.20) from the previously cut surface was taken to determine the initial chloride ion content, $C_{0}$. Another 51-mm-thick sample, to be used for the rapid chloride test, was cut from the bottom of the remaining part of the original $100 \times 200 \mathrm{~mm}$ specimen (Figure 8.20). The sides of the rapid chloride test samples were also sealed by epoxy resin. The following sections will discuss these tests and their results in detail.

\subsubsection{Acid Soluble Bulk Diffusion Test}

The acid soluble bulk diffusion test was carried out following the procedure described in ASTM C 1556-04. This test method covers the laboratory determination of the apparent chloride diffusion coefficient for cementitious mixtures by measuring the acid soluble (total) chloride content. In general, repeat samples are not required for ASTM 1156; however, to have better confidence in the results, in this study, two repeat samples (out of the three prepared) were chosen for the determination of bulk diffusion coefficient of GC.

The $100 \times 75 \mathrm{~mm}$ test specimens that were prepared for this test were immersed in a saturated $\mathrm{Ca}(\mathrm{OH})_{2}$ water bath until the mass did not change by more than $0.1 \%$ in a $24-$ 
hour period. After this condition is achieved, the saturated samples were removed from the $\mathrm{Ca}(\mathrm{OH})_{2}$ water bath, and they were rinsed with tap water. The samples were immersed in a $15.1 \%$ (by weight) sodium chloride solution in plastic containers, as illustrated in Figure $8.21 \mathrm{~b}$. All containers were sealed with plastic lids to prevent evaporation.

The test specimens were removed from the sodium chloride solution after 60 days. Then, the specimens were rinsed with tap water and were dried in laboratory conditions as specified in ASTM 1556 for 24-48 hours. From each sample, concrete powder was extracted at 8 step depths (approximately at 1,2,4,6,9,12,15, and $18 \mathrm{~mm}$ ) by grinding off material in parallel layers to the exposed surface as shown in Figure 8.21c. The powders from each depth were collected in plastic containers (Figure 8.21d). Similarly, approximately, 20 -g powders were ground off from $20-\mathrm{mm}$ thick samples that were cut out from the original $100 \times 200 \mathrm{~mm}$ cylinders to measure the initial acid and water soluble chloride concentrations of the specimens (Figure 8.20).

Collected powders were used to prepare the solutions for the acid soluble chloride content (total chloride) determination by ASTM C 1152-90 through titration using ASTM C-114-03 (ASTM, 2003). In this test method, total chloride content was determined by the potentiometric titration of chloride with silver nitrate. For each specimen, the amount of powder to prepare the solutions and the depth it is collected from can be found in the raw data presented in Appendix C. It should be noted that part of the powder at the last four depths, obtained from specimens with no SCM addition, were also used in the watersoluble chloride content determination to study the bound chlorides. This test will be described later in Section 8.5.3. 
The details of the solution preparation and the titration procedures are well known and can be found in the aforementioned standards; therefore they will not be described here in detail. Using these procedures, the total chloride concentration at each depth of each specimen were determined and are presented in Appendix C. Hereafter, the results of the acid soluble bulk diffusion test are presented.

The value of the surface chloride concentration, $C_{s}$, and apparent chloride diffusion coefficient, $D_{a}$, are calculated by fitting Eq.2.2 to the measured total chloride ion content at different depths of the samples by means of a non-linear regression analysis using the method of least squares. In order to calculate the apparent diffusion coefficient and the surface chloride concentration, the initial acid soluble chloride concentration, $C_{0}$, was determined; hence, the $C_{0}$ was measured from the powders obtained from 20-mm thick samples. The regression analysis for two repeat samples of all mixes are presented in Figures C. 1 to C.14 in Appendix C. Tables 8.9 and 8.10 present the measured acid soluble initial chloride content, the surface chloride concentration, and the apparent diffusion coefficient of all GC-MO and GC-VA specimens, respectively. It should be noted that all concentration measurements are presented as percentage of mass of concrete and are represented with $\%$.

In general, from Table 8.9 and 8.10 , it can be observed that the acid soluble initial chloride concentration for GC-MO and GC-VA specimens with RCA replacement were measured to range from $0.006 \%$ to $0.023 \%$. For GC-MO with RCA-MO replacement (i.e., $\mathrm{TRMC}=26 \%$ and $41 \%$ ), the acid soluble initial chloride concentration ranged from $0.018 \%$ (GC-MO-D-C-G41, GC-MO-D-B-E26) to 0.023\% (GC-MO-D-B-G41). The acid soluble initial chloride concentration for GC-VA with RCA-VA replacement (TRMC = 
$17 \%$ and $23 \%$ ), ranged from $0.006 \%$ (GC-VA-D-C-17E) to $0.011 \%$ (GC-VA-D-B-17E, GC-VA-D-B-G23). It is clear that the acid soluble initial chloride concentration values of GC-MO specimens with RCA replacement are 2-3 times higher than those of GC-VA specimens with RCA replacement. Although the exact origin of the concrete material from which the RCA were manufactured is not known, we believe that the higher values observed with GC-MO specimens may be attributed to higher degree of salt contamination of the residual mortar attached to RCA-MO. As it is well known, de-icing salts are widely used in the area of Montréal during winter months; however, in the Vancouver area, region from which RCA-VA was obtained from, the extent of the usage of de-icing salts is quite limited. It is also important to observe that the acid soluble initial chloride concentrations for GC-MO-D-C-NO and GC-VA-D-C-NO specimens (i.e., no RCA addition) are $0.008 \%$ and $0.007 \%$, respectively. These small quantities are in the range of the expected values that are observed in conventional concrete with no precontamination.

The limit of the acid soluble chloride concentration from the mixture ingredients specified by ACI $222 \mathrm{R}-96$ is $0.2 \%$ by weight of cement. The maximum acid soluble chloride concentration was measured for GC-MO-D-B-G41 specimens as $0.023 \%$. The percentage of cement by mass of concrete for all specimens of GC-MO and GC-VA with RCA replacement was around $15-16 \%$. Hence, the maximum acid soluble initial chloride concentration by mass of cement can be calculated as $(0.023 \times 100) / 15=0.15 \%$, which is lower than $0.2 \%$ specified by ACI $222 \mathrm{R}-96$. However, it is important to mention that the residual mortar contains additional cement that is not included in the total amount of cement for GC. This means that the $0.15 \%$ maximum acid soluble initial chloride by 
mass of cement is conservative. Hence, it is recommended for GC to specify the limit of chloride content from mixture ingredients in terms of the mass of concrete, rather than the mass of cement.

In general, the apparent chloride diffusion of all GC specimens is in the same order of magnitude $\left(\sim 10^{-12} \mathrm{~m}^{2} / \mathrm{s}\right)$ as the conventional concrete (Concrete Society, 1988). The apparent chloride diffusion coefficient for all specimens ranged from $1.8 \times 10^{-12} \mathrm{~m}^{2} / \mathrm{s}$ (GCMO-D-B-E26) to $5.58 \times 10^{-12} \mathrm{~m}^{2} / \mathrm{s}$ (GC-MO-D-C-N0). The following section discusses the effect of SCM's.

\subsubsection{Effect of SCM's}

Figure 8.22 illustrates the effect of the SCM's on the apparent diffusion coefficient of GC-MO and GC-VA specimens. It can be observed from Figure 8.22a that the apparent diffusion coefficients of GC-MO-D-F specimens designed with the general mix design or the equivalent total volume of mortar methods are (up to 1.5 times) lower than those of the GC-MO-D-C specimens (no SCM's). Similarly, it can be observed in Figure $8.22 \mathrm{~b}$ that the apparent diffusion coefficients of GC-VA-D-F specimens designed with the general mix design or the equivalent total volume of mortar methods are (up to 2 times) lower than those of GC-VA-D-C (no SCM's). It can be stated that the chloride penetration of GC is significantly improved by using fly ash as partial replacement for cement. Similar observations can be made for the addition of bfs; actually, the use of bfs reduces the apparent diffusion coefficient of all mixes designed with the general mix design and the equivalent total volume of mortar methods 1.2-2 times more than the reduction created by the addition of fly ash. 


\subsubsection{Effect of Mix Design Method, TRMC and Pre-Contamination}

It can be observed from Figure 8.22a, the apparent chloride diffusion coefficients for GC-MO-D-C (with no SCM's) specimens designed with the general mix design were found to be lower $(\sim 8 \%)$ than those designed with the equivalent total volume of mortar methods. Similarly, from Figure $8.22 \mathrm{~b}$, the apparent chloride diffusion coefficients for GC-VA-D-C (with no SCM's) specimens designed with the general mix design were lower $(\sim 10 \%)$ than of those designed with the equivalent total volume of mortar methods. It is also obvious from Figure 8.22 that the apparent diffusion coefficients of NAC specimens (with no RCA replacement) are higher than those of GC specimens with RCA replacement. There is also a general trend that the apparent diffusion coefficient for specimens produced with RCA-MO are slightly $(\sim 10$ to $\sim 30 \%)$ higher than those of specimens produced with RCA-VA.

Figure 8.23 illustrates the variation of the apparent diffusion coefficient with TRMC for GC-MO-D-C and GC-VA-D-C specimens. It can be observed from these figures that, the apparent diffusion coefficient is linearly correlated with the TRMC: an increase in TRMC results in a decrease in the apparent chloride diffusion coefficient of GC. As it can be observed from these figures, for both GC-MO-D-C and GC-VA-D-C, the linear correlations between apparent chloride diffusion coefficient and TRMC have $\mathrm{R}^{2}$ values of 0.97 . The value of the slope for the trend line for GC-MO-D-C and GC-VAD-C are $5.668 \times 10^{-13}$ and $5.768 \times 10^{-13}$, with no significant difference.

An explanation for this behaviour in the case of GC-VA specimens is not difficult to provide. These specimens, as presented in previous sections, were not found to be excessively pre-contaminated with chloride ions. Therefore, as the TRMC of the mix 
increase, it can be expected that the chloride binding capacity would also increase since there would be more mortar to bind the chloride ions. The larger binding capacity would mean that chloride ions would be hindered to penetrate inside the concrete, resulting in lower values of apparent diffusion coefficient. In Section 8.5.4, it will be demonstrated through water soluble test that the binding capacities of specimens produced with RCAVA; i.e., GC-VA-D-C-G23, GC-VA-D-E17, and GC-VA-D-C-N0 are 0.1649, 0.0201 and 0.0018 , respectively, decreasing with the decreasing TRMC values, further confirming the justification provided here.

However, the apparent diffusion coefficient results of the GC-MO specimens contradict with this thinking. It will be demonstrated in Section 8.5.4 that the binding capacities of specimens prepared by RCA-MO; i.e., GC-MO-D-C-G41, GC-MO-D-CE26, GC-MO-D-C-N0 are 0.0512, 0.1077 and 0.1142, respectively, showing an increase as the TRMC values decrease, contradicting with the previous justification. Upon this, another possible reason for the decreasing values of apparent diffusion coefficient (with increasing TRMC) was sought, and it was found that the compressive strength of GCMO-D-C-G41 specimens were slightly higher than the ones designed with the equivalent total volume of mortar method (i.e., GC-MO-D-C-E26) and with the conventional design method (i.e., GC-MO-D-C-N0), as shown in Table 8.5. This difference, possibly due to the use of water-reducing agent, is an indication that concrete designed with the general mix design method was denser, and this might be the reason for the trend observed in Figure 8.23a. 


\subsubsection{Rapid Chloride Test}

Rapid chloride test was performed following the procedure given by ASTM C 1202-97 (ASTM, 2003). In this method, the electrical conductance of concrete is determined to provide a rapid indication of the concrete resistance to the penetration of chloride ions. The test is conducted on $51-\mathrm{mm}$ thick cylindrical samples with $100 \mathrm{~mm}$ nominal diameter, whose preparation was discussed previously (Figure 8.24a). For each mixture three repeat samples were prepared. After the drying of the epoxy resin, the specimens were vacuum saturated (Figure 8.24b) and placed in the rapid chloride test setup, in which a DC potential difference of $60 \mathrm{~V}$ was maintained across the uncoated ends of the specimen, one of which was immersed in $3 \%$ (by mass) sodium chloride solution, the other in a $0.3 \mathrm{~N}$ sodium hydroxide solution (Figure $8.24 \mathrm{c}$ ). The amount of electrical current passed through the $51-\mathrm{mm}$ thick cylindrical samples with $100 \mathrm{~mm}$ nominal diameter was measured during a 6 hour testing period. ASTM C 1202-97 provides the following ranges for low, moderate and high chloride permeability for conventional concrete: the chloride ion penetrability is high when the charge passed is greater than 4000 Coulombs; it is moderate when the charge passed range from 2000 to 4000 Coulombs; and it is low when the charge passed range from 1000 to 2000 Coulombs.

Tables 8.11 and 8.12 present the charge passed and chloride ion penetrability as defined by ASTM C 1202-97 for GC-MO and GC-VA specimens, respectively. For GCMO specimens the charge passed were measured to be in the range of 1744 Coulombs (low, GC-MO-D-F-E26) to 5144 Coulombs (high, GC-MO-D-C-G41). For the GC-VA specimens, the charge passed ranged from 1302 Coulombs (low, GC-VA-D-F-G23) to 
3664 Coulombs (moderate, GC-VA-D-C-N0). Actually, the only "high" measurement was obtained for GC-MO-D-C-G41 specimens; the rest of the specimens fall in the low or moderate categories. It is also important to notice that the measurements for the NAC specimens (no RCA replacement) are very close to each other (3316 Coulombs for GCMO and 3664 Coulombs for GC-VA) in the "moderate" permeability region.

\subsubsection{Effect of SCM's}

It can be noticed from Table 8.11 and 8.12 that the rapid chloride test method provides mostly "low" rating for specimens with SCM's. The chloride ion penetrability for GC-MO-D-F-G41, GC-MO-D-F-E26, GC-VA-D-F-G23, and GC-VA-D-F-E17 specimens were graded as low. This confirms that that the use of fly ash improves the resistance of GC to chloride ion penetrability. Furthermore, the chloride ion penetrability for GC-MO-D-B-E26, GC-VA-D-B-G23, and GC-VA-D-B-E17 specimens were also found to be low. GC-MO-D-B-G41 specimens had an average current reading of 2059 Coulombs, which is only slightly above the border between low and moderate ratings; therefore it can be considered as a borderline case. This indicates that the bfs also improves the resistance of GC to chloride ion penetrability. It is important to note that the coulomb values for the fly-ash mixes were actually lower than that obtained for the slag mixes. These observations are in line with the results obtained from the acid soluble bulk diffusion test.

\subsubsection{Effect of Mix Design Method}

It can be observed from Table 8.11 that for GC-MO specimens, the mixes designed with the general mix design method $(\mathrm{TRMC}=41 \%$ ) have higher current measurements than the mixes designed with the equivalent total volume of mortar method (TRMC = 
$26 \%$ ). This also proves that the more TRMC, the higher the ion concentration per unit area; hence the higher current passed in the specimens. From Table 8.12 and for GC-VA specimens, the difference between the two design methods are not significantly apart since the TRMC values of the two mixes are quite close to each other (23\% vs. $17 \%)$.

\subsubsection{Effect of TRMC}

In general, from Table 8.11 and 8.12 , the measurements for GC-MO specimens with RCA replacement show higher current values than those of GC-VA specimens with RCA replacement. This may be attributed to the fact that the GC-MO specimens have larger TRMC values and higher values of pre-contamination with chlorides. Since rapid chloride test is a measure of electrical conductivity of the pore solution, and the conductivity of the solution increases with the increased concentration of chloride ions, due to pre-contamination effects, it is expected that the measurements for GC-MO specimens with RCA replacement are higher than those of GC-VA specimens with RCA replacement. Also, although not reported in this thesis, microscopic investigations have shown that RCA-VA contains fly-ash, and this may be another reason for the lower current values observed in GC-VA specimens than those of GC-MO specimens with RCA replacement.

\subsubsection{Bulk Diffusion vs. Rapid Chloride Test}

The typical ranges of the apparent chloride diffusion coefficient for low, moderate and high chloride permeability for conventional concrete reported by the Concrete Society (1988) can be specified as follows: (1) high chloride permeability when the

diffusion coefficient is greater than $5 \times 10^{-12} \mathrm{~m}^{2} / \mathrm{s}$; (2) moderate chloride permeability when the diffusion coefficient is between $1 \times 10^{-12}$ and $5 \times 10^{-12} \mathrm{~m}^{2} / \mathrm{s}$; and (3) low 
chloride permeability when the chloride diffusion coefficient is lower than $1 \times 10^{-12} \mathrm{~m}^{2} / \mathrm{s}$. Figure 8.25 presents the charge passed (from rapid chloride test) versus the apparent chloride diffusion coefficient (from bulk diffusion test) for GC-MO and GC-VA specimens, as well as the benchmarks for low, moderate and high chloride permeability.

It can be observed from this figure that there is a trend of increasing apparent diffusion coefficient with increasing values of current passed. The rapid chloride test and bulk diffusion test correlated relatively well for specimens with no SCM's, with the exception of GC-MO-D-C-G41 specimens, which is classified as having high chloride permeability by rapid chloride test, but having moderate permeability by the bulk diffusion test. This may be attributed to the pre-contamination of the RCA-MO by deicing salts, which would increase the conductivity of the concrete specimens, resulting in higher readings (i.e., charged passed) from the rapid chloride test. On other hand, GCMO-D-C-NO (i.e., NAC, with no RCA replacement) is classified as having high chloride permeability with the bulk diffusion and as moderate with rapid chloride test. Further contradictions exist in the specimens with SCM's, which are classified as having low chloride permeability by rapid chloride test, but having moderate permeability by the bulk diffusion test.

To summarize, it can be stated that although there is a general trend providing increasing apparent diffusion coefficient with increasing rapid chloride test measurements (i.e., charges passed), the correlation between the two provided for conventional concrete does not seem to capture the correlation for GC. Further investigation to establish this relationship will be necessary if rapid chloride test is to be used for GC reliably. However, it should also be noted that, due to possible pre- 
contamination of RCA with different ions, the rapid chloride test can provide misleading results regarding the chloride permeability of concrete; therefore even after a correlation is established, the results of the rapid chloride test should be taken cautiously.

\subsubsection{Water Soluble Test (Preliminary Study on Chloride Binding)}

The water soluble chloride content of the GC-MO and GC-VA specimens was determined following ASTM C 1218-99 (ASTM, 2003) to obtain the free chloride concentration, $C_{f}$. The water soluble test is similar to the acid soluble test with the main difference in the preparation of the solution sample from the concrete powder. The free chloride is extracted from the concrete powder sample by immersing it in water for 24 hours. The detailed procedure for the solution preparation can found in ASTM C 1218-99 (ASTM, 2003). The free chloride content for the solution was determined by the potentiometric titration of chloride with silver nitrate following ASTM C-114-03 (ASTM, 2003).

The main objective of this investigation was to carry out a preliminary study on the effect of chloride binding in GC; therefore, only the specimens without SCM's were studied. The concrete powders were obtained from four depths that ranged from $9 \mathrm{~mm}$ to $18 \mathrm{~mm}$ from surface of each specimen. The amount of powder and the depths that they were extracted from along with the results of the test are presented in Table 8.13. The bound chloride concentrations were obtained from Eq.2.3, assuming that $w_{e}=1$ for saturated conditions.

As it can be observed in Table 8.13, the water soluble initial chloride concentrations, $C_{0, w}$, were lower than $0.015 \%$ (by mass of concrete) for both GC-MO and GC-VA specimens. The water soluble initial chloride concentration for GC-MO-D-C was 
found to range from $0.002 \%$ (GC-MO-D-C-N0) to $0.015 \%$ (GC-MO-D-C-E26). The water soluble initial chloride concentration for GC-VA-D-C was in the range from $0.001 \%$ (GC-VA-D-C-N0 and GC-VA-D-C-E17) to 0.004\% (GC-VA-D-C-G23). As observed in the acid soluble initial chloride content values, the water soluble initial chloride concentrations of GC-MO specimens with $\mathrm{RCA}$ replacement (i.e., $\mathrm{TRMC}=26 \%$ and $41 \%$ ) are higher than those of GC-VA specimens with RCA replacement (i.e., TRMC $=17 \%$ and $23 \%$ ). This can possibly be attributed to the high amount of contaminated residual mortar in GC-MO specimens.

The limits of the water soluble chloride concentration from the concrete mixture ingredients specified by ACI 318M-95 and CSA A23.1-04 is 0.15\% (by weight of cement). The percentage of cement by mass in concrete for all specimens of GC-MO and GC-VA with RCA replacement was around 15-16\%. Hence, the maximum water soluble initial chloride by mass of cement $(0.015 \%$ for GC-MO-D-C-E26) can be calculated as $(0.015 \times 100) / 15=0.10 \%$, which is lower than $0.15 \%$ specified by ACI318M-95 and CSA A23.1-04. However, it is important to mention that, the residual mortar contains additional cement which is not included in the amount of total cement for GC. Therefore, as concluded for acid soluble limits, it can be stated that it is more appropriate to specify the water soluble limits in terms of percentage of concrete, rather than cement.

Figures 8.26 and 8.27 illustrate the relationship between the total, free and bound chloride measurements at four depths. From these figures, it can be observed that the bound chloride concentration is linearly correlated with the free chloride concentration, with an $\mathrm{R}^{2}$ value higher than 0.72 . Equation 8.7 can be recommended as the linear chloride isotherm for GC: 


$$
C_{b}=\alpha C_{f}+C_{b}^{0}
$$

where $C_{b c}^{0}$ is the initial bound chloride concentration as calculated in Table 8.13, and $\alpha$ is the binding capacity of GC (i.e., $\frac{\partial C_{b}}{\partial C_{f}}=\alpha$ ).

From Figure 8.26 the binding capacities of specimens prepared by RCA-MO; i.e., GC-MO-D-C-G41, GC-MO-D-C-E26, GC-MO-D-C-N0 were calculated as 0.0512 , 0.1077 and 0.1142 , respectively. The GC-MO-D-C-G41 (which was designed with the general mix design method) has the lowest binding capacity among the GC-MO specimens. This may be due to the pre-contamination of the RCA-MO with de-icing salts, which exhausted the binding capacity of the residual mortar. The binding capacity of GC-MO-D-E26 specimens (which were designed with the equivalent total volume of mortar method) is slightly lower (around 6\% lower) than the binding capacity of GCMO-D-C-N0 (i.e., NAC) mixes, further confirming that as the amount of precontaminated residual mortar decrease, the binding capacity increases. From Figure 8.27, the binding capacities for specimens produced with RCA-VA; i.e., GC-VA-D-C-G23, GC-VA-D-E17, and GC-VA-D-C-N0 were determined as 0.1649, 0.0201 and 0.0018 , respectively. GC-VA-D-C-G23 (which was designed with the general mix design method) has the highest binding capacity. This can be attributed to the fact that RCA-VA specimens were not excessively pre-contaminated with chloride ions; hence the concrete with larger TRMC would have a larger binding capacity. 


\subsubsection{Summary of the Chloride Tests Results}

- The measured acid soluble (i.e., total) and water soluble (i.e., free) initial chloride concentrations of the GC-MO and GC-VA specimens were found to be lower than the limits specified by the standards.

- Since the estimation of the total cement content of GC mixes is difficult due to the fact that the cement content in the residual mortar cannot be accurately known, it is recommended for GC that all limits for chloride content are specified in terms of the mass of concrete, rather than the mass of cement.

- The apparent chloride diffusion coefficients for all GC specimens were found to be in the same order of magnitude as the conventional structural grade concrete (i.e., $10^{-12}$ $\left.\mathrm{m}^{2} / \mathrm{s}\right)$. Specifically, the apparent chloride diffusion coefficients for all GC specimens were in the moderate chloride permeability range.

- The resistance to chloride penetration of GC is improved by using SCM's (fly ash or bfs) as partial replacement for OPC. The addition of bfs reduces the apparent diffusion coefficient of all mixes designed with both mix design methods 1.2-2 times more than the reduction created by the addition of fly ash. Both general mix design method and the equivalent total volume of mortar method provide good quality concrete against chloride penetration when SCM's are used.

- Although there is a general trend providing increasing apparent diffusion coefficient with increasing rapid chloride test measurements (i.e., charges passed), the correlation between the two provided for conventional concrete does not seem to capture the correlation for GC. Further investigation to establish this relationship will be necessary if rapid chloride test is to be used for GC reliably. However, it should also 
be noted that, due to possible pre-contamination of RCA with different ions, the rapid chloride test can provide misleading results regarding the chloride permeability of concrete; therefore even after a correlation is established, the results of the rapid chloride test should be taken cautiously.

- The investigation of the results of the GC specimens produced from different sources of RCA showed that the pre-contamination is one of the main factors affecting the apparent diffusion coefficient. If the RCA is not excessively pre-contaminated, increased amounts of residual mortar (i.e., TRMC) have the potential to increase the binding capacity of the GC; hence decreasing the diffusion of chloride ions in concrete. Therefore, when RCA is clear from pre-contamination, the use of the general mix design method improves concrete resistance to chloride ion penetration.

- In the case of pre-contaminated RCA, the increased amounts of residual mortar will not improve the binding capacity of GC, as observed in the GC-MO mixes; therefore, the use of equivalent total volume of mortar method can be considered to be more appropriate to design GC with these RCA.

\subsection{Carbonation of GC}

The carbonation test was carried out using a setup that was built based on RILEM recommendations, CPC-18, explaining the measurement of hardened concrete carbonation depth (RILEM, 1988). For this purpose, after the 28-day curing period, all prism specimens with the dimensions of $100 \times 100 \times 406 \mathrm{~mm}$ ( 3 repeat samples for each mix) were placed in a carbonation chamber, shown in Figure 8.28a, with temperature and humidity control capabilities.

The carbonation chamber was maintained at a relative humidity $(\mathrm{RH})$ of $60 \pm 5 \%$ 
and at a temperature of $23 \pm 1.7^{\circ} \mathrm{C}$. The specimens were exposed to $3 \%$ carbon dioxide concentration by volume as a mixture of oxygen and carbon dioxide gases. Figure $8.28 \mathrm{~b}$ demonstrates the relative humidity and temperature measurements during the 140 days of testing. The exposure was interrupted seven times at 7,21, 35, 56, 84, 112 and 140 days after the specimens had been exposed to the $\mathrm{CO}_{2}$ in the chamber. At each interruption, 50-mm thick slices were cut (Figure 8.28c) normal to the $(406-\mathrm{mm})$ long dimension of the specimens. The shortened specimens were placed back into the chamber for continued exposure to $\mathrm{CO}_{2}$. The freshly cut surface of each slice was cleaned and sprayed with $1-\mathrm{g}$ phenolphthalein indicator solution in $70 \%$ ethanol. The indicator turns pinkish red in the un-carbonated zone, where the $\mathrm{pH}$ is higher, and remains colorless where the concrete is carbonated (Figure 8.28d). The average carbonation depth was measured normal to four sides of each slice immediately, and also 24 hours, after spraying. The second measurement was done as per the recommendations of RILEM to check if there were any changes in 24 hours. The carbonation depths measured for each GC-MO and GC-VA specimens are presented in Appendix C3.

It should be noted that the sharp changes in the relative humidity and temperature measurements in Figure $8.28 \mathrm{~b}$ are due to the interruptions. After the specimens were placed back to the carbonation chamber, the humidifier was turned on to increase the relative humidity to $60 \%$. The $\mathrm{CO}_{2}$ concentration was boosted by allowing $100 \% \mathrm{CO}_{2}$ for 30 seconds to saturate the entire the chamber. Generally, the chamber reaches the required equilibrium conditions after 5 to 10 minutes from interruption.

Tables 8.14 and 8.15 present the average measured carbonation depths for GC-MO and GC-VA specimens, respectively. The average values presented in these tables were 
calculated for three repeat samples, whose depth measurements were taken as the mean of the four sides. It is important to note that the carbonation depths obtained from this accelerated test are expected to be higher than measurement that would have been obtained under the normal exposure conditions. It is therefore important to know the expected results from conventional concrete so that the range of the results for GC could be evaluated properly. Bouzoubaa (2007) reported that for structural-grade conventional concrete with no SCM addition, the depth of carbonation is expected to be in the range of 0-7 $\mathrm{mm}$ around 140 days, the higher end representing concrete with high w/c ratio. The range for structural-grade conventional concrete with fly ash is expected to be around 10$15 \mathrm{~mm}$ at 140 days. The samples with bfs are expected to have depths in between the two types of concrete.

The range of carbonation depth measurements after 140 days of exposure for all specimens (GC-MO and GC-VA) showed a range from $5.8 \mathrm{~mm}$ (GC-VA-D-C-G23) to 12 mm (GC-MO-D-F-G41). From Table 8.14, it can be observed that the GC-MO specimens with no SCM replacement, with fly ash, and with bfs were found to have carbonation depths in the range of 6-7 $\mathrm{mm}, 11.7-12 \mathrm{~mm}$ and 7.6-7.1 $\mathrm{mm}$, respectively. From Table 8.15 , it can be observed that the GC-VA specimens with no SCM replacement, with fly ash, and with bfs were found to have carbonation depths in the range of 5.8-6.3 $\mathrm{mm}, 11$ $\mathrm{mm}$ and 7.3-7.8 $\mathrm{mm}$, respectively. From these observations, it can be stated that the depths of all GC-MO and GC-VA specimens at 140 days fall within the expected ranges of structural-grade conventional concrete.

The carbonation coefficient, $D_{c}$, is calculated by fitting Eq.2.6 to the measured carbonation depths at different times. The details of the regression analysis can be found 
in Figures C.15 to C.19 in Appendix C. Tables 8.14 and 8.15 present the carbonation coefficient, $D_{c}$, for GC-MO and GC-VA specimens, respectively. In Table 8.14 , it can be observed that the GC-MO specimens with no SCM replacement, with fly ash, and with bfs were found to have carbonation coefficients in the range of $0.51-0.63 \mathrm{~mm} / \mathrm{day}^{0.5}$, $1.10-1.13 \mathrm{~mm} / \mathrm{day}^{0.5}$ and $0.66-0.73 \mathrm{~mm} / \mathrm{day}^{0.5}$, respectively. From Table 8.15 , it can be observed that the GC-VA specimens with no SCM replacement, with fly ash, and with bfs were found to have carbonation coefficients in the range of $0.47-0.54 \mathrm{~mm} / \mathrm{day}^{0.5}, 0.97-$ $0.99 \mathrm{~mm} /$ day $^{0.5}$ and $0.65-0.67 \mathrm{~mm} /$ day $^{0.5}$, respectively. The carbonation coefficient of GC with SCM is higher than GC with no SCM replacement. The following section will discuss the effect of the SCM.

\subsubsection{The Effect of SCM's}

Figure 8.29 illustrates the carbonation depth of the specimens with respect to the exposure time for specimens with and without SCM addition. It can be observed from this figure, as expected, specimens with no SCM addition showed the lowest level of carbonation. These specimens were followed by the specimens with bfs. Mixes with fly ash had the largest amount of carbonation depth through the 140 days duration of the test. This behaviour can be attributed to the pozzolanic action of the SCM's which consume $\mathrm{Ca}(\mathrm{OH})_{2}$ and lower the alkalinity of GC.

Figure 8.30 shows the comparison of the carbonation coefficient for GC-MO and GC-VA specimens. Similar to previous observations made in Figure 8.29, the carbonation coefficients of specimens with fly ash were the largest $\left(0.97 \mathrm{~mm} / \mathrm{day}^{0.5}\right.$ for GC-VA-D-F-G23 to $1.13 \mathrm{~mm} /$ day $^{0.5}$ for GC-MO-D-F-G41), almost $2 x$ greater than those of specimens with no SCM addition, which had the lowest carbonation coefficients from 
$0.51 \mathrm{~mm} /$ day $^{0.5}$ (for GC-MO-D-C-N0) to $0.63 \mathrm{~mm} /$ day $^{0.5}$ (for GC-MO-D-C-E26). The coefficients for mixes with bfs were in a range between the two types $0.65 \mathrm{~mm} / \mathrm{day}^{0.5}$ for GC-VA-D-B-E17 to $0.73 \mathrm{~mm} /$ day $^{0.5}$ for GC-MO-D-B-E26).

\subsubsection{The Effect of New and Residual Old Mortar}

The rate of carbonation process in concrete depends mainly on the concentration of $\mathrm{Ca}(\mathrm{OH})_{2}$ from the hydration of the OPC as well as on the other compounds such as C-SH. In $\mathrm{GC}$ the $\mathrm{Ca}(\mathrm{OH})_{2}$ can originate from the new mortar and from the residual mortar. The amount of the $\mathrm{Ca}(\mathrm{OH})_{2}$ from the hydration of cement in the new mortar is expected to be higher than that from the hydrated residual old mortar. Hence, the carbonation process in GC is mainly controlled by the total reserve alkalinity from the new mortar and residual old mortar and the cement content. To study these factors, a multi-variable regression analysis was carried out on the results of the carbonation testing presented in this section. Equation 8.7 is the outcome of this analysis:

$$
D_{C}^{G C}=40.5 D_{C}^{N A C} \sqrt{\frac{\left(\frac{V_{R M}}{V_{N M}}\right)}{C}}
$$

where $D_{c}^{G C}$ and $D_{c}^{N A C}$ are the carbonation coefficients of the GC and NAC, respectively; $V_{R M}$ and $V_{N M}$ are the volume of the residual mortar and new mortar $\left(\mathrm{m}^{3}\right)$ in $\mathrm{GC}$, respectively; and $C$ is the new cement content $\left(\mathrm{kg} / \mathrm{m}^{3}\right)$ in GC. The $\mathrm{R}^{2}$ value of the regression analyses was found to be equal to 0.90 . The standard error of estimate for carbonation coefficient was $0.051 \mathrm{~mm} /$ day $^{0.5}$. Figure 8.31 shows the measured and predicted (using Eq.8.7) values of the carbonation coefficient for GC-MO-D-C and GCVA-D-C specimens without SCM's. As it can be observed from this figure, Eq.8.7 predicts the carbonation coefficient of GC quite accurately. 


\subsubsection{The Effect of Mix Design Method}

In general, as illustrated in Figure 8.30a, the carbonation coefficient for GC-MO-DC-G41 specimens $\left(0.52 \mathrm{~mm} / \mathrm{day}^{0.5}\right)$, which were designed with the general mix design method, were lower (about $17 \%$ lower) than the carbonation coefficient of GC-MO-D-CE26 specimens $\left(0.63 \mathrm{~mm} /\right.$ day $\left.^{0.5}\right)$ designed with the equivalent total volume of mortar method. This may be attributed to the lower total cement content (new cement plus the cement from the residual mortar) in the GC-MO specimens designed with the equivalent total volume of mortar method than specimens designed with the general mix design method. Hence, GC-MO specimens with no SCM's and designed with the general mix design method have higher total reserve alkalinity in comparison with the corresponding GC-MO specimens designed with the equivalent total volume of mortar method. This difference in the results of the specimens designed with the two design methods can also be seen for the mixes with bfs ( $\sim 10 \%$ difference). However, for specimens with fly ash there is no difference. This observation can be justified by the fact that specimens with fly ash don't have reserve alkalinity; therefore, the amount of new cement is not a significant factor for these mixes. In other words, the total reserve alkalinity in GC is controlled by pozolanic action from fly ash rather than the cement content.

On the other hand, as illustrated in Figure $8.30 \mathrm{~b}$, the carbonation coefficient for GC-VA specimens which were designed with the general mix design method, were almost the same as the carbonation coefficient of specimens designed with the equivalent total volume of mortar method for all mix types (with and without SCM's). This may be attributed to the residual mortar: the TRMC values of the specimens designed with the general mix design and equivalent total volume of mortar methods are $23 \%$ and $17 \%$, 
respectively. Therefore $\mathrm{Ca}(\mathrm{OH})_{2}$ from the residual mortar will be higher in the former mixes than that of the later ones. This contribution from residual mortar compensates the larger new cement content $\left(9 \mathrm{~kg} / \mathrm{m}^{3}\right.$ higher $)$ of the mixes designed with the equivalent total volume of mortar method; hence the total amount of $\mathrm{Ca}(\mathrm{OH})_{2}$ available for carbonation will be very close in the two mix types.

Since the total volume of mortar in the specimens designed with the equivalent total volume of mortar method and the ones designed with the conventional method with no RCA addition (NAC) are the same, these two groups of specimens were also compared. From Figure 8.30a, it can be observed that the carbonation coefficient for GC-MO-D-CE26 specimens $\left(0.63 \mathrm{~mm} /\right.$ day $\left.^{0.5}\right)$ designed with equivalent total of volume method is higher than GC-MO-D-C-0 $\left(0.51 \mathrm{~mm} / \mathrm{day}^{0.5}\right)$. Since these two mixes have the same amount of total volume of mortar, this difference can be attributed to the lower total cement content in GC-MO-D-C-E26 specimens in comparison to GC-MO-D-C-NO specimens. The new cement contents of the GC-MO-D-C-E26 and GC-MO-D-C-NO specimens were $335 \mathrm{~kg} / \mathrm{m}^{3}$ and $430 \mathrm{~kg} / \mathrm{m}^{3}\left(95 \mathrm{~kg} / \mathrm{m}^{3}\right.$ difference), respectively. The addition of $\mathrm{Ca}(\mathrm{OH})_{2}$ available for carbonation from the residual mortar will not be adequate to compensate the difference in the new cement contents. Hence, the reserve alkalinity in the GC-MO-D-C-E26 specimens is lower than GC-MO-D-C-0 specimens.

Contrary to the GC-MO specimens, from Figure $8.30 \mathrm{~b}$, it can be observed that that the carbonation coefficient for GC-VA-D-C-E17 specimens $\left(0.51 \mathrm{~mm} / \mathrm{day}^{0.5}\right)$ designed with equivalent total of volume method is very close to that of GC-VA-D-C-N0 $(0.54$ $\mathrm{mm} / \mathrm{day}^{0.5}$ ). This can also be explained by the same reasoning regarding the effect of the reserve alkalinity described above. The new cement contents of the GC-VA-D-C-E17 and 
GC-VA-D-C-N0 specimens were $358 \mathrm{~kg} / \mathrm{m}^{3}$ and $424 \mathrm{~kg} / \mathrm{m}^{3}\left(66 \mathrm{~kg} / \mathrm{m}^{3}\right.$ difference), respectively, a difference smaller than the one observed in GC-MO mixes. It is postulated that the contribution of the $\mathrm{Ca}(\mathrm{OH})_{2}$ from the residual mortar compensates for this difference in new cement content; hence the reserve alkalinity of both mixes will be similar, resulting in close values for the diffusion coefficient.

\subsubsection{Summary of the Carbonation Test Results}

- The carbonation depths of GC with and without SCM's fall in the expected range for structural-grade conventional concrete. GC specimens with no SCM addition showed the lowest level of carbonation; these specimens were followed by the specimens with bfs. On the other hand, mixes with fly ash had the largest amount of carbonation depth and coefficient (low resistance to carbonation).

- The investigation of the results of the specimens designed with different mix design methods showed that the main factor affecting the carbonation of GC is the total reserve alkalinity (alkalinity from hydration of new cement content and residual old mortar. Those specimens with the high total cement content (i.e., total reserve alkalinity) have been found to give higher resistance to carbonation.

- The effect of mix design method on the carbonation of GC is not significant when fly ash is used. Therefore, when fly ash is used, both general mix design and equivalent total volume of mortar methods can be used for designing the mixes.

- When fly ash is not used, the carbonation of GC produced with RCA with high RMC (e.g. $>40 \%$ as in the case of RCA-MO) was found to be higher in mixes designed with the equivalent total volume of mortar method. Therefore, for these types of RCA, the general mix design method may be preferred. 
- When fly ash is not used, the carbonation of GC produced with RCA with low RMC (e.g. $<25 \%$ as in the case of RCA-VA) was found to be similar in all mixes designed with both mix design methods. Therefore, for these types of RCA, both methods may be used. 
Table 8.1: Mix design proportions of GC-MO mixes $\left(\mathrm{kg} / \mathrm{m}^{3}\right)$

\begin{tabular}{|c|c|c|c|c|}
\hline \multirow{3}{*}{ Materials } & \multicolumn{3}{|c|}{ RCA (\%)/ TRMC (\%) } & \multirow{3}{*}{ Sample Designation } \\
\hline & $100 / 41$ & $63 / 26$ & 0 & \\
\hline & G41 & E26 & No & \\
\hline $\mathrm{C}\left(\mathrm{kg} / \mathrm{m}^{3}\right)$ & 349 & 335 & 430 & \multirow{10}{*}{ GC-MO-D-C } \\
\hline bfs $\left(\mathrm{kg} / \mathrm{m}^{3}\right)$ & 0 & 0 & 0 & \\
\hline fly-ash $\left(\mathrm{kg} / \mathrm{m}^{3}\right)$ & 0 & 0 & 0 & \\
\hline $\mathrm{W}\left(\mathrm{kg} / \mathrm{m}^{3}\right)$ & 156 & 151 & 193 & \\
\hline $\mathrm{OVA}^{1}\left(\mathrm{~kg} / \mathrm{m}^{3}\right)$ & 0 & 414 & 835 & \\
\hline $\operatorname{RCA}^{2}\left(\mathrm{~kg} / \mathrm{m}^{3}\right)$ & 792 & 720 & 0 & \\
\hline $\operatorname{Sand}^{3}\left(\mathrm{~kg} / \mathrm{m}^{3}\right)$ & 888 & 630 & 808 & \\
\hline $\mathrm{AEA}^{4}(\mathrm{ml})$ & 35 & 33 & 86 & \\
\hline $\mathrm{WRA}^{5}\left(\mathrm{ml} / \mathrm{m}^{3}\right)$ & 1396 & 1005 & None & \\
\hline Mortar Volume $\left(\mathrm{m}^{3}\right)$ & 0.83 & 0.70 & 0.69 & \\
\hline $\mathrm{C}\left(\mathrm{kg} / \mathrm{m}^{3}\right)$ & 262 & 251 & & \multirow{10}{*}{ GC-MO-D-F } \\
\hline bfs $\left(\mathrm{kg} / \mathrm{m}^{3}\right)$ & 0 & 0 & & \\
\hline fly-ash ${ }^{6}\left(\mathrm{~kg} / \mathrm{m}^{3}\right)$ & 87 & 84 & & \\
\hline $\mathrm{W}\left(\mathrm{kg} / \mathrm{m}^{3}\right)$ & 157 & 151 & & \\
\hline $\operatorname{OVA}^{1}\left(\mathrm{~kg} / \mathrm{m}^{3}\right)$ & 0 & 414 & & \\
\hline $\operatorname{RCA}^{2}\left(\mathrm{~kg} / \mathrm{m}^{3}\right)$ & 792 & 720 & & \\
\hline $\operatorname{Sand}^{3}\left(\mathrm{~kg} / \mathrm{m}^{3}\right)$ & 888 & 630 & & \\
\hline $\mathrm{AEA}^{4}(\mathrm{ml})$ & 209 & 201 & & \\
\hline $\operatorname{WRA}^{5}\left(\mathrm{ml} / \mathrm{m}^{3}\right)$ & None & None & & \\
\hline Mortar Volume $\left(\mathrm{m}^{3}\right)$ & 0.85 & 0.72 & & \\
\hline $\mathrm{C}\left(\mathrm{kg} / \mathrm{m}^{3}\right)$ & 227 & 218 & & \multirow{10}{*}{ GC-MO-D-B } \\
\hline $\mathrm{bfs}^{7}\left(\mathrm{~kg} / \mathrm{m}^{3}\right)$ & 122 & 117 & & \\
\hline fly-ash $\left(\mathrm{kg} / \mathrm{m}^{3}\right)$ & 0 & 0 & & \\
\hline $\mathrm{W}\left(\mathrm{kg} / \mathrm{m}^{3}\right)$ & 156 & 149 & & \\
\hline $\mathrm{OVA}^{1}\left(\mathrm{~kg} / \mathrm{m}^{3}\right)$ & 0 & 414 & & \\
\hline $\operatorname{RCA}^{2}\left(\mathrm{~kg} / \mathrm{m}^{3}\right)$ & 792 & 720 & & \\
\hline $\operatorname{Sand}^{3}\left(\mathrm{~kg} / \mathrm{m}^{3}\right)$ & 888 & 630 & & \\
\hline $\mathrm{AEA}^{4}(\mathrm{ml})$ & 35 & 33 & & \\
\hline $\mathrm{WRA}^{5}\left(\mathrm{ml} / \mathrm{m}^{3}\right)$ & 523 & 1339 & & \\
\hline Mortar Volume $\left(\mathrm{m}^{3}\right)$ & 0.83 & 0.70 & & \\
\hline
\end{tabular}

${ }^{1}$ OVA used for GC-MO mixes is limestone

${ }^{2} \mathrm{RCA}$ used for GC-MO mixes is RCA-MO

${ }^{3}$ Cantley (Quebec) natural sand derived from granite

${ }^{4}$ AEA: air entraining agent Micro Air 940.

${ }^{5}$ WRA: water reducing agent low range WRA-75.

${ }^{6}$ fly-ash: fly-ash type CSA CI from Sundance (Alberta)

${ }^{7}$ bfs: blast furnace slag Grade 80

G: the general mix design method;

E: the equivalent total volume of mortar method;

$\mathrm{N}$ : NAC (conventional concrete) 
Table 8.2: Mix design proportions of GC-VA mixes $\left(\mathrm{kg} / \mathrm{m}^{3}\right)$

\begin{tabular}{|c|c|c|c|c|}
\hline \multirow{3}{*}{ Materials } & \multicolumn{3}{|c|}{ RCA (\%) / TRMC (\%) } & \multirow{3}{*}{ Sample Designation } \\
\hline & $100 / 23$ & $74 / 17$ & $\mathbf{0}$ & \\
\hline & G23 & E17 & N0 & \\
\hline $\mathrm{C}\left(\mathrm{kg} / \mathrm{m}^{3}\right)$ & 349 & $\overline{358}$ & 424 & \multirow{10}{*}{ GC-VA-D-C } \\
\hline $\mathrm{bfs}\left(\mathrm{kg} / \mathrm{m}^{3}\right)$ & 0 & 0 & 0 & \\
\hline fly-ash $\left(\mathrm{kg} / \mathrm{m}^{3}\right)$ & 0 & 0 & 0 & \\
\hline $\mathrm{W}\left(\mathrm{kg} / \mathrm{m}^{3}\right)$ & 156 & 160 & 191 & \\
\hline $\mathrm{OVA}^{1}\left(\mathrm{~kg} / \mathrm{m}^{3}\right)$ & 0 & 281 & 900 & \\
\hline $\operatorname{RCA}^{2}\left(\mathrm{~kg} / \mathrm{m}^{3}\right)$ & 867 & 813 & 0 & \\
\hline $\operatorname{Sand}^{3}\left(\mathrm{~kg} / \mathrm{m}^{3}\right)$ & 857 & 645 & 763 & \\
\hline $\mathrm{AEA}^{4}(\mathrm{ml})$ & 35 & 36 & 85 & \\
\hline $\mathrm{WRA}^{5}\left(\mathrm{ml} / \mathrm{m}^{3}\right)$ & 1047 & 1075 & None & \\
\hline Mortar Volume $\left(\mathrm{m}^{3}\right)$ & 0.77 & 0.67 & 0.67 & \\
\hline $\mathrm{C}\left(\mathrm{kg} / \mathrm{m}^{3}\right)$ & 262 & 269 & & \multirow{10}{*}{ GC-VA-D-F } \\
\hline bfs $\left(\mathrm{kg} / \mathrm{m}^{3}\right)$ & 0 & 0 & & \\
\hline fly-ash ${ }^{6}\left(\mathrm{~kg} / \mathrm{m}^{3}\right)$ & 87 & 90 & & \\
\hline $\mathrm{W}\left(\mathrm{kg} / \mathrm{m}^{3}\right)$ & 157 & 161 & & \\
\hline $\operatorname{OVA}^{1}\left(\mathrm{~kg} / \mathrm{m}^{3}\right)$ & 0 & 281 & & \\
\hline $\operatorname{RCA}^{2}\left(\mathrm{~kg} / \mathrm{m}^{3}\right)$ & 867 & 813 & & \\
\hline $\operatorname{Sand}^{3}\left(\mathrm{~kg} / \mathrm{m}^{3}\right)$ & 857 & 645 & & \\
\hline $\operatorname{AEA}^{4}(\mathrm{ml})$ & 209 & 215 & & \\
\hline $\mathrm{WRA}^{5}\left(\mathrm{ml} / \mathrm{m}^{3}\right)$ & None & None & & \\
\hline Mortar Volume $\left(\mathrm{m}^{3}\right)$ & 0.77 & 0.69 & & \\
\hline $\mathrm{C}\left(\mathrm{kg} / \mathrm{m}^{3}\right)$ & 227 & 233 & & \multirow{10}{*}{ GC-VA-D-B } \\
\hline $\mathrm{bfs}^{7}\left(\mathrm{~kg} / \mathrm{m}^{3}\right)$ & 122 & 125 & & \\
\hline fly-ash $\left(\mathrm{kg} / \mathrm{m}^{3}\right)$ & 0 & 0 & & \\
\hline $\mathrm{W}\left(\mathrm{kg} / \mathrm{m}^{3}\right)$ & 156 & 159 & & \\
\hline $\operatorname{OVA}^{1}\left(\mathrm{~kg} / \mathrm{m}^{3}\right)$ & 0 & 281 & & \\
\hline $\operatorname{RCA}^{2}\left(\mathrm{~kg} / \mathrm{m}^{3}\right)$ & 867 & 813 & & \\
\hline $\operatorname{Sand}^{3}\left(\mathrm{~kg} / \mathrm{m}^{3}\right)$ & 857 & 645 & & \\
\hline $\operatorname{AEA}^{4}(\mathrm{ml})$ & 35 & 36 & & \\
\hline $\mathrm{WRA}^{5}\left(\mathrm{ml} / \mathrm{m}^{3}\right)$ & 1047 & 1792 & & \\
\hline Mortar Volume $\left(\mathrm{m}^{3}\right)$ & 0.77 & 0.68 & & \\
\hline
\end{tabular}

OVA used for GC-VA mixes is river gravel

${ }^{2} \mathrm{RCA}$ used for GC-VA mixes is RCA-VA

${ }^{3}$ Cantley (Quebec) natural sand derived from granite

${ }^{4}$ AEA: air entraining agent Micro Air 940.

${ }^{5}$ WRA: water reducing agent low range WRA-75.

${ }^{6}$ fly-ash: fly-ash type CSA CI from Sundance (Alberta)

${ }^{7}$ bfs: blast furnace slag Grade 80

G: the general mix design method;

$E$ : the equivalent total volume of mortar method;

$\mathrm{N}$ : NAC (conventional concrete) 
Table 8.3: Fresh concrete properties for GC-MO mixes

\begin{tabular}{||c|c|c|c|c||}
\hline \multirow{2}{*}{ Fresh Property } & \multicolumn{2}{|c|}{ RCA (\%)/ TRMC (\%) } & \multirow{2}{*}{ Sample Designation } \\
\cline { 2 - 4 } & $\mathbf{1 0 0 / 4 1}$ & $\mathbf{6 3 / 2 6}$ & $\mathbf{0}$ & \\
\cline { 2 - 5 } & $\mathbf{G 4 1}$ & $\mathbf{E 2 6}$ & $\mathbf{N 0}$ & \\
\hline Slump (mm) & 70 & 105 & 175 & \multirow{2}{*}{ GC-MO-D-C } \\
Air entrained (\%) & 6.9 & 6.0 & 6.3 & \\
Fresh Density $\left(\mathrm{kg} / \mathrm{m}^{3}\right)$ & 2262 & 2305 & 2332 & \\
\hline Slump $(\mathrm{mm})$ & 130 & 120 & & \multirow{2}{*}{ GC-MO-D-F } \\
Air entrained $(\%)$ & 7.4 & 5.7 & & \\
Fresh Density $\left(\mathrm{kg} / \mathrm{m}^{3}\right)$ & 2226 & 2291 & & \\
\hline Slump $(\mathrm{mm})$ & 55 & 80 & & \multirow{2}{*}{ GC-MO-D-B } \\
Air entrained $(\%)$ & 6.0 & 5.7 & & \\
Fresh Density $\left(\mathrm{kg} / \mathrm{m}^{3}\right)$ & 2281 & 2306 & & \\
\hline
\end{tabular}

Table 8.4: Fresh concrete properties for GC-VA mixes

\begin{tabular}{|c|c|c|c|c|}
\hline \multirow{2}{*}{ Fresh Property } & \multicolumn{2}{|c|}{ RCA (\%)/ TRMC (\%) } & \multirow{2}{*}{ Sample Designation } \\
\cline { 2 - 4 } & $\mathbf{1 0 0 / 2 3}$ & $\mathbf{7 4 / 1 7}$ & $\mathbf{0}$ & \\
\cline { 2 - 4 } & $\mathbf{G 2 3}$ & $\mathbf{E 1 7}$ & $\mathbf{N 0}$ & \\
\hline Slump (mm) & 70 & 140 & 210 & \multirow{2}{*}{ GC-VA-D-C } \\
Air entrained (\%) & 7.4 & 6.0 & 6.3 & \\
Fresh Density $\left(\mathrm{kg} / \mathrm{m}^{3}\right)$ & 2306 & 2328 & 2339 & \\
\hline Slump $(\mathrm{mm})$ & 90 & 140 & & \multirow{2}{*}{ GC-VA-D-F } \\
Air entrained $(\%)$ & 6.0 & 5.5 & & \\
Fresh Density $\left(\mathrm{kg} / \mathrm{m}^{3}\right)$ & 2291 & 2321 & & \\
\hline Slump $(\mathrm{mm})$ & 110 & 150 & & \multirow{2}{*}{ GC-VA-D-B } \\
Air entrained $(\%)$ & 7.1 & 6.8 & & \\
Fresh Density $\left(\mathrm{kg} / \mathrm{m}^{3}\right)$ & 2286 & 2316 & & \\
\hline
\end{tabular}


Table 8.5: Hardened concrete properties for GC-MO specimens

\begin{tabular}{|c|c|c|c|c|c|c|c|c|c|c|}
\hline \multirow{4}{*}{ Sample \# } & \multicolumn{9}{|c|}{ RCA (\%) / TRMC (\%) } & \multirow{4}{*}{$\begin{array}{c}\text { Specimen } \\
\text { Designation }\end{array}$} \\
\hline & \multirow{2}{*}{\multicolumn{3}{|c|}{$\frac{100 / 41}{G 41}$}} & \multirow{2}{*}{\multicolumn{3}{|c|}{$\frac{63 / 26}{E 26}$}} & \multirow{2}{*}{\multicolumn{3}{|c|}{$\frac{0 / 0}{\text { No }}$}} & \\
\hline & & & & & & & & & & \\
\hline & 1 & 2 & 3 & 1 & 2 & 3 & 1 & 2 & 3 & \\
\hline fe (MPa) & 42 & 41.3 & 42.2 & 40.6 & 39.8 & 37.3 & 36.7 & 38.4 & 35.5 & \\
\hline Average & & 41.8 & & & 39.2 & & & 36.9 & & \\
\hline Std. Dev. & & 0.5 & & & 1.7 & & & 1.5 & & \\
\hline $\mathrm{CoV}(\%)$ & & 1.2 & & & 4.3 & & & 4.1 & & GC-MO-D-C \\
\hline Density $\left(\mathrm{kg} / \mathrm{m}^{3}\right)$ & 2264 & 2266 & 2263 & 2293 & 2298 & 2295 & 2301 & 2289 & 2310 & \\
\hline A verage & & 2264 & & & 2295 & & & 2300 & & \\
\hline Std. Dev. & & 1.8 & & & 2.6 & & & 10.1 & & \\
\hline $\operatorname{CoV}(\%)$ & & 0.1 & & & 0.1 & & & 0.4 & & \\
\hline fc (MPa) & 34.5 & 32.8 & 34.6 & 35.2 & 33.3 & 34.2 & & & & \\
\hline Average & & 34.0 & & & 34.2 & & & & & \\
\hline Std. Dev. & & 1.0 & & & 1.0 & & & & & \\
\hline $\operatorname{CoV}(\%)$ & & 2.9 & & & 2.9 & & & & & \\
\hline $\mathrm{fc}^{56}(\mathrm{MPa})$ & 39.5 & 37.5 & - & 39.9 & 41.9 & - & & & & \\
\hline Average & & 38.5 & & & 40.9 & & & & & GC-MO-D-F \\
\hline Std. Dev. & & 1.4 & & & 1.4 & & & & & \\
\hline $\operatorname{CoV}(\%)$ & & 3.7 & & & 3.4 & & & & & \\
\hline Density $\left(\mathrm{kg} / \mathrm{m}^{3}\right)$ & 2215 & 2221 & 2216 & 2286 & 2300 & 2292 & & & & \\
\hline Average & & 2217 & & & 2293 & & & & & \\
\hline Std. Dev. & & 3.2 & & & 6.6 & & & & & \\
\hline $\mathrm{CoV}(\%)$ & & 0.1 & & & 0.3 & & & & & \\
\hline fc (MPa) & 42 & 43 & 41 & 41.2 & 41.7 & 42.6 & & & & \\
\hline Average & & 42.0 & & & 41.8 & & & & & \\
\hline Std. Dev. & & 1.0 & & & 0.7 & & & & & \\
\hline $\operatorname{CoV}(\%)$ & & 2.4 & & & 1.7 & & & & & \\
\hline $\mathrm{fc}^{56}(\mathrm{MPa})$ & 42.5 & 47.1 & - & 48.2 & 44.3 & - & & & & \\
\hline Average & & 44.8 & & & 46.3 & & & & & GC-MO-D-B \\
\hline Std. Dev. & & 3.3 & & & 2.8 & & & & & \\
\hline $\operatorname{CoV}(\%)$ & & 7.3 & & & 6.0 & & & & & \\
\hline Density $\left(\mathrm{kg} / \mathrm{m}^{3}\right)$ & 2267 & 2270 & 2270 & 2308 & 2322 & 2327 & & & & \\
\hline Average & & 2269 & & & 2319 & & & & & \\
\hline Std. Dev. & & 1.5 & & & 10.0 & & & & & \\
\hline $\operatorname{CoV}(\%)$ & & 0.1 & & & 0.4 & & & & & \\
\hline
\end{tabular}

$\mathrm{f}_{\mathrm{c}}^{56}: 56$-day compressive strength, 2 specimens were tested. 
Table 8.6: Hardened concrete properties for GC-VA specimens

\begin{tabular}{|c|c|c|c|c|c|c|c|c|c|c|}
\hline \multirow[b]{4}{*}{ Sample \# } & \multicolumn{9}{|c|}{ RCA (\%)/TRMC (\%) } & \multirow{4}{*}{$\begin{array}{c}\text { Specimen } \\
\text { Designation }\end{array}$} \\
\hline & \multirow{2}{*}{\multicolumn{3}{|c|}{$\frac{100 / 23}{G 23}$}} & \multirow{2}{*}{\multicolumn{3}{|c|}{$\begin{array}{c}74 / 17 \\
\mathrm{E} 17 \\
\end{array}$}} & \multirow{2}{*}{\multicolumn{3}{|c|}{$\frac{0 / 0}{\text { No }}$}} & \\
\hline & & & & & & & & & & \\
\hline & 1 & 2 & 3 & 1 & 2 & 3 & 1 & 2 & 3 & \\
\hline fc (MPa) & 41.2 & 39.3 & 38.5 & 41 & 40.2 & 38.9 & 35 & 36.2 & 35.6 & \\
\hline Average & & 39.7 & & & 40.0 & & & 35.6 & & \\
\hline Std. Dev. & & 1.4 & & & 1.1 & & & 0.6 & & \\
\hline $\operatorname{CoV}(\%)$ & & 3.5 & & & 2.8 & & & 1.7 & & GC-VA-D-C \\
\hline Density $\left(\mathrm{kg} / \mathrm{m}^{\mathbf{3}}\right)$ & 2283 & 2278 & 2293 & 2316 & 2328 & 2319 & 2311 & 2317 & 2317 & \\
\hline Average & & 2285 & & & 2321 & & & 2315 & & \\
\hline Std. Dev. & & 7.6 & & & 6.5 & & & 3.1 & & \\
\hline $\operatorname{CoV}(\%)$ & & 0.3 & & & 0.3 & & & 0.1 & & \\
\hline fc (MPa) & 34.3 & 36.1 & 34.7 & 33 & 36.5 & 36.1 & & & & \\
\hline Average & & 35.0 & & & 35.2 & & & & & \\
\hline Std. Dev. & & 0.9 & & & 1.9 & & & & & \\
\hline $\operatorname{CoV}(\%)$ & & 2.6 & & & 5.4 & & & & & \\
\hline $\mathrm{fc}^{56}(\mathrm{MPa})$ & 42.20 & 39.30 & - & 43.5 & 41.1 & - & & & & \\
\hline Average & & 40.8 & & & 42.3 & & & & & GC-VA-D-F \\
\hline Std. Dev. & & 2.1 & & & 1.7 & & & & & \\
\hline $\operatorname{CoV}(\%)$ & & 5.0 & & & 4.0 & & & & & \\
\hline Density $\left(\mathrm{kg} / \mathrm{m}^{3}\right)$ & 2276 & 2277 & 2291 & 2312 & 2289 & 2310 & & & & \\
\hline Average & & 2281 & & & 2304 & & & & & \\
\hline Std. Dev. & & 8.4 & & & 12.6 & & & & & \\
\hline $\operatorname{CoV}(\%)$ & & 0.4 & & & 0.5 & & & & & \\
\hline fc (MPa) & 39.1 & 41.6 & 37.2 & 40.8 & 38.7 & 40.4 & & & & \\
\hline Average & & 39.3 & & & 40.0 & & & & & \\
\hline Std. Dev. & & 2.2 & & & 1.1 & & & & & \\
\hline $\mathrm{CoV}(\%)$ & & 5.6 & & & 2.8 & & & & & \\
\hline $\mathrm{fc}^{56}(\mathrm{MPa})$ & 42.4 & 40.1 & & 43.5 & 41.1 & - & & & & \\
\hline Average & & 41.3 & & & 42.3 & & & & & GC-VA-D-B \\
\hline Std. Dev. & & 1.6 & & & 1.7 & & & & & \\
\hline $\mathrm{CoV}(\%)$ & & 3.9 & & & 4.0 & & & & & \\
\hline Density $\left(\mathrm{kg} / \mathrm{m}^{3}\right)$ & 2273 & 2292 & 2301 & 2332 & 2320 & 2323 & & & & \\
\hline Average & & 2289 & & & 2325 & & & & & \\
\hline Std. Dev. & & 14.4 & & & 6.3 & & & & & \\
\hline $\mathrm{CoV}(\%)$ & & 0.6 & & & 0.3 & & & & & \\
\hline
\end{tabular}

$\mathrm{f}_{\mathrm{c}}^{56}$ : 56-day compressive strength, 2 specimens were tested. 
Table 8.7: Durability factor, pulse velocity, length change after 300 cycles of freeze-andthaw for GC-MO specimens

\begin{tabular}{|c|c|c|c|c|c|c|c|c|c|c|}
\hline \multirow{4}{*}{ Sample \# } & \multicolumn{9}{|c|}{ RCA (\%)/ TRMC (\%) } & \multirow{4}{*}{$\begin{array}{c}\text { Specimen } \\
\text { Designation }\end{array}$} \\
\hline & \multirow{2}{*}{\multicolumn{3}{|c|}{$\frac{100 / 41}{G 41}$}} & \multirow{2}{*}{\multicolumn{3}{|c|}{$\frac{63 / 26}{E 26}$}} & \multirow{2}{*}{\multicolumn{3}{|c|}{$\frac{0 / 0}{\text { No }}$}} & \\
\hline & & & & & & & & & & \\
\hline & 1 & 2 & 3 & 1 & 2 & 3 & 1 & 2 & 3 & \\
\hline$\overline{D F(\%)}$ & 92 & 92 & 92 & 95 & 91 & 98 & $\overline{98}$ & $\overline{95}$ & 96 & \\
\hline Average & & 92 & & & 95 & & & 97 & & \\
\hline Std. Dev. & & 0.4 & & & 3.2 & & & 1.4 & & \\
\hline $\operatorname{CoV}(\%)$ & & 0.5 & & & 3.4 & & & 1.5 & & \\
\hline$P V(\mathrm{~m} / \mathrm{s})$ & 4415 & 4415 & 4375 & 4477 & 4519 & 4466 & 4672 & 4667 & 4718 & \\
\hline Average & & 4402 & & & 4487 & & & 4686 & & GC-MO-D-C \\
\hline Std. Dev. & & 23.2 & & & 27.8 & & & 28.2 & & \\
\hline $\operatorname{CoV}(\%)$ & & 0.5 & & & 0.6 & & & 0.6 & & \\
\hline$L C(\%)$ & 0.013 & 0.010 & 0.013 & 0.022 & 0.033 & 0.025 & 0.005 & 0.007 & 0.010 & \\
\hline Average & & 0.012 & & & 0.027 & & & 0.007 & & \\
\hline Std. Dev. & & 0.002 & & & 0.006 & & & 0.002 & & \\
\hline $\operatorname{CoV}(\%)$ & & 12.8 & & & 21.2 & & & 29.8 & & \\
\hline$D F(\%)$ & 91 & 87 & 89 & 91 & 89 & 91 & & & & \\
\hline Average & & 89 & & & 90 & & & & & \\
\hline Std. Dev. & & 2.1 & & & 1.3 & & & & & \\
\hline $\operatorname{CoV}(\%)$ & & 2.3 & & & 1.5 & & & & & \\
\hline$P V(\mathrm{~m} / \mathrm{s})$ & 4167 & 4162 & 4104 & 4456 & 4400 & 4336 & & & & \\
\hline Average & & 4144 & & & 4397 & & & & & GC-MO-D-F \\
\hline Std. Dev. & & 34.7 & & & 60.3 & & & & & \\
\hline $\operatorname{CoV}(\%)$ & & 0.8 & & & 1.4 & & & & & \\
\hline$L C(\%)$ & 0.015 & 0.018 & 0.011 & 0.020 & 0.032 & 0.032 & & & & \\
\hline Average & & 0.015 & & & 0.028 & & & & & \\
\hline Std. Dev. & & 0.004 & & & 0.007 & & & & & \\
\hline $\operatorname{CoV}(\%)$ & & 25.1 & & & 24.6 & & & & & \\
\hline$D F(\%)$ & 91 & 90 & 89 & 93 & 91 & 92 & & & & \\
\hline Average & & 90 & & & 92 & & & & & \\
\hline Std. Dev. & & 0.8 & & & 1.1 & & & & & \\
\hline $\operatorname{CoV}(\%)$ & & 0.9 & & & 1.1 & & & & & \\
\hline$P V(\mathrm{~m} / \mathrm{s})$ & 4435 & 4466 & 4420 & 4551 & 4572 & 4503 & & & & \\
\hline Average & & 4441 & & & 4542 & & & & & GC-MO-D-B \\
\hline Std. Dev. & & 23.5 & & & 35.6 & & & & & \\
\hline $\operatorname{CoV}(\%)$ & & 0.5 & & & 0.8 & & & & & \\
\hline$L C(\%)$ & 0.018 & 0.026 & 0.019 & 0.021 & 0.029 & 0.022 & & & & \\
\hline Average & & 0.021 & & & 0.024 & & & & & \\
\hline Std. Dev. & & 0.004 & & & 0.004 & & & & & \\
\hline $\operatorname{CoV}(\%)$ & & 19.1 & & & 18.0 & & & & & \\
\hline
\end{tabular}


Table 8.8: Durability factor, pulse velocity, length change after 300 cycles of freeze-andthaw for GC-VA specimens

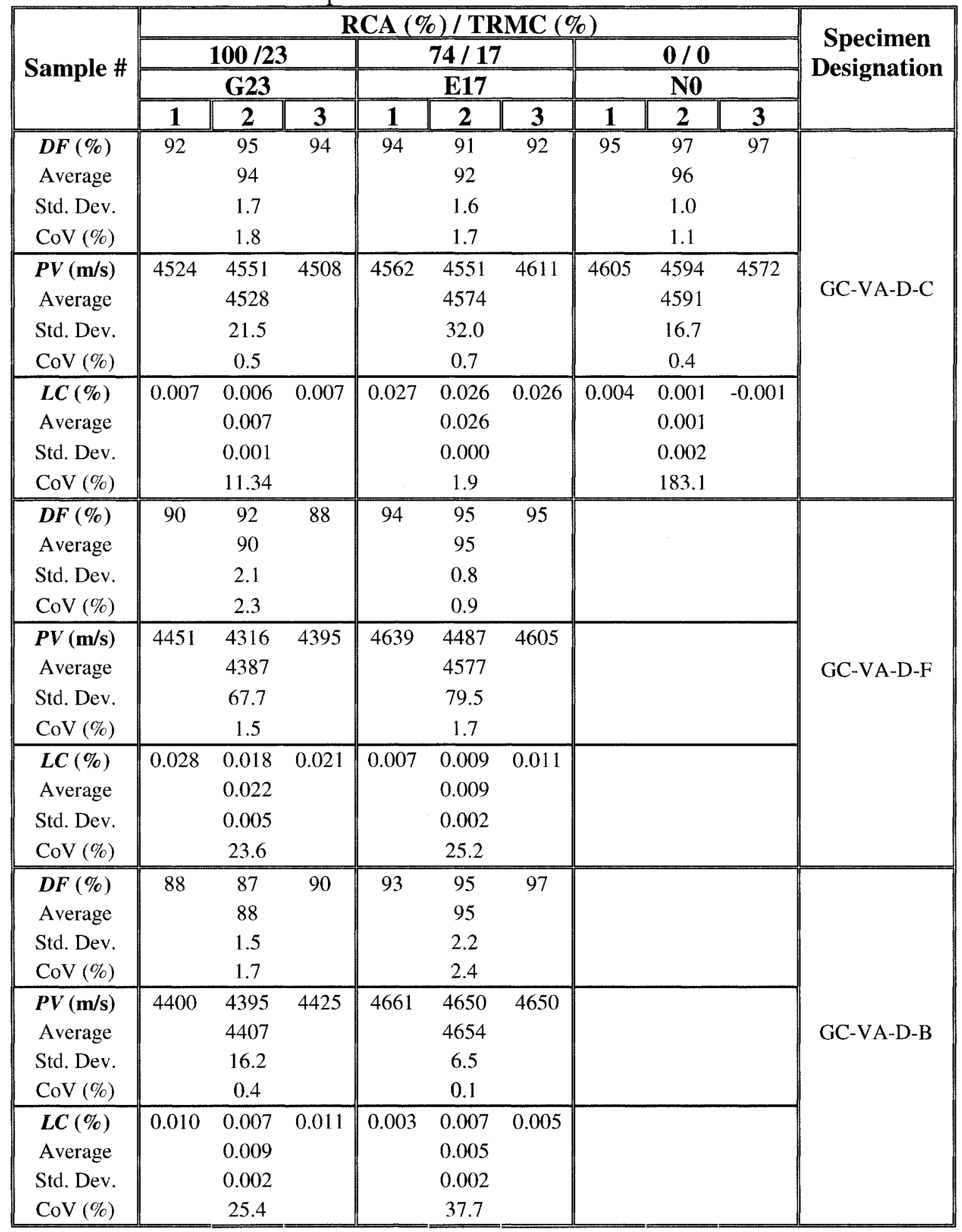


Table 8.9: Results of the acid soluble chloride content determination test (ASTM C 1556) for GC-MO specimens

\begin{tabular}{|c|c|c|c|c|}
\hline \multirow{4}{*}{ Sample \# } & \multicolumn{3}{|c|}{$\operatorname{RCA}(\%) / T R M C(\%)$} & \multirow{4}{*}{$\begin{array}{c}\text { Specimen } \\
\text { Designation }\end{array}$} \\
\hline & \multirow{2}{*}{$\frac{100 / 41}{G 41}$} & \multirow{2}{*}{$\frac{63 / 26}{\mathrm{E} 26}$} & \multirow{2}{*}{$\frac{0 / 0}{\text { No }}$} & \\
\hline & & & & \\
\hline & 2 & 2 & 2 & \\
\hline $\mathrm{C}_{0}(\%)$ & \multirow{2}{*}{$\begin{array}{cc}0.022 & 0.014 \\
0.018\end{array}$} & $\begin{array}{ll}0.027 & 0.017\end{array}$ & $0.014 \quad 0.002$ & \multirow{12}{*}{ GC-MO-D-C } \\
\hline Average & & 0.022 & 0.008 & \\
\hline Std. Dev. & 0.005 & 0.007 & \multirow{2}{*}{$\begin{array}{l}0.008 \\
101.3\end{array}$} & \\
\hline $\operatorname{CoV}(\%)$ & 30.3 & 33.5 & & \\
\hline $\mathrm{C}_{s}(\%)$ & $1.232 \quad 1.243$ & $\begin{array}{ll}1.16 & 1.26\end{array}$ & $0.99 \quad 1.00$ & \\
\hline Average & \multirow{3}{*}{$\begin{array}{c}1.238 \\
0.008 \\
0.6\end{array}$} & \multirow{3}{*}{$\begin{array}{c}1.209 \\
0.068 \\
5.6\end{array}$} & \multirow{3}{*}{$\begin{array}{c}0.996 \\
0.007 \\
0.7 \\
\end{array}$} & \\
\hline Std. Dev. & & & & \\
\hline $\operatorname{CoV}(\%)$ & & & & \\
\hline$D_{a} \times 10^{-12}\left(m^{2} / \mathrm{s}\right)$ & $3.33 \quad 5.56$ & $5.37 \quad 4.36$ & $\begin{array}{ll}5.53 & 5.63\end{array}$ & \\
\hline Average & \multirow{3}{*}{$\begin{array}{l}4.45 \\
1.57 \\
35.4 \\
\end{array}$} & \multirow{3}{*}{$\begin{array}{c}4.87 \\
0.713 \\
14.7 \\
\end{array}$} & \multirow{3}{*}{$\begin{array}{c}5.58 \\
0.070 \\
1.2 \\
\end{array}$} & \\
\hline Std. Dev. & & & & \\
\hline $\operatorname{CoV}(\%)$ & & & & \\
\hline $\mathrm{C}_{0}(\%)$ & \multirow{4}{*}{$\begin{array}{c}0.020 \quad 0.019 \\
0.019 \\
0.001 \\
4.4\end{array}$} & \multirow{4}{*}{$\begin{array}{c}0.014 \quad 0.025 \\
0.019 \\
0.008 \\
39.8\end{array}$} & & \multirow{12}{*}{ GC-MO-D-F } \\
\hline Average & & & & \\
\hline Std. Dev. & & & & \\
\hline $\operatorname{CoV}(\%)$ & & & & \\
\hline $\mathrm{C}_{\mathrm{s}}(\%)$ & $1.46 \quad 1.52$ & $1.54 \quad 1.61$ & & \\
\hline Average & 1.490 & \multirow{3}{*}{$\begin{array}{c}1.576 \\
0.050 \\
3.2 \\
\end{array}$} & & \\
\hline Std. Dev. & \multirow{2}{*}{$\begin{array}{c}0.044 \\
3.0\end{array}$} & & & \\
\hline $\operatorname{CoV}(\%)$ & & & & \\
\hline $\mathrm{D}_{\mathrm{a}} \times \mathbf{x 1 0}^{-12}\left(\mathrm{~m}^{2} / \mathrm{s}\right)$ & $3.76 \quad 3.91$ & $2.11 \quad 2.312$ & & \\
\hline Average & 3.83 & 2.21 & & \\
\hline Std. Dev. & 0.106 & 0.144 & & \\
\hline $\mathrm{CoV}(\%)$ & 2.8 & 6.5 & & \\
\hline $\mathrm{C}_{0}(\%)$ & $0.027 \quad 0.019$ & $0.018 \quad 0.018$ & & \\
\hline Average & 0.023 & 0.018 & & \\
\hline Std. Dev. & 0.005 & 0.000 & & \\
\hline $\mathrm{CoV}$ & 23.4 & 0.1 & & \\
\hline $\mathrm{C}_{\mathrm{s}}(\%)$ & $\begin{array}{ll}1.57 & 1.53\end{array}$ & $1.76 \quad 1.66$ & & \\
\hline Average & 1.553 & 1.710 & & GC-MO-D-B \\
\hline Std. Dev. & 0.026 & 0.071 & & \\
\hline $\operatorname{CoV}(\%)$ & 1.7 & 4.2 & & \\
\hline $\mathrm{D}_{\mathrm{a}} \times 10^{-12}\left(\mathrm{~m}^{2} / \mathrm{s}\right)$ & $\begin{array}{ll}2.27 & 2.04\end{array}$ & $\begin{array}{ll}1.65 & 1.95\end{array}$ & & \\
\hline Average & 2.15 & 1.80 & & \\
\hline Std. Dev. & 0.162 & 0.207 & & \\
\hline $\mathrm{CoV}(\%)$ & 7.5 & 11.5 & & \\
\hline
\end{tabular}


Table 8.10: Results of the acid soluble chloride content determination test (ASTM C 1556) for GC-VA specimens

\begin{tabular}{|c|c|c|c|c|}
\hline \multirow[b]{4}{*}{ Sample \# } & \multicolumn{3}{|c|}{ RCA (\%) / TRMC (\%) } & \multirow{4}{*}{$\begin{array}{c}\text { Specimen } \\
\text { Designation }\end{array}$} \\
\hline & \multirow{2}{*}{$\frac{100 / 23}{G 23}$} & \multirow{2}{*}{$74 / 17$} & \multirow{2}{*}{$\begin{array}{l}0 / 0 \\
\text { No }\end{array}$} & \\
\hline & & & & \\
\hline & 1 & 1 & 1 & \\
\hline $\mathrm{C}_{0}(\%)$ & \multirow{2}{*}{$\begin{array}{cc}0.007 & 0.009 \\
0.008\end{array}$} & \multirow{2}{*}{$\begin{array}{cc}0.007 & 0.004 \\
0.006\end{array}$} & $0.010 \quad 0.005$ & \multirow{12}{*}{ GC-VA-D-C } \\
\hline Average & & & 0.007 & \\
\hline Std. Dev. & \multirow{2}{*}{$\begin{array}{c}0.001 \\
12.6\end{array}$} & \multirow{2}{*}{$\begin{array}{c}0.002 \\
28.8\end{array}$} & \multirow{2}{*}{$\begin{array}{c}0.004 \\
53.2\end{array}$} & \\
\hline $\mathrm{CoV}(\%)$ & & & & \\
\hline $\mathrm{C}_{\mathrm{s}}(\%)$ & \multirow{2}{*}{$\begin{array}{c}1.12 \quad 1.12 \\
1.120\end{array}$} & \multirow{2}{*}{$1.71{ }_{1.703}^{1.69}$} & $\begin{array}{ll}1.08 & 1.29\end{array}$ & \\
\hline Average & & & 1.183 & \\
\hline Std. Dev. & 0.003 & 0.014 & 0.152 & \\
\hline $\mathrm{CoV}(\%)$ & 0.3 & 0.8 & 12.8 & \\
\hline $\mathrm{D}_{\mathrm{a}} \times 10^{-12}\left(\mathrm{~m}^{2} / \mathrm{s}\right)$ & $\begin{array}{ll}4.24 & 3.45\end{array}$ & $\begin{array}{ll}4.57 \quad 3.95\end{array}$ & $4.84 \quad 5.16$ & \\
\hline Average & \multirow{3}{*}{$\begin{array}{c}3.84 \\
0.557 \\
14.5\end{array}$} & \multirow{3}{*}{$\begin{array}{r}4.26 \\
0.439 \\
10.3 \\
\end{array}$} & \multirow{3}{*}{$\begin{array}{c}5.00 \\
0.222 \\
4.4\end{array}$} & \\
\hline Std. Dev. & & & & \\
\hline $\operatorname{CoV}(\%)$ & & & & \\
\hline $\mathrm{C}_{0}(\%)$ & \multirow{4}{*}{$\begin{array}{c}0.003 \quad 0.009 \\
0.006 \\
0.004 \\
68.3\end{array}$} & \multirow{4}{*}{$\begin{array}{c}0.0096 \quad 0.0069 \\
0.008 \\
0.002 \\
22.5\end{array}$} & & \\
\hline Average & & & & \\
\hline Std. Dev. & & & & \\
\hline $\mathrm{CoV}(\%)$ & & & & \\
\hline $\mathrm{C}_{\mathrm{s}}(\%)$ & $\begin{array}{ll}1.36 & 1.50\end{array}$ & $1.658 \quad 1.634$ & & \\
\hline Average & 1.432 & 1.646 & & GC-VA-D-F \\
\hline Std. Dev. & 0.101 & 0.018 & & \\
\hline $\mathrm{CoV}(\%)$ & 7.1 & 1.1 & & \\
\hline $\mathbf{D}_{\mathrm{a}} \times 10^{-12}\left(\mathrm{~m}^{2} / \mathrm{s}\right)$ & $\begin{array}{ll}2.87 & 2.37\end{array}$ & $\begin{array}{ll}2.39 & 2.73\end{array}$ & & \\
\hline Average & 2.62 & 2.56 & & \\
\hline Std. Dev. & 0.353 & 0.245 & & \\
\hline $\operatorname{CoV}(\%)$ & 13.4 & 9.6 & & \\
\hline $\mathrm{C}_{0}(\%)$ & $0.006 \quad 0.015$ & $0.009 \quad 0.012$ & & \\
\hline Average & 0.011 & 0.011 & & \\
\hline Std. Dev. & 0.007 & 0.001 & & \\
\hline $\mathrm{CoV}(\%)$ & 64.4 & 13.6 & & \\
\hline $\mathrm{C}_{\mathrm{s}}(\%)$ & $1.30 \quad 1.44$ & $\begin{array}{ll}1.23 & 1.46\end{array}$ & & \\
\hline Average & 1.374 & 1.344 & & GC-VA-D-B \\
\hline Std. Dev. & 0.099 & 0.161 & & \\
\hline $\operatorname{CoV}(\%)$ & 7.2 & 11.9 & & \\
\hline $\mathbf{D}_{\mathrm{a}} \times 10^{-12}\left(\mathrm{~m}^{2} / \mathrm{s}\right)$ & $\begin{array}{ll}2.076 & 1.82\end{array}$ & 1.94 & & \\
\hline Average & 1.95 & 1.85 & & \\
\hline Std. Dev. & 0.176 & 0.129 & & \\
\hline $\mathrm{CoV}(\%)$ & 9.1 & 7.0 & & \\
\hline
\end{tabular}


Table 8.11: Charge passed (Coulombs) for GC-MO specimens

\begin{tabular}{|c|c|c|c|c|c|c|c|c|c|c|}
\hline \multirow[b]{4}{*}{ Sample \# } & \multicolumn{9}{|c|}{ RCA (\%) / TRMC (\%) } & \multirow{4}{*}{$\begin{array}{c}\text { Specimen } \\
\text { Designation }\end{array}$} \\
\hline & \multirow{2}{*}{\multicolumn{3}{|c|}{$\frac{100 / 41}{G 41}$}} & \multirow{2}{*}{\multicolumn{3}{|c|}{$\frac{63 / 26}{E 26}$}} & \multirow{2}{*}{\multicolumn{3}{|c|}{$\begin{array}{l}0 / 0 \\
\text { No }\end{array}$}} & \\
\hline & & & & & & & & & & \\
\hline & 1 & 2 & 3 & 1 & 2 & $\overline{3}$ & 1 & 2 & $\overline{3}$ & \\
\hline Charge passed & 5301 & 5081 & 5050 & 2183 & 4389 & 44112 & 3509 & 3051 & $\overline{3389}$ & \\
\hline Average & & 5144 & & & 3561 & & & 3316 & & GC-MO-D-C \\
\hline Std. Dev. & & 137 & & & 196 & & & 237 & & \\
\hline $\operatorname{CoV}(\%)$ & & 2.7 & & & 5.5 & & & 7.2 & & \\
\hline $\mathrm{Cl}^{-}$Penetrability & & High & & & Toderat & & & Moderate & & \\
\hline Charge passed & 2057 & 1894 & - & 1745 & 1134 & 1742 & & & & \\
\hline Average & & 1976 & & & 1744 & & & & & $G C:$ \\
\hline Std. Dev. & & 115 & & & 2 & & & & & UC-WIU-D-F \\
\hline $\operatorname{CoV}(\%)$ & & 5.8 & & & 0.1 & & & & & \\
\hline $\mathrm{Cl}$ Penetrability & & Low & & & Low & & & & & \\
\hline Charge passed & 2125 & 1919 & 2134 & 2169 & 2091 & 1172 & & & & \\
\hline Average & & 2059 & & & 1811 & & & & & GC-MO-D-B \\
\hline Std. Dev. & & 122 & & & 554 & & & & & U-1VIU-D-D \\
\hline $\operatorname{CoV}(\%)$ & & 5.9 & & & 30.6 & & & & & \\
\hline $\mathrm{Cl}^{-}$Penetrability & & Moderate & & & Low & & & & & \\
\hline
\end{tabular}

Table 8.12: Charge passed (Coulombs) for GC-VA specimens

\begin{tabular}{|c|c|c|c|c|c|c|c|c|c|c|}
\hline \multirow{4}{*}{ Sample \# } & \multicolumn{9}{|c|}{ RCA (\%)/ TRMC (\%) } & \multirow{4}{*}{$\begin{array}{l}\text { Specimen } \\
\text { Designation }\end{array}$} \\
\hline & \multirow{2}{*}{\multicolumn{3}{|c|}{$\frac{100 / 23}{G 23}$}} & \multirow{2}{*}{\multicolumn{3}{|c|}{$\frac{74 / 17}{\text { E17 }}$}} & \multirow{2}{*}{\multicolumn{3}{|c|}{$\frac{0 / 0}{\text { No }}$}} & \\
\hline & & & & & & & & & & \\
\hline & 1 & 2 & 3 & 1 & 2 & 3 & 1 & 2 & 3 & \\
\hline Charge passed & 3345 & 3395 & 4070 & 2731 & 315 & 2974 & 4307 & 3021 & $\ldots$ & \\
\hline Average & & 3603 & & & 295 & & & 3664 & & GC-VA-D-C \\
\hline Std. Dev. & & 405 & & & 21 & & & 909 & & \\
\hline $\operatorname{CoV}(\%)$ & & 11.2 & & & 7.2 & & & 24.8 & & \\
\hline $\mathrm{Cl}^{-}$Penetrability & & Toderat & & & Mode & & & Moderate & & \\
\hline Charge passed & 1323 & 1569 & 1015 & 1519 & 138 & 1336 & & & & \\
\hline Average & & 1302 & & & 141 & & & & & \\
\hline Std. Dev. & & 278 & & & 95 & & & & & GC-VA-D-F \\
\hline $\operatorname{CoV}(\%)$ & & 21.3 & & & 6.7 & & & & & \\
\hline $\mathrm{Cl}^{-}$Penetrability & & Low & & & Lo & & & & & \\
\hline Charge passed & 1654 & 1651 & 1612 & 1962 & 197 & 1471 & & & & \\
\hline Average & & 1639 & & & 180 & & & & & $G C \mathrm{VAD} \mathrm{B}$ \\
\hline Std. Dev. & & 23 & & & 28 & & & & & $A-7 A-D-D$ \\
\hline $\operatorname{CoV}(\%)$ & & 1.4 & & & 16. & & & & & \\
\hline $\mathrm{Cl}^{-}$Penetrability & & Low & & & Lo & & & & & \\
\hline
\end{tabular}


Table 8.13: The results of the water soluble chloride test results (\%)

\begin{tabular}{|c|c|c|c|c|c|c|}
\hline \multirow{2}{*}{ Property } & \multicolumn{5}{|c|}{ Steps of powdering ( $D=$ depth) } & \multirow{2}{*}{$\begin{array}{c}\text { Specimen } \\
\text { Designation }\end{array}$} \\
\hline & $\mathrm{C}_{0 \mathrm{w}}$ & D5 & D6 & D7 & D8 & \\
\hline Depth (mm) & & 9.34 & 12.30 & 15.36 & 19.31 & \multirow{6}{*}{ GC-MO-D-C-G41 } \\
\hline Amount of powder $(\mathrm{g})$ & 10.07 & 10.01 & 10.02 & 9.69 & 10.06 & \\
\hline $\mathrm{C}_{\mathrm{f}}(\%)$ & 0.011 & 0.277 & 0.172 & 0.101 & 0.041 & \\
\hline $\mathrm{C}_{\mathrm{t}}(\%)$ & 0.022 & 0.300 & 0.193 & 0.120 & 0.051 & \\
\hline $\mathrm{C}_{\mathrm{b}}(\%)$ & 0.0103 & 0.023 & 0.021 & 0.019 & 0.010 & \\
\hline Binding Capacity & \multicolumn{5}{|c|}{0.0512} & \\
\hline Depth (mm) & & 8.88 & 11.87 & 14.79 & 18.80 & \multirow{6}{*}{ GC-MO-D-C-E26 } \\
\hline Amount of powder $(\mathrm{g})$ & 10.08 & 10.02 & 10.05 & 9.50 & 10.01 & \\
\hline $\mathrm{C}_{\mathrm{f}}(\%)$ & 0.015 & 0.379 & 0.223 & 0.138 & 0.053 & \\
\hline $\mathrm{C}_{\mathrm{t}}(\%)$ & 0.027 & 0.424 & 0.267 & 0.146 & 0.068 & \\
\hline $\mathrm{C}_{\mathrm{b}}(\%)$ & 0.012 & 0.046 & 0.044 & 0.008 & 0.015 & \\
\hline Binding Capacity & \multicolumn{5}{|c|}{0.1077} & \\
\hline Depth $(\mathrm{mm})$ & & 9.34 & 12.23 & $\overline{15.36}$ & 19.24 & \multirow{6}{*}{ GC-MO-D-C-NO } \\
\hline Amount of powder (g) & 10.01 & 10.08 & 9.98 & 9.83 & 10.00 & \\
\hline $\mathrm{C}_{\mathrm{f}}(\%)$ & 0.002 & 0.296 & 0.177 & 0.089 & 0.016 & \\
\hline $\mathrm{C}_{\mathrm{t}}(\%)$ & 0.014 & 0.341 & 0.201 & 0.104 & 0.026 & \\
\hline $\mathrm{C}_{\mathrm{b}}(\%)$ & 0.012 & 0.045 & 0.024 & 0.015 & 0.010 & \\
\hline Binding Capacity & \multicolumn{5}{|c|}{0.1142} & \\
\hline Depth $(\mathrm{mm})$ & & 9.55 & 12.63 & 15.56 & $\overline{19.51}$ & \multirow{6}{*}{ GC-VA-D-C-G23 } \\
\hline Amount of powder $(\mathrm{g})$ & 10.01 & 9.47 & 10.04 & 9.39 & 10.02 & \\
\hline $\mathrm{C}_{\mathrm{f}}(\%)$ & 0.004 & 0.301 & 0.170 & 0.078 & 0.018 & \\
\hline $\mathrm{C}_{\mathrm{t}}(\%)$ & 0.007 & 0.351 & 0.214 & 0.091 & 0.029 & \\
\hline $\mathrm{C}_{\mathrm{b}}(\%)$ & 0.003 & 0.051 & 0.044 & 0.013 & 0.011 & \\
\hline Binding Capacity & \multicolumn{5}{|c|}{0.1649} & \\
\hline Depth (mm) & & 8.91 & 12.15 & 14.97 & 18.42 & \multirow{6}{*}{ GC-VA-D-C-E17 } \\
\hline Amount of powder (g) & 10.01 & 9.04 & 10.07 & 8.51 & 10.00 & \\
\hline $\mathrm{C}_{\mathrm{f}}(\%)$ & 0.001 & 0.468 & 0.270 & 0.128 & 0.051 & \\
\hline $\mathrm{C}_{\mathrm{t}}(\%)$ & 0.007 & 0.482 & 0.279 & 0.143 & 0.053 & \\
\hline $\mathrm{C}_{\mathrm{b}}(\%)$ & 0.006 & 0.014 & 0.009 & 0.015 & 0.003 & \\
\hline Binding Capacity & \multicolumn{5}{|c|}{0.0201} & \\
\hline Depth (mm) & & 9.46 & 12.38 & 15.39 & 19.36 & \multirow{6}{*}{ GC-VA-D-C-NO } \\
\hline Amount of powder $(\mathrm{g})$ & 9.34 & 9.73 & 10.03 & 8.09 & 10.04 & \\
\hline $\mathrm{C}_{\mathrm{f}}(\%)$ & 0.001 & 0.302 & - & 0.092 & 0.030 & \\
\hline $\mathrm{C}_{\mathrm{t}}(\%)$ & 0.010 & 0.302 & - & 0.101 & 0.040 & \\
\hline $\mathrm{C}_{\mathrm{b}}(\%)$ & 0.009 & 0.000 & - & 0.009 & 0.009 & \\
\hline Binding Capacity & \multicolumn{5}{|c|}{0.0018} & \\
\hline
\end{tabular}


Table 8.14: Average carbonation depth and carbonation coefficient for GC-MO

\begin{tabular}{|c|c|c|c|c|c|c|c|c|c|}
\hline \multirow{2}{*}{\multicolumn{2}{|c|}{ Specimens Designation }} & \multirow{2}{*}{\multicolumn{7}{|c|}{\begin{tabular}{|c|} 
Average Depth of Carbonation (mm) \\
Time (day) \\
\end{tabular}}} & \multirow{3}{*}{$\begin{array}{c}\mathbf{D}_{\mathbf{c}} \\
\left(\frac{\mathrm{mm}}{\sqrt{\text { day }}}\right) \\
\end{array}$} \\
\hline & & & & & & & & & \\
\hline & & \multirow{2}{*}{$\frac{7}{0.1}$} & \multirow{2}{*}{$\frac{21}{2.0}$} & \multirow{2}{*}{$\frac{35}{3.0}$} & \multirow{2}{*}{$\frac{56}{4.0}$} & \multirow{2}{*}{$\frac{84}{4.8}$} & \multirow{2}{*}{$\frac{112}{5.5}$} & \multirow{2}{*}{$\frac{140}{6.6}$} & \\
\hline \multirow{3}{*}{ GC-MO-D-C } & G41 & & & & & & & & 0.52 \\
\hline & E26 & 1.3 & 3.3 & 4.3 & 5.0 & 5.9 & 6.5 & 7.0 & 0.63 \\
\hline & No & 0.4 & 2.3 & 3.1 & 4.1 & 4.6 & 5.4 & 6.0 & 0.51 \\
\hline \multirow{2}{*}{ GC-MO-D-F } & G41 & 3.2 & 6.2 & 7.8 & 9.9 & 10.7 & 11.0 & 12.0 & 1.13 \\
\hline & E26 & 2.9 & 5.8 & 7.6 & 9.5 & 10.3 & 11.1 & 11.7 & 1.10 \\
\hline \multirow{2}{*}{ GC-MO-D-B } & G41 & 1.7 & 3.4 & 4.6 & 5.2 & 6.0 & 6.6 & 7.6 & 0.66 \\
\hline & E26 & 2.3 & 3.9 & 5.6 & 5.9 & 6.3 & 7.3 & 8.1 & 0.73 \\
\hline
\end{tabular}

Table 8.15: Average carbonation depth and carbonation coefficient for GC-VA

\begin{tabular}{|c|c|c|c|c|c|c|c|c|c|}
\hline \multirow{2}{*}{\multicolumn{2}{|c|}{ Specimens Designation }} & \multirow{2}{*}{\multicolumn{7}{|c|}{$\frac{\text { Average Depth of Carbonation (mm) }}{\text { Time (day) }}$}} & \multirow{3}{*}{$\begin{array}{c}\mathbf{D}_{\mathbf{c}} \\
\left(\frac{\mathrm{mm}}{\sqrt{\text { day }}}\right) \\
\end{array}$} \\
\hline & & & & & & & & & \\
\hline & & \multirow{2}{*}{$\frac{7}{0.7}$} & \multirow{2}{*}{$\frac{21}{1.4}$} & \multirow{2}{*}{$\frac{35}{3.1}$} & \multirow{2}{*}{$\frac{56}{3.6}$} & \multirow{2}{*}{$\frac{84}{4.2}$} & \multirow{2}{*}{$\frac{112}{5.2}$} & \multirow{2}{*}{$\frac{140}{5.8}$} & \\
\hline \multirow{3}{*}{ GC-VA-D-C } & G23 & & & & & & & & 0.47 \\
\hline & E17 & 0.7 & 2.4 & 3.3 & 3.8 & 4.2 & 4.8 & 5.9 & 0.48 \\
\hline & No & 0.1 & 2.1 & 3.8 & 4.4 & 4.9 & 5.6 & 6.3 & 0.54 \\
\hline \multirow{2}{*}{ GC-VA-D-F } & G23 & 2.6 & 4.9 & 7.0 & 7.5 & 8.5 & 10.1 & 11.0 & 0.97 \\
\hline & E17 & 2.9 & 4.8 & 6.0 & 8.0 & 9.3 & 10.3 & 11.0 & 0.99 \\
\hline \multirow{2}{*}{ GC-VA-D-B } & G23 & 2.5 & 3.6 & 4.5 & 5.2 & 6.0 & 6.7 & 7.8 & 0.67 \\
\hline & E17 & 0.3 & 3.3 & 4.8 & 5.0 & 6.0 & 6.7 & 7.3 & 0.65 \\
\hline
\end{tabular}




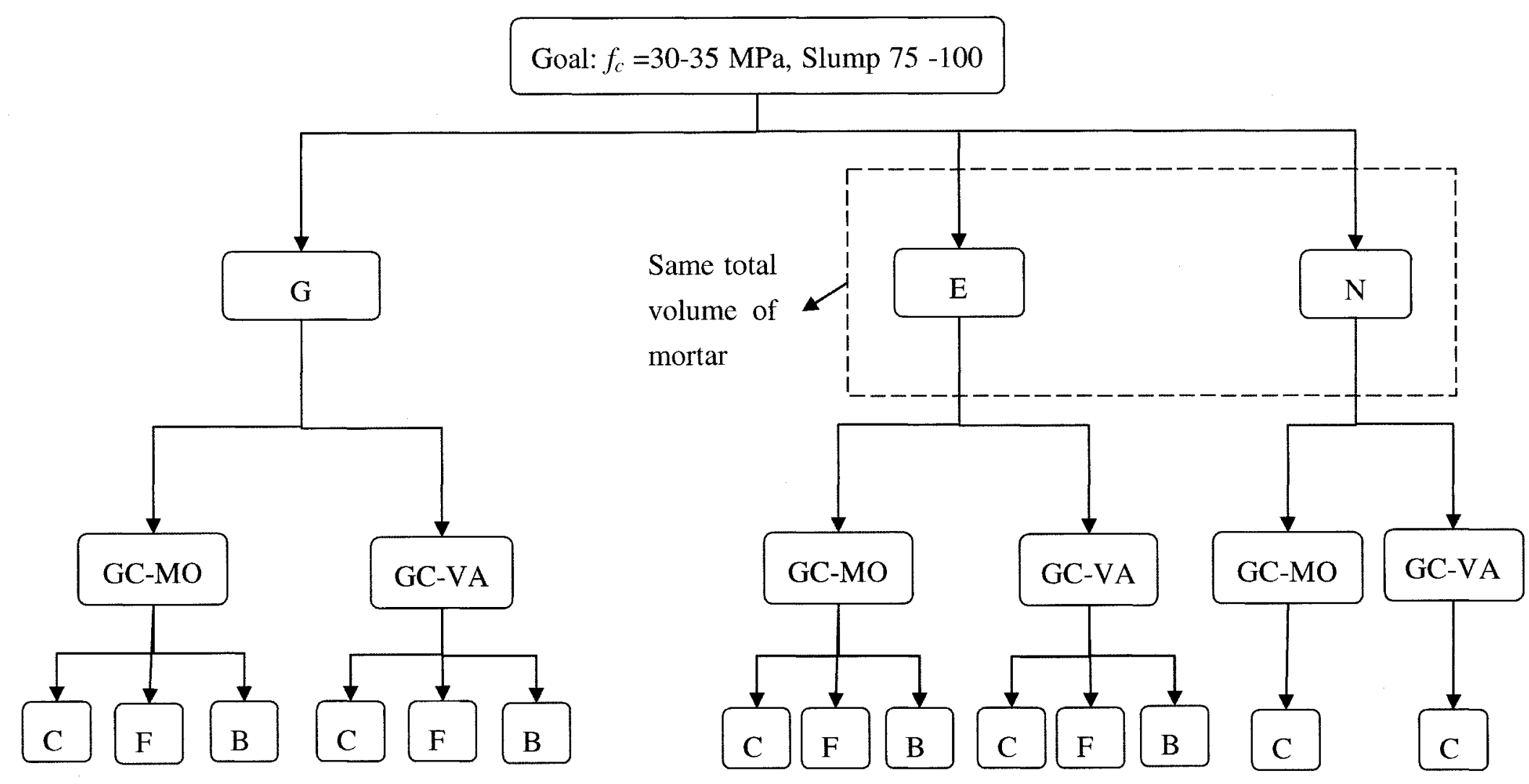

G: the general mix design method;

E: the equivalent total volume of mortar method;

$\mathrm{N}$ : NAC (conventional concrete);

C: $100 \%$ OPC; F: $75 \%$ OPC $+25 \%$ fly ash; B: $65 \%$ OPC $+35 \%$ bfs

Figure 8.1: The test grid for the durability study of GC 


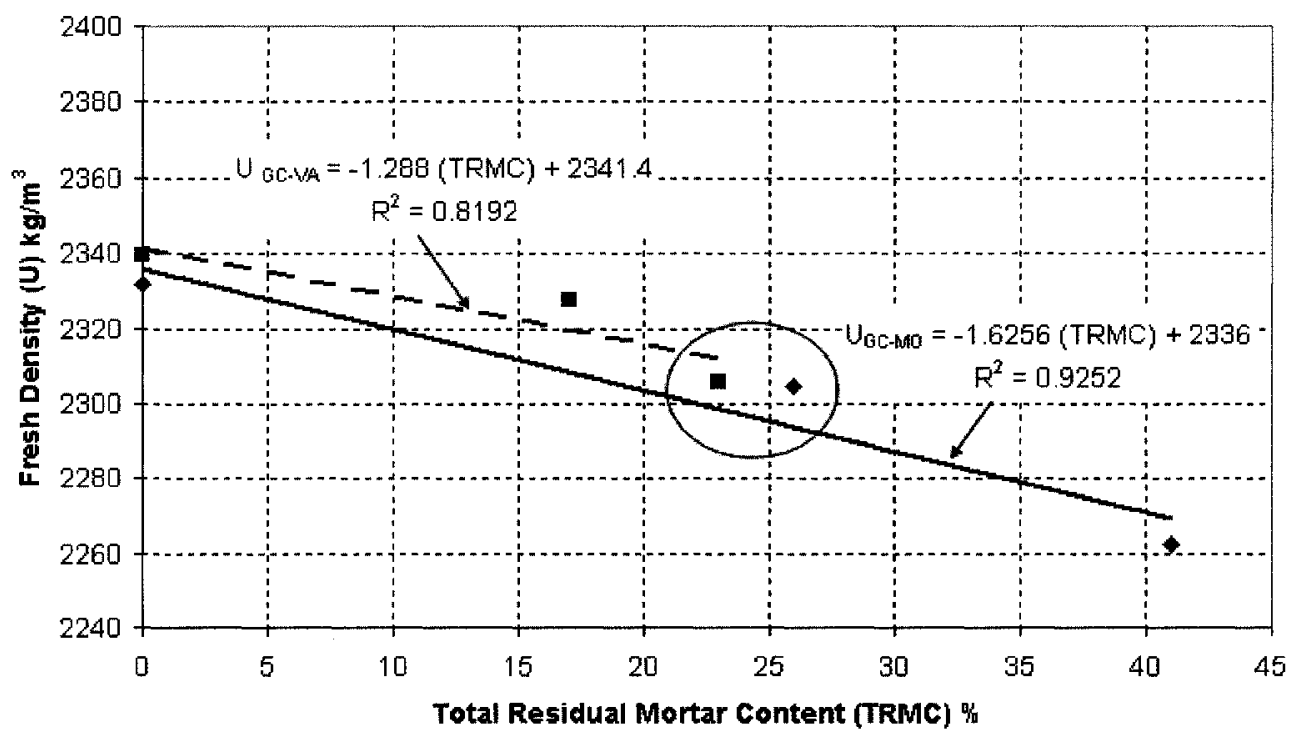

- GC-MO-D-C $\approx$ GC-VA-D-C

Figure 8.2: Variation of fresh density with TRMC for GC-MO-D-C and GC-VA-D-C mixes 


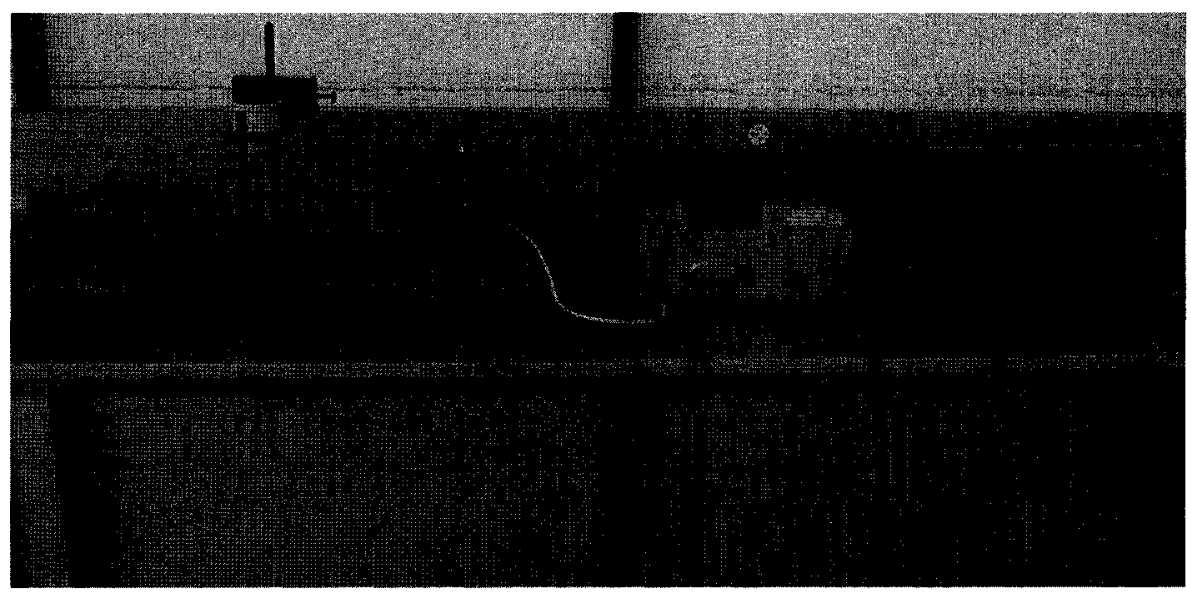

(a) Transverse frequency apparatus

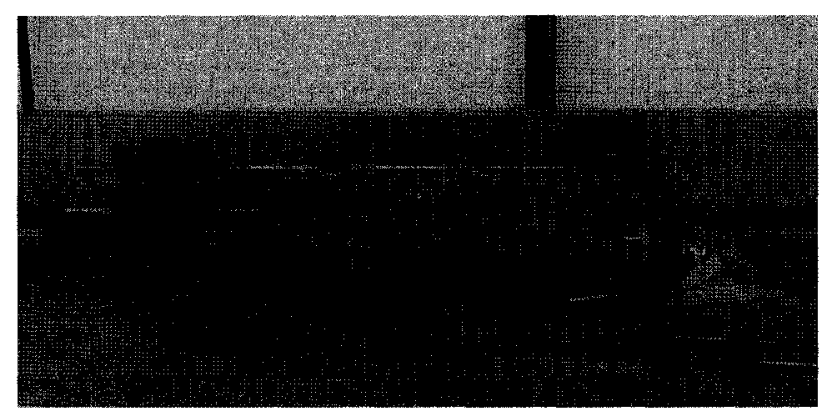

(b) Length change comparator

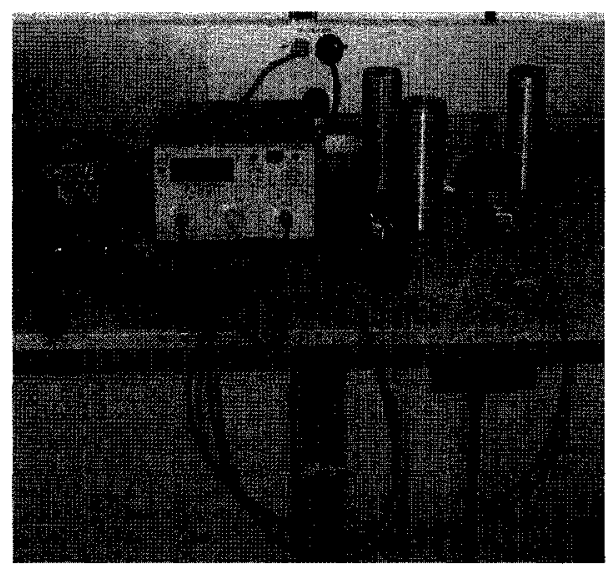

(c) Pulse velocity apparatus

Figure 8.3: Apparatus for measuring properties in freeze-and-thaw tests 

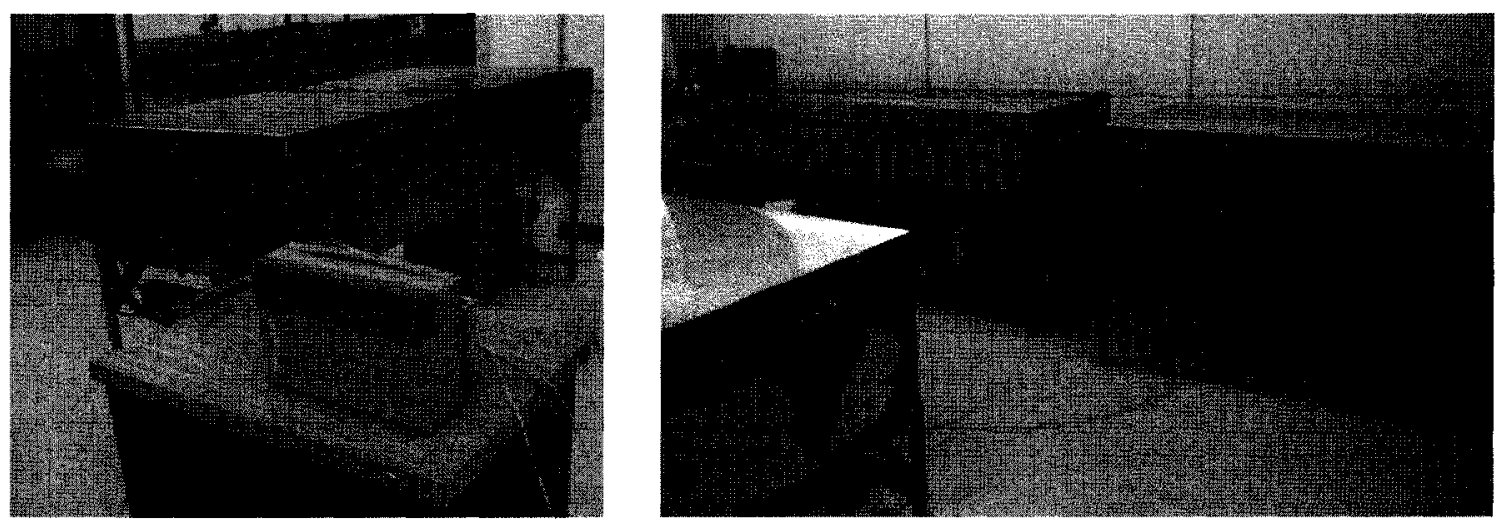

(a) Freezers A1, A2 and A3

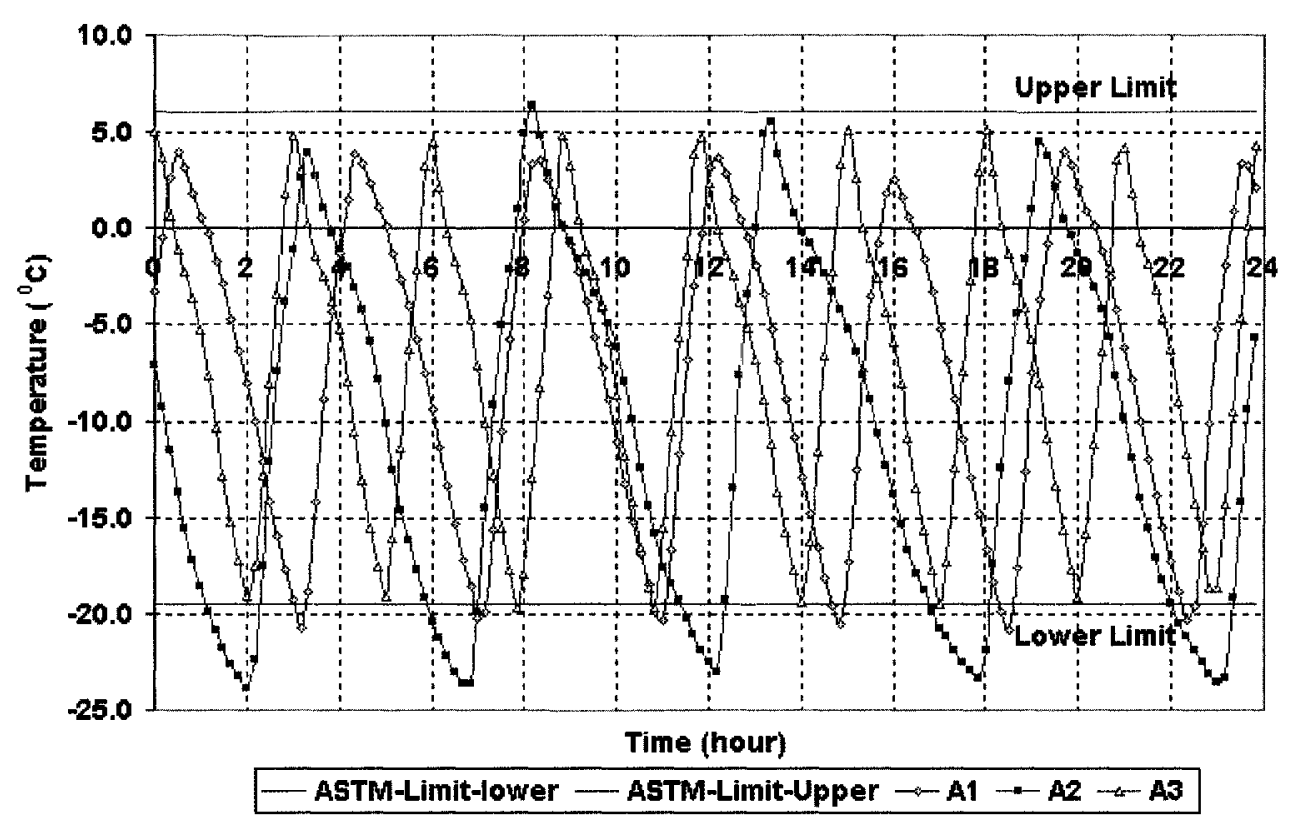

(b) Typical 24-hour freeze-and-thaw cycles for freezers A1, A2 and A3

Figure 8.4: Freezers used in the freeze-and-thaw tests 


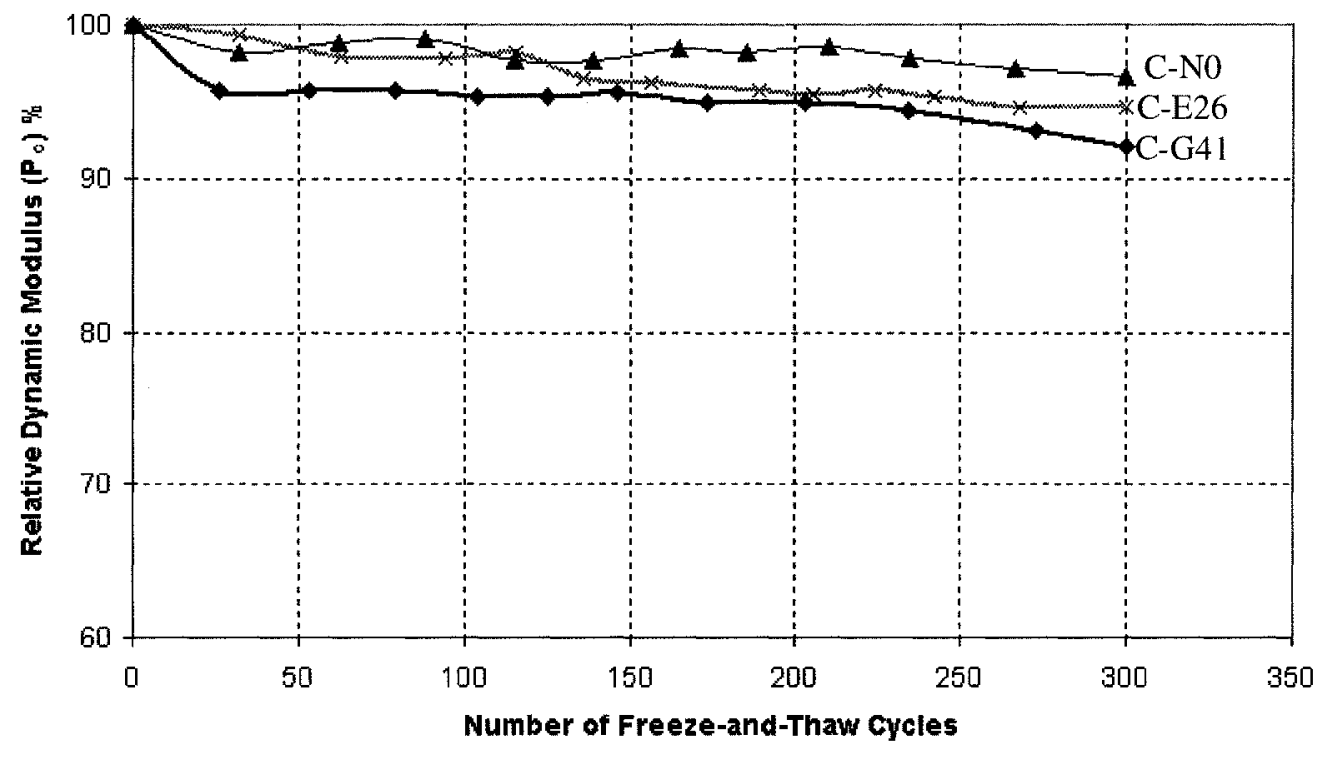

$\leadsto-$ GC-MO-D-C-G41 $\cdots$ GC-MO-D-C-E26 —-GC-MO-D-C-NO

(a) GC-MO-D-C specimens

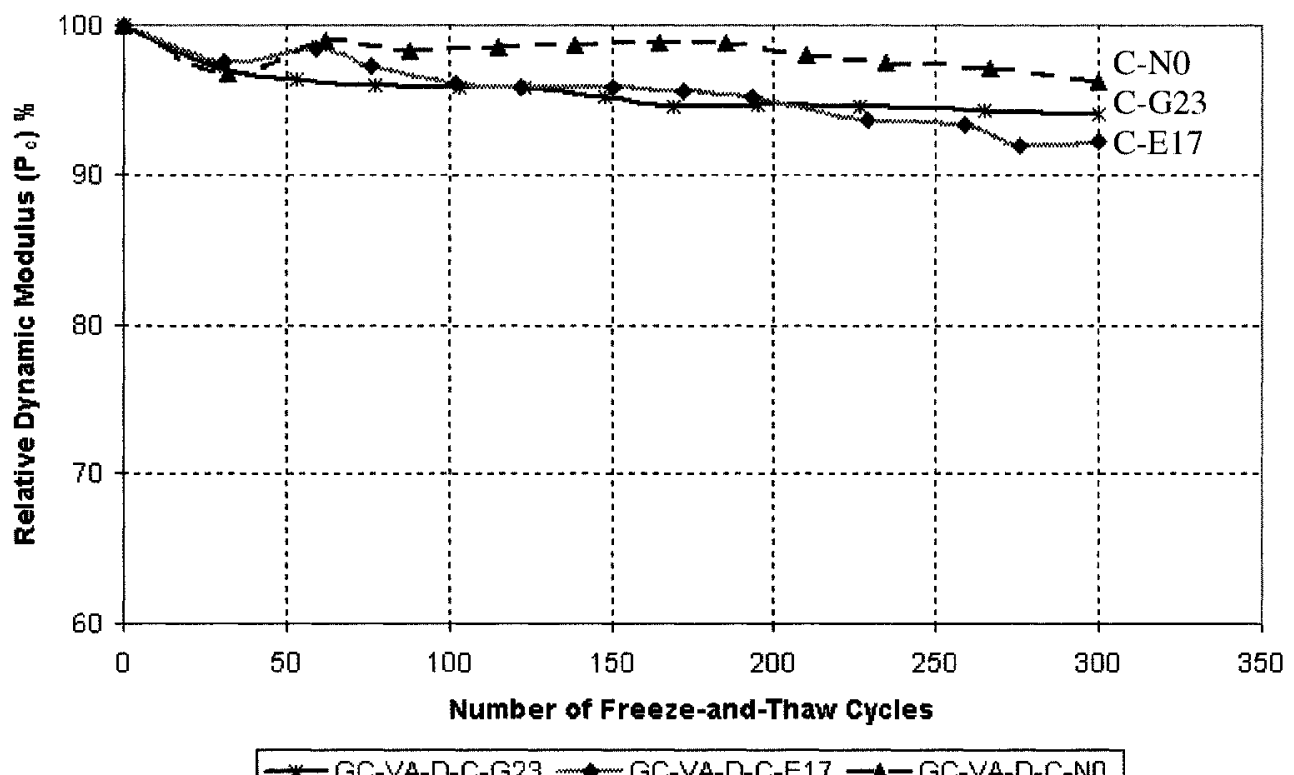

(b) GC-VA-D-C specimens

Figure 8.5: Variation of the relative dynamic modulus with number of freeze-and-thaw cycles for specimens with no SCM's 


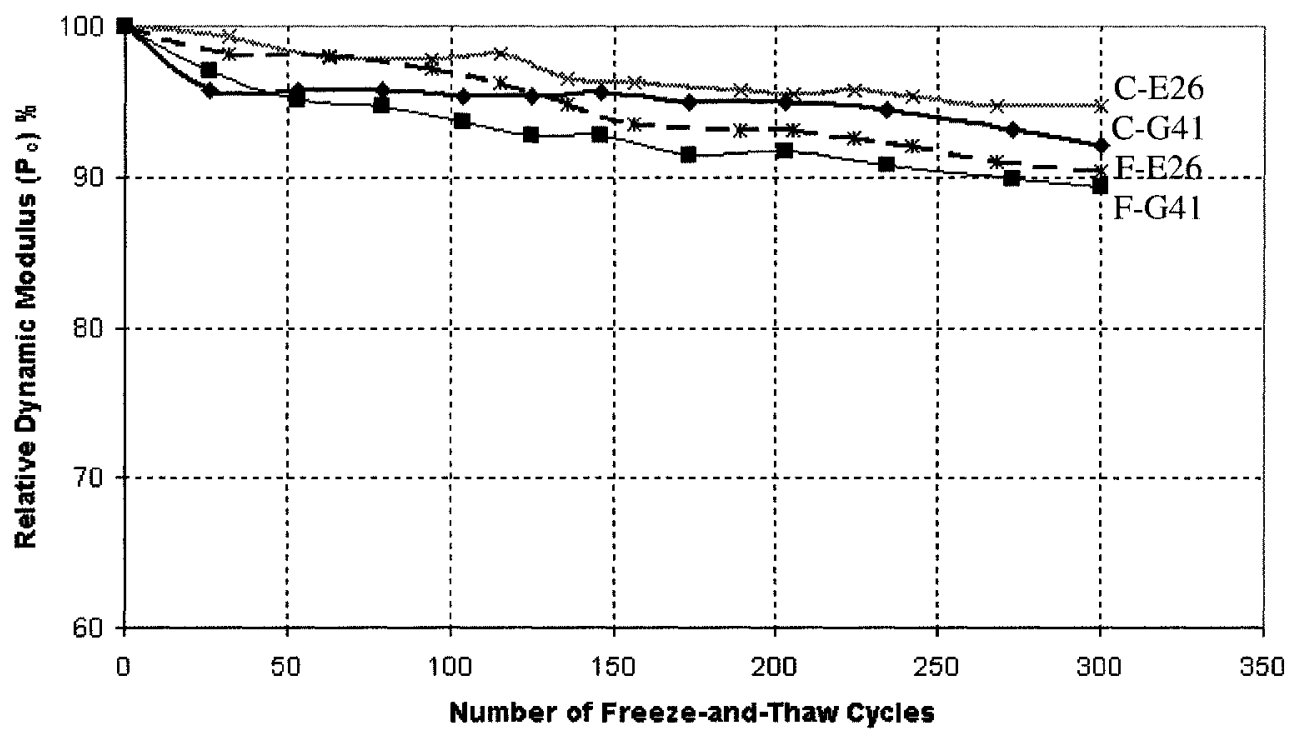

GC-MO-D-C-G41 - GC-MO-D-F-G41 GC-MO-D-C-E26-

(a) GC-MO-D specimens

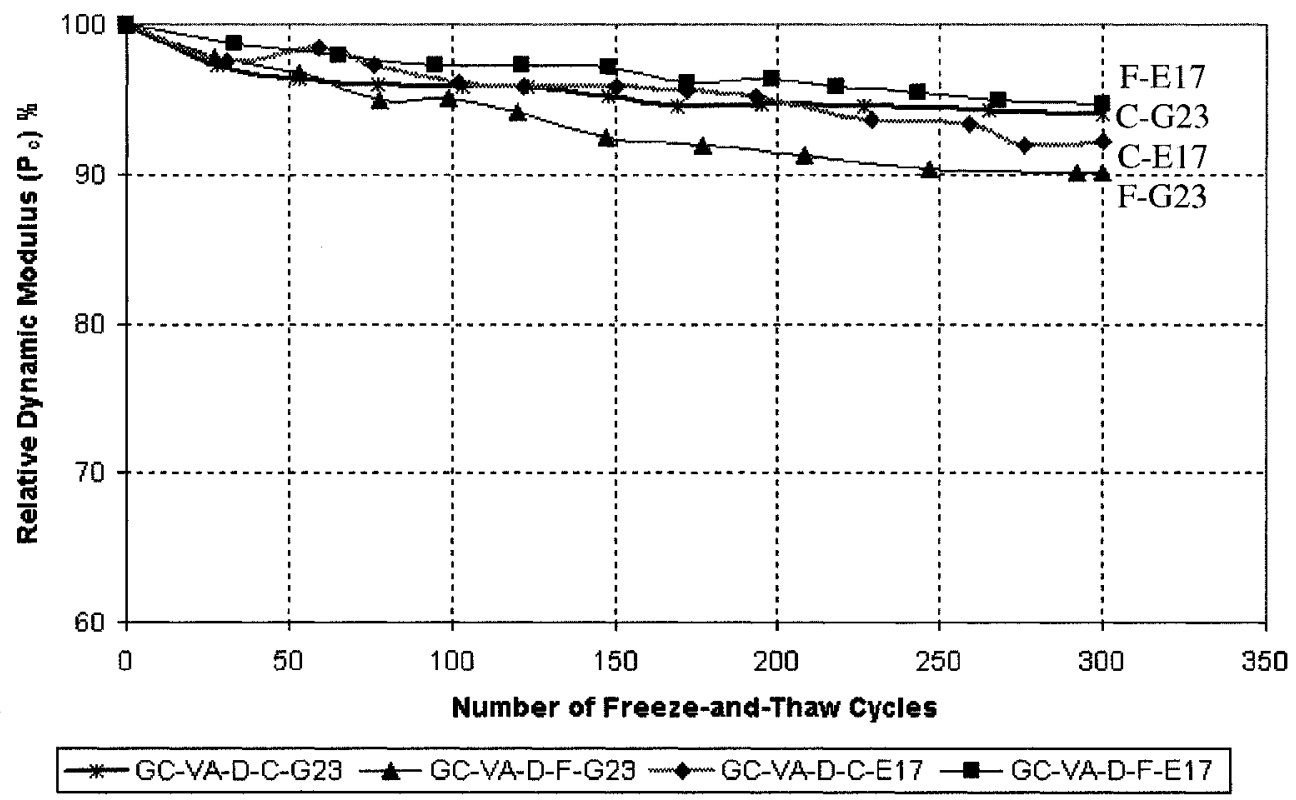

(b) GC-VA-D specimens

Figure 8.6: Variation of the relative dynamic modulus with number of freeze-and-thaw cycles for specimens with fly ash 


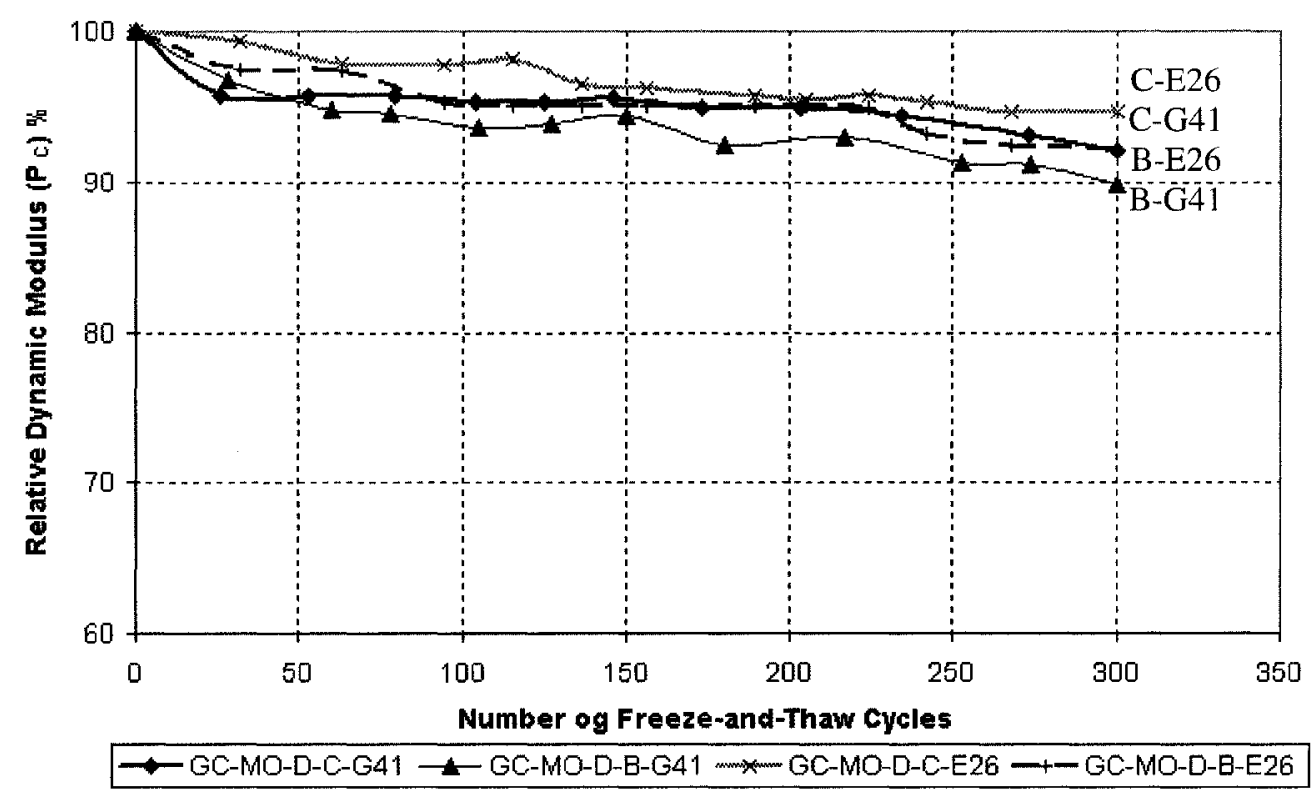

(a) GC-MO-D specimens

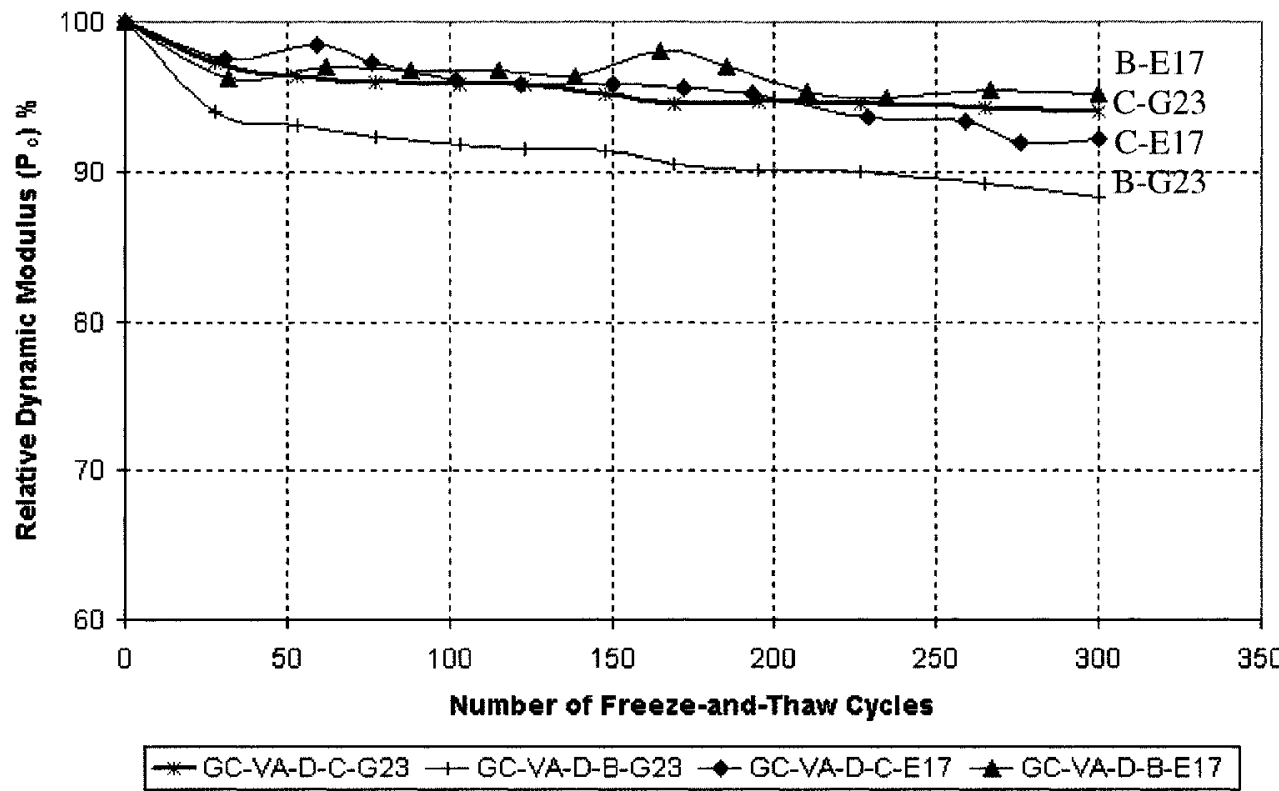

(b) GC-VA-D specimens

Figure 8.7: Variation of the relative dynamic modulus with number of freeze-and- thaw cycles for specimens with bfs 


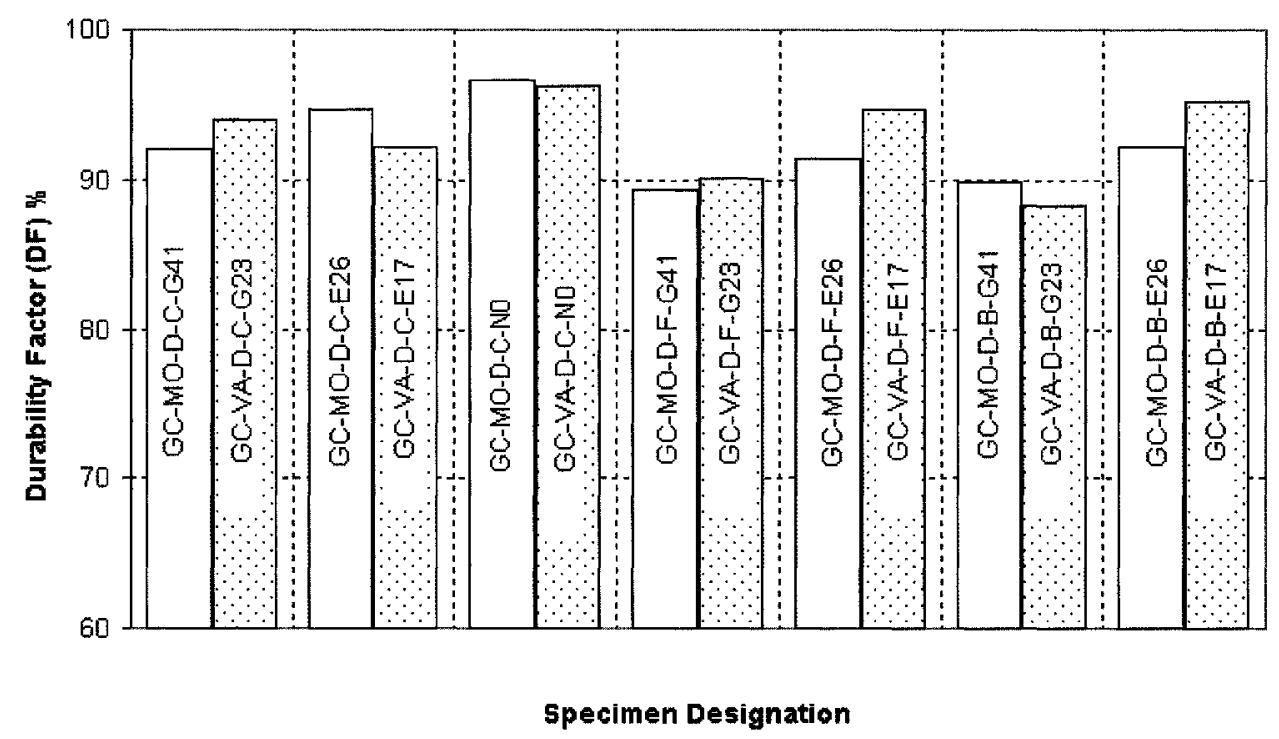

Figure 8.8: Summary of the $D F$ measurements of the freeze-and-thaw specimens 


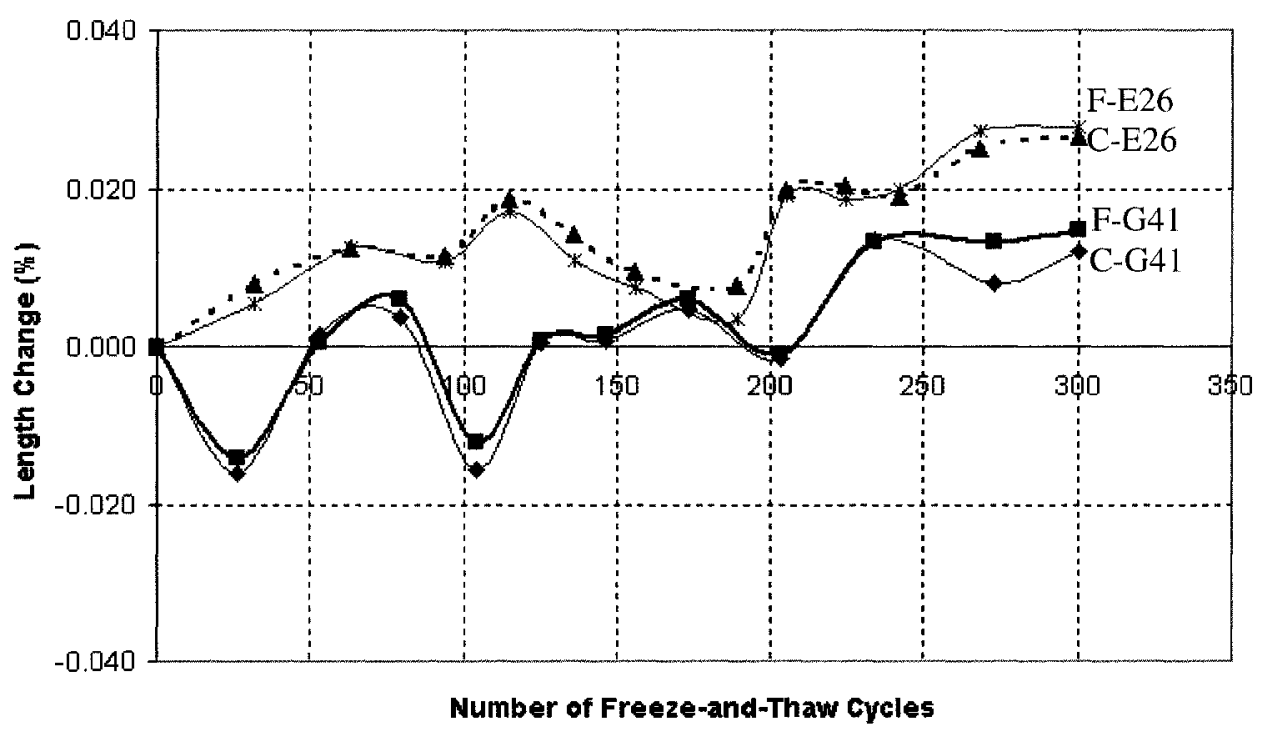

$\rightarrow-$ GC-MO-D-C-G41 - GC-MO-D-F-G41 - $\neq$ GC-MO-D-C-E26 * GC-MO-D-F-E26

(a) GC-MO-D

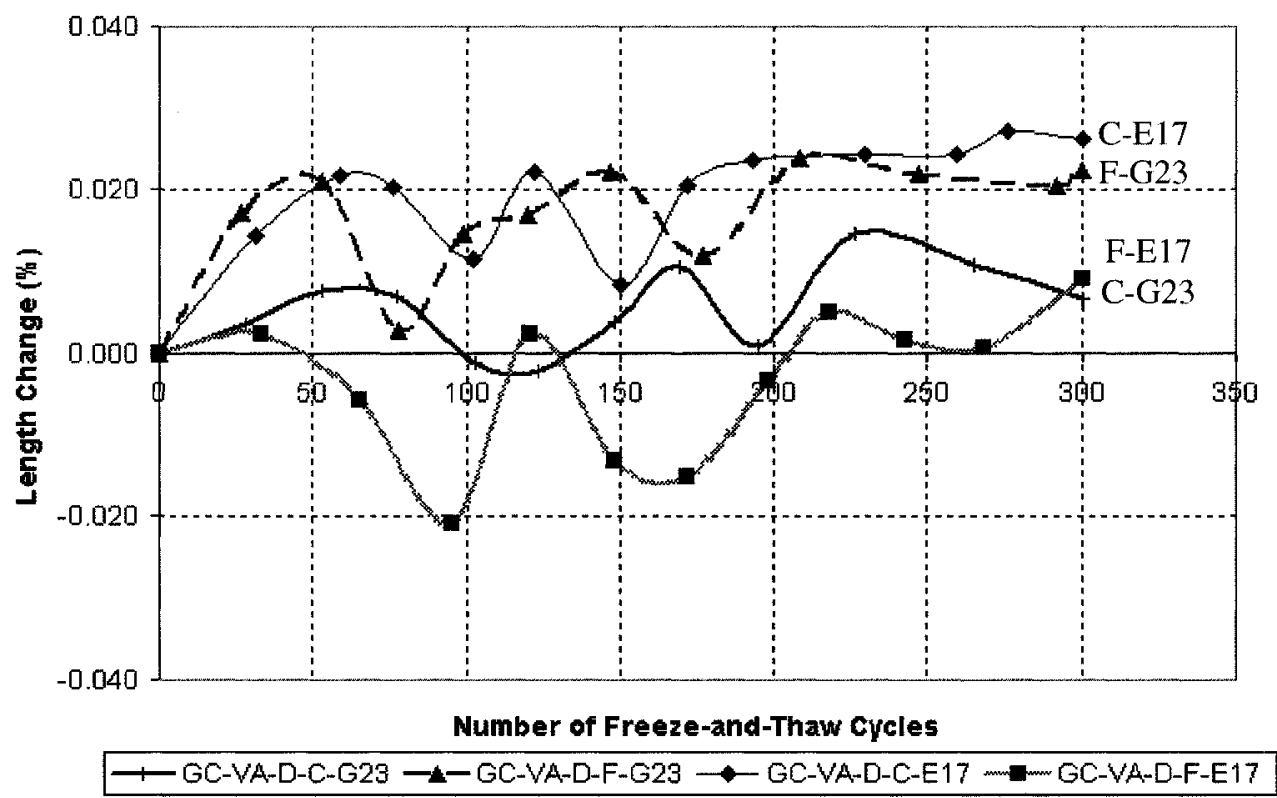

(b) GC-VA-D

Figure 8.9: Variation of the length change with number of freeze-and-thaw cycles for specimens with fly ash 


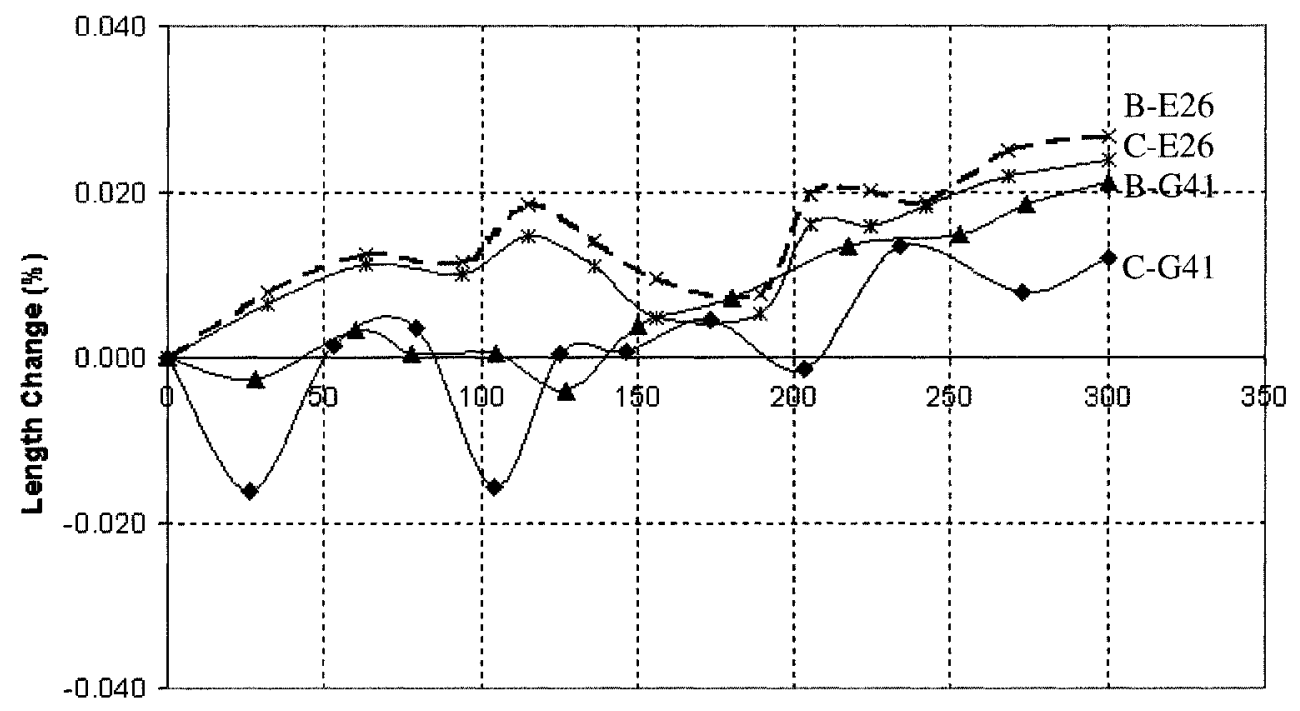

Number of Freeze-and-Thaw Cycles

$\rightarrow-$ GC-MO-D-C-G41 — GC-MO-D-B-G41 - - GC-MO-D-C-E26 * GC-MO-D-B-E26

(a) GC-MO-D

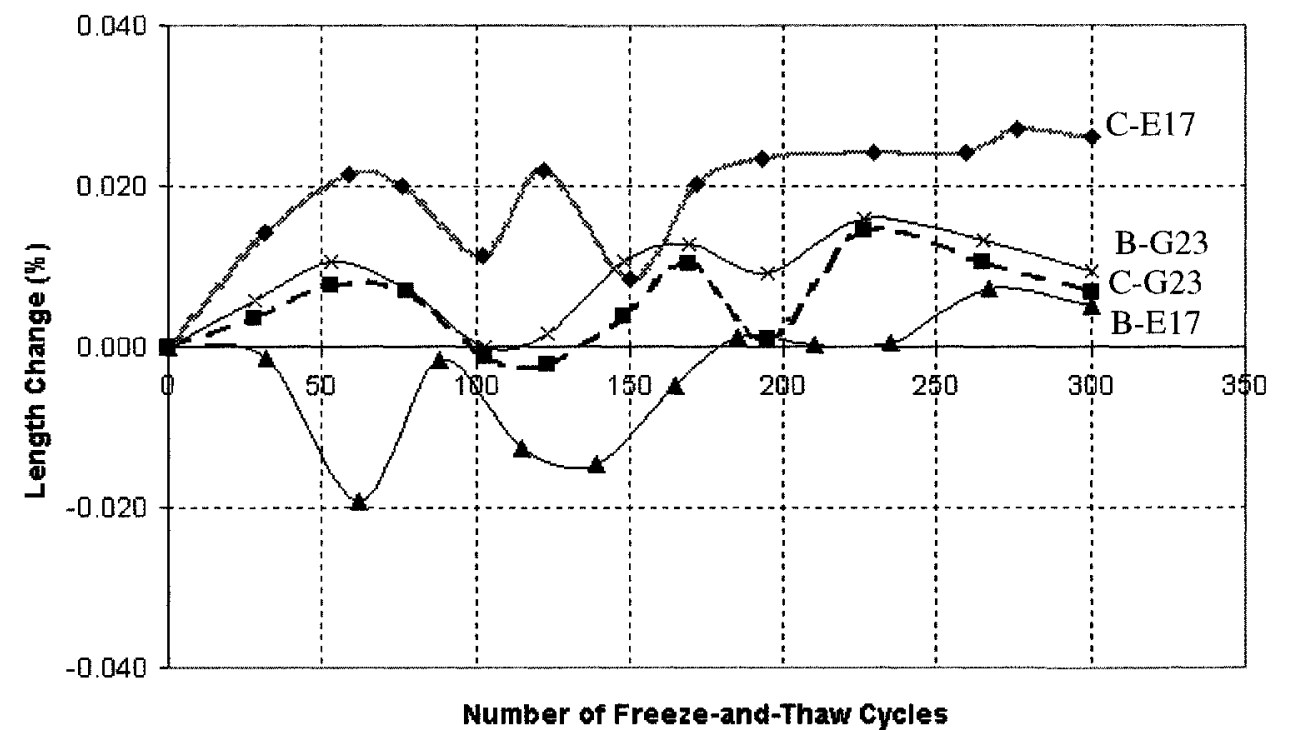

GC-VA-D-C-G23 $\rightarrow$ GC-VA-D-B-G23 $\rightarrow \cdots$ GC-VA-D-C-E17 $\rightarrow$ GC-VA-D-B-E17

(b) GC-VA-D

Figure 8.10: Variation of the length change with number of freeze-and-thaw cycles for specimens with bfs 


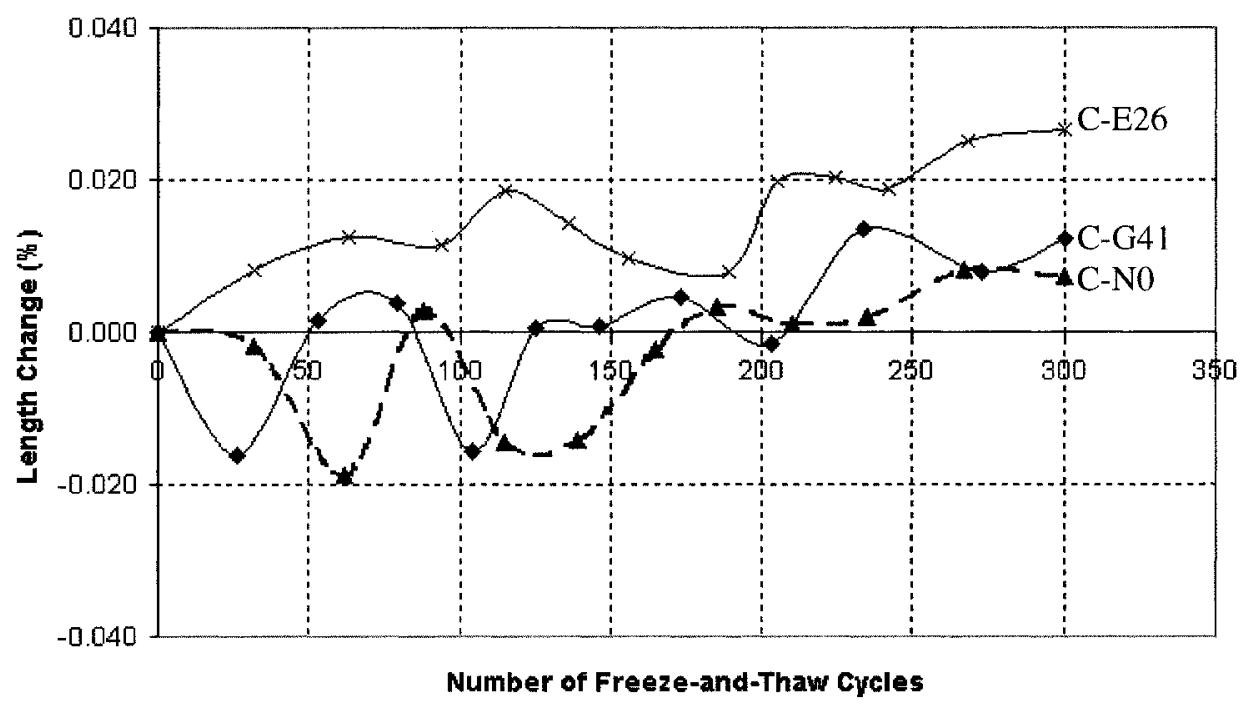

$\rightarrow-$ GC-MO-D-C-G41 $\rightarrow$ GC-MO-D-C-E26 - GC-MO-D-C-NO

(a) GC-MO-D-C

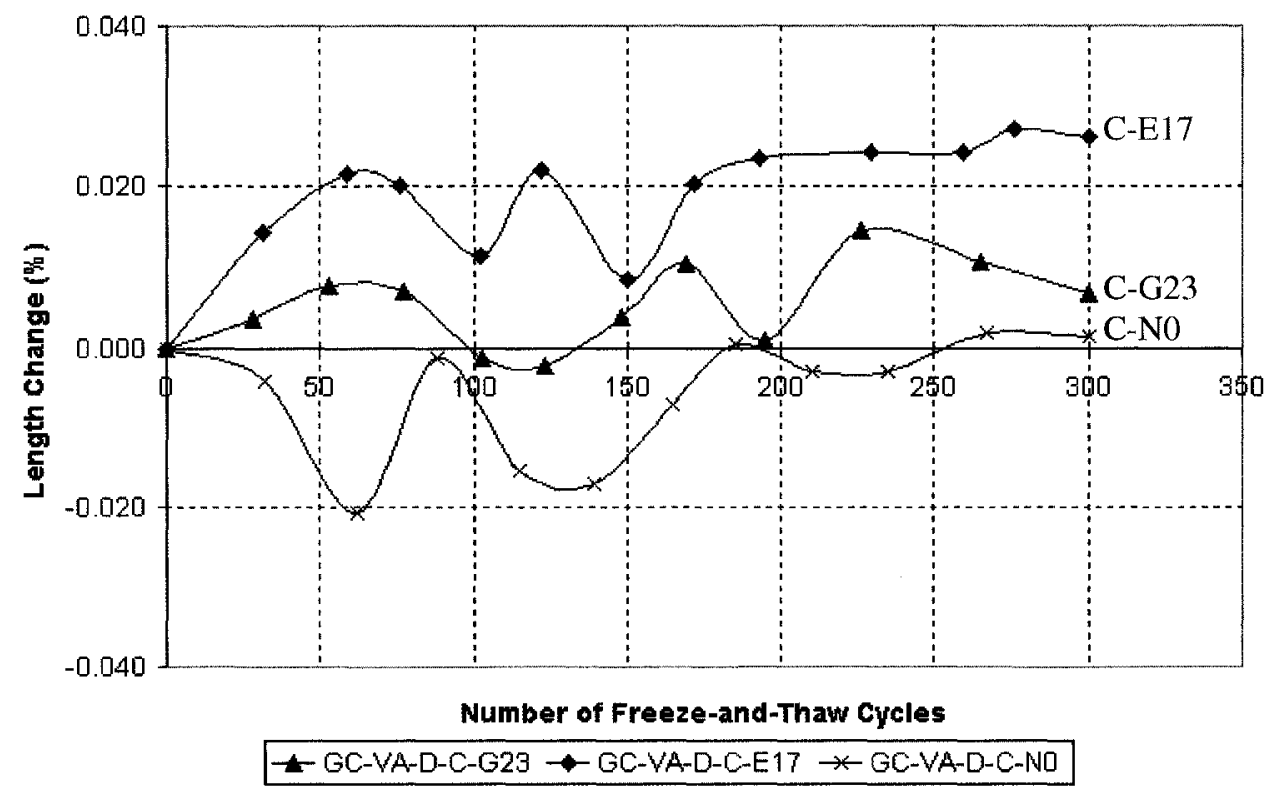

(b) GC-VA-D-C

Figure 8.11: Variation of the length change with number of freeze-and-thaw cycles for specimens with no SCM's 


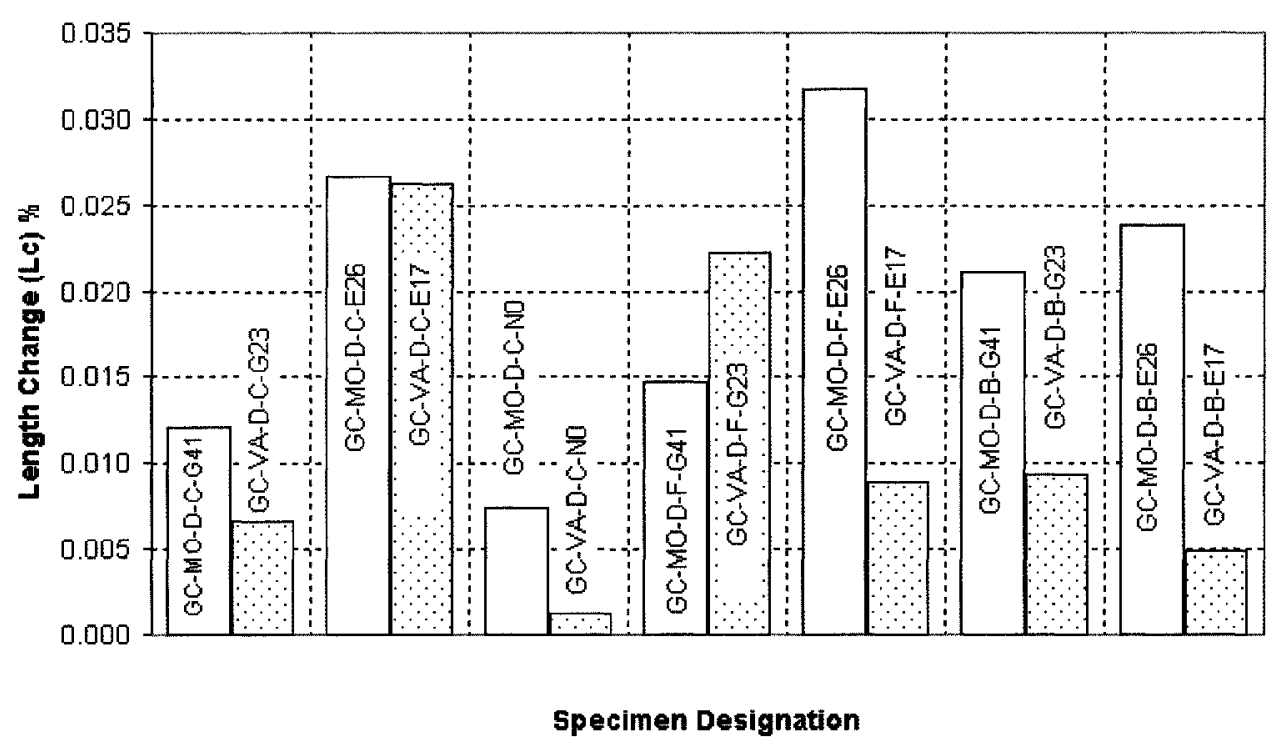

Figure 8.12: Summary of the length change measurements of the freeze-and-thaw specimens 


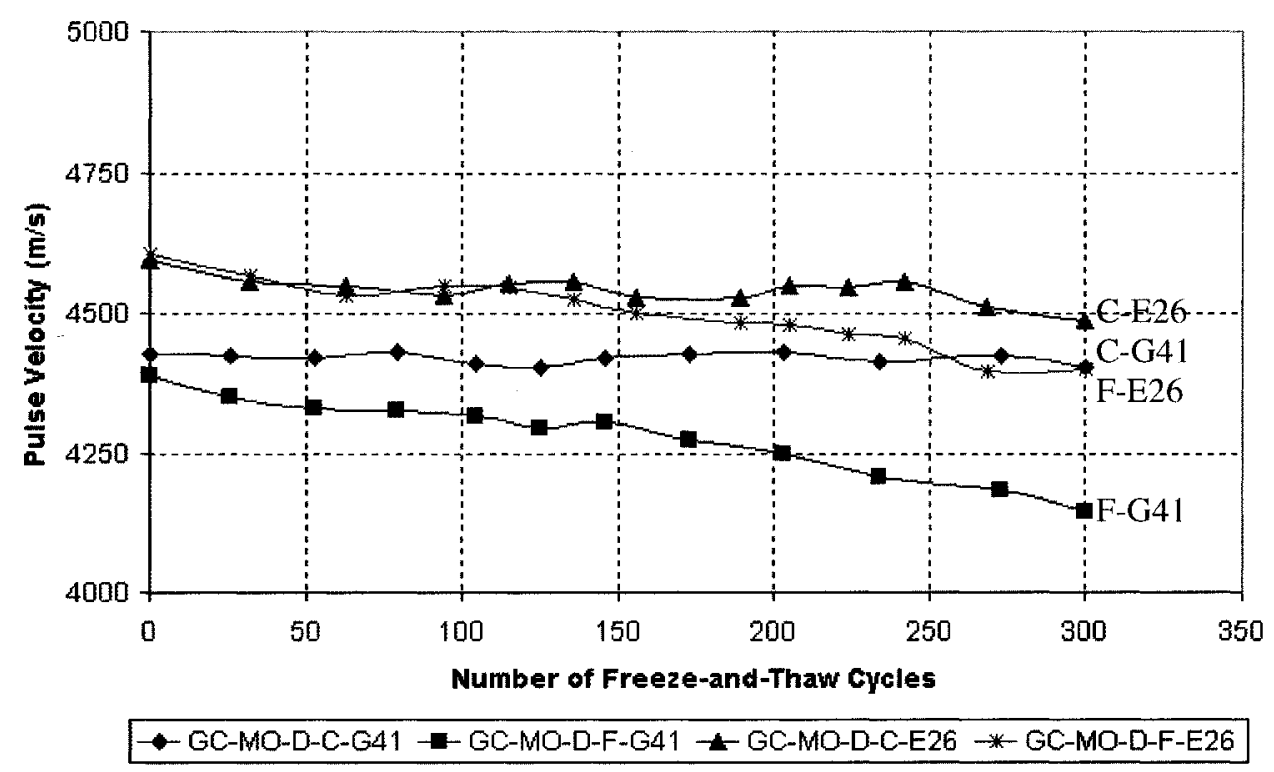

(a) GC-MO-D

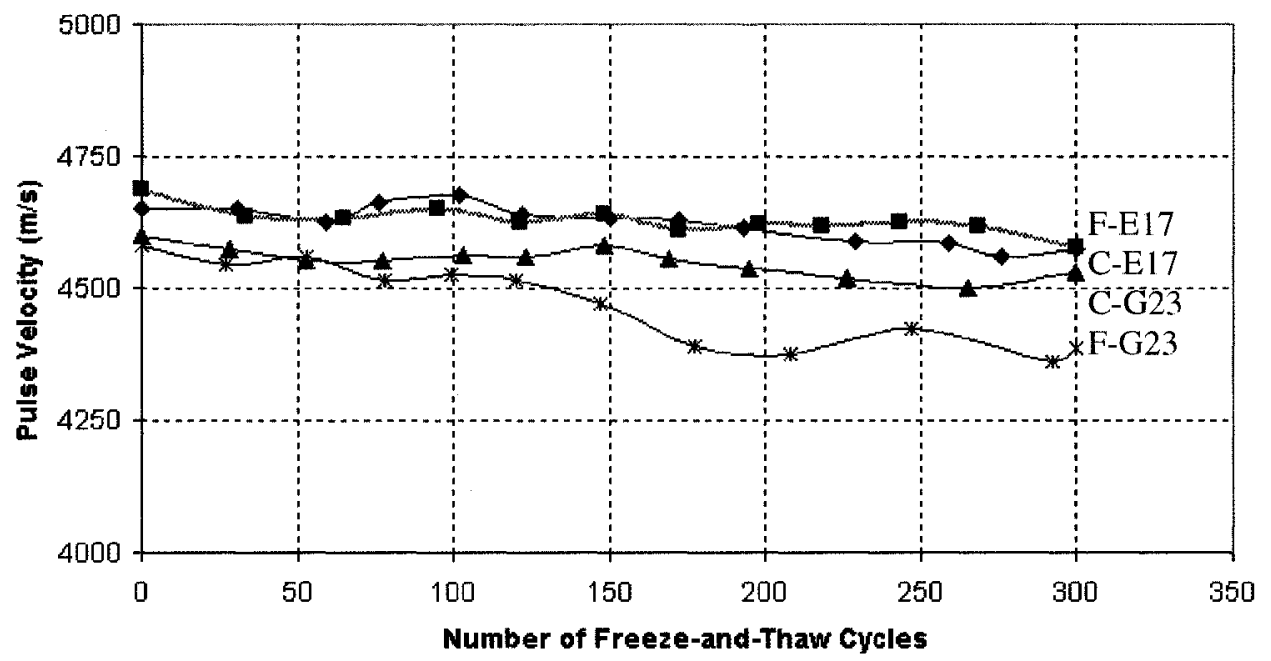

A GC-VA-D-C-G23 *-GC-VA-D-F-G23 $\rightarrow$ GC-VA-D-C-E17 $m$ GC-VA-D-F-E17

(b) GC-VA-D

Figure 8.13: Variation of the pulse velocity with number of freeze-and-thaw cycles for specimens with fly ash 


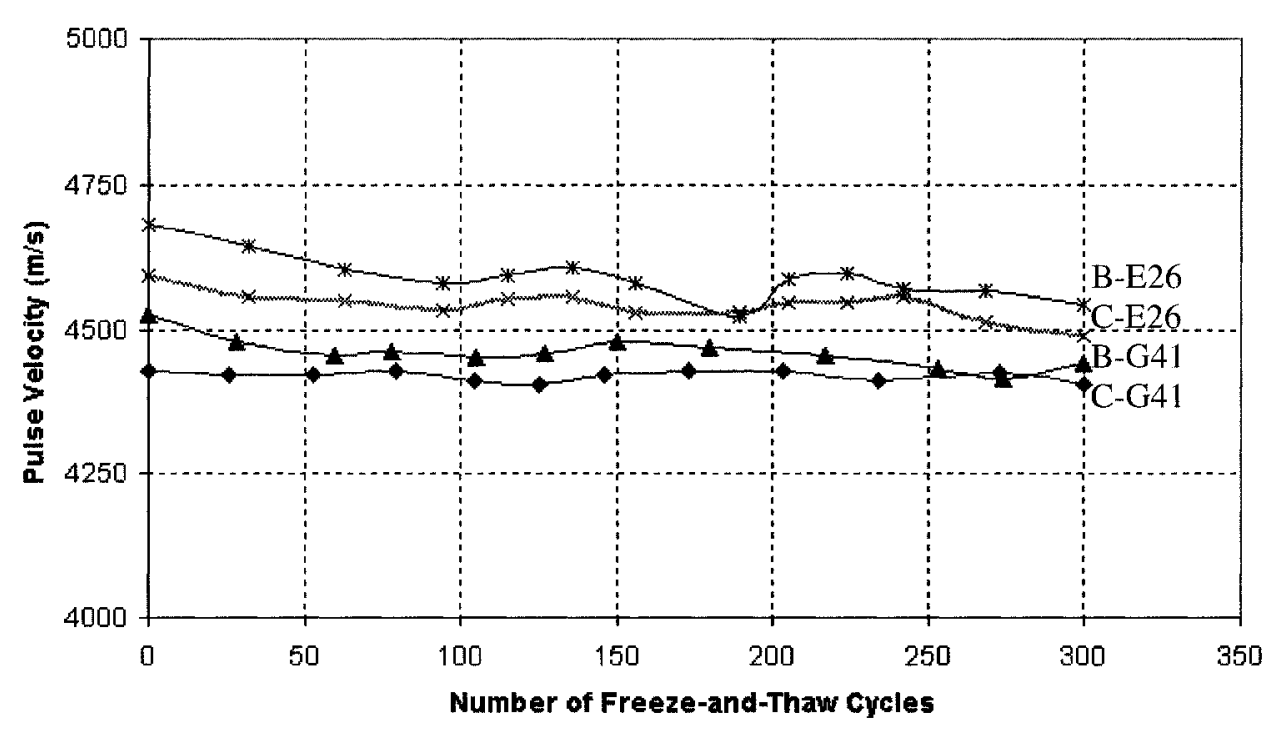

$\rightarrow-$ GC-MO-D-C-G41 —-GC-MO-D-B-G41 m* GC-MO-D-C-E26 * GC-MO-D-B-E26

(a) GC-MO-D

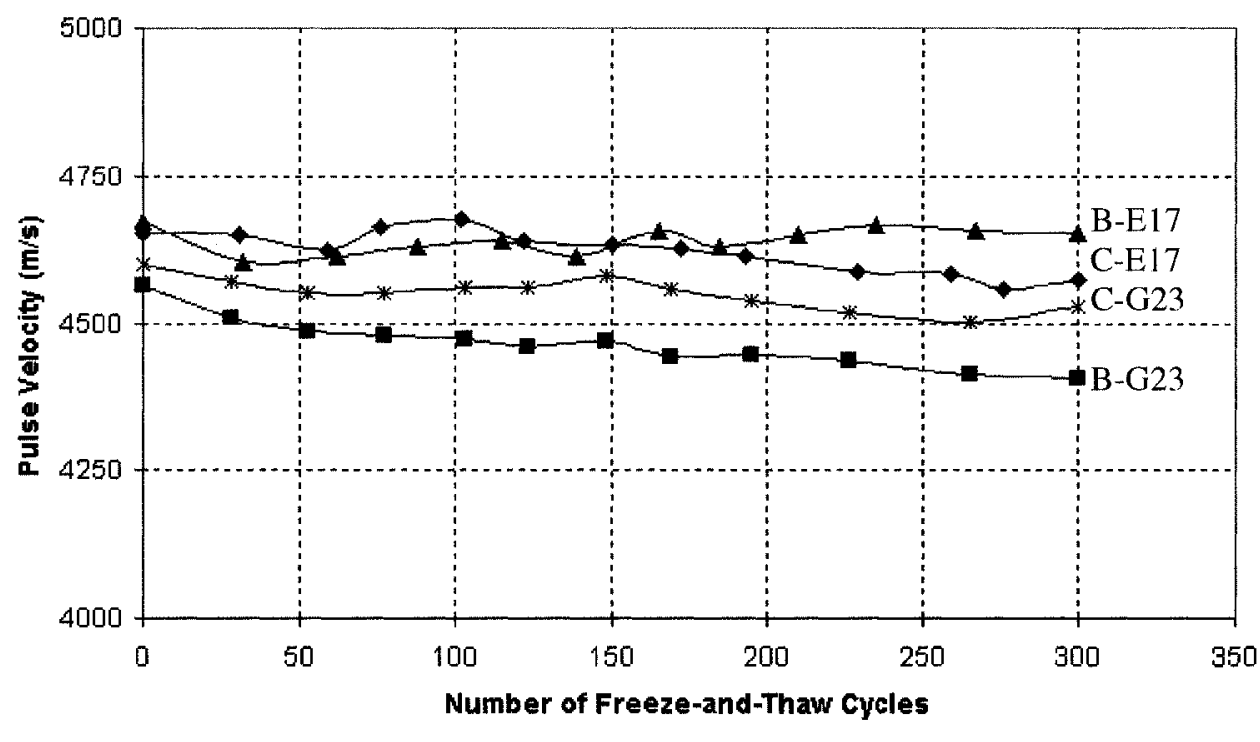

* GC-VA-D-C-G23 - GC-VA-D-B-G23 - -GC-VA-D-C-E17 —GC-VA-D-B-E17

(b) GC-VA-D

Figure 8.14: Variation of the pulse velocity with number of freeze-and-thaw cycles for specimens with bfs 


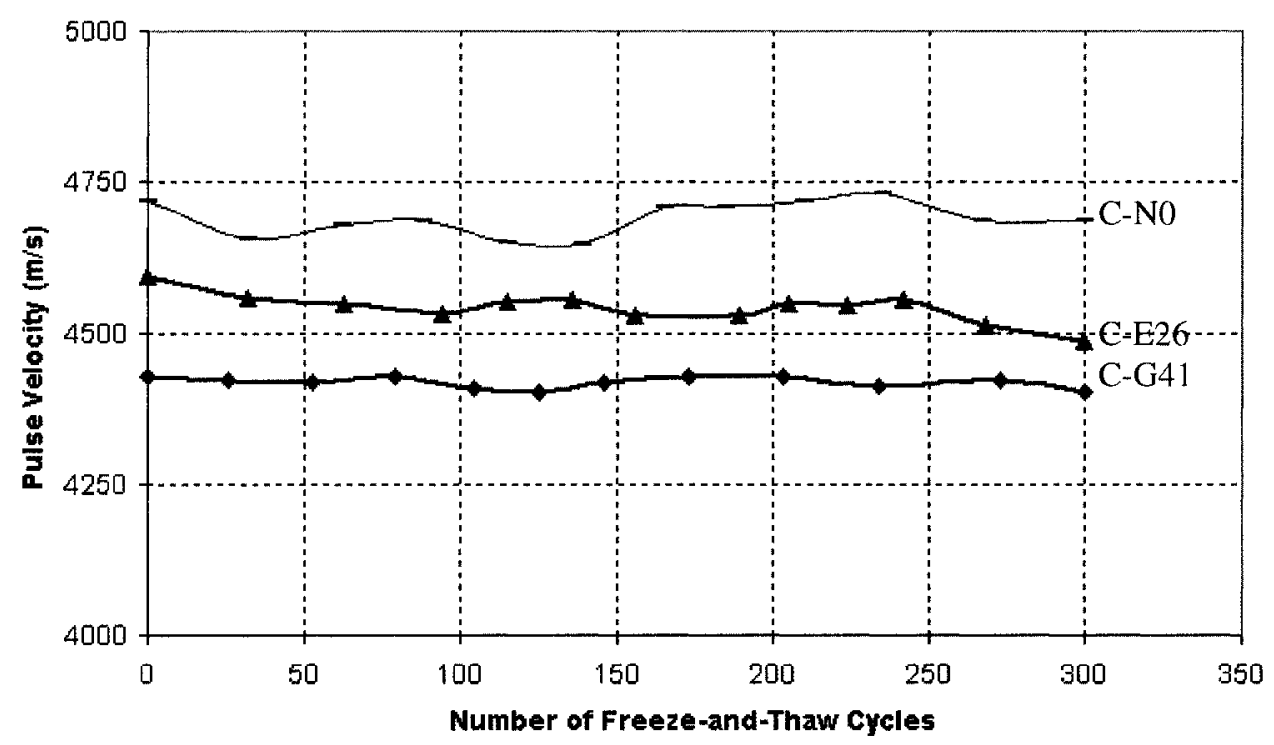

$\bullet-$ GC-MO-D-C-G41 $\rightarrow$ GC-MO-D-C-E26 - GC-MO-D-C-NO

(a) GC-MO-D-C

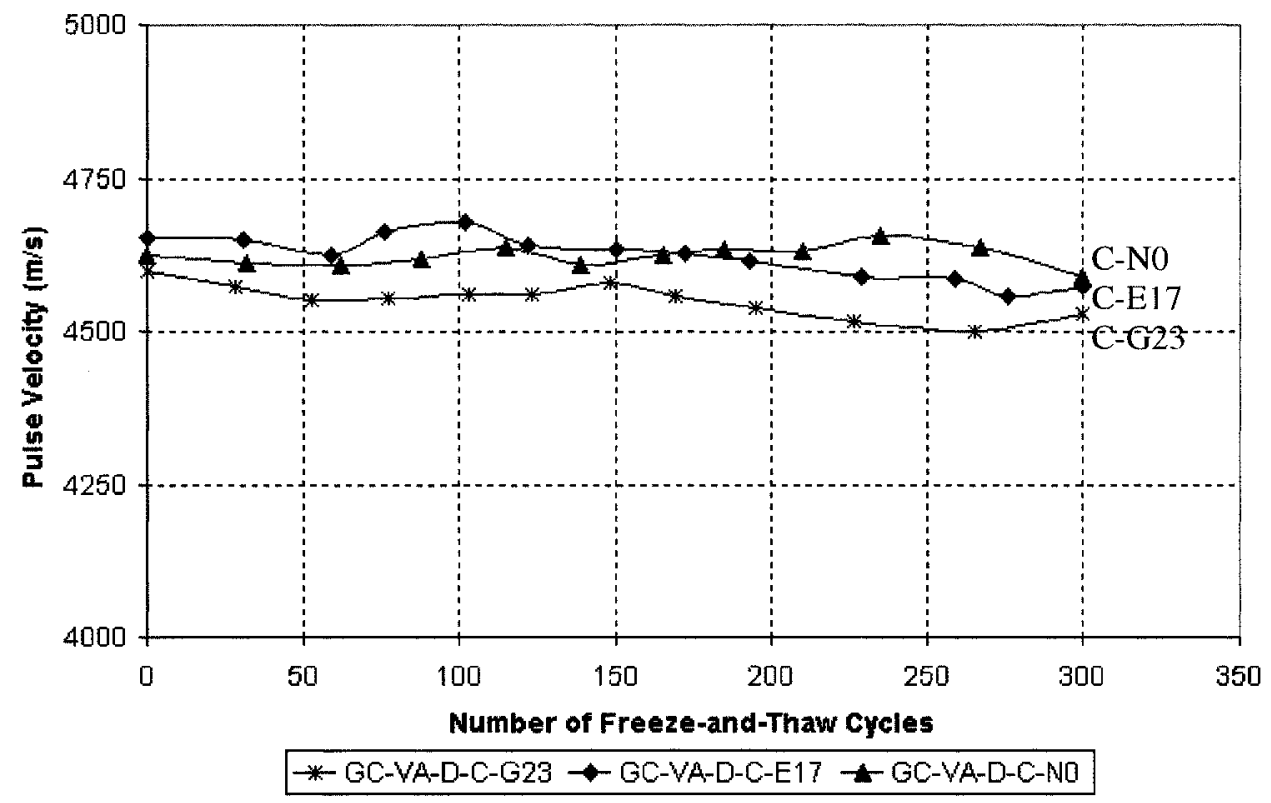

(b) GC-VA-D-C

Figure 8.15: Variation of the pulse velocity with number of freeze-and-thaw cycles for specimens with no SCM's 


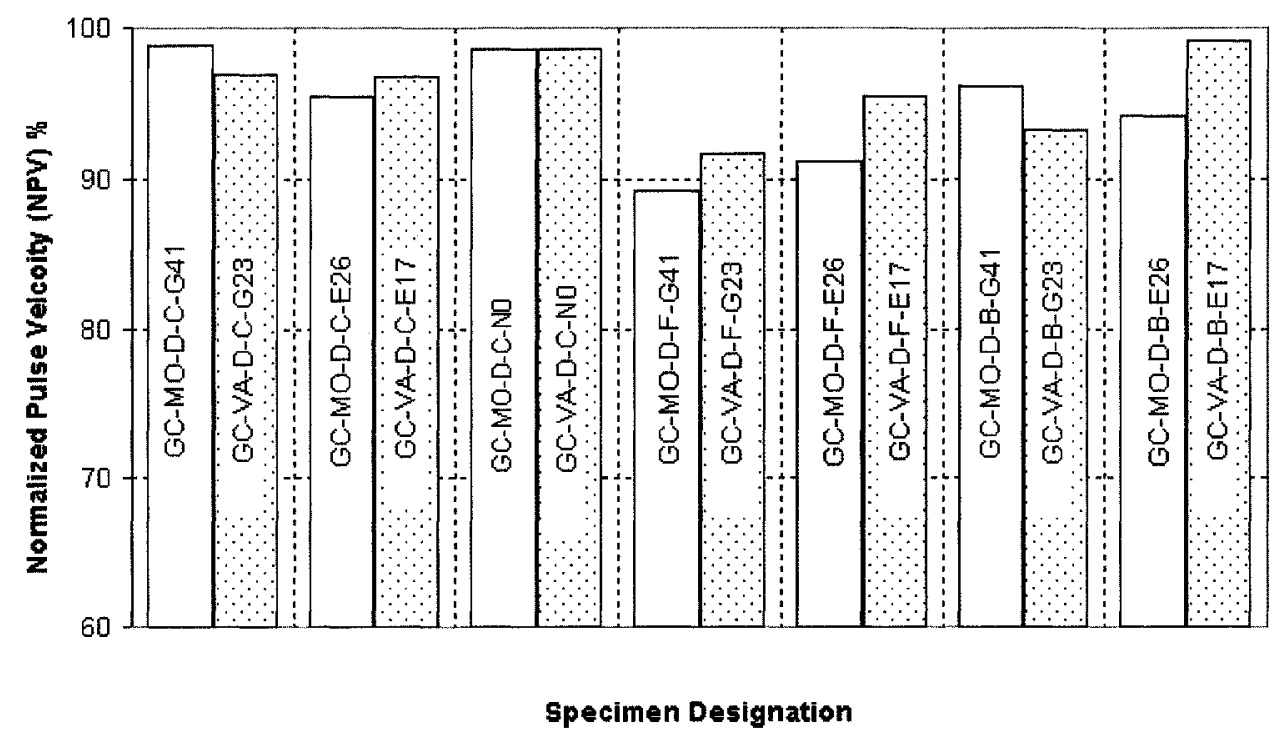

Figure 8.16: Summary of the normalized PV 


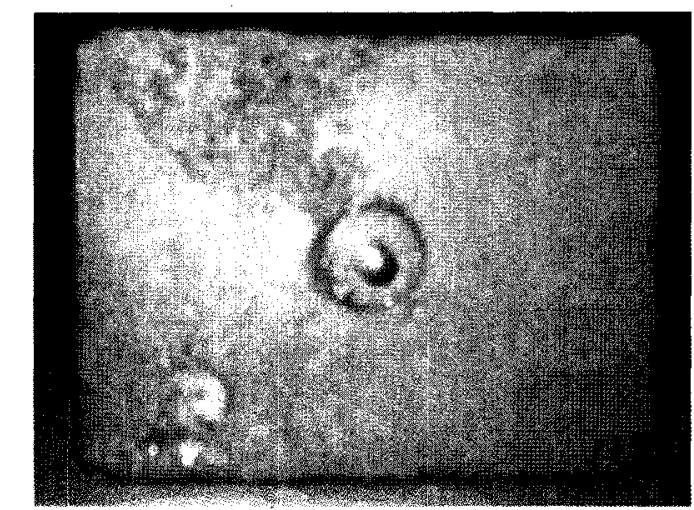

Scaling in one specimen at the right corner ( after 156 freeze-and-thaw cycles)

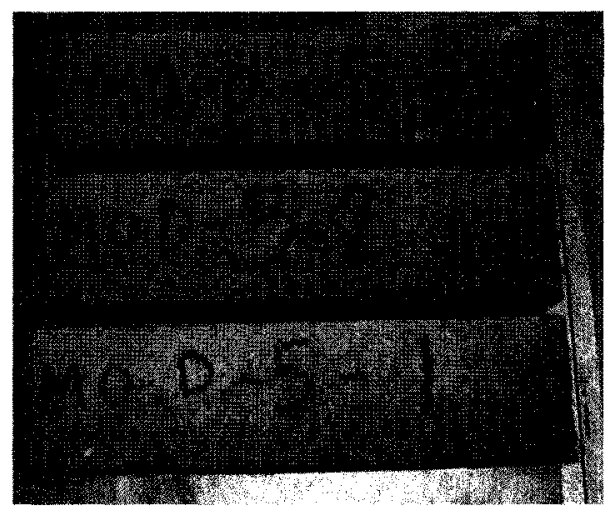

After 300 freeze-and-thaw cycles

(a) GC-MO-D-F-E26 specimens

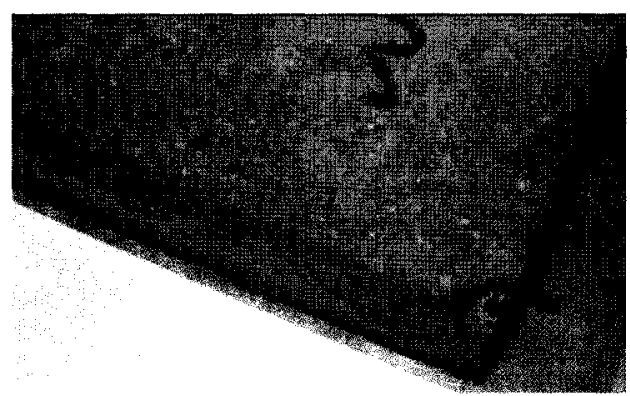

Pop out at the corner of one specimen after 273 freeze-and-thaw cycles

(b) GC-MO-D-C-G41 specimens

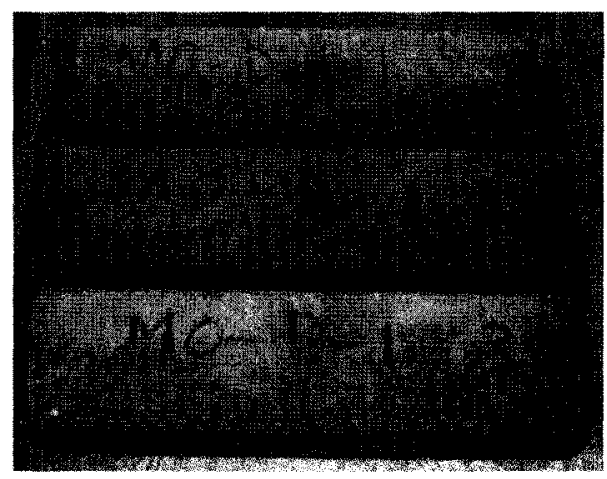

After 300 freeze-and-thaw cycles

Figure 8.17: Visual appearance of GC-MO specimens during the freeze-and-thaw tests 


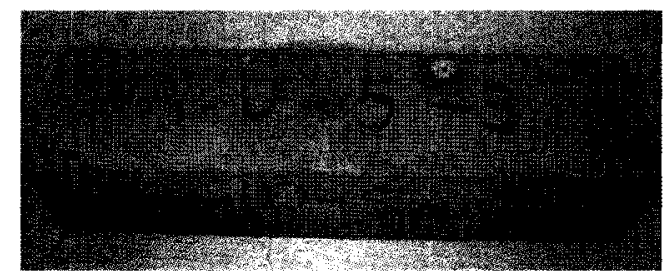

Pop outs at the top of one speceimen after 95 freeze-and-thaw cycles

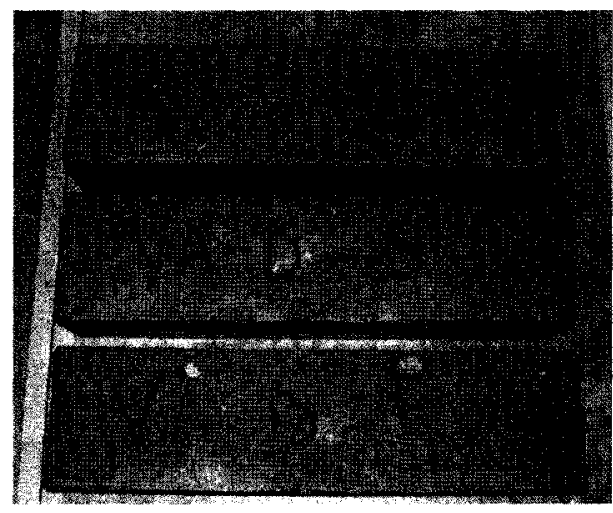

Appearance after 300 freeze-and-thaw cycles

(a) GC-VA-D-F-E17 specimens

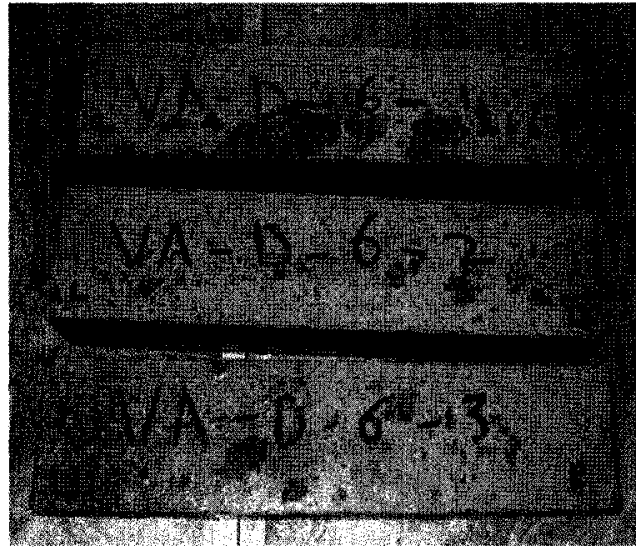

Light scaling

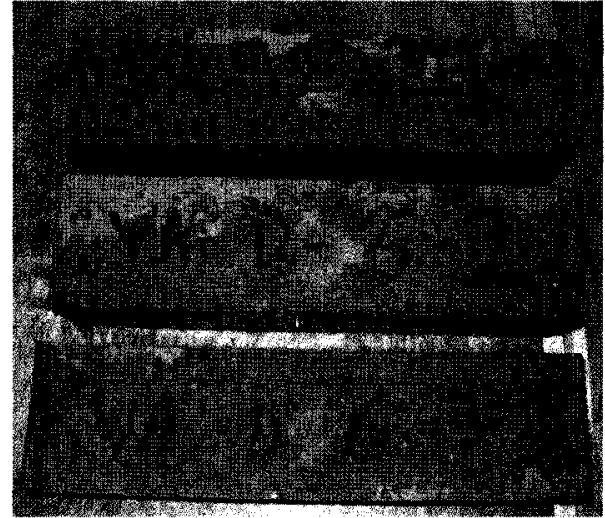

Appearance after 300 freeze-and-thaw cycles

after 88 freeze-and-thaw cycles

(b) GC-VA-D-B-E17 specimens

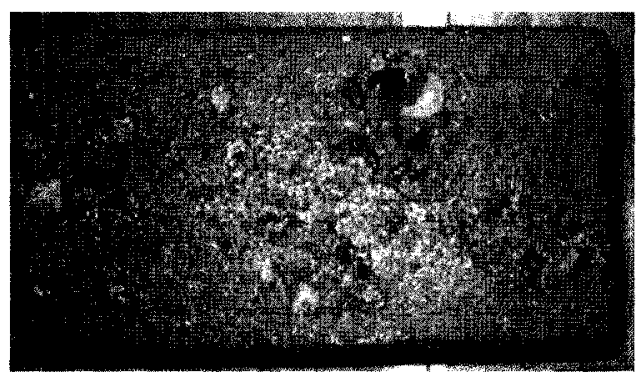

Pop outs at the bottom of one specimen after 147 freeze-and-thaw cycles

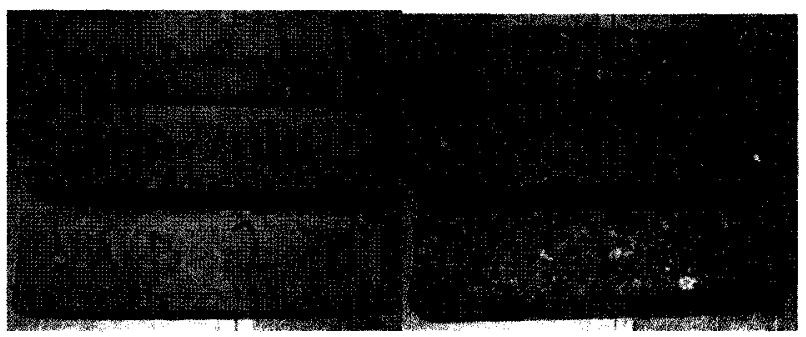

Scaling at the bottom after 300 freeze-andthaw cycles

(c) GC-VA-D-F-G23 specimens

Figure 8.18: Visual appearance of GC-VA specimens during the freeze-and-thaw tests 


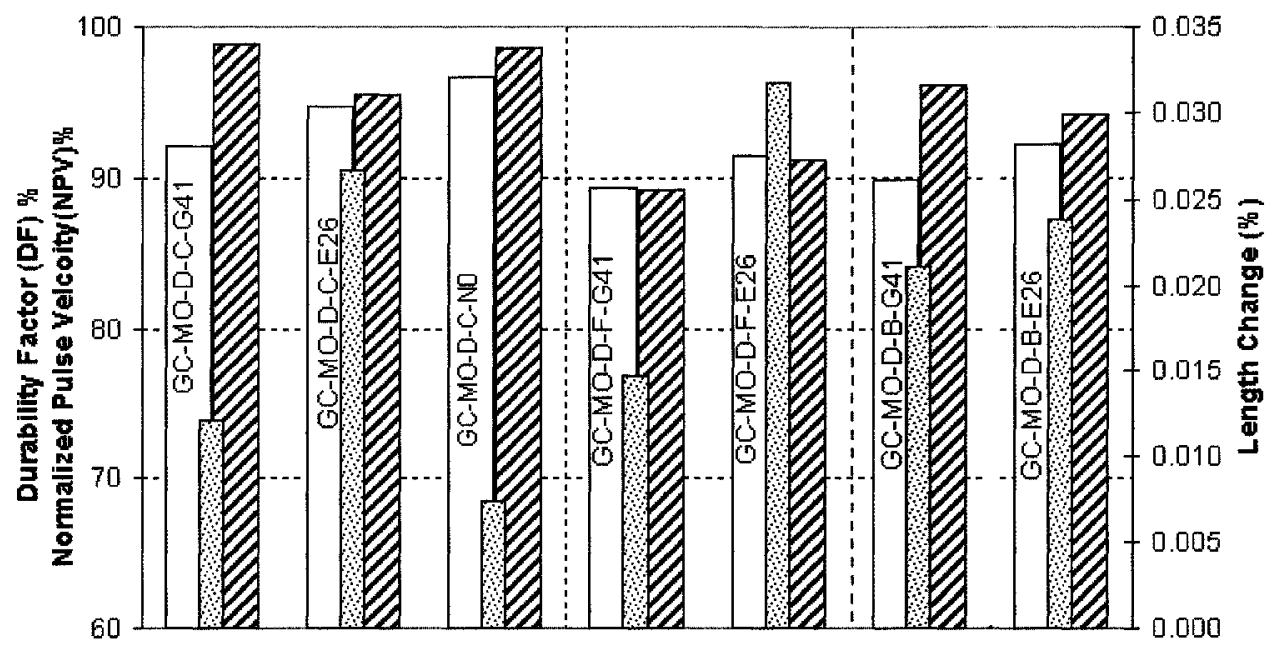

Specimen Designation

QDurability Factor (DF) D Normalized Pulse Velcolty (NPV) 을 Length Change (LC)

(a) GC-MO specimens

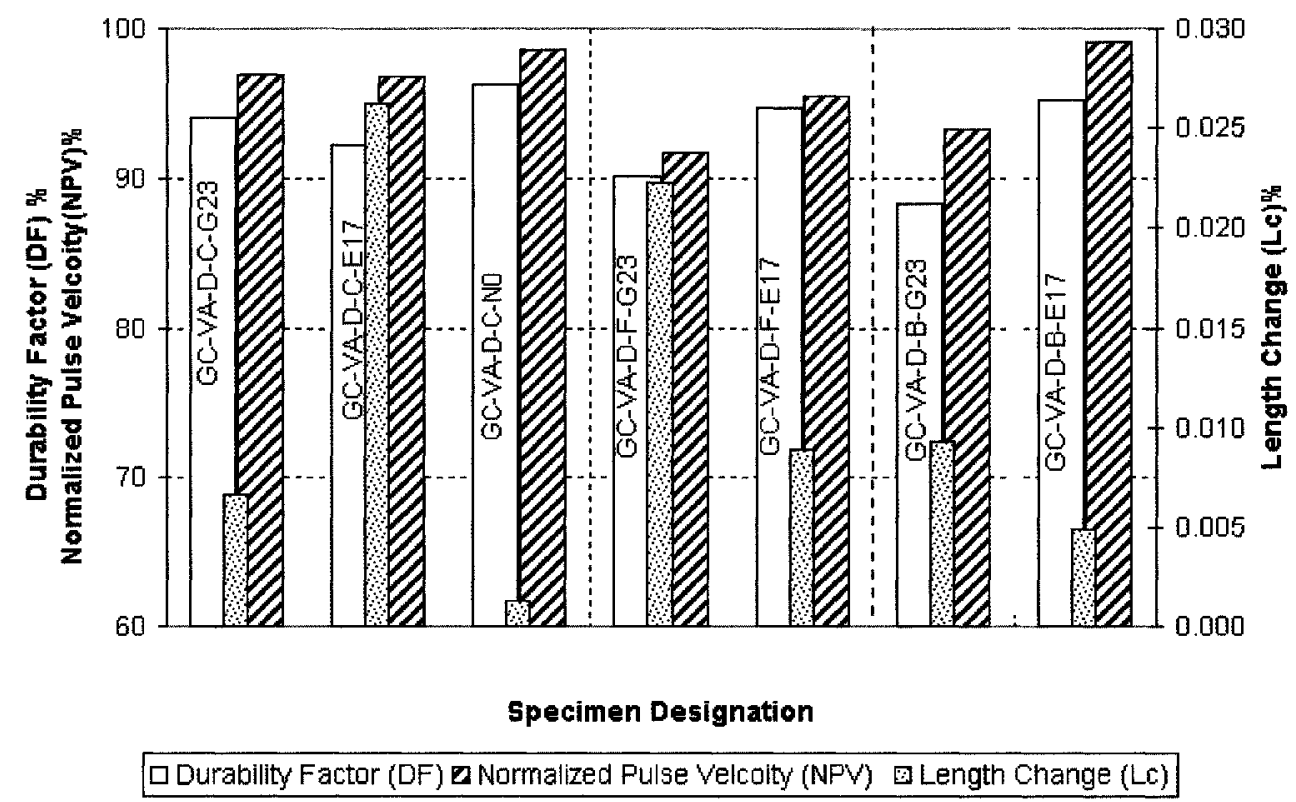

(b) GC-VA specimens

Figure 8.19: Summary of the performance of GC 


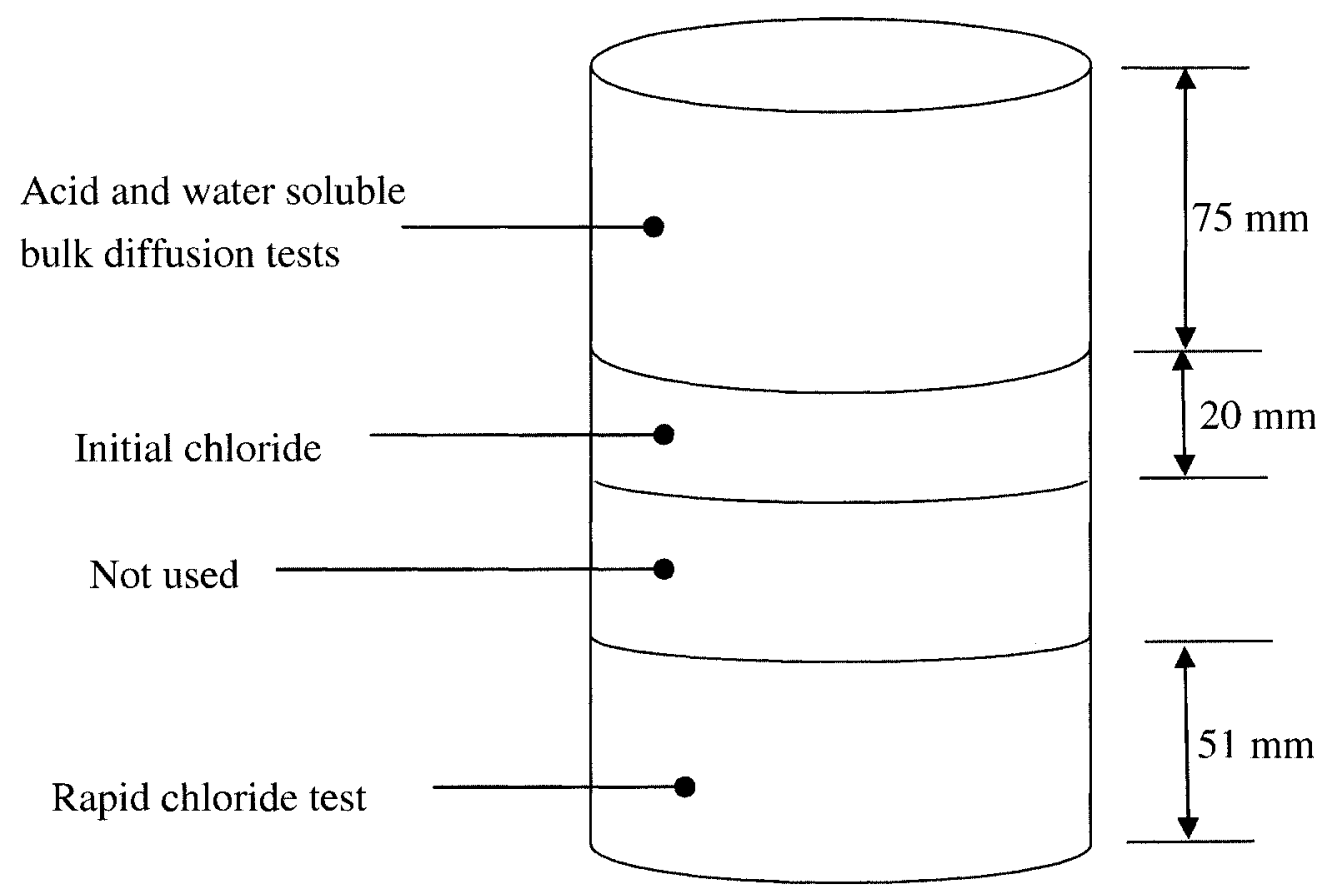

Figure 8.20: Samples for chloride penetration tests 


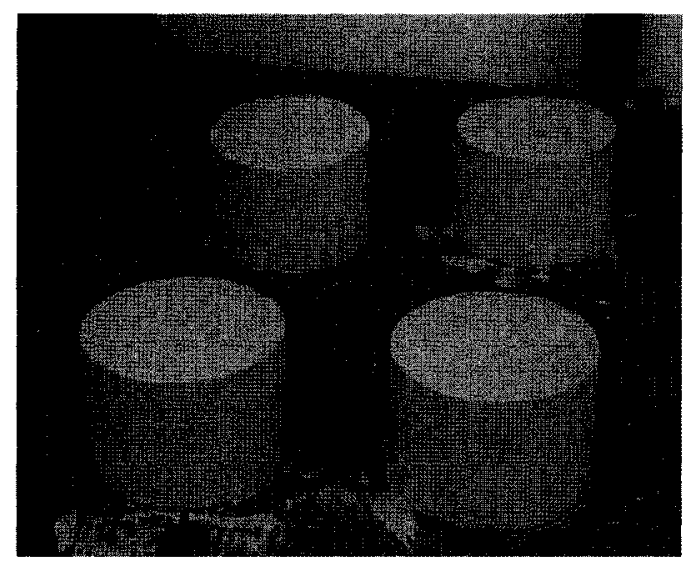

(a) Sealing of specimens

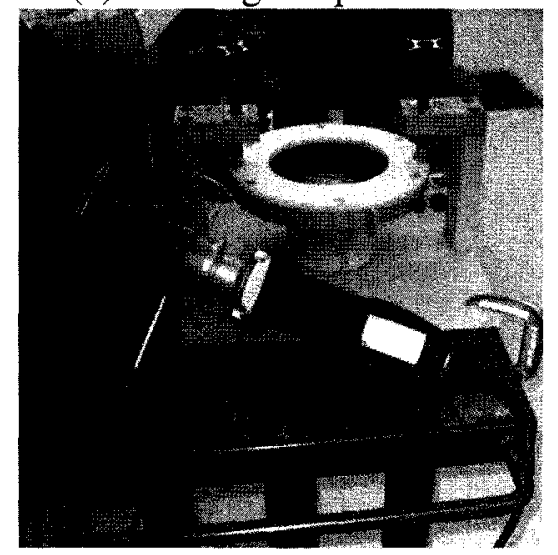

(c) Grinding of the specimens

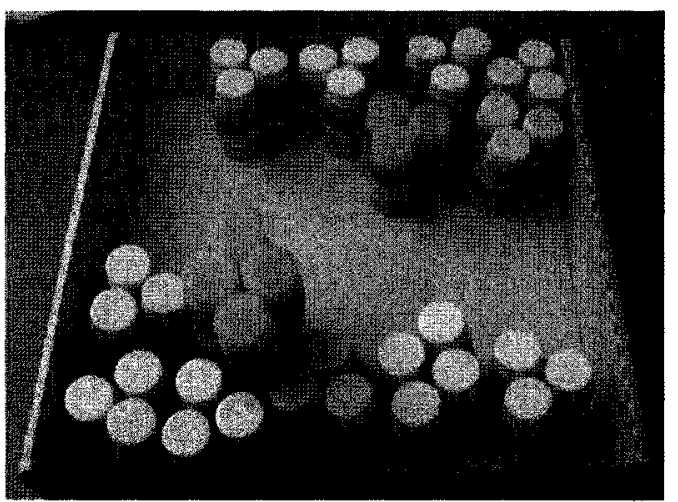

(d) concrete powder in container
(d) concrete powder in container

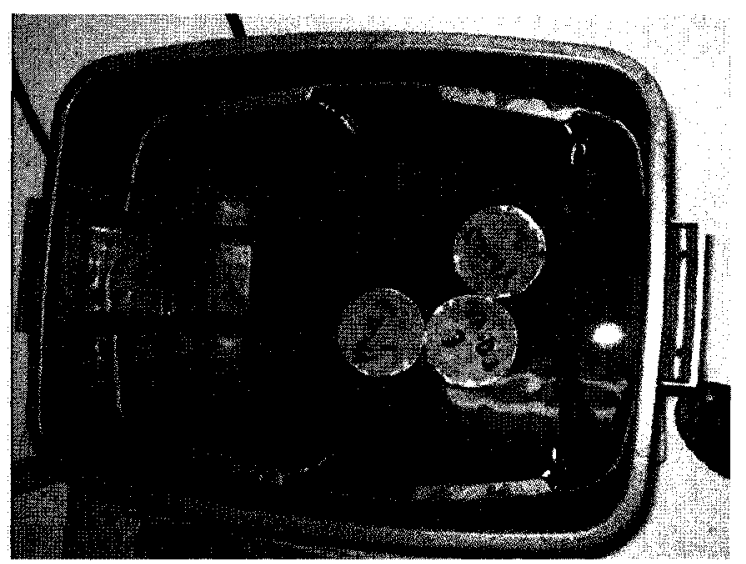

(b) Specimens in sodium chloride solution

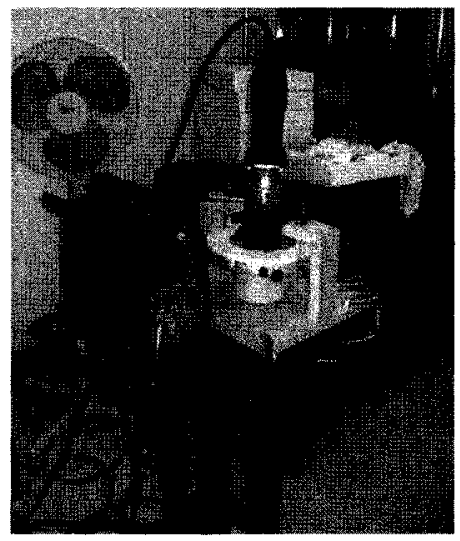

Figure 8.21: Preparation of the concrete powder for bulk diffusion test 


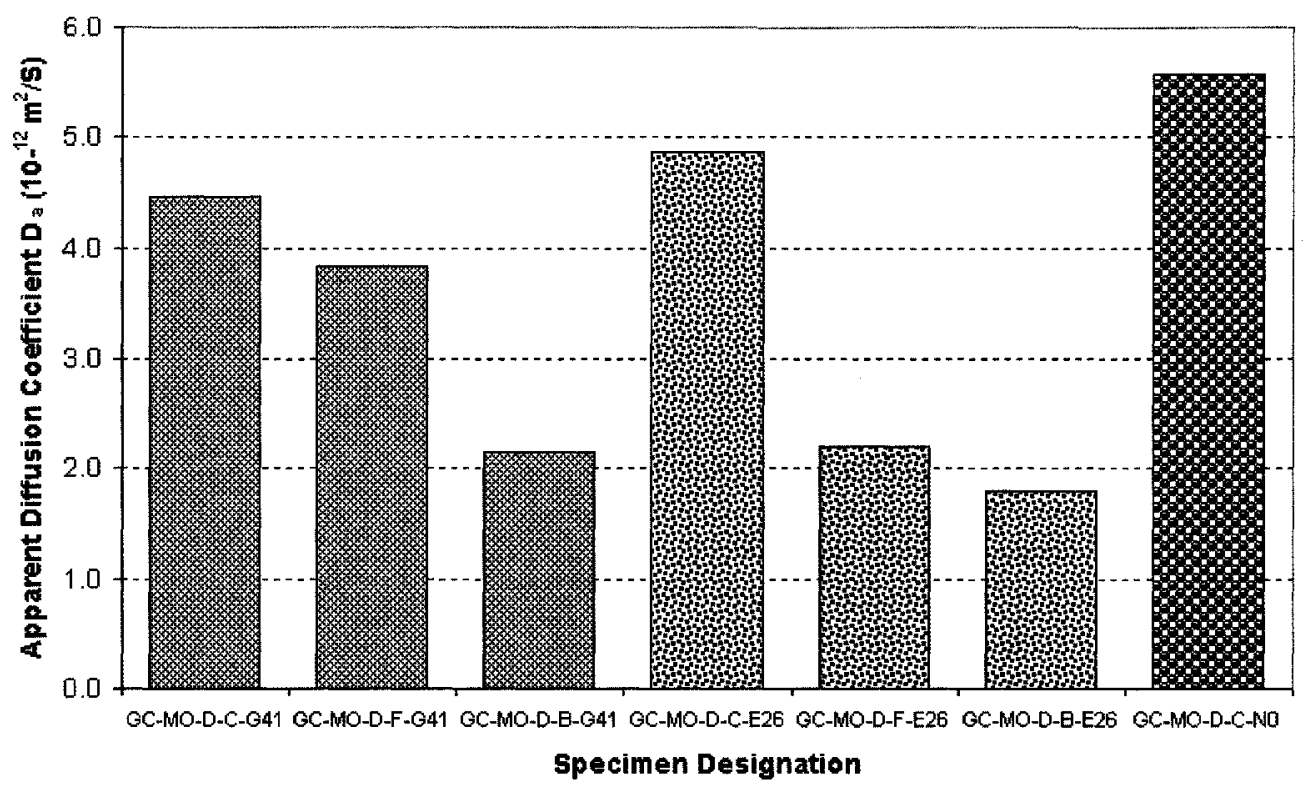

(a) GC-MO Specimens

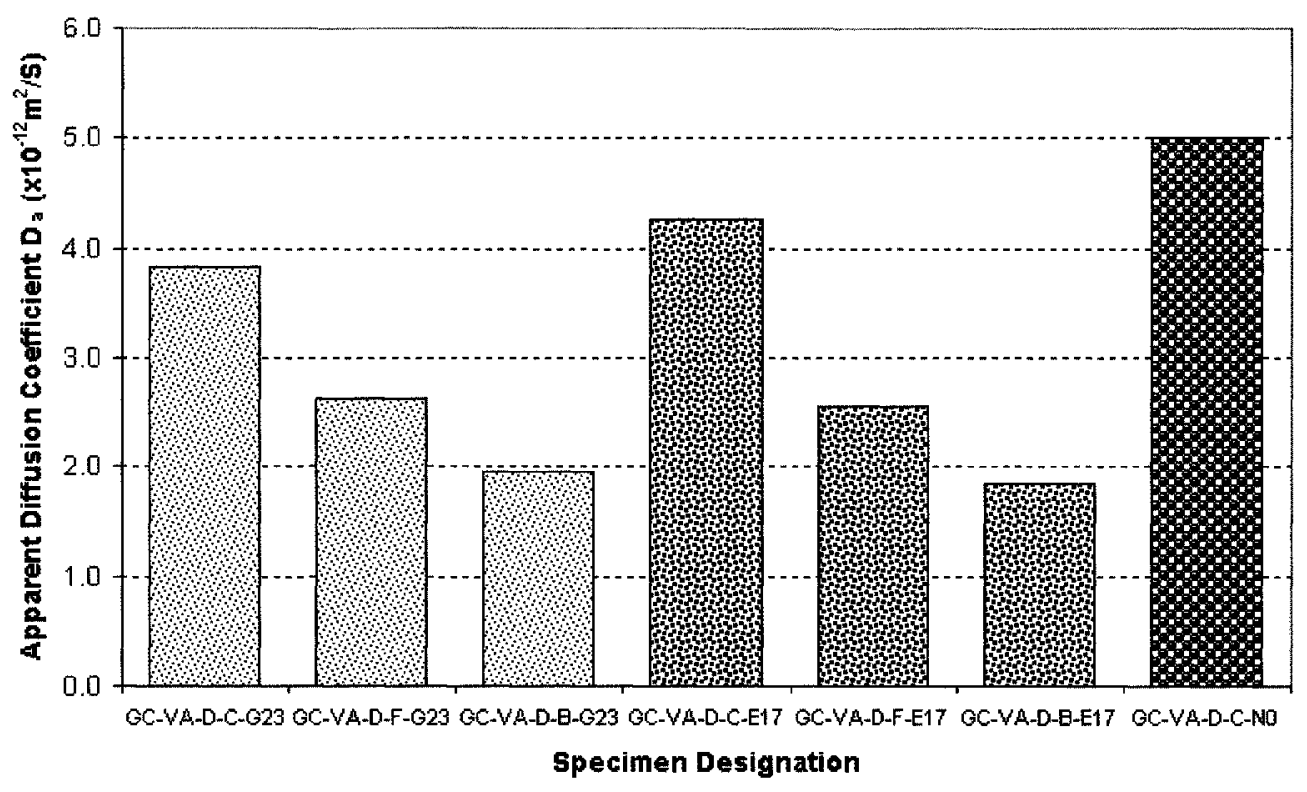

(b) GC-VA Specimens

Figure 8.22: Variation of the apparent diffusion coefficient 


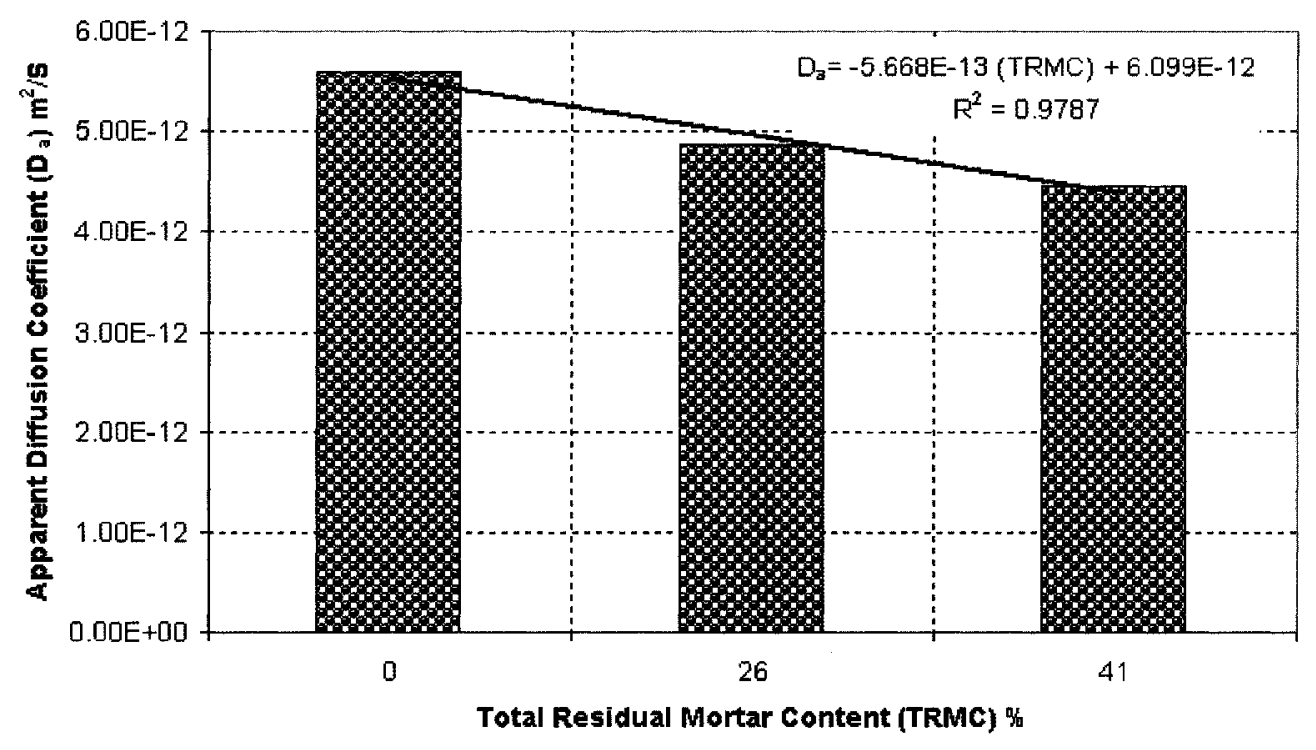

(a) GC-MO-D-C

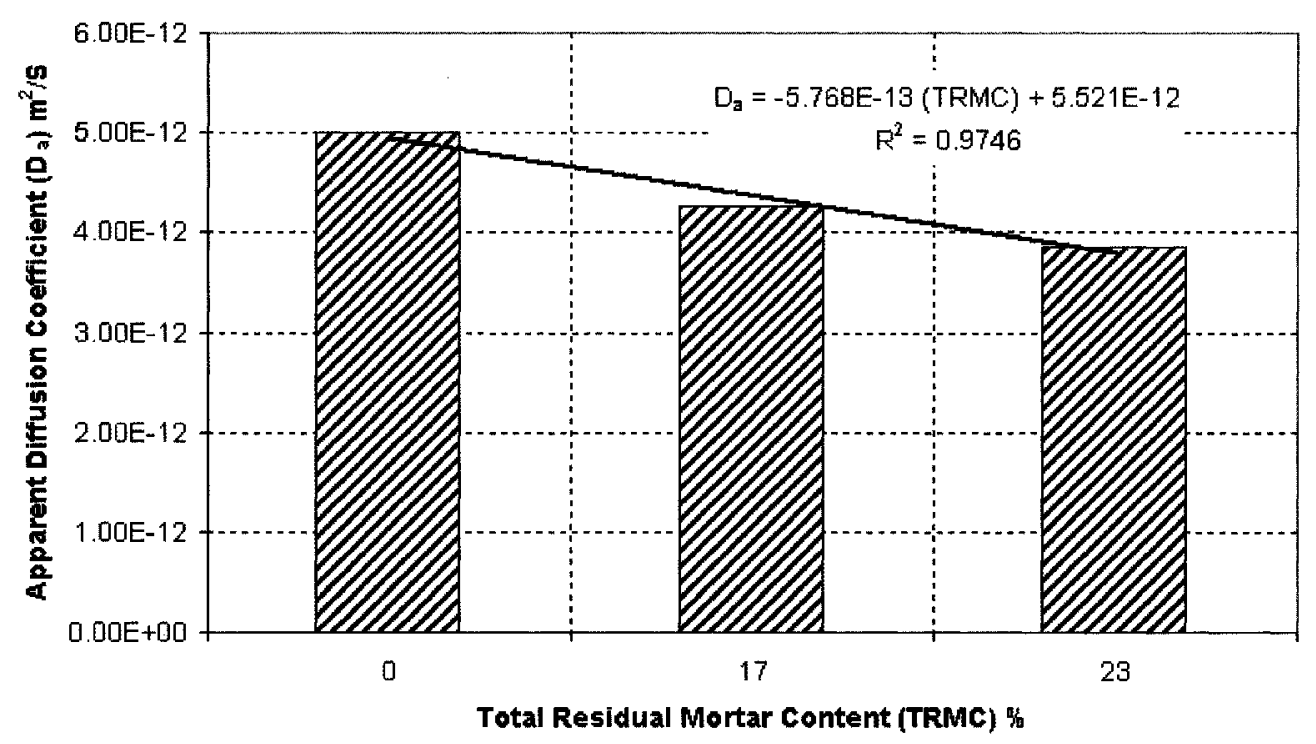

(b) GC-VA-D-C

Figure 8.23: Variation of the apparent diffusion coefficient with TRMC for GC specimens 


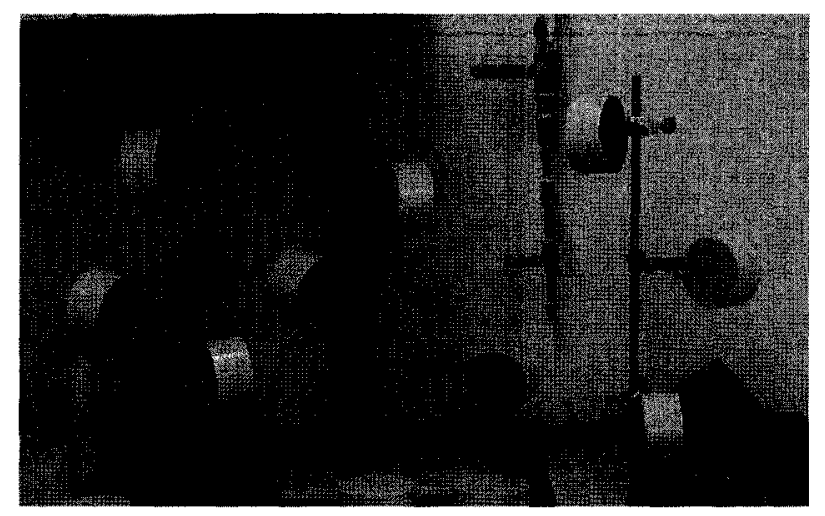

(a) Preparation and painting the specimens

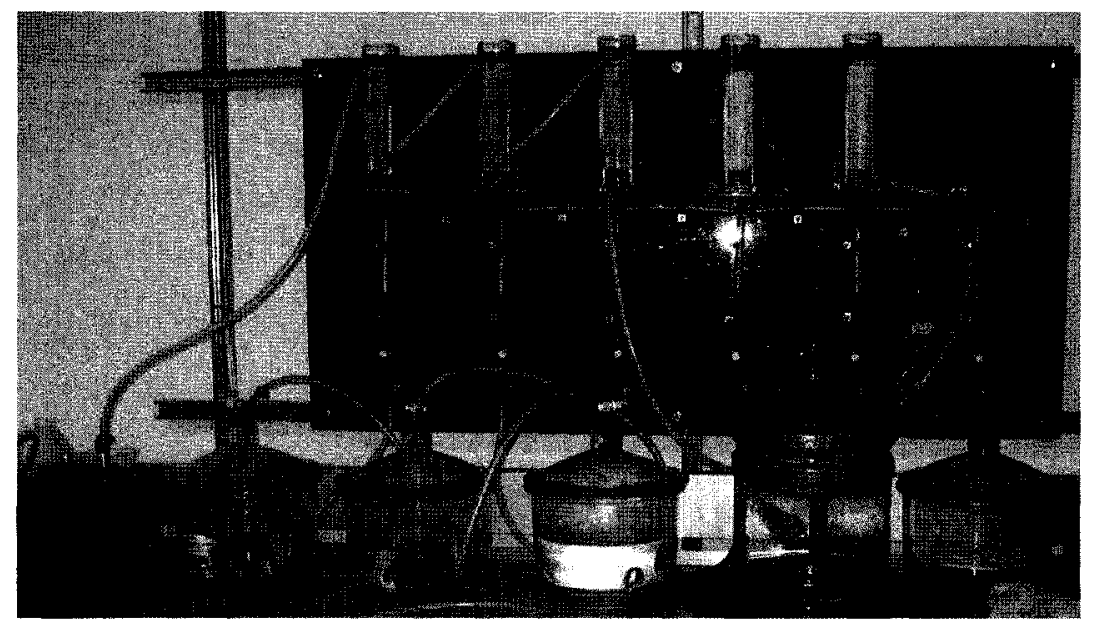

(b) Vacuum saturation the specimen
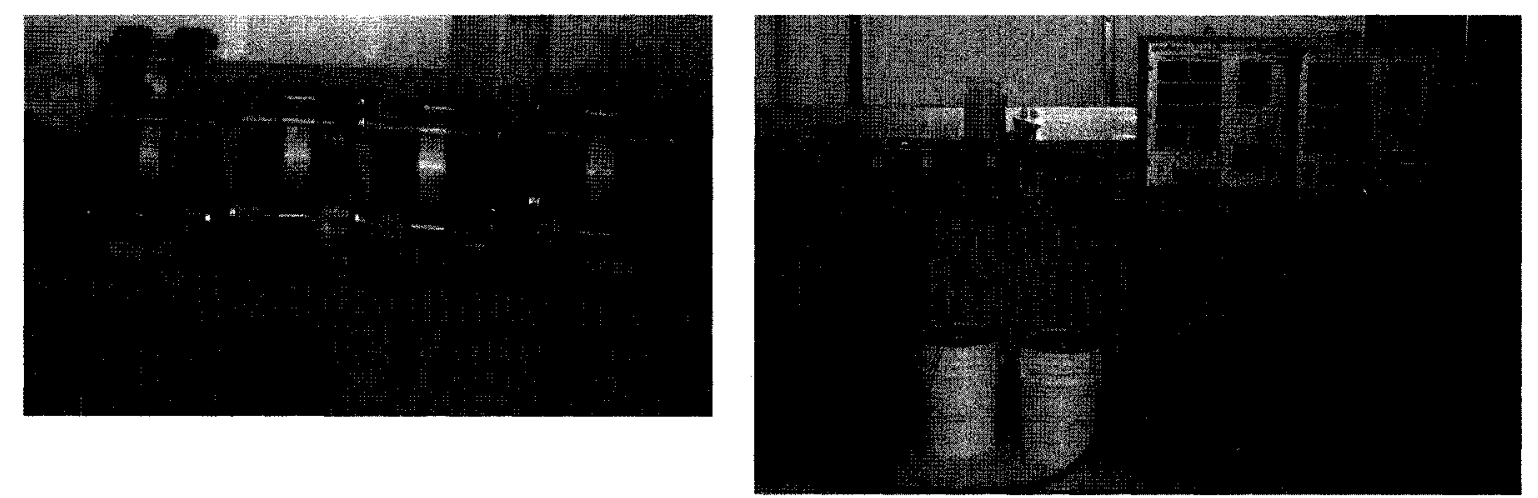

(c) Testing the specimens

Figure 8.24: Rapid chloride test setup 


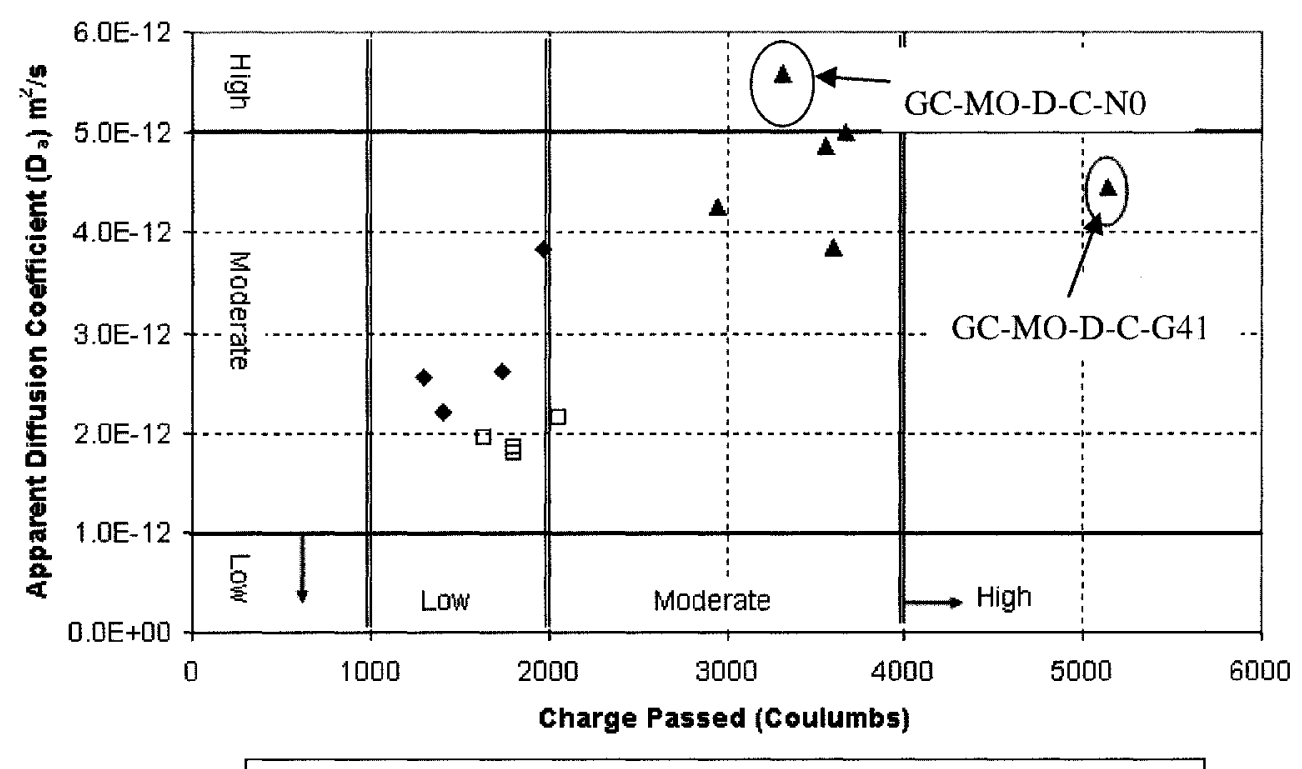

- Specimens with Fly-ash $\square$ Specimens with bfs $\propto$ Specimens with no SCM

Figure 8.25: Charge passed vs. apparent chloride diffusion coefficient 


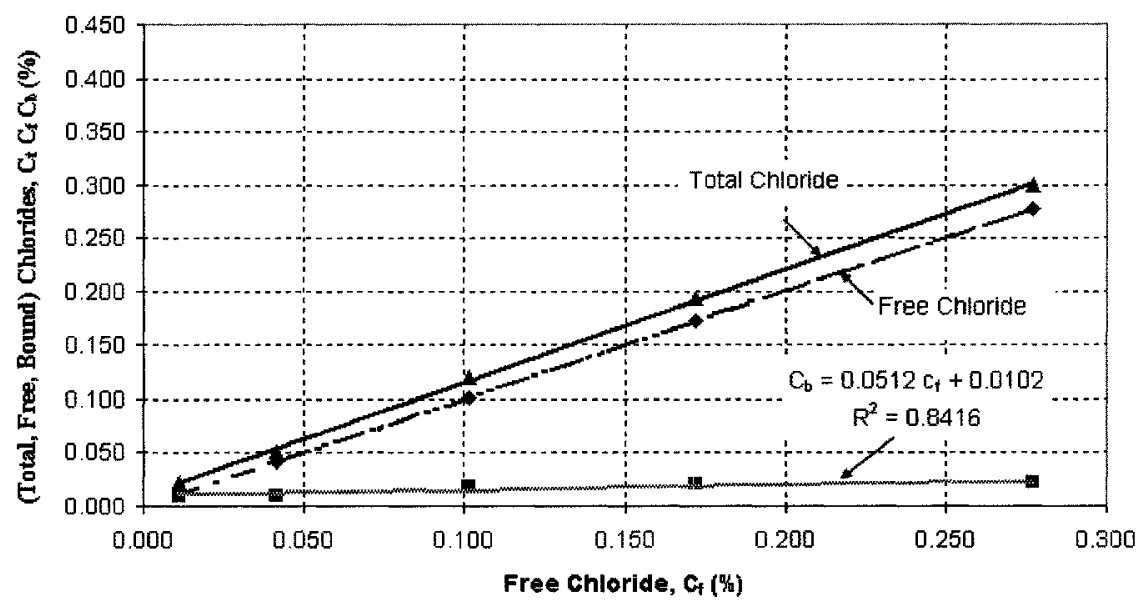

(a) GC-MO-D-C-G41

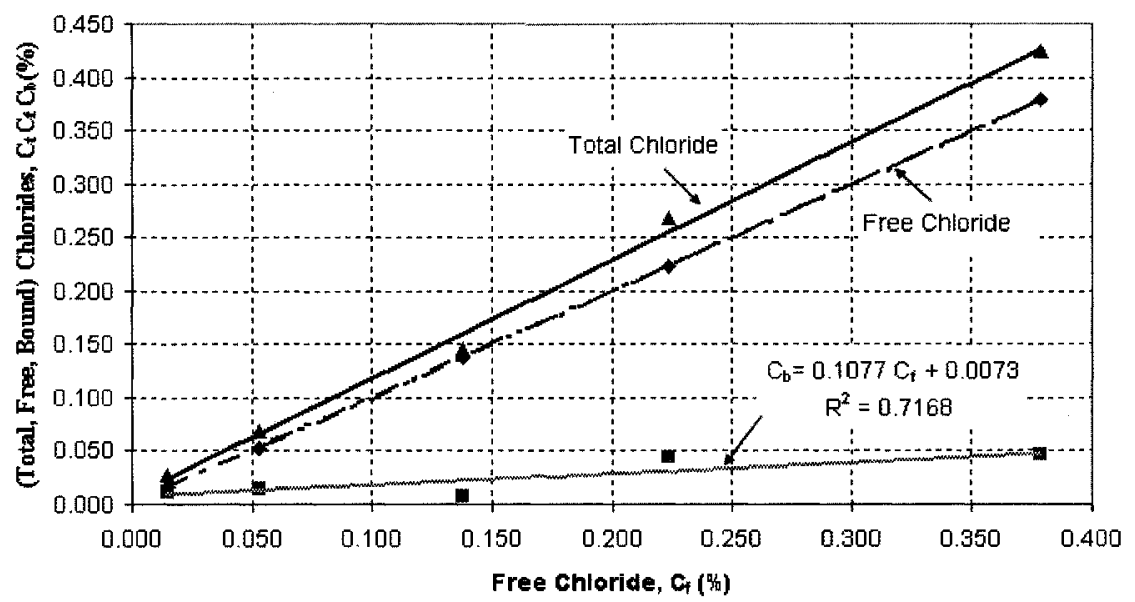

(b) GC-MO-D-C-E26

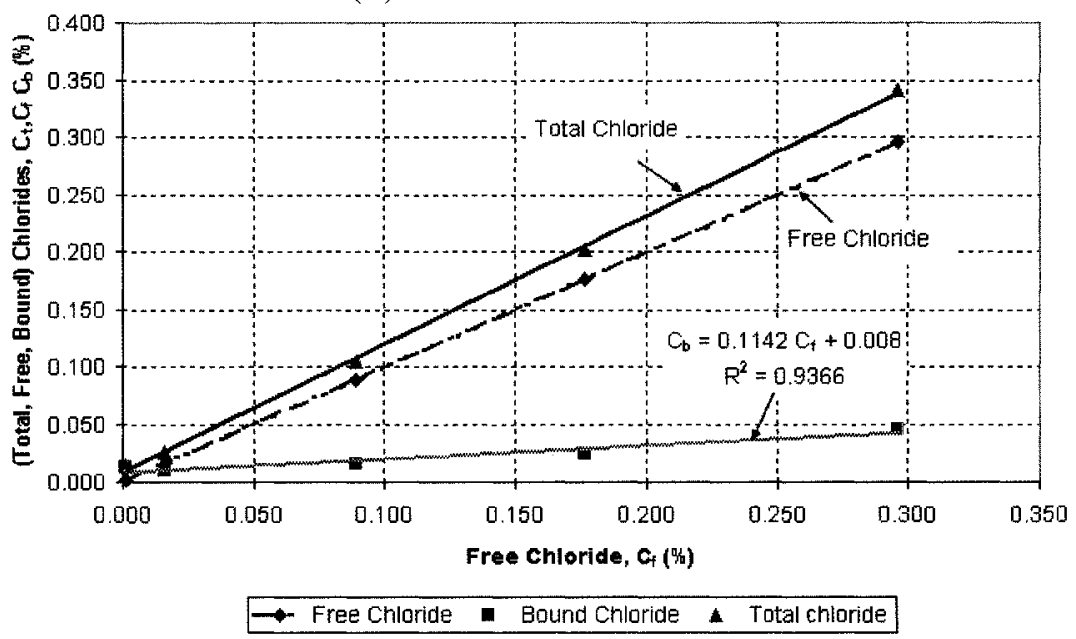

(c) GC-MO-D-C-NO

Figure 8.26: Total, free and bound chloride (\%) measurements for GC-MO specimens 


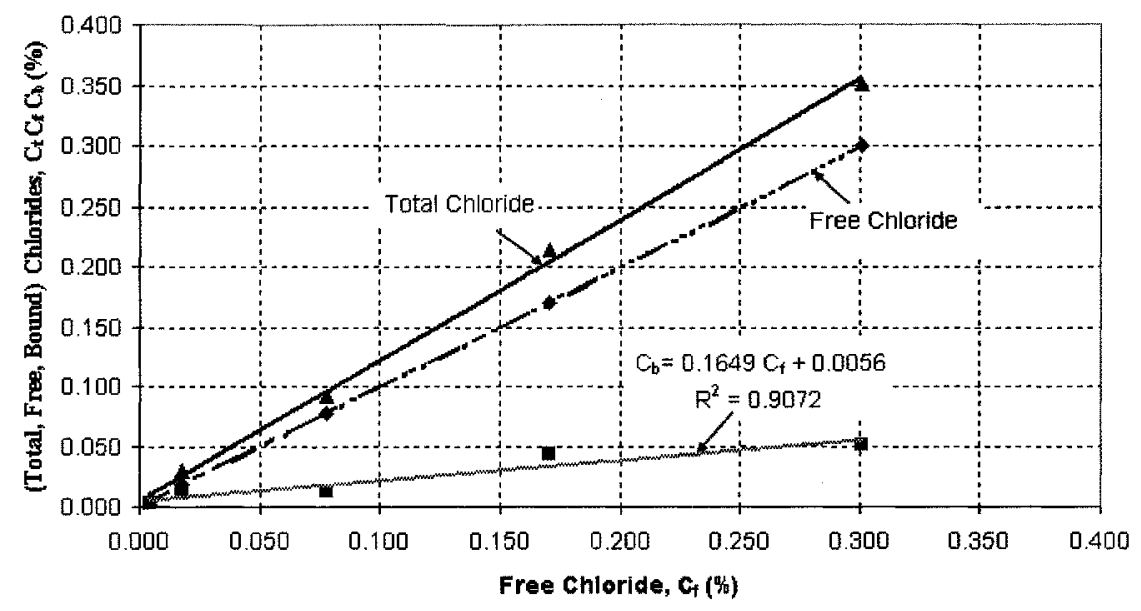

(a) GC-VA-D-C-G23

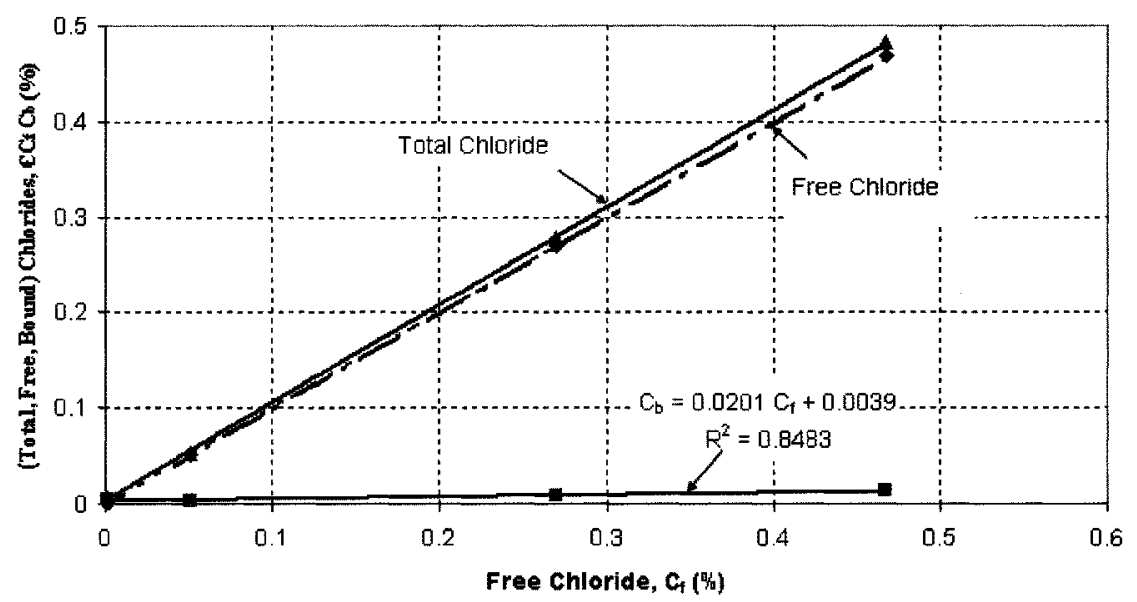

(b) GC-VA-D-C-E17

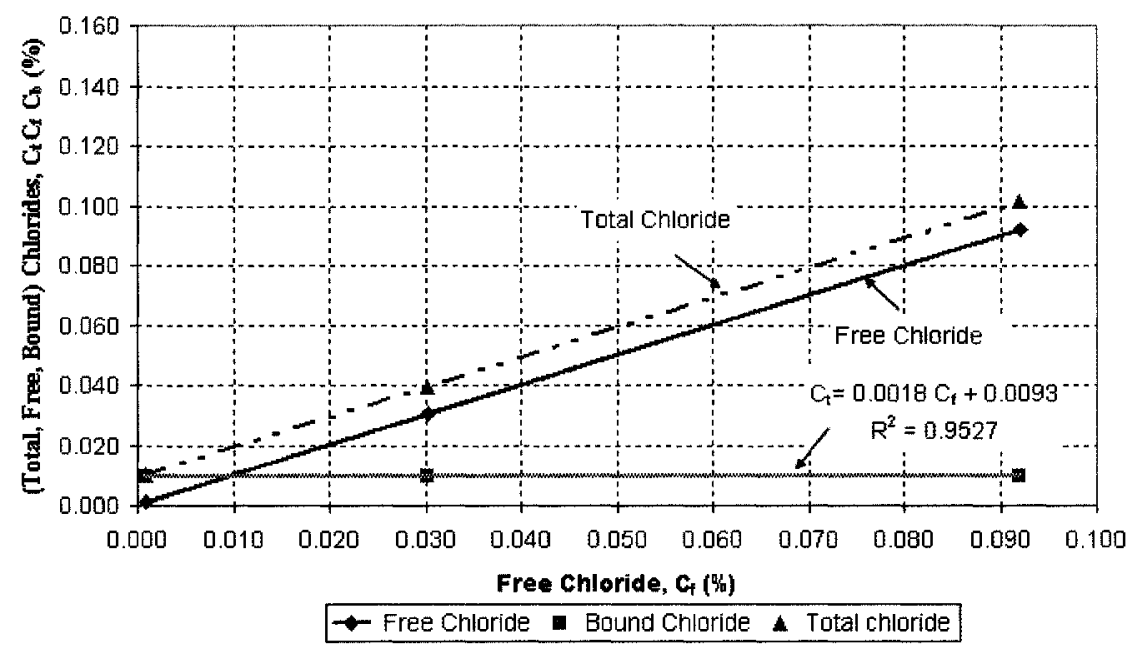

(c) GC-VA-D-C-NO

Figure 8.27: Total, free and bound chloride $(\%)$ measurements for GC-VA specimens 


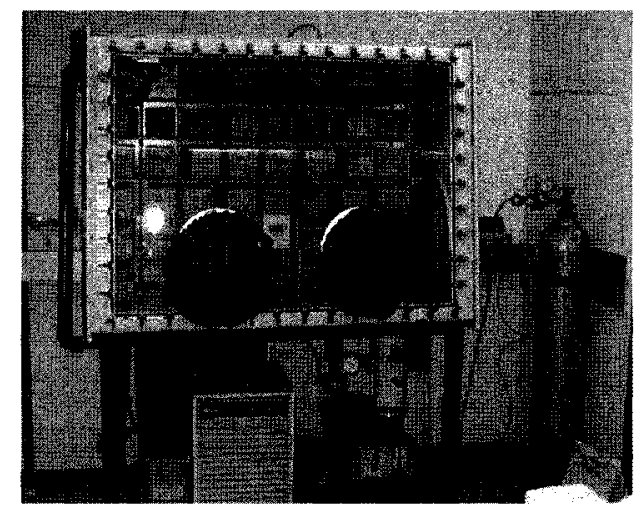

(a) Carbonation chamber

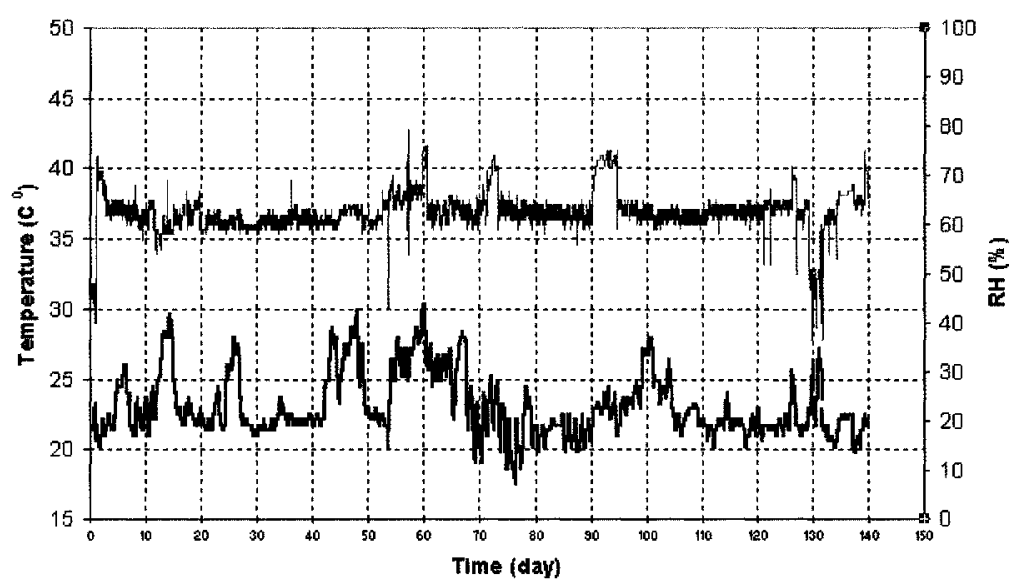

(b) Relative humidity and temperature measurement in the carbonation chamber.

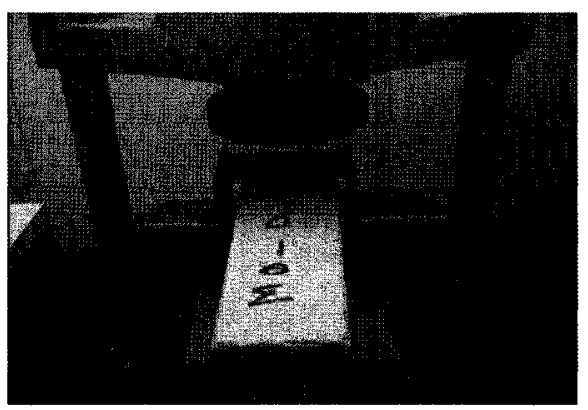

(c) Cutting the specimen
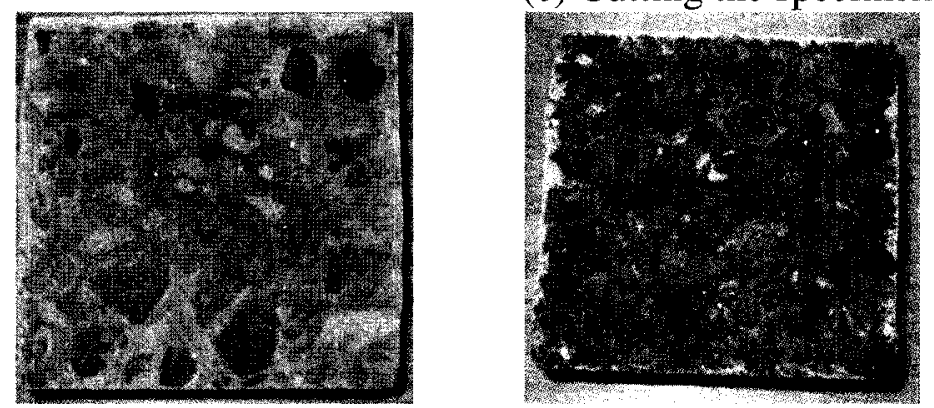

After spray (7-days)

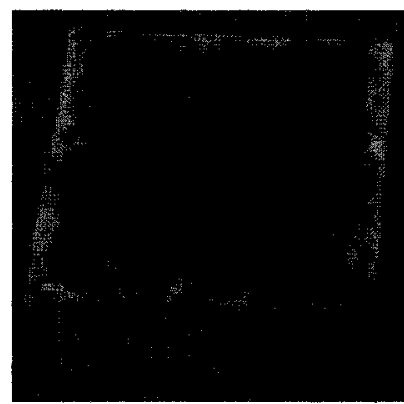

After (84 days)

(d) Spray with phenolphthalein

Figure 8.28: Carbonation test apparatus and process 

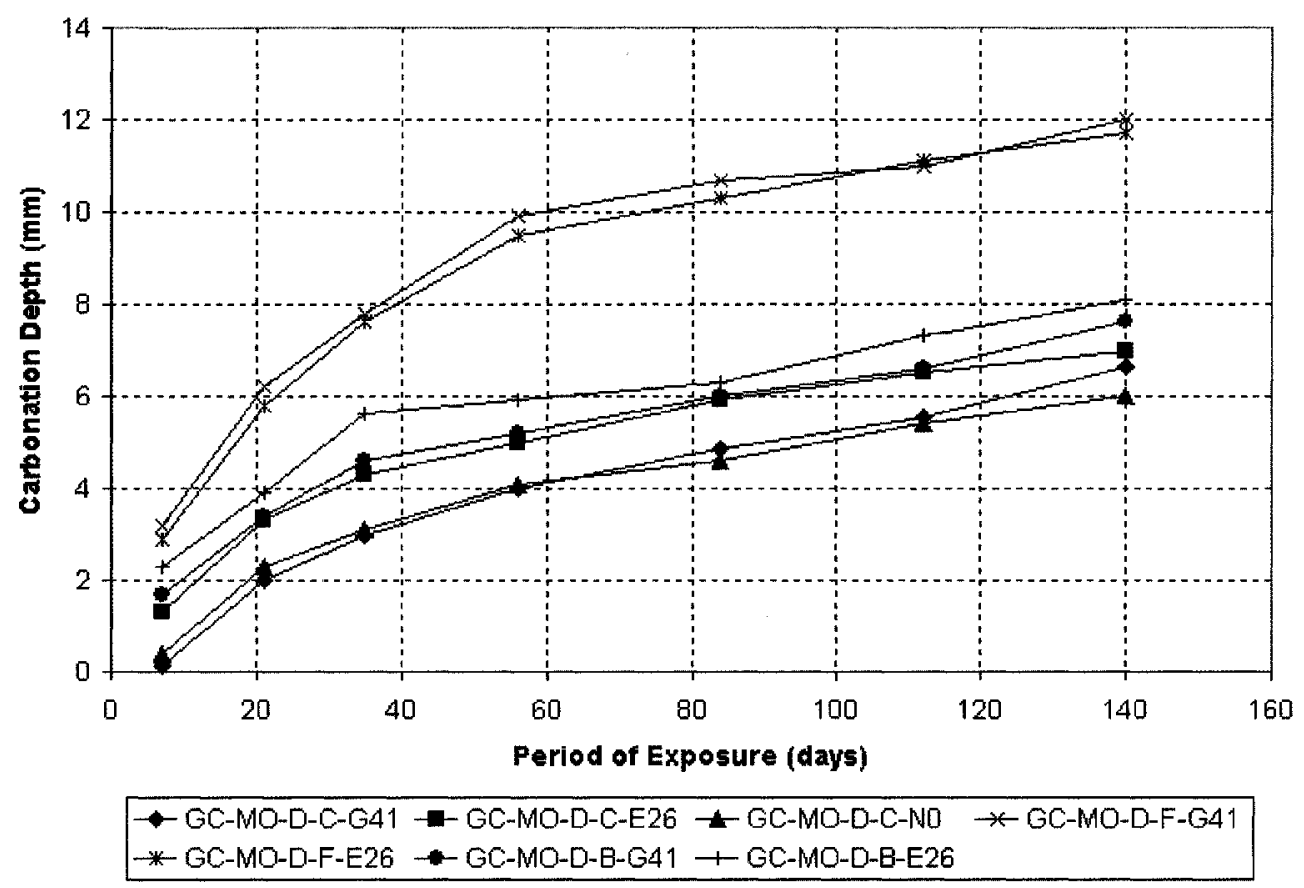

(a) GC-MO Specimens

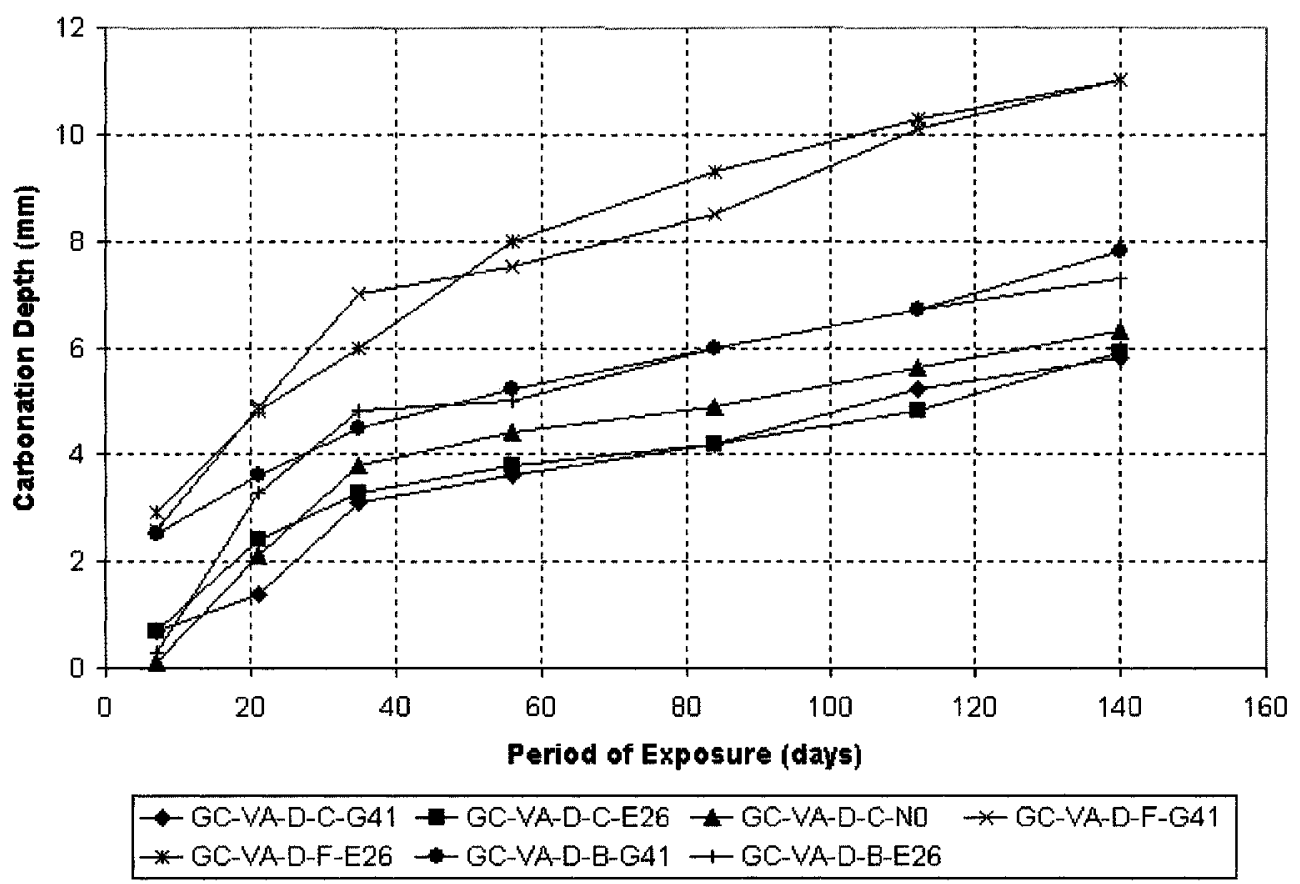

(b) GC-VA Specimens

Figure 8.29: Variation of the carbonation depth with exposure time 


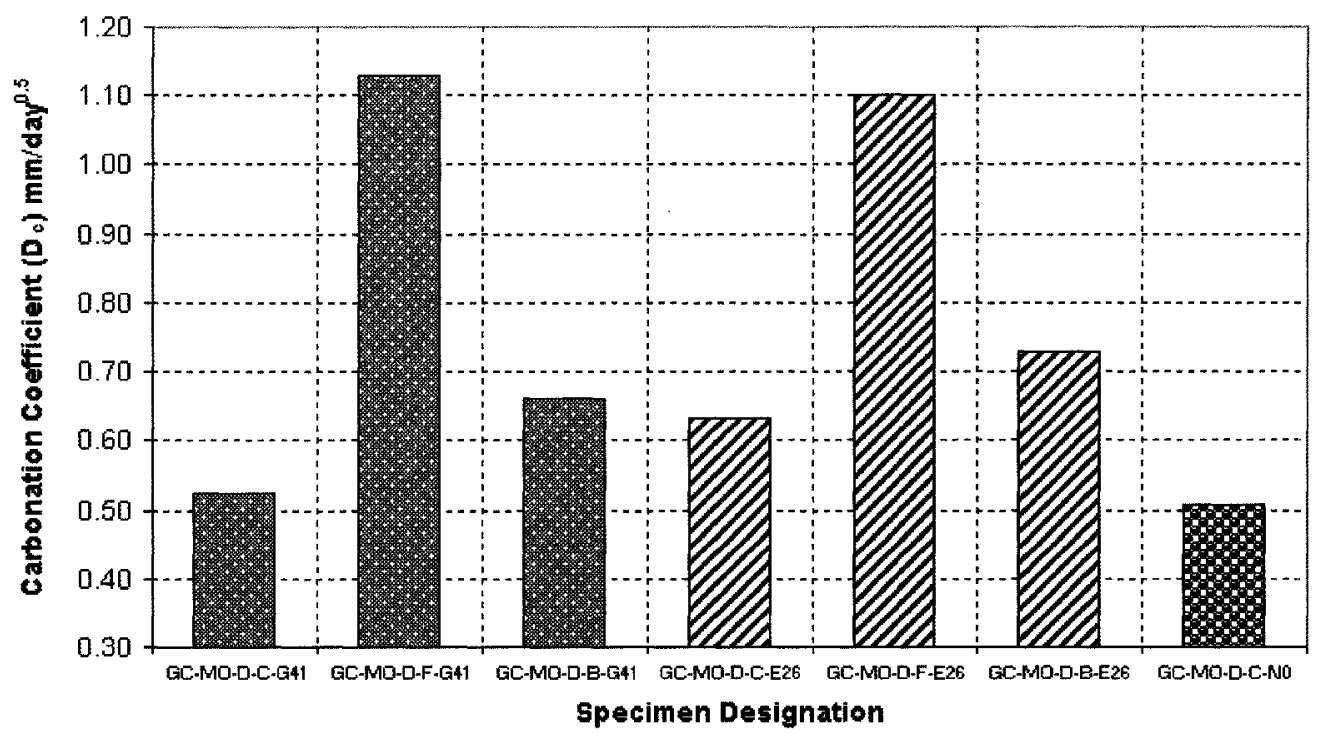

(a) GC-MO Specimens

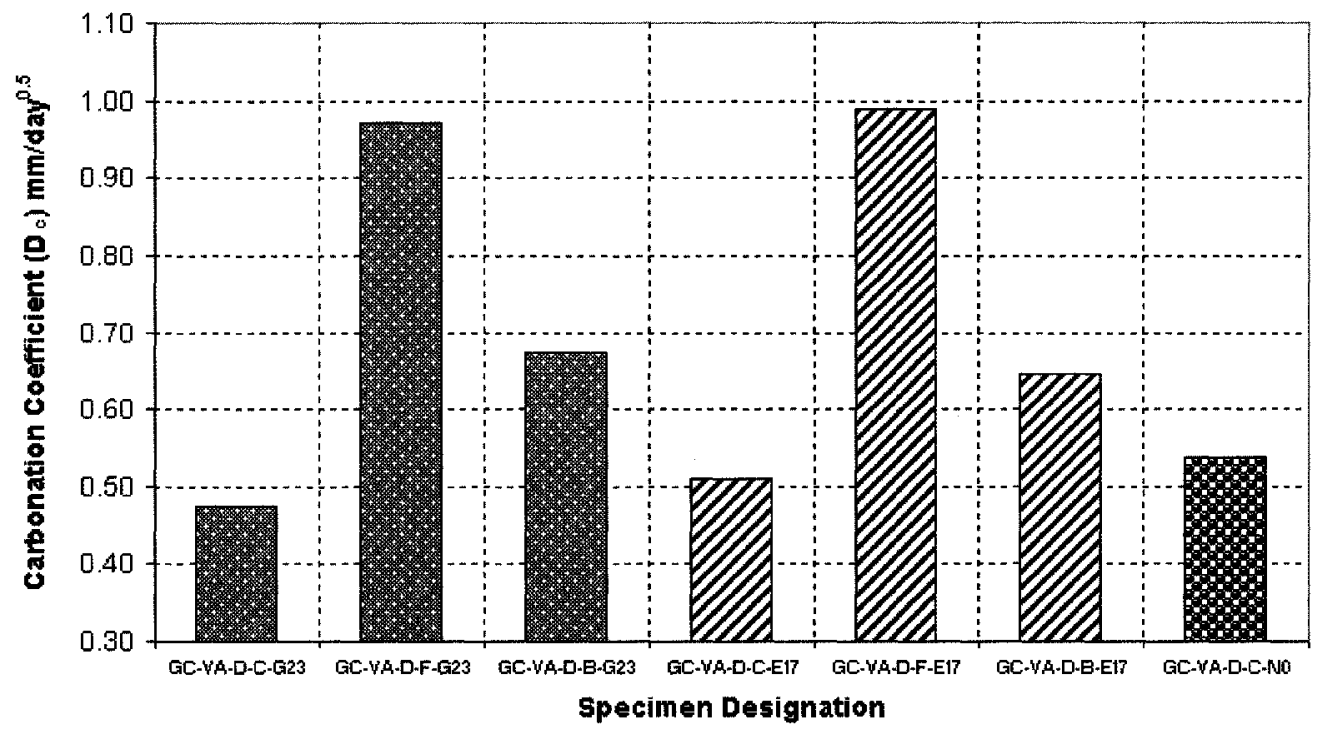

(b) GC-VA Specimens

Figure 8.30: Variation of the carbonation coefficient with specimens with/without SCM's 


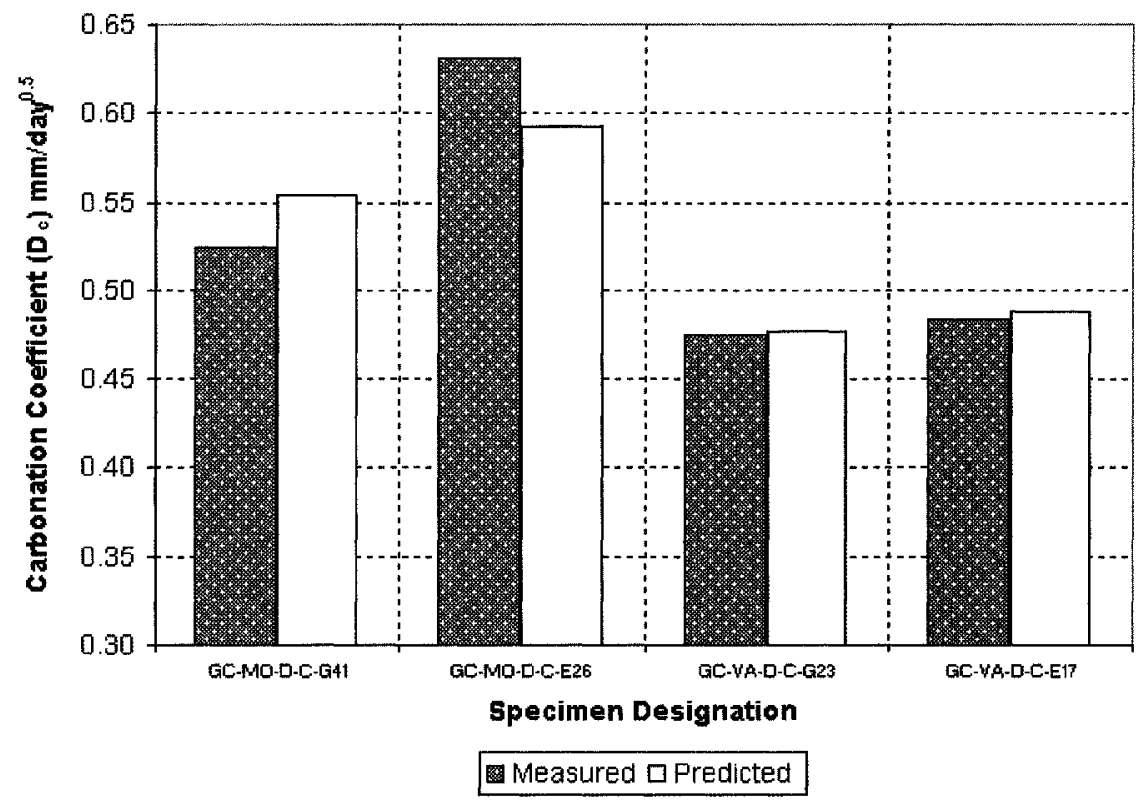

Figure 8.31: Measured and predicted values for the carbonation coefficient for specimens without SCM's 


\section{CHAPTER 9: CONCLUSIONS AND FUTURE WORK}

\subsection{Conclusions}

A comprehensive study investigating the durability of GC as a structural material is presented in this thesis. The research program was developed around the identified gaps in the literature regarding the structural-grade concrete produced with RCA. The research was carried out in stages including: (1) the material characterization of two sources of RCA obtained from different geographical locations of Canada and showing significantly different properties; (2) the preliminary study investigating the fresh and hardened properties of concrete produced with these two types of RCA; (3) the development of mix design procedures for GC based on the results obtained from the preliminary study; (4) the investigation of the durability properties, more specifically, the resistance to freezeand-thaw action, chloride penetration and carbonation, of $\mathrm{GC}$ produced with the developed mix design procedures. The following sections provide the conclusions of the research by subject.

\subsubsection{Material Characterization}

- For the two sources used in this study (RCA-MO and RCA-VA), the main factor affecting the RCA properties, such as absorption capacity, specific gravity, porosity, and Los Angeles abrasion value, was found to be the RMC.

- The relationships between the RCA properties and RMC have been shown to be independent of the source of RCA; i.e., for the two sources used in the study, the main factor affecting the RCA properties was found to be the RMC, not the source. This 
conclusion is reinforced by the analytical expressions developed through a rational approach relating the RCA properties to the RMC.

- The rational approach developed in this study can be used to determine the properties of different types of RCA with minimum amount of experimental work, thus reducing the uncertainty and the cost associated with the material characterization process of RCA before they are used in GC mixes.

- A new experimental method to quickly determine the RMC of RCA was developed. The results obtained through image analysis confirmed that the quick laboratory test provides very accurate measurements of RMC of RCA.

- Both RCA determination test and the image analysis showed that RMC of RCA-MO and RCA-VA showed large difference: RMC for RCA-MO and RCA-VA were determined to be $41 \%$ and $23 \%$ (by weight). Volume-based measurements were $49.6 \%$ and $31 \%$, respectively.

- Los Angeles abrasion value of RCA was lower than 50\%, which is the allowable abrasion value for coarse aggregates to be used in structural concrete specified by ASTM C33-93. However, it was noted that most of the abrasion loss was due to the separation of residual mortar from the OVA particles.

- It has been observed that the Los Angeles test removes the vast majority of the residual mortar from the OVA particles. Visual examination of the samples after each test confirmed that OVA particles were mostly free of residual mortar. Actually, the Los Angeles abrasion values of RCA-MO and RCA-VA were found to be very close to the weight-based RMC of these aggregates; therefore, there is a possibility that the 
Los Angeles test could be used as a quick test method to rapidly assess the RMC of RCA. However, this observation needs to be investigated further.

- There is no significant difference between the specific gravity and absorption capacity for RCA-MO and RCA-VA aggregate material immersed in water for 24, 48 and 72 hours. Therefore, the immersion of the RCA in water for 24 hours can be considered as a practical time period for the calculation of the specific gravity and absorption capacity of RCA.

- The soundness of RCA was studied using two standard test procedures: (1) ASTM C 88-90 (ASTM, 2003) using sodium sulfate and magnesium sulfate solutions; (2) MTO unconfined freeze-and-thaw test method LS-614 (MTO, 2001) using repeated freezeand-thawing for aggregate sample immersed for $24 \mathrm{hr}$ in a sodium chloride solution. The test based on the sodium sulfate solution failed to satisfy the ASTM standards for both RCA-MO and RCA-VA. On the other hand, using magnesium sulfate solution, both RCA-MO and RCA-VA satisfied the ASTM standard. These controversial results show that ASTM soundness test is not conclusive and require further investigation so that it is calibrated for RCA.

- MTO soundness test, which is based on applying mechanical stresses on the aggregates through freeze-and-thaw cycles in sodium chloride solution, revealed that only RCA-VA satisfied the soundness requirements.

- In general, it can be concluded from the inconsistencies of the results of different methods that the determination of the soundness of RCA is a topic that requires further investigation 


\subsubsection{Preliminary Study: Fresh Properties of GC}

- It has been demonstrated that the ACI guidelines for achieving workable structuralgrade concrete (i.e., Table 4.1) are applicable to GC produced with RCA.

- It has been observed that the main factors affecting the workability of the GC mixes are the shape and surface characteristics of the RCA.

- TRMC does not seem to be correlated with the slump of the fresh mix for GC-MO samples. This observation was expected since the shape and the texture of the RCAMO and NA (limestone) particles are quite similar to each other. Since the amount of residual mortar that is attached to NA (limestone) does not change the shape and texture of the aggregates significantly, the TRMC does not have an effect on the slump for these mixes.

- In the case of GC-VA mixes, on the other hand, there is a slight, but recognizable decrease in the slump of the mixes as the TRMC increases. This observation is true for both air-entrained and non-air-entrained samples and can be explained by the difference between the RCA-VA and NA (river gravel) particles in terms of surface texture and the shape. River gravel has smooth surface and round shape. The attached residual mortar on the river gravel particles makes the surface texture of the aggregates rougher and their shape more irregular and angular.

- The TRMC has clear effect on the fresh density of the concrete produced with RCA. The increases in the TRMC decrease the fresh density of GC. General expressions that relate the fresh density of air-entrained and non-air-entrained concrete to the TRMC and $w / c$ ratio were developed. 


\subsubsection{Preliminary Study: Mechanical Properties of GC}

- The hardened density of the specimens tested has been observed to be in the range of normal density concrete.

- The hardened density of GC has been shown to decrease by the increasing values of TRMC.

- As a good quality control practice, it might be necessary to carry out larger number of compressive tests than the usual two when GC has large TRMC values (TRMC> $40 \%)$.

- The main factors affecting the compressive strength and elastic modulus of GC are the TRMC, w/c ratio and air content. General expressions relating the compressive strength and elastic modulus of air-entrained and non-air-entrained GC to the TRMC and $w / c$ ratio were developed.

- The main factor affecting the mechanical properties of $\mathrm{GC}$ is the residual mortar content of the RCA, and not the RCA content.

- A relationship between the modulus of elasticity and compressive strength for GC was developed, in which the effect of the TRMC was also incorporated. It was found that the CSA standard equation for conventional concrete that relate the compressive strength to its elastic modulus can be used for GC instead of the developed equation when the TRMC values are not large (e.g. lower than $25 \%$ ).

- The CSA equation for conventional concrete that relates the compressive strength and the splitting tensile strength can also be used for GC. 


\subsubsection{Durability of GC}

\subsubsection{Freeze-and-Thaw Action}

- The visual examination of GC-MO and GC-VA specimens was carried out throughout 300 freeze-and-thaw cycles. The defects observed during and at the end of the freezeand-thaw tests for GC-MO and GC-VA specimens ranged from minor scaling to pop outs.

- The performance of GC against freeze-and-thaw action was represented by the durability factor, normalized pulse velocity and length change values. The durability factors for all GC specimens were higher than $88 \%$, higher than $60 \%$ specified by the ASTM C 666-97 to terminate the freeze-and-thaw test. The maximum length change for all specimens was lower than $0.032 \%$. The normalized pulse velocity measurements were also found to be higher than $88 \%$.

- The performance indicators, the relative dynamic modulus and pulse velocity are correlated well with each other; therefore, either method can be used to study the effect of freeze and-thaw action in GC. However, since the relative dynamic modulus technique is standardized, and the pulse velocity measurements do not provide additional information, it can be stated that ASTM C 666-97 (ASTM, 2003) method is more appropriate to study the freeze-and-the resistance of GC.

- Length change measurements can be used to have a general idea about the freeze-andthaw resistance of GC; however, since the length changes are very small and are sensitive to the measurement conditions, it can be stated that it should be used cautiously. 
- It can be stated that, when the same cementitious material is used, the freeze-and-thaw resistance of GC decreases with increasing values of TRMC. Furthermore, the mixes with no RCA replacement (NAC, TRMC $=0$ ) showed higher durability factor than GC specimens designed with the equivalent total volume of mortar method; this is attributed to the presence of residual mortar in GC.

- The durability factor of GC was not improved by using SCM's as a partial replacement for OPC. However, as mentioned earlier, it should be noted that these tests were carried out at 28 days, and it is expected that the properties of the mixes with SCM's would improve as the curing period for these specimens becomes longer.

- From all performance indicators, it can be concluded that GC produced with either general mix design method (100\% RCA replacement) or with the equivalent total volume of mortar method (partial RCA replacement) have strong resistance against freeze-and-thaw action, a resistance expected from structural-grade concrete. However, since lower TRMC values can be achieved using the equivalent total volume of mortar method, this method could be preferred for RCA with very large RMC values and when freeze-and-thaw resistance is considered to be a serious problem for the structure under consideration.

\subsubsection{Chloride Penetration}

- The measured acid soluble (i.e., total) and water soluble (i.e., free) initial chloride concentrations of the GC-MO and GC-VA specimens were found to be lower than the limits specified by the standards.

- Since the estimation of the total cement content of GC mixes is difficult due to the fact that the cement content in the residual mortar cannot be accurately known, it is 
recommended for GC that all limits for chloride content are specified in terms of the mass of concrete, rather than the mass of cement.

- The apparent chloride diffusion coefficients for all GC specimens were found to be in the same order of magnitude as the conventional structural grade concrete (i.e., $10^{-12}$ $\left.\mathrm{m}^{2} / \mathrm{s}\right)$. Specifically, the apparent chloride diffusion coefficients for all GC specimens were in the moderate chloride permeability range.

- The resistance to chloride penetration of GC is improved by using SCM's (fly ash or bfs) as partial replacement for OPC. The use of bfs reduces the apparent diffusion coefficient of all mixes designed with both mix design methods 1.2-2 times more than the reduction created by the addition of fly ash. Both general mix design method and the equivalent total volume of mortar method provide good quality concrete against chloride penetration when SCM's are used.

- Although there is a general trend providing increasing apparent diffusion coefficient with increasing rapid chloride test measurements (i.e., charges passed), the correlation between the two provided for conventional concrete does not seem to capture the correlation for GC. Further investigation to establish this relationship will be necessary if rapid chloride test is to be used for GC reliably. However, it should also be noted that, due to possible pre-contamination of RCA with different ions, the rapid chloride test can provide misleading results regarding the chloride permeability of concrete; therefore, even after a correlation is established, the results of the rapid chloride test should be taken cautiously.

- The investigation of the results of the GC specimens produced from different sources of RCA showed that the pre-contamination is one of the main factors affecting the 
apparent diffusion coefficient. If the RCA is not excessively pre-contaminated, increased amounts of residual mortar (i.e., TRMC) have the potential to increase the binding capacity of the GC; hence decreasing the diffusion of chloride ions in concrete. Therefore, when RCA is clear from pre-contamination, the use of the general mix design method improves concrete resistance to chloride ion penetration.

- In the case of pre-contaminated RCA, the increased amounts of residual mortar will not improve the binding capacity of GC, as observed in the GC-MO mixes; therefore, the use of equivalent total volume of mortar method can be considered to be more appropriate to design GC with these RCA.

\subsubsection{Carbonation}

- The carbonation depths of GC with and without SCM's fall in the expected range for structural-grade conventional concrete. GC specimens with no SCM addition showed the lowest level of carbonation; these specimens were followed by the specimens with bfs. On the other hand, mixes with fly ash had the largest amount of carbonation depth and coefficient (low resistance to carbonation).

- The investigation of the results of the specimens designed with different mix design methods showed that the main factor affecting the carbonation of GC is the total reserve alkalinity (alkalinity from hydration of new cement content and residual old mortar). Those specimens with the high total cement content (i.e., total reserve alkalinity) have been found to give higher resistance to carbonation.

- The effect of mix design method on the carbonation of GC is not significant when fly ash is used. Therefore, when fly ash is used, both general mix design and equivalent total volume of mortar methods can be used for designing the mixes. 
- When fly ash is not used, the carbonation of GC produced with RCA with high RMC (e.g. $>40 \%$ as in the case of RCA-MO) was found to be higher in mixes designed with the equivalent total volume of mortar method. Therefore, for these types of RCA, the general mix design method may be preferred.

- When fly ash is not used, the carbonation of GC produced with RCA with low RMC (e.g. $<25 \%$ as in the case of RCA-VA) was found to be similar in all mixes designed with both mix design methods. Therefore, for these types of RCA, both methods may be used.

\subsection{Recommendation for Future Work}

- Although RCA-MO and RCA-VA show significantly different properties, and the results of this study can be extrapolated to other RCA sources, it is beneficial to expand the study to cover other types of RCA from different sources.

- While covering other types of RCA, different durability properties, such as resistance to sulfate and acid attacks and alkali-aggregate reactions, should also be studied.

- Because of the inconsistencies of the soundness results of different methods, the determination of the soundness of RCA is a topic that requires further investigation.

- The possible use of the Los Angeles abrasion test as a quick-and-dirty method to determine the RMC of RCA should be investigated.

- Although a procedure is provided in this research for the determination of the specific gravity and absorption capacity of residual mortar, further studies are necessary to standardize this process.

- The chloride binding in GC should be investigated further to cover mixes with SCM's.

- Since there is a possibility of the release of bound chlorides with carbonation, the 
combined effect of chloride penetration and carbonation should be investigated for GC.

- Although there is a general trend providing increasing apparent diffusion coefficient with increasing rapid chloride test measurements (i.e., charges passed), the correlation between the two provided for conventional concrete does not seem to capture the correlation for GC. Further investigation to establish this relationship will be necessary if rapid chloride test is to be used for GC reliably. 


\section{REFERENCES}

Abbas, A., Fathifazl, G., Isgor, O.B., Razaqpur, A.G., Fouriner, B., and Foo, S. 2006a. Environmental Benefits of Green Concrete. Climate Change Conference, Ottawa, Ontario.

ACI Committee 555. 2002.Removal and reuse of hardened concrete. Report ACI 555R-01, American Concrete Institute, Farmington Hills, Michigan.

ACI Committee 211, 1997. Standard practice for selecting proportions for Normal, Heavyweight and Mass concrete, ACI 211.1-97, American Concrete Institute, Farmington Hills, Michigan.

ACI Committee 222. 1996. Corrosion of metal in concrete. Report ACI 222R-96, American Concrete Institute, Farmington Hills, Michigan.

ACI Committee 318M. 1995. Building code requirements for structural concrete. Report ACI 318RM-95, American Concrete Institute, Farmington Hills, Michigan.

ASTM . 2003. Concrete and concrete aggregates. Annual Book of American Society for Testing and Materials, Volume 4.02, ASTM, Philadelphia.

Ajdukiewicz, A., and Kliszczewicz, A. 2002. Influence of recycled aggregates on mechanical properties of HS/HPC. Cement \& Concrete Composites, 24: 269 -279.

Bairagi, N.K., Vidyadhara, H.S. 1990. Mix design procedure for recycled aggregate Concrete. Construction and Building Materials, 4 (4): 188-193.

Barra de Oliveira, M., and Vazquez E. 1996. The influence of retained moisture in aggregates from recycling on the properties of new hardened concrete. Waste Management, 16 (1-3): $113-117$.

Bertolini L., Elsener B., Pedeferri P., and Polder R. 2004. Corrosion of steel in 
concrete- Prevention, Diagnosis, Repair. Wiley-VCH, Weinheim.

Bouzoubaa, N. 2007. Private communication. Natural Resources Canada, CANMET, Ottawa.

Bureau de normalization du Quebec (BNQ). 2002. Classification and characteristic of recycled material from concrete construction debris coated with bitumen and bricks. Recycle material specifications. Report NQ2560-600/2002, Bureau de normalization du Quebec, Quebec.

Chen, H., Yen, T., and Chen, K. 2003. Use of building rubbles as recycle aggregates. Cement and Concrete Research, 33:125-132.

Chini, A.R., Kuo, S.S., Armaghani, J.M., and Duxbury, J.P. 2001. Test of recycled concrete aggregate in accelerated test track. Journal of Transportation Engineering, 127 (6): 486- 492 .

Concrete Society. 1988. Permeability testing of site concrete- A Review of Methods and experience, Report of concrete society working party, concrete society technical report, 31: 95

Collins, R. 1998. Recycled aggregate in ready mix - Dr. Rod Collins describes two practical applications of RCA in concrete and evaluates their success. Concrete Engineering International, 2 (2): 49 -54.

Corinaldesi, V., Giuggiolini, M., and Moriconi, G. 2002. Use of rubble from building demolition in mortars. Waste Management, 22 (8): 893-900.

CSA Standards A23.1. 2004. Concrete materials and methods of concrete construction, Canadian Standards Association, Toronto. 
CSA Standards A23.3. 2004. Concrete Design hand book, Canadian Standards Association, Toronto.

Dhir, R.K., Limbachiya, M.C., and Leelawat, T. 1999. Suitability of recycled concrete aggregate for use in BS 5328 designated mixes. Proceedings of the Institution of Civil Engineers - Structures and Buildings, 134 (3): 257 -274.

Dhir, R. , Paine, K. , and Dyer, T. 2004. Recycling construction and demolition wastes in concrete. Concrete - Crowthorne, 38(3): 25-28.

Environmental Council of Concrete Organization, ECCO. 1999. Recycling Concrete and Masonry. [online]. Available from http://ecco.org/. [cited 15 August, 2004].

Environment Canada, Government Canada, 2006c. [Online]. Available: http://www.ec.gc.ca/press/2001/010711 b_e.htm, July issue [cited 20 January 2006].

Fathifazi, G. 2007a. Private communication. Carleton University, Department of Civil and Environmental Engineering.

Fathifazi, G., Abbas, A., Razaqpur, A.G., Isgor, O.B., Fouriner, B., and Foo, S. 2007b. Proposed mixture proportioning procedure for recycled aggregate concrete. Submitted to ACI material Journals February 2007.

George, J.V and Michael, N. 2001. Waste streams from building construction and demolition, with a specific focus on concrete reuse and recycling report submitted to public works Government Services Canada, Gatineau, Quebec, Canada.

Gokce, A., Nagataki, S., Saeki, T., and Hisada, M. 2004. Freezing and thawing resistance of air-entrained concrete incorporating recycled coarse aggregate: The role of air content in demolished concrete. Cement and Concrete Research, 34:799-806.

Gomez-Soberon, J.M., V. 2002. Porosity of recycled concrete with substitution of 
recycled concrete aggregate - An experimental study. Cement and Concrete Research, 32 (8): $1301-1312$.

Gomez-Soberon, J.M., V. 2003. Relationship between gas adsorption and the shrinkage and creep of recycled aggregate concrete. Cement Concrete and Aggregates, 25 (2): 42-48.

Ho, D. W. S., and Lewis, R., K. 1987. Carbonation of concrete and its prediction. Cement and Concrete Research, 17: 489.

Intergovernmental Panel on Climate Change (IPCC), Government of Canada, 2005a. [Online]. Available:

www.climatechange.gc.ca/english/climate change/factsheet en.pdf [cited 20 January 2006].

Katz, A. 2003. Properties of concrete made with recycled aggregate from partially hydrated old concrete. Cement and Concrete Research, 33 (5): 703-712.

Krezel, Z.A., and McManus, K.J. 2000. Recycling Demolition Easte to Fight Noise Polution. 4th Annual Australian Environmental Engineering Research Event, Victor Harbor.

Levy, S. M., Helene, P. 2004. Durability of recycled aggregates concrete: a safe way to sustainable development. Cement and Concrete Research, 34 (2004): 1975-1980.

Limbachiya, M.C., Leelawat, T., and Dhir, R.K., 2000. Technical Reports - Use of recycled concrete aggregate in high-strength concrete. Materials and Structures, 33(233): $574-580$.

Lydon, F. D., 1982 Concrete mix design. 2nd edition, Applied Science Publisher, London and New York 
Malhotra, V.M and Carino, N.J. 2004. Hand book on nondestructive testing of concrete, second edition, CRC Press LLC, New York

Malhotra, V.M and Mehta P.K., 2005. High performance, high-volume fly-ash concrete: Materials, mixture proportioning, properties, construction practice, and case histories, second edition. Supplementary cementing materials for sustainable development Inc., Ottawa, Canada

Mailvaganam, N. P.1992. Repair and protection of concrete structures, Boca Raton Anna Arbor London, CRC press.

Mandal, S., Chakraborty, S., and Gupta, A. 2002. Some studies on durability of recycled aggregate concrete. Indian Concrete Journal, 76(6): 385-388.

Martin-Perez, B.1999. Service life modeling of R.C. Highway structures exposed to chlorides. Ph.D. Thesis, Toronto University

Mehta P.K, and Monterio P.J.M. 2006. Concrete, microstructure, properties, and materials, third edition, The McGraw-Hill companies, Inc.

Mehta, P.K. 2001. Reducing the environmental impact of concrete. Concrete International, 61-66.

Ministry of Transportation-Ontario. 2001. Method of test for freezing and thawing of coarse aggregate, Laboratory testing Manual, Test Method LS-614,Rev.No. 21

MOTH. 1999. Construction aggregates sector trends. Bristish Columbia Ministry of Transport and Highways.

Nagataki, S., Gokce, A. and Saeki, T., 1999. Effects of recycled aggregate characteristics on performance parameters of recycled aggregate concrete. American Concrete Institute (ACI) special publication: SP 192-4: 51-71. 
Nagataki, S., Gokce, A., Saeki, T., and Hisada, M. 2004. Assessment of recycling process induced damage sensitivity of recycled concrete aggregates. Cement and Concrete Research, 34: 965-971.

Neville, A. M. 1995a. Properties of Concrete. $4^{\text {th }}$ Edition, Longman Group Limited, Essex, England

Neville, A. M. 1995b. Chloride attack of reinforced concrete: an Overview. Materials and Structures, 28: 63-70

Nilsson, L., -O., Massat, M., and Tang, L. 1994. The Effect of Non-Linear Chloride Binding on Prediction of Chloride Penetration into Concrete Structures. In Durability of Concrete, Edited by V. Malhotra, ACI SP-145, pp. 469-486, Detroit.

Noguchi, T., and Tamura, M., 2001. Concrete design towards complete recycling. Structural Concrete, 2 (3): 155-167

Olorunsogo, F.T., and Padayachee, N., 2002. Performance of recycled aggregate concrete monitored by durability indexes. Cement and Concrete Research, 32 (2): 179186.

Otsuki, N., Miyazato, S.S., and Yodsudjai, W. 2003. Influence of Recycled Aggregate on Interfacial Transition Zone, Strength, Chloride Penetration and Carbonation of Concrete. Journal of Materials in Civil Engineering, 15(5): 443-551.

Panagapko, D. 2003. Canadian Minerals Yearbook, Natural Resources Canada.Takenaka Corporation, $1999 . \quad$ [online]. Available: www.takenaka.co.jp/takenaka e/news e/pr9912/m9912 01.htm [cited 20 May 2004]

Poon, C.S., Shui, Z.H., Lam, L., Fok, H., and Kou, S.C. 2004. Influence of moisture states of natural and recycled aggregates on the slump and compressive strength 
of concrete. Cement and Concrete Research, 34: 31-36.

Popovics, S.1998. Strength and related properties of concrete, a quantitative approach. John Wiley \& Sons Inc., Toronto

Rakshvir, M. and Barai, S.V. 2006. Studies on recycled aggregates-based concrete. Waste management and research, 24: 225-233.

Ramamurthy, K., and Gumaste, K.S. 1998. Properties of recycled aggregate concrete. Indian Concrete Journal, 72(1): 49-56.

Ravindrarajah, S.R. and Tam, C.T. 1985. Properties of concrete made with Crushed concrete as coarse aggregate. Magazine of Concrete Research, 37 (130): 29-38.

RILEM recommendation CPC 18. 1988. Measurement of hardened concrete carbonation depth, Material Structure, 21 (126): 435

Ryu, J.S. 2002a. An experimental study on the effect of recycled aggregate on concrete properties. Magazine of Concrete Research, 54 (1): 7-12.

Ryu, J.S. 2002b. Improvement on strength and impermeability of recycled concrete made from crushed concrete coarse aggregate. Journal of Materials Science Letters, 21 (20): $1565-1568$.

Sagoe-Crentsil, K., and Brown, T. 1998. Guide for specification of recycle concrete aggregates (RCA) for concrete production, EcoRecycled Victoria, CSIRO, Building Construction and Engineering,Victoria.

Sagoe-Crentsil, K.K., Brown, T., and Taylor, A.H. 2001. Performance of concrete made with commercially produced coarse recycled concrete aggregate. Cement and Concrete Research, 31(5): 707-712.

Salem, R.M., and Burdette, E.G. 1998. Role of chemical and mineral admixtures on 
physical properties and frost resistance of recycled aggregate concrete. ACI Materials Journal - American Concrete Institute, 95 (5): 558-563.

Salem, R.M., Burdette, E.G., and Jackson, N.M. 2003. Resistance to freezing and thawing of recycled aggregate concrete. ACI Materials Journal - American Concrete Institute, 100 (3): 216-221.

Sanchez de Juan, M. and Alaejos Gutierrez, P. 2004. Influence of Attached Mortar Content on the Properties of Recycled Concrete Aggregate. Proceedings (PRO40) of the International RILEM Conference on the Use of Recycled Materials in Buildings and Structures, November 8-11 2004, Barcelona (Spain), Vol. 1: 536-544.

Sergi, G., Yu, S., and Page, C. 1992. Diffusion of chloride and hydroxyl ions in cementious materials exposed to a saline environment. Magazine of Concrete Research, 44(158):63-69.

Shayan, A., and $\mathrm{Xu}, \mathrm{A} .2003$. Performance and properties of structural concrete made with recycled concrete aggregate. ACI Materials Journal - American Concrete Institute, 100 (5): 371-380.

Stueck W. 2006. B.C. Company's hunt finds its quary. Newspaper article, The Globe and Mail issue April 13.

Takenaka Corporation, 1999.High-quality recycled aggregate "Cyclite" Acquires first approval of innovative building technologies from building center of Japan [online]. Available: www.takenaka.co.jp/takenaka_e/news_e/pr9912/m9912 01.htm [cited 15 May, 2004]

Tang, L., and Nilsson, L.,-O.1993. Chloride binding capacity and binding isotherms of OPC pastes and mortars. Cement and Concrete Research, (23):247-253. 
Tavakoli, M., and Soroushian, P. 1996a. Strength of recycled aggregate concrete made using field-demolished concrete as aggregate. ACI Materials Journal - American Concrete Institute, 93(2): 182-190.

Taylor, P.c., and Shrinivas B.B., 2004. PCA develops guide specifications for HPC bridge elements" Roads \& Bridges :(42) 8 [Online]. Available: http://www.roadsbridges.com/Bridges-With-More-article5387 [cited 10 March 2007]

Topcu, I.B. 1997. Physical and mechanical properties of concrete produced with waste concrete. Cement and Concrete Research 27(12), 1817-1823.

Wilburn, D.R., and Goonan, T.G., 1998. Aggregates from natural and recycled sources, Economic assessments for construction applications, A materials flow analysis, U.S. Geological survey circular 1176 .

Winfield, M.S., Taylor, A. (2005). Replacing the load: The need for an aggregates conservation strategy for Ontario, Report published by Pembina Institute for Appropriate Development, ISBN 0-921719-71-x.

Zaharieva, R., Buyle-Bodin, F., Skoczylas, F., and Wirquin, E. 2003. Assessment of the surface permeation properties of recycled aggregate concrete. Cement and Concrete Composites, 25 (2): 223-232

Zaharieva, R., Buyle-Bodin, F., and Wirquin, E. 2004. Frost resistance of recycled aggregate concrete. Cement and Concrete Research, 34 (2004): 1927-1932 


\section{APPENDIX A: MATERIAL CHARACTERIZATION METHODS}

\section{A.1. Determination of the Residual Mortar Content}

\section{Scope}

This test method covers the testing of coarse RCA to determine the RMC. This test is based on repeated freezing and thawing of RCA in sodium sulfate solution.

\section{Apparatus}

2.1. Freezer: a freezer capable of maintaining a temperature of $-18 \pm 2{ }^{\circ} \mathrm{C}$.

2.2. Sieves: With square openings and with suitable sizes to prepare the test sample as in Section 4 and to separate the residual mortar from the OVA during washing as in step 5.4.

2.3. Containers: Containers for immersing the samples of RCA in the solution, plastic container with $2.3 \mathrm{~L}$ capacity is preferable.

2.4. Balance: A balance or scale accurate to within $0.1 \%$ of the sample mass, or $1 \mathrm{~g}$, whichever is greater, over the range required for the test.

2.5. Oven: Oven capable of maintaining a temperature of $80^{\circ} \mathrm{C}$.

\section{Preparation of Solution}

3.1. Prepare sufficient $26 \%$ sodium sulfate solution by dissolving $350 \mathrm{~g}$ of anhydrous sodium sulfate in $1000 \mathrm{~g}(1 \mathrm{~L})$ of water. It is preferable to prepare the solutions in small quantities (e.g. 2 L of water). 


\section{Preparation of Samples}

4.1. Separate the sample in the fraction distribution as shown in Table A.1 by mechanical sieving. For each sample prepare minimum weight quantities as indicated in Table A.1. Prepare at least three samples from each fraction.

Table A1: Weight of test samples

\begin{tabular}{|c||c|c||}
\hline Pass & Retained & Min. Weight (g) \\
\hline $25 \mathrm{~mm}$ & $19 \mathrm{~mm}$ & 2000 \\
$19 \mathrm{~mm}$ & $12.7 \mathrm{~mm}$ & 1500 \\
$12.7 \mathrm{~mm}$ & $9.5 \mathrm{~mm}$ & 1250 \\
$9.5 \mathrm{~mm}$ & $4.75 \mathrm{~mm}$ & 1000 \\
\hline
\end{tabular}

\section{Procedure}

5.1. Dry the RCA sample to constant weight at a temperature of $110^{\circ} \mathrm{C}$ and record the weight.

5.2. Immerse the RCA samples in sodium sulfate solution for $24 \mathrm{~h}$ in such a manner that the solution covers them.

5.3. Place the immersed samples in the freezer for $16 \pm 4 \mathrm{~h}$ overnight, and remove them in the morning to allow thawing for at least $5 \pm 1 \mathrm{~h}$ in $80-110^{\circ} \mathrm{C}$ oven. Perform at least 3 and not more than 5 freeze-and-thaw cycles.

5.4. After the completion of the final cycle and after the sample has cooled, drain the solution and wash the RCA. Place the sample in $2.36 \mathrm{~mm}$ sieve and drain the sodium sulfate solution and wash it by water at $45 \pm 5^{\circ} \mathrm{C}$. If the sample is $1500 \mathrm{~g}$ or higher wash small quantities at a time until the entire sample is washed. During the washing, do not lose any particle. At the end of the wash sieve by letting the water flush from the bottom of the sieve.

5.5. Oven dry the sample to constant weight at a temperature of $1100 \mathrm{C}$. 
5.6. Visually inspect the sample after the sample has cooled down. Pick any particle with visible residual mortar (if present) and remove them by knife or hitting the particle gently by a hammer or a rod. Retain the OVA and weigh the residual mortar and record its weight.

\section{Quantitative Examination}

7.1. After the removing of the residual mortar, weigh each sample fraction and record the weight.

7.2. Record the weight of the residual mortar removed in the visual inspection for the qualitative examination and the acceptance of the test.

\section{Calculation}

8.1. Calculate the RMC for each fraction as follows:

$$
\mathrm{RMC}=\left(\frac{W_{R C A}-W_{O V A}}{W_{R C A}}\right) 100
$$

where, $W_{R C A}$ is the weight of the original or initial weight of RCA before test (g), $W_{\text {OVA }}$ is weight of OVA after removal from the residual mortar $(\mathrm{g})$. 


\section{A.2. Determination of Impurities of RCA}

\section{Scope}

This test method covers testing of RCA to determine the amount of impurities in RCA, such as bricks, tiles, asphalt or bitumen coating, wood, glass, rubber, metal plastic, etc.

\section{Apparatus}

2.1. Containers: Containers for the sampling of RCA.

2.2. Balance: A balance or scale accurate to within $0.1 \%$ of the sample mass, or $1 \mathrm{~g}$, whichever is greater, over the range required for the test.

2.3. Oven: Oven capable of maintaining a temperature of $110^{\circ} \mathrm{C}$.

\section{Preparation of samples}

3.1.Separate the sample in the fraction distribution as shown in Table A.2 by mechanical sieving .For each sample prepare minimum weight quantities as indicated in Table A.2. Prepare at least three samples from each fraction.

Table A2: Weight of test samples

\begin{tabular}{|c|c|c|}
\hline Pass & Retained & Min. Weight $(\mathrm{g})$ \\
\hline $19 \mathrm{~mm}$ & $12.7 \mathrm{~mm}$ & 1500 \\
$12.7 \mathrm{~mm}$ & $9.5 \mathrm{~mm}$ & 1250 \\
$9.5 \mathrm{~mm}$ & $4.75 \mathrm{~mm}$ & 1000 \\
\hline
\end{tabular}

\section{Procedure}

5.1. Dry and heat the RCA sample to constant weight at a temperature of $110^{\circ} \mathrm{C}$ and record the weight. The heat was found to help detecting the bitumen coating visually.

5.2. Make visual inspection on the sample after the sample has cooled down, and pick up the impurities such as, asphalt or bitumen coating, wood, glass, rubber, metal plastic, etc.

5.3. Record the weight and the number of each type of impurity. 


\section{Calculation}

Calculate the percentage of impurities as follows:

$$
\text { Impurities content }=\left(\frac{W_{I M}}{W_{R C A}}\right) 100
$$

where: $W_{R C A}$ : The original or initial weight of RCA before test $(\mathrm{g}), . W_{I M}$ is the weight of the impurities in RCA sample.

Tables A.3 and A.4 show the data collected for the impurities of RCA-MO and RCA-VA, respectively. 
Table A3: Data collection and calculation of impurities for RCA-MO

\begin{tabular}{|c|c|c|c|c|c|c|c|c|c|c|c|c|c|c|}
\hline \multirow{2}{*}{$\begin{array}{c}\text { RCA-size } \\
\text { (mm) }\end{array}$} & \multirow{2}{*}{ Sample } & \multirow{2}{*}{$\begin{array}{c}\text { Weight } \\
\text { OD } \\
(\mathrm{g}) \\
\end{array}$} & \multicolumn{6}{|c|}{ Impurities weight (g) and particles number ( ) } & \multicolumn{6}{|c|}{ Percentage by weight } \\
\hline & & & Bitumen & Tiles & Brick & Glass & Wood & Other & Bitumen & Tiles & Brick & Glass & Wood & Other \\
\hline \multirow{4}{*}{4.75} & 1 & 967 & $1(2)$ & & & & & $3(3)$ & 0.10 & 0.0 & 0.0 & & 0.00 & 0.3 \\
\hline & 2 & 1274 & 0 & & & & & $0(2)$ & 0.00 & 0.0 & 0.0 & & 0.00 & 0.0 \\
\hline & 3 & 1082 & 0 & & & & & $0(2)$ & 0.00 & 0.0 & 0.0 & & 0.00 & 0.0 \\
\hline & Average & & & & & & & & 0.03 & 0.0 & 0.0 & & 0.0 & 0.1 \\
\hline \multirow{4}{*}{9.5} & 1 & 1665 & 0 & & 0 & & 0 & $1(3)$ & 0.00 & 0.0 & 0.0 & 0 & 0.00 & 0.1 \\
\hline & 2 & 1568 & 0 & & 3 & & 1 & 0 & 0.00 & 0.0 & 0.2 & 0 & 0.06 & 0.00 \\
\hline & 3 & 1849 & 0 & & 0 & & 0 & 0 & 0.00 & 0.0 & 0.0 & 0.0 & 0.00 & 0.0 \\
\hline & Average & & & & & & & & 0.00 & 0.0 & 0.1 & 0.00 & 0.02 & 0.02 \\
\hline \multirow{4}{*}{12.7} & 1 & 2481 & & & & & 0 & 0 & 0.00 & 0.0 & 0.0 & & & 0.0 \\
\hline & 2 & 2162 & & & & & $0(2)$ & $0(1)$ & 0.00 & 0.0 & 0.0 & & & 0.0 \\
\hline & 3 & 2571 & & & & & 0 & 0 & 0.00 & 0.0 & 0.0 & & & 0.0 \\
\hline & Average & & & & & & & & 0.00 & 0.0 & 0.0 & & & \\
\hline
\end{tabular}


Table A4: Data collection and calculation of impurities for RCA-VA

\begin{tabular}{|c|c|c|c|c|c|c|c|c|c|c|c|c|c|c|c|c|}
\hline \multirow{2}{*}{$\begin{array}{c}\text { RCA-size } \\
(\mathbf{m m}) \\
\end{array}$} & \multirow{2}{*}{$\begin{array}{c}\text { Sample } \\
\# \\
\end{array}$} & \multirow{2}{*}{$\begin{array}{l}\text { Weight } \\
\text { OD (g) } \\
\end{array}$} & \multicolumn{7}{|c|}{ Impurities weight (g) and particles number ( ) } & \multicolumn{7}{|c|}{ Percentage by weight } \\
\hline & & & Bitumen & Tiles & Brick & Glass & Wood & Metal & Other & Bitumen & Tiles & Brick & Glass & Wood & Metal & Other \\
\hline \multirow{4}{*}{4.75} & 1 & 1332 & $42(50)$ & & $3(9)$ & & $1(3)$ & $17(1)$ & & 3 & 0.0 & 0.2 & & 0.08 & 1.28 & 0.0 \\
\hline & 2 & 1363 & $35(39)$ & & & & 1(3) & & & 3 & 0.0 & 0.0 & & 0.07 & 0.00 & 0.0 \\
\hline & 3 & 1824 & $41(47)$ & & $4(5)$ & & $2(6)$ & & $5(7)$ & 2 & 0.0 & 0.2 & & 0.11 & 0.00 & 0.3 \\
\hline & Average & & & & & & & & & 3 & 0.0 & 0.1 & & 0.1 & 0.4 & 0.1 \\
\hline \multirow{4}{*}{9.5} & 1 & 1683 & $26(19)$ & $2(2)$ & & & $1(2)$ & & & 2 & 0.1 & 0.0 & 0 & 0.06 & 0.00 & 0.0 \\
\hline & 2 & 2005 & $41(21)$ & $2(1)$ & $7(3)$ & & $1(1)$ & $8(1)$ & $0(1)$ & 2 & 0.1 & 0.3 & 0 & 0.05 & 0.40 & 0.00 \\
\hline & 3 & 1701 & $49(22)$ & $1(1)$ & $5(3)$ & & & & & 3 & 0.1 & 0.3 & 0.0 & 0.00 & 0.00 & 0.0 \\
\hline & Average & & & & & & & & & 2 & 0.1 & 0.2 & 0.00 & 0.04 & 0.13 & 0.00 \\
\hline \multirow{4}{*}{12.7} & 1 & 2843 & $35(8)$ & & $23(2)$ & & $1(3)$ & & $0(1)$ & 1 & 0.0 & 0.8 & & 0.04 & 0.00 & 0.0 \\
\hline & 2 & 2207 & $27(6)$ & $8(1)$ & $8(2)$ & & & $15(1)$ & $0(1)$ & 1 & 0.4 & 0.4 & & 0.00 & 0.68 & 0.0 \\
\hline & 3 & 2419 & $20(5)$ & & & & $0(1)$ & & & 1 & 0.0 & 0.0 & & 0.00 & 0.00 & 0.0 \\
\hline & Average & & & & & & & & & 1 & 0.1 & 0.4 & & 0.0 & 0.2 & 0.0 \\
\hline
\end{tabular}




\section{A.3. Properties of Cementitious Materials}

Table A5: Chemical analysis results of cement, fly-ash and bfs

\begin{tabular}{|cc|c|c|c|}
\hline \multirow{2}{*}{ Chemical Compound } & \multicolumn{3}{|c|}{ Cementitious Material } \\
\cline { 3 - 5 } & $\%$ & Cement & Fly-ash & Bfs \\
\hline $\mathrm{SiO}_{2}$ & $\%$ & 4.89 & 23.6 & 9.77 \\
\hline $\mathrm{Al}_{2} \mathrm{O}_{3}$ & $\%$ & 2.29 & 3.65 & 0.59 \\
\hline $\mathrm{Fe}_{2} \mathrm{O}_{3}$ & $\%$ & 2.38 & 1.06 & 11.45 \\
\hline $\mathrm{MgO}$ & $\%$ & 62.5 & 10.9 & 36.3 \\
\hline $\mathrm{CaO}$ & $\%$ & 0.22 & 3.17 & 0.42 \\
\hline $\mathrm{Na}_{2} \mathrm{O}$ & $\%$ & 0.75 & 0.64 & 0.54 \\
\hline $\mathrm{K}_{2} \mathrm{O}$ & $\%$ & 0.21 & 0.62 & 0.68 \\
\hline $\mathrm{TiO}_{2}$ & $\%$ & 0.25 & 0.09 & $<0.01$ \\
\hline $\mathrm{P}_{2} \mathrm{O}_{2}$ & $\%$ & 0.05 & 0.05 & 0.76 \\
\hline $\mathrm{MgO}_{\mathrm{LOI}}$ & $\%$ & 3.15 & 0.64 & -1.28 \\
\hline $\mathrm{Sum}_{\mathrm{SO}}$ & $\%$ & 95.8 & 98.2 & 95.8 \\
\hline $\mathrm{CO}_{3}(\mathrm{t})$ & $\%$ & 3.81 & 0.22 & 3.35 \\
\hline $\mathrm{Cl}$ & $\%$ & 0.54 & 0.38 & 0.14 \\
\hline $\mathrm{G} / \mathrm{T}$ & 47 & 31 & 111 \\
\hline$\%$ & 62 & & \\
\hline$\left(3 \mathrm{CaO} . \mathrm{SiO}_{2}\right) /\left(\mathrm{C}_{3} \mathrm{~S}\right)^{*}$ & $\%$ & 8 & & \\
\hline$\left(2 \mathrm{CaO} . \mathrm{SiO}_{2}\right) /\left(\mathrm{C}_{2} \mathrm{~S}\right)^{*}$ & $\%$ & 9 & & \\
\hline$\left(3 \mathrm{CaO} . \mathrm{Al}_{2} \mathrm{O}_{3}\right) /\left(\mathrm{C}_{3} \mathrm{~A}\right)^{*}$ & $\%$ & 7 & & \\
\hline$\left(3 \mathrm{CaO} . \mathrm{Al}_{2} \mathrm{O}_{3} \cdot \mathrm{Fe}_{2} \mathrm{O}_{3}\right)\left(\mathrm{C}_{3} \mathrm{AF}\right)^{*}$ & $\%$ & & \\
\hline
\end{tabular}

* Calculated from equation provided by ASTM C 150-00 (ASTM Committee C1, 2003) 
Table A6: Specific gravity of cement, fly-ash and bfs

\begin{tabular}{|c|c|c|c|c|c|c|}
\hline \multirow{2}{*}{$\begin{array}{c}\text { Cementitious } \\
\text { material }\end{array}$} & \multirow{2}{*}{ Sample } & \multirow{2}{*}{$\begin{array}{l}\mathbf{W} \\
(\mathrm{g})\end{array}$} & \multicolumn{2}{|c|}{$\begin{array}{c}\text { Reading of } \\
\text { Kerosine level in Le } \\
\text { Chatelier flask }\end{array}$} & \multirow{2}{*}{$\begin{array}{l}\text { Displaced } \\
\text { Volume } \\
\left(\mathrm{cm}^{3}\right)\end{array}$} & \multirow[t]{2}{*}{$\begin{array}{l}\text { Specific } \\
\text { Gravity }\end{array}$} \\
\hline & & & $\begin{array}{c}\text { Initial } \\
\left(\mathbf{R}_{1}\right)\end{array}$ & $\begin{array}{c}\text { Final } \\
\left(\mathbf{R}_{2}\right)\end{array}$ & & \\
\hline \multirow{4}{*}{ Cement } & 1 & 64.04 & 0.35 & 20.60 & 20.25 & 3.16 \\
\hline & 2 & 64.03 & 0.60 & 20.60 & 20.00 & 3.20 \\
\hline & 3 & 64.02 & 0.40 & 20.65 & 20.25 & 3.16 \\
\hline & 4 & 64.05 & 0.40 & 21.10 & 20.70 & 3.09 \\
\hline Average & & & & & & 3.15 \\
\hline Std. Dev. & & & & & & 0.04 \\
\hline $\mathrm{CoV}$ & & & & & & 0.01 \\
\hline \multirow{3}{*}{ Fly-ash } & 1 & 40.00 & 0.40 & 20.50 & 20.10 & 1.99 \\
\hline & 2 & 40.03 & 0.70 & 20.50 & 19.80 & 2.02 \\
\hline & 3 & 40.00 & 0.50 & 20.40 & 19.90 & 2.01 \\
\hline Average & & & & & & 2.01 \\
\hline Std. Dev. & & & & & & 0.02 \\
\hline $\mathrm{CoV}$ & & & & & & 0.01 \\
\hline \multirow{3}{*}{ bfs } & 1 & 55.00 & 0.20 & 18.50 & 18.30 & 3.01 \\
\hline & 2 & 55.06 & 0.60 & 18.80 & 18.20 & 3.03 \\
\hline & 3 & 55.00 & 0.20 & 18.90 & 18.70 & 2.94 \\
\hline Average & & & & & & 2.99 \\
\hline Std. Dev. & & & & & & 0.04 \\
\hline $\mathrm{CoV}$ & & & & & & 0.01 \\
\hline
\end{tabular}




\section{APPENDIX B: MIX DESIGN EXAMPLES}

\section{B.1.General Mix Design Examples}

\section{B.1.1. Example 1: Design of 100\% RCA Concrete for Severe Exposure}

Required compressive strength $=30-35 \mathrm{MPa}$.

Required slump $=75-100 \mathrm{~mm}$.

Nominal maximum size of the $\mathrm{RCA}=19 \mathrm{~mm}$

Average $\mathrm{RMC}$ of $\mathrm{RCA}=41 \%$.

Unit weight of RCA $=1372 \mathrm{~kg} / \mathrm{m}^{3}$

Bulk specific gravity of $\mathrm{RCA}=2.30$

Absorption capacity of $\mathrm{RCA}=5.4 \%$

Moisture content of RCA $=1.1 \%$.

SSD specific gravity of fine aggregate $=2.70$

Absorption capacity of fine aggregate $=0.8 \%$

Fineness modulus of fine aggregate $=2.6$.

Specific gravity for $\mathrm{OPC}=3.15$

Step 1: For severe weather air-entrained GC with $6 \%$ air content will be used (Table 4.1Section 4.2). The required slump is in the range of 75 to $100 \mathrm{~mm}$, from Table 4.1 Section 4.2 , water content is $184 \mathrm{~kg} / \mathrm{m}^{3}$.

Step 2: From Figure 7.2, the w/c ratio needed to produce compressive strength of 31 MPa in air-entrained GC with TRMC of $41 \%$ is 0.45 . 
Step 3: From step 1 and step 2 the cement content is equal to $184 / 0.45=409 \mathrm{~kg} / \mathrm{m}^{3}$.

Step 4: The aggregate content can be calculated using the analytical approach as follows:

By using Eq.7.1a, the estimated fresh concrete density $(U)$ is equal to:

$$
U=70.4\left(\frac{1}{0.45}\right)-2.9(41)+2154.2=2192 \mathrm{~kg} / \mathrm{m}^{3}
$$

From Table 7.1, for fineness modulus of 2.6 and $41 \%$ RMC of RCA, the oven dry-rodded volume of RCA per unit volume of concrete $v_{R C A}^{G C}$ is 0.61 . The ratio of the compacted or absolute volume of coarse aggregate to the absolute volume of concrete $\left(V_{a}\right)$ can be calculated by Eq.7.4 as follows:

$$
V_{a}=\left(\frac{1372}{1000 \times 2.31}\right) \times 0.61=0.363
$$

The weight of the SSD RCA can be found using Eq.7.2 as:

$$
W_{S S D-R C A}^{G C}=\frac{0.363 \times\left[409\left(\frac{1}{3.15}-\frac{1}{2.70}\right)+\frac{(2192-184)}{2.70}+184\right]}{\left[\frac{1}{2.42}-\left(\frac{1}{2.42}-\frac{1}{2.70}\right) \times 0.363\right]}=827 \mathrm{~kg} / \mathrm{m}^{3}
$$

The fine natural aggregate can be calculated by using Eq.7.4:

$$
W_{S S D-F A}^{G C}=2192-409-827-184=772 \mathrm{~kg} / \mathrm{m}^{3}
$$

The absorption capacity and the moisture content of RCA are $5.4 \%$ and $1.1 \%$, respectively. Therefore the air-dry weight of the RCA is calculated as follows:

$$
\text { Weight of dry RCA }=\frac{827 \times\left(1+\frac{1.1}{100}\right)}{\left(1+\frac{5.4}{100}\right)}=793 \mathrm{~kg} / \mathrm{m}^{3}
$$

The absorption capacity for the fine aggregate is $0.8 \%$. The dry weight of the fine aggregate is calculated as follows:

$$
\text { Weight of dry sand }=\frac{772}{\left(1+\frac{0.8}{100}\right)}=766 \mathrm{~kg} / \mathrm{m}^{3}
$$




\section{B.1.2. Example 2: Design of 50\% RCA Concrete for Moderate Exposure}

Required compressive strength $=44 \mathrm{MPa}$.

Required slump $=75-100 \mathrm{~mm}$.

Nominal maximum size of the RCA $=19 \mathrm{~mm}$

Average $\mathrm{RMC}$ of $\mathrm{RCA}=41 \%$.

Unit weight of RCA $=1372 \mathrm{~kg} / \mathrm{m}^{3}$

Bulk specific gravity of $\mathrm{RCA}=2.31$

Absorption capacity of RCA $=5.4 \%$

Moisture content of RCA $=1.1 \%$

Unit weight of $\mathrm{NA}=1612 \mathrm{~kg} / \mathrm{m}^{3}$

Bulk specific gravity of $\mathrm{NA}=2.70$

Absorption capacity of NA $=0.34 \%$

Moisture content of NA $=0.2 \%$.

SSD specific gravity of fine aggregate $=2.70$

Absorption capacity of fine aggregate $=0.8 \%$

Fineness modulus of fine aggregate $=2.6$.

Specific gravity for $\mathrm{OPC}=3.15$

The unit weight, the specific gravity and the absorption capacity of the blended coarse aggregate $(50 \% \mathrm{RCA}$ and $50 \% \mathrm{NA})$ can be calculated as follows:

$$
M=\frac{50}{100} \times 1372+\frac{50}{100} \times 1612=1492 \mathrm{~kg} / \mathrm{m}^{3}
$$




$$
\begin{aligned}
& S G_{b}=\frac{1}{\frac{50}{100(2.31)}+\frac{50}{100(2.7)}}=2.49 \\
& A C=\frac{50}{100} \times 5.4+\frac{50}{100} \times 0.34=2.87 \% \\
& S G_{S S D}=2.49 \times\left(1+\frac{2.87}{100}\right)=2.56
\end{aligned}
$$

The TRMC for GC with (50\% RCA and 50\% NA) $=21 \%$.

Step 1: For moderate weather, non-air entrained GC can be used. The required slump is in the range of 75 to $100 \mathrm{~mm}$, from Table 4.1 of Section 4.2 , water content is $205 \mathrm{~kg} / \mathrm{m}^{3}$.

Step 2: From Figure 7.2, for compressive strength of $44 \mathrm{MPa}$, the w/c ratio for non-air entrained GC with TRMC of $21 \%$ is 0.45 .

Step 3: From step 1 and step 2 the cement content is equal to $205 / 0.45=456 \mathrm{~kg} / \mathrm{m}^{3}$

Step 4: The aggregate content can be calculated using the analytical approach as follows:

By using Eq. 7.1b, the estimated fresh concrete density $(U)$

$$
U_{G C}=12.4\left(\frac{1}{0.45}\right)-2.3(21)+2394.6=2373 \mathrm{~kg} / \mathrm{m}^{3}
$$

From Table 7.1, and for fineness modulus of 2.6 and by reducing the oven dry-rodded volume of RCA per unit volume of concrete by the absorption capacity of the blended aggregate $(2.87 \%), v_{R C A}^{G C}$ is calculated as $0.64 \times(1-0.0287)=0.62$. The ratio of the compacted or absolute volume of coarse aggregate to the absolute volume of concrete $\left(V_{a}\right)$ can be calculated by Eq. 7.4 as follows:

$$
V_{a}=\left(\frac{1492}{1000 \times 2.49}\right) \times 0.62=0.37
$$

The weight of the SSD RCA can be calculated using Eq. 7.2 as follows:

$$
W_{S S D-b l e n d e d C A}^{G C}=\frac{0.37 \times\left[456\left(\frac{1}{3.15}-\frac{1}{2.70}\right)+\frac{(2373-205)}{2.70}+205\right]}{\left[\frac{1}{2.56}-\left(\frac{1}{2.56}-\frac{1}{2.70}\right) \times 0.37\right]}=950 \mathrm{~kg} / \mathrm{m}^{3}
$$

The fine natural aggregate can be calculated by using Eq.7.4: 


$$
W_{S S D-F A}^{G C}=2373-456-950-205=762 \mathrm{~kg} / \mathrm{m}^{3}
$$

The weight of dry coarse blended aggregate is calculated as:

$$
\text { Weight of blended aggregate }=\frac{950}{\left(1+\frac{2.56}{100}\right)}=926 \mathrm{~kg} / \mathrm{m}^{3}
$$

Hence, the weight of the oven dry RCA $=0.5 \times 926=463 \mathrm{~kg} / \mathrm{m}^{3}$

The weight of the oven dry NA $=0.5 \times 926=463 \mathrm{~kg} / \mathrm{m}^{3}$

The moisture content of RCA and NA are $1.1 \%$ and $0.2 \%$, respectively.

Hence, the weight of the air dry RCA $=463 \times(1+1.1 / 100)=468 \mathrm{~kg} / \mathrm{m}^{3}$

The weight of air dry NA $=463 \times(1+0.2 / 100)=464 \mathrm{~kg} / \mathrm{m}^{3}$

The absorption capacity for the fine aggregate is $0.8 \%$. The dry weight of the fine aggregate is calculated as:

$$
\text { Weight of dry sand }=\frac{762}{\left(1+\frac{0.8}{100}\right)}=756 \mathrm{~kg} / \mathrm{m}^{3}
$$

Therefore, the mix proportions of the GC with 50\%RCA and 50\% NA are:

$$
\begin{array}{ll}
\text { Cement content }=456 \mathrm{~kg} / \mathrm{m}^{3} & \mathrm{NA}=464 \mathrm{~kg} / \mathrm{m}^{3} \\
\text { Water content }=205 \mathrm{~kg} / \mathrm{m}^{3} & \mathrm{RCA}=468 \mathrm{~kg} / \mathrm{m}^{3} \\
\text { Sand }=756 \mathrm{~kg} / \mathrm{m}^{3} &
\end{array}
$$

It should be noted that, trial batches using this mix (50\%RCA and 50\% NA) was carried out, and the results of the measured fresh and mechanical properties of GC were determined as:
Slump $=140 \mathrm{~mm}$
Elastic modulus $=31.7 \mathrm{GPa}$
Air content $=1.8 \%$
Compressive strength $=46.2 \mathrm{MPa}$
Fresh density $=2352 \mathrm{~kg} / \mathrm{m}^{3}$
Splitting tensile strength $=4 \mathrm{MPa}$ 


\section{B.2.Equivalent Total Volume of Mortar Method}

\section{B.2.1 Derivation the Equation of Equivalent Total Volume of Mortar Method}

The first step in the equivalent total volume of mortar method is the determination of the coarse aggregate content for GC. The second step is the determination of the water, cement and fine aggregate content. The following sections will discuss the proportioning of the GC by using equivalent total volume of mortar method.

\section{B.2.1.1. Coarse Aggregate Content}

In order to satisfy the condition of the equivalent total volume of mortar in GC and in NAC, the total volume of coarse natural aggregate (OVA plus NA) in GC needs to be equal to the volume of NA in NAC.

$$
V_{T N A}^{G C}=V_{N A}^{N A C}
$$

where $V_{T N A}^{G C}$ is the volume of total natural aggregate in $\mathrm{GC}$, and $V_{N A}^{N A C}$ is the volume of natural aggregate in NAC. The volume of total natural aggregate in GC $\left(V_{T N A}^{G C}\right)$ consists of two parts: the volume of OVA $\left(V_{O V A}^{G C}\right)$, and the volume of NA $\left(V_{N A}^{G C}\right)$ in GC. Therefore, $\left(V_{T N A}^{G C}\right)$ can be written using Eq. B.2:

$$
V_{T N A}^{G C}=V_{O V A}^{G C}+V_{N A}^{G C}
$$

By substituting $\left(V_{T N A}^{G C}\right)$ from Eq.B.1 in Eq. B.2:

$$
V_{N A}^{N A C}=V_{O V A}^{G C}+V_{N A}^{G C}
$$

Furthermore, the ( $V_{O V A}^{G C}$ ) can be calculated using Eq. B.4 as follows:

$$
V_{O V A}^{G C}=\frac{W_{O D}^{O V A}}{1000 S G_{b}^{O V A}}
$$

where $\left(S G_{b}^{O V A}\right)$ is the bulk specific gravity of OVA, $\left(W_{O D}^{O V A}\right)$ is the OD weight of RCA. 
The $W_{O D}^{O V A}$ can be calculated using the definition of the RMC presented in Eq.3.1 (Chapter 3, Section 3.3.1) as follows:

$$
W_{O D}^{O V A}=(1-R M C) W_{O D}^{R C A}=(1-R M C) V_{R C A} S G_{b}^{R C A}
$$

where $\left(S G_{b}^{R C A}\right.$ ) is the bulk specific gravity of RCA. By substituting ( $W_{O D}^{O V A}$ ) from Eq.B.5

in Eq.B.4, ( $\left.V_{O V A}^{G C}\right)$ can be determined using Eq.B.6:

$$
V_{O V A}^{G C}=V_{R C A}^{G C}(1-R M C) \frac{S G_{b}^{R C A}}{S G_{b}^{\text {OVA }}}
$$

Substitution of ( $V_{\text {OVA }}^{G C}$ ) from Eq.B.6 in Eq.B.7 yields:

$$
V_{N A}^{N A C}=V_{R C A}^{G C}(1-R M C) \frac{S G_{b}^{R C A}}{S G_{b}^{O V A}}+V_{N A}^{G C}
$$

To satisfy the condition stated in Eq.B.1, the residual mortar volume in GC should be replaced by natural aggregate volume, which is given by:

$$
V_{R M}^{G C}=V_{N A}^{G C}
$$

By substituting Eq.B.8 in Eq.B.3, the volume of the RCA in GC ( $\left.V_{R C A}^{G C}\right)$ should be equal to the volume of natural aggregate in NAC, which can be calculated by:

$$
V_{R C A}^{G C}=V_{N A}^{N A C}
$$

Division of Eq.B.8 by Eq.B.9 yields:

$$
\frac{V_{R M}^{G C}}{V_{R C A}^{G C}}=\frac{V_{N A}^{G C}}{V_{N A}^{N A C}}
$$

The left-hand side of Eq.B.10 is the definition of the RMC by volume $\left(R M C_{V}\right)$ given by Eq.3.2 (Section 3.3.1, Chapter 3). Therefore, Eq.B.10 can be presented as follows:

$$
\frac{V_{N A}^{C C}}{V_{N A}^{N A C}}=R M C_{V}
$$

By substituting $\left(V_{N A}^{G C}\right)$ from Eq.B.11 in Eq.B.7 and rearranging $V_{R C A}$ :

$$
V_{R C A}^{G C}=\frac{V_{N A}^{N A C}\left(1-R M C_{V}\right)}{(1-R M C) \frac{S G_{b}^{R C A}}{S G_{b}^{O V A}}}
$$


Recalling Eq.3.1, the $\left(R M C_{V}\right)$ is given as follows:

$$
R M C_{V}=\frac{S G_{b}^{R C A}}{S G_{b}^{R M}} R M C
$$

where $\left(S G_{b}^{R M}\right)$ is the bulk specific gravity of residual mortar. Substitution of $\left(R M C_{V}\right)$ from Eq.B.13 in Eq.B.12, $V_{R C A}^{G C}$ can be written in the following weight base format:

$$
V_{R C A}^{G C}=\frac{\frac{W_{O D-N A}^{N A C}}{S G_{b}^{N A}}\left(1-\frac{S G_{b}^{R C A}}{S G_{b}^{R M}} R M C\right)}{(1-R M C) \frac{S G_{b}^{R C A}}{S G_{b}^{O V A}}}
$$

where $W_{O D-N A}^{N A C}$ is the oven dry weight of NA in NAC. When the type of NA used in NAC is similar to the OVA in the RCA, (i.e., the bulk specific gravity for NA and OVA are identical), Eq. B.14 can be written in the following format:

$$
V_{R C A}^{G C}=\frac{W_{O D-N A}^{N A C}\left(\frac{1}{S G_{b}^{R C A}}-\frac{1}{S G_{b}^{R M}} R M C\right)}{(1-R M C)}
$$

By knowing the volume of RCA and bulk specific gravities of RCA and NA, the coarse RCA and NA proportions can be calculated using Eqs.B.16 and B.17 as:

$$
\begin{aligned}
& W_{O D-R C A}^{G C}=1000 V_{R C A}^{G C} S G_{b}^{R C A} \\
& W_{O D-N A}^{G C}=1000 V_{N A}^{N A C} R M C_{V} S G_{b}^{N A}
\end{aligned}
$$

where $W_{O D-R C A}^{G C}$ and $W_{O D-N A}^{G C}$ are OD weight in GC for RCA and NA, respectively. Substitution of $\left(V_{R C A}^{G C}\right)$ from Eq.B.15 in Eq.B.16, and $\left(R M C_{V}\right)$ from Eq.B.13 in Eq.B.17 yields:

$$
\begin{gathered}
W_{O D-R C A}^{G C}=\frac{W_{O D-N A}^{N A C}\left(1-\frac{S G_{b}^{R C A}}{S G_{b}^{R M}} R M C\right)}{(1-R M C)} \\
W_{O D-N A}^{G C}=W_{O D-N A}^{N A C} \frac{S G_{b}^{R C A}}{S G_{b}^{R M}} R M C
\end{gathered}
$$

where $\left(S G_{b}^{R M}\right)$ is the bulk specific gravity of residual mortar and can be calculated from 
Eq.3.3 (Chapter 3) as follows:

$$
S G_{b}^{R M}=\frac{R M C}{\frac{1}{S G_{b}^{R C A}}-\frac{1-R M C}{S G_{b}^{O V A}}}
$$

The coarse aggregate content (RCA and NA) of the GC by the total volume equivalent method can be determined by Eqs. B.18 and B.19. The proportioning of the cement, sand, and water content, which represents the new mortar content of the GC will be explained in the following section.

\section{B.2.1.2.New Mortar Content}

As mentioned before, GC mortar consists of new mortar and residual mortar. In the equivalent total volume method the new mortar is proportioned so that the total volume of mortar in GC is equivalent to the total volume mortar in NAC. Therefore, to produce GC with equivalent total volume of mortar as the NAC, the following condition has to be satisfied:

$$
V_{T M}^{G C}=V_{M}^{N A C}
$$

where $V_{T M}^{G C}$ is the volume of total mortar in GC, $V_{M}^{N A C}$ is the volume of mortar in NAC. However, the volume of total mortar $\left(V_{T M}^{G C}\right)$ in GC consists of two parts, the volume of residual mortar $\left(V_{R M}^{G C}\right)$, and the volume of new mortar $\left(V_{N M}^{G C}\right)$ such that:

$$
V_{T M}^{G C}=V_{R M}^{G C}+V_{N M}^{G C}
$$

By substituting $V_{T M}^{G C}$ from Eq.B.22 in Eq.B.21 and rearranging $\left(V_{N M}^{G C}\right)$ :

$$
V_{N M}^{G C}=V_{M}^{N A C}-V_{R M}^{G C}
$$

$V_{M}^{N A C}$ can be calculated from the mix proportions of the NAC. $V_{R M}^{G C}$ can be calculated from: 


$$
V_{R M}^{G C}=V_{R C A}^{G C}\left(\frac{S G_{b}^{R C A}}{S G_{b}^{R M}} R M C\right)
$$

where the last term represents $R M C_{V}$. Therefore, the volume of new mortar in $\mathrm{GC}$ can be calculated from Eq.B.23. The mix proportions of the new mortar such as cement, sand and water can be found from the corresponding ingredients of the mortar in NAC by $V_{N M}^{G C} / V_{M}^{N A C}$ ratio. The mix proportions of water, cement, and fine aggregate in the new mortar of GC can be found by Eqs.B.25, B.26, and B.27:

$$
W_{W}^{G C}=W_{W}^{N A C}\left(\frac{V_{N M}^{G C}}{V_{M}^{N A C}}\right)
$$

where $W_{w}^{G C}$ and $W_{w}^{N A C}$ are the water contents $\left(\mathrm{kg} / \mathrm{m}^{3}\right)$ for GC and NAC, respectively. Also:

$$
W_{C}^{G C}=W_{C}^{N A C}\left(\frac{V_{N M}^{G C}}{V_{M}^{N A C}}\right)
$$

where $W_{C}^{G C}$ and $W_{C}^{N A C}$ are the cement contents $\left(\mathrm{kg} / \mathrm{m}^{3}\right)$ for GC and NAC, respectively. Similarly:

$$
W_{F A}^{G C}=W_{F A}^{N A C}\left(\frac{V_{N M}^{G C}}{V_{M}^{N A C}}\right)
$$

where $W_{F A}^{G C}$ and $W_{F A}^{N A C}$ are the natural fine aggregate contents $\left(\mathrm{kg} / \mathrm{m}^{3}\right)$ for $\mathrm{GC}$ and NAC, respectively. The ratio of $V_{N M}^{G C} / V_{M}^{N A C}$ can also be derived in the weight base format. Substitution of $V_{N M}^{G C}$ from Eq.B.23 in the $\frac{V_{N M}^{G C}}{V_{M}^{N A C}}$ ratio yields:

$$
\frac{V_{N M}^{G C}}{V_{M}^{N A C}}=\frac{V_{M}^{N A C}-V_{R M}^{G C}}{V_{M}^{N A C}}=1-\frac{V_{R M}^{G C}}{V_{M}^{N A C}}
$$

Substitution of $V_{R M}^{G C}$ from Eq.B.24 in Eq.B.28 gives:

$$
\frac{V_{N M}^{G C}}{V_{M}^{N A C}}=1-\frac{W_{O D}^{R C A} R M C}{1000 S G_{b}^{R M} V_{M}^{N A C}}
$$


The volume of mortar in NAC $\left(V_{M}^{N A C}\right)$ is the sum of the volume of water $\left(V_{W}^{N A C}\right)$, volume of cement $\left(V_{C}^{N A C}\right)$, volume of natural fine aggregate $\left(V_{F A}^{N A C}\right)$, and volume of air $\left(V_{A}^{N A C}\right)$, which can be converted to weight as per Eq.B.30.:

$$
\begin{aligned}
& V_{M}^{N A C}=V_{W}^{N A C}+V_{F A}^{N A C}+V_{C}^{N A C}+V_{A}^{N A C} \\
& =\left[\left(\frac{W_{W}^{N A C}}{1}+\frac{W_{F A}^{N A C}}{S G_{b}^{F A}}+\frac{W_{C}^{N A C}}{S G^{C}}\right) \frac{1}{1000}+A\right]
\end{aligned}
$$

Substitution of $V_{M}^{N A C}$ in Eq.B.29 yields:

$$
\frac{V_{N M}^{G C}}{V_{M}^{N A C}}=\left[1-\left(\frac{\frac{W_{O D-R C A}^{G C}}{1000 S G_{b}^{R M}} R M C}{\left(\frac{W_{W}^{N A C}}{1}+\frac{W_{F A}^{N A C}}{S G_{b}^{F A}}+\frac{W_{C}^{N A C}}{S G^{C}}\right) \frac{1}{1000}+A}\right)\right]
$$

\section{B.2.1.3.RCA Content}

The RCA content for GC is the weight of RCA divided by the total weight of coarse aggregate as per:

$$
\text { RCA Content }=\left(\frac{W_{R C A}^{G C}}{W_{R C A}^{G C}+W_{N A}^{G C}}\right) 100
$$

As mentioned in Section 7.3.1, and from Eq.B.8, to have equivalent volume of NA in GC and in the NAC, the volume of the residual mortar in GC is replaced by the volume of NA (i.e., $V_{R M}^{G C}=V_{N A}^{G C}$ ). However, the volume of the residual mortar in GC can be calculated by Eq.B.24. Combined Eqs.B.8, B.24 yields:

$$
V_{N A}^{G C}=\frac{S G_{b}^{R C A}}{S G_{b}^{R M}} R M C \cdot\left(\frac{W_{R C A}^{G C}}{S G_{b}^{R C A}}\right)
$$

Hence, the weight of NA in GC can be calculated using:

$$
W_{N A}^{G C}=\frac{S G_{b}^{N A}}{S G_{b}^{R M}} R M C . W_{R C A}^{G C}
$$

Combining Eqs.B.34 and B.32, the RCA content is given by: 


$$
\text { RCA Content }=\left(\frac{1}{1+\frac{S G_{b}^{N A}}{S G_{b}^{R M}} R M C}\right) 100
$$

\section{B.2.2Equivalent Total Volume of Mortar Method Example}

Mix batch of NAC is prepared by the following proportions: Cement $=409 \mathrm{~kg} / \mathrm{m}^{3}$, Mixing Water $=184 \mathrm{~kg} / \mathrm{m}^{3}$, Air dry natural aggregate $=794 \mathrm{~kg} / \mathrm{m}^{3}$, Dry Natural Sand $=$ $769 \mathrm{Kg} / \mathrm{kg} / \mathrm{m}^{3}$. The measurement of the air content for the NAC is $6.4 \%$. Using the RCA and NA properties provided in Example 1 of the general mix design method, calculate the mix proportions of GC that has the same equivalent total volume of mortar as the NAC.

By using Eq.B.20, the specific gravity of the residual mortar is calculated as:

$$
S G_{b}^{R M}=\frac{0.41}{\frac{1}{2.31}-\frac{1-0.41}{2.701}}=1.91
$$

Firstly, the mix proportion will be adjusted to $1 \mathrm{~m}^{3}$. The volume of the NAC produced with $6.4 \%$ air content can be calculated as follows:

$$
\text { Volume }=\left[\frac{409}{3.15}+\frac{184}{1}+\frac{794 \times\left(1+\frac{0.34}{100}\right)}{\left[1+\left(\frac{0.2}{100}\right)\right] \times 2.71}+\frac{769\left(1+\frac{0.8}{100}\right)}{2.70}\right] \times \frac{1.064}{1000}=0.9513
$$

Division of the given mix proportions by 0.9513 will give the adjusted mix proportions for NAC as follows: Cement $=430 \mathrm{~kg} / \mathrm{m}^{3}$, Mixing Water $=193 \mathrm{~kg} / \mathrm{m}^{3}$ and Dry Natural Sand $=808 \mathrm{~kg} / \mathrm{m}^{3}$. Also:

$$
\text { Weight of coarse natural aggregate }=\frac{794}{\left(1+\frac{0.2}{100}\right) \times 0.9513}=833 \mathrm{~kg} / \mathrm{m}^{3}
$$

The weight of RCA and NA in GC are calculated by using Eqs. B.18 and B.19: 


$$
\begin{aligned}
& W_{O D-R C A}^{G C}=\frac{833\left(1-\frac{2.31}{1.91} \times 0.41\right)}{(1-0.41)}=712 \mathrm{~kg} / \mathrm{m}^{3} \\
& W_{O D-N A}^{G C}=833 \times \frac{2.31}{1.91} \times 0.41=413 \mathrm{~kg} / \mathrm{m}^{3}
\end{aligned}
$$

The ratio of $\frac{V_{N M}^{G C}}{V_{M}^{N A C}}$ can be calculated using Eq.B.31 as:

$$
\frac{V_{N M}^{G C}}{V_{M}^{N A C}}=\left[1-\left(\frac{\frac{712}{1.91 \times 1000} \times 0.41}{\left.\left(\frac{193}{1}+\frac{808}{2.68}+\frac{430}{3.15}\right) \times \frac{1}{1000}+0.064\right)}\right]=0.78\right.
$$

The weight of water, cement, and fine aggregate, in new mortar content of GC can be calculated by Eqs.B.25, B.26, and B.27 as:

The weight of water for GC: $W_{W}^{G C}=0.78 \times 193=151 \mathrm{~kg} / \mathrm{m}^{3}$

The weight of cement for GC: $W_{C}^{G C}=0.78 \times 430=335 \mathrm{~kg} / \mathrm{m}^{3}$

The weight of sand for GC: $W_{F A}^{G C}=0.78 \times 808=630 \mathrm{~kg} / \mathrm{m}^{3}$

The weight of RCA in GC (Air dry): $W_{A D-R C A}^{G C}=712 \times\left(1+\frac{1.11}{100}\right)=720 \mathrm{~kg} / \mathrm{m}^{3}$

The weight of coarse NA in GC: $W_{A D-N A}^{G C}=413 \times\left(1+\frac{0.2}{100}\right)=414 \mathrm{~kg} / \mathrm{m}^{3}$. 


\title{
APPENDIX C: DURABILITY EXPERIMENTS (RAW DATA)
}

\author{
C.1.Freeze-and-Thaw Test (RAW DATA)
}




\begin{tabular}{|c|c|c|c|c|c|c|c|c|c|c|c|c|c|}
\hline \multirow{2}{*}{$\#$} & \multirow{2}{*}{ Measured Property } & \multicolumn{12}{|c|}{ Number of Freeze-and-Thaw Cycles } \\
\hline & & $\mathbf{0}$ & 26 & 53 & 79 & 104 & 125 & 146 & 173 & 203 & 234 & 273 & 300 \\
\hline \multirow{6}{*}{1} & Width (mm) & 101.81 & 101.77 & 101.85 & 101.75 & 101.71 & 101.82 & 101.8 & 101.78 & 101.95 & 101.87 & 101.88 & 101.85 \\
\hline & Depth (mm) & 77.34 & 77.25 & 77.52 & 77.42 & 77.56 & 77.55 & 77.6 & 77.3 & 77.49 & 77.43 & 77.47 & 77.24 \\
\hline & Weight $(\mathrm{Kg})$ & 6.857 & 6.829 & 6.799 & 6.863 & 6.856 & 6.88 & 6.868 & 6.869 & 6.86 & 6.841 & 6.842 & 6.817 \\
\hline & Time for pulse velocity (us) & 87.2 & 87.3 & 87.3 & 86.8 & 87.6 & 87.6 & 87 & 87 & 87 & 87.4 & 87.1 & 87.2 \\
\hline & Length Comparator reading (in) & 0.2371 & 0.2351 & 0.2372 & 0.2376 & 0.2351 & 0.2373 & 0.2373 & 0.2378 & 0.2366 & 0.239 & 0.2381 & 0.2388 \\
\hline & Transverse Frequency $(\mathrm{Hz})$ & 1886 & 1845 & 1845 & 1848 & 1843 & 1843 & 1845 & 1838 & 1839 & 1836 & 1824 & 1813 \\
\hline \multirow{6}{*}{2} & Width (mm) & 101.74 & 101.59 & 101.81 & 101.62 & 101.77 & 101.59 & 101.83 & 101.83 & 101.93 & 101.88 & 101.87 & 101.74 \\
\hline & Depth (mm) & 76.13 & 76.28 & 76.19 & 76.3 & 76.11 & 76.3 & 76.14 & 76.11 & 76.18 & 75.75 & 75.99 & 76.05 \\
\hline & Weight $(\mathrm{Kg})$ & 6.904 & 6.888 & 6.842 & 6.913 & 6.906 & 6.948 & 6.918 & 6.919 & 6.911 & 6.89 & 6.894 & 6.868 \\
\hline & Time for pulse velocity ( $\mu s)$ & 86.8 & 86.7 & 86.8 & 86.7 & 86.5 & 87.2 & 86.8 & 86.5 & 86.6 & 87 & 86.6 & 87.2 \\
\hline & Length Comparator reading (in) & 0.2946 & 0.292 & 0.2947 & 0.2947 & 0.2921 & 0.2941 & 0.2943 & 0.295 & 0.2943 & 0.2961 & 0.2955 & 0.296 \\
\hline & Transverse Frequency $(\mathrm{Hz})$ & 1892 & 1851 & 1850 & 1850 & 1851 & 1848 & 1848 & 1842 & 1840 & 1838 & 1821 & 1818 \\
\hline \multirow{6}{*}{3} & Width (mm) & 101.9 & 101.63 & 101.69 & 101.75 & 101.78 & 101.9 & 101.75 & 101.85 & 101.95 & 101.74 & 102.01 & 101.9 \\
\hline & Depth (mm) & 76.72 & 76.5 & 76.45 & 76.53 & 76.66 & 76.52 & 76.42 & 76.52 & 76.24 & 76.36 & 76.47 & 76.32 \\
\hline & Weight $(\mathrm{Kg})$ & 6.898 & 6.873 & 6.833 & 6.909 & 6.898 & 6.925 & 6.906 & 6.903 & 6.894 & 6.871 & 6.868 & 6.844 \\
\hline & Time for pulse velocity ( $\mu s)$ & 86.9 & 87.2 & 87.2 & 87.3 & 87.8 & 87.6 & 87.5 & 87.4 & 87.2 & 87.4 & 87.4 & 88 \\
\hline & Length Comparator reading (in) & 0.2841 & 0.2821 & 0.2845 & 0.285 & 0.2822 & 0.2846 & 0.2845 & 0.2849 & 0.2843 & 0.2862 & 0.2854 & 0.2859 \\
\hline & Transverse Frequency $(\mathrm{Hz})$ & 1890 & 1849 & 1849 & 1847 & 1840 & 1842 & 1848 & 1842 & 1844 & 1834 & 1822 & 1809 \\
\hline
\end{tabular}

Length of the specimens $=385 \mathrm{~mm}$ 


\begin{tabular}{|c|c|c|c|c|c|c|c|c|c|c|c|c|c|}
\hline \multirow{2}{*}{$\#$} & \multirow{2}{*}{ Measured Property } & \multicolumn{12}{|c|}{ Number of Freeze-and-Thaw Cycles } \\
\hline & & $\mathbf{0}$ & 26 & 53 & 79 & 104 & 125 & 146 & 173 & 203 & 234 & 273 & 300 \\
\hline \multirow{6}{*}{1} & Width (mm) & 101.54 & 101.28 & 101.5 & 101.55 & 101.47 & 101.58 & 101.46 & 101.73 & 101.53 & 101.51 & 101.56 & 102.41 \\
\hline & Depth (mm) & 77.93 & 78.05 & 77.94 & 77.66 & 77.71 & 77.87 & 77.76 & 77.43 & 77.37 & 77.63 & 77.12 & 76.94 \\
\hline & Weight $(\mathrm{Kg})$ & 6.758 & 6.736 & 6.691 & 6.756 & 6.751 & 6.778 & 6.745 & 6.729 & 6.705 & 6.671 & 6.648 & 6.62 \\
\hline & Time for pulse velocity (us) & 87.6 & 88.5 & 89 & 89 & 89.6 & 89.6 & 89.3 & 89.9 & 90.1 & 91.3 & 91 & 92.4 \\
\hline & Length Comparator reading (in) & 0.1883 & 0.1864 & 0.1885 & 0.1889 & 0.1865 & 0.1883 & 0.1888 & 0.1891 & 0.1884 & 0.1904 & 0.1903 & 0.1903 \\
\hline & Transverse Frequency $(\mathrm{Hz})$ & 1821 & 1783 & 1784 & 1785 & 1780 & 1780 & 1781 & 1755 & 1764 & 1752 & 1749 & 1740 \\
\hline \multirow{6}{*}{2} & Width (mm) & 101.49 & 101.5 & 101.54 & 101.67 & 101.85 & 101.83 & 101.86 & 102.48 & 101.92 & 102.04 & 102.36 & 102.32 \\
\hline & Depth $(\mathrm{mm})$ & 76.08 & 76.08 & 76.16 & 76.23 & 75.7 & 76.52 & 75.23 & 76.31 & 75.85 & 75.67 & 76.03 & 76.06 \\
\hline & Weight $(\mathrm{Kg})$ & 6.722 & 6.698 & 6.663 & 6.722 & 6.711 & 6.722 & 6.681 & 6.673 & 6.641 & 6.605 & 6.585 & 6.553 \\
\hline & Time for pulse velocity (us) & 87.5 & 88.1 & 88.5 & 88.8 & 89.2 & 89.7 & 89.7 & 89.9 & 90 & 91.3 & 92.7 & 92.5 \\
\hline & Length Comparator reading (in) & 0.2739 & 0.2727 & 0.2745 & 0.2755 & 0.2733 & 0.275 & 0.2745 & 0.2754 & 0.2745 & 0.2761 & 0.2759 & 0.2764 \\
\hline & Transverse Frequency $(\mathrm{Hz})$ & 1820 & 1785 & 1760 & 1754 & 1740 & 1730 & 1730 & 1725 & 1725 & 1720 & 1703 & 1699 \\
\hline \multirow{6}{*}{3} & Width (mm) & 101.48 & 101.46 & 101.56 & 101.68 & 101.92 & 101.86 & 101.7 & 101.56 & 101.73 & 102 & 101.52 & 101.72 \\
\hline & Depth (mm) & 77.77 & 77.58 & 77.6 & 77.49 & 77.56 & 77.76 & 77.64 & 76.8 & 76.9 & 76.89 & 77.2 & 77.04 \\
\hline & Weight $(\mathrm{Kg})$ & 6.771 & 6.756 & 6.709 & 6.776 & 6.755 & 6.769 & 6.737 & 6.724 & 6.697 & 6.659 & 6.639 & 6.605 \\
\hline & Time for pulse velocity (us) & 88.1 & 88.9 & 89.3 & 89.2 & 88.9 & 89.6 & 89.4 & 90.5 & 91.9 & 91.9 & 92.5 & 93.8 \\
\hline & Length Comparator reading (in) & 0.3262 & 0.3235 & 0.3256 & 0.3264 & 0.3236 & 0.3254 & 0.3257 & 0.3263 & 0.3251 & 0.3273 & 0.3276 & 0.3277 \\
\hline & Transverse Frequency $(\mathrm{Hz})$ & 1829 & 1820 & 1787 & 1783 & 1771 & 1755 & 1756 & 1748 & 1748 & 1740 & 1734 & 1729 \\
\hline
\end{tabular}

Length of the specimens $=385 \mathrm{~mm}$ 


\begin{tabular}{|c|c|c|c|c|c|c|c|c|c|c|c|c|c|}
\hline \multirow{2}{*}{ \# } & \multirow{2}{*}{ Measured Property } & \multicolumn{12}{|c|}{ Number of Freeze-and-Thaw Cycles } \\
\hline & & $\mathbf{0}$ & 28 & 60 & 78 & 105 & 127 & 150 & 180 & 217 & 253 & 274 & 300 \\
\hline \multirow{6}{*}{1} & Width (mm) & 101.78 & 101.92 & 102 & 101.85 & 102.28 & 102.01 & 102.09 & 101.92 & 101.97 & 101.92 & 102 & 102.24 \\
\hline & Depth (mm) & 77.13 & 77.13 & 77.07 & 77.04 & 76.48 & 77.36 & 77.04 & 76.84 & 77.01 & 77.16 & 77.09 & 76.87 \\
\hline & Weight $(\mathrm{Kg})$ & 6.814 & 6.804 & 6.864 & 6.851 & 6.865 & 6.886 & 6.859 & 6.868 & 6.845 & 6.84 & 6.849 & 6.822 \\
\hline & Time for pulse velocity ( $u s)$ & 85 & 86.4 & 86.6 & 86.5 & 86.7 & 86.5 & 86.1 & 86.5 & 86.9 & 87.2 & 87.3 & 86.8 \\
\hline & Length Comparator reading (in) & 0.2318 & 0.2316 & 0.2321 & 0.2317 & 0.2319 & 0.2311 & 0.2323 & 0.2325 & 0.2336 & 0.2337 & 0.2343 & 0.2343 \\
\hline & Transverse Frequency $(\mathrm{Hz})$ & 1905 & 1868 & 1868 & 1868 & 1867 & 1873 & 1866 & 1850 & 1849 & 1835 & 1835 & 1814 \\
\hline \multirow{6}{*}{2} & Width (mm) & 101.82 & 101.85 & 102.04 & 101.82 & 101.96 & 102.36 & 101.95 & 101.93 & 101.83 & 101.78 & 101.74 & 101.88 \\
\hline & Depth (mm) & 76.53 & 76.76 & 76.54 & 76.58 & 76.31 & 76.75 & 76.57 & 76.71 & 76.17 & 76.46 & 76.29 & 76.29 \\
\hline & Weight $(\mathrm{Kg})$ & 6.803 & 6.794 & 6.868 & 6.85 & 6.866 & 6.884 & 6.856 & 6.856 & 6.845 & 6.829 & 6.838 & 6.807 \\
\hline & Time for pulse velocity ( $u s)$ & 85 & 85.7 & 86.1 & 85.8 & 86.2 & 85.9 & 85.9 & 85.9 & 85.8 & 86.5 & 86.9 & 86.2 \\
\hline & Length Comparator reading (in) & 0.2831 & 0.2827 & 0.2835 & 0.2832 & 0.2838 & 0.2829 & 0.2839 & 0.2846 & 0.2853 & 0.2856 & 0.2861 & 0.2866 \\
\hline & Transverse Frequency (Hz) & 1901 & 1870 & 1853 & 1848 & 1838 & 1844 & 1844 & 1820 & 1848 & 1821 & 1806 & 1800 \\
\hline \multirow{6}{*}{3} & Width (mm) & 101.91 & 101.93 & 101.97 & 101.95 & 101.5 & 102.27 & 102.06 & 102.14 & 101.94 & 101.75 & 102.03 & 102.23 \\
\hline & Depth $(\mathrm{mm})$ & 77.76 & 77.77 & 77.82 & 77.66 & 78.32 & 77.87 & 77.93 & 77.75 & 77.16 & 77.96 & 78.05 & 77.76 \\
\hline & Weight $(\mathrm{Kg})$ & 6.888 & 6.874 & 6.94 & 6.932 & 6.944 & 6.96 & 6.931 & 6.925 & 6.912 & 6.899 & 6.908 & 6.871 \\
\hline & Time for pulse velocity $(u s)$ & 85.2 & 85.8 & 86.6 & 86.6 & 86.6 & 86.8 & 86 & 86.1 & 86.7 & 87 & 87.5 & 87.1 \\
\hline & Length Comparator reading (in) & 0.2796 & 0.2791 & 0.2803 & 0.2798 & 0.279 & 0.2788 & 0.2799 & 0.2803 & 0.2811 & 0.2813 & 0.2817 & 0.2822 \\
\hline & Transverse Frequency (Hz) & 1937 & 1910 & 1868 & 1868 & 1849 & 1848 & 1868 & 1850 & 1841 & 1831 & 1841 & 1829 \\
\hline
\end{tabular}

Length of the specimens $=385 \mathrm{~mm}$ 
Specimen Designation: GC-MO-D-C-E26

\begin{tabular}{|c|c|c|c|c|c|c|c|c|c|c|c|c|c|c|}
\hline \multirow{2}{*}{ \# } & \multirow{2}{*}{ Measured Property } & \multicolumn{13}{|c|}{ Number of Freeze-and-Thaw Cycles } \\
\hline & & $\mathbf{0}$ & 32 & 63 & 94 & 115 & 136 & 156 & 189 & 205 & 224 & 242 & 268 & 300 \\
\hline \multirow{6}{*}{1} & Width (mm) & 102 & 101.8 & 101.9 & 101.9 & 101.8 & 101.7 & 102 & 101.8 & 102.3 & 101.9 & 101.9 & 101.8 & 102.0 \\
\hline & Depth $(\mathrm{mm})$ & 77.29 & 77.43 & 77.19 & 77.11 & 77.96 & 77.15 & 77.28 & 77.37 & 77.49 & 77.34 & 77.24 & 76.86 & 76.99 \\
\hline & Weight $(\mathrm{Kg})$ & 7.052 & 7.03 & 6.996 & 6.983 & 7.058 & 7.049 & 7.066 & 7.09 & 7.063 & 7.049 & 7.063 & 7.047 & 7.027 \\
\hline & Time for pulse velocity ( $\mu \mathrm{s})$ & 82.9 & 83 & 83.8 & 84.2 & 83.8 & 83.8 & 84.8 & 84.8 & 84.3 & 84.1 & 84.1 & 85.6 & 86 \\
\hline & Length Comparator reading & 0.328 & 0.329 & 0.330 & 0.330 & 0.331 & 0.330 & 0.329 & 0.329 & 0.330 & 0.330 & 0.330 & 0.331 & 0.331 \\
\hline & Transverse Frequency $(\mathrm{Hz})$ & 1894 & 1905 & 1886 & 1875 & 1879 & 1873 & 1870 & 1856 & 1850 & 1860 & 1848 & 1843 & 1846 \\
\hline \multirow{6}{*}{2} & Width (mm) & 101.5 & 101.5 & 101.6 & 101.6 & 101.5 & 101.6 & 101.8 & 101.6 & 102.1 & 101.9 & 101.9 & 102.1 & 101.6 \\
\hline & Depth (mm) & 78.31 & 78.33 & 78.53 & 78.41 & 78.46 & 78.39 & 78.6 & 78.36 & 78.33 & 78.65 & 78.45 & 78.14 & 78.2 \\
\hline & Weight $(\mathrm{Kg})$ & 7.024 & 7.008 & 6.979 & 6.959 & 7.033 & 7.025 & 7.046 & 7.048 & 7.025 & 7.012 & 7.014 & 7.011 & 6.978 \\
\hline & Time for pulse velocity $(\mu \mathrm{s})$ & 84.4 & 85.4 & 85 & 85.4 & 85.3 & 85.3 & 85.4 & 85.4 & 84.9 & 84.8 & 84.6 & 85.5 & 85.2 \\
\hline & Length Comparator reading & 0.357 & 0.359 & 0.359 & 0.359 & 0.360 & 0.360 & 0.359 & 0.359 & 0.361 & 0.361 & 0.361 & 0.362 & 0.362 \\
\hline & Transverse Frequency $(\mathrm{Hz})$ & 1890 & 1874 & 1850 & 1850 & 1850 & 1821 & 1816 & 1816 & 1814 & 1815 & 1814 & 1808 & 1805 \\
\hline \multirow{6}{*}{3} & Width $(\mathrm{mm})$ & 101.4 & 101.4 & 101.4 & 101.5 & 101.5 & 101.5 & 101.6 & 101.7 & 101.6 & 101.6 & 101.6 & 101.3 & 101.6 \\
\hline & Depth $(\mathrm{mm})$ & 78.32 & 77.91 & 78.11 & 78.48 & 78.52 & 78.6 & 78.81 & 78.04 & 78.49 & 78.36 & 78.23 & 77.66 & 77.77 \\
\hline & Weight (Kg) & 7.038 & 7.012 & 6.98 & 6.972 & 7.04 & 7.032 & 7.051 & 7.067 & 7.042 & 7.027 & 7.037 & 7.027 & 7.002 \\
\hline & Time for pulse velocity ( $u$ s) & 84.2 & 85.1 & 85.2 & 85.2 & 84.6 & 84.5 & 84.8 & 84.8 & 84.8 & 85.2 & 84.9 & 84.9 & 86.2 \\
\hline & Length Comparator reading & 0.305 & 0.306 & 0.307 & 0.306 & 0.307 & 0.307 & 0.306 & 0.306 & 0.307 & 0.307 & 0.307 & 0.308 & 0.308 \\
\hline & Transverse Frequency $(\mathrm{Hz})$ & 1854 & 1842 & 1844 & 1851 & 1857 & 1844 & 1843 & 1843 & 1843 & 1840 & 1840 & 1833 & 1832 \\
\hline
\end{tabular}

Length of the specimens $=385 \mathrm{~mm}$ 


\begin{tabular}{|c|c|c|c|c|c|c|c|c|c|c|c|c|c|c|}
\hline & \multirow{2}{*}{ Measured Property } & \multicolumn{13}{|c|}{ Number of Freeze-and-Thaw Cycles } \\
\hline & & $\mathbf{0}$ & 32 & 63 & 94 & 115 & 136 & 156 & 189 & 205 & 224 & 242 & 268 & 300 \\
\hline & Width (mm) & 102.0 & 101.9 & 102.0 & 102.0 & 102.1 & 102.0 & 102.0 & 102.1 & 102.1 & 102.0 & 102.1 & 102.0 & 102.1 \\
\hline & Depth (mm) & 77.99 & 78.01 & 77.98 & 77.95 & 78.04 & 77.93 & 77.87 & 77.89 & 77.94 & 77.9 & 77.94 & 77.98 & 77.76 \\
\hline & Weight $(\mathrm{Kg})$ & 7.071 & 7.054 & 7.012 & 7.007 & 7.081 & 7.079 & 7.09 & 7.092 & 7.072 & 7.061 & 7.069 & 7.043 & 7.04 \\
\hline & Time for pulse velocity $(\mu \mathrm{s})$ & 83.3 & 84.3 & 84.5 & 84.1 & 83.7 & 84.5 & 85.5 & 84.9 & 85.2 & 84.8 & 85.1 & 87.6 & 86.4 \\
\hline & Length Comparator reading & 0.298 & 0.298 & 0.299 & 0.299 & 0.300 & 0.299 & 0.298 & 0.297 & 0.300 & 0.299 & 0.300 & 0.301 & 0.300 \\
\hline & Transverse Frequency $(\mathrm{Hz})$ & 1911 & 1880 & 1895 & 1870 & 1874 & 1852 & 1850 & 1848 & 1847 & 1850 & 1838 & 1819 & 1820 \\
\hline & Width (mm) & 101.5 & 101.5 & 101.5 & 101.5 & 101.6 & 101.8 & 101.7 & 102.0 & 101.9 & 102.0 & 102.0 & 101.4 & 101.1 \\
\hline & Depth (mm) & 77.5 & 78.63 & 78.6 & 78.54 & 79.34 & 77.86 & 78.94 & 79.45 & 79.98 & 78.32 & 78.1 & 77.82 & 77.96 \\
\hline & Weight (Kg) & 7.203 & 7.178 & 7.147 & 7.134 & 7.208 & 7.203 & 7.213 & 7.193 & 7.169 & 7.154 & 7.15 & 7.111 & 7.09 \\
\hline & Time for pulse velocity $(\mu s)$ & 83.3 & 83.9 & 85.1 & 84.8 & 85 & 85.2 & 85.6 & 87.2 & 87.4 & 87.9 & 87.5 & 87.4 & 87.5 \\
\hline & Length Comparator reading & 0.300 & 0.301 & 0.301 & 0.301 & 0.302 & 0.302 & 0.301 & 0.301 & 0.303 & 0.303 & 0.303 & 0.304 & 0.304 \\
\hline & Transverse Frequency $(\mathrm{Hz})$ & 1944 & 1923 & 1907 & 1902 & 1901 & 1882 & 1874 & 1865 & 1863 & 1846 & 1843 & 1846 & 1832 \\
\hline & Width (mm) & 101.9 & 101.7 & 101.8 & 101.7 & 101.9 & 101.8 & 102.0 & 101.8 & 102.3 & 101.7 & 102.0 & 102.0 & 102.1 \\
\hline & Depth (mm) & 78 & 78 & 78 & 78 & 78.2 & 79.41 & 79.65 & 79.6 & 79.65 & 79.73 & 79.56 & 79.16 & 79.25 \\
\hline & Weight $(\mathrm{Kg})$ & 7.103 & 7.081 & 7.041 & 7.035 & 7.104 & 7.097 & 7.113 & 7.097 & 7.079 & 7.063 & 7.069 & 7.037 & 7.018 \\
\hline & Time for pulse velocity $(\mu \mathrm{s})$ & 84.2 & 84.8 & 85.2 & 85.1 & 85.4 & 85.5 & 85.6 & 85.6 & 85.4 & 86.3 & 86.8 & 87.8 & 88.8 \\
\hline & Length Comparator reading & 0.266 & 0.266 & 0.267 & 0.267 & 0.268 & 0.267 & 0.267 & 0.266 & 0.268 & 0.268 & 0.268 & 0.27 & 0.270 \\
\hline & Transverse Frequency $(\mathrm{Hz})$ & 1904 & 1903 & 1901 & 1903 & 1875 & 1873 & 1843 & 1843 & 1847 & 1846 & 1843 & 1827 & 1820 \\
\hline
\end{tabular}

Length of the specimens $=385 \mathrm{~mm}$ 


\begin{tabular}{|c|c|c|c|c|c|c|c|c|c|c|c|c|c|c|}
\hline \multirow{2}{*}{ \# } & \multirow{2}{*}{ Measured Property } & \multicolumn{13}{|c|}{ Number of Freeze-and-Thaw Cycles } \\
\hline & & $\mathbf{0}$ & 32 & 63 & 94 & 115 & 136 & 156 & 189 & 205 & 224 & 242 & 268 & 300 \\
\hline \multirow{6}{*}{1} & Width (mm) & 101.9 & 101.7 & 101.6 & 101.7 & 101.7 & 101.8 & 101.9 & 101.9 & 102 & 102.1 & 102.0 & 102.4 & 101.8 \\
\hline & Depth (mm) & 77.39 & 77.05 & 77.25 & 77.16 & 77.23 & 77.06 & 77.71 & 77.93 & 77.37 & 77.95 & 77.35 & 77.45 & 77.43 \\
\hline & Weight $(\mathrm{Kg})$ & 7.152 & 7.126 & 7.081 & 7.07 & 7.143 & 7.139 & 7.165 & 7.161 & 7.143 & 7.134 & 7.142 & 7.134 & 7.123 \\
\hline & Time for pulse velocity $(\mu \mathrm{s})$ & 82.4 & 82.8 & 83.4 & 83.9 & 84.5 & 84 & 85 & 85 & 84 & 84.3 & 84.5 & 84.6 & 84.6 \\
\hline & Length Comparator reading & 0.319 & 0.32 & 0.320 & 0.320 & 0.321 & 0.320 & 0.319 & 0.319 & 0.321 & 0.321 & 0.321 & 0.321 & 0.322 \\
\hline & Transverse Frequency $(\mathrm{Hz})$ & 1910 & 1907 & 1911 & 1877 & 1867 & 1870 & 1872 & 1871 & 1870 & 1873 & 1846 & 1845 & 1844 \\
\hline \multirow{6}{*}{2} & Width (mm) & 101.6 & 101.6 & 101.6 & 101.6 & 101.6 & 101.7 & 102.2 & 101.6 & 101.8 & 101.9 & 101.8 & 101.8 & 101.6 \\
\hline & Depth (mm) & 77.5 & 77.36 & 77.36 & 77.33 & 77.58 & 77.25 & 77.14 & 77.7 & 77.37 & 77.34 & 77.46 & 77.13 & 77.11 \\
\hline & Weight $(\mathrm{Kg})$ & 7.075 & 7.061 & 7.016 & 7.01 & 7.078 & 7.072 & 7.165 & 7.084 & 7.067 & 7.051 & 7.055 & 7.039 & 7.037 \\
\hline & Time for pulse velocity ( $u s)$ & 81.8 & 82.5 & 83.3 & 84.2 & 82.7 & 82.9 & 83.4 & 84.5 & 84.5 & 83.2 & 83.7 & 83.9 & 84.2 \\
\hline & Length Comparator reading & 0.289 & 0.290 & 0.291 & 0.291 & 0.291 & 0.291 & 0.291 & 0.291 & 0.292 & 0.292 & 0.293 & 0.293 & 0.293 \\
\hline & Transverse Frequency $(\mathrm{Hz})$ & 1931 & 1905 & 1904 & 1877 & 1881 & 1879 & 1878 & 1878 & 1878 & 1880 & 1871 & 1850 & 1843 \\
\hline \multirow{6}{*}{3} & Width (mm) & 101.3 & 100.9 & 100.9 & 101.0 & 101.1 & 101.3 & 101.0 & 101.4 & 101.0 & 101.2 & 101.4 & 101.0 & 101.1 \\
\hline & Depth (mm) & 77.4 & 77.9 & 77.33 & 77.51 & 77.47 & 77.27 & 77.25 & 77.39 & 77.4 & 77.48 & 77.15 & 77.58 & 76.92 \\
\hline & Weight $(\mathrm{Kg})$ & 7.039 & 7.015 & 6.975 & 6.962 & 7.031 & 7.023 & 7.049 & 7.045 & 7.029 & 7.019 & 7.027 & 7.002 & 6.998 \\
\hline & Time for pulse velocity $(\mu \mathrm{s})$ & 82.5 & 83.5 & 84.3 & 84.1 & 84.3 & 83.8 & 83.9 & 86 & 83.4 & 83.8 & 84.5 & 84.5 & 85.5 \\
\hline & Length Comparator reading & 0.185 & 0.186 & 0.187 & 0.187 & 0.188 & 0.187 & 0.186 & 0.186 & 0.187 & 0.187 & 0.187 & 0.188 & 0.188 \\
\hline & Transverse Frequency $(\mathrm{Hz})$ & 1924 & 1880 & 1873 & 1873 & 1872 & 1871 & 1871 & 1870 & 1874 & 1864 & 1848 & 1848 & 1847 \\
\hline
\end{tabular}

Length of the specimens $=385 \mathrm{~mm}$ 


\begin{tabular}{|c|c|c|c|c|c|c|c|c|c|c|c|c|c|}
\hline \multirow{2}{*}{$\#$} & \multirow{2}{*}{ Measured Property } & \multicolumn{12}{|c|}{ Number of Freeze-and-Thaw Cycles } \\
\hline & & $\mathbf{0}$ & 32 & 62 & 88 & 115 & 139 & 165 & 185 & 210 & 235 & 267 & 300 \\
\hline \multirow{6}{*}{1} & Width (mm) & 101.47 & 101.43 & 101.5 & 101.61 & 101.54 & 101.58 & 101.91 & 101.6 & 101.91 & 101.56 & 101.34 & 101.46 \\
\hline & Depth $(\mathrm{mm})$ & 78.04 & 78.07 & 78.33 & 77.95 & 78.67 & 78.36 & 78.56 & 78.85 & 78.5 & 78.48 & 78.08 & 77.93 \\
\hline & Weight $(\mathrm{Kg})$ & 7.02 & 7.001 & 6.958 & 6.943 & 7.019 & 7.01 & 7.045 & 7.035 & 7.004 & 7 & 6.992 & 6.974 \\
\hline & Time for pulse velocity ( $u s)$ & 82.1 & 83.2 & 82.8 & 82.5 & 83.2 & 83.5 & 82.3 & 82 & 82 & 81.7 & 82.4 & 82.4 \\
\hline & Length Comparator reading (in) & 0.2979 & 0.2976 & 0.2951 & 0.2984 & 0.2959 & 0.2955 & 0.2974 & 0.2978 & 0.2978 & 0.298 & 0.2988 & 0.2986 \\
\hline & Transverse Frequency $(\mathrm{Hz})$ & 1934 & 1935 & 1960 & 1943 & 1929 & 1925 & 1935 & 1937 & 1937 & 1927 & 1921 & 1916 \\
\hline \multirow{6}{*}{2} & Width (mm) & 101.74 & 101.61 & 101.55 & 101.63 & 101.82 & 101.69 & 101.69 & 101.55 & 101.57 & 101.7 & 101.86 & 101.62 \\
\hline & Depth (mm) & 77.37 & 77.42 & 77.6 & 77.45 & 77.6 & 77.41 & 77.67 & 77.71 & 77.5 & 77.36 & 77.59 & 76.77 \\
\hline & Weight $(\mathrm{Kg})$ & 6.934 & 6.918 & 6.879 & 6.864 & 6.942 & 6.936 & 6.972 & 6.972 & 6.948 & 6.946 & 6.936 & 6.92 \\
\hline & Time for pulse velocity (us) & 81.7 & 82.5 & 82. & 82.2 & 82.9 & 82.8 & 81.6 & 82 & 81.6 & 81.7 & 82.5 & 82.5 \\
\hline & Length Comparator reading (in) & 0.2142 & 0.2139 & 0.2117 & 0.2145 & 0.212 & 0.2124 & 0.214 & 0.2148 & 0.2143 & 0.2148 & 0.2154 & 0.2152 \\
\hline & Transverse Frequency $(\mathrm{Hz})$ & 1930 & 1907 & 1932 & 1930 & 1906 & 1907 & 1909 & 1905 & 1911 & 1907 & 1896 & 1885 \\
\hline \multirow{6}{*}{3} & Width (mm) & 101.85 & 102.1 & 101.86 & 101.94 & 102.02 & 101.83 & 102.12 & 102 & 101.86 & 101.98 & 102.49 & 102.1 \\
\hline & Depth $(\mathrm{mm})$ & 77.26 & 76.29 & 76.08 & 76.42 & 76.55 & 76.27 & 76.61 & 76.37 & 76.06 & 76.47 & 76.28 & 76.09 \\
\hline & Weight (Kg) & 7.003 & 6.991 & 6.946 & 6.928 & 7.011 & 7 & 7.033 & 7.029 & 7.007 & 7.007 & 6.992 & 6.97 \\
\hline & Time for pulse velocity (us) & 81 & 82.3 & 82 & 81.9 & 82.3 & 82.3 & 81.4 & 81.4 & 81.2 & 80.8 & 81.6 & 81.6 \\
\hline & Length Comparator reading (in) & 0.3222 & 0.3221 & 0.3199 & 0.3225 & 0.3205 & 0.3206 & 0.322 & 0.323 & 0.3226 & 0.3223 & 0.3234 & 0.3235 \\
\hline & Transverse Frequency $(\mathrm{Hz})$ & 1938 & 1905 & 1874 & 1904 & 1900 & 1900 & 1911 & 1908 & 1913 & 1902 & 1900 & 1900 \\
\hline
\end{tabular}

Length of the specimens $=385 \mathrm{~mm}$ 


\begin{tabular}{|c|c|c|c|c|c|c|c|c|c|c|c|c|c|}
\hline \multirow{2}{*}{$\#$} & \multirow{2}{*}{ Measured Property } & \multicolumn{12}{|c|}{ Number of Freeze-and-Thaw Cycles } \\
\hline & & $\mathbf{0}$ & 28 & 53 & 77 & 103 & 123 & 148 & 169 & 195 & 226 & 265 & 300 \\
\hline \multirow{6}{*}{1} & Width (mm) & 101.28 & 101.19 & 101.23 & 101.34 & 101.31 & 101.47 & 101.76 & 101.83 & 101.18 & 101.18 & 101.79 & 102.21 \\
\hline & Depth (mm) & 76.87 & 76.94 & 76.82 & 76.98 & 77.01 & 77.62 & 76.86 & 77.3 & 76.66 & 76.78 & 76.72 & 76.59 \\
\hline & Weight $(\mathrm{Kg})$ & 7.015 & 6.964 & 6.945 & 7.021 & 7.031 & 7.039 & 7.012 & 6.958 & 7.01 & 6.997 & 6.988 & 6.944 \\
\hline & Time for pulse velocity ( $u s)$ & 83.5 & 84.2 & 84.6 & 84.6 & 84.3 & 84.5 & 83.7 & 83.7 & 84.3 & 85 & 85.9 & 85.1 \\
\hline & Length Comparator reading (in) & 0.2702 & 0.2704 & 0.2712 & 0.2712 & 0.2701 & 0.2697 & 0.2708 & 0.272 & 0.2703 & 0.272 & 0.2715 & 0.2711 \\
\hline & Transverse Frequency $(\mathrm{Hz})$ & 1958 & 1906 & 1907 & 1900 & 1902 & 1900 & 1890 & 1881 & 1885 & 1884 & 1881 & 1879 \\
\hline \multirow{6}{*}{2} & Width (mm) & 101.95 & 101.71 & 101.77 & 101.86 & 101.83 & 101.91 & 101.85 & 101.44 & 101.89 & 101.74 & 101.84 & 102.11 \\
\hline & Depth (mm) & 76.6 & 76.61 & 76.46 & 76.55 & 76.63 & 76.8 & 76.89 & 76.86 & 76.52 & 76.35 & 76.5 & 76.16 \\
\hline & Weight $(\mathrm{Kg})$ & 6.968 & 6.909 & 6.894 & 6.969 & 6.978 & 6.985 & 6.956 & 7.016 & 6.952 & 6.936 & 6.929 & 6.882 \\
\hline & Time for pulse velocity ( $\mu \mathrm{s})$ & 83.6 & 83.9 & 84.1 & 84.3 & 84.3 & 84.2 & 84.2 & 84.8 & 84.8 & 84.6 & 85.4 & 84.6 \\
\hline & Length Comparator reading (in) & 0.3495 & 0.3502 & 0.3504 & 0.3503 & 0.3494 & 0.3492 & 0.3498 & 0.3509 & 0.3493 & 0.3515 & 0.3507 & 0.3503 \\
\hline & Transverse Frequency $(\mathrm{Hz})$ & 1904 & 1904 & 1880 & 1875 & 1875 & 1877 & 1875 & 1874 & 1874 & 1865 & 1864 & 1860 \\
\hline \multirow{6}{*}{3} & Width (mm) & 101.8 & 101.38 & 101.81 & 102.07 & 101.93 & 102.47 & 101.97 & 101.85 & 101.95 & 102.06 & 102.1 & 101.91 \\
\hline & Depth (mm) & 76.05 & 76.02 & 75.97 & 76.01 & 75.91 & 75.89 & 76.06 & 75.98 & 76.08 & 75.6 & 75.92 & 75.81 \\
\hline & Weight $(\mathrm{Kg})$ & 6.91 & 6.859 & 6.841 & 6.918 & 6.936 & 6.941 & 6.914 & 6.922 & 6.916 & 6.892 & 6.884 & 6.845 \\
\hline & Time for pulse velocity ( $u s)$ & 84 & 84.5 & 85.1 & 84.8 & 84.6 & 84.6 & 84.3 & 85 & 85.4 & 86.1 & 85.3 & 85.4 \\
\hline & Length Comparator reading (in) & 0.2837 & 0.2842 & 0.2849 & 0.2847 & 0.2834 & 0.2836 & 0.2843 & 0.2847 & 0.2841 & 0.2858 & 0.2855 & 0.2847 \\
\hline & Transverse Frequency $(\mathrm{Hz})$ & 1904 & 1874 & 1874 & 1873 & 1868 & 1868 & 1858 & 1852 & 1852 & 1855 & 1851 & 1850 \\
\hline
\end{tabular}

Length of the specimens $=385 \mathrm{~mm}$ 


\begin{tabular}{|c|c|c|c|c|c|c|c|c|c|c|c|c|c|}
\hline \multirow{2}{*}{$\#$} & \multirow{2}{*}{ Measured Property } & \multicolumn{12}{|c|}{ Number of Freeze-and-Thaw Cycles } \\
\hline & & $\mathbf{0}$ & 27 & 53 & 78 & 99 & 120 & 147 & 177 & 208 & 247 & 292 & 300 \\
\hline \multirow{6}{*}{1} & Width (mm) & 101.42 & 101.47 & 101.47 & 101.58 & 101.58 & 101.86 & 101.63 & 101.76 & 102.09 & 102.09 & 102.01 & 101.91 \\
\hline & Depth (mm) & 76.85 & 76.82 & 76.88 & 76.91 & 76.71 & 76.94 & 76.95 & 76.92 & 76.45 & 76.88 & 76.82 & 76.76 \\
\hline & Weight $(\mathrm{Kg})$ & 6.902 & 6.859 & 6.939 & 6.922 & 6.955 & 6.93 & 6.93 & 6.91 & 6.87 & 6.822 & 6.769 & 6.773 \\
\hline & Time for pulse velocity ( $\mu s$ ) & 83.6 & 84.7 & 84.7 & 85.3 & 85.4 & 85.3 & 85.3 & 86.7 & 87.1 & 85.5 & 87.4 & 86.5 \\
\hline & Length Comparator reading (in) & 0.3068 & 0.3094 & 0.3099 & 0.3078 & 0.3091 & 0.3097 & 0.3103 & 0.3094 & 0.3105 & 0.3104 & 0.3102 & 0.3106 \\
\hline & Transverse Frequency (Hz) & 1880 & 1839 & 1827 & 1822 & 1821 & 1820 & 1811 & 1800 & 1798 & 1793 & 1789 & 1787 \\
\hline \multirow{6}{*}{2} & Width $(\mathrm{mm})$ & 101.17 & 101.36 & 101.45 & 101.74 & 101.33 & 101.21 & 101.2 & 101.18 & 101.43 & 101.32 & 101.29 & 101.28 \\
\hline & Depth (mm) & 77.49 & 77.34 & 77.45 & 77.15 & 77.12 & 77.65 & 77.64 & 76.59 & 76.64 & 77.1 & 76.66 & 76.78 \\
\hline & Weight $(\mathrm{Kg})$ & 6.86 & 6.819 & 6.888 & 6.883 & 6.91 & 6.881 & 6.858 & 6.852 & 6.795 & 6.781 & 6.725 & 6.731 \\
\hline & Time for pulse velocity ( $\mu \mathrm{s})$ & 84.5 & 85.2 & 85 & 85.5 & 85.4 & 85.8 & 86.9 & 89.7 & 89.7 & 87.7 & 89.8 & 89.2 \\
\hline & Length Comparator reading (in) & 0.3467 & 0.349 & 0.3493 & 0.3467 & 0.3484 & 0.3484 & 0.3492 & 0.3476 & 0.3493 & 0.3489 & 0.3489 & 0.3491 \\
\hline & Transverse Frequency (Hz) & 1904 & 1880 & 1866 & 1867 & 1850 & 1848 & 1845 & 1844 & 1833 & 1831 & 1827 & 1827 \\
\hline \multirow{6}{*}{3} & Width $(\mathrm{mm})$ & 101.71 & 101.84 & 101.66 & 101.99 & 101.79 & 101.81 & 101.82 & 101.84 & 101.97 & 101.85 & 102.04 & 102.17 \\
\hline & Depth (mm) & 77.5 & 77.52 & 77.01 & 78.33 & 77.8 & 77.51 & 77.6 & 77.16 & 77.03 & 77.01 & 76.82 & 77.36 \\
\hline & Weight $(\mathrm{Kg})$ & 6.944 & 6.897 & 6.969 & 6.968 & 6.996 & 6.968 & 6.956 & 6.901 & 6.843 & 6.829 & 6.773 & 6.783 \\
\hline & Time for pulse velocity (us) & 84 & 84.3 & 83.6 & 85 & 84.5 & 84.8 & 86.2 & 86.7 & 87.3 & 88 & 87.7 & 87.6 \\
\hline & Length Comparator reading (in) & 0.3743 & 0.3764 & 0.3772 & 0.3744 & 0.3762 & 0.3766 & 0.3773 & 0.3756 & 0.3777 & 0.3774 & 0.3771 & 0.3772 \\
\hline & Transverse Frequency (Hz) & 1903 & 1903 & 1900 & 1852 & 1873 & 1849 & 1812 & 1808 & 1800 & 1783 & 1783 & 1784 \\
\hline
\end{tabular}

Length of the specimens $=385 \mathrm{~mm}$ 
Specimen Designation: GC-VA-D-B-G23

\begin{tabular}{|c|c|c|c|c|c|c|c|c|c|c|c|c|c|}
\hline \multirow{2}{*}{ \# } & \multirow{2}{*}{ Measured Property } & \multicolumn{12}{|c|}{ Number of Freeze-and-Thaw Cycles } \\
\hline & & $\mathbf{0}$ & 28 & 53 & 77 & 103 & 123 & 148 & 169 & 195 & 226 & 265 & 300 \\
\hline \multirow{6}{*}{1} & Width (mm) & 102 & 102.1 & 101.93 & 102.14 & 101.93 & 102 & 102.07 & 102.06 & 102.4 & 102.33 & 102.17 & 102.19 \\
\hline & Depth (mm) & 76.43 & 76.81 & 76.8 & 76.4 & 76.72 & 77.15 & 77.15 & 77.04 & 76.71 & 77.19 & 76.68 & 76.24 \\
\hline & Weight (Kg) & 7.002 & 6.942 & 6.922 & 6.998 & 7.008 & 7.012 & 6.98 & 6.994 & 6.988 & 6.966 & 6.96 & 6.897 \\
\hline & Time for pulse velocity (us) & 83.9 & 85.2 & 85.6 & 85.6 & 85.9 & 85.9 & 85.9 & 85.8 & 86.7 & 86.8 & 87.4 & 87.5 \\
\hline & Length Comparator reading (in) & 0.3146 & 0.3153 & 0.3162 & 0.3157 & 0.3146 & 0.3145 & 0.3161 & 0.3161 & 0.3158 & 0.3167 & 0.3166 & 0.316 \\
\hline & Transverse Frequency $(\mathrm{Hz})$ & 1923 & 1847 & 1846 & 1845 & 1833 & 1829 & 1829 & 1822 & 1816 & 1814 & 1803 & 1799 \\
\hline \multirow{6}{*}{2} & Width (mm) & 101.38 & 101.2 & 101.31 & 101.29 & 101.49 & 101.48 & 101.98 & 101.27 & 101.36 & 101.34 & 101.35 & 101.43 \\
\hline & Depth $(\mathrm{mm})$ & 77.5 & 77.59 & 77.62 & 77.76 & 77.6 & 77.92 & 77.58 & 77.73 & 77.39 & 77.77 & 77.45 & 77.95 \\
\hline & Weight (Kg) & 6.958 & 6.907 & 6.884 & 6.959 & 6.973 & 6.977 & 6.95 & 6.948 & 6.944 & 6.92 & 6.908 & 6.85 \\
\hline & Time for pulse velocity $(\mu \mathrm{s})$ & 84.9 & 85.4 & 86.2 & 86.3 & 86 & 86.5 & 86.5 & 87.5 & 86.5 & 86.5 & 87.1 & 87.6 \\
\hline & Length Comparator reading (in) & 0.32 & 0.3207 & 0.3214 & 0.3207 & 0.3198 & 0.3197 & 0.321 & 0.3211 & 0.3211 & 0.3222 & 0.3214 & 0.3209 \\
\hline & Transverse Frequency $(\mathrm{Hz})$ & 1915 & 1840 & 1827 & 1821 & 1822 & 1820 & 1814 & 1810 & 1809 & 1809 & 1800 & 1790 \\
\hline \multirow{6}{*}{3} & Width (mm) & 101.4 & 101.53 & 101.77 & 101.66 & 101.54 & 101.71 & 101.95 & 101.59 & 101.95 & 101.348 & 101.97 & 101.72 \\
\hline & Depth (mm) & 78 & 77.89 & 77.71 & 77.79 & 77.84 & 77.89 & 77.83 & 77.83 & 77.67 & 77.57 & 77.49 & 77.46 \\
\hline & Weight (Kg) & 6.918 & 6.869 & 6.843 & 6.917 & 6.928 & 6.933 & 6.9 & 6.899 & 6.9 & 6.865 & 6.852 & 6.79 \\
\hline & Time for pulse velocity $(\mu \mathrm{s})$ & 84.3 & 85.6 & 85.8 & 86 & 86.3 & 86.6 & 86.1 & 86.7 & 86.7 & 87 & 87.2 & 87 \\
\hline & Length Comparator reading (in) & 0.3329 & 0.3338 & 0.3342 & 0.334 & 0.3331 & 0.3339 & 0.3347 & 0.3355 & 0.3343 & 0.3351 & 0.3349 & 0.3344 \\
\hline & Transverse Frequency $(\mathrm{Hz})$ & 1895 & 1869 & 1856 & 1840 & 1838 & 1834 & 1838 & 1823 & 1815 & 1814 & 1811 & 1798 \\
\hline
\end{tabular}

Length of the specimens $=385 \mathrm{~mm}$ 
Specimen Designation: GC-VA-D-C-E17

\begin{tabular}{|c|c|c|c|c|c|c|c|c|c|c|c|c|c|c|}
\hline \multirow{2}{*}{$\#$} & \multirow{2}{*}{ Measured Property } & \multicolumn{13}{|c|}{ Number of Freeze-and-Thaw Cycles } \\
\hline & & $\mathbf{0}$ & 31 & 59 & 76 & 102 & 122 & 150 & 172 & 193 & 229 & 259 & 276 & 300 \\
\hline \multirow{6}{*}{1} & Width (mm) & 101.1 & 101.2 & 101.1 & 101.2 & 101.2 & 101.3 & 101.4 & 101.4 & 101.5 & 101.7 & 101.5 & 101.2 & 101.4 \\
\hline & Depth (mm) & 77.02 & 77.11 & 77.24 & 77.1 & 77.02 & 76.84 & 77.86 & 76.9 & 77.49 & 76.97 & 77.28 & 77.08 & 76.65 \\
\hline & Weight (Kg) & 6.994 & 6.963 & 6.94 & 7.022 & 7.025 & 7.039 & 7.042 & 7.022 & 7.039 & 7.009 & 6.998 & 6.994 & 6.982 \\
\hline & Time for pulse velocity ( $\mu \mathrm{s})$ & 83.6 & 83 & 83.1 & 82.6 & 82.6 & 83.8 & 83 & 83 & 83.8 & 83.8 & 83.6 & 84.4 & 84.4 \\
\hline & Length Comparator reading & 0.262 & 0.263 & 0.265 & 0.264 & 0.263 & 0.265 & 0.263 & 0.264 & 0.265 & 0.265 & 0.265 & 0.265 & 0.265 \\
\hline & Transverse Frequency $(\mathrm{Hz})$ & 1904 & 1881 & 1904 & 1896 & 1876 & 1873 & 1873 & 1872 & 1861 & 1858 & 1846 & 1846 & 1846 \\
\hline \multirow{6}{*}{2} & Width (mm) & 101.5 & 101.4 & 101.4 & 101.6 & 102.1 & 101.5 & 101.7 & 101.8 & 101.6 & 101.6 & 101.7 & 101.7 & 101.7 \\
\hline & Depth (mm) & 76.58 & 76.85 & 76.74 & 76.78 & 77.31 & 76.72 & 76.97 & 76.99 & 76.78 & 76.89 & 76.85 & 76.96 & 76.65 \\
\hline & Weight $(\mathrm{Kg})$ & 6.958 & 6.93 & 6.908 & 6.987 & 6.99 & 7 & 7.01 & 6.988 & 7.007 & 6.97 & 6.96 & 6.954 & 6.944 \\
\hline & Time for pulse velocity ( $u$ s) & 82.2 & 82.6 & 83.2 & 82.5 & 82.6 & 82.1 & 83.2 & 83.5 & 83.4 & 84.5 & 84.8 & 85.5 & 84.6 \\
\hline & Length Comparator reading & 0.342 & 0.345 & 0.345 & 0.344 & 0.343 & 0.345 & 0.343 & 0.345 & 0.345 & 0.345 & 0.345 & 0.346 & 0.345 \\
\hline & Transverse Frequency $(\mathrm{Hz})$ & 1933 & 1900 & 1891 & 1893 & 1877 & 1880 & 1878 & 1874 & 1872 & 1850 & 1857 & 1846 & 1845 \\
\hline \multirow{6}{*}{3} & Width (mm) & 101.8 & 101.7 & 101.8 & 101.9 & 101.4 & 101.3 & 101.9 & 101.9 & 101.9 & 102.0 & 101.8 & 102.1 & 102.2 \\
\hline & Depth $(\mathrm{mm})$ & 76.59 & 76.64 & 76.69 & 76.39 & 76.88 & 76.81 & 76.88 & 76.92 & 77.04 & 76.67 & 76.38 & 76.41 & 76.48 \\
\hline & Weight $(\mathrm{Kg})$ & 7.032 & 7.002 & 6.984 & 7.065 & 7.063 & 7.079 & 7.095 & 7.074 & 7.093 & 7.061 & 7.051 & 7.045 & 7.033 \\
\hline & Time for pulse velocity $(\mu \mathrm{s})$ & 82.5 & 82.8 & 83.5 & 82.6 & 81.7 & 83 & 83.1 & 83.1 & 83.1 & 83.4 & 83.5 & 83.5 & 83.5 \\
\hline & Length Comparator reading & 0.313 & 0.314 & 0.316 & 0.316 & 0.314 & 0.316 & 0.314 & 0.316 & 0.316 & 0.316 & 0.316 & 0.317 & 0.317 \\
\hline & Transverse Frequency $(\mathrm{Hz})$ & 1908 & 1891 & 1905 & 1877 & 1879 & 1870 & 1871 & 1871 & 1871 & 1849 & 1849 & 1815 & 1826 \\
\hline
\end{tabular}

Length of the specimens $=385 \mathrm{~mm}$ 


\begin{tabular}{|c|c|c|c|c|c|c|c|c|c|c|c|c|c|}
\hline \multirow{2}{*}{ \# } & \multirow{2}{*}{ Measured Property } & \multicolumn{12}{|c|}{ Number of Freeze-and-Thaw Cycles } \\
\hline & & $\mathbf{0}$ & 33 & 65 & 95 & 121 & 148 & 172 & 198 & 218 & 243 & 268 & 300 \\
\hline \multirow{6}{*}{1} & Width (mm) & 101.71 & 101.53 & 101.45 & 101.49 & 101.62 & 101.51 & 101.66 & 101.43 & 101.52 & 101.52 & 101.55 & 101.69 \\
\hline & Depth (mm) & 75.23 & 75.29 & 75.49 & 75.44 & 75.48 & 75.5 & 75.44 & 75.88 & 75.6 & 75.2 & 74.5 & 74.5 \\
\hline & Weight $(\mathrm{Kg})$ & 6.973 & 6.964 & 6.95 & 6.911 & 6.886 & 6.963 & 6.954 & 6.797 & 6.962 & 6.95 & 6.889 & 6.87 \\
\hline & Time for pulse velocity ( $u$ s) & 82.3 & 82.8 & 82.9 & 82.5 & 83.3 & 82.9 & 83.3 & 83.6 & 83.1 & 83.2 & 82.6 & 83 \\
\hline & Length Comparator reading (in) & 0.2309 & 0.2305 & 0.2293 & 0.2272 & 0.2306 & 0.2283 & 0.2282 & 0.2301 & 0.2307 & 0.2301 & 0.2305 & 0.2318 \\
\hline & Transverse Frequency $(\mathrm{Hz})$ & 1894 & 1884 & 1875 & 1877 & 1877 & 1876 & 1853 & 1847 & 1846 & 1840 & 1838 & 1833 \\
\hline \multirow{6}{*}{2} & Width (mm) & 101.44 & 101.41 & 101.55 & 101.51 & 101.41 & 101.51 & 101.83 & 101.61 & 101.5 & 101.74 & 101.57 & 101.56 \\
\hline & Depth (mm) & 74.46 & 75.1 & 74.96 & 74.58 & 74.78 & 74.61 & 75.05 & 74.83 & 74.76 & 74.48 & 75.18 & 73.68 \\
\hline & Weight $(\mathrm{Kg})$ & 6.95 & 6.95 & 6.939 & 6.894 & 6.871 & 6.937 & 6.919 & 6.937 & 6.919 & 6.876 & 6.872 & 6.849 \\
\hline & Time for pulse velocity ( $\mu \mathrm{s})$ & 81.6 & 83.3 & 83.2 & 82.8 & 83.3 & 82.6 & 83.8 & 83.3 & 83.1 & 83.4 & 83.6 & 85.8 \\
\hline & Length Comparator reading (in) & 0.2838 & 0.284 & 0.2828 & 0.2809 & 0.2842 & 0.282 & 0.2816 & 0.2831 & 0.2846 & 0.2842 & 0.2839 & 0.285 \\
\hline & Transverse Frequency $(\mathrm{Hz})$ & 1895 & 1892 & 1884 & 1875 & 1868 & 1872 & 1866 & 1863 & 1865 & 1858 & 1848 & 1848 \\
\hline \multirow{6}{*}{3} & Width (mm) & 102.12 & 102.26 & 102.07 & 102.08 & 102.08 & 102.2 & 102.44 & 102.75 & 102.5 & 102.14 & 102.2 & 101.56 \\
\hline & Depth (mm) & 76.41 & 76.76 & 76.24 & 76.65 & 76.84 & 76.23 & 76.86 & 76.33 & 76.83 & 76.86 & 75.85 & 73.68 \\
\hline & Weight $(\mathrm{Kg})$ & 7.035 & 7.035 & 7.019 & 6.976 & 6.95 & 7.018 & 7.004 & 7.031 & 7.016 & 6.974 & 6.968 & 6.945 \\
\hline & Time for pulse velocity ( $\mu \mathrm{s})$ & 82.5 & 83 & 83.2 & 83 & 83.2 & 83.4 & 83.4 & 83.1 & 83.9 & 83.1 & 83.9 & 83.6 \\
\hline & Length Comparator reading (in) & 0.1813 & 0.1824 & 0.1815 & 0.1794 & 0.1821 & 0.1803 & 0.18 & 0.1814 & 0.1827 & 0.1823 & 0.1818 & 0.1828 \\
\hline & Transverse Frequency $(\mathrm{Hz})$ & 1896 & 1871 & 1866 & 1854 & 1863 & 1856 & 1853 & 1871 & 1854 & 1856 & 1851 & 1849 \\
\hline
\end{tabular}

Length of the specimens $=385 \mathrm{~mm}$ 


\begin{tabular}{|c|c|c|c|c|c|c|c|c|c|c|c|c|c|}
\hline \multirow{2}{*}{ \# } & \multirow{2}{*}{ Measured Property } & \multicolumn{12}{|c|}{ Number of Freeze-and-Thaw Cycles } \\
\hline & & $\mathbf{0}$ & 32 & 62 & 88 & 115 & 139 & 165 & 185 & 210 & 235 & 267 & 300 \\
\hline \multirow{6}{*}{1} & Width $(\mathrm{mm})$ & 101.5 & 101.59 & 101.49 & 101.72 & 101.49 & 101.54 & 102.21 & 101.56 & 101.67 & 101.63 & 101.64 & 101.55 \\
\hline & Depth (mm) & 78.22 & 78.47 & 78.2 & 78.46 & 78.44 & 78.25 & 78.61 & 78.31 & 78.57 & 78.49 & 78.35 & 77.95 \\
\hline & Weight $(\mathrm{Kg})$ & 7.174 & 7.158 & 7.115 & 7.088 & 7.163 & 7.165 & 7.187 & 7.193 & 7.161 & 7.153 & 7.137 & 7.115 \\
\hline & Time for pulse velocity ( $\boldsymbol{\mu} \mathrm{s})$ & 82.1 & 83.1 & 82.7 & 82.6 & 82.4 & 83.1 & 81.7 & 82.6 & 82.4 & 82.1 & 82.6 & 82.6 \\
\hline & Length Comparator reading (in) & 0.3284 & 0.3281 & 0.326 & 0.328 & 0.3267 & 0.3263 & 0.3278 & 0.3285 & 0.3281 & 0.3284 & 0.3293 & 0.3288 \\
\hline & Transverse Frequency $(\mathrm{Hz})$ & 1953 & 1920 & 1911 & 1910 & 1910 & 1908 & 1926 & 1904 & 1902 & 1892 & 1890 & 1883 \\
\hline \multirow{6}{*}{2} & Width (mm) & 101.58 & 101.52 & 101.36 & 101.54 & 101.44 & 101.7 & 102.34 & 102.03 & 101.89 & 101.77 & 101.66 & 101.47 \\
\hline & Depth (mm) & 76.92 & 76.78 & 76.83 & 77.12 & 76.97 & 76.64 & 77.23 & 77.12 & 77.07 & 76.9 & 76.69 & 76.71 \\
\hline & Weight $(\mathrm{Kg})$ & 7.094 & 7.083 & 7.029 & 7.012 & 7.089 & 7.087 & 7.109 & 7.113 & 7.084 & 7.082 & 7.084 & 7.063 \\
\hline & Time for pulse velocity ( $\mu \mathrm{s})$ & 82.7 & 84 & 83.6 & 83.7 & 83.4 & 84 & 83.2 & 83.5 & 83.5 & 82.7 & 82.8 & 82.8 \\
\hline & Length Comparator reading (in) & 0.3624 & 0.3621 & 0.3596 & 0.3624 & 0.3604 & 0.3603 & 0.362 & 0.3625 & 0.3626 & 0.3622 & 0.3635 & 0.3633 \\
\hline & Transverse Frequency $(\mathrm{Hz})$ & 1940 & 1890 & 1898 & 1888 & 1893 & 1880 & 1907 & 1902 & 1880 & 1883 & 1893 & 1892 \\
\hline \multirow{6}{*}{3} & Width (mm) & 101.15 & 101.1 & 101.37 & 101.26 & 101.08 & 100.98 & 102.46 & 101.25 & 101.41 & 101.3 & 101.28 & 101.28 \\
\hline & Depth $(\mathrm{mm})$ & 79.59 & 79.49 & 79.51 & 79.45 & 79.55 & 79.45 & 79.6 & 79.76 & 79.86 & 79.64 & 79.62 & 79.72 \\
\hline & Weight $(\mathrm{Kg})$ & 7.147 & 7.135 & 7.085 & 7.072 & 7.148 & 7.152 & 7.169 & 7.171 & 7.137 & 7.141 & 7.139 & 7.119 \\
\hline & Time for pulse velocity ( $\mu \mathrm{s})$ & 82.3 & 83.8 & 84 & 83.2 & 83.1 & 83.3 & 83.1 & 83.3 & 82.5 & 82.7 & 82.6 & 82.8 \\
\hline & Length Comparator reading (in) & 0.3757 & 0.3757 & 0.373 & 0.3754 & 0.3742 & 0.3739 & 0.3747 & 0.3759 & 0.3759 & 0.376 & 0.3766 & 0.3764 \\
\hline & Transverse Frequency $(\mathrm{Hz})$ & 1936 & 1907 & 1931 & 1936 & 1931 & 1932 & 1937 & 1933 & 1908 & 1905 & 1912 & 1911 \\
\hline
\end{tabular}

Length of the specimens $=385 \mathrm{~mm}$ 


\begin{tabular}{|c|c|c|c|c|c|c|c|c|c|c|c|c|c|}
\hline \multirow{2}{*}{$\#$} & \multirow{2}{*}{ Measured Property } & \multicolumn{12}{|c|}{ Number of Freeze-and-Thaw Cycles } \\
\hline & & $\mathbf{0}$ & 32 & 62 & 88 & 115 & 139 & 165 & 185 & 210 & 235 & 267 & 300 \\
\hline \multirow{6}{*}{1} & Width (mm) & 101.92 & 101.86 & 102.05 & 101.88 & 101.84 & 101.97 & 102.1 & 102.05 & 102.25 & 101.95 & 101.88 & 102.02 \\
\hline & Depth (mm) & 78.42 & 78.3 & 78.59 & 78.23 & 78.37 & 78.28 & 78.4 & 78.34 & 78.23 & 77.92 & 77.68 & 77.05 \\
\hline & Weight $(\mathrm{Kg})$ & 7.085 & 7.079 & 7.025 & 7.011 & 7.091 & 7.083 & 7.105 & 7.1 & 7.069 & 7.067 & 7.053 & 7.037 \\
\hline & Time for pulse velocity (us) & 82.6 & 82.7 & 82.9 & 82.1 & 82.5 & 82.7 & 82.4 & 82 & 82.7 & 82 & 82.2 & 83.6 \\
\hline & Length Comparator reading (in) & 0.2565 & 0.2562 & 0.2539 & 0.2568 & 0.2547 & 0.2544 & 0.2558 & 0.2569 & 0.2564 & 0.2564 & 0.2574 & 0.257 \\
\hline & Transverse Frequency (Hz) & 1951 & 1904 & 1938 & 1932 & 1931 & 1937 & 1938 & 1938 & 1930 & 1919 & 1919 & 1902 \\
\hline \multirow{6}{*}{$\mid 2$} & Width (mm) & 101.72 & 101.79 & 101.77 & 101.8 & 101.74 & 101.82 & 102.28 & 101.88 & 101.94 & 101.86 & 101.79 & 101.5 \\
\hline & Depth (mm) & 76.64 & 76.19 & 76.22 & 76.31 & 76.13 & 76.15 & 76.78 & 77.03 & 76.42 & 76.03 & 75.92 & 75.55 \\
\hline & Weight $(\mathrm{Kg})$ & 7.013 & 7.004 & 6.953 & 6.939 & 7.023 & 7.016 & 7.041 & 7.043 & 7.019 & 7.018 & 7.014 & 7 \\
\hline & Time for pulse velocity $(\mu \mathrm{s})$ & 83.3 & 83.6 & 83.6 & 83.8 & 83.6 & 83.7 & 83.6 & 83.6 & 83 & 83 & 82.7 & 83.8 \\
\hline & Length Comparator reading (in) & 0.1817 & 0.181 & 0.1789 & 0.1814 & 0.1795 & 0.1793 & 0.1807 & 0.1818 & 0.1814 & 0.1814 & 0.182 & 0.1818 \\
\hline & Transverse Frequency $(\mathrm{Hz})$ & 1946 & 1923 & 1933 & 1927 & 1928 & 1929 & 1930 & 1930 & 1918 & 1925 & 1927 & 1916 \\
\hline \multirow{6}{*}{3} & Width (mm) & 101.44 & 101.44 & 101.2 & 101.35 & 101.16 & 101.27 & 101.3 & 101.64 & 101.39 & 101.28 & 101.68 & 101.18 \\
\hline & Depth (mm) & 75.57 & 75.53 & 75.85 & 75.74 & 75.78 & 75.75 & 76.18 & 75.77 & 75.76 & 75.9 & 75.85 & 75.44 \\
\hline & Weight $(\mathrm{Kg})$ & 6.942 & 6.929 & 6.883 & 6.867 & 6.949 & 6.943 & 6.966 & 6.964 & 6.936 & 6.934 & 6.924 & 6.915 \\
\hline & Time for pulse velocity ( $u s)$ & 83.9 & 84.2 & 84.1 & 84.3 & & 84.3 & 83.8 & 83.6 & 83.8 & 83.1 & 84.2 & 84.2 \\
\hline & Length Comparator reading (in) & 0.2546 & 0.2539 & 0.2516 & 0.2541 & 0.2523 & 0.2521 & 0.2534 & 0.2542 & 0.2538 & 0.2538 & 0.2541 & 0.2545 \\
\hline & Transverse Frequency (Hz) & 1908 & 1883 & 1903 & 1896 & 1903 & 1902 & 1902 & 1902 & 1898 & 1888 & 1876 & 1876 \\
\hline
\end{tabular}

Length of the specimens $=385 \mathrm{~mm}$ 


\section{C.2.Chloride Penetration Test (RAW DATA)}


Specimen Designation: GC-MO-D-C-G41

\begin{tabular}{|c|c|c|c|c|c|c|c|c|c|c|}
\hline \multirow{2}{*}{ Repeat Sample } & \multirow{2}{*}{$\begin{array}{l}\text { \# of measurements } \\
\text { around the perimeter }\end{array}$} & \multicolumn{9}{|c|}{ Steps of Powdering ( $D=$ Depth) } \\
\hline & & Ref & D1 & $\overline{\mathrm{D} 2}$ & D3 & D4 & D5 & D6 & D7 & $\overline{\mathrm{D} 8}$ \\
\hline \multirow{11}{*}{1} & 1 & 16.54 & 17.31 & 18.56 & 20.62 & 22.48 & 25.46 & 28.48 & 31.51 & 35.43 \\
\hline & 2 & 16.22 & 17.46 & 18.51 & 20.89 & 22.63 & 25.62 & 28.67 & 31.78 & 35.68 \\
\hline & 3 & 15.88 & 17.51 & 18.44 & 20.4 & 22.49 & 25.67 & 28.54 & 31.67 & 35.59 \\
\hline & 4 & 15.7 & 17.17 & 18.4 & 20.35 & 22.12 & 25.27 & 28.24 & 31.25 & 35.38 \\
\hline & 5 & 16.28 & 16.98 & 18.32 & 20.28 & 22.24 & 25.31 & 28.19 & 31.21 & 35.11 \\
\hline & Average $(\mathrm{mm})$ & 16.12 & 17.29 & 18.45 & 20.51 & 22.39 & 25.47 & 28.42 & 31.48 & 35.44 \\
\hline & Exact depth, $\mathrm{x}_{0}(\mathrm{~mm})$ & & 1.16 & 2.32 & 4.38 & 6.27 & 9.34 & 12.30 & 15.36 & 19.31 \\
\hline & Depth to the middle, $\mathrm{x}(\mathrm{mm})$ & & 0.58 & 1.74 & 3.35 & 5.33 & 7.81 & 10.82 & 13.83 & 17.34 \\
\hline & Thickness, $\Delta \mathrm{x}(\mathrm{mm})$ & & 1.16 & 1.16 & 2.06 & 1.88 & 3.07 & 2.96 & 3.06 & 3.95 \\
\hline & Powder weight for test $(\mathrm{g})$ & & 6.84 & 6.22 & 10.02 & 10.11 & 10.01 & 10.03 & 10.05 & 10.02 \\
\hline & Chloride Concentration (\%) & & 1.237 & 0.872 & 0.609 & 0.415 & 0.300 & 0.193 & 0.120 & 0.051 \\
\hline \multirow{11}{*}{2} & 1 & 15.69 & 16.55 & 17.73 & 19.64 & 21.59 & 24.63 & 27.77 & 30.46 & 34.66 \\
\hline & 2 & 14.95 & 16.56 & 17.67 & 19.75 & 21.56 & 24.77 & 27.73 & 30.58 & 34.7 \\
\hline & 3 & 14.6 & 16.51 & 17.53 & 19.68 & 21.47 & 24.67 & 27.43 & 30.42 & 34.5 \\
\hline & 4 & 14.48 & 16.2 & 17.37 & 19.27 & 21.33 & 24.27 & 27.09 & 30.33 & 34.31 \\
\hline & 5 & 15.27 & 16.24 & 17.39 & 19.29 & 21.2 & 24.15 & 27.09 & 30.13 & 34.21 \\
\hline & Average (mm) & 15.00 & 16.41 & 17.54 & 19.53 & 21.43 & 24.50 & 27.42 & 30.38 & 34.48 \\
\hline & Exact depth, $\mathrm{x}_{0}(\mathrm{~mm})$ & & 1.41 & 2.54 & 4.53 & 6.43 & 9.50 & 12.42 & 15.39 & 19.48 \\
\hline & Depth to the middle, $x(\mathrm{~mm})$ & & 0.71 & 1.98 & 3.53 & 5.48 & 7.97 & 10.96 & 13.91 & 17.43 \\
\hline & Thicknesș, $\Delta \mathrm{x}(\mathrm{mm})$ & & 1.41 & 1.13 & 1.99 & 1.90 & 3.07 & 2.92 & 2.96 & 4.09 \\
\hline & Powder weight for test $(\mathrm{g})$ & & 5.67 & 6.81 & 10.06 & 10.03 & 10.1 & 10.06 & 10.02 & 10.07 \\
\hline & Chloride Concentration (\%) & & 1.294 & 0.933 & 0.689 & 0.508 & 0.399 & 0.278 & 0.190 & 0.088 \\
\hline
\end{tabular}


Specimen Designation: GC-MO-D-F-G41

\begin{tabular}{|c|c|c|c|c|c|c|c|c|c|c|}
\hline \multirow{2}{*}{ Repeat Sample } & \multirow{2}{*}{$\begin{array}{l}\text { \# of measurements } \\
\text { around the perimeter }\end{array}$} & \multicolumn{9}{|c|}{ Steps of Powdering } \\
\hline & & Ref & D1 & D2 & D3 & D4 & D5 & D6 & D7 & $\overline{\text { D8 }}$ \\
\hline \multirow{11}{*}{1} & 1 & 15.39 & 16.49 & 17.41 & 19.42 & 21.52 & 24.54 & 27.31 & 30.69 & 34.58 \\
\hline & 2 & 15.63 & 16.56 & 17.59 & 19.54 & 21.65 & 24.6 & 27.44 & 30.79 & 34.59 \\
\hline & 3 & 15.48 & 16.67 & 17.63 & 19.61 & 21.85 & 24.68 & 27.88 & 30.63 & 34.68 \\
\hline & 4 & 15 & 16.55 & 17.43 & 19.58 & 21.65 & 24.36 & 27.51 & 30.55 & 34.46 \\
\hline & 5 & 15.11 & 16.48 & 17.7 & 19.66 & 21.69 & 24.58 & 27.52 & 30.48 & 34.45 \\
\hline & \multirow{4}{*}{$\begin{array}{c}\text { Average }(\mathrm{mm}) \\
\text { Exact depth, } \mathrm{x}_{0}(\mathrm{~mm}) \\
\text { Depth to the middle, } \mathrm{x}(\mathrm{mm}) \\
\text { Thickness, } \Delta \mathrm{x}(\mathrm{mm})\end{array}$} & 15.32 & 16.55 & 17.55 & 19.56 & 21.67 & 24.55 & 27.53 & 30.63 & 34.55 \\
\hline & & & 1.23 & 2.23 & 4.24 & 6.35 & 9.23 & 12.21 & 15.31 & 19.23 \\
\hline & & & 0.61 & 1.73 & 3.24 & 5.30 & 7.79 & 10.72 & 13.76 & 17.27 \\
\hline & & & 1.23 & 1.00 & 2.01 & 2.11 & 2.88 & 2.98 & 3.10 & 3.92 \\
\hline & Powder weight for test $(\mathrm{g})$ & & 5.98 & 6.14 & 10.03 & 10.11 & 10.17 & 10.06 & 10.02 & 10.07 \\
\hline & Chloride Concentration (\%) & & 1.346 & 1.157 & 0.854 & 0.611 & 0.355 & 0.118 & 0.024 & 0.021 \\
\hline \multirow{11}{*}{2} & 1 & 15.45 & 16.45 & 17.58 & 19.46 & 21.64 & 24.47 & 27.38 & 30.73 & 34.45 \\
\hline & 2 & 15.35 & 16.7 & 17.81 & 19.74 & 21.54 & 24.7 & 27.7 & 30.89 & 34.7 \\
\hline & 3 & 15.31 & 16.63 & 17.87 & 19.59 & 21.71 & 24.58 & 27.61 & 30.85 & 34.76 \\
\hline & 4 & 15.44 & 16.18 & 17.48 & 19.22 & 21.33 & 24.27 & 27.45 & 30.35 & 34.39 \\
\hline & 5 & 15.05 & 16.23 & 17.31 & 19.21 & 21.38 & 24.25 & 27.43 & 30.35 & 34.36 \\
\hline & \multirow{4}{*}{$\begin{array}{c}\text { Average }(\mathrm{mm}) \\
\text { Exact depth, } \mathrm{x}_{0}(\mathrm{~mm}) \\
\text { Depth to the middle, } \mathrm{x}(\mathrm{mm}) \\
\text { Thickness, } \Delta \mathrm{x}(\mathrm{mm}) \\
\end{array}$} & 15.32 & 16.44 & 17.61 & 19.44 & 21.52 & 24.45 & 27.51 & 30.63 & 34.53 \\
\hline & & & 1.12 & 2.29 & 4.12 & 6.20 & 9.13 & 12.19 & 15.31 & 19.21 \\
\hline & & & 0.56 & 1.70 & 3.21 & 5.16 & 7.67 & 10.66 & 13.75 & 17.26 \\
\hline & & & 1.12 & 1.17 & 1.83 & 2.08 & 2.93 & 3.06 & 3.12 & 3.90 \\
\hline & Powder weight for test $(\mathrm{g})$ & & 4.36 & 6.02 & 10.03 & 10.04 & 10.08 & 10.04 & 10.06 & 10.06 \\
\hline & Chloride Concentration (\%) & & 1.353 & 1.292 & 0.935 & 0.632 & 0.37 & 0.15 & 0.04 & 0.02 \\
\hline
\end{tabular}


Specimen Designation: GC-MO-D-B-G41

\begin{tabular}{|c|c|c|c|c|c|c|c|c|c|c|}
\hline \multirow{2}{*}{ Repeat Sample } & \multirow{2}{*}{$\begin{array}{l}\# \text { of measurements } \\
\text { around the perimeter }\end{array}$} & \multicolumn{9}{|c|}{ Steps of Powdering } \\
\hline & & Ref & D1 & D2 & D3 & D4 & D5 & D6 & D7 & D8 \\
\hline \multirow{11}{*}{1} & 1 & 15.05 & 16.78 & 17.79 & 19.98 & 21.91 & 24.74 & 27.7 & 30.73 & 33.9 \\
\hline & 2 & 15 & 16.92 & 17.79 & 19.73 & 21.66 & 24.92 & 27.8 & 30.95 & 34.05 \\
\hline & 3 & 15.47 & 16.77 & 17.59 & 19.65 & 21.55 & 24.58 & 27.61 & 30.83 & 33.64 \\
\hline & 4 & 15.49 & 16.56 & 17.55 & 19.63 & 21.32 & 24.41 & 27.26 & 30.27 & 33.47 \\
\hline & 5 & 15.18 & 16.45 & 17.51 & 19.42 & 21.5 & 24.49 & 27.57 & 30.39 & 33.33 \\
\hline & Average $(\mathrm{mm})$ & 15.24 & 16.70 & 17.65 & 19.68 & 21.59 & 24.63 & 27.59 & 30.63 & 33.68 \\
\hline & Exact depth, $x_{0}(\mathrm{~mm})$ & & 1.46 & 2.41 & 4.44 & 6.35 & 9.39 & 12.35 & 15.40 & 18.44 \\
\hline & Depth to the middle, $x(\mathrm{~mm})$ & & 0.73 & 1.93 & 3.43 & 5.40 & 7.87 & 10.87 & 13.87 & 16.92 \\
\hline & Thickness, $\Delta \mathrm{x}(\mathrm{mm})$ & & 1.46 & 0.95 & 2.04 & 1.91 & 3.04 & 2.96 & 3.05 & 3.04 \\
\hline & Powder weight for test $(\mathrm{g})$ & & 8.03 & 6.02 & 10.02 & 10.03 & 10.06 & 10.03 & 10.08 & 10.02 \\
\hline & Chloride Concentration (\%) & & 1.436 & 1.052 & 0.714 & 0.449 & 0.252 & 0.081 & 0.025 & 0.015 \\
\hline \multirow{11}{*}{2} & 1 & 15.24 & 15.97 & 17.08 & 19.3 & 21.1 & 24 & 27.13 & 29.98 & 33.28 \\
\hline & 2 & 14.2 & 16.17 & 17.24 & 19.29 & 21.21 & 24.46 & 27.29 & 30.31 & 33.34 \\
\hline & 3 & 14.78 & 16.45 & 17.28 & 19.17 & 21.21 & 24.25 & 27.01 & 30.01 & 33.4 \\
\hline & 4 & 15.14 & 16 & 16.96 & 19.08 & 21.14 & 24.05 & 26.95 & 29.73 & 32.93 \\
\hline & 5 & 15.37 & 15.92 & 16.92 & 18.76 & 20.86 & 23.87 & 27.06 & 29.75 & 32.99 \\
\hline & Average $(\mathrm{mm})$ & 14.95 & 16.10 & 17.10 & 19.12 & 21.10 & 24.13 & 27.09 & 29.96 & 33.19 \\
\hline & Exact depth, $x_{0}(\mathrm{~mm})$ & & 1.16 & 2.15 & 4.17 & 6.16 & 9.18 & 12.14 & 15.01 & 18.24 \\
\hline & Depth to the middle, $x(\mathrm{~mm})$ & & 0.58 & 1.65 & 3.16 & 5.17 & 7.67 & 10.66 & 13.58 & 16.63 \\
\hline & Thickness, $\Delta \mathrm{x}(\mathrm{mm})$ & & 1.16 & 0.99 & 2.02 & 1.98 & 3.02 & 2.96 & 2.87 & 3.23 \\
\hline & Powder weight for test $(\mathrm{g})$ & & 6.14 & 6.58 & 10.01 & 10.08 & 10.07 & 10.02 & 10.07 & 10.08 \\
\hline & Chloride Concentration (\%) & & 1.437 & 1.071 & 0.698 & 0.430 & 0.232 & 0.077 & 0.025 & 0.023 \\
\hline
\end{tabular}


Specimen Designation: GC-MO-D-C-E26

\begin{tabular}{|c|c|c|c|c|c|c|c|c|c|c|}
\hline \multirow{2}{*}{ Repeat Sample } & \multirow{2}{*}{$\begin{array}{l}\text { \# of measurements } \\
\text { around the perimeter }\end{array}$} & \multicolumn{9}{|c|}{ Steps of Powdering } \\
\hline & & Ref & D1 & D2 & D3 & $\mathrm{D} 4$ & D5 & D6 & D7 & D8 \\
\hline \multirow{11}{*}{1} & 1 & 16.39 & 16.19 & 18 & 19.9 & 21.81 & 24.86 & 27.99 & 30.99 & 34.82 \\
\hline & 2 & 16.65 & 16.81 & 17.9 & 19.96 & 21.98 & 25.16 & 28.21 & 31.13 & 35.06 \\
\hline & 3 & 16.09 & 17.08 & 17.98 & 20.04 & 22.08 & 25.44 & 28.12 & 30.95 & 35.08 \\
\hline & 4 & 15.84 & 16.82 & 18.01 & 19.83 & 22.02 & 24.88 & 27.98 & 30.88 & 34.93 \\
\hline & 5 & 15.83 & 16.74 & 17.95 & 19.87 & 21.98 & 24.88 & 27.87 & 30.78 & 34.92 \\
\hline & Average $(\mathrm{mm})$ & 16.16 & 16.73 & 17.97 & 19.92 & 21.97 & 25.04 & 28.03 & 30.95 & 34.96 \\
\hline & Exact depth, $\mathrm{x}_{0}(\mathrm{~mm})$ & & 0.57 & 1.81 & 3.76 & 5.81 & 8.88 & 11.87 & 14.79 & 18.80 \\
\hline & Depth to the middle, $x(\mathrm{~mm})$ & & 0.28 & 1.19 & 2.78 & 4.79 & 7.35 & 10.38 & 13.33 & 16.79 \\
\hline & Thickness, $\Delta \mathrm{x}(\mathrm{mm})$ & & 0.57 & 1.24 & 1.95 & 2.05 & 3.07 & 2.99 & 2.91 & 4.02 \\
\hline & Powder weight for test $(\mathrm{g})$ & & 3.88 & 6.51 & 10.04 & 10.01 & 10.07 & 10.07 & 10 & 10.1 \\
\hline & Chloride Concentration (\%) & & 1.221 & 1.003 & 0.730 & 0.564 & 0.424 & 0.267 & 0.146 & 0.068 \\
\hline \multirow{11}{*}{2} & 1 & 16.34 & 17.3 & 18.27 & 20.31 & 22.29 & 25.25 & 28.14 & 31.37 & 34.94 \\
\hline & 2 & 15.77 & 17.11 & 18.35 & 20.35 & 22.34 & 25.44 & 28.48 & 31.49 & 35.02 \\
\hline & 3 & 15.85 & 17.09 & 18.15 & 20.13 & 22.08 & 24.94 & 28.2 & 31.23 & 35.28 \\
\hline & 4 & 15.48 & 16.73 & 17.68 & 19.78 & 21.77 & 24.66 & 27.76 & 30.69 & 34.8 \\
\hline & 5 & 15.38 & 16.66 & 17.7 & 19.68 & 21.65 & 24.7 & 27.67 & 30.79 & 34.56 \\
\hline & Average $(\mathrm{mm})$ & 15.76 & 16.98 & 18.03 & 20.05 & 22.03 & 25.00 & 28.05 & 31.11 & 34.92 \\
\hline & Exact depth, $\mathrm{x}_{0}(\mathrm{~mm})$ & & 1.21 & 2.27 & 4.29 & 6.26 & 9.23 & 12.29 & 15.35 & 19.16 \\
\hline & Depth to the middle, $x$ (mm) & & 0.61 & 1.74 & 3.28 & 5.27 & 7.75 & 10.76 & 13.82 & 17.25 \\
\hline & Thicknesș, $\Delta \mathrm{x}(\mathrm{mm})$ & & 1.21 & 1.05 & 2.02 & 1.98 & 2.97 & 3.05 & 3.06 & 3.81 \\
\hline & Powder weight for test $(\mathrm{g})$ & & 6.5 & 5.93 & 10.05 & 10 & 10 & 10.01 & 10.08 & 10.05 \\
\hline & Chloride Concentration (\%) & & 1.256 & 1.000 & 0.663 & 0.515 & 0.36 & 0.23 & 0.13 & 0.05 \\
\hline
\end{tabular}


Specimen Designation: GC-MO-D-F-E26

\begin{tabular}{|c|c|c|c|c|c|c|c|c|c|c|}
\hline \multirow{2}{*}{ Repeat Sample } & \multirow{2}{*}{$\begin{array}{l}\text { \# of measurements } \\
\text { around the perimeter }\end{array}$} & \multicolumn{9}{|c|}{ Steps of Powdering } \\
\hline & & Ref & $\overline{\overline{D D 1}}$ & $\overline{\mathrm{D} 2}$ & $\overline{\mathrm{D} 3}$ & $\overline{\mathrm{D} 4}$ & $\overline{\text { D5 }}$ & D6 & $\overline{\mathrm{D} 7}$ & D8 \\
\hline \multirow{11}{*}{1} & 1 & 16.43 & 16.86 & 17.97 & 20.17 & 21.8 & 25.08 & 28.06 & 30.78 & 35.06 \\
\hline & 2 & 15.55 & 16.78 & 17.93 & 20.08 & 22.02 & 25.07 & 27.96 & 31.06 & 35.05 \\
\hline & 3 & 15.75 & 16.95 & 17.86 & 19.99 & 21.92 & 24.74 & 27.72 & 30.85 & 34.69 \\
\hline & 4 & 15.22 & 16.53 & 17.58 & 19.59 & 21.53 & 24.62 & 27.56 & 30.72 & 34.49 \\
\hline & 5 & 16.05 & 16.51 & 17.57 & 19.54 & 21.63 & 24.55 & 27.61 & 30.47 & 34.51 \\
\hline & Average $(\mathrm{mm})$ & 15.80 & 16.73 & 17.78 & 19.87 & 21.78 & 24.81 & 27.78 & 30.78 & 34.76 \\
\hline & Exact depth, $x_{0}(\mathrm{~mm})$ & & 0.93 & 1.98 & 4.07 & 5.98 & 9.01 & 11.98 & 14.98 & 18.96 \\
\hline & Depth to the middle, $x(\mathrm{~mm})$ & & 0.46 & 1.45 & 3.03 & 5.03 & 7.50 & 10.50 & 13.48 & 16.97 \\
\hline & Thickness, $\Delta \mathrm{x}(\mathrm{mm})$ & & 0.93 & 1.06 & 2.09 & 1.91 & 3.03 & 2.97 & 2.99 & 3.98 \\
\hline & Powder weight for test (g) & & 5.17 & 5.71 & 10.01 & 10.02 & 10.01 & 10.03 & 10.02 & $\overline{10.03}$ \\
\hline & Chloride Concentration (\%) & & 1.481 & 1.126 & 0.733 & 0.473 & 0.231 & 0.075 & 0.021 & $\overline{0.015}$ \\
\hline \multirow{11}{*}{2} & $\overline{11}$ & 15.34 & 16.42 & 17.56 & 19.51 & 21.51 & 24.52 & 27.59 & 30.35 & 34.58 \\
\hline & 2 & 14.78 & 16.4 & 17.67 & 19.5 & 21.56 & 24.75 & 27.72 & 30.71 & 34.61 \\
\hline & 3 & 15.15 & 16.77 & 17.57 & 19.46 & 21.53 & 24.69 & 27.5 & 30.42 & 34.66 \\
\hline & 4 & 15.45 & 16.49 & 17.43 & 19.48 & 21.59 & 24.55 & 27.58 & 30.41 & 34.63 \\
\hline & 5 & 15.32 & 16.46 & 17.49 & 19.53 & 21.5 & 24.28 & 27.51 & 30.3 & 34.46 \\
\hline & Average $(\mathrm{mm})$ & 15.21 & 16.51 & 17.54 & 19.50 & 21.54 & 24.56 & 27.58 & 30.44 & 34.59 \\
\hline & Exact depth, $\mathrm{x}_{0}(\mathrm{~mm})$ & & 1.30 & 2.34 & 4.29 & 6.33 & 9.35 & 12.37 & 15.23 & 19.38 \\
\hline & Depth to the middle, $x(\mathrm{~mm})$ & & 0.65 & 1.82 & 3.31 & 5.31 & 7.84 & 10.86 & 13.80 & 17.31 \\
\hline & Thickness, $\Delta \mathrm{x}(\mathrm{mm})$ & & 1.30 & 1.04 & 1.95 & 2.04 & 3.02 & 3.02 & 2.86 & 4.15 \\
\hline & Powder weight for test $(\mathrm{g})$ & & 5.73 & 6.33 & 10.05 & 10.01 & 10.01 & 10.03 & 10.07 & 10.01 \\
\hline & . Chloride Concentration (\%) & & 1.438 & 1.188 & 0.770 & 0.461 & 0.242 & 0.056 & 0.020 & $\overline{0.015}$ \\
\hline
\end{tabular}


Specimen Designation: GC-MO-D-B-E26

\begin{tabular}{|c|c|c|c|c|c|c|c|c|c|c|}
\hline \multirow{2}{*}{ Repeat Sample } & \multirow{2}{*}{$\begin{array}{c}\text { \# of measurements } \\
\text { around the perimeter }\end{array}$} & \multicolumn{9}{|c|}{ Steps of Powdering } \\
\hline & & Ref & D1 & $\overline{\mathrm{D} 2}$ & D3 & D4 & D5 & D6 & D7 & $\overline{\mathrm{D} 8}$ \\
\hline \multirow{11}{*}{1} & 1 & 15.64 & 16.45 & 17.65 & 19.45 & 21.6 & 24.54 & 27.44 & 30.38 & 34.55 \\
\hline & 2 & 15.39 & 16.69 & 17.58 & 19.57 & 21.43 & 24.87 & 27.8 & 30.34 & 34.84 \\
\hline & 3 & 15.37 & 16.71 & 17.73 & 19.43 & 21.49 & 24.78 & 27.83 & 30.81 & 34.75 \\
\hline & 4 & 15.63 & 16.68 & 17.57 & 19.42 & 20.51 & 24.53 & 27.65 & 30.49 & 34.61 \\
\hline & 5 & 15.43 & 16.73 & 17.59 & 19.7 & 21.59 & 24.53 & 27.79 & 30.72 & 34.56 \\
\hline & Average & 15.49 & 16.65 & 17.62 & 19.51 & 21.32 & 24.65 & 27.70 & 30.55 & 34.66 \\
\hline & $\mathrm{X}_{0}=$ & & 1.16 & 2.13 & 4.02 & 5.83 & 9.16 & 12.21 & 15.06 & 19.17 \\
\hline & $X=$ & & 0.58 & 1.65 & 3.08 & 4.93 & 7.50 & 10.68 & 13.63 & 17.11 \\
\hline & $\Delta \mathrm{X}=$ & & 1.16 & 0.97 & 1.89 & 1.81 & 3.33 & 3.05 & 2.85 & 4.11 \\
\hline & Powder weight for test $(\mathrm{g})$ & & 5.46 & 5.52 & 10.03 & 10.03 & 10.03 & 10.07 & 10.01 & 10.07 \\
\hline & Chloride Concentration (\%) & & 1.622 & 1.185 & 0.739 & 0.437 & 0.240 & 0.104 & 0.022 & 0.017 \\
\hline \multirow{11}{*}{2} & 1 & 15.66 & 16.46 & 17.49 & $\mid 19.47$ & 21.65 & 24.62 & 27.67 & 30.69 & 34.45 \\
\hline & 2 & 14.98 & 16.72 & 17.82 & 19.98 & 22.06 & 24.88 & 28.12 & 30.77 & 34.47 \\
\hline & 3 & 15.56 & 16.79 & 17.53 & 19.6 & 21.6 & 24.66 & 27.68 & 30.44 & 34.78 \\
\hline & 4 & 15.51 & 16.43 & 17.41 & $\mid 19.38$ & 21.52 & 24.38 & 27.34 & 30.46 & 34.33 \\
\hline & 5 & 15.76 & 16.53 & 17.36 & 19.42 & 21.42 & 24.19 & 27.33 & 30.49 & 34.45 \\
\hline & Average $(\mathrm{mm})$ & 15.49 & 16.59 & 17.52 & 19.57 & 21.65 & 24.55 & 27.63 & 30.57 & 34.50 \\
\hline & Exact depth, $x_{0}(\mathrm{~mm})$ & & 1.09 & 2.03 & 4.08 & 6.16 & 9.05 & 12.13 & 15.08 & 19.00 \\
\hline & Depth to the middle, $\mathrm{x}(\mathrm{mm})$ & & 0.55 & 1.56 & 3.05 & 5.12 & 7.60 & 10.59 & 13.61 & 17.04 \\
\hline & Thickness, $\Delta \mathrm{x}(\mathrm{mm})$ & & 1.09 & 0.94 & 2.05 & 2.08 & 2.90 & 3.08 & 2.94 & 3.93 \\
\hline & Powder weight for test $(\mathrm{g})$ & & 5.43 & 5.31 & 10.03 & 10.02 & 10.06 & 10.11 & 10.02 & 10.01 \\
\hline & . Chloride Concentration (\%) & & 1.488 & 1.266 & 0.773 & 0.433 & 0.21 & 0.06 & 0.02 & 0.02 \\
\hline
\end{tabular}


Specimen Designation: GC-MO-D-C-NO

\begin{tabular}{|c|c|c|c|c|c|c|c|c|c|c|}
\hline \multirow{2}{*}{ Repeat Sample } & \multirow{2}{*}{$\begin{array}{l}\text { \# of measurements } \\
\text { around the perimeter }\end{array}$} & \multicolumn{9}{|c|}{ Steps of Powdering } \\
\hline & & Ref & $\overline{\mathrm{D} 1}$ & $\overline{\mathrm{D} 2}$ & $\overline{\mathrm{D} 3}$ & D4 & D5 & D6 & $\overline{\mathrm{D} 7}$ & $\overline{\mathrm{D} 8}$ \\
\hline \multirow{11}{*}{1} & 1 & 15.79 & 16.85 & 18.17 & 20.04 & 22.25 & 24.98 & 28.05 & 31.09 & 35.04 \\
\hline & 2 & 15.22 & 17.18 & 17.92 & 20.17 & 22.25 & 25.26 & 28.19 & 31.15 & 35.14 \\
\hline & 3 & 15.98 & 17.26 & 18.08 & 20.16 & 22.22 & 25.54 & 28.32 & 31.5 & 35.26 \\
\hline & 4 & 15.75 & 17.02 & 17.97 & 20.07 & 21.84 & 25.08 & 27.97 & 31.12 & 34.99 \\
\hline & 5 & 16.46 & 17.1 & 18.23 & 20.08 & 22.01 & 25.02 & 27.8 & 31.13 & 34.95 \\
\hline & \multirow{4}{*}{$\begin{array}{c}\text { Average }(\mathrm{mm}) \\
\text { Exact depth, } \mathrm{x}_{0}(\mathrm{~mm}) \\
\text { Depth to the middle, } \mathrm{x}(\mathrm{mm}) \\
\text { Thickness, } \Delta \mathrm{x}(\mathrm{mm})\end{array}$} & 15.84 & 17.08 & 18.07 & 20.10 & 22.11 & 25.18 & 28.07 & 31.20 & 35.08 \\
\hline & & & 1.24 & 2.23 & 4.26 & 6.27 & 9.34 & 12.23 & 15.36 & 19.24 \\
\hline & & & 0.62 & 1.74 & 3.25 & 5.27 & 7.81 & 10.78 & 13.79 & 17.30 \\
\hline & & & 1.24 & 0.99 & 2.03 & 2.01 & 3.06 & 2.89 & 3.13 & 3.88 \\
\hline & Powder weight for test $(\mathrm{g})$ & & 7.82 & 5.41 & 10.05 & 10.02 & 10.01 & 10.00 & 10.01 & 10.03 \\
\hline & Chloride Concentration (\%) & & 0.991 & 0.790 & 0.613 & 0.448 & 0.341 & 0.201 & 0.104 & 0.026 \\
\hline \multirow{11}{*}{2} & 1 & 15.35 & 16.22 & 17.22 & 19.01 & 21.11 & 24.18 & 26.99 & 30.32 & 34.43 \\
\hline & 2 & 14.61 & 16.37 & 17.32 & 19.3 & 20.98 & 24.31 & 27.23 & 30.56 & 34.66 \\
\hline & 3 & 15.31 & 16.55 & 17.42 & 19.52 & 21.28 & 24.23 & 27.71 & 30.59 & 34.53 \\
\hline & 4 & 15.41 & 16.39 & 17.24 & 19.58 & 21.48 & 24.29 & 27.68 & 30.36 & 34.44 \\
\hline & 5 & 16.5 & 16.32 & 17.43 & 19.43 & 21.11 & 24.27 & 27.18 & 30.19 & 34.27 \\
\hline & \multirow{4}{*}{$\begin{array}{c}\text { Average }(\mathrm{mm}) \\
\text { Exact depth, } \mathrm{x}_{0}(\mathrm{~mm}) \\
\text { Depth to the middle, } \mathrm{x}(\mathrm{mm}) \\
\text { Thickness, } \Delta \mathrm{x}(\mathrm{mm})\end{array}$} & 15.44 & 16.37 & 17.33 & 19.37 & 21.19 & 24.26 & 27.36 & 30.40 & 34.47 \\
\hline & & & 0.93 & 1.89 & 3.93 & 5.76 & 8.82 & 11.92 & 14.97 & 19.03 \\
\hline & & & 0.47 & 1.41 & 2.91 & 4.84 & 7.29 & 10.37 & 13.45 & 17.00 \\
\hline & & & 0.93 & 0.96 & 2.04 & 1.82 & 3.06 & 3.10 & 3.05 & 4.06 \\
\hline & Powder weight for test $(\mathrm{g})$ & & 5.09 & 5.91 & 10.01 & 10.05 & 10.05 & 10.02 & 10.04 & 10.01 \\
\hline & Chloride Concentration $(\%)$ & & 0.930 & $\overline{0.892}$ & 0.690 & 0.530 & 0.332 & 0.188 & 0.081 & 0.018 \\
\hline
\end{tabular}


Specimen Designation: GC-VA-D-C-G23

\begin{tabular}{|c|c|c|c|c|c|c|c|c|c|c|}
\hline \multirow{2}{*}{ Repeat Sample } & \multirow{2}{*}{$\begin{array}{l}\text { \# of measurements } \\
\text { around the perimeter }\end{array}$} & \multicolumn{9}{|c|}{ Steps of Powdering } \\
\hline & & Ref & $\overline{\mathrm{D} 1}$ & $\mathrm{D} 2$ & $\overline{\mathrm{D} 3}$ & $\overline{\mathrm{D} 4}$ & $\overline{\mathrm{D} 5}$ & $\overline{\mathrm{D} 6}$ & $\overline{\mathrm{D} 7}$ & $\overline{\mathrm{D} 8}$ \\
\hline \multirow{11}{*}{1} & 1 & 15.77 & 17.11 & 18.02 & 20.3 & 22.27 & 25.33 & 28.37 & 31.34 & $\overline{35.34}$ \\
\hline & 2 & 15.97 & 17.33 & 18.3 & 20.55 & 22.42 & 25.73 & 28.42 & 31.64 & 35.55 \\
\hline & 3 & 15.81 & 17.18 & 18.31 & 20.09 & 22.14 & 25.25 & 28.54 & 31.22 & 35.31 \\
\hline & 4 & 15.63 & 17.13 & 18.26 & 20.14 & 21.99 & 25.14 & 28.3 & 31.13 & 35.1 \\
\hline & 5 & 15.55 & 17.38 & 18.22 & 20.36 & 22.05 & 25.05 & 28.25 & 31.2 & 34.99 \\
\hline & Average $(\mathrm{mm})$ & 15.75 & 17.23 & 18.22 & 20.29 & 22.17 & 25.30 & 28.38 & 31.31 & 35.26 \\
\hline & Exact depth, $x_{0}(\mathrm{~mm})$ & & 1.48 & 2.48 & 4.54 & 6.43 & 9.55 & 12.63 & 15.56 & 19.51 \\
\hline & Depth to the middle, $x(\mathrm{~mm})$ & & 0.74 & 1.98 & 3.51 & 5.49 & 7.99 & 11.09 & 14.10 & 17.54 \\
\hline & Thickness, $\Delta \mathrm{x}(\mathrm{mm})$ & & 1.48 & 1.00 & 2.07 & 1.89 & 3.13 & 3.08 & 2.93 & 3.95 \\
\hline & Powder weight for test $(\mathrm{g})$ & & 5.09 & 5 & 10.09 & 10.01 & 10.16 & 10.09 & 10.05 & 10.14 \\
\hline & Chloride Concentration (\%) & & 1.101 & 0.859 & 0.635 & 0.430 & 0.351 & 0.214 & 0.091 & 0.029 \\
\hline \multirow{11}{*}{2} & $\overline{1}$ & 15.29 & 16.57 & 177.48 & 19.39 & 21.64 & 24.72 & 27.27 & 30.58 & 34.34 \\
\hline & 2 & 15.28 & 16.97 & 17.82 & 20.03 & 21.92 & 25.06 & 27.95 & 30.85 & 34.96 \\
\hline & 3 & 15.55 & 16.79 & 17.57 & 19.81 & 21.75 & 24.72 & 27.83 & 31.06 & 34.43 \\
\hline & 4 & 15.56 & 16.37 & 17.46 & 19.29 & 21.41 & 24.26 & 27.38 & 30.45 & 34.32 \\
\hline & 5 & 15.55 & 16.51 & 17.26 & 19.28 & 21.32 & 24.6 & 27.47 & 30.44 & 34.39 \\
\hline & Average $(\mathrm{mm})$ & 15.45 & 16.64 & 17.52 & 19.56 & 21.61 & 24.67 & 27.58 & 30.68 & 34.49 \\
\hline & Exact depth, $x_{0}(\mathrm{~mm})$ & & 1.20 & 2.07 & 4.11 & 6.16 & 9.23 & 12.13 & 15.23 & 19.04 \\
\hline & Depth to the middle, $\mathrm{x}(\mathrm{mm})$ & & 0.60 & 1.63 & 3.09 & 5.14 & 7.69 & 10.68 & 13.68 & 17.14 \\
\hline & Thickness, $\Delta \mathrm{x}(\mathrm{mm})$ & & 1.20 & 0.88 & 2.04 & 2.05 & 3.06 & 2.91 & 3.10 & 3.81 \\
\hline & Powder weight for test $(\mathrm{g})$ & & 5.1 & 4.4 & 10.02 & 10.07 & 10.04 & 10.02 & 10.01 & 10.05 \\
\hline & Chloride Concentration (\%) & & 1.144 & 0.829 & 0.590 & 0.391 & 0.272 & 0.203 & 0.105 & 0.030 \\
\hline
\end{tabular}


Specimen Designation: GC-VA-D-F-G23

\begin{tabular}{|c|c|c|c|c|c|c|c|c|c|c|}
\hline \multirow{2}{*}{ Repeat Sample } & \multirow{2}{*}{$\begin{array}{l}\# \text { of measurements } \\
\text { around the perimeter }\end{array}$} & \multicolumn{9}{|c|}{ Steps of Powdering } \\
\hline & & Ref & D1 & D2 & D3 & D4 & D5 & D6 & D7 & D8 \\
\hline \multirow{11}{*}{1} & 1 & 15.92 & 16.38 & 17.31 & 19.24 & 21.49 & 24.25 & 27.28 & 30.27 & 33.88 \\
\hline & 2 & 15.44 & 16.26 & 17.52 & 19.36 & 21.39 & 24.4 & 27.52 & 30.39 & 33.87 \\
\hline & 3 & 15.42 & 16.26 & 17.18 & 19.25 & 21.25 & 24.13 & 27.31 & 30.13 & 33.53 \\
\hline & 4 & 14.48 & 16.37 & 17.26 & 19.17 & 21.26 & 24.11 & 27.38 & 30.28 & 33.71 \\
\hline & 5 & 15.45 & 16.23 & 17.58 & 19.3 & 21.29 & 24.07 & 27.29 & 30.07 & 33.81 \\
\hline & Average $(\mathrm{mm})$ & & 0.96 & 2.03 & 3.92 & 5.99 & 8.85 & 12.01 & 14.89 & 18.42 \\
\hline & Exact depth, $\mathrm{x}_{0}(\mathrm{~mm})$ & & 0.48 & 1.49 & 2.98 & 4.96 & 7.42 & 10.43 & 13.45 & 16.65 \\
\hline & Depth to the middle, $x(\mathrm{~mm})$ & & 0.96 & 1.07 & 1.89 & 2.07 & 2.86 & 3.16 & 2.87 & 3.53 \\
\hline & Thickness, $\Delta \mathrm{x}(\mathrm{mm})$ & & 1.228 & 1.125 & 0.803 & 0.449 & 0.261 & 0.088 & 0.015 & 0.014 \\
\hline & Powder weight for test $(\mathrm{g})$ & & 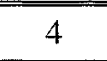 & 5.25 & 10.08 & 10.11 & 10.02 & 10.03 & 10.05 & 10.06 \\
\hline & . Chloride Concentration (\%) & & 0.96 & 2.03 & 3.92 & 5.99 & 8.85 & 12.01 & 14.89 & $\overline{18.42}$ \\
\hline \multirow{11}{*}{2} & 1 & 15.07 & 17.05 & 18.15 & 20.35 & 22.09 & 24.58 & 27.09 & 29.88 & 33.9 \\
\hline & 2 & 15.36 & 17.14 & 18.14 & 20.2 & 22.18 & 24.76 & 27.35 & 30.19 & 34 \\
\hline & 3 & 15.26 & 16.94 & 17.96 & 20.03 & 21.91 & 24.45 & 27.05 & 29.84 & 39.84 \\
\hline & 4 & 16.08 & 16.95 & 17.96 & 19.9 & 22 & 24.42 & 27.04 & 29.88 & 33.65 \\
\hline & 5 & 15.87 & 16.91 & 18.02 & 19.82 & 21.87 & 24.31 & 26.97 & 29.8 & 33.75 \\
\hline & Average $(\mathrm{mm})$ & 15.53 & 17.00 & 18.05 & 20.06 & 22.01 & 24.50 & 27.10 & 29.92 & 35.03 \\
\hline & Exact depth, $\mathrm{x}_{0}(\mathrm{~mm})$ & & 1.47 & 2.52 & 4.53 & 6.48 & 8.98 & 11.57 & 14.39 & 19.50 \\
\hline & Depth to the middle, $x(\mathrm{~mm})$ & & 0.73 & 1.99 & 3.53 & 5.51 & 7.73 & 10.27 & 12.98 & 16.95 \\
\hline & Thickness, $\Delta \mathrm{x}(\mathrm{mm})$ & & 1.47 & 1.05 & 2.01 & 1.95 & 2.49 & 2.60 & 2.82 & 5.11 \\
\hline & Powder weight for test $(\mathrm{g})$ & & 5.17 & 5.11 & 10.01 & 10.05 & 10.02 & 10.1 & 10.06 & 10.05 \\
\hline & Chloride Concentration (\%) & & 1.394 & 0.976 & 0.663 & 0.422 & 0.250 & 0.097 & 0.025 & 0.013 \\
\hline
\end{tabular}


Specimen Designation: GC-VA-D-B-G23

\begin{tabular}{|c|c|c|c|c|c|c|c|c|c|c|}
\hline \multirow{2}{*}{ Repeat Sample } & \multirow{2}{*}{$\begin{array}{l}\text { \# of measurements } \\
\text { around the perimeter }\end{array}$} & \multicolumn{9}{|c|}{ Steps of Powdering } \\
\hline & & Ref & D1 & $\overline{\mathrm{D} 2}$ & D3 & D4 & $\overline{\mathrm{D} 5}$ & D6 & D7 & $\overline{\text { D8 }}$ \\
\hline \multirow{11}{*}{1} & 1 & 15.22 & 16.52 & 17.78 & 19.67 & 21.7 & 24.79 & 27.88 & 30.78 & 34.56 \\
\hline & 2 & 14.55 & 16.53 & 17.93 & 19.83 & 21.84 & 25.35 & 27.79 & 31.05 & 34.8 \\
\hline & 3 & 15.11 & 16.68 & 17.78 & 19.72 & 21.57 & 24.63 & 27.84 & 30.65 & 34.69 \\
\hline & 4 & 15.49 & 16.59 & 17.57 & 19.4 & 21.46 & 24.64 & 27.81 & 30.33 & 34.73 \\
\hline & 5 & 15.8 & 16.46 & 17.48 & 19.34 & 21.4 & 24.41 & 27.44 & 30.55 & 34.85 \\
\hline & Average $(\mathrm{mm})$ & 15.23 & 16.56 & 17.71 & 19.59 & 21.59 & 24.76 & 27.75 & 30.67 & 34.73 \\
\hline & Exact depth, $\mathrm{x}_{0}(\mathrm{~mm})$ & & 1.32 & 2.47 & 4.36 & 6.36 & 9.53 & 12.52 & 15.44 & 19.49 \\
\hline & Depth to the middle, $x(\mathrm{~mm})$ & & 0.66 & 1.90 & 3.42 & 5.36 & 7.95 & 11.02 & 13.98 & 17.47 \\
\hline & Thickness, $\Delta \mathrm{x}(\mathrm{mm})$ & & 1.32 & 1.15 & 1.88 & 2.00 & 3.17 & 2.99 & 2.92 & 4.05 \\
\hline & Powder weight for test $(\mathrm{g})$ & & 6.2 & 5.82 & 10.03 & 10.07 & 10.03 & 10.03 & & 10.06 \\
\hline & Chloride Concentration (\%) & & 1.144 & 0.909 & 0.607 & 0.327 & 0.097 & 0.041 & 0.018 & 0.011 \\
\hline \multirow{11}{*}{2} & 1 & 15.36 & 16.66 & 17.42 & 19.68 & 21.48 & 24.64 & 27.54 & 30.6 & 34.58 \\
\hline & 2 & 15.37 & 16.59 & 17.84 & 19.75 & 21.67 & 24.7 & 28 & 30.74 & 34.62 \\
\hline & 3 & 16.03 & 16.06 & 17.79 & 19.58 & 21.39 & 24.64 & 27.63 & 30.68 & 34.49 \\
\hline & 4 & 15.29 & 16.44 & 17.36 & 19.22 & 21.4 & 24.22 & 27.4 & 30.2 & 34.26 \\
\hline & 5 & 15.67 & 16.48 & 17.32 & 19.36 & 21.38 & 24.28 & 27.42 & 30.24 & 34.27 \\
\hline & Average (mm) & 15.54 & 16.45 & 17.55 & 19.52 & 21.46 & 24.50 & 27.60 & 30.49 & 34.44 \\
\hline & Exact depth, $x_{0}(\mathrm{~mm})$ & & 0.90 & 2.00 & 3.97 & 5.92 & 8.95 & 12.05 & 14.95 & 18.90 \\
\hline & Depth to the middle, $x(\mathrm{~mm})$ & & 0.45 & 1.45 & 2.99 & 4.95 & 7.44 & 10.50 & 13.50 & 16.92 \\
\hline & Thicknesș, $\Delta \mathrm{x}(\mathrm{mm})$ & & 0.90 & 1.10 & 1.97 & 1.95 & 3.03 & 3.10 & 2.89 & 3.95 \\
\hline & Powder weight for test $(\mathrm{g})$ & & 4.93 & 5.36 & 10.01 & 10 & 10 & 10.03 & 10.07 & 10.05 \\
\hline & Chloride Concentration (\%) & & 1.340 & 1.081 & 0.664 & 0.388 & 0.183 & 0.053 & 0.015 & 0.014 \\
\hline
\end{tabular}


Specimen Designation: GC-VA-D-C-E17

\begin{tabular}{|c|c|c|c|c|c|c|c|c|c|c|}
\hline \multirow{2}{*}{ Repeat Sample } & \multirow{2}{*}{$\begin{array}{l}\text { \# of measurements } \\
\text { around the perimeter }\end{array}$} & \multicolumn{9}{|c|}{ Steps of Powdering } \\
\hline & & Ref & D1 & D2 & D3 & $\mathrm{D} 4$ & D5 & D6 & D7 & D8 \\
\hline \multirow{11}{*}{1} & 1 & 15.13 & 16.24 & 17.09 & 19.02 & 21.31 & 24.11 & 27.88 & 30.18 & 33.52 \\
\hline & 2 & 15.33 & 16.69 & 17.51 & 19.56 & 21.47 & 24.52 & 27.62 & 30.64 & 34.05 \\
\hline & 3 & 15.26 & 16.35 & 17.34 & 19.63 & 21.36 & 24.48 & 27.45 & 30.46 & 33.98 \\
\hline & 4 & 15.47 & 16.35 & 17.25 & 19.25 & 21.19 & 24.17 & 27.32 & 30.23 & 33.66 \\
\hline & 5 & 15.46 & 16.22 & 17.16 & 19.13 & 21.06 & 23.9 & 27.14 & 30 & 33.56 \\
\hline & Average (mm) & 15.33 & 16.37 & 17.27 & 19.32 & 21.28 & 24.24 & 27.48 & 30.30 & 33.75 \\
\hline & Exact depth, $x_{0}(\mathrm{~mm})$ & & 1.04 & 1.94 & 3.99 & 5.95 & 8.91 & 12.15 & 14.97 & 18.42 \\
\hline & Depth to the middle, $x(\mathrm{~mm})$ & & 0.52 & 1.49 & 2.96 & 4.97 & 7.43 & 10.53 & 13.56 & 16.70 \\
\hline & Thickness, $\Delta \mathrm{x}(\mathrm{mm})$ & & 1.04 & 0.90 & 2.05 & 1.96 & 2.96 & 3.25 & 2.82 & 3.45 \\
\hline & Powder weight for test (g) & & 2.46 & 5.58 & 10.04 & 10.01 & 10.01 & 10.05 & 10.08 & $\overline{10.05}$ \\
\hline & Chloride Concentration (\%) & & 1.659 & & 1.097 & 0.76 & 0.482 & 0.279 & 0.143 & 0.053 \\
\hline \multirow{11}{*}{2} & 1 & 16.01 & 17.23 & 17.89 & 19.9 & 22.08 & 24.74 & 27.93 & 31.14 & 34.28 \\
\hline & 2 & 16.43 & 17.41 & 18.15 & 19.91 & 22.27 & 25.27 & 28.03 & 31.26 & 34.22 \\
\hline & 3 & 16 & 17.03 & 18.13 & 20.19 & 22.29 & 25.16 & 28.21 & 31.09 & 34.04 \\
\hline & 4 & 15.8 & 16.38 & 17.56 & 19.85 & 22.17 & 24.95 & 28.1 & 30.68 & 33.91 \\
\hline & 5 & 16.66 & 17.03 & 17.53 & 19.56 & 21.97 & 24.44 & 28.06 & 30.3 & 33.25 \\
\hline & Average $(\mathrm{mm})$ & 16.18 & 17.02 & 17.85 & 19.88 & 22.16 & 24.91 & 28.07 & 30.89 & 33.94 \\
\hline & Exact depth, $x_{0}(\mathrm{~mm})$ & & 0.84 & 1.67 & 3.70 & 5.98 & 8.73 & 11.89 & 14.71 & 17.76 \\
\hline & Depth to the middle, $x(\mathrm{~mm})$ & & 0.42 & 1.25 & 2.69 & 4.84 & 7.35 & 10.31 & 13.30 & 16.24 \\
\hline & Thickness, $\Delta \mathrm{x}(\mathrm{mm})$ & & 0.84 & 0.84 & 2.03 & 2.27 & 2.76 & 3.15 & 2.83 & 3.05 \\
\hline & Powder weight for test (g) & & 4.42 & 5.07 & 10.03 & 10.16 & 10.13 & 10.1 & 10.13 & 10.09 \\
\hline & Chloride Concentration (\%) & & 1.703 & 1.439 & 1.028 & 0.668 & 0.446 & 0.290 & 0.179 & 0.087 \\
\hline
\end{tabular}


Specimen Designation: GC-VA-D-F-E17

\begin{tabular}{|c|c|c|c|c|c|c|c|c|c|c|}
\hline \multirow{2}{*}{ Repeat Sample } & \multirow{2}{*}{$\begin{array}{l}\text { \# of measurements } \\
\text { around the perimeter }\end{array}$} & \multicolumn{9}{|c|}{ Steps of Powdering } \\
\hline & & Ref & $\overline{\mathrm{D} 1}$ & $\overline{\mathrm{D} 2}$ & $\overline{\mathrm{D} 3}$ & $\overline{\mathrm{D} 4}$ & $\overline{\mathrm{D} 5}$ & $\overline{\mathrm{D} 6}$ & $\overline{\mathrm{D} 7}$ & $\overline{\mathrm{D} 8}$ \\
\hline \multirow{11}{*}{1} & 1 & 16.58 & 18.3 & 22.7 & 23.91 & 26.8 & 29.86 & 32.65 & 36.55 & 40.8 \\
\hline & 2 & 16.64 & 18.07 & 21.7 & 24.15 & 26.35 & 29.63 & 32.06 & 36.42 & 41 \\
\hline & 3 & 16.86 & 18.2 & 22.4 & 24.03 & 27.08 & 30.03 & 32.95 & 36.85 & 40.84 \\
\hline & 4 & 16.68 & 18.05 & 22.37 & 23.61 & 26.43 & 29.71 & 32.32 & 36.61 & 40.88 \\
\hline & 5 & 16.37 & 18.32 & 22.68 & 24.47 & 26.79 & 29.49 & 32.45 & 36.89 & 40.9 \\
\hline & Average $(\mathrm{mm})$ & 16.63 & 18.19 & 22.37 & 24.03 & 26.69 & 29.74 & 32.49 & 36.66 & 40.88 \\
\hline & Exact depth, $x_{0}(\mathrm{~mm})$ & & 1.56 & 5.74 & 7.41 & 10.06 & 13.12 & 15.86 & 20.04 & 24.26 \\
\hline & Depth to the middle, $x(\mathrm{~mm})$ & & 0.78 & 3.65 & 6.58 & 8.74 & 11.59 & 14.49 & 17.95 & 22.15 \\
\hline & Thickness, $\Delta \mathrm{x}(\mathrm{mm})$ & & 1.56 & 4.18 & 1.66 & 2.66 & 3.05 & 2.74 & 4.18 & 4.22 \\
\hline & Powder weight for test $(\mathrm{g})$ & & 7.11 & 10.10 & 5.58 & 10.04 & $10 . \overline{08}$ & 10.14 & 10.02 & $\overline{10.06}$ \\
\hline & . Chloride Concentration (\%) & & 1.56 & 5.74 & 7.41 & 10.06 & 13.12 & 15.86 & 20.04 & 24.26 \\
\hline \multirow{11}{*}{2} & 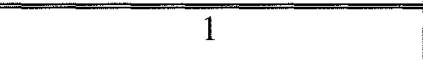 & 15.46 & 16.88 & 18 & 20.26 & 22.82 & 25.72 & 27.95 & 31.67 & 35.62 \\
\hline & 2 & 15.85 & 17.09 & 18.31 & 20.29 & 23.17 & 25.88 & 28.19 & 31.61 & 36.12 \\
\hline & 3 & 15.35 & 17.02 & 18.42 & 20.41 & 23.12 & 25.98 & 28.9 & 31.91 & 36.47 \\
\hline & 4 & 15.76 & 17.46 & 18.35 & 20.48 & 22.61 & 26.05 & 28.89 & 31.93 & 36.47 \\
\hline & 5 & 15.47 & 17.69 & 18.81 & 20.88 & 23.19 & 26.29 & 28.92 & 32.15 & 36.62 \\
\hline & Average (mm) & 15.58 & 17.23 & 18.38 & 20.46 & 22.98 & 25.98 & 28.57 & 31.85 & 36.26 \\
\hline & Exact depth, $\mathrm{x}_{0}(\mathrm{~mm})$ & & 1.65 & 2.80 & 4.89 & 7.40 & 10.41 & 12.99 & 16.28 & 20.68 \\
\hline & Depth to the middle, $x(\mathrm{~mm})$ & & 0.82 & 2.23 & 3.84 & 6.15 & 8.91 & 11.70 & 14.63 & 18.48 \\
\hline & Thickness, $\Delta \mathrm{x}(\mathrm{mm})$ & & 1.65 & 1.15 & 2.09 & 2.52 & 3.00 & 2.59 & 3.28 & 4.41 \\
\hline & Powder weight for test (g) & & 5.33 & 5.21 & 10.1 & 10.05 & 10.1 & 10.03 & 10.03 & 10.07 \\
\hline & Chloride Concentration (\%) & & 1.439 & 1.112 & 0.749 & 0.415 & 0.184 & 0.040 & 0.018 & 0.014 \\
\hline
\end{tabular}


Specimen Designation: GC-VA-D-B-E17

\begin{tabular}{|c|c|c|c|c|c|c|c|c|c|c|}
\hline \multirow{2}{*}{ Repeat Sample } & \multirow{2}{*}{$\begin{array}{l}\# \text { of measurements } \\
\text { around the perimeter }\end{array}$} & \multicolumn{9}{|c|}{ Steps of Powdering } \\
\hline & & Ref & D1 & D2 & D3 & D4 & D5 & D6 & D7 & D8 \\
\hline \multirow{11}{*}{1} & 1 & 14.7 & 16.5 & 17.21 & 19.53 & 21.41 & 24.36 & 27.44 & 30.38 & 34.39 \\
\hline & 2 & 15.69 & 16.52 & 17.16 & 19.41 & 21.22 & 24.34 & 27.42 & 30.48 & 34.46 \\
\hline & 3 & 15.42 & 16.6 & 17.66 & 19.64 & 21.9 & 24.91 & 27.9 & 30.8 & 34.82 \\
\hline & 4 & 15.14 & 16.95 & 17.85 & 19.74 & 21.75 & 24.71 & 27.8 & 30.69 & 34.69 \\
\hline & 5 & 15.22 & 16.94 & 17.83 & 19.83 & 21.89 & 24.63 & 27.69 & 30.82 & 34.85 \\
\hline & Average (mm) & 15.23 & 16.70 & 17.54 & 19.63 & 21.63 & 24.59 & 27.65 & 30.63 & 34.64 \\
\hline & Exact depth, $\mathrm{x}_{0}(\mathrm{~mm})$ & & 1.47 & 2.31 & 4.40 & 6.40 & 9.36 & 12.42 & 15.40 & 19.41 \\
\hline & Depth to the middle, $\mathrm{x}(\mathrm{mm})$ & & 0.73 & 1.89 & 3.35 & 5.40 & 7.88 & 10.89 & 13.91 & 17.40 \\
\hline & Thickness, $\Delta \mathrm{x}(\mathrm{mm})$ & & 1.47 & 0.84 & 2.09 & 2.00 & 2.96 & 3.06 & 2.98 & 4.01 \\
\hline & Powder weight for test $(\mathrm{g})$ & & 7.46 & 6.12 & 10.01 & 10.02 & 10.03 & 10.06 & 10.07 & 10.04 \\
\hline & Chloride Concentration (\%) & & 1.081 & 0.811 & 0.491 & 0.286 & 0.118 & 0.026 & 0.012 & 0.007 \\
\hline \multirow{11}{*}{2} & 1 & 15.58 & 15.95 & 17.07 & 19.02 & 20.91 & 24.04 & 27.06 & 30.11 & 33.97 \\
\hline & 2 & 15.43 & 15.99 & 17.14 & 18.95 & 20.75 & 23.86 & 26.91 & 29.9 & 33.91 \\
\hline & 3 & 15.61 & 16.07 & 17.42 & 19.26 & 21.08 & 24.21 & 27.04 & 30.32 & 34.24 \\
\hline & 4 & 14.88 & 16.4 & 17.22 & 19.22 & 20.95 & 24.1 & 26.99 & 30.16 & 34.03 \\
\hline & 5 & 14.68 & 16.33 & 17.29 & 19.38 & 21.3 & 24.29 & 27.3 & 30.17 & 33.99 \\
\hline & Average (mm) & 15.24 & 16.15 & 17.23 & 19.17 & 21.00 & 24.10 & 27.06 & 30.13 & 34.03 \\
\hline & Exact depth, $x_{0}(\mathrm{~mm})$ & & 0.91 & 1.99 & 3.93 & 5.76 & 8.86 & 11.82 & 14.90 & 18.79 \\
\hline & Depth to the middle, $x(\mathrm{~mm})$ & & 0.46 & 1.45 & 2.96 & 4.85 & 7.31 & 10.34 & 13.36 & 16.84 \\
\hline & Thickness, $\Delta \mathrm{x}(\mathrm{mm})$ & & 0.91 & 1.08 & 1.94 & 1.83 & 3.10 & 2.96 & 3.07 & 3.90 \\
\hline & Powder weight for test (g) & & 4.37 & 5.66 & 10.02 & 10.01 & 10.02 & 10.03 & 10.02 & 10.02 \\
\hline & Chloride Concentration (\%) & & 1.276 & 1.221 & 0.676 & 0.395 & 0.200 & 0.049 & 0.017 & 0.012 \\
\hline
\end{tabular}


Specimen Designation: GC-VA-D-C-NO

\begin{tabular}{|c|c|c|c|c|c|c|c|c|c|c|}
\hline \multirow{2}{*}{ Repeat Sample } & \multirow{2}{*}{$\begin{array}{l}\# \text { of measurements } \\
\text { around the perimeter }\end{array}$} & \multicolumn{9}{|c|}{ Steps of Powdering } \\
\hline & & Ref & $\mathrm{D} 1$ & $\overline{\mathrm{D} 2}$ & $\overline{\mathrm{D} 3}$ & $\overline{\mathrm{D} 4}$ & D5 & $\overline{\mathrm{D} 6}$ & D7 & $\overline{\mathrm{D} 8}$ \\
\hline \multirow{11}{*}{1} & 1 & 15.74 & 16.94 & 17.74 & 19.79 & 21.58 & 24.68 & 27.82 & 30.8 & 34.85 \\
\hline & 2 & 15.21 & 17.39 & 17.9 & 20.03 & 21.77 & 25.32 & 27.99 & 31.16 & 35.18 \\
\hline & 3 & 15.74 & 17.25 & 17.86 & 20.22 & 21.81 & 25.15 & 28.26 & 31.32 & 35.18 \\
\hline & 4 & 15.35 & 17.09 & 17.95 & 19.8 & 21.5 & 25.09 & 27.8 & 30.82 & 34.93 \\
\hline & 5 & 15.83 & 16.74 & 17.65 & 19.7 & 21.55 & 24.91 & 27.9 & 30.73 & 34.53 \\
\hline & Average $(\mathrm{mm})$ & 15.57 & 17.08 & 17.82 & 19.91 & 21.64 & 25.03 & 27.95 & 30.97 & 34.93 \\
\hline & Exact depth, $x_{0}(\mathrm{~mm})$ & & 1.51 & 2.25 & 4.33 & 6.07 & 9.46 & 12.38 & 15.39 & 19.36 \\
\hline & Depth to the middle, $x(\mathrm{~mm})$ & & 0.75 & 1.88 & 3.29 & 5.20 & 7.76 & 10.92 & 13.89 & 17.38 \\
\hline & Thickness, $\Delta \mathrm{x}(\mathrm{mm})$ & & 1.51 & 0.74 & 2.09 & 1.73 & 3.39 & 2.92 & 3.01 & 3.97 \\
\hline & Powder weight for test $(\mathrm{g})$ & & 5.24 & 4.5 & 10.11 & 10.01 & 10.03 & 10.01 & 10.2 & 10.04 \\
\hline & Chloride Concentration (\%) & & 1.008 & 0.853 & 0.674 & 0.480 & 0.302 & 0.155 & 0.101 & 0.040 \\
\hline \multirow{11}{*}{2} & 1 & 15.02 & 16.23 & 17.2 & 19.33 & 21.3 & 24.44 & 27.19 & 30.12 & 34.11 \\
\hline & 2 & 15.5 & 16.51 & 17.27 & 19.33 & 21.42 & 24.23 & 27.4 & 30.24 & 34.41 \\
\hline & 3 & 15.16 & 16.15 & 17.38 & 19.01 & 21.17 & 24.4 & 27.46 & 30.34 & 34.65 \\
\hline & 4 & 15.75 & 16.32 & 17.52 & 19.04 & 21.36 & 24.09 & 27.35 & 30.17 & 34.5 \\
\hline & 5 & 14.62 & 16.2 & 17.08 & 19.19 & 21.27 & 23.88 & 27.29 & 30.36 & 34.17 \\
\hline & Average $(\mathrm{mm})$ & 15.21 & 16.28 & 17.29 & 19.18 & 21.30 & 24.21 & 27.34 & 30.25 & 34.37 \\
\hline & Exact depth, $\mathrm{x}_{0}(\mathrm{~mm})$ & & 1.07 & 2.08 & 3.97 & 6.09 & 9.00 & 12.13 & 15.04 & 19.16 \\
\hline & Depth to the middle, $x(\mathrm{~mm})$ & & 0.54 & 1.58 & 3.03 & 5.03 & 7.55 & 10.56 & 13.58 & 17.10 \\
\hline & Thicknesș, $\Delta \mathrm{x}(\mathrm{mm})$ & & 1.07 & 1.01 & 1.89 & 2.12 & 2.90 & 3.13 & 2.91 & 4.12 \\
\hline & Powder weight for test $(\mathrm{g})$ & & 5.47 & 5.18 & 10.05 & 10.06 & 10.05 & 10.18 & 10.01 & 10.18 \\
\hline & Chloride Concentration (\%) & & 1.278 & 1.121 & 0.750 & 0.560 & 0.422 & 0.265 & 0.145 & 0.048 \\
\hline
\end{tabular}



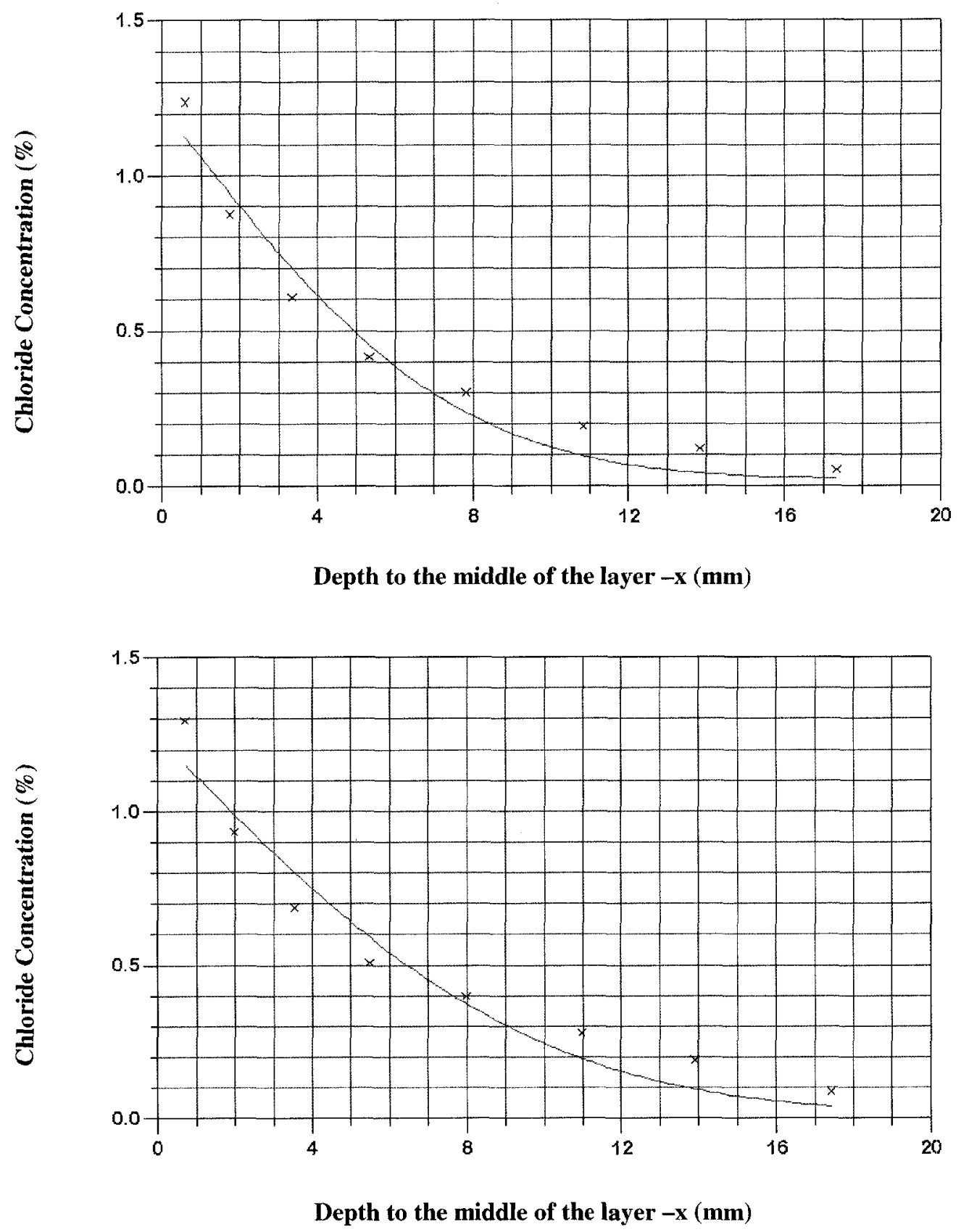

Figure C.1: Regression analysis curve for GC-MO-D-C-G4l specimens 

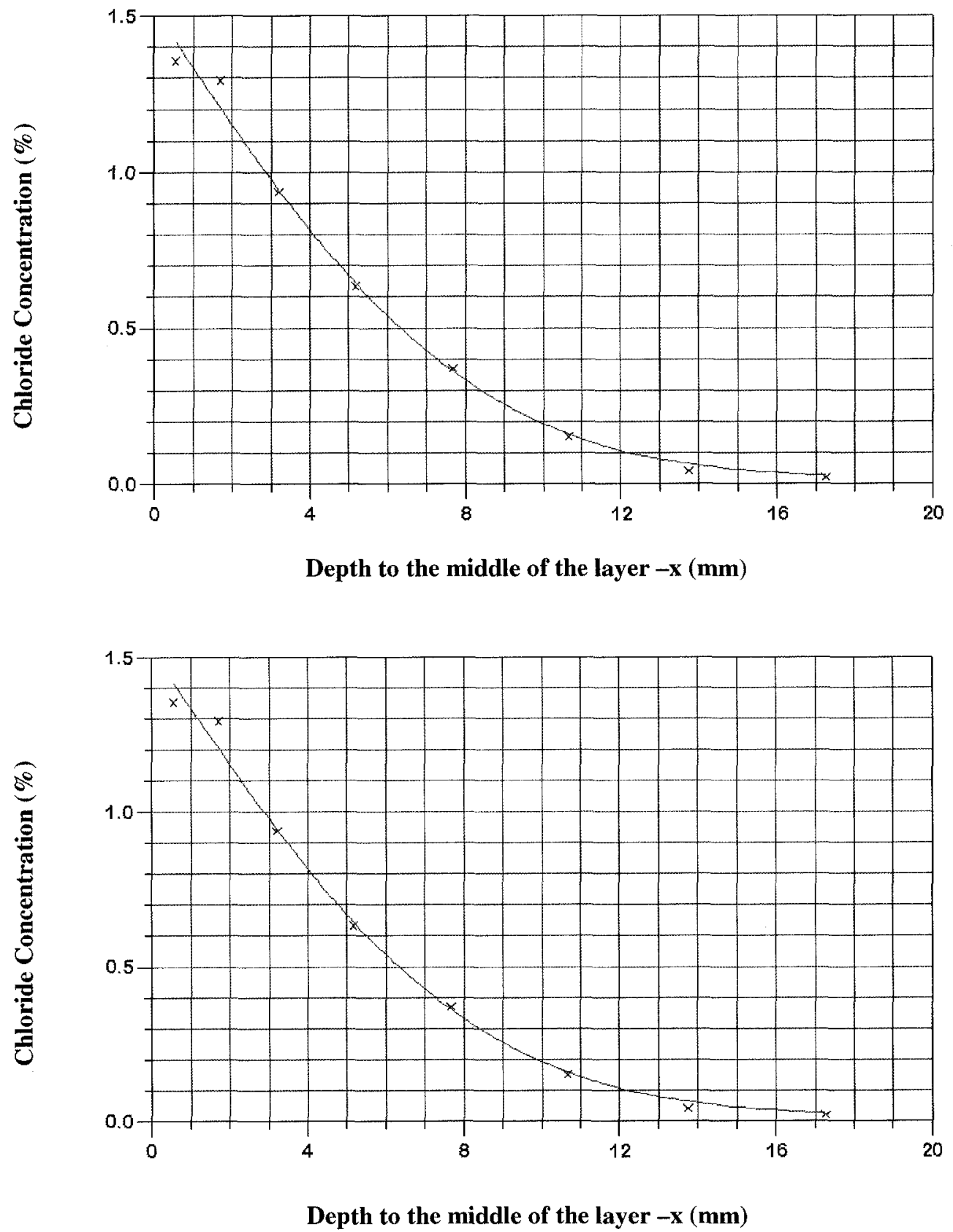

Figure C.2: Regression analysis curve for GC-MO-D-F-G41 specimens 


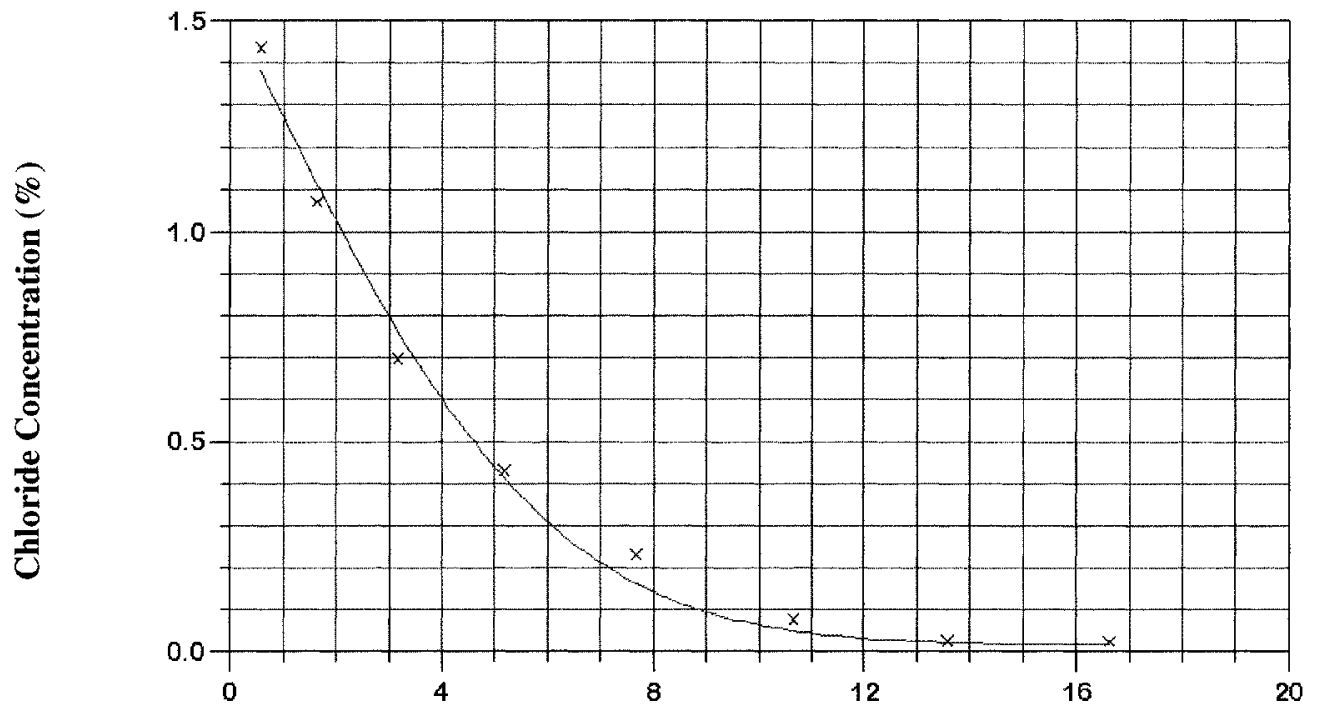

Depth to the middle of the layer $-x(\mathrm{~mm})$

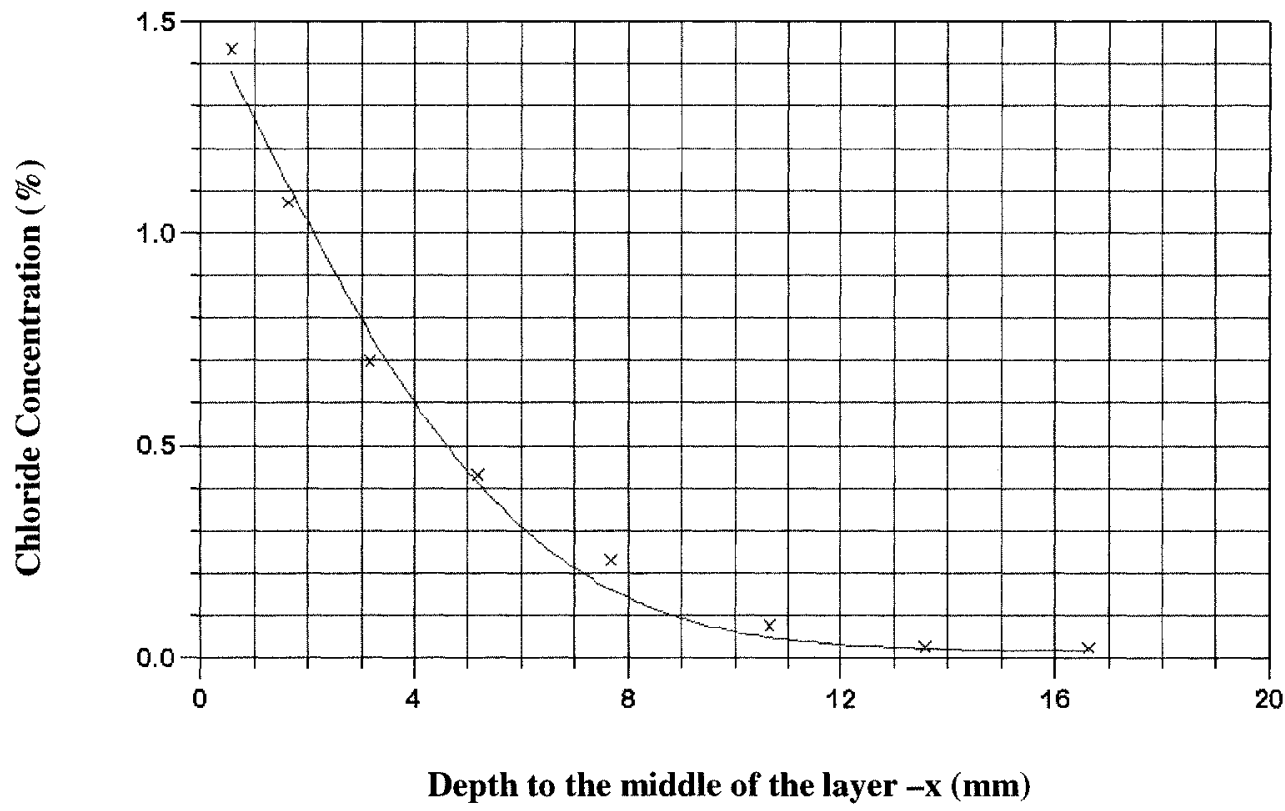

Figure C.3: Regression analysis curve for GC-MO-D-B-G41 specimens 


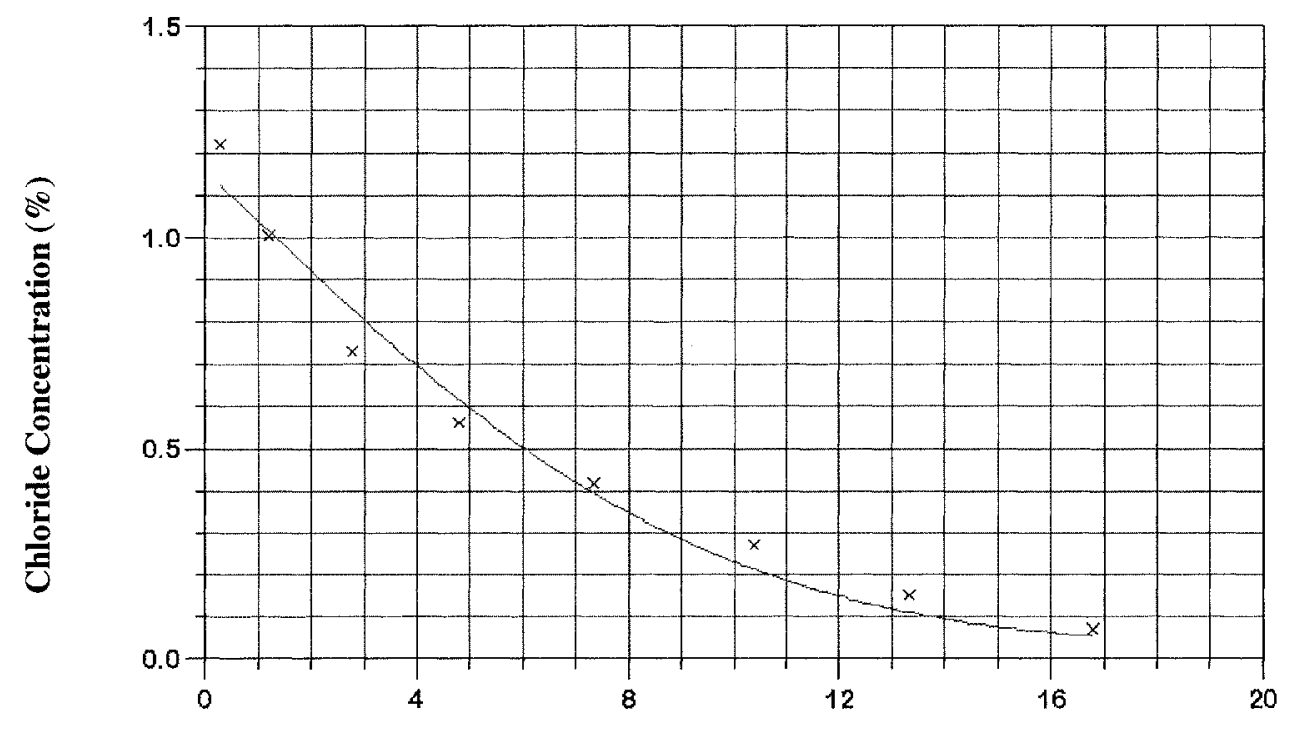

Depth to the middle of the layer $-\mathrm{x}(\mathrm{mm})$

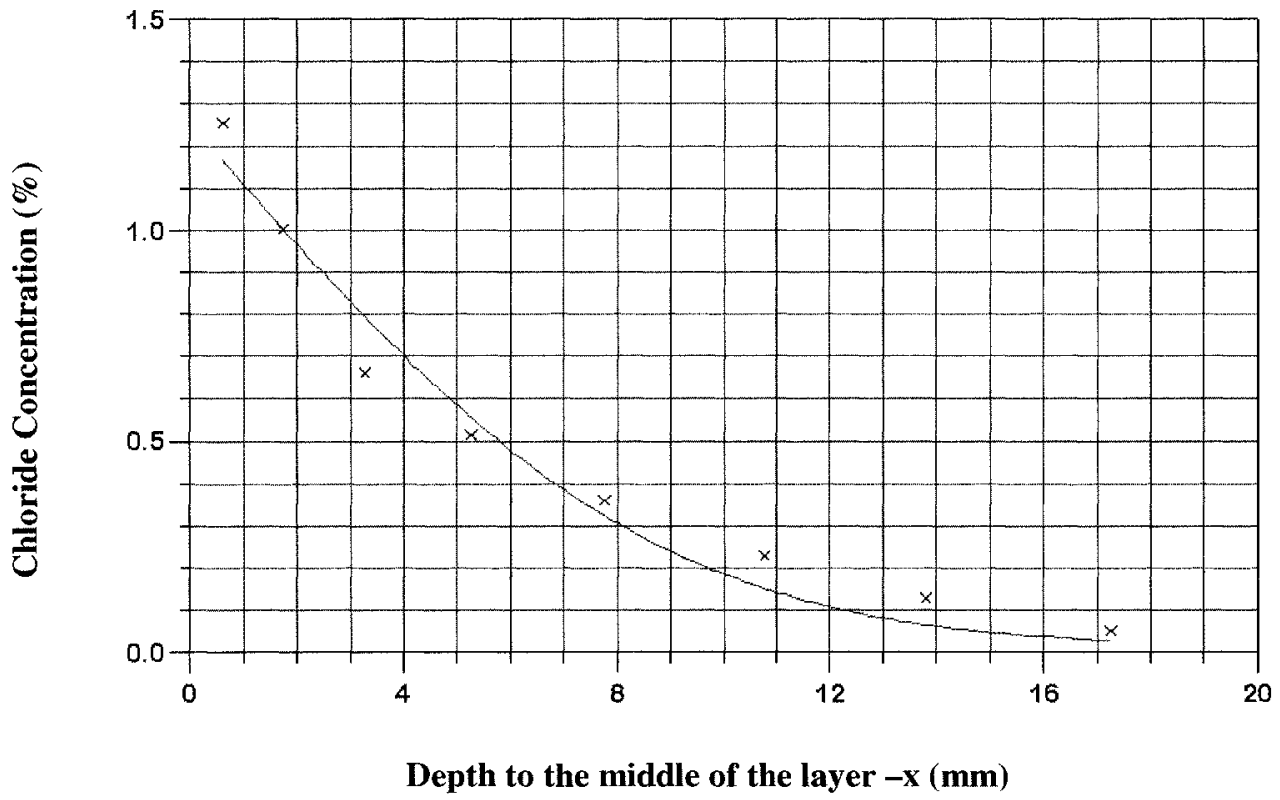

Figure C.4: Regression analysis curve for GC-MO-D-C-E26 specimens 


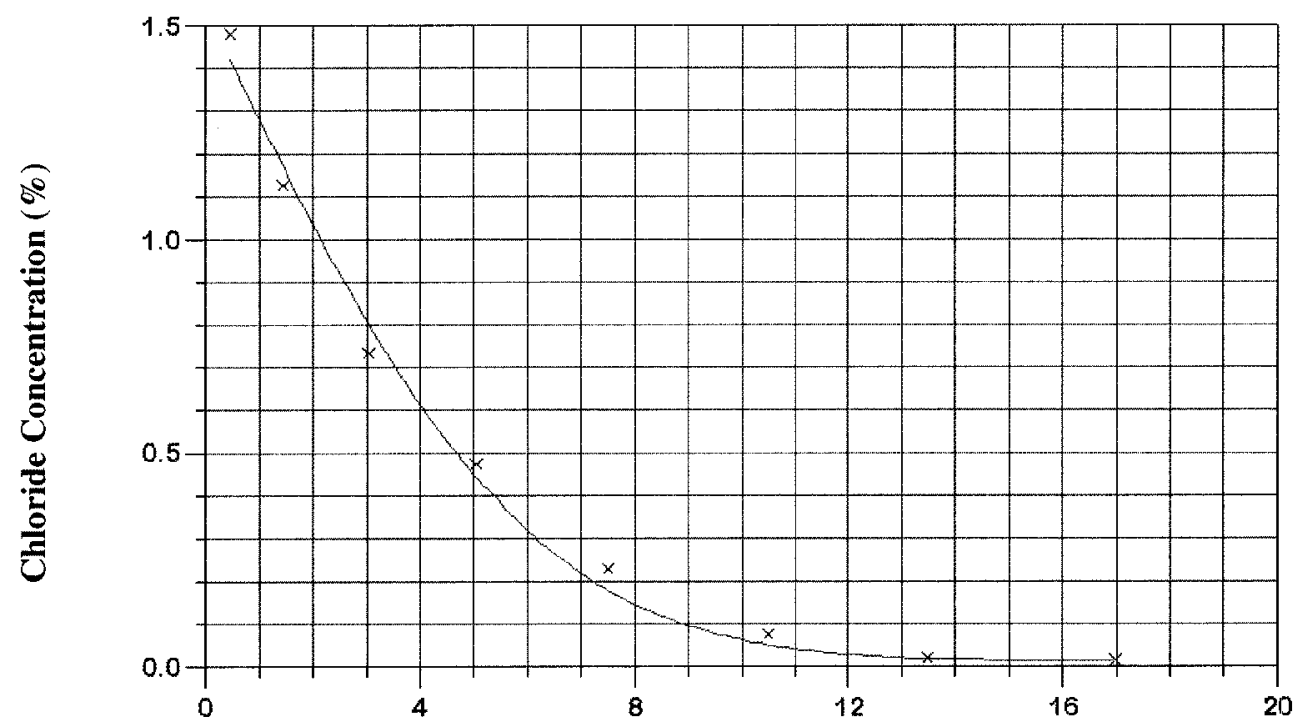

Depth to the middle of the layer $-x(\mathbf{m m})$

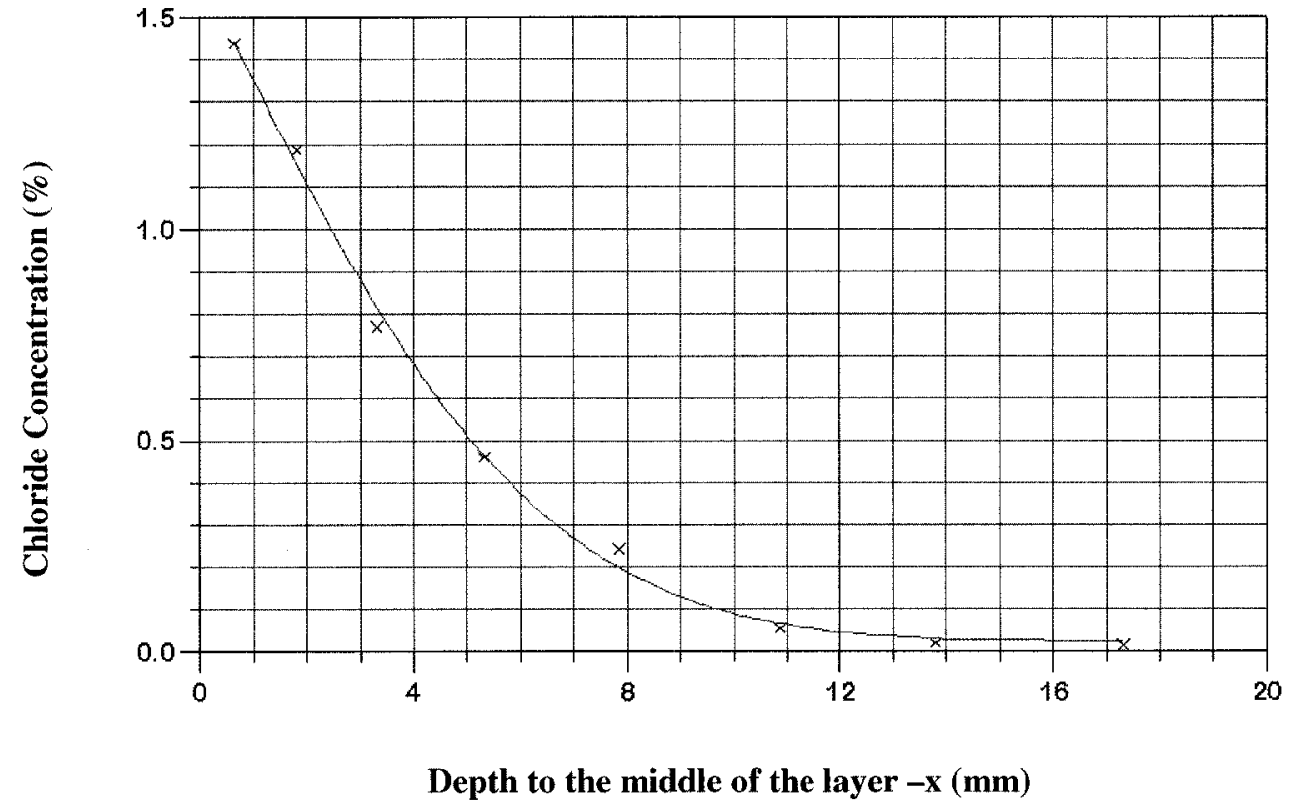

Figure C.5: Regression analysis curve for GC-MO-D-F-E26 specimens 


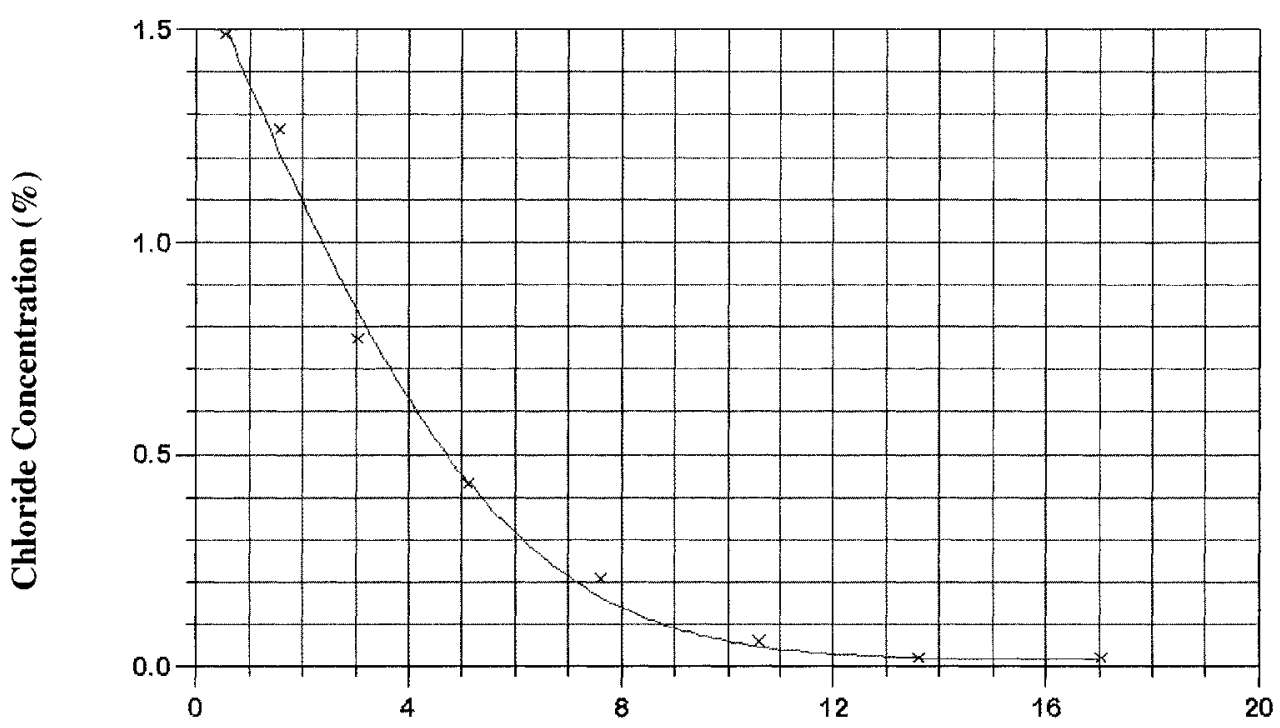

Depth to the middle of the layer $-x(\mathrm{~mm})$

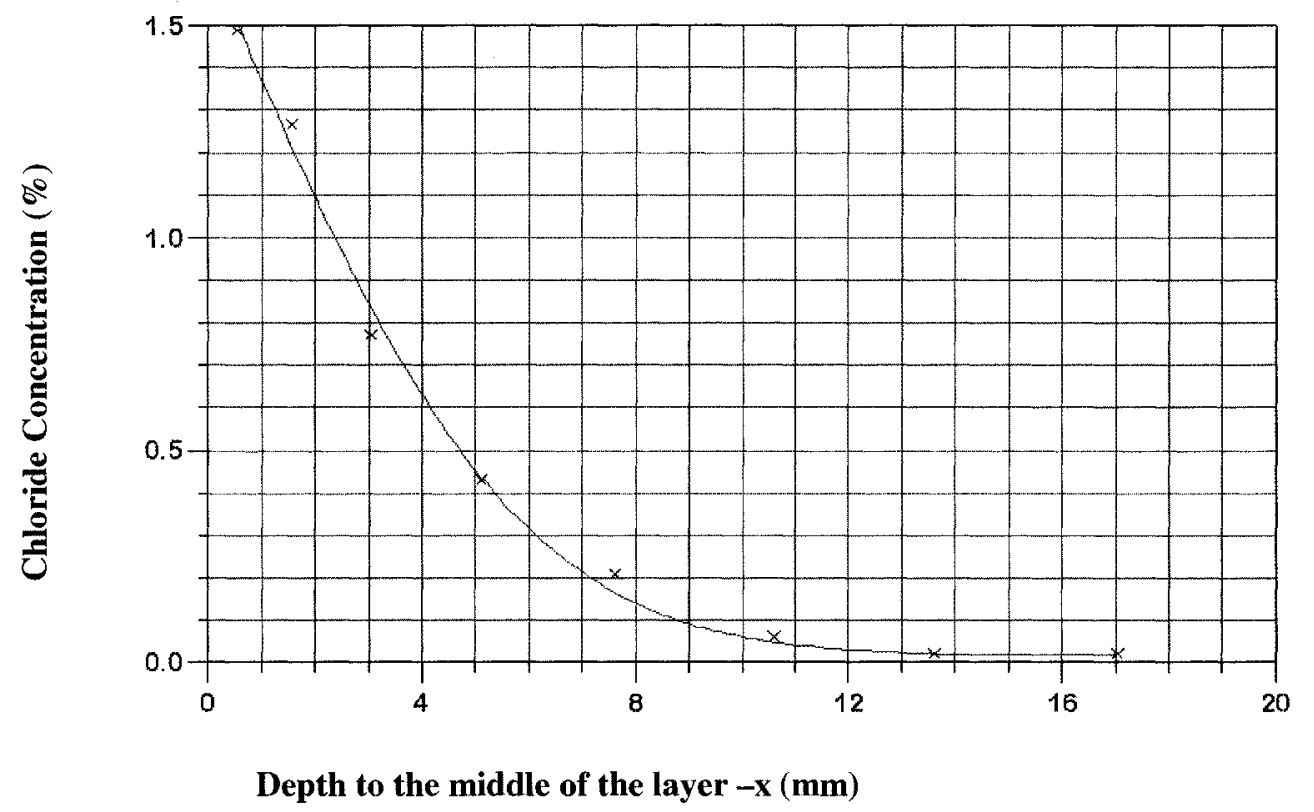

Figure C.6: Regression analysis curve for GC-MO-D-B-E26 specimens 

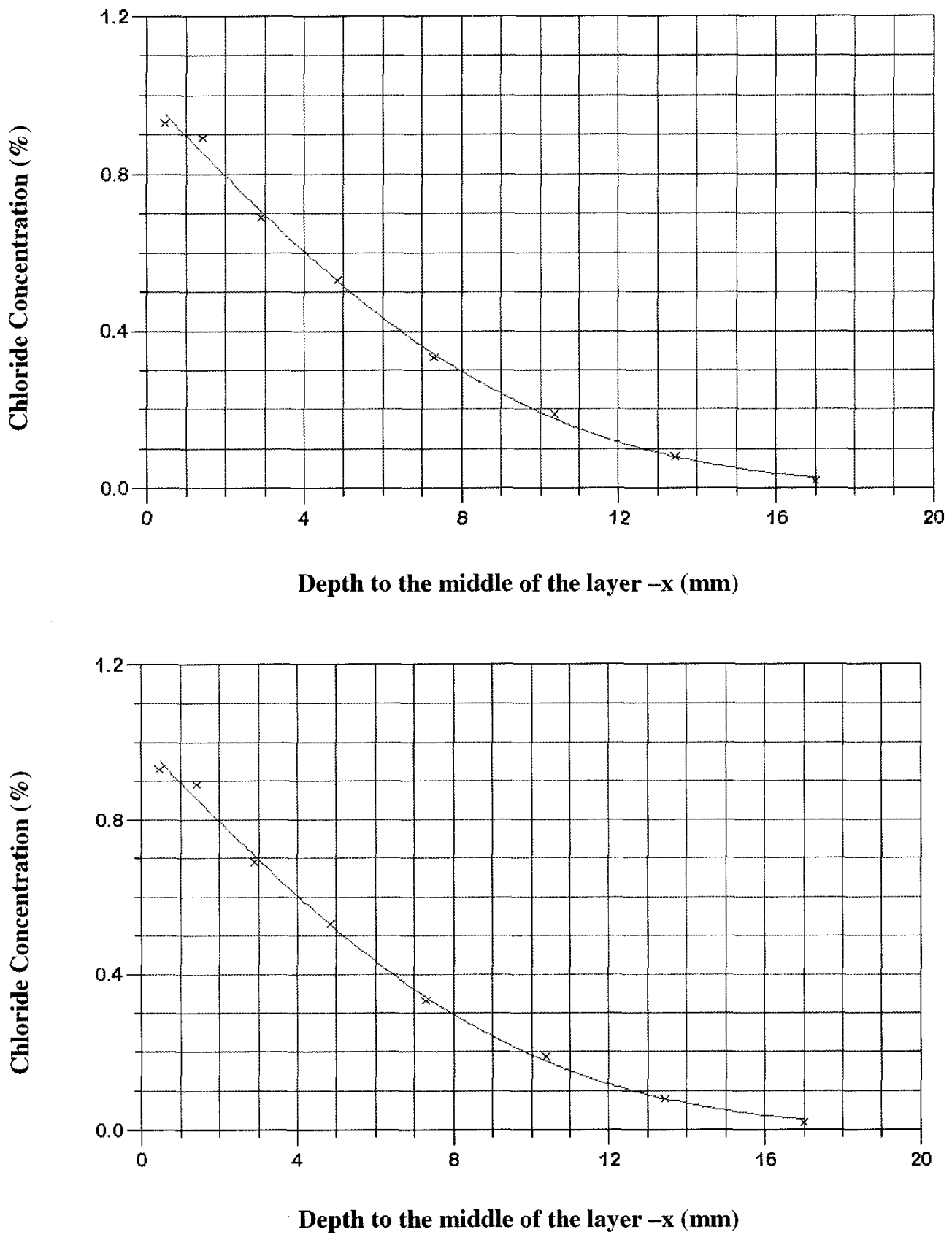

Figure C.7: Regression analysis curve for GC-MO-D-C-N0 specimens 


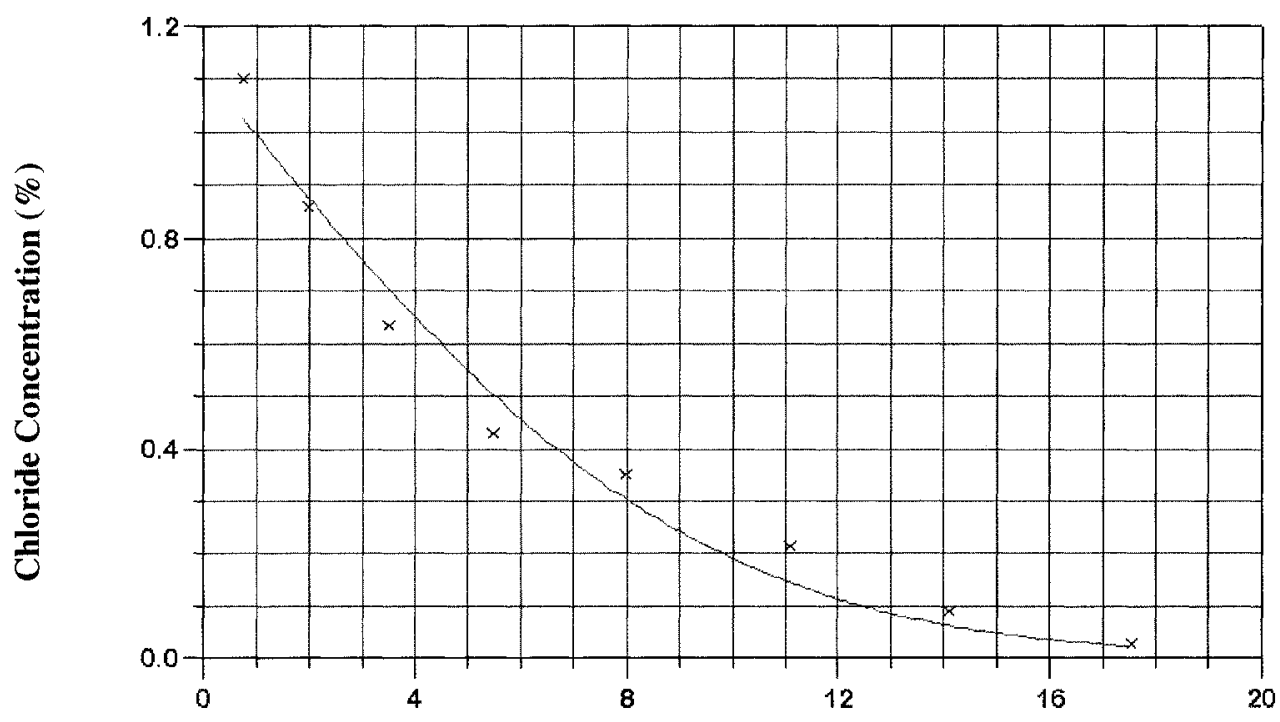

Depth to the middle of the layer $-x(\mathbf{m m})$

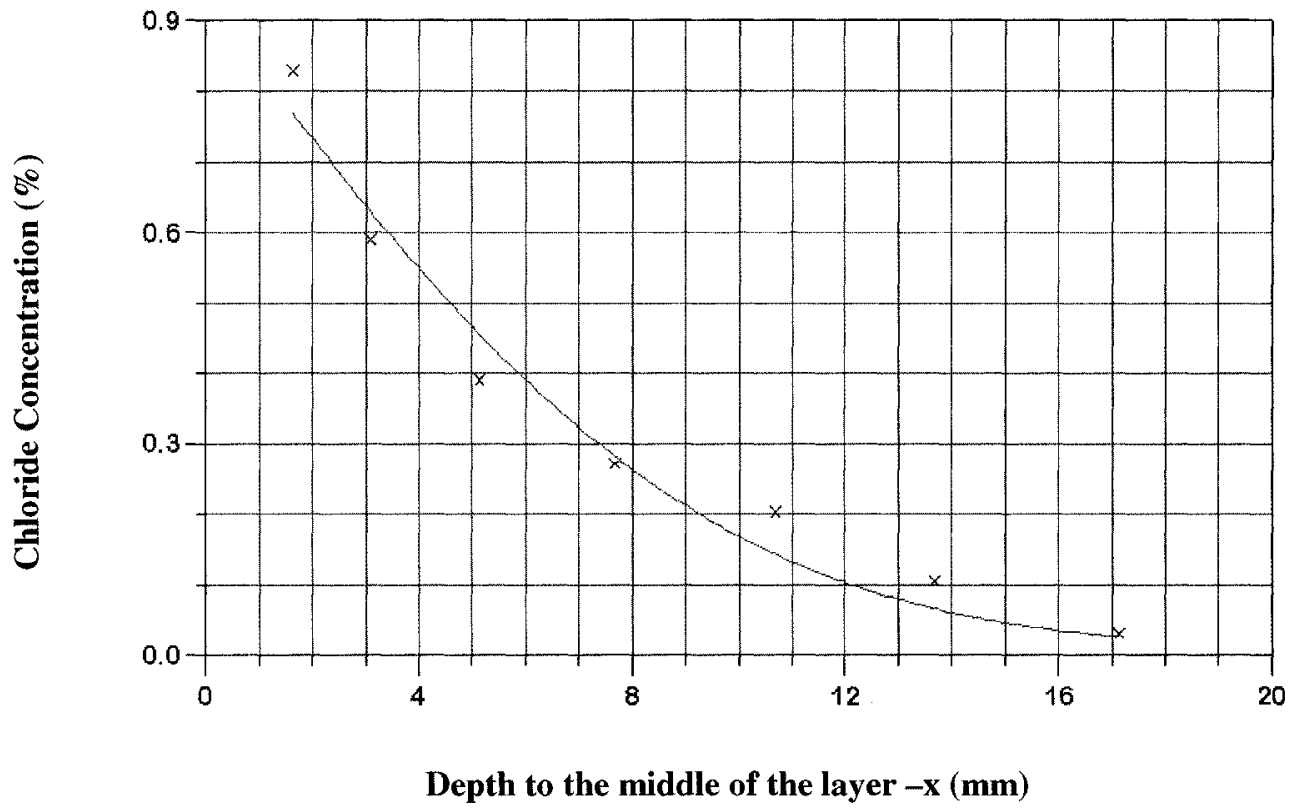

Figure C.8: Regression analysis curve for GC-VA-D-C-G23 specimens 

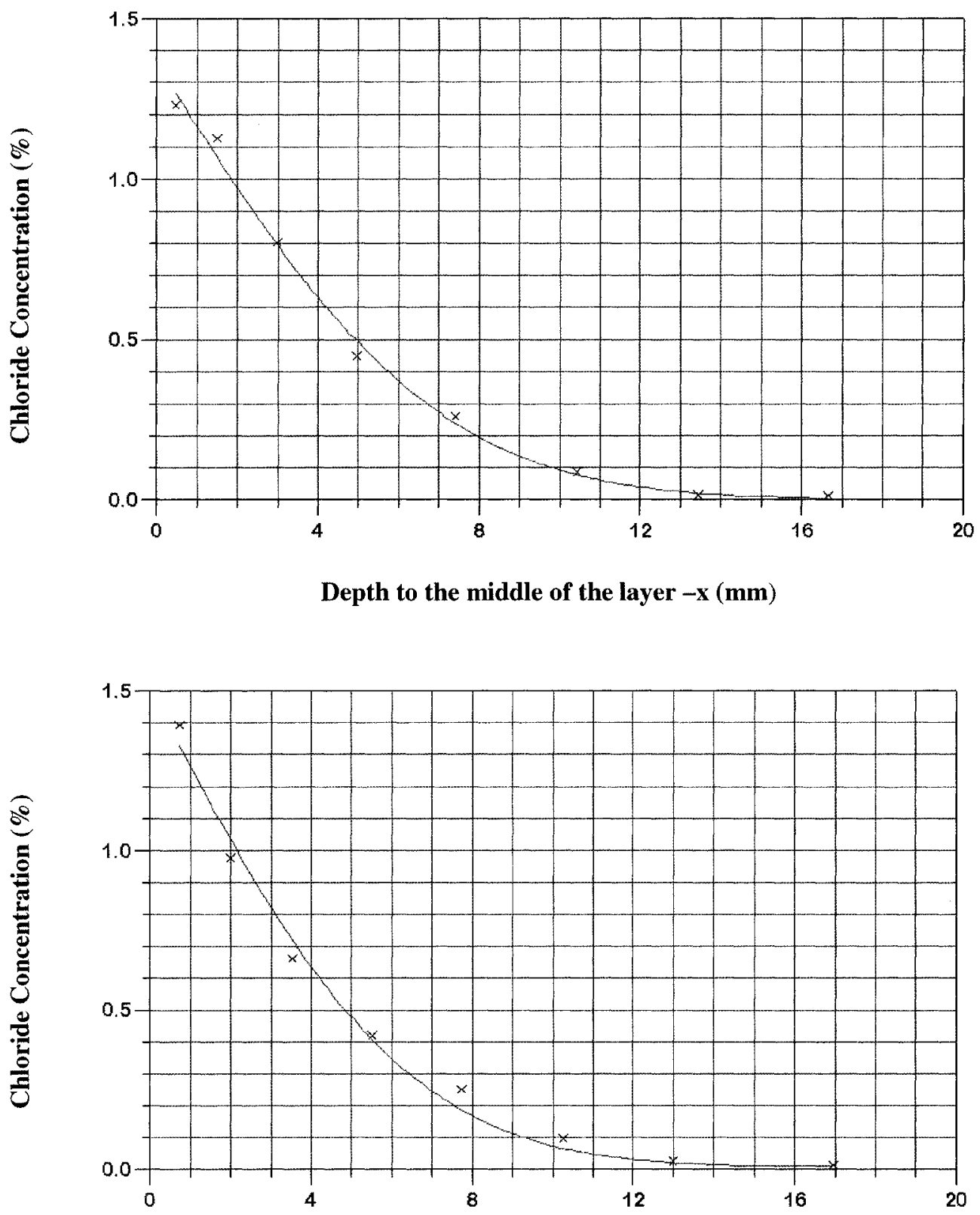

Depth to the middle of the layer $-x(\mathrm{~mm})$

Figure C.9: Regression analysis curve for GC-VA-D-F-G23 specimens 


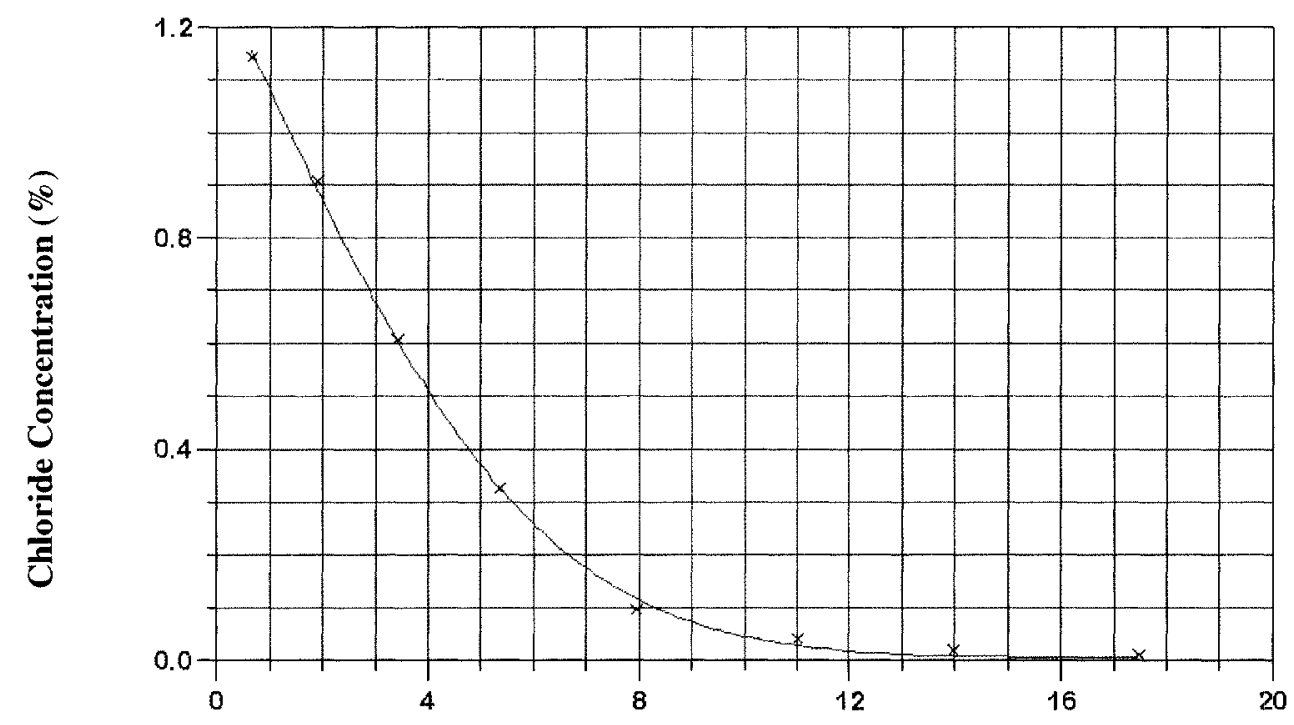

Depth to the middle of the layer $-x(\mathrm{~mm})$

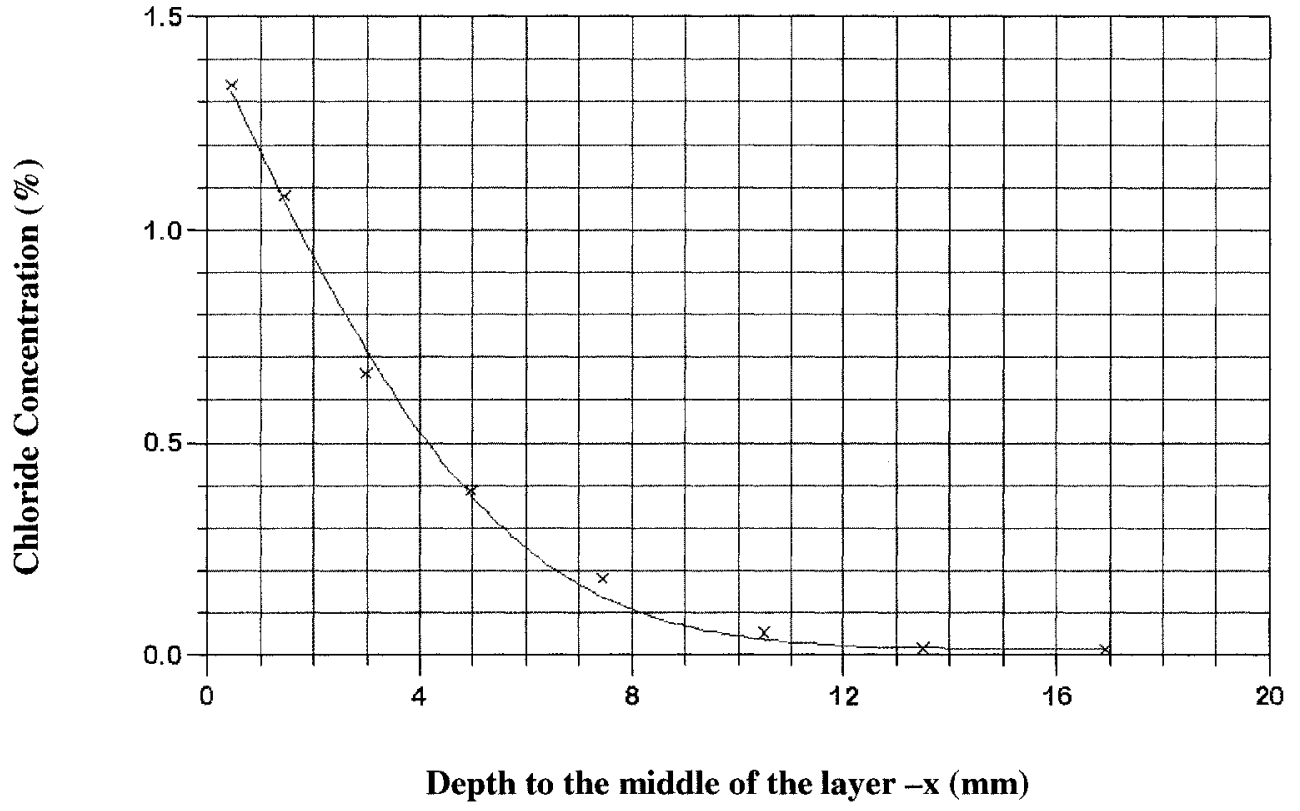

Figure C.10: Regression analysis curve for GC-VA-D-B-G23 specimens 


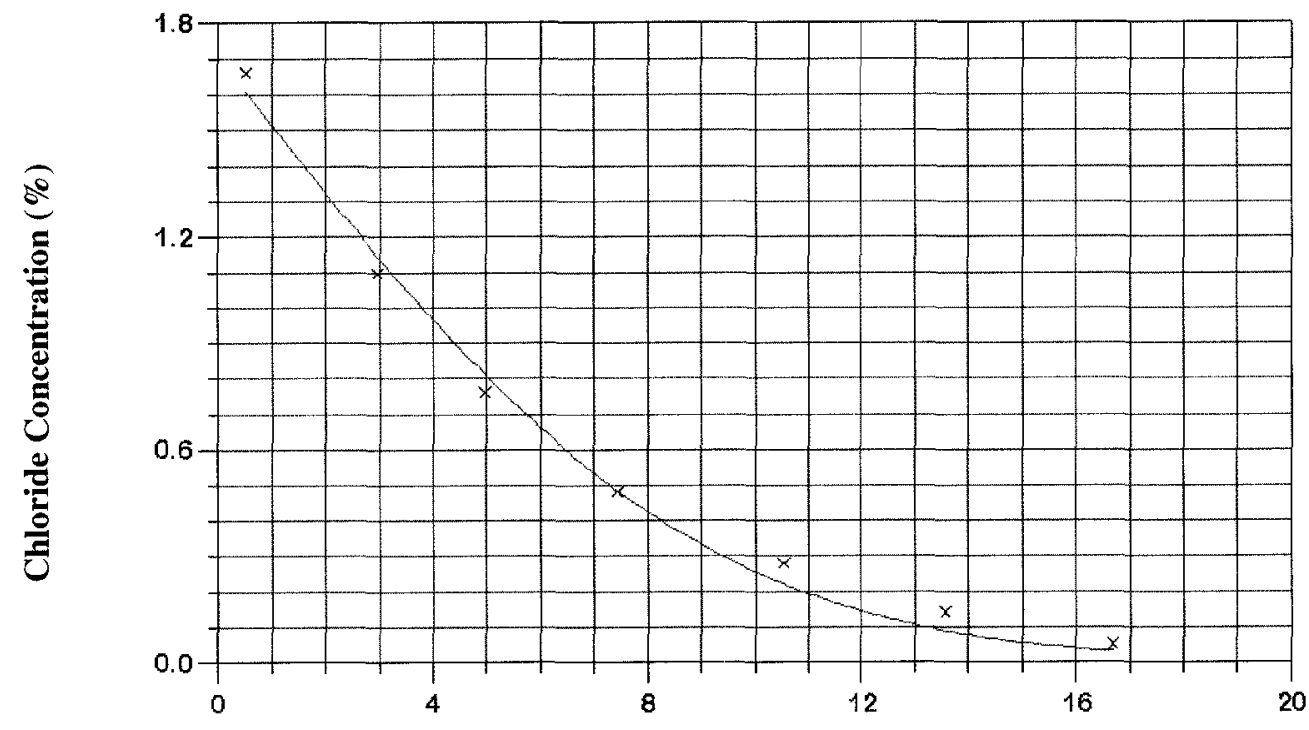

Depth to the middle of the layer $-\mathrm{x}(\mathrm{mm})$

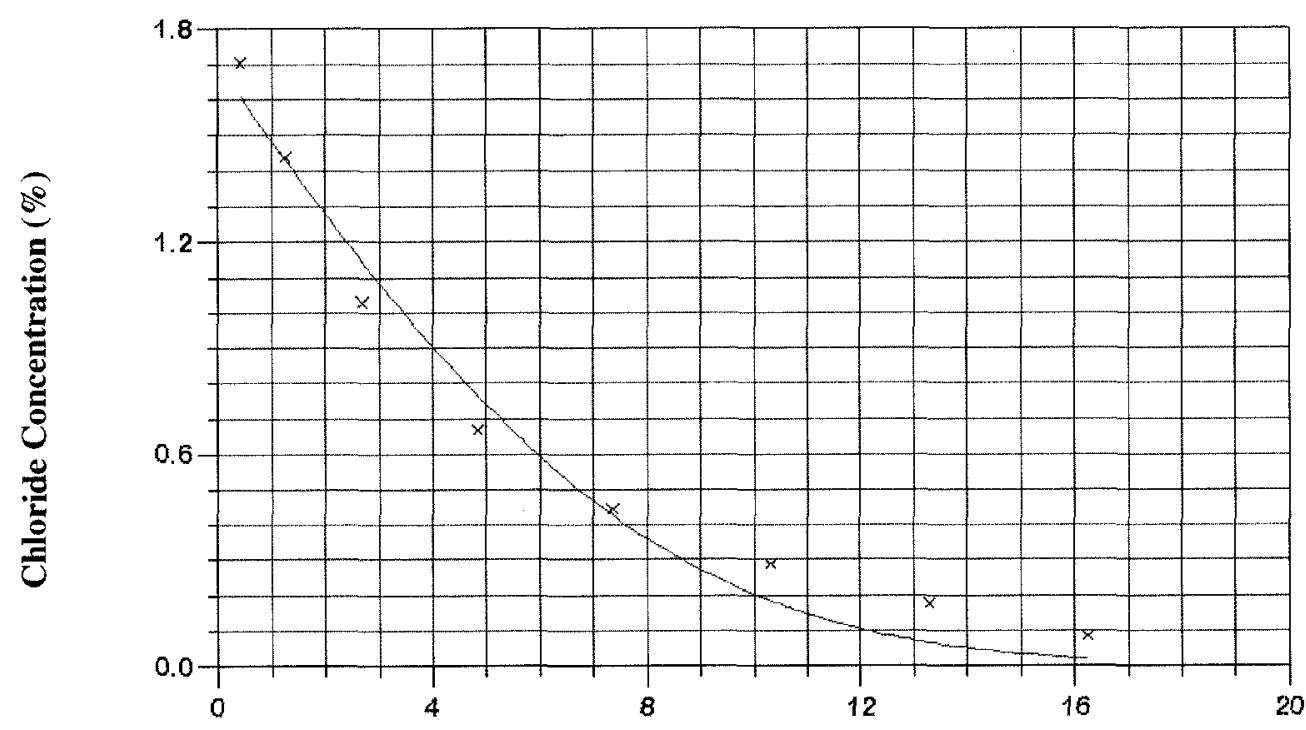

Depth to the middle of the layer $-x(\mathrm{~mm})$

Figure C.11: Regression analysis curve for GC-VA-D-C-E17 specimens 


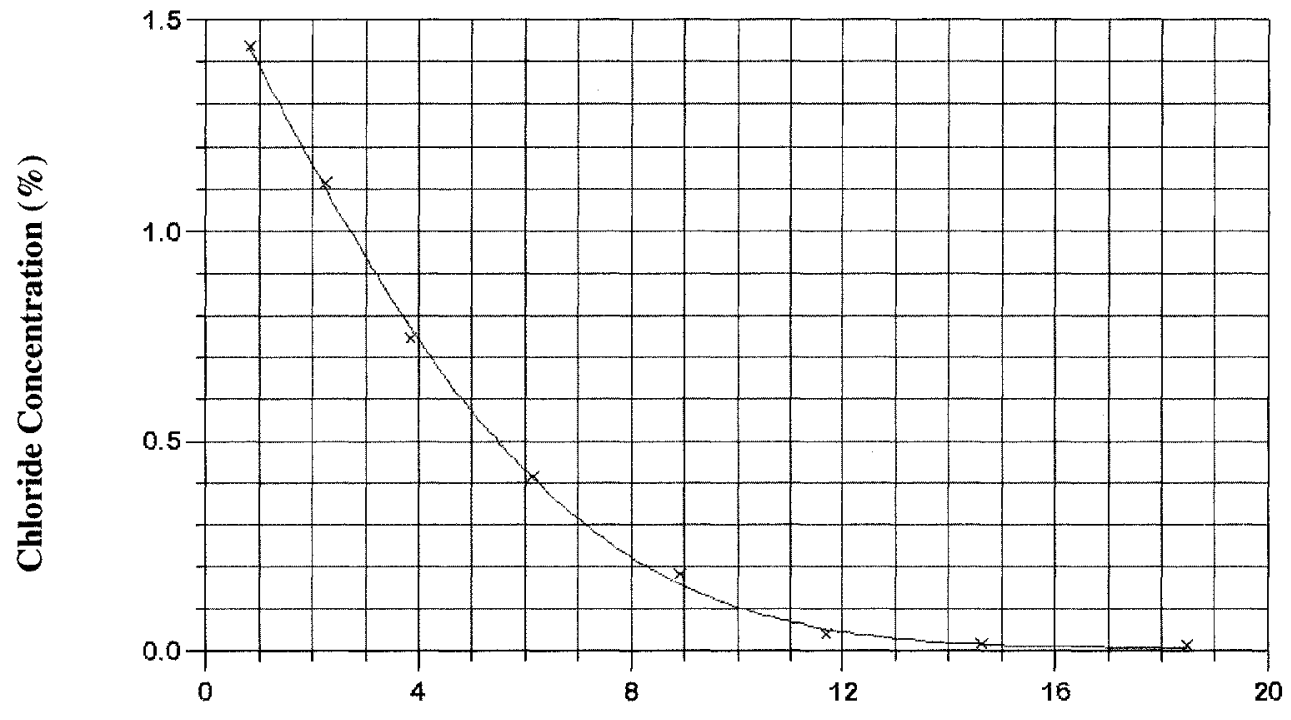

Depth to the middle of the layer $-x(\mathrm{~mm})$

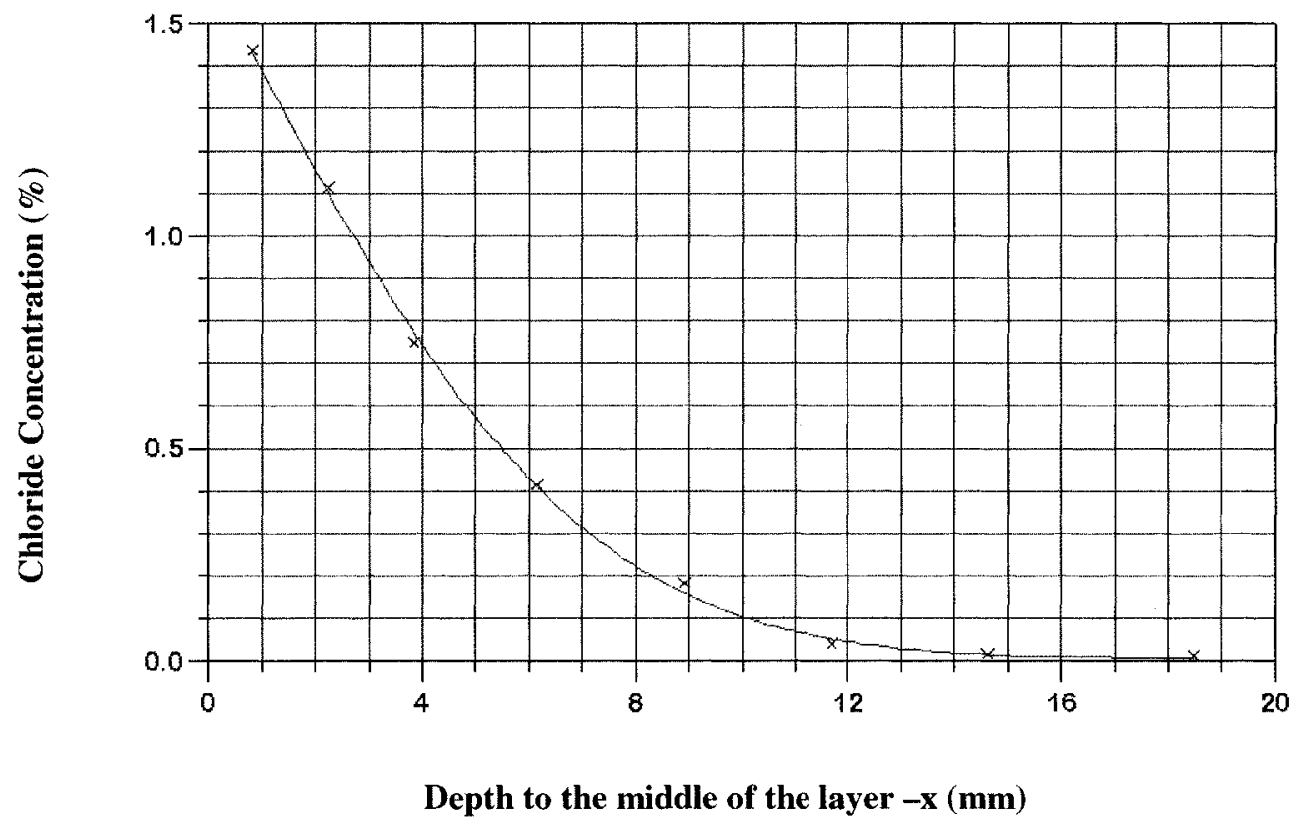

Figure C.12: Regression analysis curve for GC-VA-D-F-E17 specimens 

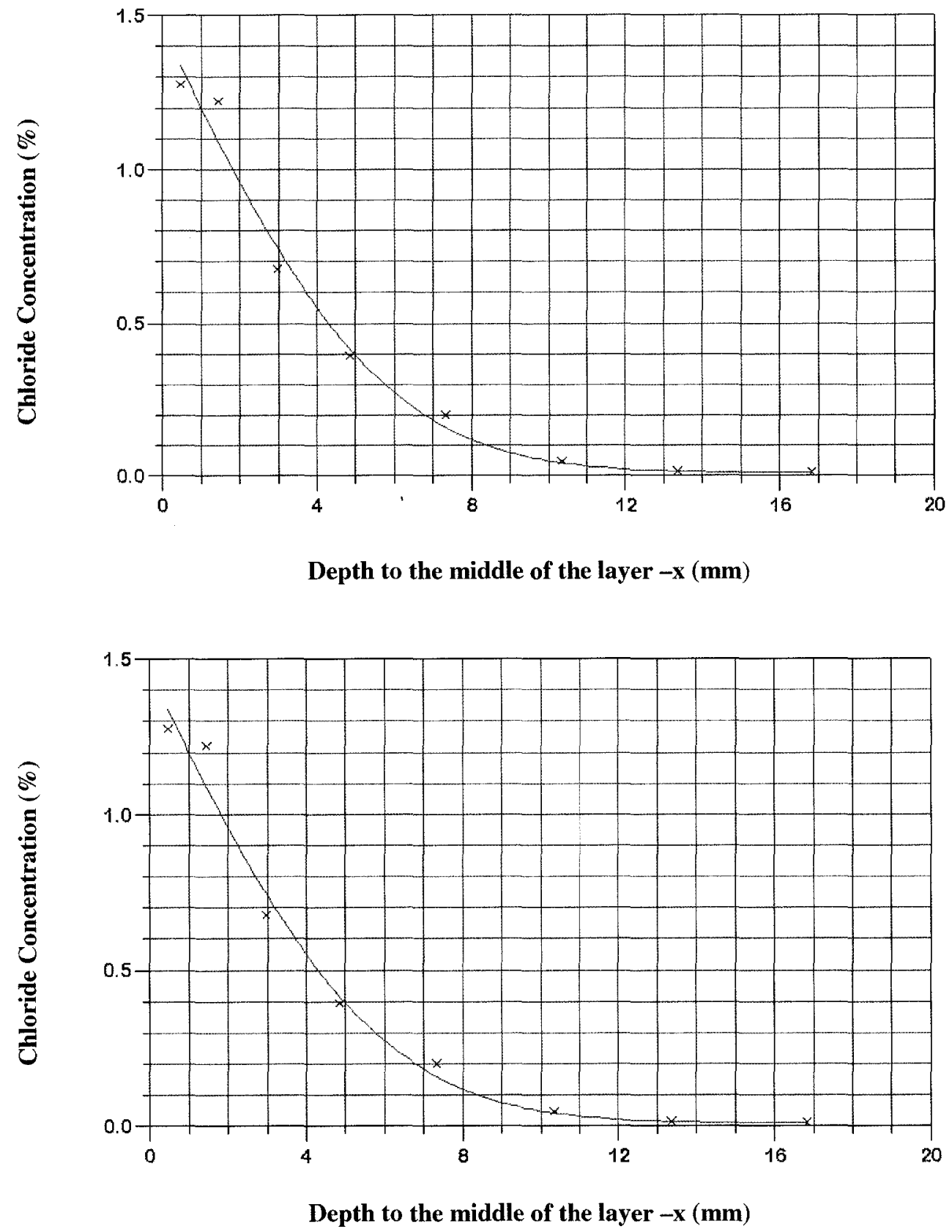

Figure C.13: Regression analysis curve for GC-VA-D-B-E17 specimens 


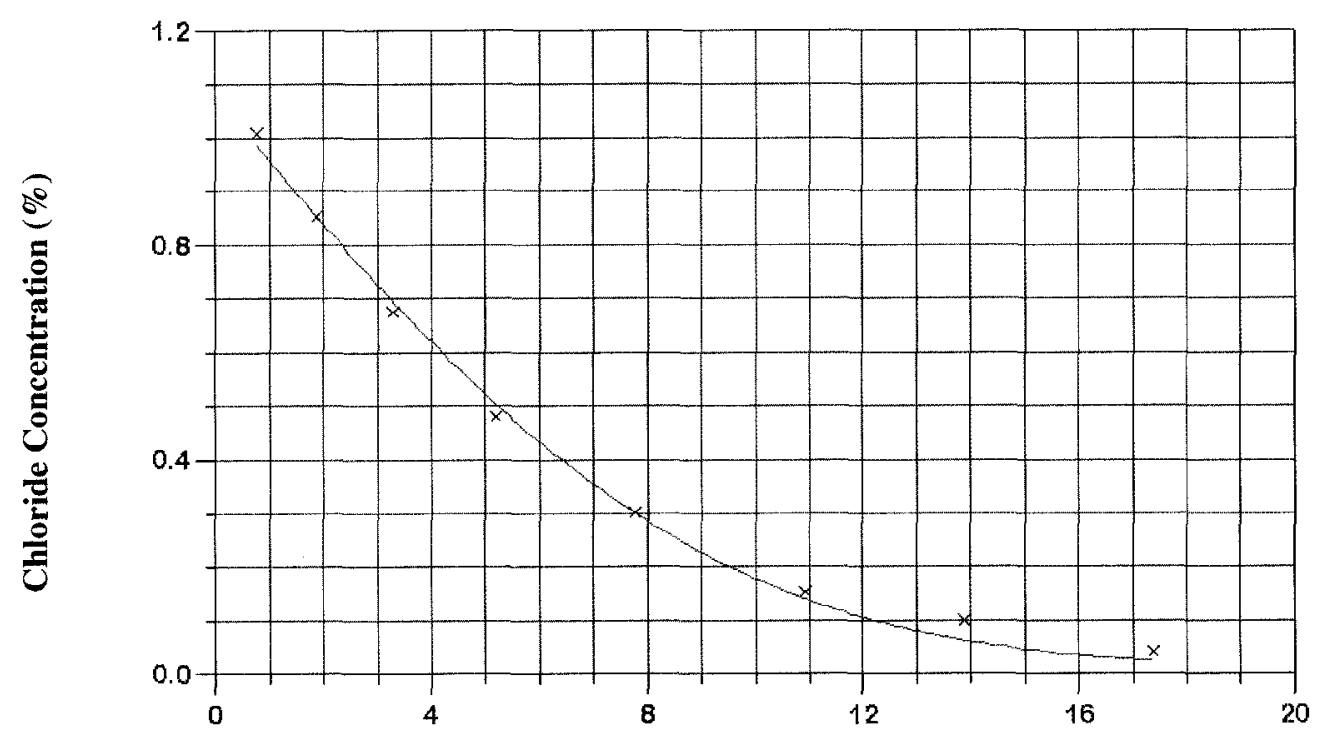

Depth to the middle of the layer $-x(\mathbf{m m})$

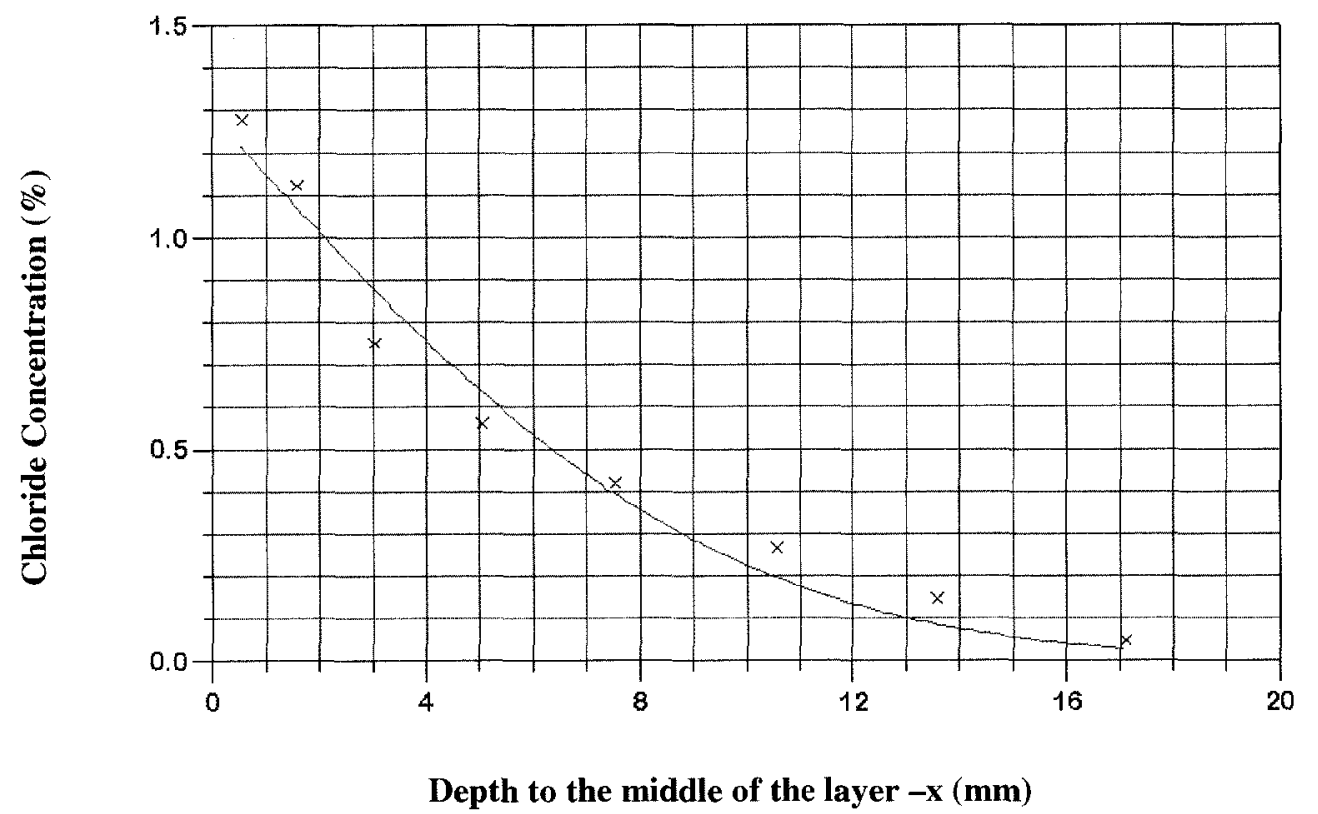

Figure C.14: Regression analysis curve for GC-VA-D-C-N0 specimens 


\section{C.3.Carbonation Test (RAW DATA)}


Specimen Designation: GC-MO-D-C-G41

\begin{tabular}{|c|c|c|c|c|c|c|c|c|}
\hline \multirow{3}{*}{ \# } & \multicolumn{8}{|c|}{ Depth of Carbonation (mm) } \\
\hline & \multirow{2}{*}{ Sides } & \multicolumn{7}{|c|}{ Numbers of day } \\
\hline & & 7 & 21 & 35 & 56 & 84 & 112 & 140 \\
\hline \multirow{5}{*}{1} & Top & 0.0 & 3.0 & 3.0 & 5.0 & 5.0 & 5.5 & 8.0 \\
\hline & Bottom & 0.0 & 0.0 & 3.0 & 0.0 & 5.0 & 5.0 & 7.0 \\
\hline & Right & 0.0 & 2.0 & 3.0 & 5.0 & 5.0 & 6.0 & 5.0 \\
\hline & Left & 0.0 & 2.0 & 3.0 & 4.0 & 4.5 & 5.0 & 7.0 \\
\hline & Average & 0.0 & 2.3 & 3.0 & 3.5 & 4.9 & 5.4 & 6.8 \\
\hline \multirow{5}{*}{2} & Top & 0.0 & 2.0 & 3.0 & 4.0 & 5.0 & 5.5 & 7.0 \\
\hline & Bottom & 0.0 & 0.0 & 3.0 & 4.0 & 5.0 & 6.0 & 7.0 \\
\hline & Right & 1.0 & 2.0 & 3.0 & 4.0 & 5.0 & 5.5 & 6.0 \\
\hline & Left & 0.0 & 2.0 & 3.0 & 4.0 & 5.0 & 6.0 & 7.0 \\
\hline & Average & 0.3 & 2.0 & 3.0 & 4.0 & 5.0 & 5.8 & 6.8 \\
\hline \multirow{5}{*}{3} & Top & 0.5 & 2.0 & 3.0 & 5.0 & 5.0 & 5.5 & 7.0 \\
\hline & Bottom & 0.0 & 0.0 & 3.0 & 4.0 & 4.0 & 5.0 & 6.0 \\
\hline & Right & 0.0 & 1.5 & 3.0 & 4.0 & 5.0 & 5.5 & 6.0 \\
\hline & Left & 0.0 & 1.5 & 3.0 & 5.0 & 4.5 & 6.0 & 6.5 \\
\hline & Average & 0.1 & 1.7 & 3.0 & 4.5 & 4.6 & 5.5 & 6.4 \\
\hline
\end{tabular}


Specimen Designation: GC-MO-D-F-G41

\begin{tabular}{|c|c|c|c|c|c|c|c|c|}
\hline \multirow{3}{*}{ \# } & \multicolumn{8}{|c|}{ Depth of Carbonation (mm) } \\
\hline & \multirow{2}{*}{ Sides } & \multicolumn{7}{|c|}{ Numbers of day } \\
\hline & & 7 & 21 & 35 & 56 & 84 & 112 & 140 \\
\hline \multirow{5}{*}{1} & Top & 3.5 & 7.0 & 8.5 & 12.0 & 12.0 & 12.0 & 13.0 \\
\hline & Bottom & 0.0 & 0.0 & 8.0 & 0.0 & 10.0 & 10.0 & 10.0 \\
\hline & Right & 3.5 & 7.0 & 8.0 & 9.0 & 10.0 & 11.0 & 12.0 \\
\hline & Left & 4.0 & 5.0 & 8.0 & 10.0 & 11.0 & 11.0 & 12.0 \\
\hline & Average & 3.7 & 6.3 & 8.1 & 10.3 & 10.8 & 11.0 & 11.8 \\
\hline \multirow{5}{*}{2} & Top & 3.0 & 6.5 & 8.0 & 11.0 & 11.5 & 12.0 & 13.0 \\
\hline & Bottom & 0.0 & 0.5 & 6.0 & 8.0 & 10.0 & 9.0 & 11.0 \\
\hline & Right & 2.0 & 5.0 & 7.5 & 10.0 & 11.0 & 12.0 & 13.0 \\
\hline & Left & 3.5 & 5.0 & 8.0 & 10.0 & 10.0 & 11.0 & 12.0 \\
\hline & Average & 2.8 & 5.5 & 7.4 & 9.8 & 10.6 & 11.0 & 12.3 \\
\hline \multirow{5}{*}{3} & Top & 3.0 & 7.5 & 8.0 & 9.0 & 11.0 & 12.0 & 13.0 \\
\hline & Bottom & 0.0 & 1.0 & 7.0 & 9.0 & 10.0 & 9.0 & 11.0 \\
\hline & Right & 2.5 & 5.5 & 8.0 & 10.0 & 10.5 & 12.0 & 12.0 \\
\hline & Left & 3.5 & 7.5 & 9.0 & 10.0 & 11.0 & 11.0 & 12.5 \\
\hline & Average & 3.0 & 6.8 & 8.0 & 9.5 & 10.6 & 11.0 & 12.1 \\
\hline
\end{tabular}


Specimen Designation: GC-MO-D-B-G41

\begin{tabular}{|c|c|c|c|c|c|c|c|c|}
\hline \multirow{3}{*}{$\#$} & \multicolumn{8}{|c|}{ Depth of Carbonation (mm) } \\
\hline & \multirow{2}{*}{ Sides } & \multicolumn{7}{|c|}{ Numbers of day } \\
\hline & & 7 & 21 & 35 & 56 & 84 & 112 & 140 \\
\hline \multirow{5}{*}{1} & Top & 2.0 & 4.0 & 4.0 & 5.5 & 6.5 & 7.0 & 8.0 \\
\hline & Bottom & 0.0 & 0.0 & 4.0 & 4.0 & 6.0 & 6.0 & 7.0 \\
\hline & Right & 1.0 & 3.0 & 4.0 & 6.0 & 6.5 & 7.0 & 8.0 \\
\hline & Left & 2.0 & 3.0 & 4.5 & 5.0 & 7.0 & 7.0 & 8.0 \\
\hline & $\begin{array}{c}\text { Averag } \\
\mathrm{e} \\
\end{array}$ & 1.7 & 3.3 & 4.1 & 5.1 & 6.5 & 6.8 & 7.8 \\
\hline \multirow{5}{*}{2} & Top & 2.0 & 4.0 & 5.0 & 5.0 & 5.5 & 7.0 & 7.0 \\
\hline & Bottom & 0.0 & 0.0 & 5.0 & 5.0 & 6.0 & 6.0 & 7.0 \\
\hline & Right & 1.0 & 3.0 & 5.0 & 5.0 & 6.0 & 7.0 & 8.5 \\
\hline & Left & 2.0 & 3.0 & 5.0 & 5.0 & 5.5 & 6.0 & 8.0 \\
\hline & $\begin{array}{c}\text { Averag } \\
\mathrm{e} \\
\end{array}$ & 1.7 & 3.3 & 5.0 & 5.0 & 5.8 & 6.5 & 7.6 \\
\hline \multirow{5}{*}{3} & Top & 2.0 & 4.0 & 4.0 & 5.0 & 5.5 & 6.0 & 7.5 \\
\hline & Bottom & 0.0 & 3.0 & 5.0 & 5.0 & 5.5 & 6.0 & 6.0 \\
\hline & Right & 1.0 & 3.0 & 4.5 & 5.0 & 5.5 & 6.0 & 7.0 \\
\hline & Left & 2.0 & 4.0 & 5.0 & 7.0 & 7.0 & 8.0 & 9.0 \\
\hline & $\begin{array}{c}\text { Averag } \\
\mathrm{e}\end{array}$ & 1.7 & 3.7 & 4.6 & 5.5 & 5.9 & 6.5 & 7.4 \\
\hline
\end{tabular}


Specimen Designation: GC-MO-D-C-E26

\begin{tabular}{|c|c|c|c|c|c|c|c|c|}
\hline \multirow{3}{*}{ \# } & \multicolumn{8}{|c|}{ Depth of Carbonation ( $\mathrm{mm}$ ) } \\
\hline & \multirow{2}{*}{ Sides } & \multicolumn{7}{|c|}{ Numbers of day } \\
\hline & & 7 & 21 & 35 & 56 & 84 & 112 & 140 \\
\hline \multirow{5}{*}{1} & Top & 2.0 & 3.5 & 4.0 & 5.0 & 5.5 & 6.0 & 7.0 \\
\hline & Bottom & 0.0 & 0.0 & 5.0 & 5.0 & 6.0 & 6.5 & 6.0 \\
\hline & Right & 2.5 & 4.0 & 4.0 & 5.0 & 6.0 & 6.5 & 8.0 \\
\hline & Left & 1.0 & 3.0 & 5.0 & 5.0 & 6.0 & 7.0 & 8.0 \\
\hline & $\begin{array}{c}\text { Averag } \\
\mathrm{e} \\
\end{array}$ & 1.8 & 3.5 & 4.5 & 5.0 & 5.9 & 6.5 & 7.3 \\
\hline \multirow{5}{*}{2} & Top & 1.0 & 3.0 & 4.0 & 5.0 & 6.0 & 6.5 & 7.0 \\
\hline & Bottom & 0.0 & 0.0 & 4.0 & 5.0 & 6.0 & 6.0 & 7.0 \\
\hline & Right & 0.5 & 3.0 & 5.0 & 5.0 & 5.5 & 6.5 & 7.0 \\
\hline & Left & 0.5 & 3.0 & 4.0 & 5.0 & 6.0 & 7.0 & 7.5 \\
\hline & $\begin{array}{c}\text { Averag } \\
\mathrm{e} \\
\end{array}$ & 0.7 & 3.0 & 4.3 & 5.0 & 5.9 & 6.5 & 7.1 \\
\hline \multirow{5}{*}{3} & Top & 1.5 & 4.0 & 4.0 & 5.0 & 6.0 & 6.0 & 7.0 \\
\hline & Bottom & 0.0 & 1.0 & 4.0 & 5.0 & 6.0 & 6.0 & 7.0 \\
\hline & Right & 1.5 & 3.0 & 5.0 & 5.0 & 6.0 & 6.5 & 7.0 \\
\hline & Left & 1.0 & 3.0 & 4.0 & 5.0 & 6.0 & 7.0 & 6.0 \\
\hline & $\begin{array}{c}\text { Averag } \\
\mathrm{e}\end{array}$ & 1.3 & 3.3 & 4.3 & 5.0 & 6.0 & 6.4 & 6.8 \\
\hline
\end{tabular}


Specimen Designation: GC-MO-D-F-E26

\begin{tabular}{|c|c|c|c|c|c|c|c|c|}
\hline \multirow{3}{*}{ \# } & \multicolumn{8}{|c|}{ Depth of Carbonation $(\mathrm{mm})$} \\
\hline & \multirow{2}{*}{ Sides } & \multicolumn{7}{|c|}{ Numbers of day } \\
\hline & & 7 & 21 & 35 & 56 & 84 & 112 & 140 \\
\hline \multirow{5}{*}{1} & Top & 3.0 & 6.5 & 8.0 & 10.0 & 11.0 & 12.0 & 12.0 \\
\hline & Bottom & 0.0 & 3.5 & 6.0 & 8.0 & 9.0 & 10.0 & 10.0 \\
\hline & Right & 2.5 & 6.0 & 10.0 & 10.0 & 11.0 & 10.0 & 12.0 \\
\hline & Left & 2.5 & 5.5 & 10.0 & 10.0 & 11.0 & 11.5 & 12.0 \\
\hline & Average & 2.7 & 6.0 & 8.5 & 9.5 & 10.5 & 10.9 & 11.5 \\
\hline \multirow{5}{*}{2} & Top & 3.0 & 6.0 & 8.0 & 9.0 & 10.0 & 12.0 & 12.5 \\
\hline & Bottom & 0.5 & 3.0 & 6.5 & 9.0 & 10.0 & 11.0 & 11.5 \\
\hline & Right & 2.5 & 5.5 & 6.5 & 10.0 & 11.0 & 11.0 & 12.0 \\
\hline & Left & 2.5 & 5.0 & 7.0 & 10.0 & 9.5 & 11.0 & 11.5 \\
\hline & Average & 2.7 & 5.5 & 7.0 & 9.5 & 10.1 & 11.3 & 11.9 \\
\hline \multirow{5}{*}{3} & Top & 3.5 & 6.0 & 7.0 & 10.0 & 10.0 & 12.0 & 12.0 \\
\hline & Bottom & 0.5 & 5.0 & 6.5 & 8.0 & 10.0 & 10.0 & 11.0 \\
\hline & Right & 3.5 & 6.0 & 8.0 & 9.5 & 10.5 & 11.5 & 12.0 \\
\hline & Left & 3.5 & 6.0 & 8.0 & 10.0 & 10.0 & 11.0 & 12.0 \\
\hline & Average & 3.5 & 6.0 & 7.4 & 9.4 & 10.1 & 11.1 & 11.8 \\
\hline
\end{tabular}


Specimen Designation: GC-MO-D-B-E26

\begin{tabular}{|c|c|c|c|c|c|c|c|c|}
\hline \multirow{3}{*}{$\#$} & \multicolumn{8}{|c|}{ Depth of Carbonation (mm) } \\
\hline & \multirow{2}{*}{ Sides } & \multicolumn{7}{|c|}{ Numbers of day } \\
\hline & & 7 & 21 & 35 & 56 & 84 & 112 & 140 \\
\hline \multirow{5}{*}{1} & Top & 2.0 & 4.0 & 4.5 & 5.0 & 6.5 & 7.0 & 9.0 \\
\hline & Bottom & 0.0 & 4.0 & 5.0 & 5.0 & 6.0 & 7.0 & 7.5 \\
\hline & Right & 2.5 & 4.0 & 4.5 & 5.0 & 5.0 & 6.0 & 7.0 \\
\hline & Left & 3.0 & 3.5 & 6.5 & 7.0 & 7.0 & 8.0 & 7.0 \\
\hline & Average & 2.5 & 3.8 & 5.1 & 5.5 & 6.1 & 7.0 & 7.6 \\
\hline \multirow{5}{*}{2} & Top & 2.0 & 4.0 & 6.0 & 6.0 & 6.0 & 7.0 & 9.0 \\
\hline & Bottom & 0.0 & 3.0 & 5.0 & 6.0 & 6.0 & 6.0 & 8.0 \\
\hline & Right & 3.0 & 4.0 & 6.0 & 6.0 & 6.0 & 7.0 & 7.0 \\
\hline & Left & 2.0 & 3.0 & 6.0 & 6.0 & 6.5 & 7.0 & 8.0 \\
\hline & Average & 2.3 & 3.7 & 5.8 & 6.0 & 6.1 & 6.8 & 8.0 \\
\hline \multirow{5}{*}{3} & Top & 2.5 & 4.0 & 5.0 & 6.0 & 6.5 & 7.0 & 8.0 \\
\hline & Bottom & 0.0 & 1.0 & 6.0 & 6.0 & 6.0 & 8.0 & 8.0 \\
\hline & Right & 1.5 & 4.0 & 6.5 & 6.5 & 7.0 & 9.0 & 10.0 \\
\hline & Left & 2.5 & 4.5 & 6.5 & 6.0 & 7.0 & 8.0 & 9.0 \\
\hline & Average & 2.2 & 4.2 & 6.0 & 6.1 & 6.6 & 8.0 & 8.8 \\
\hline
\end{tabular}


Specimen Designation: GC-MO-D-C-N0

\begin{tabular}{|c|c|c|c|c|c|c|c|c|}
\hline \multirow{3}{*}{ \# } & \multicolumn{8}{|c|}{ Depth of Carbonation (mm) } \\
\hline & \multirow{2}{*}{ Sides } & \multicolumn{7}{|c|}{ Numbers of day } \\
\hline & & 7 & 21 & 35 & 56 & 84 & 112 & 140 \\
\hline \multirow{5}{*}{1} & Top & 1.0 & 3.0 & 3.0 & 4.0 & 4.0 & 5.0 & 6.0 \\
\hline & Bottom & 0.0 & 0.0 & 3.0 & 4.0 & 4.0 & 4.5 & 5.0 \\
\hline & Right & 0.5 & 1.5 & 3.0 & 3.5 & 4.0 & 4.5 & 6.0 \\
\hline & Left & 0.0 & 2.0 & 3.0 & 4.0 & 4.5 & 5.0 & 6.0 \\
\hline & Average & 0.5 & 2.2 & 3.0 & 3.9 & 4.1 & 4.8 & 5.8 \\
\hline \multirow{5}{*}{2} & Top & 1.0 & 3.0 & 3.0 & 4.0 & 4.0 & 5.0 & 5.0 \\
\hline & Bottom & 0.0 & 0.0 & 2.0 & 4.0 & 5.0 & 6.0 & 6.0 \\
\hline & Right & 1.0 & 1.5 & 3.0 & 3.5 & 4.0 & 5.0 & 6.0 \\
\hline & Left & 0.0 & 2.0 & 4.0 & 4.0 & 5.0 & 6.0 & 6.5 \\
\hline & Average & 0.7 & 2.2 & 3.0 & 3.9 & 4.5 & 5.5 & 5.9 \\
\hline \multirow{5}{*}{3} & Top & 0.0 & 3.0 & 4.0 & 5.0 & 5.0 & 5.5 & 6.0 \\
\hline & Bottom & 0.0 & 0.0 & 3.0 & 4.0 & 5.0 & 6.0 & 6.0 \\
\hline & Right & 0.0 & 2.0 & 3.0 & 5.0 & 5.5 & 6.0 & 6.5 \\
\hline & Left & 0.0 & 3.0 & 3.0 & 4.5 & 5.5 & 6.0 & 6.5 \\
\hline & Average & 0.0 & 2.7 & 3.3 & 4.6 & 5.3 & 5.9 & 6.3 \\
\hline
\end{tabular}


Specimen Designation: GC-VA-D-C-G23

\begin{tabular}{|c|c|c|c|c|c|c|c|c|}
\hline \multirow{3}{*}{ \# } & \multicolumn{8}{|c|}{ Depth of Carbonation ( $\mathrm{mm}$ ) } \\
\hline & \multirow{2}{*}{ Sides } & \multicolumn{7}{|c|}{ Numbers of day } \\
\hline & & 7 & 21 & 35 & 56 & 84 & 112 & 140 \\
\hline \multirow{5}{*}{1} & Top & 2.0 & 3.0 & 4.0 & 4.0 & 5.0 & 6.0 & 7.0 \\
\hline & Bottom & 0.0 & 0.5 & 3.0 & 3.0 & 3.5 & 5.0 & 5.0 \\
\hline & Right & 0.0 & 2.0 & 3.0 & 3.5 & 3.5 & 5.0 & 6.0 \\
\hline & Left & 0.0 & 0.5 & 3.0 & 3.5 & 4.0 & 6.0 & 6.0 \\
\hline & Average & 0.7 & 1.8 & 3.3 & 3.5 & 4.0 & 5.5 & 6.0 \\
\hline \multirow{5}{*}{2} & Top & 2.0 & 3.0 & 3.5 & 4.0 & 4.5 & 5.0 & 6.0 \\
\hline & Bottom & 0.0 & 0.0 & 3.0 & 4.0 & 4.0 & 4.0 & 5.0 \\
\hline & Right & 0.0 & 0.5 & 3.0 & 3.5 & 4.0 & 5.0 & 5.5 \\
\hline & Left & 0.5 & 0.5 & 3.0 & 3.5 & 4.0 & 5.0 & 5.0 \\
\hline & Average & 0.8 & 1.3 & 3.1 & 3.8 & 4.1 & 4.8 & 5.4 \\
\hline \multirow{5}{*}{3} & Top & 2.0 & 3.0 & 3.0 & 4.0 & 5.0 & 5.0 & 6.0 \\
\hline & Bottom & 0.0 & 0.0 & 0.0 & 3.0 & 4.0 & 5.0 & 5.0 \\
\hline & Right & 0.0 & 0.0 & 3.0 & 4.0 & 4.5 & 5.0 & 6.0 \\
\hline & Left & 0.0 & 0.5 & 3.0 & 3.0 & 4.0 & 6.0 & 6.5 \\
\hline & Average & 0.7 & 1.2 & 3.0 & 3.5 & 4.4 & 5.3 & 5.9 \\
\hline
\end{tabular}


Specimen Designation: GC-VA-D-F-G23

\begin{tabular}{|c|c|c|c|c|c|c|c|c|}
\hline \multirow{3}{*}{ \# } & \multicolumn{8}{|c|}{ Depth of Carbonation (mm) } \\
\hline & \multirow{2}{*}{ Sides } & \multicolumn{7}{|c|}{ Numbers of day } \\
\hline & & 7 & 21 & 35 & 56 & 84 & 112 & 140 \\
\hline \multirow{5}{*}{$\mathbf{1}$} & Top & 4.0 & 5.0 & 7.0 & 7.0 & 9.0 & 11.0 & 12.0 \\
\hline & Bottom & 0.0 & 0.0 & 7.0 & 8.0 & 8.5 & 9.0 & 10.0 \\
\hline & Right & 1.5 & 5.0 & 7.0 & 7.0 & 8.0 & 10.0 & 11.0 \\
\hline & Left & 3.0 & 5.0 & 7.0 & 7.5 & 8.5 & 11.0 & 12.0 \\
\hline & Average & 2.8 & 5.0 & 7.0 & 7.4 & 8.5 & 10.3 & 11.3 \\
\hline \multirow{5}{*}{2} & Top & 3.5 & 4.5 & 7.0 & 8.0 & 9.0 & 10.0 & 11.0 \\
\hline & Bottom & 0.0 & 0.0 & 7.0 & 7.0 & 8.0 & 10.0 & 10.5 \\
\hline & Right & 2.0 & 5.0 & 7.0 & 7.5 & 9.0 & 10.0 & 12.0 \\
\hline & Left & 2.0 & 5.0 & 7.0 & 9.0 & 9.0 & 10.5 & 11.0 \\
\hline & Average & 2.5 & 4.8 & 7.0 & 7.9 & 8.8 & 10.1 & 11.1 \\
\hline \multirow{5}{*}{3} & Top & 3.0 & 5.0 & 7.0 & 7.5 & 8.5 & 9.5 & 11.0 \\
\hline & Bottom & 0.0 & 5.0 & 7.0 & 7.0 & 8.0 & 10.0 & 10.0 \\
\hline & Right & 2.0 & 5.0 & 7.0 & 7.0 & 8.0 & 10.0 & 11.0 \\
\hline & Left & 2.0 & 4.5 & 7.0 & 7.5 & 8.0 & 10.0 & 11.0 \\
\hline & Average & 2.3 & 4.8 & 7.0 & 7.3 & 8.1 & 9.9 & 10.8 \\
\hline
\end{tabular}


Specimen Designation: GC-VA-D-B-G23

\begin{tabular}{|c|c|c|c|c|c|c|c|c|}
\hline \multirow{3}{*}{ \# } & \multicolumn{8}{|c|}{ Depth of Carbonation ( $\mathrm{mm}$ ) } \\
\hline & \multirow{2}{*}{ Sides } & \multicolumn{7}{|c|}{ Numbers of day } \\
\hline & & 7 & 21 & 35 & 56 & 84 & 112 & 140 \\
\hline \multirow{5}{*}{$\mathbf{1}$} & Top & 2.0 & 4.0 & 5.0 & 6.5 & 6.5 & 7.0 & 8.0 \\
\hline & Bottom & 0.0 & 0.0 & 4.0 & 5.0 & 6.0 & 0.0 & 7.5 \\
\hline & Right & 2.0 & 4.0 & 4.5 & 5.0 & 6.0 & 7.0 & 8.0 \\
\hline & Left & 3.0 & 3.0 & 4.0 & 5.0 & 6.0 & 7.0 & 8.0 \\
\hline & Average & 2.3 & 3.7 & 4.4 & 5.4 & 6.1 & 7.0 & 7.9 \\
\hline \multirow{5}{*}{2} & Top & 2.5 & 3.0 & 4.0 & 5.0 & 6.0 & 6.0 & 7.5 \\
\hline & Bottom & 0.0 & 0.0 & 4.0 & 5.0 & 6.0 & 7.0 & 8.0 \\
\hline & Right & 2.0 & 3.0 & 4.0 & 5.0 & 6.5 & 7.0 & 8.0 \\
\hline & Left & 3.5 & 4.5 & 5.0 & 5.0 & 6.0 & 6.5 & 8.0 \\
\hline & Average & 2.7 & 3.5 & 4.3 & 5.0 & 6.1 & 6.6 & 7.9 \\
\hline \multirow{5}{*}{3} & Top & 2.5 & 3.0 & 5.0 & 5.0 & 6.0 & 7.0 & 7.0 \\
\hline & Bottom & 0.0 & 0.0 & 3.0 & 4.0 & 5.0 & 6.0 & 7.0 \\
\hline & Right & 2.0 & 4.0 & 5.0 & 5.0 & 6.0 & 7.0 & 8.0 \\
\hline & Left & 3.0 & 4.0 & 7.0 & 7.0 & 6.0 & 6.0 & 8.0 \\
\hline & Average & 2.5 & 3.7 & 5.0 & 5.3 & 5.8 & 6.5 & 7.5 \\
\hline
\end{tabular}


Specimen Designation: GC-VA-D-C-E17

\begin{tabular}{|c|c|c|c|c|c|c|c|c|}
\hline \multirow{3}{*}{$\#$} & \multicolumn{8}{|c|}{ Depth of Carbonation (mm) } \\
\hline & \multirow{2}{*}{ Sides } & \multicolumn{7}{|c|}{ Numbers of day } \\
\hline & & 7 & 21 & 35 & 56 & 84 & 112 & 140 \\
\hline \multirow{5}{*}{1} & Top & 1.0 & 3.0 & 4.0 & 4.5 & 5.0 & 5.0 & 6.0 \\
\hline & Bottom & 0.0 & 0.0 & 3.0 & 3.5 & 4.0 & 4.0 & 6.0 \\
\hline & Right & 0.0 & 2.5 & 4.0 & 4.5 & 4.5 & 5.0 & 6.0 \\
\hline & Left & 0.5 & 2.0 & 3.0 & 3.0 & 3.5 & 5.0 & 6.0 \\
\hline & Average & 0.5 & 2.5 & 3.5 & 3.9 & 4.3 & 4.8 & 6.0 \\
\hline \multirow{5}{*}{2} & Top & 2.0 & 3.0 & 4.0 & 4.0 & 5.0 & 5.0 & 6.0 \\
\hline & Bottom & 0.0 & 0.0 & 3.0 & 4.0 & 4.0 & 5.0 & 6.0 \\
\hline & Right & 0.5 & 3.0 & 4.0 & 4.5 & 5.0 & 5.0 & 7.0 \\
\hline & Left & 0.5 & 2.0 & 3.0 & 4.0 & 4.0 & 4.0 & 7.0 \\
\hline & Average & 1.0 & 2.7 & 3.5 & 4.1 & 4.5 & 4.8 & 6.5 \\
\hline \multirow{5}{*}{3} & Top & 0.5 & 3.0 & 3.0 & 3.0 & 3.5 & 5.0 & 5.5 \\
\hline & Bottom & 0.0 & 0.5 & 3.0 & 3.5 & 4.0 & 5.0 & 5.0 \\
\hline & Right & 0.5 & 2.0 & 3.0 & 3.0 & 3.0 & 4.0 & 5.0 \\
\hline & Left & 0.5 & 1.0 & 3.0 & 4.0 & 4.5 & 5.0 & 5.5 \\
\hline & Average & 0.5 & 2.0 & 3.0 & 3.4 & 3.8 & 4.8 & 5.3 \\
\hline
\end{tabular}


Specimen Designation: GC-VA-D-F-E17

\begin{tabular}{|c|c|c|c|c|c|c|c|c|}
\hline \multirow{3}{*}{$\#$} & \multicolumn{8}{|c|}{ Depth of Carbonation ( $\mathrm{mm}$ ) } \\
\hline & \multirow{2}{*}{ Sides } & \multicolumn{7}{|c|}{ Numbers of day } \\
\hline & & 7 & 21 & 35 & 56 & 84 & 112 & 140 \\
\hline \multirow{5}{*}{1} & Top & 2.5 & 3.5 & 6.0 & 8.0 & 8.5 & 10.0 & 10.0 \\
\hline & Bottom & 0.0 & 0.0 & 6.0 & 8.0 & 9.0 & 10.0 & 10.0 \\
\hline & Right & 3.0 & 5.0 & 6.0 & 8.0 & 9.5 & 10.0 & 11.0 \\
\hline & Left & 4.0 & 5.0 & 6.0 & 8.0 & 9.5 & 11.0 & 13.0 \\
\hline & Average & 3.2 & 4.5 & 6.0 & 8.0 & 9.1 & 10.3 & 11.0 \\
\hline \multirow{5}{*}{2} & Top & 3.0 & 5.0 & 6.0 & 8.0 & 10.0 & 11.0 & 11.5 \\
\hline & Bottom & 0.0 & 0.0 & 6.0 & 8.0 & 9.0 & 10.0 & 10.0 \\
\hline & Right & 3.0 & 5.0 & 6.5 & 7.5 & 8.0 & 10.0 & 11.5 \\
\hline & Left & 2.5 & 5.0 & 6.0 & 8.0 & 10.0 & 11.0 & 11.0 \\
\hline & Average & 2.8 & 5.0 & 6.1 & 7.9 & 9.3 & 10.5 & 11.0 \\
\hline \multirow{5}{*}{3} & Top & 3.0 & 5.0 & 6.0 & 8.0 & 10.0 & 10.0 & 11.5 \\
\hline & Bottom & 0.0 & 0.0 & 6.0 & 8.0 & 9.0 & 10.0 & 10.0 \\
\hline & Right & 2.0 & 5.0 & 5.5 & 8.0 & 10.0 & 11.0 & 12.0 \\
\hline & Left & 3.0 & 5.0 & 6.0 & 8.0 & 9.0 & 10.0 & 11.0 \\
\hline & Average & 2.7 & 5.0 & 5.9 & 8.0 & 9.5 & 10.3 & 11.1 \\
\hline
\end{tabular}


Specimen Designation: GC-VA-D-B-E17

\begin{tabular}{|c|c|c|c|c|c|c|c|c|}
\hline \multirow{3}{*}{$\#$} & \multicolumn{7}{|c|}{ Depth of Carbonation (mm) } \\
\cline { 2 - 10 } & \multirow{3}{*}{ Sides } & \multicolumn{7}{|c|}{ Numbers of day } \\
\cline { 2 - 10 } & & $\mathbf{7}$ & $\mathbf{2 1}$ & $\mathbf{3 5}$ & $\mathbf{5 6}$ & $\mathbf{8 4}$ & $\mathbf{1 1 2}$ & $\mathbf{1 4 0}$ \\
\hline \multirow{4}{*}{1} & Top & 0.5 & 3.0 & 5.0 & 5.0 & 6.0 & 7.0 & 7.0 \\
\cline { 2 - 10 } & Bottom & 0.0 & 0.0 & 0.0 & 5.0 & 6.0 & 6.0 & 7.0 \\
\cline { 2 - 10 } & Right & 0.0 & 4.0 & 5.0 & 5.0 & 6.0 & 5.0 & 7.0 \\
\cline { 2 - 10 } & Left & 0.0 & 2.0 & 5.0 & 5.0 & 6.0 & 6.5 & 7.0 \\
\cline { 2 - 10 } & Average & 0.2 & 3.0 & 5.0 & 5.0 & 6.0 & 6.1 & 7.0 \\
\hline \multirow{4}{*}{2} & Top & 0.5 & 2.5 & 4.0 & 5.0 & 6.0 & 7.0 & 7.5 \\
\cline { 2 - 10 } & Bottom & 0.0 & 0.0 & 0.0 & 5.0 & 6.0 & 7.0 & 7.0 \\
\cline { 2 - 10 } & Right & 0.0 & 3.0 & 4.0 & 5.0 & 7.0 & 7.0 & 7.0 \\
\cline { 2 - 10 } & Left & 0.5 & 3.0 & 5.0 & 5.5 & 6.0 & 7.5 & 8.0 \\
\cline { 2 - 10 } & Average & 0.3 & 2.8 & 4.3 & 5.1 & 6.3 & 7.1 & 7.4 \\
\hline \multirow{3}{*}{3} & Top & 0.5 & 4.0 & 5.0 & 5.0 & 5.5 & 6.0 & 7.0 \\
\cline { 2 - 9 } & Bottom & 0.0 & 0.0 & 5.0 & 5.0 & 6.0 & 7.0 & 8.0 \\
\cline { 2 - 9 } & Right & 0.0 & 5.0 & 5.0 & 5.0 & 6.0 & 7.0 & 7.5 \\
\cline { 2 - 9 } & Left & 0.5 & 3.0 & 5.0 & 5.0 & 6.0 & 7.0 & 8.0 \\
\cline { 2 - 9 } & Average & 0.3 & 4.0 & 5.0 & 5.0 & 5.9 & 6.8 & 7.6 \\
\hline
\end{tabular}


Specimen Designation: GC-VA-D-C-N0

\begin{tabular}{|c|c|c|c|c|c|c|c|c|}
\hline \multirow{3}{*}{$\#$} & \multicolumn{8}{|c|}{ Depth of Carbonation ( $\mathrm{mm}$ ) } \\
\hline & \multirow{2}{*}{ Sides } & \multicolumn{7}{|c|}{ Numbers of day } \\
\hline & & 7 & 21 & 35 & 56 & 84 & 112 & 140 \\
\hline \multirow{5}{*}{1} & Top & 0.0 & 3.5 & 4.0 & 5.0 & 5.0 & 5.0 & 5.5 \\
\hline & Bottom & 0.0 & 0.0 & 3.0 & 0.0 & 5.0 & 5.5 & 5.0 \\
\hline & Right & 0.0 & 0.5 & 3.0 & 4.5 & 5.0 & 6.0 & 6.0 \\
\hline & Left & 0.0 & 2.0 & 4.0 & 5.0 & 5.0 & 6.0 & 6.5 \\
\hline & Average & 0.0 & 2.0 & 3.5 & 3.6 & 5.0 & 5.6 & 5.8 \\
\hline \multirow{5}{*}{2} & Top & 0.5 & 3.0 & 4.0 & 4.0 & 4.5 & 5.0 & 6.5 \\
\hline & Bottom & 0.0 & 0.0 & 4.0 & 4.0 & 4.5 & 5.0 & 6.0 \\
\hline & Right & 0.0 & 0.5 & 4.0 & 5.0 & 5.0 & 5.5 & 7.0 \\
\hline & Left & 0.0 & 2.0 & 4.0 & 5.0 & 5.0 & 6.0 & 6.5 \\
\hline & Average & 0.2 & 1.8 & 4.0 & 4.5 & 4.8 & 5.4 & 6.5 \\
\hline \multirow{5}{*}{3} & Top & 0.5 & 3.0 & 4.0 & 5.0 & 5.0 & 6.0 & 7.0 \\
\hline & Bottom & 0.0 & 0.0 & 4.0 & 5.0 & 5.0 & 6.0 & 6.0 \\
\hline & Right & 0.0 & 2.0 & 4.0 & 5.0 & 5.0 & 5.5 & 6.0 \\
\hline & Left & 0.0 & 2.0 & 4.0 & 5.0 & 5.0 & 6.0 & 7.0 \\
\hline & Average & 0.2 & 2.3 & 4.0 & 5.0 & 5.0 & 5.9 & 6.5 \\
\hline
\end{tabular}




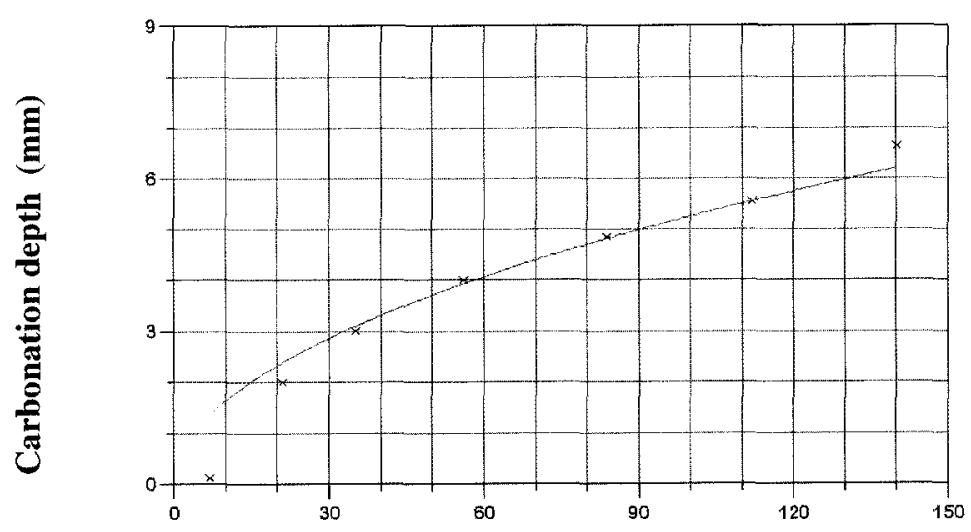

Time (day)

(a) GC-MO-D-C-G41

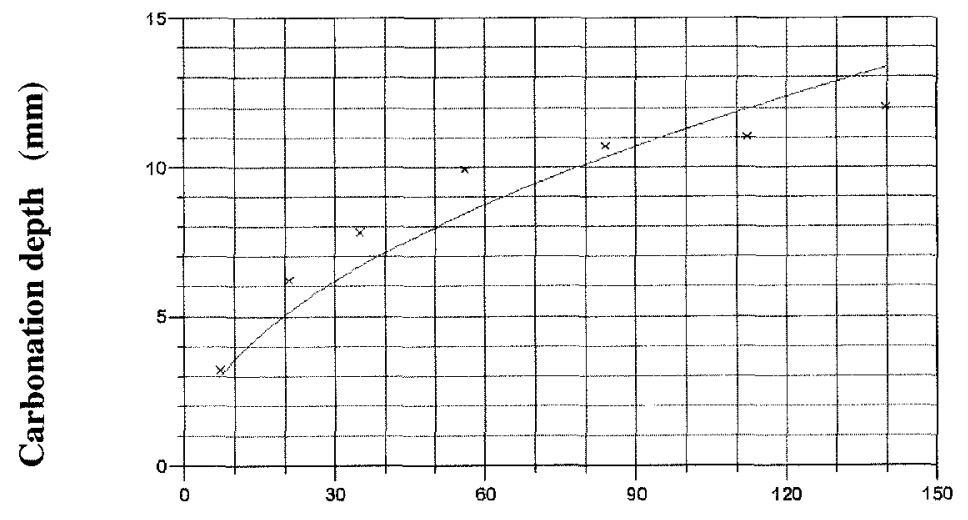

Time (day)

(b) GC-MO-D-F-G41

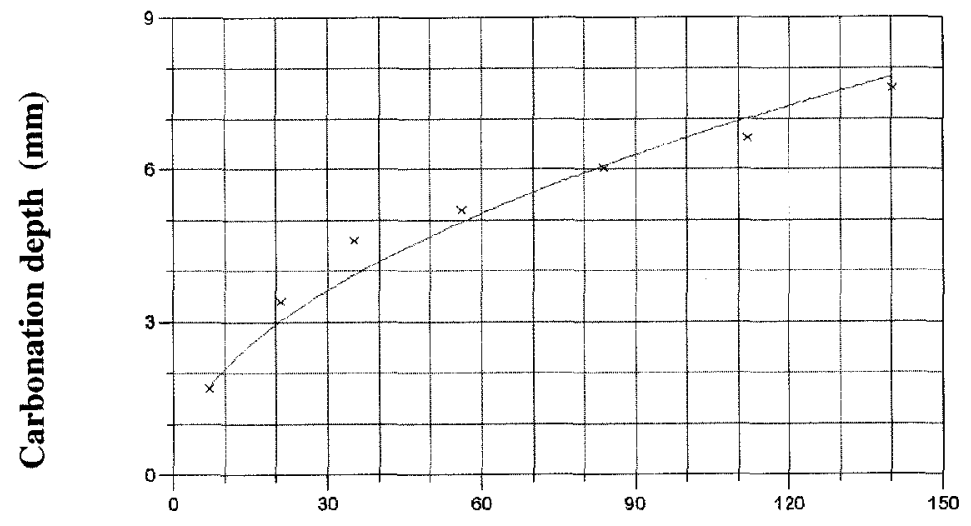

Time (day)

(c) GC-MO-D-B-G41

Figure C.15: Regression analysis to determine $D_{c}$ for GC-MO specimens designed with (G) method 


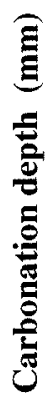

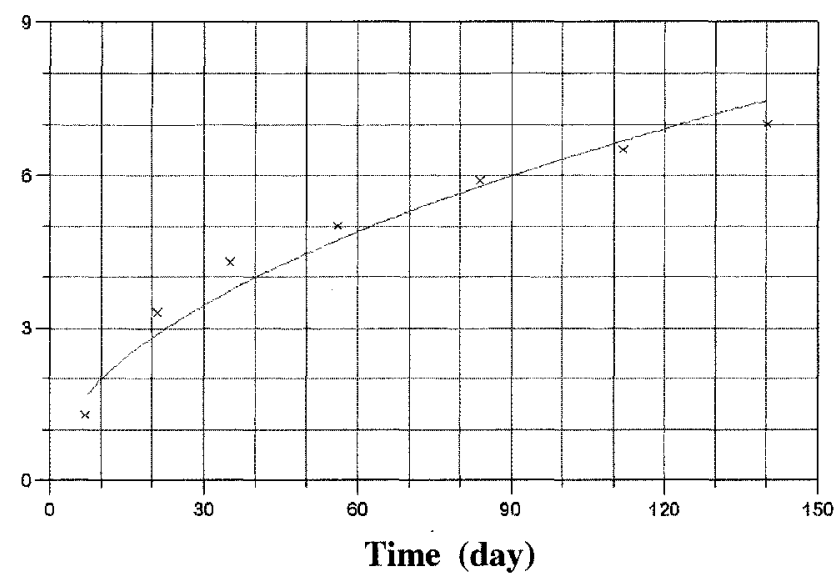

(a) GC-MO-D-C-E26

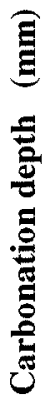

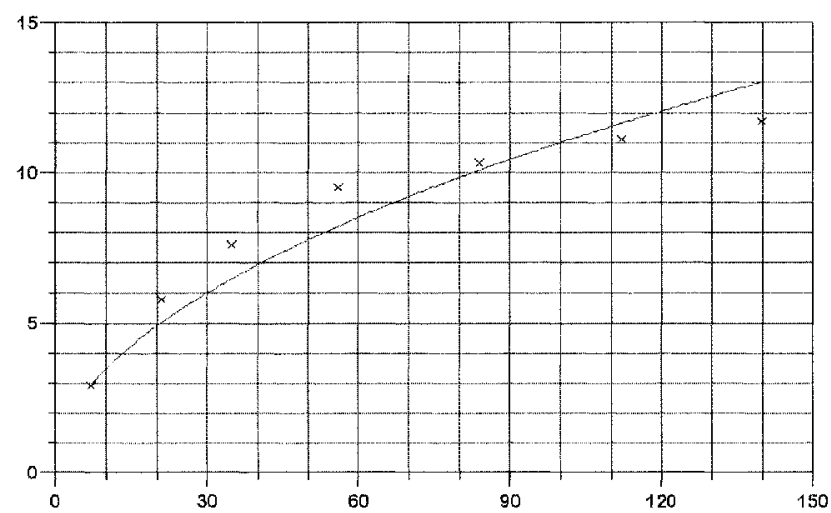

Time (day)

(b) GC-MO-D-F-E26

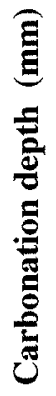

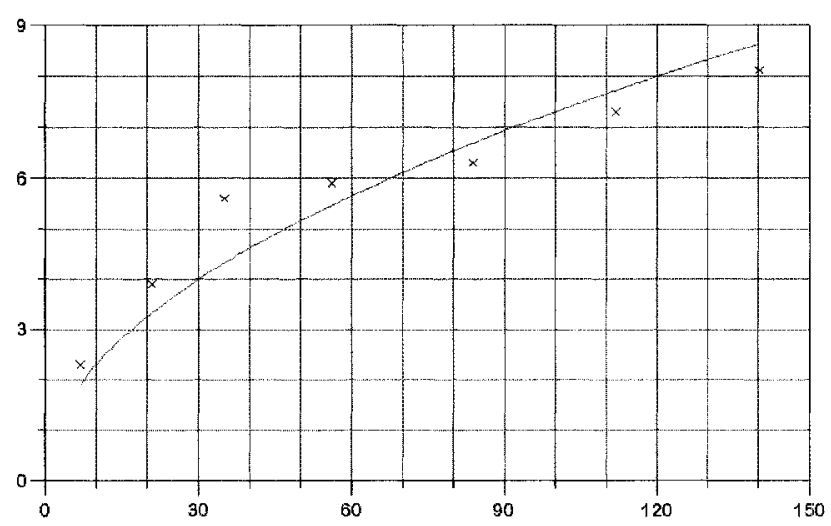

Time (day)

(c) GC-MO-D-B-E26

Figure C.16: Regression analysis to determine $D_{c}$ for GC-MO specimens designed with

(E) method 


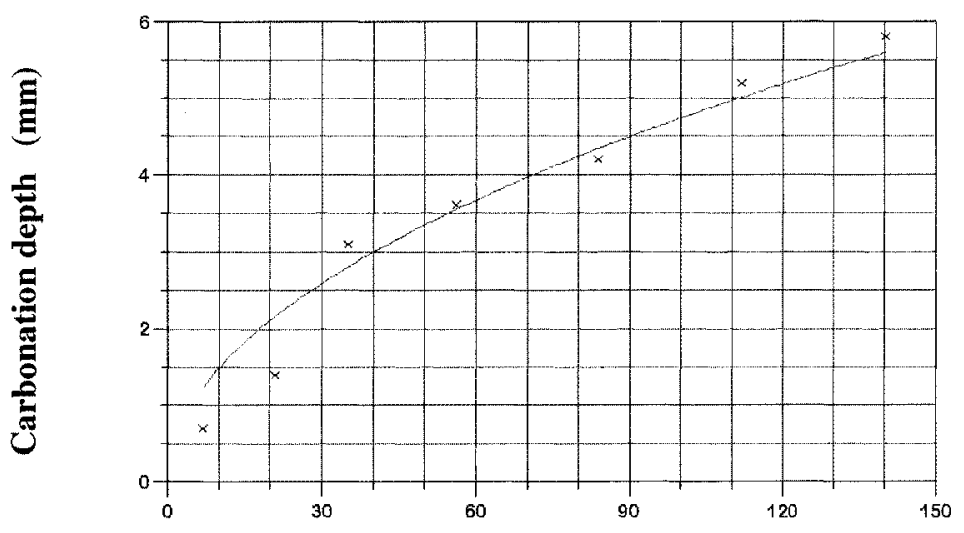

Time (day)

(a) GC-VA-D-C-G23

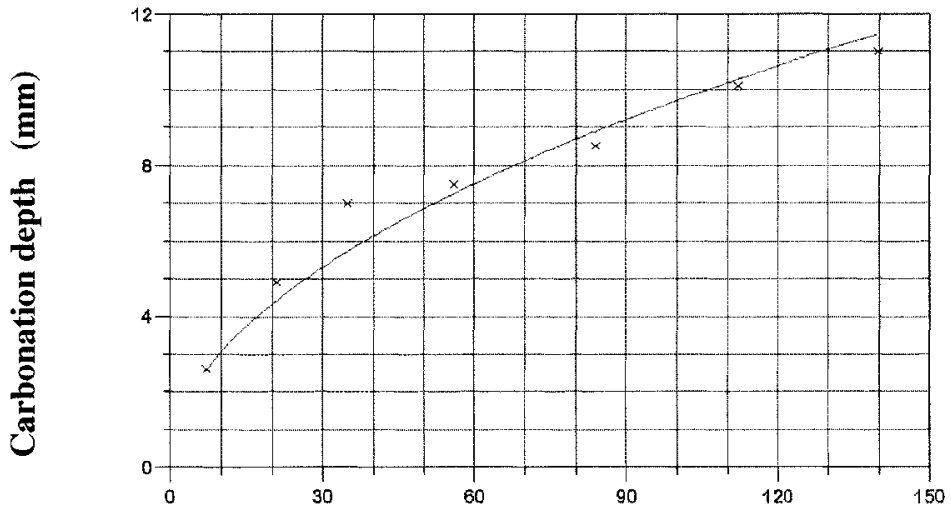

Time (day)

(b) GC-VA-D-F-G23

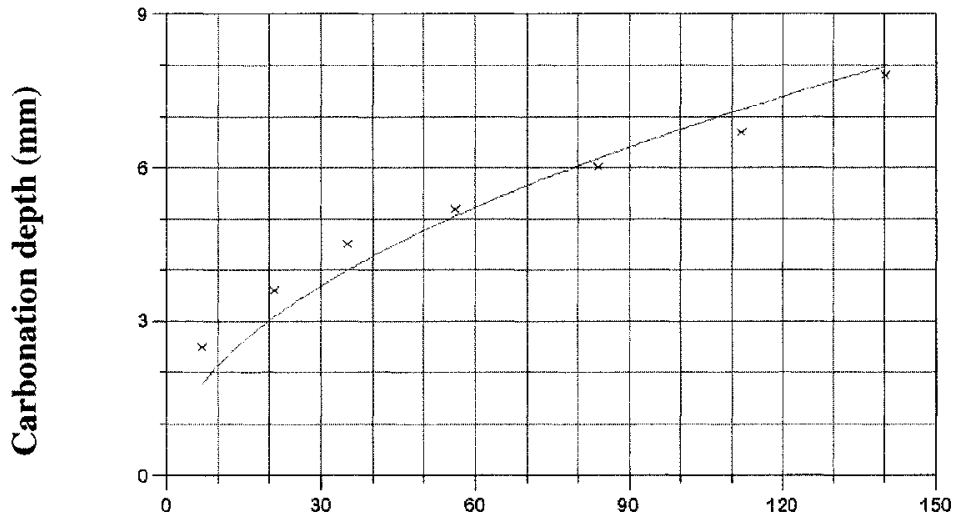

Time (day)

(c) GC-VA-D-B-G23

Figure C.17: Regression analysis to determine $D_{c}$ for GC-VA specimens designed with general mix design method 


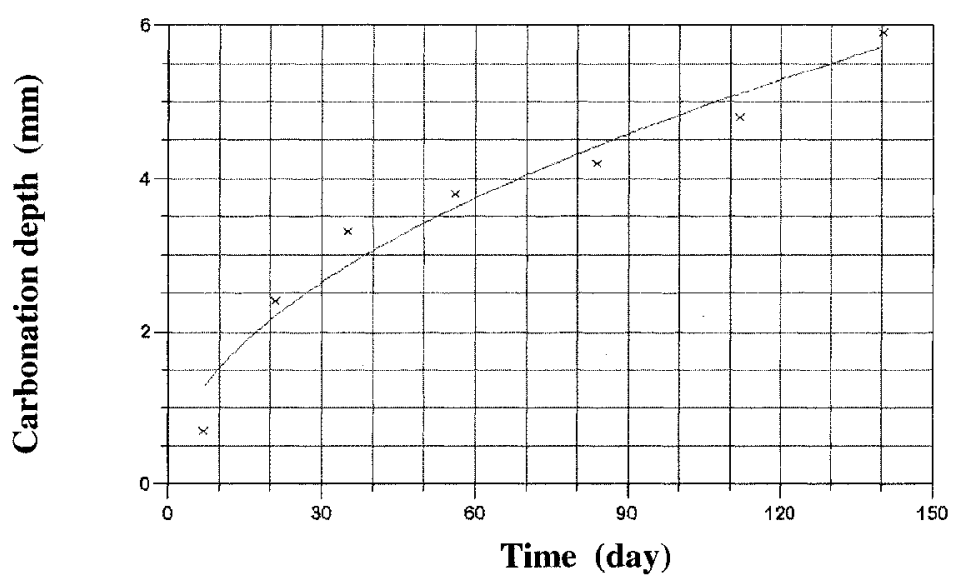

(a) GC-VA-D-C-E17

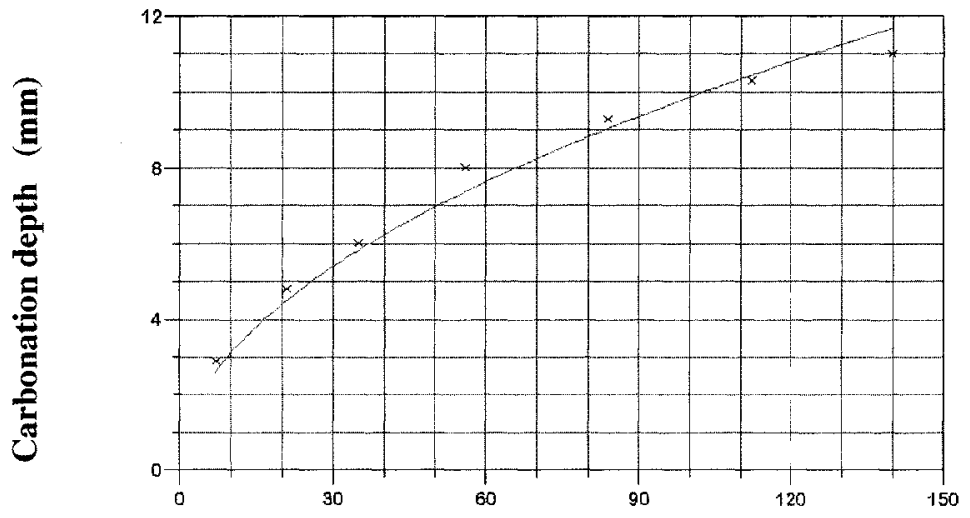

Time (day)

(b) GC-VA-D-F-E17

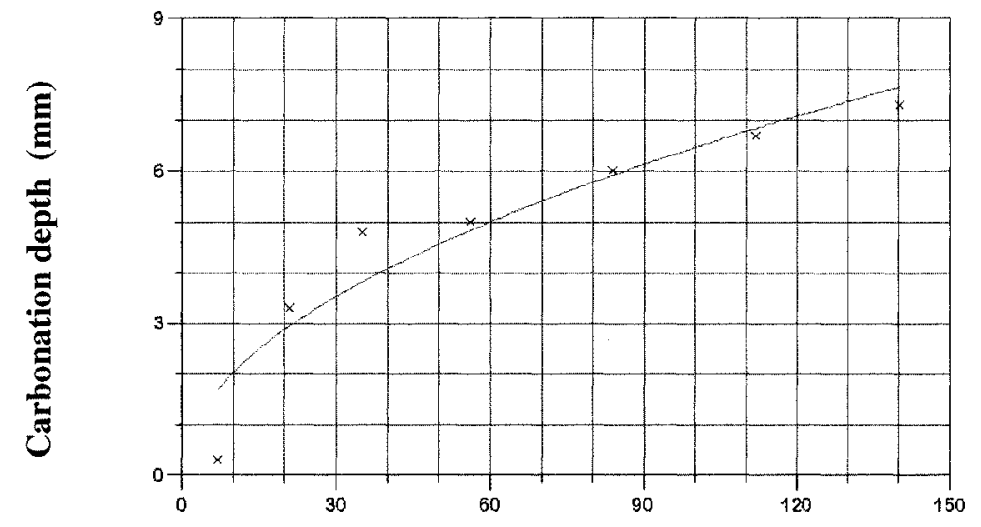

Time (day)

(c) GC-VA-D-B-E17

Figure C.18: Regression analysis to determine $D_{c}$ for GC-VA specimens designed with equivalent total volume method 


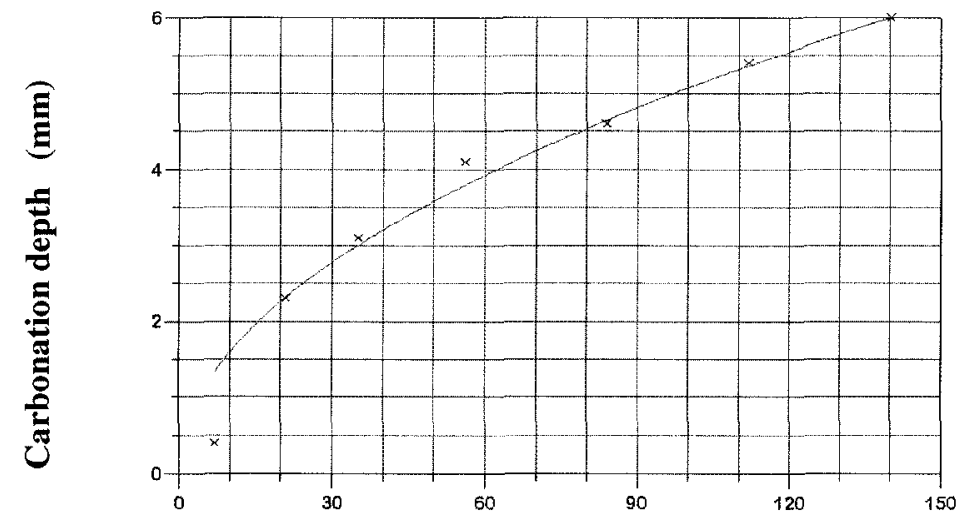

Time (day)

(a) GC-MO-D-C-NO

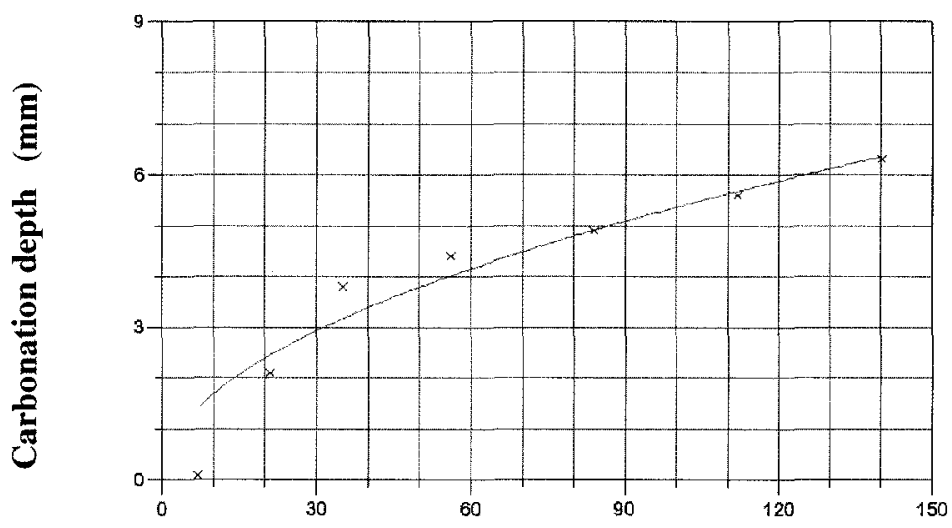

Time (day)

(b) GC-VA-D-C-NO

Figure C.19: Regression analysis to determine $D_{c}$ for NAC 